UNIVERSITAT JAUME I

Departament de Química Inorgànica i Orgànica

UNIVERSITAT Àrea Química Inorgànica

JAUME•I

\title{
MULTIFUNCTIONAL N-HETEROCYCLIC CARBENE METAL COMPLEXES FOR THE DESIGN OF IMPROVED CATALYTIC PROCESSES
}

\author{
Doctoral Thesis \\ Sara Sabater López
}

PhD. supervisors: José A. Mata and Eduardo Peris Castellón de la Plana, May 2015 



\section{Prof. Dr. Eduardo V. Peris Fajarnés, Catedràtic de l'àrea de Química Inorgànica i Dr. José A. Mata Martínez, professor titular de la mateixa àrea, pertanyents al Departament de Química Inorgànica i Orgànica de la Universitat Jaume I.}

Certifiquen: Que la tesi Doctoral amb títol "Multifunctional N-heterocyclic carbene metal complexes for the design of improved catalytic processes" ha sigut desenvolupada sota la seua direcció, en l'àrea de Química Inorgànica del Departament de Química Inorgànica i Orgànica de la Universitat Jaume I, per Sara Sabater López.

Castelló de la Plana, a 21 d'abril de 2015

Sign. Prof. Dr. Eduardo V. Peris Fajarnés

Sign. Dr. José A. Mata Martínez 



\section{AGRADECIMIENTOS}

En fin, después de cuatro años aquí estoy, intentando resumir en unas cuantas palabras todos mis sentimientos de gratitud hacia aquellas personas que han formado parte de este largo proceso. Primero que todo gracias a los mayores responsables de sacar todo el trabajo adelante, mis directores de tesis: Eduardo y José. Gracias por haberme dejado formar parte de este grupo de investigación sin apenas conocerme. Gracias Eduardo por estar siempre tan pendiente, en todos los aspectos. Por toda tu ayuda, tus consejos cuando las cosas no van bien y sobre todo por haberme transmitido tu pasión por la investigación. Gràcies José per la teua més que infinita paciència, per tots els teus consells, per sempre interessar-te per mi, per la teua ajuda sempre amb un somriure, encara que els meus dubtes foren per a pegar-me palaes al cap!

A mis compañeros de laboratorio, Maca, por tu ayuda siempre incondicional. Elena, porque aunque a veces me marees tanto siempre estás ahí. Goyo, porque es siempre un placer hablar contigo pero aun lo es más poder meterme contigo sin que te enfades. Miriam, fue poco tiempo pero gracias por tu amabilidad. Alex, menos tiempo si cabe, pero de ti heredé el gran "ditz". Susana, te ha tocado mi parte más dura, gracias por tus palabras de ánimo. Amparito, por entenderme tan bien cuando nadie más puede hacerlo. Arthur, ay! mi wey favorito, por aguantarme tanto, creo que eres el que más sufría con mi preciosa voz. Cande, gràcies per tants bons moments que hem passat juntes, tant en Espanya com en Dublín, sense tu no haguera segut el mateix. Sergio, qui ens ho anava a dir!16 anys...se diu prompte, un plaer compartir amb tu tants moments. Hugo, porque lo solucionas todo con una sonrisa, ves preparando el tequila para cuando acabe! Sheila, que paciencia has tenido conmigo, aunque yo no siempre la corresponda, gracias por quererme tanto! Carmencita, mi medio limón! Me alegro infinito de haberte conocido. Als xiquets, David i Victor. David, gràcies per estar sempre tan pendent, tranquil que anirà tot bé! Martínez! Tu si que saps de música!! A todos los que han ido pasando por el laboratorio: Alberto, Roberto, Lisa, Diego, Jo, Marco, George and Ian. Un placer conoceros! Prof. Gusev, your stays in the group are always a pleasure.

A Zoel, Cristina, Manolo, Ángel y Javi, con quienes compartí tantos buenos momentos durante el máster. Fue todo un placer conoceros, ojalá no perdamos el contacto! 
A Bancaixa per la beca d'investigació que vaig gaudir només acabar la carrera. A la Generalitat Valenciana, per la beca FPI que he gaudit aquest últims tres anys i m'ha permès dur a terme aquesta tesi doctoral.

A todos los técnicos de Servicios Centrales, y cuando digo todos son todos, porque creo que no hay ni uno al que no haya ido a marear con mis muestras: Cristian, Gabriel, José, Cris, Maru, Javi, Laura y Pere. A las técnicas de QIO Silvia, Inma y Alicia, porque a vosotras también he ido a marearos mil veces.

A tota la gent de QIO, en especial als companys de la planta 0, som un big team, encara que ens vam mudar les nostres festes sagrades no les perdonem per res. A la gent del nou edifici, tant físics com químics analítics, perquè alguna liadeta bona sempre ens hem pegat!

Thanks to Prof. Martin Albrecht for giving me the opportunity to join his group. Thank you for your unconditional help before, during and after my stay. Also thanks to the Albies!! You are amazing guys! Especially to Miquel, Kevin and Cristiana. It was only four months, but all of you will be in my heart forever.

A mis amigas, mis nenas, Toya, Mayte y Alba, que aunque no entendéis mucho que hago, siempre me habéis apoyado llenas de orgullo. A mis químicas del alma, os quiero con locura. Aunque sean pocas las veces que estemos todas juntas, esos momentos valen oro. A mis Dignas/Rafaelas/\#adictasalJäger, por todos esas fiestas, que sin ellas me hubiera vuelto loca hace mil, y más aun por las reuniones de domingos al sol!

A por último a mi familia, qué orgullosa me siento de formar parte de ella! podría escribir otra tesis hablando sólo de ello. Es el claro ejemplo que la distancia no significa nada cuando de verdad se quiere. Gracias por todo vuestro apoyo y vuestro constante interés. A Santi, que te vull més que a la meua ànima encara que sigues un punxa de cuidado sempre saps com fer-me riure. A Alba, que contenta estoy que seas de la familia, personas como tu sois muy difíciles de encontrar. Y por supuesto a los más importantes, mis padres, Sonia y Rodrigo, gracias a vosotros soy todo lo que soy, por enseñarme todo, por entender todo lo que hago, por querer sin más, absolutamente todo os lo debo a vosotros. GRACIAS, GRACIAS Y GRACIAS. 


\section{INDEX}

Chapter 1: NHC-based bimetallic complexes for multimetallic catalysis

1. Introduction

2. Results and discussion

2.1. Synthesis of bimetallic complexes

2.1.1. Synthesis of heterodimetallic complexes

$\begin{array}{ll}\text { 2.1.2. Synthesis of homodimetallic complexes } & 17\end{array}$

2.2. Metal-to-metal electronic communication studies 21

$\begin{array}{ll}2.3 \text { Catalytic activity: multimetallic processes } & 26\end{array}$

2.3.1. $\mathrm{Ir}(\mathrm{III}) / \mathrm{Au}(\mathrm{I})$ complex 3A: Reduction of nitroarenes by transfer hydrogenation using primary alcohols

2.3.2. $\mathrm{Ir}(\mathrm{III}) / \mathrm{Ru}$ (II) complex 4A: Chelation-assisted $\mathrm{C}-\mathrm{H}$ activation of arylpyridines and Oppenauer oxidation of alcohols

2.3.3. $\mathrm{Ru}(\mathrm{II}) / \mathrm{Pd}(\mathrm{II})$ complex 5A: Carbon-Fluor bond activation via hydrodefluorination

3. Conclusions

4. References

Chapter 2: Asymmetric catalysis with NHC complexes bearing enantiopure ortho-palladated amines

1. Introduction

2. Results and discussion $\quad 65$

2.1. Synthesis of ortho-metalated palladacycles $\quad 65$

2.2. Synthesis of chiral palladacycles with NHC ligands 68

2.2.1. Synthesis of NHC-palladacycles with tertiary amines 68

2.2.2. Synthesis of NHC-palladacycles with primary amines 74

2.3. Synthesis of heterodimetallic Ir-Pd* chiral complexes 77 
2.4. Catalytic studies $\quad 83$

2.4.1. Asymmetric hydrophosphination using chiral palladacycles with NHC ligands

2.4.2.Sequential isomerization of propargylic alcohols/ hydrophosphination using Ir/Pd* chiral complexes

3. Conclusions

4. References $\quad 90$

Chapter 3: Iridium complexes with chiral ortho-metalated amines: 95 Applications in transfer hydrogenation

1. Introduction $\quad 97$

2. Results and discussion 102

2.1. Synthesis of Ir-NH-NHC complexes 102

2.2. Catalytic studies 108

$\begin{array}{ll}\text { 2.2.1. Transfer hydrogenation of ketones } & 108\end{array}$

2.2.2. Asymmetric transfer hydrogenation of ketones 110

$\begin{array}{ll}\text { 2.3. Mechanistic studies } & 111\end{array}$

3. Conclusions 119

4. References $\quad 120$

Chapter 4: Ruthenium complexes with functionalized MIC ligands: 125 Synthesis and catalytic applications

$\begin{array}{ll}\text { 1. Introduction } & 127\end{array}$

2. Results and discussion $\quad 129$

2.1. Synthesis and characterization of the triazolium salts 129

2.2. Synthesis of $\mathrm{Ru}($ triazolylidene) complexes via decarboxylation $\quad 130$

2.3. Synthesis of ' $\mathrm{Ru}(p$-cymene)' triazolylidene-based complexes 135

2.4. Synthesis of solvento Ru-triazolylidene complexes 141

2.5. Catalytic studies 145

$\begin{array}{ll}\text { 2.5.1. Transfer hydrogenation } & 145\end{array}$ 
2.5.2. Base-free dehydrogenation of benzyl alcohol 147

3. Conclusions 150

4. References 151

Chapter 5: Immobilization of pyrene-tagged catalysts onto pyrene- 153 type surfaces

1. Introduction

2. Results and discussion $\quad 157$

2.1. Synthesis of metal complexes bearing a pyrene-tagged NHC ligand 157

2.2. Synthesis and characterization of hybrid M-rGO materials 162

2.3. Synthesis and characterization of hybrid bimetallic Ru-Pd-rGO material 5rGO

$\begin{array}{ll}\text { 2.4. Catalytic studies } & 168\end{array}$

2.4.1. Hydrogenation of unsaturated substrates using molecular hydrogen $\quad 168$

$\begin{array}{ll}\text { 2.4.2. Dehydrogenative oxidation of alcohols } & 175\end{array}$

$\begin{array}{lr}\text { 2.4.3. Hydrodefluorination of fluoroarenes } & 180\end{array}$

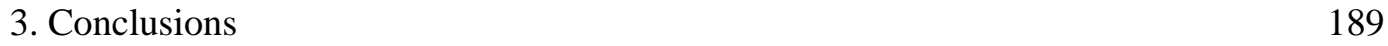

$\begin{array}{ll}\text { 4. References } & 190\end{array}$

Chapter 6: Experimental Section 195

1. Analytical techniques 197

2. Synthesis and characterization 200

2.1. Synthesis and characterization of azolium salts 200

2.2. Synthesis and characterization of bimetallic complexes 203

2.2.1. Synthesis and characterization of heterodimetallic Ir-Pd chiral 207 complexes

2.3. Synthesis and characterization of chiral palladacycles with NHC ligands 210

2.4. Synthesis and characterization of iridium complexes with ortho-metalated primary amines 
2.5. Synthesis and characterization of metal complexes bearing a pyrene-tagged NHC ligand

2.6. Synthesis and characterization of functionalized Ru-triazolylidene complexes

2.7. Synthesis and characterization of hybrid M-rGO materials

3. Catalytic experiments

3.1. Reduction of nitroarenes by transfer hydrogenation using primary alcohols using bimetallic $\mathrm{Au} / \mathrm{Ir}$ complexes

3.2. Chelation assisted $\mathrm{C}-\mathrm{H}$ activation of arylpyridines and Oppenauer oxidation using bimetallic $\mathrm{Ru} / \mathrm{Ir}$ complexes

3.3. Carbon-Fluor activation via Hydrodefluorination using bimetallic $\mathrm{Ru} / \mathrm{Pd}$ complexes

3.4. Asymmetric hydrophosphination using chiral palladacycles with NHC ligands

3.5. Sequential isomerization of propargylic alcohols/hydrophosphination using $\mathrm{Ir} / \mathrm{Pd} *$ chiral complexes

3.6. Transfer hydrogenation using Ir-NH-NHC complexes 246

3.7. Catalytic experiments using Ru-MIC complexes 247

3.8. Catalytic experiments and recyclability using hybrid materials 247

4. DFT Studies 250

5. X-ray Diffraction 251

$\begin{array}{ll}\text { 6. References } & 260\end{array}$

Chapter 7: Compuestos multifuncionales con ligandos NHC para el 263 diseño de procesos catalíticos

1. Introducción

2. Resultados y discusión $\quad 267$

2.1. Síntesis y aplicaciones catalíticas de compuestos bimetálicos 267

2.1.1. Reducción de nitroarenos mediante transferencia de hidrogeno usando alcoholes primarios 
2.1.2. Activación C-H asistida por quelación y oxidación Oppenauer de alcoholes

2.1.3. Activación de en laces C-F via hidrodefluoración

2.2. Catálisis asimétrica usando complejos NHC con aminas orto-paladadas enantiopuras

2.2.1. Catalizadores quirales de paladio con ligandos NHC

2.2.2. Complejos heterobimetálicos de $\mathrm{Ir} / \mathrm{Pd} *$ quirales

2.3. Complejos de iridio con aminas orto-metaladas. Aplicaciones en transferencia de hidrogeno

2.4. Síntesis y aplicaciones catalíticas de complejos de Ru con ligandos carbeno mesoiónicos (MIC) funcionalizados

2.5. Inmovilización de catalizadores sobre superficies de grafeno 


\section{List of abbreviations}

$\Delta$

$\eta$

$\lambda$

$\varepsilon_{\mathrm{r}}$

v

$\mathrm{Ar}$

AFM

ATR

BET

$\mathrm{Bn}$

BSE

cat.

Conv.

$\mathrm{Cp}^{*}$

CP/MAS

CV

DCE

DFT

DMF

DPV

DMSO

E
Refluxing temperature

Ligand hapticity

Wavelength

Relative error

Frequency

Aromatic

Atomic Force Microscopy

Attenuated Total Reflection

Brunauer-Emmett-Teller

Benzyl

Back-Scattered Electrons

Catalyst

Conversion

1,2,3,4,5-pentamethylcyclopentadienyl

Cross-Polarization Magnetic Angle Spinning

Cyclic Voltammetry

1,2-dichloroethane

Density Functional Theory

Dimethylformamide

Differential Pulse Voltammetry

Dimethylsulfoxide

Potential 


\begin{tabular}{|c|c|}
\hline EA & Elemental Analysis \\
\hline$e e$ & Enantiomeric excess \\
\hline EDS & Energy-Dispersive X-ray Spectroscopy \\
\hline Et & Ethyl \\
\hline EtOAc & Ethyl acetate \\
\hline $\mathrm{EtOH}$ & Ethanol \\
\hline $\mathrm{Et}_{2} \mathrm{O}$ & Diethyl ether \\
\hline ESI-MS & Electrospray Ionization Mass Spectrometry \\
\hline ESI-TOF-MS & Electrospray Ionization Time-of-Flight Mass Spectrometry \\
\hline FBZ & Fluorobenzene \\
\hline FT-IR & Fourier Transform Infrared \\
\hline GC & Gas Chromatography \\
\hline GO & Graphene Oxide \\
\hline $\mathrm{h}$ & Hour \\
\hline HAADF & High-Angle Annular Dark-Field \\
\hline HDF & Hydrodefluorination \\
\hline HRMS & High Resolution Mass Spectrometry \\
\hline HSQC & Heteronuclear Single Quantum Coherence \\
\hline I & Current intensity \\
\hline ICP-MS & Inductively Coupled Plasma Mass Spectrometry \\
\hline$i \mathrm{PrOH}$ & 2-propanol \\
\hline KIE & Kinetic Isotopic Effect \\
\hline $\mathrm{L}$ & Ligand \\
\hline
\end{tabular}




$\begin{array}{lll}\text { Me } & \text { Metal } \\ \text { MeOH } & \text { Methyl } \\ \text { MeCN } & \text { Acetonitrile } \\ \text { MIC } & \text { Mesoionic carbene } \\ n \mathrm{Bu} & & \text { 1-butyl } \\ \mathrm{NHC} & & \text { N-heterocyclic carbene } \\ \mathrm{NMP} & & \text { N-methyl-2-pyrrolidone } \\ \mathrm{NMR} & & \text { Nuclear Magnetic Resonance } \\ & \delta & \text { Chemical shift } \\ & \text { br } & \text { broad } \\ & \mathrm{d} & \text { doublet } \\ & J & \text { coupling constant } \\ & \text { m } & \text { multiplet } \\ & \text { ppm } & \text { parts per milion } \\ & \mathrm{s} & \text { singlet } \\ & \text { sp } & \text { septuplet } \\ & \text { triplet }\end{array}$

$\begin{array}{ll}\text { OAc } & \text { Acetate } \\ \mathrm{O} t \mathrm{Bu} & \text { tert-butoxide } \\ \text { OTf } & \text { Triflate } \\ \text { p-cymene } & \text { 1-methyl-4-(isopropyl)benzene } \\ \mathrm{Ph} & \text { Phenyl }\end{array}$




$\begin{array}{ll}\text { Py } & \text { Pyridine } \\ \text { rGO } & \text { Reduced Graphene Oxide } \\ \text { RT } & \text { Room Temperature } \\ \text { SE } & \text { Secondary Electrons } \\ \text { SEM } & \text { Scanning Electron Microscopy } \\ \text { STEM } & \text { Scanning Transmission Electron Microscopy } \\ \text { t } & \text { Time } \\ \text { T } & \text { Temperature } \\ t \text { Am } & \text { tert-amyl or 1,1-dimethylpropyl } \\ t \text { Bu } & \text { tert-butyl } \\ \text { TEP } & \text { Tolman Electronic Parameter } \\ \text { TEM } & \text { Transmission Electron Microscopy } \\ \text { TGA } & \text { TermoGravimetric Analysis } \\ \text { THF } & \text { Tetrahydrofuran } \\ t z & \text { Triazole } \\ \text { UV/Vis } & \text { Ultraviolet/Visible }\end{array}$





\section{CHAPTER 1}

\section{NHC-BASED BIMETALLIC COMPLEXES FOR MULTIMETALLIC CATALYSIS}

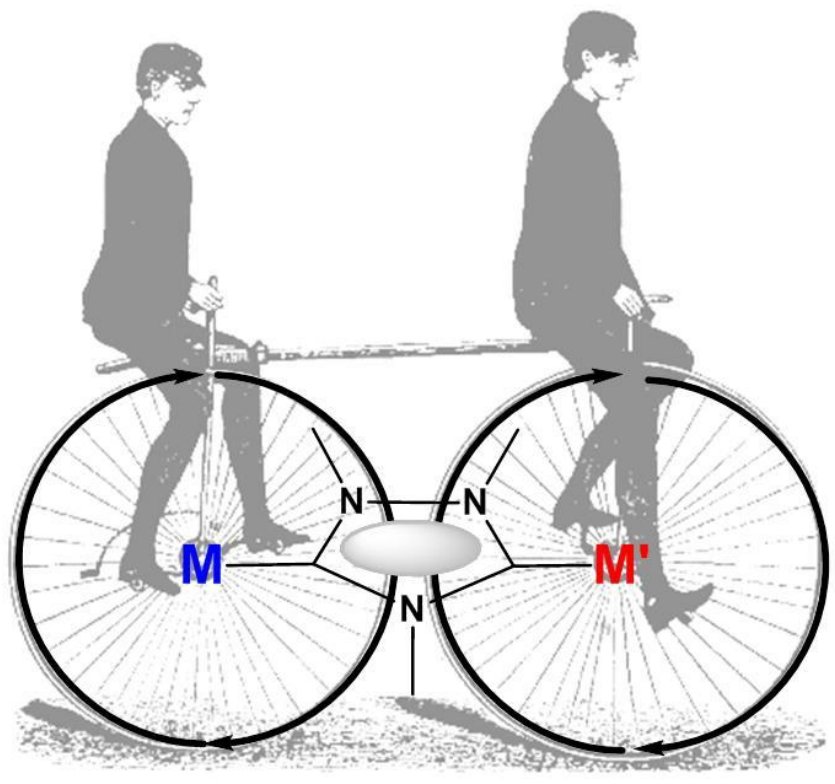





\section{INTRODUCTION}

The increasing demand for environmentally benign and economical synthetic processes is triggering the development of one-pot multiple catalytic transformations that facilitate the production of the desired materials in more efficient ways. ${ }^{1}$ Within this context, clickchemistry methodologies, ${ }^{2-4}$ or tandem catalytic processes, ${ }^{5-8}$ have appeared as useful synthetic tools for improving the efficient use of materials and energy. In the development of new approaches towards the synthesis of value added organic molecules, the design of effective catalysts plays a decisive role. In general, well-defined catalysts are preferred over in-situ generated ones, because the formers often facilitate the investigation of the reaction mechanism and further improvement of the activity by rational tuning of the properties of the catalyst. Moreover, in-situ generated catalysts often need a preactivation time after the addition of an excess of ligand in order to allow the active catalyst to be formed.

In the search for more efficient ways for preparing complex organic architectures, there has been an increasing effort in finding multimetallic catalysts that may be active for a wide set of organic reactions. The combination of fundamental catalytic steps starting from simple and readily available substrates can lead to sophisticated molecules. In fact, in nature, it has been shown that many enzymes contain two metal ions that operate cooperatively. ${ }^{9}$ As a result, complexes containing two metals in close proximity have become subject of extensive investigations in order to mimic nature in the design of effective catalysts. ${ }^{10,11}$

A straightforward approach for the design of tandem processes is to combine two different metal complexes, aiming that each of the metal facilitates a mechanistically independent catalytic cycle. ${ }^{1,5,12,13}$ In such type of process, each complex is supposed to promote an individual catalytic step. However, despite the appropriate combination of two metal centers, the synergism is difficult to achieve. On the contrary, pre-organized single-framed heterometallic complexes are preferred because they may facilitate the cooperative behavior between the active metal centers, especially when the two metal centers are at a close through-space distance, of typically 3-6 $\AA^{14}$ 
The great topological versatility of $\mathrm{N}$-heterocyclic carbenes (NHCs), ${ }^{15}$ their wide range of associated electronic properties, ${ }^{16}$ and their ability to form stable complexes with a large variety of metal fragments, ${ }^{17}$ have raised NHCs to a privileged position in the design of all kinds of homogeneous catalysts, including those having more than one metal. ${ }^{11}$ Polymetallic N-heterocyclic carbene complexes are normally supported by di- or trisNHCs. ${ }^{11,18}$ Specifically, di-NHCs that can be bound to two different metals may allow the preparation of NHC-based heterodimetallic complexes, and this may constitute the basis for the design of sophisticated orthogonal tandem catalysis. ${ }^{11}$

In 1972, Curphey and co-workers described the synthesis the dicationic 1,2,4-trisalkylated triazolium salt $\left[\operatorname{ditz} \mathrm{H}_{2}\right]\left(\mathrm{BF}_{4}\right)_{2}{ }^{19}$ The high potential of this molecule to behave as an NHC-based bridging ligand was demonstrated in 1997, when Bertrand and co-workers described a polymeric silver triazole-di-ylidene (ditz) compound. ${ }^{20}$ Based on these two pioneering works, the Peris' group at the Universitat Jaume I (QOMCAT group), started to describe a number of homo- and heterodimetallic complexes with ditz (Scheme 1.1), which were applied to a wide set of catalytic transformations. ${ }^{21-28}$
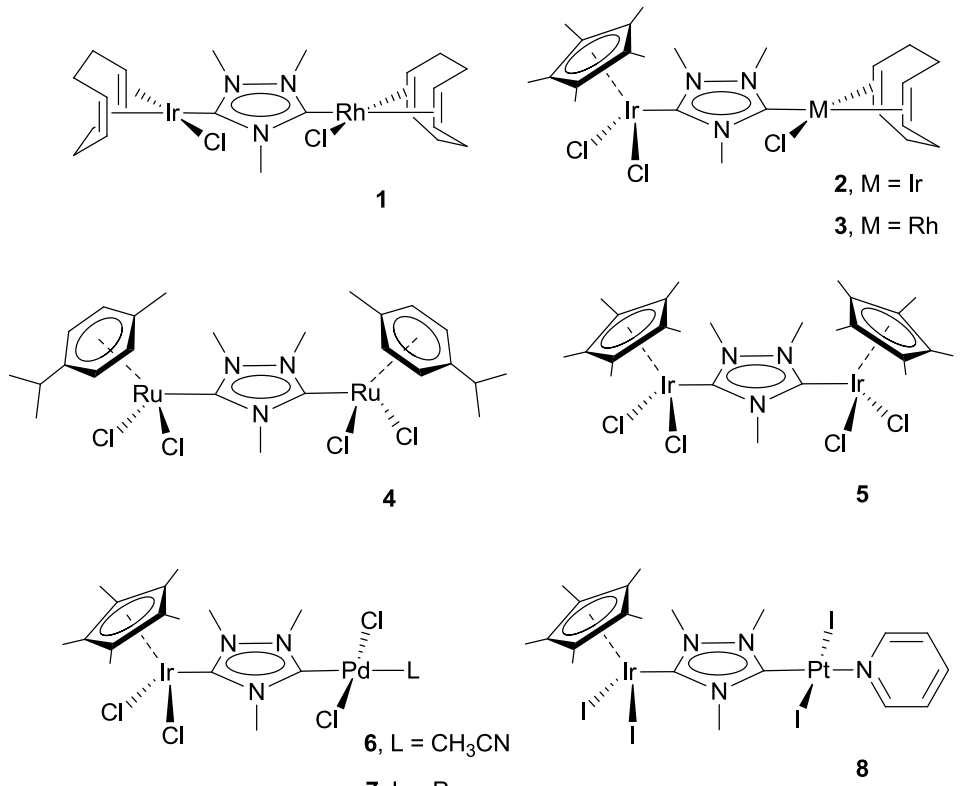

7, $L=P y$

Scheme 1.1 Homo- and heterodimetallic complexes based on ditz ligand 
The idea of using two different metals bound by a single-frame ligand to promote a tandem process is extremely simple, and consists of the use of each of the metals to facilitate mechanistically distinct (orthogonal) cycles, which may be combined to compose the overall tandem process (Scheme 1.2).

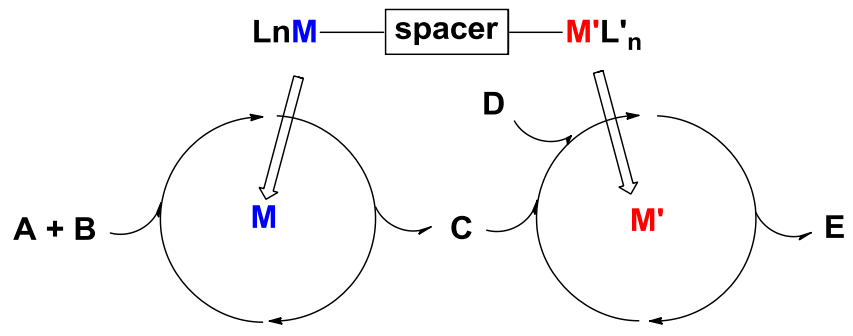

Scheme 1.2 Scheme of a tandem catalytic process

In the Group of Organometallic Chemistry and Catalysis (QOMCAT) at the UJI, the applicability of heterodimetallic complexes was extensively demonstrated in the design of different tandem catalytic processes. ${ }^{11}$ Some of the processes studied included the combination of the following reactions:

a) Cyclization of 2-aminophenyl ethyl alcohol/alkylation of the resulting indole ${ }^{23}$
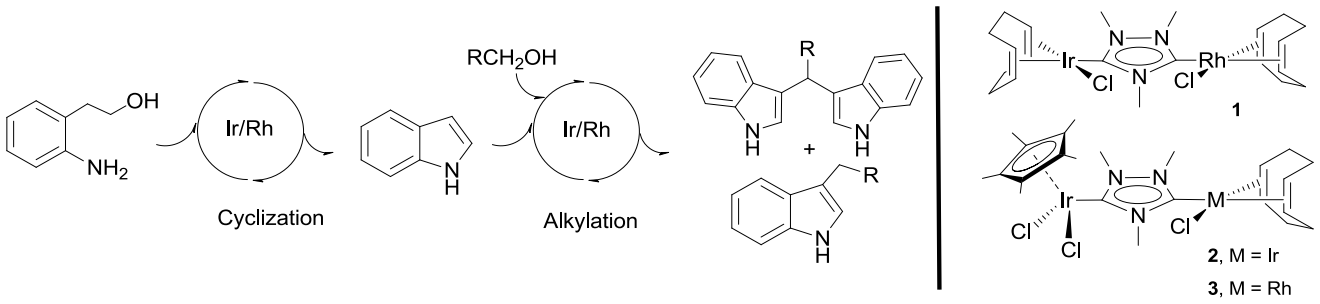

Scheme 1.3

b) Dehalogenation/transfer hydrogenation of haloacetophenones ${ }^{24}$
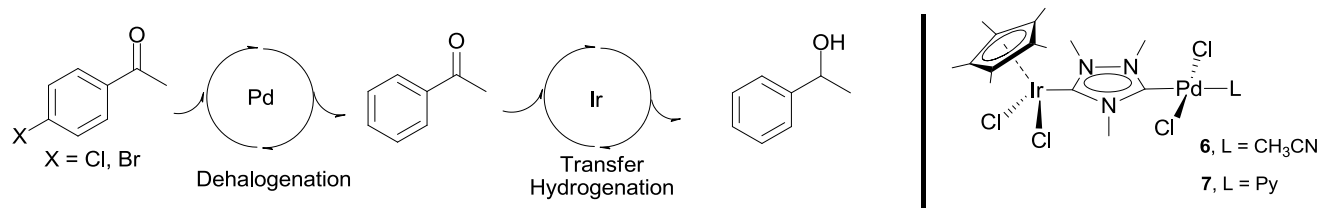

Scheme 1.4 
c) Suzuki-Miyaura C-C coupling/transfer hydrogenation ${ }^{24}$

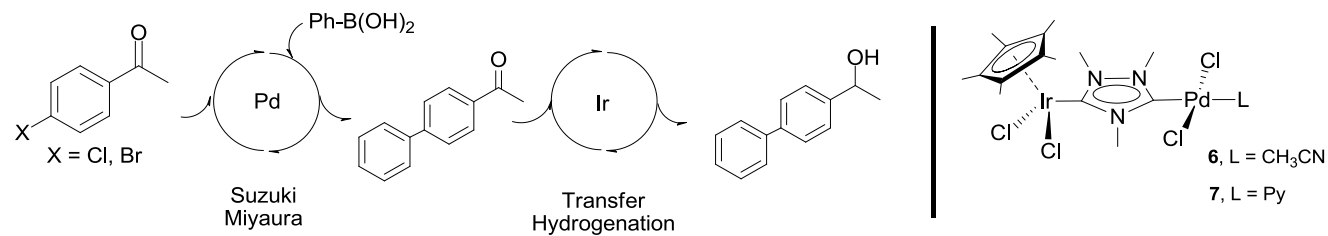

Scheme 1.5

d) Suzuki-Miyaura C-C coupling/ $\alpha$-alkylation of haloacetophenones ${ }^{24}$

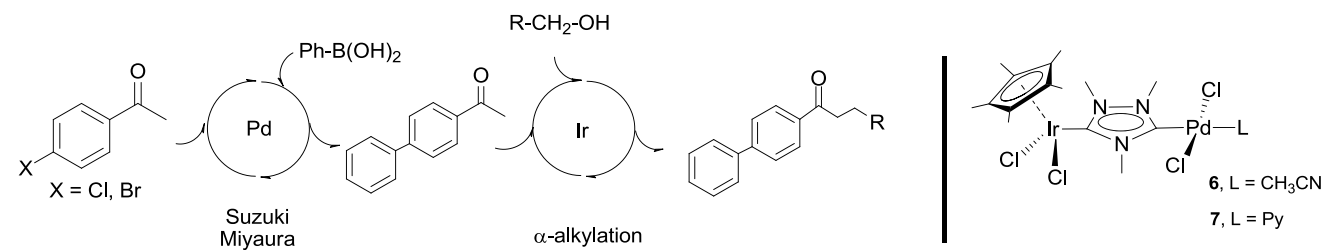

\section{Scheme 1.6}

e) Cyclization of 2-aminophenyl ethyl alcohol/intramolecular hydroalkoxylation of an alkynyl alcohol/indole addition to an enol ether ${ }^{27}$

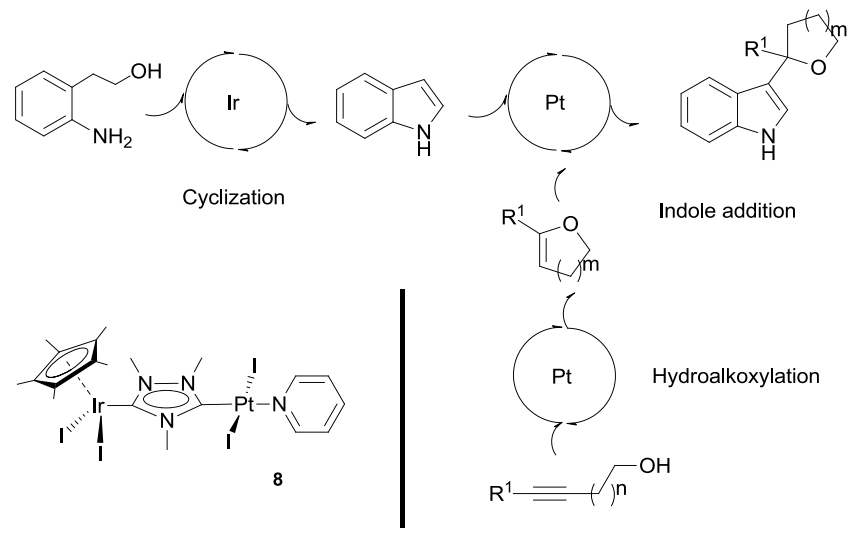

Scheme 1.7

The detailed study of the results indicated an improvement of the catalytic activities of the heterodimetallic species, when compared to those shown by mixtures of the 
homodimetallic analogue catalysts, therefore suggesting that the two different metals are mutually cooperating in the heterodimetallic complex. This 'catalytic cooperativity' may need further study from electrochemical and computational approaches, but its existence is undeniable from the experimental point of view. This seminal research opened a very innovative conceptual advance in the field of homogeneous catalysis, and many research groups are now on the run for the preparation of heterometallic species for tandem catalysis.

Based on the previous achievements developed by the QOMCAT group in the field of tandem catalysis, this chapter deals with the preparation of new ditz-based homo- and heterodimetallic complexes, and the study of their catalytic properties. The results obtained will be compared with previous results by our group, and with recent examples that appeared recently in the bibliography. 


\section{RESULTS AND DISCUSSION}

\subsection{Synthesis of bimetallic complexes}

The general procedure for the preparation of dicationic triazolium salts is described in Scheme 1.8, which merely reproduces the synthetic protocol described by Curphey in 1972. ${ }^{19}$ These azoles are precursors of triazole-di-ylidenes, which will be used all along this chapter. In principle, the two salts $\mathbf{A H}_{2}$ and $\mathbf{B} \mathbf{H}_{2}$ are similar, since they only differ in the N4-alkyl group (N-Me in $\mathbf{A H}_{\mathbf{2}}$, and N-Et in $\mathbf{B} \mathbf{H}_{\mathbf{2}}$ ). Although this modification may seem minor, we thought that the presence of the ethyl group in $\mathbf{B H}_{2}$ may increase the solubility of the metal complexes derived from the use of this ligand precursor.

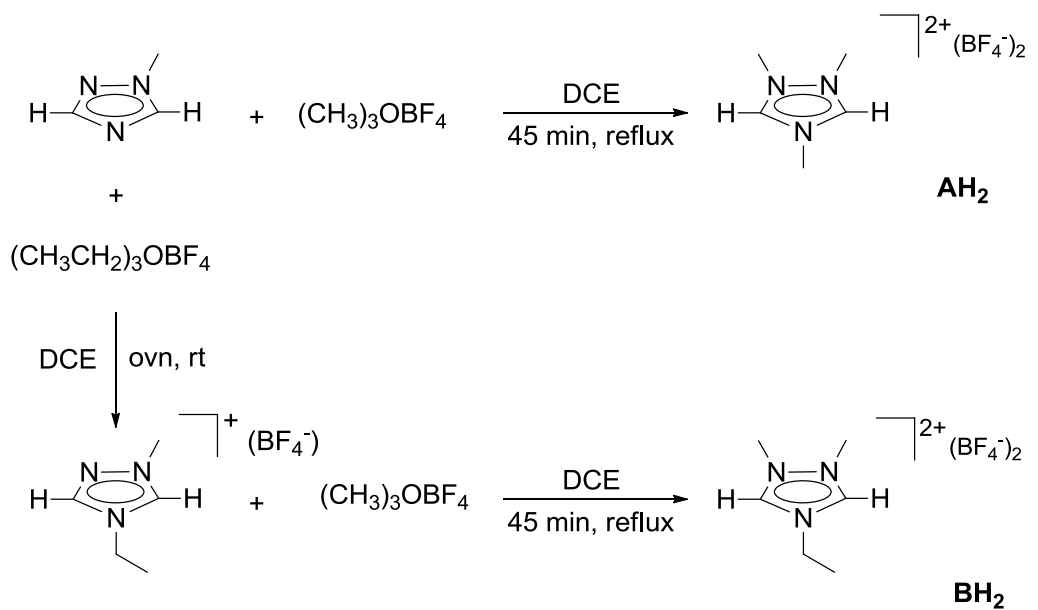

Scheme 1.8 Synthesis of $\mathbf{L H}_{2}$ salts

The reaction implies a two-step double $\mathrm{N}$-alkylation of 1-methyl-triazole. The first alkylation (either with trimethyloxonium or triethyloxonium tetrafluoroborate), is produced at the N4 position. The second step of the reaction involves the alkylation of the remaining nitrogen $(\mathrm{N} 2)$ with trimethyloxonium tetrafluoroborate.

At this point it is important to mention, that we tried to modify the substituents at the triazolium salt, in order to get a wider variety of ligands. The $\mathrm{N}$-alkylation at the $\mathrm{N} 4$ position was achieved using different alkylating agents, such as 1-iodobutane (c) or 
benzyl chloride (d) (Scheme 1.9). Unfortunately, all attempts for the subsequent alkylation at the remaining $\mathrm{N} 2$ position were unsuccessful, even when increasing the temperature or using longer reaction times. For reasons that we are unable to explain, the alkylation at the $\mathrm{N} 2$ position could only be achieved with trimethyloxonium tetrafluoroborate, when N4-Me (a) and N4-Et (b) were used as starting materials, affording $\mathbf{A H}_{2}$ and $\mathbf{B H}_{2}$, respectively.

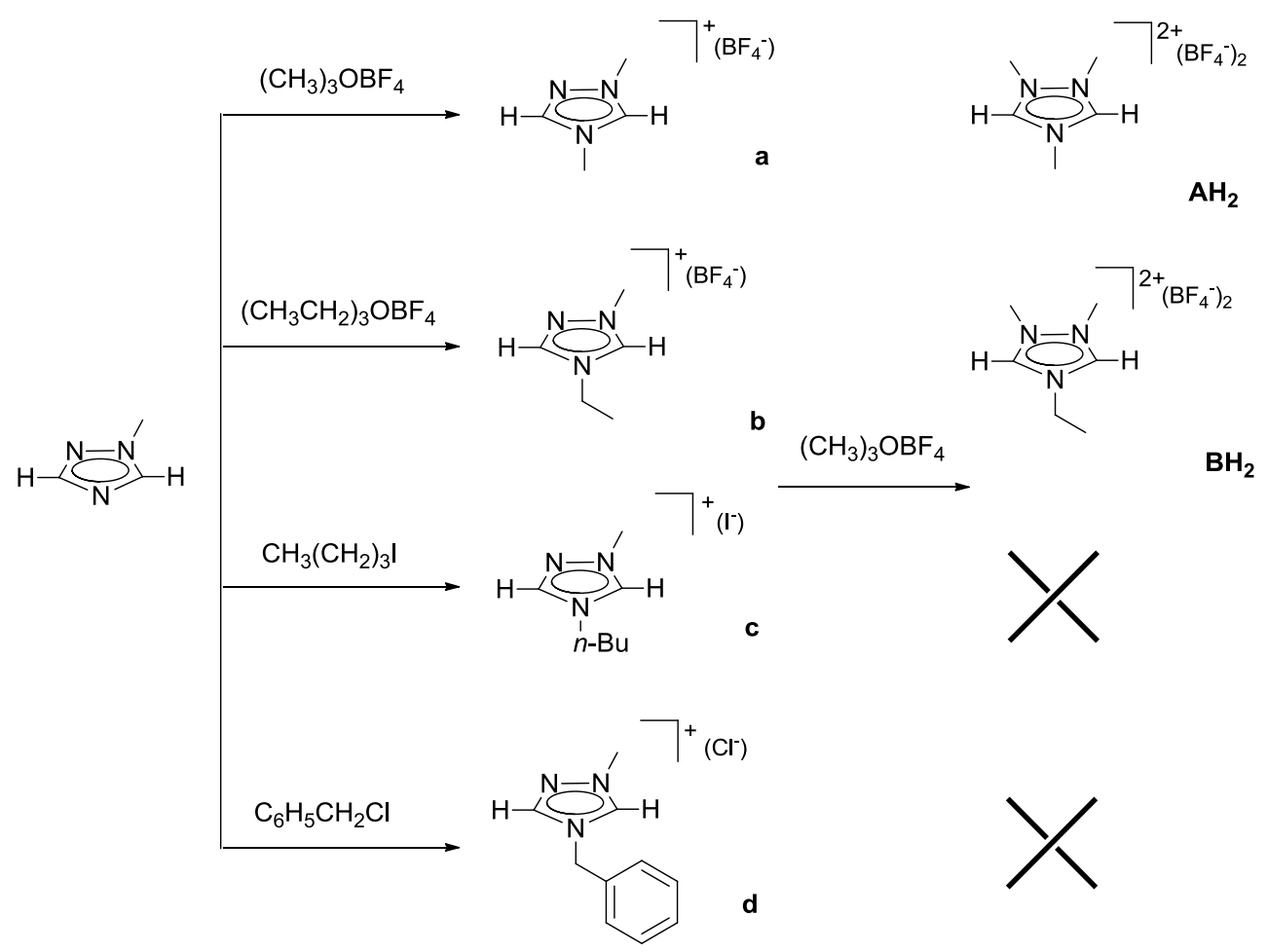

Scheme 1.9 Attempts to modify the substituents at the triazolium ring

\subsubsection{Synthesis of heterodimetallic complexes}

\section{Synthesis and characterization of metal-azolium-ylidene precursors (1AH and $2 \mathrm{AH}$ )}

The general strategy for the preparation of NHC-based hetero-metallic complexes consists of the sequential coordination of the different metal fragments to the triazole-diylidene (ditz) ligand. This general protocol allowed for the preparation of a wide variety 
of heterodimetallic complexes, as already mentioned in the introduction of this chapter (Scheme 1.1). ${ }^{21,23,24,27}$

The reaction of $\mathbf{A H}_{2}$ with an equimolecular amount of $\mathrm{NaH}$ in methanol, affords the methanolic adduct $[\mathbf{A H}-\mathbf{M e O H}]$ in quantitative yield. ${ }^{29}$ This adduct can react with a metal source to afford a monometallic triazolium-ylidene metal complex, which can be subsequently deprotonated and coordinated to a second metal fragment of choice. This method was already used in our group, for the preparation of the iridium-triazoliumylidene 1AH. ${ }^{23}$ For the preparation of this iridium complex, $1 / 2$ equivalents of $\left[\mathrm{IrCp}^{*} \mathrm{Cl}_{2}\right]_{2}$ reacted with $[\mathbf{A H}-\mathbf{M e O H}]$ in refluxing methanol during two hours (Scheme 1.10).

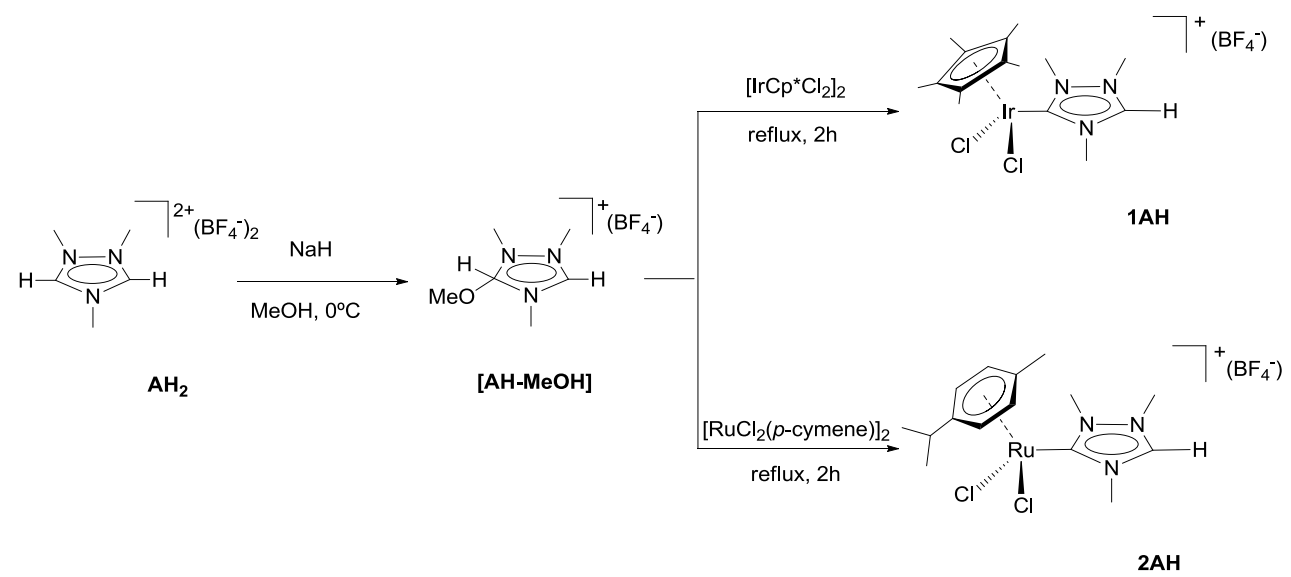

Scheme 1.10 Synthesis of complexes $1 \mathrm{AH}$ and $2 \mathrm{AH}$

The ruthenium complex $\mathbf{2} \mathbf{A H}$ was obtained under similar reaction conditions, by addition of $\left[\mathrm{RuCl}_{2}(p \text {-cymene })\right]_{2}$ to $[\mathrm{AH}-\mathrm{MeOH}]$, which was formed in situ. The product was isolated as an orange solid after crystallization in acetonitrile/methanol in $80 \%$ yield. Complex 2AH was characterized by NMR spectroscopy, High Resolution Mass Spectrometry (HRMS), elemental analysis and X-ray diffraction studies.

NMR spectroscopic characterization of $\mathbf{2 A H}$

The ${ }^{1} \mathrm{H}$ NMR spectrum of $\mathbf{2 A H}$ (Figure 1.1) shows a singlet at $9.54 \mathrm{ppm}$, due to the $\mathrm{NCHN}$ proton. The signals assigned to the protons of the methyl groups appear at 4.38 , 4.21 and $4.17 \mathrm{ppm}$, thus confirming the loss of symmetry of the ligand upon coordination 
by one of its edges. Further confirmation of metalation was given by the appearance of the resonances corresponding to the protons of the $p$-cymene group bound to the ruthenium center, as two doublets at 5.66 and $5.43 \mathrm{ppm}\left({ }^{3} \mathrm{~J}_{\mathrm{H}, \mathrm{H}}=6 \mathrm{~Hz}\right)$.
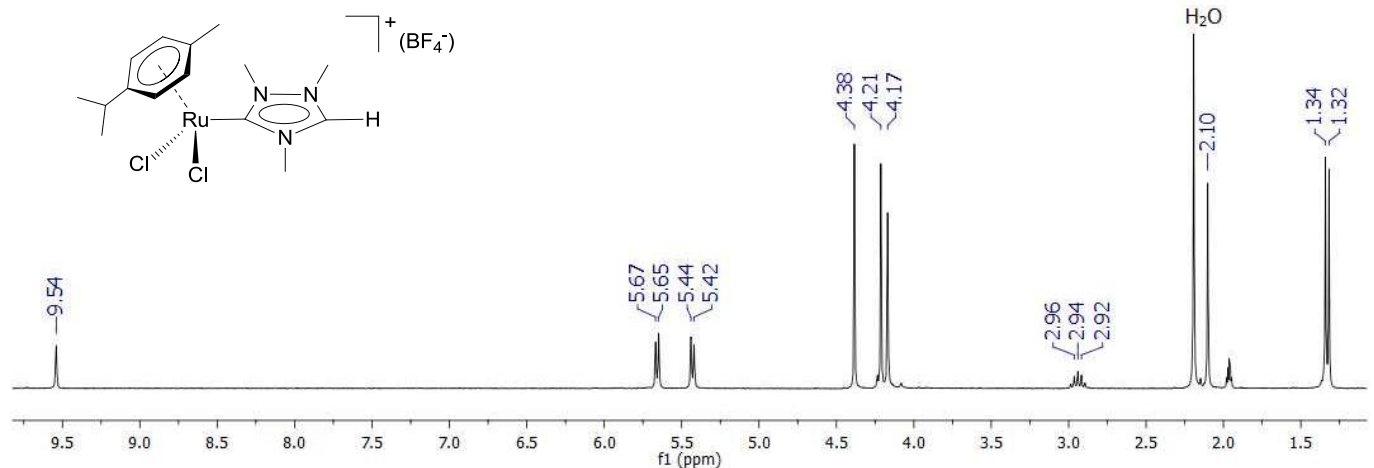

Figure 1.1 ${ }^{1} \mathrm{H}$ NMR spectrum of complex $2 \mathrm{AH}$ in $\mathrm{CD}_{3} \mathrm{CN}$

Figure 1.2 shows the ${ }^{13} \mathrm{C}\left\{{ }^{1} \mathrm{H}\right\}$ NMR spectrum of complex 2AH. The most relevant feature is the presence of the signal at $192.3 \mathrm{ppm}$ corresponding to the carbene carbon, which provides evidence of the metalation to the ruthenium center. The resonance due to the $\mathrm{NCHN}$ of the triazole ring appears at $145.7 \mathrm{ppm}$. The signals attributed to the protons of the methyls of the triazole ring appear at 40.1, 39.6 and $38.1 \mathrm{ppm}$.

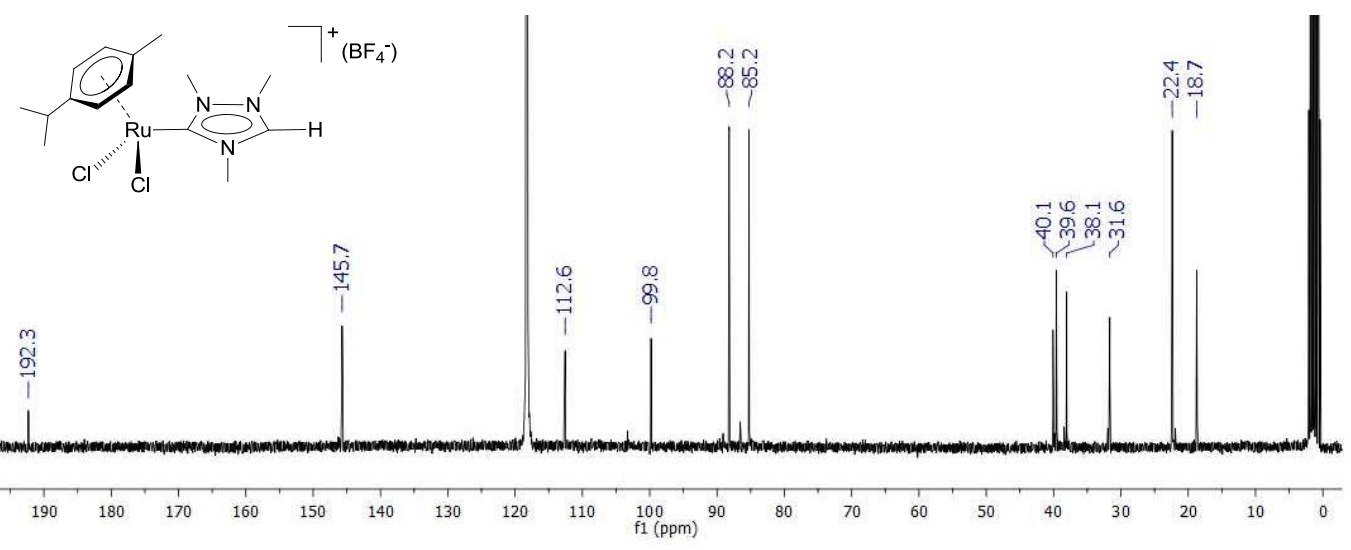

Figure 1.2 ${ }^{13} \mathrm{C}\left\{{ }^{1} \mathrm{H}\right\}$ NMR spectrum of complex $2 \mathrm{AH}$ in $\mathrm{CD}_{3} \mathrm{CN}$ 
Further confirmation of the nature of the complex was given by means of High Resolution Mass Spectrometry, which showed a main peak assigned to the $[\mathrm{M}]^{+}$fragment at $\mathrm{m} / \mathrm{z}=418.0385$.

\section{Molecular structure of $\mathbf{2 A H}$}

The molecular structure of $\mathbf{2 A H}$ (Figure 1.3) was unambiguously confirmed by X-ray diffraction studies. Crystals of $\mathbf{2 A H}$ suitable for analysis were obtained by slow evaporation of a concentrated solution of the compound in acetonitrile/methanol. The molecule can be regarded as a three-legged piano-stool, with a $p$-cymene ligand, two chlorides and a triazolium-ylidene bound to the $\mathrm{Ru}(\mathrm{II})$ center. The cationic nature of the complex is confirmed by the presence of a $\mathrm{BF}_{4}{ }^{-}$counterion. The $\mathrm{Ru}-\mathrm{C}_{\text {carbene }}$ bond length is $2.058(4) \AA$, in the same range of other Ru complexes containing a bridging ditz ligand. ${ }^{22}$

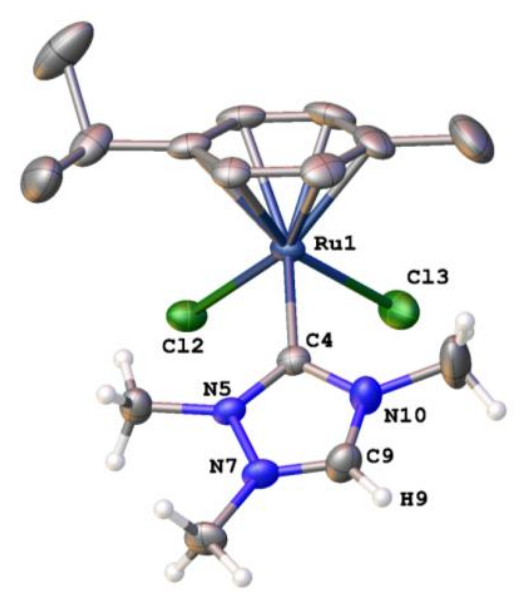

Figure 1.3 Molecular diagram of complex 2AH. Ellipsoids are shown at 50\% probability level. Hydrogen atoms of $p$-cymene ligand and counteranion $\left(\mathrm{BF}_{4}^{-}\right)$are omitted for clarity

\section{Synthesis and characterization of heterodimetallic complexes (3A-5A)}

As already mentioned, the monometallic triazolium-ylidene metal complexes (1AH and 2AH) are highly valuable synthons for the preparation of heterometallic dicarbene species. Both compounds have a remaining $\mathrm{CH}$ bond at the azole ring that can be further activated/deprotonated to generate a second metal-carbene bond. Starting from $\mathbf{1 A H}$ and 
2AH, we synthesized three different heterodimetallic complexes of $\operatorname{Ir} / \mathrm{Au}(\mathbf{3 A}), \mathrm{Ir} / \mathrm{Ru}$ (4A) and $\mathrm{Ru} / \mathrm{Pd}(\mathbf{5 A})$, as depicted in Scheme 1.11.

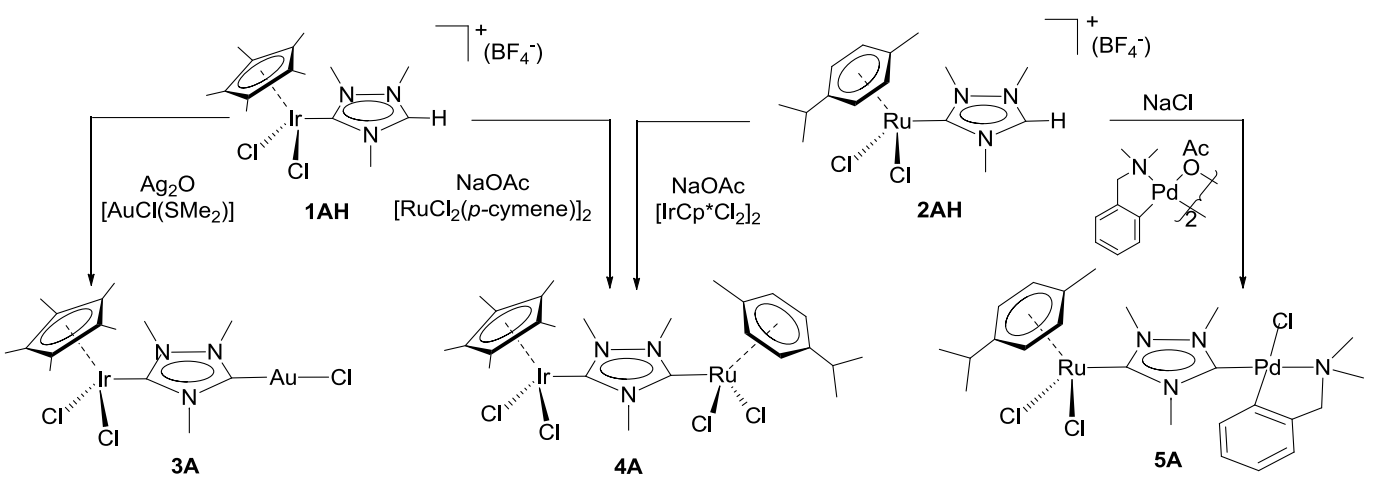

Scheme 1.11 Synthesis of heterodimetallic complexes 3A, 4A and 5A

The Ir/Au heterodimetallic complex 3A was prepared by transmetalating a preformed silver-NHC complex that was used in situ. The reaction of iridiumtriazolium-ylidene complex $(\mathbf{1} \mathbf{A H})$ with $\mathrm{Ag}_{2} \mathrm{O}$ in acetonitrile at room temperature afforded a silver-NHC intermediate, which was subsequently transmetalated to $\left[\mathrm{AuCl}\left(\mathrm{SMe}_{2}\right)\right]$. The mixture was filtered through celite, and the solvent was removed under reduced pressure. The resulting crude solid was purified by crystallization in acetonitrile/diethyl ether, to give the heterodimetallic complex of $\mathrm{Ir} / \mathrm{Au}(\mathbf{3 A})$ as a yellow crystalline solid in $70 \%$ yield.

The $\mathrm{Ru} / \mathrm{Ir}$ complex $\mathbf{4 A}$ was prepared by reaction of $\mathbf{1 A H}$ and $\left[\mathrm{RuCl}_{2}(p \text {-cymene })\right]_{2}$ in the presence of $\mathrm{NaOAc}$ in acetone at $50^{\circ} \mathrm{C}$ overnight. The mixture was filtered through celite, and the solvent was removed under reduced pressure. Crystallization from a mixture of dichloromethane/diethyl ether yielded compound $\mathbf{4 A}$, as an orange crystalline solid in $70 \%$ yield. Noteworthy, compound $\mathbf{4 A}$ could also be obtained starting from the ruthenium-triazolyl complex $(\mathbf{2 A H})$ by addition of $\left[\mathrm{IrCp}^{*} \mathrm{Cl}_{2}\right]_{2}$ in the presence of $\mathrm{NaOAc}$. By following the same purification work-up, compound $\mathbf{4 A}$ was obtained in $81 \%$ yield, therefore this procedure is more convenient than the one starting from $\mathbf{1 A H}$, which only afforded $70 \%$ of $\mathbf{4 A}$. 
The $\mathrm{Ru} / \mathrm{Pd}$ complex $\mathbf{5 A}$ was obtained by reaction of the ruthenium-triazolium-ylidene complex $2 \mathbf{A H}$ and $[\mathrm{Pd}(\mathrm{OAc})(\mathrm{dmba})]_{2}(\mathrm{dmba}=$ dimethylbenzylamine $)$, in acetone at $50^{\circ} \mathrm{C}$ in the presence of $\mathrm{NaCl}$, which was used to supply the chloride ligands to the final product, for $15 \mathrm{~h}$. The mixture was filtered through celite, and the solvent was removed under reduced pressure. The crude solid was purified by column chromatography and crystallized from a mixture of dichloromethane/hexane to give compound $\mathbf{5 A}$ as an orange crystalline solid in $73 \%$ yield.

In general, for all the complexes described in this section, the first indication of coordination of the second metal fragment is the disappearance of the signal corresponding to the $\mathrm{NCHN}$ proton in the ${ }^{1} \mathrm{H}$ NMR spectrum, which appeared in the starting complexes ( $\delta 9.51$ for $\mathbf{1 A H}$, and $\delta 9.54$ for $\mathbf{2 A H}$ ). Another significant feature comes from the observation in the ${ }^{13} \mathrm{C}$ NMR spectra of two signals, which are assigned to the metalated carbene-carbons. For example, for the Ir/Au complex $\mathbf{3 A}$, the ${ }^{13} \mathrm{C} N M R$ spectrum displays two signals at 177.0 and $162.5 \mathrm{ppm}$, for the metalated carbene-carbons bound to $\mathrm{Au}$ and $\mathrm{Ir}$, respectively. In the case of the $\mathrm{Ir} / \mathrm{Ru}$ complex $\mathbf{4 A}$, the metalated carbene carbons appear at 186.4 and $168.1 \mathrm{ppm}$ for $C_{\text {carbene- }}-\mathrm{Ru}$ and $C_{\text {carbene }}-\mathrm{Ir}$, respectively. Finally, for the heterodimetallic Ru/Pd complex $\mathbf{5 A}$, the signals due to the carbene-carbons appear at 186.4 and $182.4 \mathrm{ppm}$, attributed to the $C_{\text {carbene }}-\mathrm{Ru}$ and $C_{\text {carbene- }}$ $\mathrm{Pd}$, respectively.

All three complexes were also characterized by means of elemental analysis and HRMS. This latter technique revealed as a very useful tool for determining the heterometallic nature of all new species. The main peaks were at $\mathrm{m} / \mathrm{z}=706.0634\left([\mathrm{M}-\mathrm{Cl}]^{+}, \mathbf{3 A}\right)$, $780.0796\left([\mathrm{M}-\mathrm{Cl}]^{+}, \mathbf{4 A}\right)$, and $780.0796\left([\mathrm{M}-\mathrm{Cl}]^{+}, \mathbf{5 A}\right)$.

As an example of the characterization of these complexes, a detailed description of the ${ }^{1} \mathrm{H}$ NMR and ${ }^{13} \mathrm{C}$ NMR of complex $\mathbf{5 A}$ is given. All the details regarding the NMR spectroscopic characterization of the rest of the complexes are given in the Experimental Section (Chapter 6). 


\section{${ }^{I} H$ NMR spectrum of complex $\mathbf{5 A}$}

The first evidence of coordination to the second metal center is the disappearance of the signal of the $\mathrm{NCHN}$ proton. Figure 1.4 shows the ${ }^{1} \mathrm{H}$ NMR spectrum of the heterodimetallic complex 5A. The three resonances at $4.41,4.35$ and $4.30 \mathrm{ppm}$ are due to the protons of the methyl groups at the azole bridge indicating the lack of the twofold symmetry of the ligand. The two doublets at 4.01 and $3.85 \mathrm{ppm}\left({ }^{3} \mathrm{~J}_{\mathrm{AB}}=13.5 \mathrm{~Hz}\right)$ are due to the non-equivalent geminal protons of the ortho-metalated amine. The signals attributed to the aromatic protons of the $p$-cymene ligand are displayed as four doublets at 5.56, 5.53, 5.31 and $5.18 \mathrm{ppm}$.

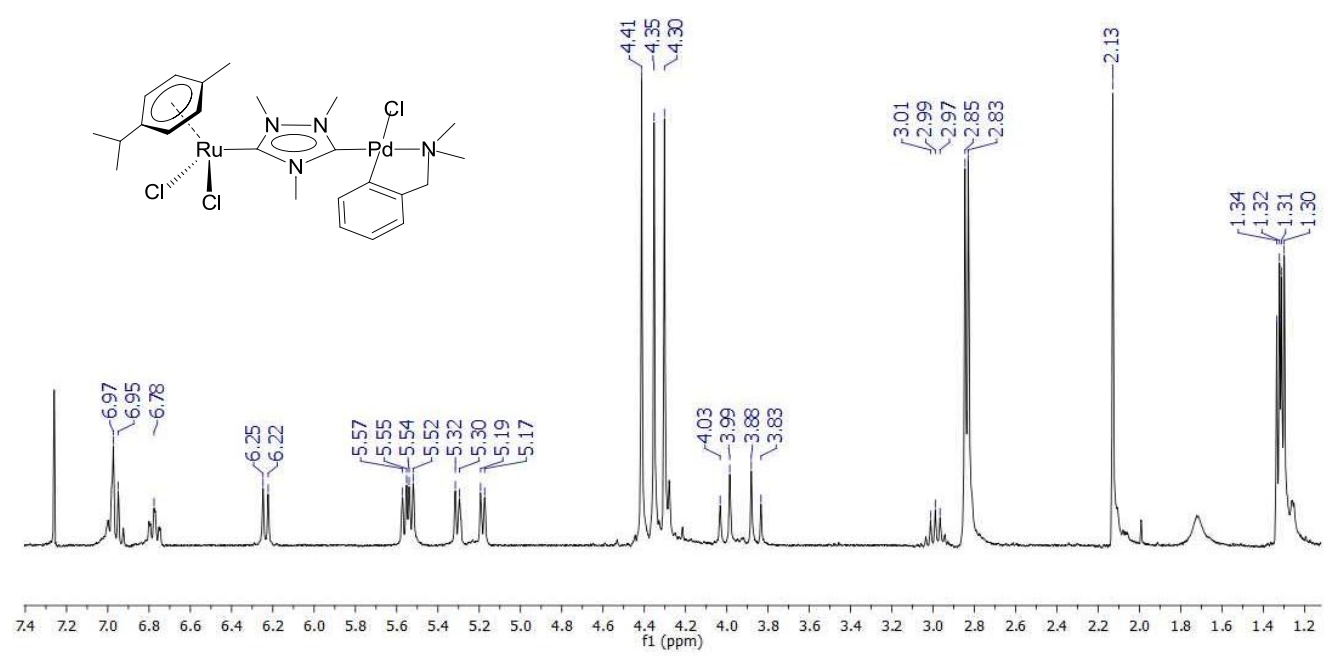

Figure 1.4 ${ }^{1} \mathrm{H}$ NMR spectrum of $\mathbf{5 A}$ in $\mathrm{CDCl}_{3}$

\section{${ }^{13} C\left\{{ }^{1} H\right\}$ NMR spectrum of complex $\mathbf{5 A}$}

Figure 1.5 shows the ${ }^{13} \mathrm{C}\left\{{ }^{1} \mathrm{H}\right\}$ NMR spectrum of complex 5A. The most relevant signals are the ones shown at 186.4 and $182.4 \mathrm{ppm}$, attributed to the carbene-carbons bound to $\mathrm{Ru}$ and $\mathrm{Pd}$, respectively. The signals due to the carbons of the methyl groups at the triazole ring appear at 42.2, 38.6 and $37.9 \mathrm{ppm}$. The signal assigned to the $\mathrm{CH}_{2}$ of the ortho-metalated amine appears at $72.3 \mathrm{ppm}$. The resonances due to the carbons of the methyl groups of the amine are shown at 50.8 and $50.4 \mathrm{ppm}$. 

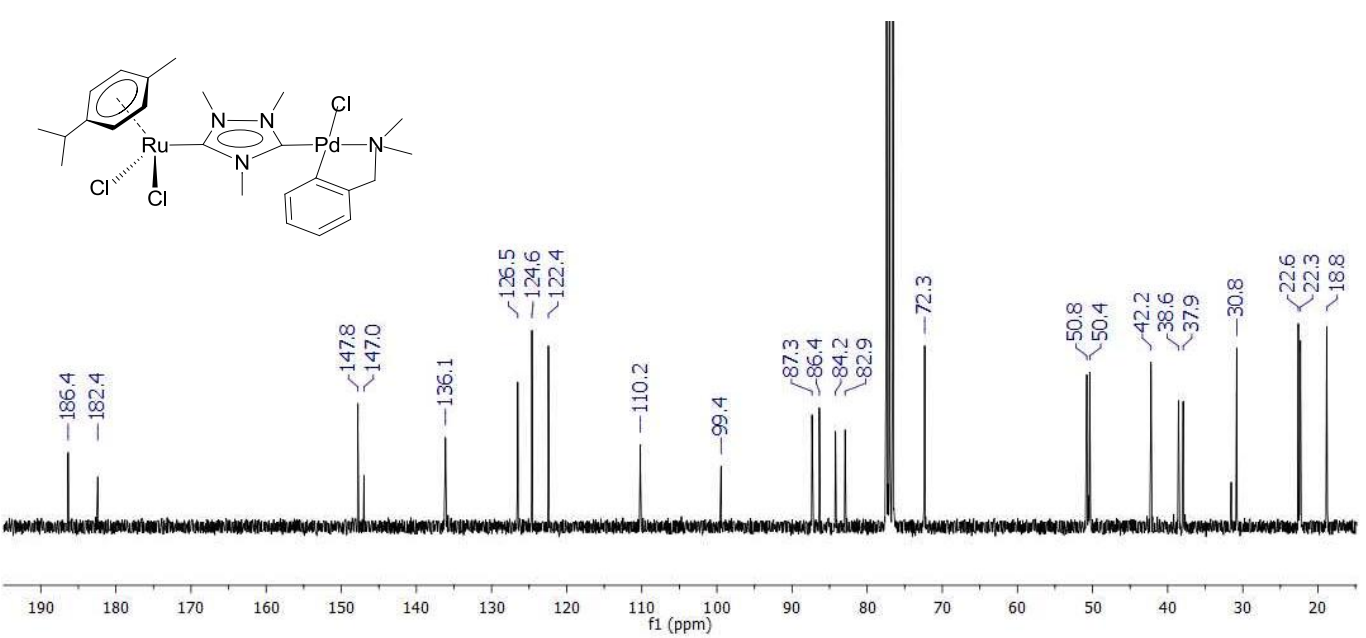

Figure $1.5{ }^{13} \mathrm{C}\left\{{ }^{1} \mathrm{H}\right\}$ NMR spectrum of complex $\mathbf{5 A}$ in $\mathrm{CDCl}_{3}$

\section{$X$-ray molecular structure of $\mathbf{3 A}$}

Crystals of 3A suitable for X-ray diffraction were obtained by slow diffusion of diethyl ether into a concentrated solution of the compound in acetonitrile. The molecular structure of complex $\mathbf{3 A}$ contains an " $\mathrm{ICCp}^{*} \mathrm{Cl}_{2}$ " fragment connected to a "AuCl" unit by the triazole-di-ylidene bridge, thus confirming the bimetallic nature of the complex (Figure 1.6, top). The iridium bond lengths and angles about the iridium sphere lie in the expected range, when compared to similar complexes reported by us for the same metal fragment containing the triazole-di-ylidene ligand. For example, the $\mathrm{Ir}-\mathrm{C}_{\text {carbene }}$ bond length is $2.027(14) \AA$, which is very similar to the $\mathrm{Ir}_{-} \mathrm{C}_{\text {carbene }}$ bond length shown by $\mathbf{1 A H}(2.048(11) \AA)^{23}$ The coordination about the gold center is linear with an angle of $177.3(4)^{\circ}$ and a Au-C bond length of 1.962(13) A.

The crystal packing of complex $\mathbf{3 A}$ shows the formation of dimers. Two molecules, in an anti disposition, show a hydrogen bonding interaction between one chloride and one of the protons of a methyl group of the triazole-di-ylidene (Figure 1.6, bottom). In general, this type of chloride-hydrogen interactions is not very strong, but in this particular case the $\mathrm{Cl}-\mathrm{C}_{\text {methyl }}$ bond length of $3.52 \AA$ is significantly short. ${ }^{30}$ 

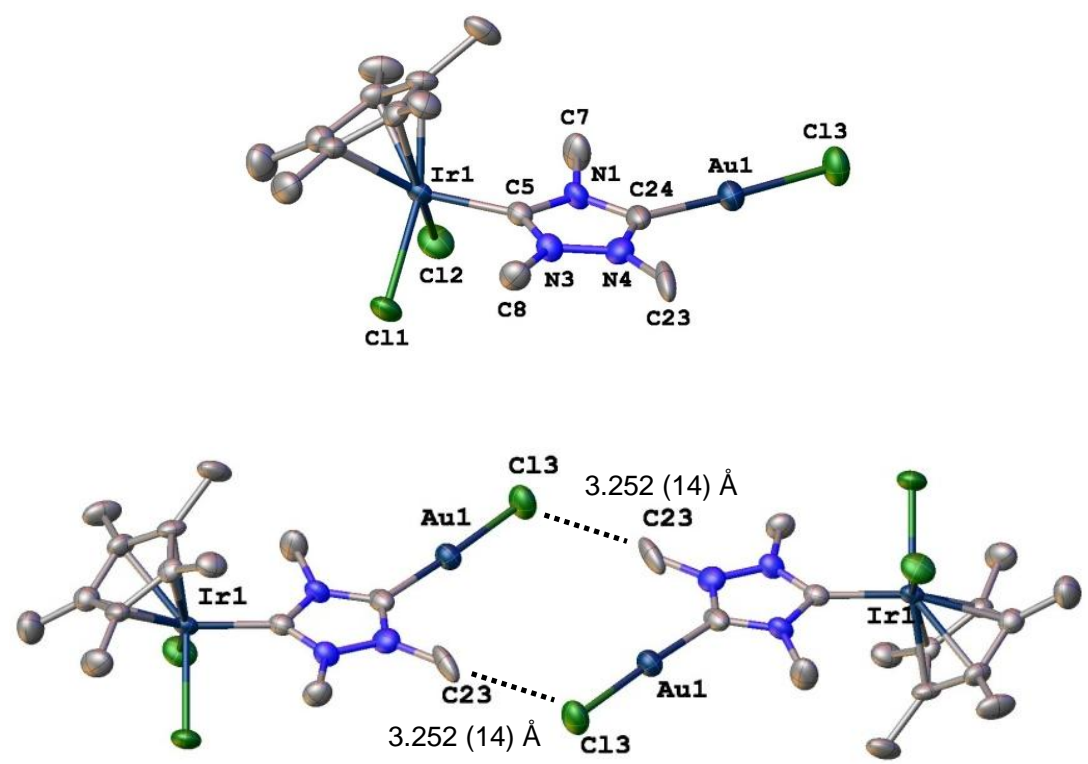

Figure 1.6 Molecular diagram (top) and crystal packing showing the dimmers form in the solid state (bottom) of complex 3A. Ellipsoids are shown at 50\% probability level. Hydrogens omitted for clarity. Selected bond lengths ( $⿱$ $)$ and angles $\left(^{\circ}\right)$ : $\operatorname{Ir}(1)-\mathrm{C}(1) 2.027(14), \operatorname{Ir}(1)-\mathrm{Cl}(1) 2.407(3)$, $\operatorname{Ir}(1)-\mathrm{Cl}(2)$ 2.413(3), $\mathrm{Ir}(1)-\mathrm{C}_{\text {centr }} 1.807, \mathrm{Au}(2)-\mathrm{C}(2)$ 1.962(13), $\mathrm{Au}(2)-\mathrm{Cl}(3)$ 2.273(4), $\mathrm{Cl}(1)-$ $\operatorname{Ir}(1)-\mathrm{C}(1)$ 89.4(3), C(2) - $\mathrm{Au}(2)-\mathrm{Cl}(3)$ 177.3(4)

\subsubsection{Synthesis of homodimetallic complexes}

For the study of the catalytic activity of the heterodimetallic complexes described in the previous section, it is important to ascertain whether the two metal fragments are cooperating or, in other words, if the heterometallic nature of the complex provides any benefits compared to the use of similar homodimetallic complexes. For this reason, we were also interested in obtaining the homodimetallic analogues to the heterometallic complexes described in the previous section.

In general, the coordination of two metals to the dicationic salts $\left(\mathbf{A H}_{\mathbf{2}}\right.$ or $\left.\mathbf{B} \mathbf{H}_{2}\right)$ is achieved by a one-pot procedure. The homodimetallic complexes of iridium ${ }^{23}(\mathbf{6 A})$ and ruthenium $^{22}$ (7A) were previously obtained in our group (Scheme 1.12). 

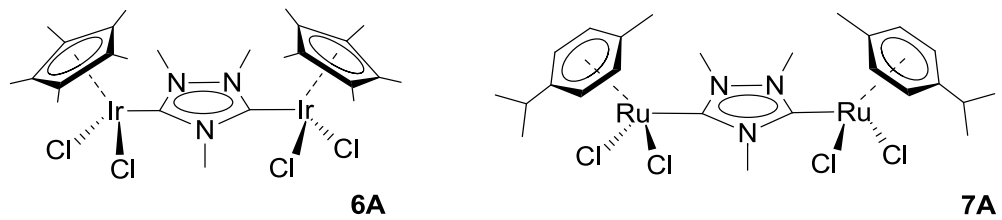

Scheme 1.12

The digold (1B) and dipalladium $(\mathbf{8 A})$ were synthesized following the procedures shown in Scheme 1.13.

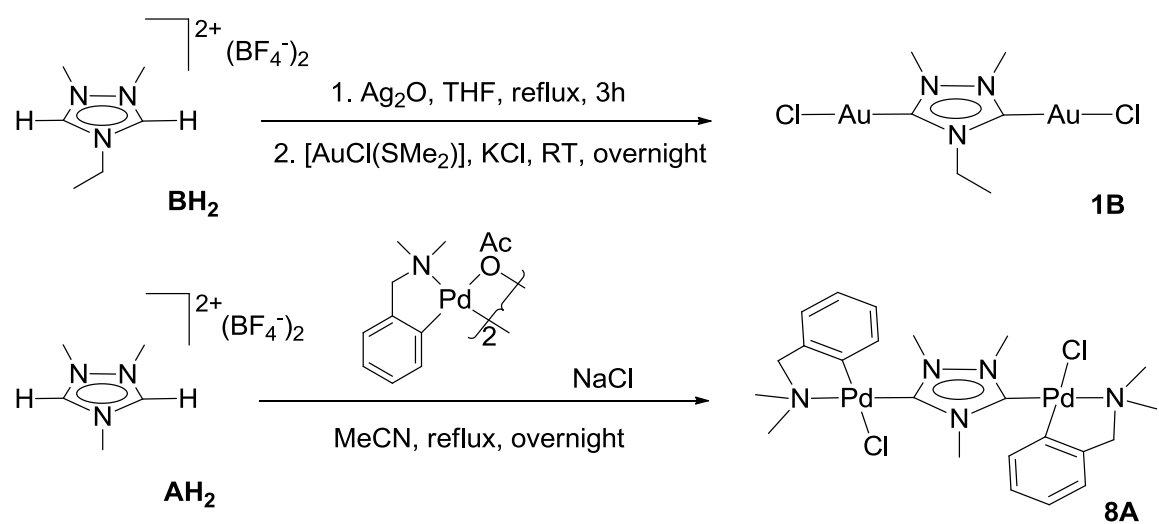

Scheme 1.13 Synthesis of homodimetallic complexes $\mathbf{1 B}$ and $\mathbf{8 A}$

The digold complex 1B was prepared starting from the dicationic salt $\mathbf{B H}_{2}$, by transmetalating the di-carbene ligand from the in situ prepared, pre-formed silver carbene compound. $^{20}$ The synthesis consists of the reaction of the ligand precursor with two equivalents of $\mathrm{AgOAc}$ in refluxing tetrahydrofuran for $3 \mathrm{~h}$, in exclusion of light, followed by the addition of $\left[\mathrm{AuCl}\left(\mathrm{SMe}_{2}\right)\right]$ and $\mathrm{KCl}$ in acetonitrile at room temperature overnight. The suspension was filtered over celite and the solvents were concentrated under reduced pressure. Precipitation from acetonitrile/diethyl ether afforded 1B as a white crystalline solid, in $80 \%$ yield. We also tried to obtain the related bimetallic complex of Au using the trimethylated triazolium $\mathbf{A H}_{2}$, but for reasons that we could not explain all our attempts were unsuccessful. 
The synthesis of the dipalladium complex $\mathbf{8 A}$ was accomplished by reaction of the ligand precursor $\left(\mathbf{A H}_{2}\right)$ with one equivalent of $[\mathrm{Pd}(\mathrm{OAc})(\mathrm{dmba})]_{2}$ in the presence of $\mathrm{NaCl}$. The reaction was taken to reflux in acetonitrile overnight. The final suspension was filtered through celite and the solvent was evaporated under reduced pressure. Crystallization from dichloromethane/hexane afforded pure compound $\mathbf{8 A}$ as an off-white crystalline solid, in $82 \%$ yield.

Both 1B and 8A were characterized by NMR spectroscopy, High Resolution Mass Spectrometry (HRMS) and elemental analysis.

The coordination of the two metal centers to the dicarbene ligand was confirmed by NMR spectroscopy. As for the rest of the heterodimetallic complexes described above, the first indication of the successful coordination is the disappearance of the signal attributed to the $\mathrm{NCHN}$ proton of the bisazolium salts (10.66 ppm for $\mathbf{A} \mathbf{H}_{2}$ and $10.03 \mathrm{ppm}$ for $\mathbf{B} \mathbf{H}_{2}$ ) in the ${ }^{1} \mathrm{H}$ NMR spectrum. Further confirmation of the metalation is given by the ${ }^{13} \mathrm{C}$ NMR spectrum, where complex 1B exhibited a characteristic resonance at $174.5 \mathrm{ppm}$ for the carbene-carbon. An interesting feature of complex $\mathbf{8 A}$ is the appearance of two isomers, due to the restricted rotation about the $\mathrm{Pd}-\mathrm{C}$ bond. The presence of these two atropisomers is evidenced by the ${ }^{1} \mathrm{H}$ NMR spectrum, which shows two different sets of signals corresponding to the sin and anti conformations, as depicted in Scheme 1.14. All the details about the spectroscopic characterization of $\mathbf{1 B}$ and $\mathbf{8 A}$ are given in the Experimental Section (Chapter 6).

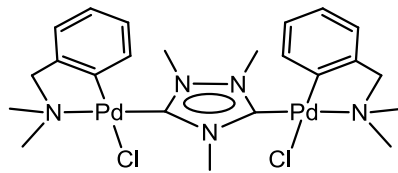

$\sin$

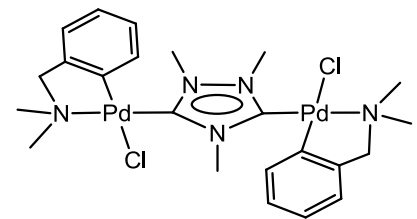

anti

Scheme 1.14 Sin and anti isomers for complex 8A

\section{Molecular structure of $\mathbf{1 B}$}

The molecular structure of 1B (Figure 1.7) was confirmed by means of X-ray diffraction studies. Crystals of $\mathbf{1 B}$ were obtained by slow diffusion of diethyl ether into a 
concentrated solution of the compound in acetonitrile. The molecular structure consists of two facially opposed Au-Cl fragments connected by the 4-ethyl-1, 2-dimethyltriazol-3,5diylidene ligand. The coordination about the two metal centers is linear, with $\mathrm{C}-\mathrm{Au}-\mathrm{Cl}$ angles of $178.1^{\circ}$ for $\mathrm{Au}(1)$, and $176.9^{\circ}$ for $\mathrm{Au}(2)$. The intramolecular distance between the gold atoms is $6.02 \AA$, and the average Au-C distance is $1.97 \AA .{ }^{31,32}$

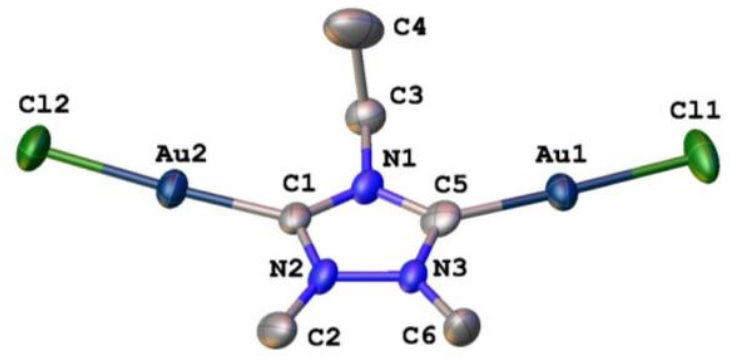

Figure 1.7 Molecular diagram of complex 1B. Ellipsoids are shown at 50\% probability level. Hydrogens omitted for clarity. Selected bond lengths $(\AA)$ and angles $\left({ }^{\circ}\right)$ : $\mathrm{Au}(1)-\mathrm{C}(5)$ 1.953(11), $\mathrm{Au}(2)-\mathrm{C}(1)$ 1.982(10), $\mathrm{Au}(1)-\mathrm{Cl}(1) 2.270(3), \mathrm{Au}(2)-\mathrm{Cl}(2) 2.258(3), \mathrm{C}(5)-\mathrm{Au}(1)-\mathrm{Cl}(1)$ 178.1(3), $\mathrm{C}(1)-\mathrm{Au}(2)-\mathrm{Cl}(2) 176.9(3)$

The molecule is essentially planar, except for the methyl of the ethyl group. Interestingly, the crystal packing of complex $\mathbf{1 B}$ shows an intermolecular $\mathrm{Au}^{\mathrm{I}}-\mathrm{Au}^{\mathrm{I}}$ interaction contact of $3.26 \AA .^{33,34}$ This aurophilic interaction justifies the formation of polymeric zig-zag chains in the crystal packing (Figure 1.8). The ethyl group is pointing outside the polymeric chain, preventing a strong chain-to-chain aurophilic interaction, although the $\mathrm{Au}-\mathrm{Au}$ distance between adjacent chains is $3.50 \AA$, which still can be considered as a weak aurophilic interaction. ${ }^{35}$

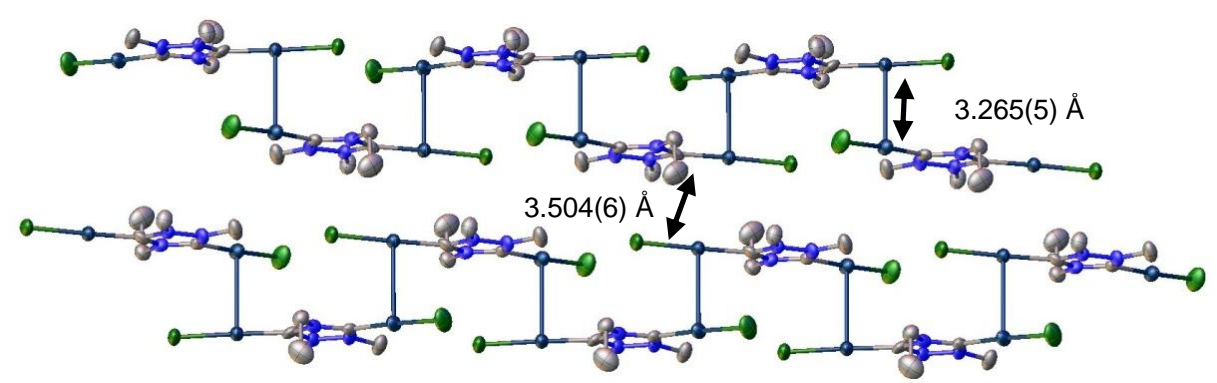

Figure 1.8 Crystal packing of complex 1B showing the zig-zag polymeric chain 


\subsection{Metal-to-metal electronic communication studies}

Despite the experimental evidences, the nature of the cooperative behavior between metal centers linked by a single-frame ligand is still unclear and needs further studies. Some authors have proposed that a plausible explanation may come from metal-to-metal electronic communication in bimetallic complexes, which may influence the reactivity of each individual metal fragment either enhancing ${ }^{24,27,28}$ or interrupting ${ }^{36}$ the overall catalytic activity of the bimetallic complex. Electrochemical and computational approaches have revealed that factors such as the intermetallic distance, the rigid three dimensional geometry of the scaffold, and the integrity of the multimetallic structure during the catalytic cycle should play important roles. ${ }^{37}$

Cyclic voltammetry (CV) studies have been proposed as a valuable tool for studying the electronic communication degree across a bridging ligand. ${ }^{38,39}$ Albrecht and co-workers described the electrochemical and spectrochemical analysis of a series of iron and ruthenium complexes linked by a benzobis(imidazolylidene) ligand (Scheme 1.15, top). ${ }^{38}$ The electrochemical analyses, and comparative studies using analogue monometallic carbene complexes, revealed that despite the $\pi$-delocalized nature of the bridging ligand, the two metal centers are moderately coupled in the case of the iron complex $\left(\Delta \mathrm{E}_{1 / 2}=80\right.$ $\mathrm{mV})$, and nearly electronically uncoupled in the case of the ruthenium complex $\left(\Delta \mathrm{E}_{1 / 2}=42\right.$ $\mathrm{mV}$ ). Bielawski and co-workers also found a similar behavior in the metal-to-metal interaction in di-iridium complexes bridged by similar benzobis(imidazolylidenes), as deduced from spectroscopic and electrochemical studies. ${ }^{39}$

In a more recent study, Albrecht and co-workers described the synthesis and the electronic properties of a diruthenium complex linked to a benzobis(imidazolylidene) ligand (Scheme 1.15, bottom). ${ }^{40}$ The electrochemical studies showed that the two metals centers are electronically decoupled when the ruthenium is in a pseudo-tetrahedral geometry imparted by a $p$-cymene spectator ligand. Interestingly, ligand exchange, and consequent modification of the coordination geometry to octahedral, leads to an electronically coupled system, as evidenced by two distinctly different metal-centered oxidation processes separated by $134 \mathrm{mV}$. The intermetallic communication is critically boosted by the appropriate alignment of the metal $4 d$ and the ligand $p$ orbitals, thus in this 
case, the rigid octahedral geometry induces sufficiently large $d_{M}-\pi_{L}$ overlap for imparting a high degree of intermetallic communication. These results also support the relevance of the $\pi$-contributions to the metal-NHC bond.
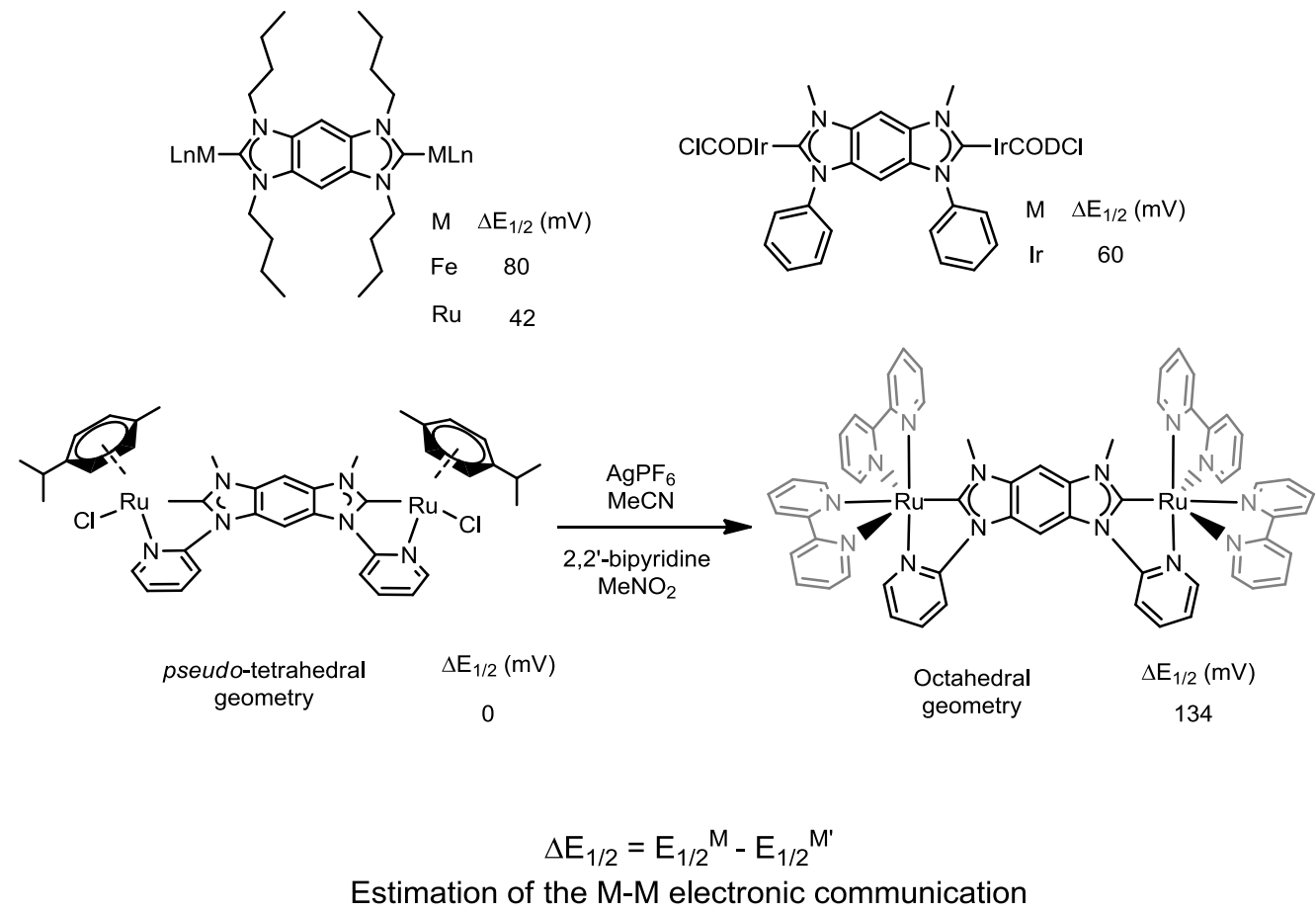

Scheme 1.15 Top: Bimetallic complexes described by Albrecht (left) and Bielawsky (right). Bottom: Diruthenium complexes described by Albrecht

As an alternative to electrochemical studies, the variations in the Tolman electronic parameter (TEP) may also be an effective way for determining the electronic communication between metals in bimetallic compounds. ${ }^{41}$ The M-M coupling is measured as the variation of the TEP value by changing the nature of the metal fragment attached to one of the edges of a Janus di-NHC, while maintaining the other edge bound to $\mathrm{Ni}(\mathrm{CO})_{3}$ (Scheme 1.16$)$. DFT studies suggest that $\pi$-interactions apparently play no significant role in these systems, so the M-M coupling is independent of the presence of an appropriate $\pi$-delocalized system. 

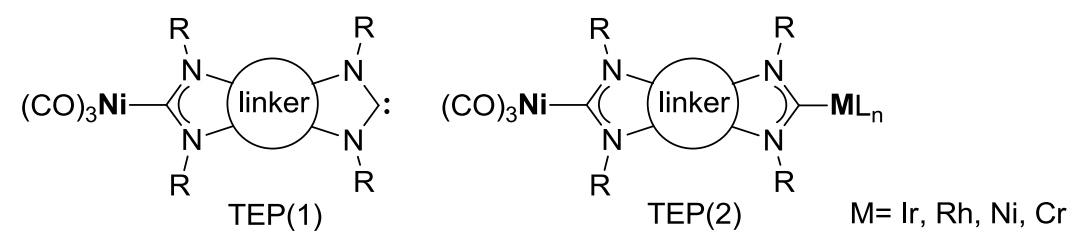

$\Delta T E P=$ Estimation of the M-M coupling

Scheme 1.16 Estimation of the M-M coupling as the variation of the TEP value

\section{Cyclic Voltammetry studies}

The electronic communication in the heterodimetallic complex $\mathbf{4 A}$ was evaluated by Cyclic Voltammetry (CV) and Differential Pulse Voltammetry (DPV) studies. For comparative purposes the electrochemical behavior of 6A, 7A, 1C and $2 \mathrm{C}$ (Scheme 1.17) was also studied.
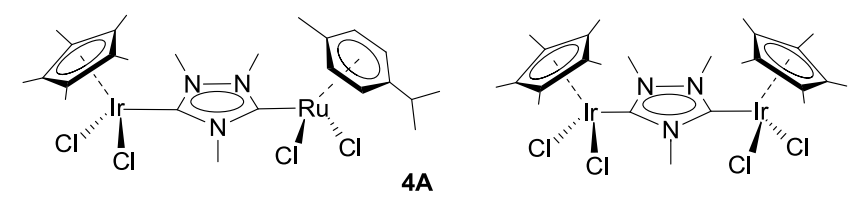

6A
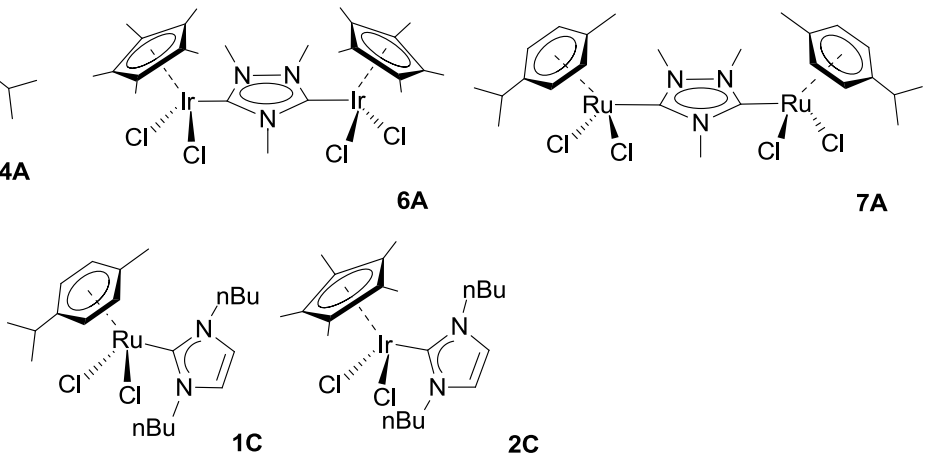

Scheme 1.17 Mono- and bi-metallic complexes used in the electrochemical studies

The $\mathrm{CV}$ diagrams of the monometallic $\mathrm{Ru}$ and $\mathrm{Ir}$ compounds $\mathbf{1 C}$ and $\mathbf{2 C}$ (Figure 1.9) showed reversible waves assigned to the $\mathrm{E}\left(\mathrm{Ru}^{\mathrm{II}} / \mathrm{Ru}^{\mathrm{III}}\right)$ and $\mathrm{E}\left(\mathrm{Ir}^{\mathrm{III}} / \mathrm{Ir}^{\mathrm{IV}}\right)$ processes, at $\mathrm{E}_{1 / 2}=$ $1.13 \mathrm{~V}$ and $1.17 \mathrm{~V}$, respectively. These values can be compared to the ones shown by the bimetallic complexes $\mathbf{4 A}, \mathbf{6 A}$ and $7 \mathrm{~A}$. 

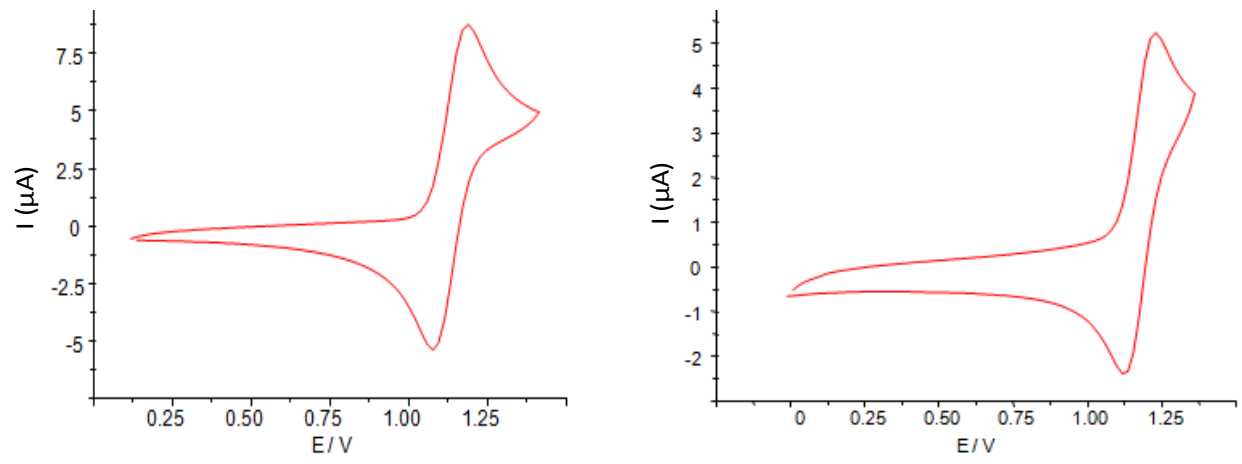

Figure 1.9 CV plots of $1 \mathrm{C}$ (left) and $2 \mathrm{C}$ (right). Measurements performed on $1 \mathrm{mM}$ analyte in dichloromethane, $0.1 \mathrm{M} \mathrm{TBAPF}{ }_{6}$ electrolyte, calibrated to $\mathrm{Fc}^{+} / \mathrm{Fc}\left(\mathrm{E}_{1 / 2}=0.446 \mathrm{~V} v\right.$ s. SCE $)$ at 100 $\mathrm{mV} \mathrm{s-}{ }^{1}$

The cyclic voltagram of the heterodimetallic complex 4A displays two independent redox processes referred to the $\mathrm{Ru}$ and $\mathrm{Ir}$ centers, at 1.29 and $1.41 \mathrm{~V}$, respectively (Figure 1.10, left). The DPV analysis allowed detecting two peaks separated by $120 \mathrm{mV}$ (Figure 1.10, right). Plenio and co-workers proposed $\mathrm{CV}$ studies as a valuable means to study the electronic properties of NHC ligands. ${ }^{42,43}$ These values confirm that the triazole-diylidene ligand, in $\mathbf{4 A}$, has a lower electrondonating character than the monocarbenes in $\mathbf{1 C}$ and $2 \mathrm{C}$.
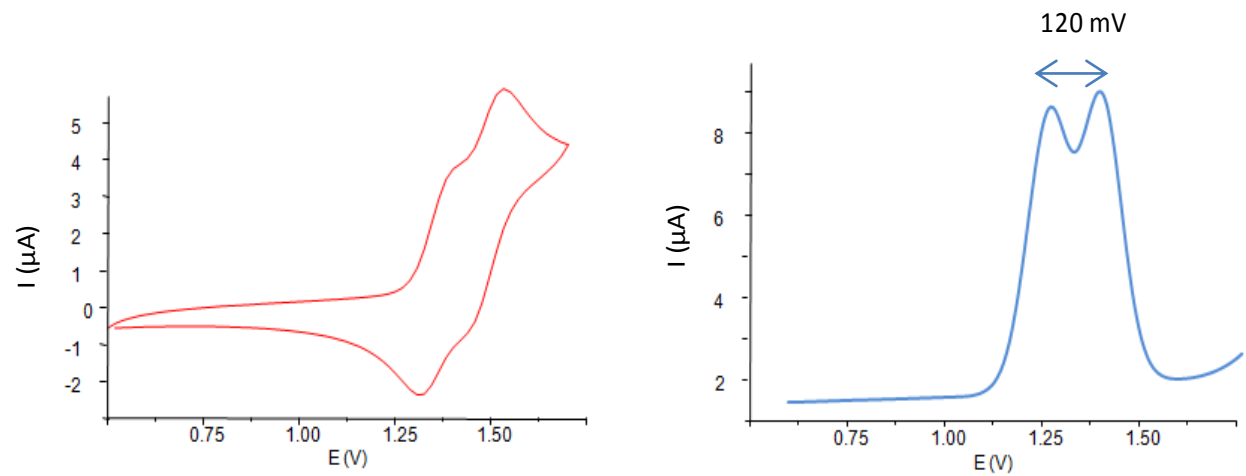

Figure 1.10 CV and DPV plots of 4A. Measurements performed on $1 \mathrm{mM}$ analyte in dichloromethane ${ }_{2}, 0.1 \mathrm{M} \mathrm{TBAPF}{ }_{6}$ electrolyte, calibrated to $\mathrm{Fc}^{+} / \mathrm{Fc}\left(\mathrm{E}_{1 / 2}=0.446 \mathrm{~V} v s\right.$. SCE $)$ at 100 $\mathrm{mV} \mathrm{s}^{-1}$ 
Additionally, taking into account that the redox values corresponding to the monometallic complexes $1 \mathrm{C}$ and $2 \mathrm{C}$ are very similar $(\Delta \mathrm{E}=40 \mathrm{mV})$, the separation of the two redox processes by $120 \mathrm{mV}$ in $\mathbf{4 A}$ is probably due to the influence of the $\mathrm{Ru}$ center over the oxidation of the Ir center, suggesting some electronic coupling between the two metal centers, albeit weak.

The CV measurements of the diruthenium complex 7A (Figure 1.11, left), indicate that the complex undergoes a reversible oxidation at $\mathrm{E}_{1 / 2}=1.40 \mathrm{mV}$. The DPV analysis reveals that the oxidation wave is significantly broader than that of the monometallic $\mathrm{Ru}$ complex 1C (Figure 1.11, right).
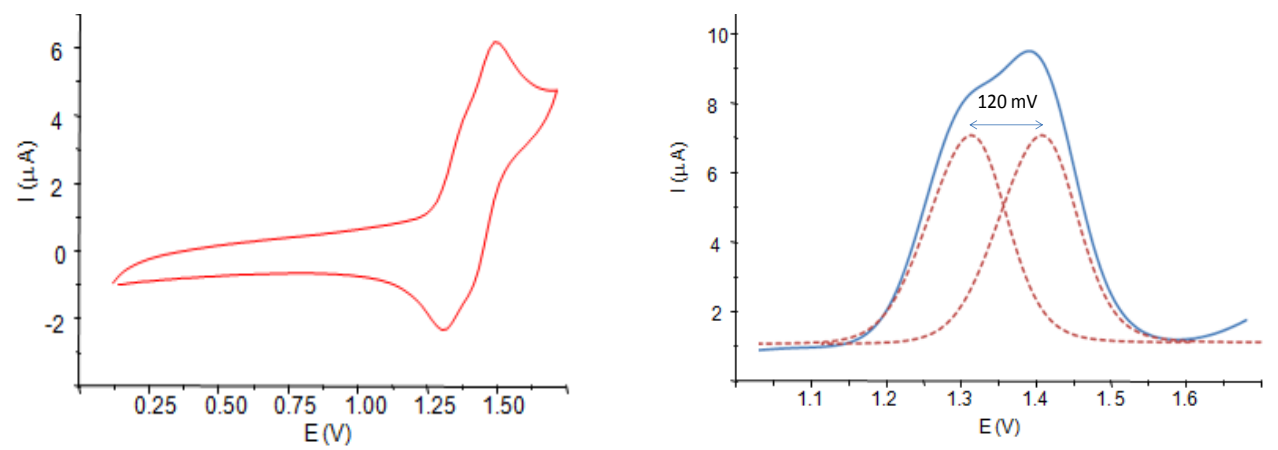

Figure 1.11 CV and DPV plots of 7A. Measurements performed on $1 \mathrm{mM}$ analyte in dichloromethane, $0.1 \mathrm{M} \mathrm{TBAPF} 6$ electrolyte, calibrated to $\mathrm{Fc}^{+} / \mathrm{Fc}\left(\mathrm{E}_{1 / 2}=0.446 \mathrm{~V} v s\right.$. SCE) at 100 $\mathrm{mV} \mathrm{s}^{-1}$. The convoluted curves were obtained from adding two potential-shifted and weighed signals of the monometallic complex $\mathbf{1 C}$

Taking the DPV-signal of the monometallic complex $\mathbf{1 C}$ as a reference, the deconvolution of the DPV curve for 7A yielded two oxidations bands separated by 120 $\mathrm{mV}$. This separation corresponds to a class II system, according the Robin and Day classification, ${ }^{44}$ and is consistent with the presence of two mutually dependent redox sites. Although this interaction can be classified as weak, it has to be taken into account that it lies among the highest electronic coupling measured among all other Janus-type bis-NHC related ligands (couplings ranging from $60-80 \mathrm{mV}$ ). ${ }^{38,39,45,46}$

The first oxidation peak in 7A appears at $1.34 \mathrm{~V}$, at a value slightly higher than the same process referred to the $\mathrm{Ru}$ center in $\mathbf{4 A}(1.29 \mathrm{~V})$. This moderate increment of the 
oxidation potential suggests that the ' $\mathrm{Ru}(p$-cymene $) \mathrm{Cl}_{2}$ ' fragment reduces the electrondonating character of the part of the ligand bound to the ' $\mathrm{Ru}(p$-cymene $) \mathrm{Cl}_{2}$ ', compared to the ' $\mathrm{IrCp}^{*} \mathrm{Cl}_{2}$ ' fragment, albeit this effect is small. Unfortunately, the di-iridium complex 6A, gave an irreversible CV wave and a non-defined DPV curve, so we could not obtain suitable data for analysis.

\subsection{Catalytic activity: multimetallic processes}

Our main interest in the design of our complexes is their use as multifunctional catalysts. In theory, a heterometallic catalyst may be appropriate for the design of many multimetallic processes, by simply combining the individual catalytic capabilities of each metal site, thus providing a great advantage over traditional monometallic catalysts. Despite the simplicity of this idea, the practical approach to the design of efficient heterometallic complexes for multimetallic processes suffers from some important complications, derived from:

- The compatibility of the two (or more) catalytic active sites with the reaction conditions needed for each individual catalytic process.

- The possibility of mutual deactivation of the two metals.

- The lack of stability of the ligand needed to maintain the heterometallic nature of the catalyst during the whole catalytic process.

- The possibility that the two metals display similar reactivity patterns, and therefore are able to facilitate either of the cycles comprised in the overall tandem reaction.

As mentioned earlier in this chapter, the synthesis of heterodimetallic complexes has allowed the QOMCAT group the design of tandem catalytic processes by combining the activity of two metal centers in a sequence of catalytic reactions, performed in one-pot manner. In the following sections of this chapter, new processes for which we used our bimetallic complexes will be described. At this stage, it is worth pointing out that multimetallic catalysis is formally based on the combined action of different metals in a chemical transformation and therefore, it 
may include either tandem or dual processes. Each metal center may be specifically responsible for elementary steps/transformations contributing to the overall transformation under investigation. ${ }^{47}$

For the design of these processes, typically, what we first did is to search catalytic reactions for each of the metals comprised in the heterodimetallic complex, and the reactions that we finally tested were those that gave more apriori chances of compatibility in terms of solvents, temperatures, bases or additives. By doing so, we finally decided to study the following reactions, which were specifically combined for the use of each of our new three heterodimetallic complexes:

a) $\operatorname{Ir}(\mathrm{III}) / \mathrm{Au}(\mathrm{I})$ complex 3A: Reduction of nitroarenes by transfer hydrogenation using primary alcohols

b) $\operatorname{Ir}(\mathrm{III}) / \mathrm{Ru}(\mathrm{II})$ complex 4A: Chelation assisted $\mathrm{C}-\mathrm{H}$ activation of arylpyridines and Oppenauer oxidation

c) $\mathrm{Ru}(\mathrm{II}) / \mathrm{Pd}(\mathrm{II})$ complex 5A: Carbon-fluor activation via hydrodefluorination

\subsubsection{Ir(III)/Au(I) complex 3A: Reduction of nitroarenes by transfer hydrogenation using primary alcohols}

The development of an efficient and general method for the synthesis of imines is highly desirable because of its potential versatility and wide scope. ${ }^{48}$ Imines are considered as an important class of carbon-nitrogen compounds. They are versatile synthetic intermediates for the preparation of nitrogen-containing compounds, many of which are useful building blocks for biological and pharmaceutical applications, and are widely used as electrophiles in organic transformations. ${ }^{49,50}$ For all these reasons, the production of imines from readily available alcohols and amines is a very convenient procedure, and it is currently the aim of many investigations.

Gold and iridium metal centers have been widely used in the reduction of nitroarenes ${ }^{28,51,52}$ and in the oxidation of alcohols. ${ }^{28,53,54}$ The use of two transition metals to simultaneously catalyze a reaction can afford an interesting opportunity to achieve new 
reactivity and selectivity patterns when compared to the use of a single-metal catalytic system. In this work, we were especially interested in testing whether there is any influence of using two metals with distinct reactivity patterns (Ir and $\mathrm{Au}$ in $\mathbf{3 A}$ ) on the distribution of reaction products, rather than designing a new tandem process by the combination of reactions typically catalyzed by $\operatorname{Ir}(\mathrm{III})$ and $\mathrm{Au}(\mathrm{I})$.

The reduction of nitroarenes to anilines is a mechanistically complex process and many intermediates are involved. A generally accepted mechanistic pathway is described in Scheme 1.18, in which the aniline can be obtained via the Direct or the Condensation route.

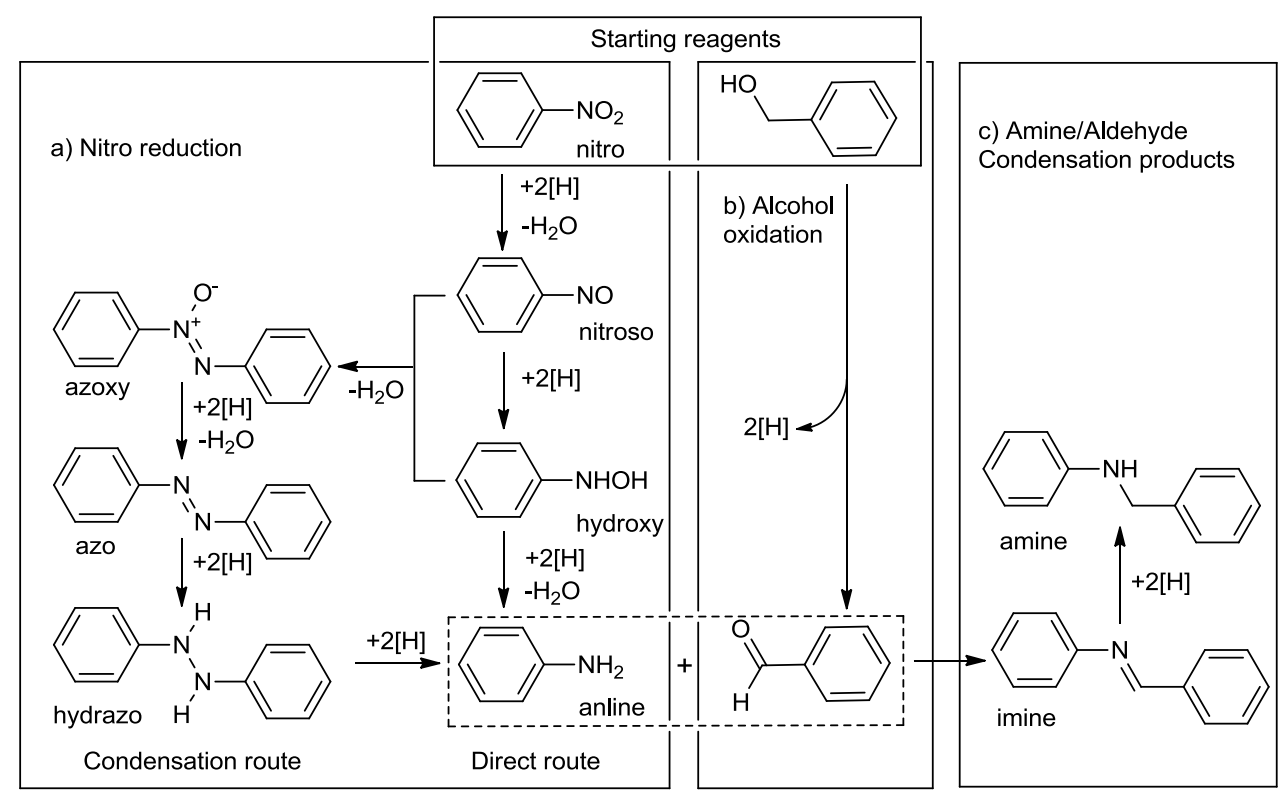

Scheme 1.18 Different mechanistic pathways for the nitroarenes reduction

The Direct route implies the sequential hydrogenation/dehydration process via nitroso and hydroxy intermediates. On the other hand, the Condensation route implies the condensation between nitroso and hydroxy products, leading to azoxy compounds. The latter are hydrogenated/dehydrated to afford azo compounds. Finally, by subsequent hydrogenation, hydrazo and amine are obtained. 
Taking all this into account, we decided to test our $\operatorname{Ir}(\mathrm{III}) / \mathrm{Au}(\mathrm{I})$ complex $\mathbf{3 A}$, in this important transformation in order to assess the catalytic outcomes given by 3A, and the homodimetallic versions, $\mathbf{1 B}$ and $\mathbf{6} \mathbf{A}^{23}$ (Scheme 1.19).

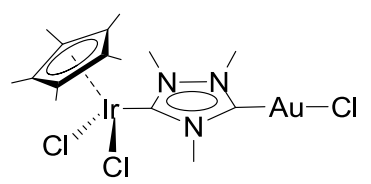

$3 \mathrm{~A}$

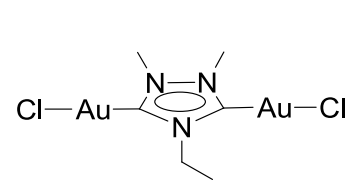

$1 B$

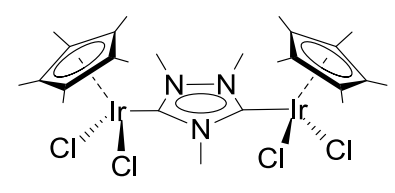

6A

Scheme 1.19 Bimetallic complexes tested

The catalytic experiments were carried out with a catalyst loading of $1 \mathrm{~mol} \%$ (based on the amount of each metal), in the presence of $\mathrm{Cs}_{2} \mathrm{CO}_{3}$, and benzyl alcohol, at $100^{\circ} \mathrm{C}$ in a capped vessel under aerobic conditions. The reactions produced different products depending on the catalysts used. The results shown in Table 1.1 indicate that the iridiumcontaining complexes, $\mathbf{3 A}$ and $\mathbf{6 A}$, provided good to excellent yields to the formation of the imine. On the other hand, the digold catalyst $\mathbf{1 B}$, afforded moderate to good yields to the hydroxy and azo compounds (entries 1 and 4).

The differences in activity are rather significant in the case of $p$-substituted nitrobenzene (entries 7-12), where the digold complex 1B results inactive (entries 7 and 10) while the iridium-containing complexes afforded selectively the imine in moderate to good yields (entries 8, 9, 11 and 12). At this stage, it is worth pointing out that the overall reaction implies the concomitant formation of water, thus the reaction yields were improved by adding molecular sieves to the reaction media (compare entries 4-6 with 1-3).

The most important feature that we can observe in this catalytic system is the better catalytic outcomes displayed by the Ir-Au heterodimetallic complex 3A, regardless the substrate used (see entries 2, 5, 8 and 11). Interestingly, the heterodimetallic complex $\mathbf{3 A}$ is in all cases more effective than the only-iridium complex $\mathbf{6 A}$, either in terms of yield or selectivity, and using the same catalyst loading. This result provides a clear example of a dual catalytic system in which both, gold and iridium centers, are cooperatively contributing to the overall transformation. 
Table 1.1 Catalyst screening for the reaction of nitrobenzene and benzyl alcohol

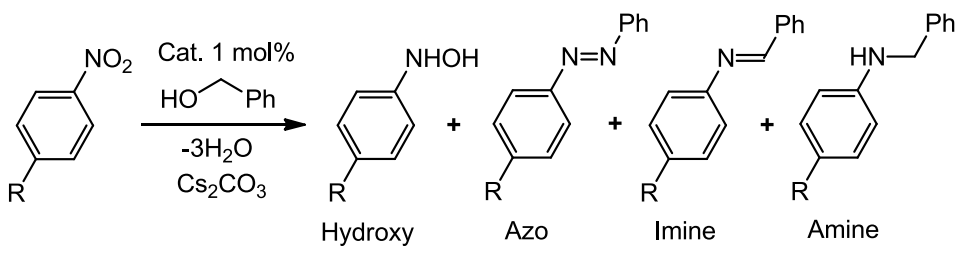

\begin{tabular}{|c|c|c|c|c|c|c|c|}
\hline Entry & Catalyst & $\mathbf{R}$ & $\mathbf{t}(\mathbf{h})$ & $\operatorname{Hydroxy}(\%)^{\mathrm{a}}$ & $\operatorname{Azo}(\%)^{a}$ & $\operatorname{Imine}(\%)^{\mathrm{a}}$ & $\operatorname{Amine}(\%)^{\mathrm{a}}$ \\
\hline 1 & 1B & $\mathrm{H}$ & 22 & 55 & 17 & 4 & -- \\
\hline 2 & 3A & $\mathrm{H}$ & 22 & -- & -- & 96 & 2 \\
\hline 3 & $6 A$ & $\mathrm{H}$ & 22 & -- & -- & 60 & -- \\
\hline $4^{\mathrm{b}}$ & 1B & $\mathrm{H}$ & 15 & 81 & 9 & -- & -- \\
\hline $5^{\mathrm{b}}$ & $3 \mathbf{A}$ & $\mathrm{H}$ & 15 & -- & -- & 99 & -- \\
\hline $6^{\mathrm{b}}$ & 6A & $\mathrm{H}$ & 15 & -- & -- & 86 & 12 \\
\hline 7 & 1B & $\mathrm{Me}$ & 15 & -- & -- & -- & -- \\
\hline 8 & $\mathbf{3 A}$ & $\mathrm{Me}$ & 15 & -- & -- & 93 & -- \\
\hline 9 & $6 A$ & $\mathrm{Me}$ & 15 & -- & -- & 48 & -- \\
\hline 10 & 1B & $\mathrm{OMe}$ & 15 & -- & -- & -- & -- \\
\hline 11 & $\mathbf{3 A}$ & $\mathrm{OMe}$ & 15 & -- & -- & 41 & -- \\
\hline 12 & $6 A$ & $\mathrm{OMe}$ & 15 & -- & -- & 7 & -- \\
\hline $13^{\mathrm{b}}$ & $1 B+6 A$ & $\mathrm{H}$ & 15 & -- & -- & 81 & 9 \\
\hline
\end{tabular}

Reaction conditions: Catalyst ( $1 \mathrm{~mol} \%$ based on metal), nitrobenzene $(0.3 \mathrm{mmol})$, benzyl alcohol (10 mmol) used as solvent and reagent, $\mathrm{Cs}_{2} \mathrm{CO}_{3}(0.3 \mathrm{mmol})$, anisole as internal standard $(0.3 \mathrm{mmol})$. The solution was heated at $100^{\circ} \mathrm{C}$ under aerobic conditions. [a] Yields determined by GC. [b] Using molecular sieves 4 Å.

In order to evaluate if there is any benefits in having the two metal centers bound to a single-frame ligand, we studied the catalytic activity of the mixture of the related homodimetallic complexes (1B and $\mathbf{6 A})$. For the reaction carried out with this mixture of compounds, we observed that the activity was lower than that shown by the 
heterodimetallic complex 3A (compare entries 5 and 13). Despite the slight difference in activity, it is interesting to point out that this is a reproducible pattern that can be observed in other series of triazole-di-ylidene-based heterodimetallic complexes, where the heterodimetallic complexes always afforded better catalytic outcomes than the related mixtures of homodimetallic ones. ${ }^{23,24,27,28}$

Regarding the mechanistic insights, from the results of Table 1.1 it seems that iridiumcontaining catalysts are following the Direct route (Scheme 1.18), as we could not detect any of the possible intermediates. In the case of the digold complex 1B, we do not have any concluding evidences for postulating any mechanisms, because the reaction is interrupted at the hydroxyl and azo intermediates without formation of the imine.

In order to shed some light on this point, we decided to test the catalytic activity starting from the azoxybenzene, which is the condensation intermediate (Scheme 1.20). In this catalytic experiment, complexes $\mathbf{3 A}$ and $\mathbf{6 A}$ yielded the same product; although the activities were different (6A was more active than $\mathbf{3 A})$. The reactions were stopped at azobenzene intermediate and did not yield any traces of imine. This result suggests that iridium-containing complexes are likely following the Direct route.

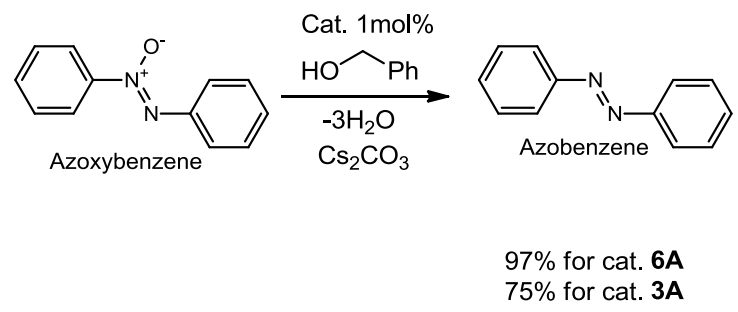

Scheme 1.20 Catalytic activity of $6 \mathrm{~A}$ and $3 \mathrm{~A}$ starting from azoxybenzene intermediate

\subsection{2. $\operatorname{Ir}(\mathrm{III}) / \mathrm{Ru}(\mathrm{II}) \quad$ complex $4 \mathrm{~A}$ : Chelation-assisted $\mathrm{C}-\mathrm{H}$ activation of arylpyridines and Oppenauer oxidation of alcohols}

Chelation-assisted arylation of arenes (Scheme 1.21) consists of a C-H activation/C-C bond formation process, in which the presence of a heteroatom in the substrate acts as a directing group allowing the approach of the metal to a specific $\mathrm{C}-\mathrm{H}$ bond. 


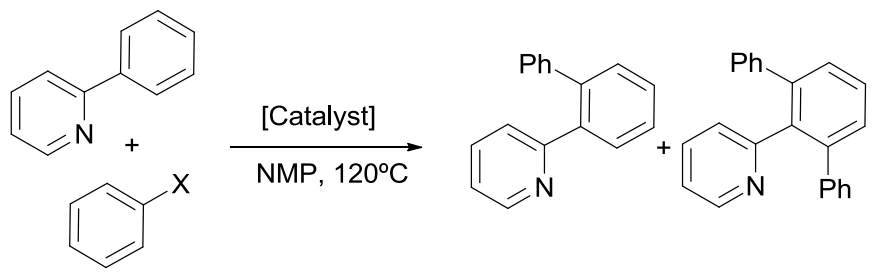

Scheme 1.21 Chelation-assisted arylation of arenes: Arylation of 2-phenylpyridine with aryl

halides

Since the pioneer work by $\mathrm{Oi}$ and Inoue,${ }^{55}$ many efforts have been made in order to develop efficient catalysts for this valuable transformation, as exemplified by the publication of a number of interesting review articles by Ackermann, ${ }^{56,57}$ Bruneau and Dixneuf. ${ }^{58}$ The QOMCAT group also contributed to the field with the description of a series of $\left[\mathrm{RuCl}_{2}\right.$ (arene)NHC], which were used in the arylation of a wide set of arylpyridines. ${ }^{59}$

With the aim to design new tandem catalytic processes, using our heterodimetallic $\operatorname{Ir}(\mathrm{III}) / \mathrm{Ru}(\mathrm{II})$ complex $\mathbf{4 A}$, we decided to combine the chelation-assisted arylation of arylpyridines with aryl halides and the Oppenauer oxidation of alcohols (Scheme 1.22). Despite this tandem transformation presumably can be catalyzed by only one metal $(\mathrm{Ru})$, we wanted to explore the effect of the two metal centers in the bimetallic complexes on the final outcome of this overall process.

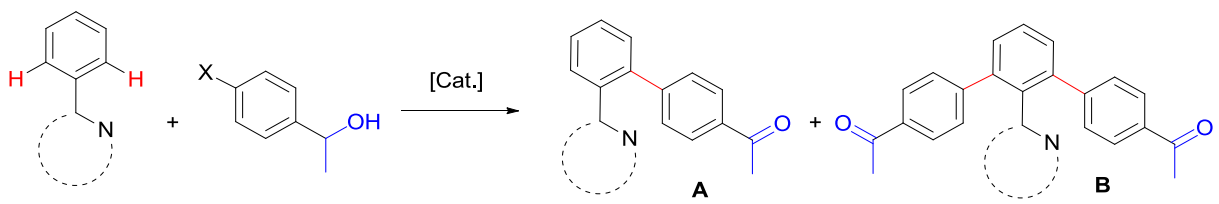

Scheme 1.22 Arylation of arylpyridines and Oppenauer oxidation in tandem reaction

For comparative purposes, and in order to evaluate the effect of each metal, we also used the homodimetallic complexes $\mathbf{6 A}$ and $\mathbf{7 A}$, as well as the monometalic ruthenium complex 1C. The latter is an effective catalyst for the arylation of a wide set of substantially different pyridines ${ }^{59}$ (Scheme 1.23 ). 

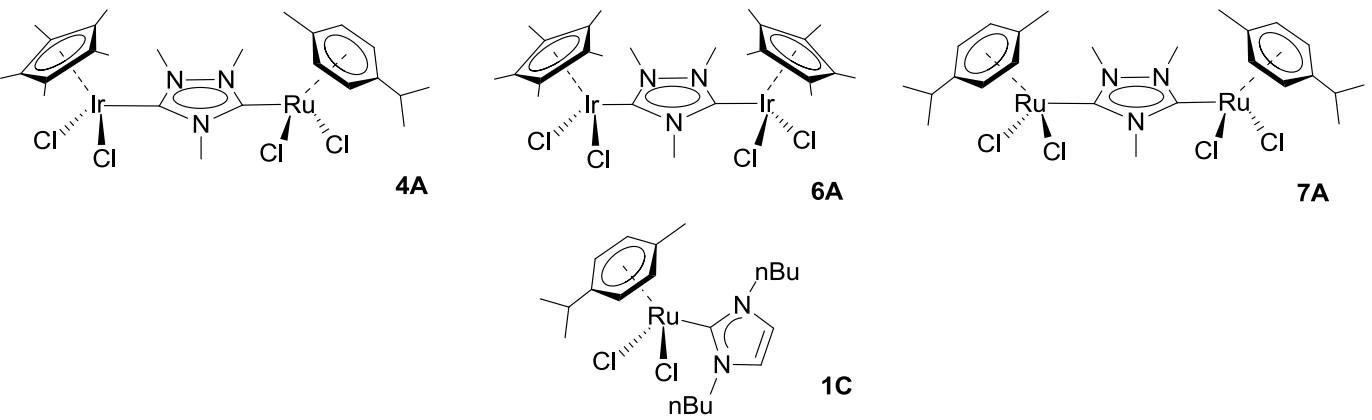

Scheme 1.23 Complexes tested in the tandem chelation-assisted arylation/Oppenauer oxidation

We first studied separately each individual step of the reaction. We began by studying the arylation of 2-phenyl-pyridine with chlorobenzene. The reactions were carried out at $120^{\circ} \mathrm{C}$ in $\mathrm{N}$-methyl-2-pyrrolidone (NMP) using a catalyst loading of $5 \mathrm{~mol} \%$, in the presence of a mixture of $\mathrm{KOAc}$ and $\mathrm{K}_{2} \mathrm{CO}_{3}$.

The results are summarized in Table 1.2. In general, it can be observed that the diruthenium complex 7A proved to be the most active catalyst, both in terms of conversion and selectivity, toward the bisarylated product (entry 2). Reduction of the catalyst loading of 7A to $2.5 \mathrm{~mol} \%$ (entry $4,5 \mathrm{~mol} \%$ based on metal) lowers the yields and selectivity, and the outcomes are identical to those shown by the monometallic catalyst 1C. This is actually logical if we take into account that in both cases the catalyst loading based on metal is the same (5 mol\%).

Interestingly, under these reaction conditions, catalyst $\mathbf{7 A}$ is far more effective than the Ir-Ru catalyst 4A (compare entries 3 and 4), despite the fact that the reaction was carried out with the same concentration of $\mathrm{Ru}$. This better catalytic outcome is likely due to the presence of two ruthenium centers in $\mathbf{7 A}$ compared to $\mathbf{4 A}$, which only contains one. This result not only indicates that the iridium fragment is formally inactive in this reaction, but also that the two metals in $\mathbf{7 A}$ behave as active catalytic centers, meaning that we can discard a situation in which one ruthenium is acting as a spectator center while the other is catalyzing the process. Besides, taking into account the inactivity of the iridium fragment, this result also suggests a cooperative behavior between the two ruthenium centers. 
Table 1.2 Chelation-assisted arylation of 2-phenyl-pyridine with chlorobenzene

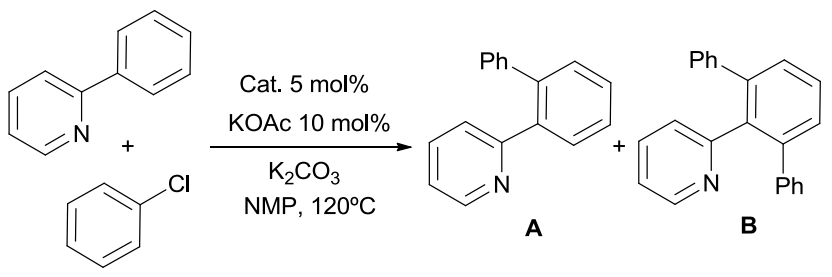

\begin{tabular}{ccccc}
\hline Entry & Catalyst & Time $(\mathbf{h})$ & $\mathbf{A}(\boldsymbol{\%})^{\mathbf{a}}$ & $\mathbf{B}(\boldsymbol{\%})^{\mathbf{a}}$ \\
\hline 1 & $\mathbf{1 C}$ & 12 & 25 & 74 \\
2 & $\mathbf{7 A}$ & 5 & -- & 99 \\
3 & $\mathbf{4 A}$ & 12 & 56 & 18 \\
4 & $\mathbf{7 A}^{\mathrm{b}}$ & 12 & 25 & 74 \\
\hline
\end{tabular}

Reaction conditions: KOAc (10 mol\%), catalyst (5 mol\%), $2 \mathrm{~mL} \mathrm{NMP} \mathrm{as} \mathrm{solvent,} \mathrm{room} \mathrm{temperature} \mathrm{for} 1 \mathrm{~h}$, then 2-phenylpyridine $(0.5 \mathrm{mmol})$, chlorobenzene $(1.25 \mathrm{mmol}), \mathrm{K}_{2} \mathrm{CO}_{3}(1.5 \mathrm{mmol}), 120^{\circ} \mathrm{C}$. [a] Yields and ratios determined by GC (internal standard: anisole) and by ${ }^{1} \mathrm{H}$ NMR. [b] 5 mol\% of metal.

In the study of the second process, the Oppenauer oxidation of alcohols, all complexes were tested under identical conditions. The reactions were performed using a catalyst loading of $1 \mathrm{~mol} \%$, 1-phenylethanol as model substrate, in the presence of $\mathrm{K}_{2} \mathrm{CO}_{3}$, at $100^{\circ} \mathrm{C}$ in acetone.

Table 1.3 Oppenauer oxidation of 1-phenylethanol

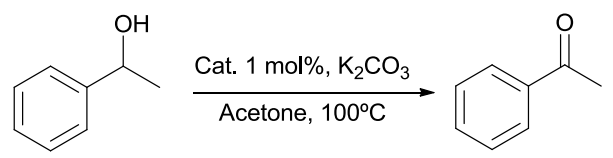

\begin{tabular}{cccc}
\hline Entry & Catalyst & Time(h) & Yield(\%) $^{\mathbf{a}}$ \\
\hline 1 & $\mathbf{1 C}$ & 1 & 99 \\
2 & $\mathbf{7 A}$ & 9 & 76 \\
3 & $\mathbf{4 A}$ & 9 & 88 \\
4 & $\mathbf{6 A}^{\mathrm{b}}$ & 9 & 94 \\
5 & $\mathbf{7 A}^{\mathrm{b}}$ & 9 & 37 \\
\hline
\end{tabular}

Reaction conditions: catalyst $(0.01 \mathrm{mmol}), 1 \mathrm{mmol}$ phenyl ethanol, $1.5 \mathrm{mmol} \mathrm{K}_{2} \mathrm{CO}_{3}, 2 \mathrm{~mL}$ of acetone as solvent, $100^{\circ} \mathrm{C}$. [a] Yields and ratios determined by GC (internal standard: anisole). [b] $1 \mathrm{~mol} \%$ of metal. 
Table 1.3 shows that the monometallic ruthenium complex $\mathbf{1 C}$ gave the best catalytic outcomes, affording quantitative yields in $1 \mathrm{~h}$ of reaction (entry 1). The rest of the catalysts tested showed lower catalytic activity, both in terms of conversions and reaction times. As expected, all the NHC-based catalysts were active in the catalytic process, with 6A being the best among all the bimetallic complexes (entry 4).

Once we proved the activity of all complexes in both separate individual reactions, the arylation of arylpyridines and the oxidation of alcohols with acetone, we combined both processes into a one-pot tandem reaction. We tested all the bimetallic complexes, as well as the monoruthenium catalyst $\mathbf{1 C}$, in the arylation of 2-phenylpyridine with 1-(4bromophenyl)ethanol) (Scheme 1.24). The reaction conditions used were the same as the single-arylation of 2-phenylpyridine, but using a mixture of NMP and acetone (1:1) as solvent, in order to promote the oxidation of the alcohol.

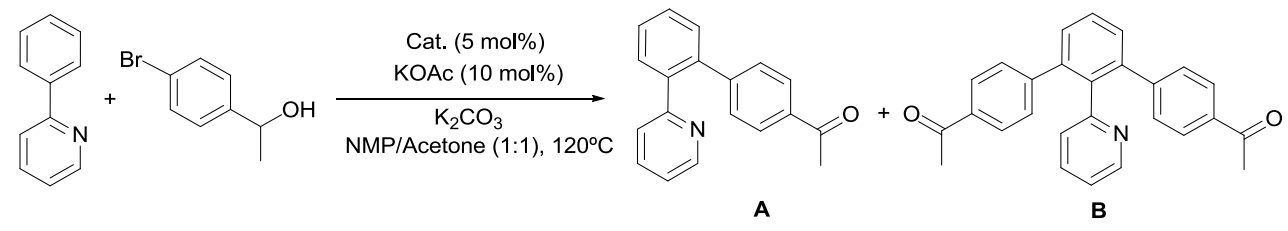

Scheme 1.24 Arylation of 2-phenylpyridine with 1-(4-bromophenyl)ethanol)

In our preliminary studies, the monitoring of this reaction allowed us to observe that only the starting substrates and the final product are detected along the reaction time course, indicating that once the oxidation of the alcohol had taken place, the arylation step is fast. In a parallel experiment, we decided to perform the reaction in the absence of acetone, so that we avoided the oxidation of the alcohol. Under these reaction conditions, the arylation of 1-(4-bromophenyl)ethanol did not occur, therefore indicating that the arylbromide is activated by the oxidation of the alcohol functionality to the ketone. In this regard, we can consider the catalytic system as a multistep cascade process in which the first reaction taking place is the Opennauer oxidation of the alcohol yielding the corresponding ketone, and the subsequent arylation, which is a fast step as indicated by the absence of any detectable non-arylated ketone intermediate. 
The results in Table 1.4 show that the homodimetallic Ru complex 7A was an excellent catalyst, and performed better than the Ir-Ru heterodimetallic complex $\mathbf{4 A}$, under the same reaction conditions (compare entries 1 and 2). However, the best catalyst, from all those that we used, was compound $\mathbf{1 C}$, when a catalyst loading of $5 \mathrm{~mol} \%$ (based on metal) was used (compare entries 3 and 4). The best outcome shown by catalyst $\mathbf{1 C}$ may be understood if we take into account that the oxidation of the alcohol is the rate determining step of the process, a step in which $\mathbf{1 C}$ is more active, as seen from the results shown in Table 1.3. As expected, the di-iridium catalyst $6 \mathbf{A}$ was inactive in the overall process, because it could not facilitate the second step of the reaction (arylation of the pyridine) (entry 5).

Table 1.4 Catalyst screening for the arylation of 2-phenylpyridine with 1-(4bromophenyl)ethanol
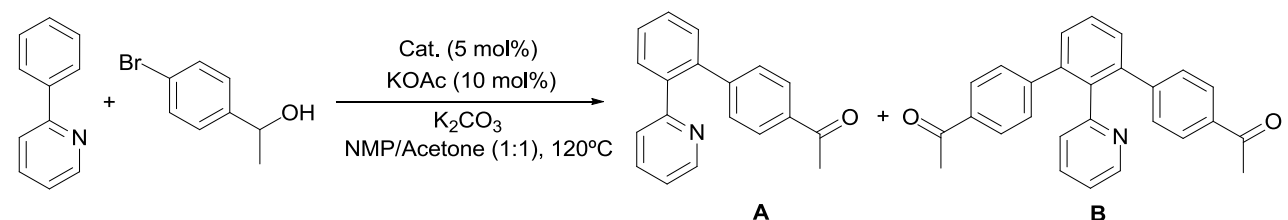

\begin{tabular}{ccccc}
\hline Entry & Catalyst & Time(h) & $\mathbf{A}(\boldsymbol{\%})^{\mathbf{a}}$ & $\mathbf{B}(\boldsymbol{\%})^{\mathbf{a}}$ \\
\hline 1 & $\mathbf{4 A}$ & 9 & -- & 76 \\
2 & $\mathbf{7 A}$ & 9 & -- & 98 \\
3 & $\mathbf{7 A}^{[\mathrm{c}]}$ & 9 & -- & 72 \\
4 & $\mathbf{1 C}$ & 9 & -- & 90 \\
5 & $\mathbf{6 A}$ & 9 & 0 & 0 \\
\hline
\end{tabular}

Reaction conditions: KOAc (10 mol\%), catalyst (5 mol\%), $2 \mathrm{~mL}$ NMP/Acetone (1:1) as solvent, room temperature for $1 \mathrm{~h}$, then arylpyridine $(0.5 \mathrm{mmol}), \mathrm{Ar}-\mathrm{X}(1.25 \mathrm{mmol}), \mathrm{K}_{2} \mathrm{CO}_{3}(2.5 \mathrm{mmol}), 120^{\circ} \mathrm{C}$. [a] Yields and ratios determined by GC (internal standard: anisole) and by ${ }^{1} \mathrm{H}$ NMR. [b] $5 \mathrm{~mol} \%$ of metal

Once we confirmed that catalyst 1C was the most active one in the overall tandem process, we decided to study its performance using different substrates. The results are summarized in Table 1.5. 
Table 1.5 Tandem oxidation of 1-(4-halophenyl)ethanol and arylation of arylpyridine

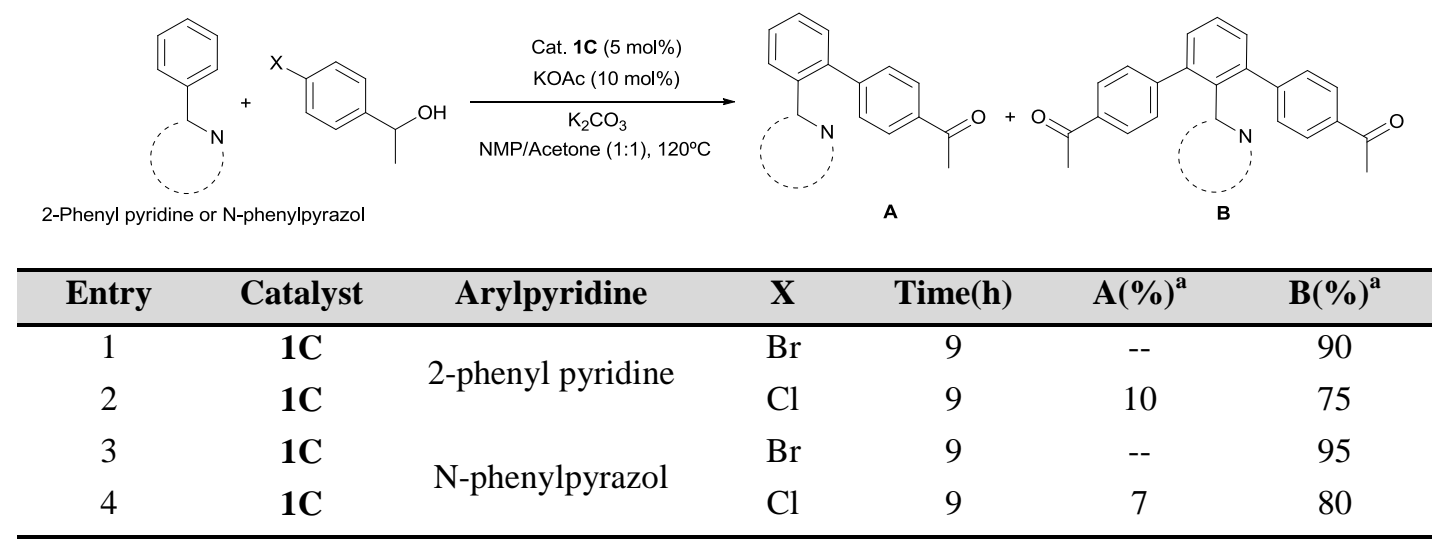

Reaction conditions: KOAc (10 mol\%), catalyst (5 mol\%), $2 \mathrm{~mL} \mathrm{NMP/Acetone} \mathrm{(1:1)} \mathrm{as} \mathrm{solvent,} \mathrm{room}$ temperature for $1 \mathrm{~h}$, then arylpyridine $(0.5 \mathrm{mmol})$, $\mathrm{Ar}-\mathrm{X}(1.25 \mathrm{mmol}), \mathrm{K}_{2} \mathrm{CO}_{3}(2.5 \mathrm{mmol}), 120^{\circ} \mathrm{C}$. [a] Yields and ratios determined by GC (internal standard: anisole) and by ${ }^{1} \mathrm{H}$ NMR.

As shown in Table 1.5, catalyst $\mathbf{1 C}$ was active in the bisarylation of 2-phenylpyridine and N-phenyl-pyrazol, both when 1-(4-chlorophenyl)ethanol and 1-(4-bromophenyl)ethanol were used as arylating agents, therefore proving its versatility.

\subsection{3. $\quad \mathrm{Ru}(\mathrm{II}) / \mathrm{Pd}(\mathrm{II})$ complex 5A: Carbon-fluor bond activation via hydrodefluorination}

The importance of organofluorine compounds in all aspects of the chemical industry is striking, but the fact that 'as many as $30-40 \%$ of agrochemicals and $25 \%$ of pharmaceuticals on the market are estimated to contain fluorine, including three of the top five drugs sold in $2013,{ }^{60}$ helps to explain why the demand of fluorinated molecules continues growing at tremendous speed. Since the properties of organic molecules, including stability, lipophilicity and bioavailability, can be dramatically altered by the introduction of one, or a small number of fluorine substituents, ${ }^{61}$ a range of diverse synthetic strategies have been developed for the introduction of fluorine into biologically relevant molecules. The reverse reaction, the selective cleavage and functionalization of a C-F bond, can also be considered as a pathway to the synthesis of fluororoganic molecules, ${ }^{62}$ for example, by transforming polyfluorinated species into partially 
fluorinated analogues. As simple perfluorinated substrates are readily available on an industrial scale, their use as synthons for the formation of lower fluorine-containing products is a highly attractive proposition. The study of processes allowing the substitution of fluorine by other functional groups represents not only an intellectual challenge, but may also provide knowledge to more effectively approach the reverse reaction, the fluorination of organic molecules.

Fluorine forms the strongest single $\sigma$-bond to carbon. In addition to the thermodynamic stability of fluorocarbons, kinetic issues also explain the inertness of $\mathrm{C}-\mathrm{F}$ bonds, thus making C-F bonds among the most unreactive functionalities in chemistry. ${ }^{63,64}$ The chemical inertness of the C-F bond makes the chemistry of fluorocarbons a very attractive field of research in different areas of chemistry, such as materials science, catalysis, medicine, agrochemistry and biochemistry. ${ }^{62,65}$ Also, the stability imparted by the C-F bonds leads to the long atmospheric lifetimes of chlorofluorocarbons (CFCs), considered as 'supergreenhouse gases'. ${ }^{66-70}$ Therefore, there is a growing demand for the development of new routes to transform efficiently these bonds, because while interests in these molecules are high, the number of available methods for their efficient activation is limited.

Hydrodefluorination (HDF) (Scheme 1.25) is the simplest C-F activation process, but it features a surprising mechanistic diversity. ${ }^{62,65,71,72}$ The reaction formally implies the activation of a carbon-fluorine bond followed by the introduction of hydrogen to form the final hydrodefluorinated molecule.

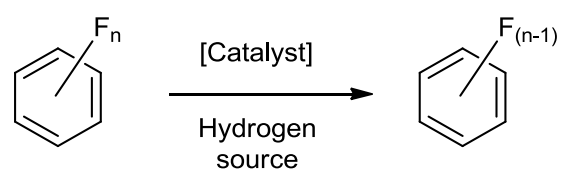

Scheme 1.25 HDF of fluoroarenes

Milstein and co-workers were the first to report an homogeneous catalytic system based on a $\mathrm{Rh}$ (I) complex for the hydrodefluorination of fluoroarenes using silanes as hydrogen source. ${ }^{73}$ The driving force of the reaction is the formation of the stable Si-F bond. Since this seminal work, many efforts are currently being made to generate efficient catalytic 
systems for this challenging transformation. ${ }^{74}$ Despite the valuable results obtained from a variety of catalytic systems in the activation, in both $\mathrm{Csp}^{2}-\mathrm{F}$ and $\mathrm{Csp}^{3}-\mathrm{F}$ bonds, based on different transition metal complexes, ${ }^{75-82}$ and silylium carborane complexes, ${ }^{63}$ most of them suffer from the disadvantage of using strong reductants such as silanes, aluminum hydrides or high hydrogen pressures in order to form the C-H bond. HDF in milder conditions avoiding strong reductants and high pressures of hydrogen are desirable in order to get a more attractive and environmental benign process. Aiming this goal, recent advances in HDF by transfer hydrogenation methodologies have emerged as convenient alternatives in homogeneously ${ }^{83,84,85}$ and heterogeneously ${ }^{86,87}$ catalyzed processes.

Under this scenario, we thought that our heterodimetallic complexes may play an interesting role in the activation of $\mathrm{C}-\mathrm{F}$ bonds through HDF. In particular, the heterodimetallic $\mathrm{Ru}(\mathrm{II}) / \mathrm{Pd}$ (II) complex $\mathbf{5 A}$ may gather the properties of the palladium fragment to activate $\mathrm{C}-\mathrm{F}$ bonds, together with the properties conferred by the ruthenium fragment to facilitate the introduction of the hydrogen atom by a reductive transfer hydrogenation process. On the basis of this initial hypothesis, the catalytic hydrodefluorination of a series of substrates was tested. The next section describes our results in this regard.

\section{Activation of $\mathrm{C}_{\mathrm{sp} 2}-\mathrm{X}$ bonds}

In a first catalyst screening, we studied the dehalogenation of bromo-, chloro- and fluorobenzene. The reactions were performed in 2-propanol in the presence of $\mathrm{NaO} t \mathrm{Bu}$ at $80^{\circ} \mathrm{C}$, with a catalyst loading of $1 \mathrm{~mol} \%$ (Scheme 1.26). Yields and conversions were determined by gas chromatography using anisole as internal standard.

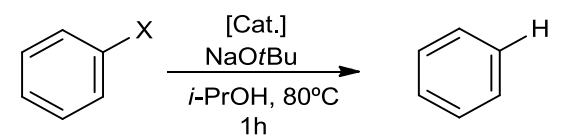

Scheme 1.26 Dehalogenation of phenyl halides

For comparative purposes the homodimetallic $\mathbf{7 A}$ and $\mathbf{8 A}$ complexes were also tested (Scheme 1.27). 


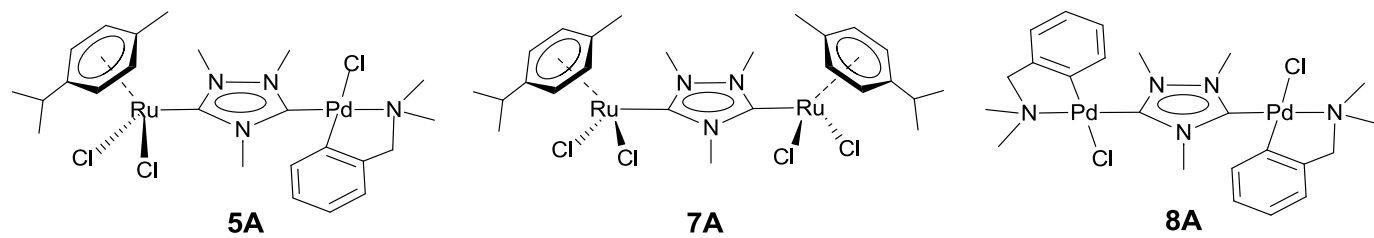

Scheme 1.27 Bimetallic complexes under study

The results shown in Table 1.6 indicate that the diruthenium complex (7A) is completely ineffective in the dehalogenation of bromobenzene, chlorobenzene and fluorobenzene (entries 2, 6, and 10). This is actually logical, if we take into account that the ruthenium fragment is unable to activate the $\mathrm{C}$-X bond under these reaction conditions. The dipalladium complex $(\mathbf{8 A})$ showed moderate to good activity towards the activation of bromobenzene and chlorobenzene (entries 3 and 7), but almost negligible activity in the case of fluorobenzene (entry 11). This result may be interpreted as a consequence of the stability of the palladium-fluoride formed after the cleavage of the C-F bond, thus suggesting that it may be the second step of the overall reaction (the introduction of hydrogen into the aromatic molecule), the one which is failing. Noteworthy, equimolecular mixtures of the ruthenium (7A) and palladium (8A) complexes, afforded moderate yields of benzene coming from the HDF of fluorobenzene (entry 13), therefore suggesting that the two different metals play a role in the overall process. This result is very interesting, because it confirms our initial hypothesis that mixtures of $\mathrm{Pd}$ and $\mathrm{Ru}$ complexes may have benefits in the overall HDF process.

The heterodimetallic complex (5A) showed the best catalytic outcomes in the dehalogenation of all substrates, independently of the starting halide used, affording quantitative conversions to benzene (entries 4, 8 and 12). Finally, the comparison of the results given by the by the mixture of the two homodimetallic complexes $7 \mathbf{A}$ and $\mathbf{8 A}$, and the heterodimetallic complex $\mathbf{5 A}$ in the hydrodefluorination of fluorobenzene, suggests that there must be some type of catalytic cooperativity between the two metals comprised in the bimetallic unit (compare entries 12 and 13). We also confirmed that the dehalogenation without catalyst does not proceed (entries 1,5 and 9), thus discarding that the $\mathrm{C}-\mathrm{X}$ activation via nucleophilic aromatic substitution. ${ }^{88}$ 
Table 1.6 Catalytic dehalogenation of phenyl halides

\begin{tabular}{|c|c|c|c|}
\hline Entry & Catalyst & $\mathbf{X}$ & Yield $(\%)^{\mathrm{a}}$ \\
\hline 1 & - & $\mathrm{Br}$ & 0 \\
\hline 2 & $7 \mathrm{~A}$ & $\mathrm{Br}$ & 0 \\
\hline 3 & $8 \mathbf{A}$ & $\mathrm{Br}$ & 80 \\
\hline 4 & $5 \mathrm{~A}$ & $\mathrm{Br}$ & 100 \\
\hline 5 & - & $\mathrm{Cl}$ & 0 \\
\hline 6 & 7A & $\mathrm{Cl}$ & 0 \\
\hline 7 & $8 A$ & $\mathrm{Cl}$ & 65 \\
\hline 8 & 5A & $\mathrm{Cl}$ & 100 \\
\hline 9 & - & $\mathrm{F}$ & 0 \\
\hline 10 & 7A & $\mathrm{F}$ & 0 \\
\hline 11 & $8 A$ & $\mathrm{~F}$ & 3 \\
\hline $12^{\mathrm{b}}$ & 5A & $\mathrm{F}$ & 100 \\
\hline $13^{\mathrm{c}}$ & $7 A+8 A$ & $\mathrm{~F}$ & 49 \\
\hline
\end{tabular}

Reaction conditions: Fluoroarene $(0.3 \mathrm{mmol}), \mathrm{NaO} t \mathrm{Bu}(0.3 \mathrm{mmol})$, catalyst $(1 \mathrm{~mol} \%), 2 \mathrm{~mL}$ of 2-propanol for 1 hour at $80^{\circ} \mathrm{C}$. [a] Yields determined by GC analysis using anisole as internal standard. [b] The mercurydrop experiment under the same reaction conditions resulted in exactly the same reaction outcome. [c] Catalyst 7A $(0.5 \mathrm{~mol} \%)+$ catalyst $\mathbf{8 A}(0.5 \mathrm{~mol} \%)$.

In order to prove that the reaction is homogeneously catalyzed, we performed the HDF of fluorobenzene using $\mathbf{5} \mathbf{A}$ in the presence of a mercury drop (entry 12), ${ }^{89}$ and observed that the activity of the catalyst did not change. Despite we are aware that the $\mathrm{Hg}(0)$ may occasionally lead to inconclusive results, ${ }^{90}$ we believe that this experiment supports the idea that the process is homogeneous.

In order to widen the scope of the heterodimetallic catalyst in the hydrodeflurination of other aromatic substrates, we decided to extend the study to other fluoroarenes (Table 1.7), such as $p$-fluorotoluene, $p$-fluoroaniline and $p$-fluoroacetophenone. Again, it is observed that the homodimetallic ruthenium and palladium catalysts are completely ineffective in the HDF process (entries 1, 2, 5, 6, 9, 10, 13 and 14). Mixtures of the 
homodimetallic complexes 7A and $\mathbf{8 A}$ afforded low to moderate yields of the final compounds (entries 4, 8, 12 and 16), and the heterodimetallic complex 5A always provided the best catalytic outcomes (entries 3, 7, 11 and 15). These results indicate, once more, that the two metal fragments in $\mathbf{5 A}$ are mutually cooperating, and therefore we have a new example of catalytic cooperativity between two metals that are comprised in a heterodimetallic unit.

Interestingly, under the reaction conditions used, the substrates containing carbonyl groups are quantitatively reduced to the corresponding alcohols, as a consequence of the hydrogen transfer conditions used (entries 15 and 16).

Table 1.7 Hydrodefluorination of fluoroarenes

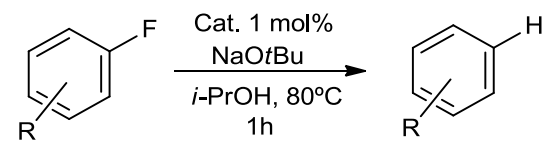

\begin{tabular}{|c|c|c|c|c|}
\hline Entry & Catalyst & Substrate & Product & Yield (\%) \\
\hline 1 & $7 \mathrm{~A}$ & & & 0 \\
\hline 2 & $8 \mathrm{~A}$ & & & 3 \\
\hline 3 & $\mathbf{5 A}$ & & & 100 \\
\hline $4^{\mathrm{a}}$ & $7 \mathrm{~A}+8 \mathrm{~A}$ & & & 49 \\
\hline 5 & $7 \mathrm{~A}$ & & & 0 \\
\hline 6 & $8 A$ & & & 3 \\
\hline 7 & $5 \mathrm{~A}$ & & & $70(96)^{\mathrm{b}}$ \\
\hline $8^{\mathrm{a}}$ & $7 \mathrm{~A}+8 \mathrm{~A}$ & & & 68 \\
\hline 9 & 7A & & & 0 \\
\hline 10 & $8 \mathbf{A}$ & & & 15 \\
\hline 11 & $5 \mathrm{~A}$ & & & 93 \\
\hline $12^{\mathrm{a}}$ & $7 \mathrm{~A}+8 \mathrm{~A}$ & & & 45 \\
\hline 13 & $7 \mathrm{~A}$ & & & 0 \\
\hline 14 & $8 \mathrm{~A}$ & & & 0 \\
\hline 15 & $\mathbf{5 A}$ & & & 63 \\
\hline $16^{\mathrm{a}}$ & $7 \mathrm{~A}+8 \mathrm{~A}$ & & & 13 \\
\hline 17 & $5 \mathrm{~A}$ & & & 85 \\
\hline
\end{tabular}




(1)

Reaction condition: fluoroarene $(0.3 \mathrm{mmol}), \mathrm{NaO} t \mathrm{Bu}(0.3 \mathrm{mmol})$, catalyst $(1 \mathrm{~mol} \%), 2 \mathrm{~mL}$ of 2-propanol for 1 hour at $80^{\circ} \mathrm{C}$. Yields determined by GC analysis using anisole as internal standard. [a] Catalyst 7A $(0.5$ $\mathrm{mol} \%)+$ catalyst $\mathbf{8 A}(0.5 \mathrm{~mol} \%)$. [b] Yields in parenthesis are after 2 hours. [c] Isolated yield after 3 hours. [d] 2 Equivalents of $\mathrm{NaO} t \mathrm{Bu}(0.6 \mathrm{mmol})$ and 2 hours of reaction. [e] 3 equivalents of $\mathrm{NaO} t \mathrm{Bu}(0.9 \mathrm{mmol})$ and 2 hours of reaction.

The results given in Table 1.7 show that $\mathbf{5 A}$ is highly active in the hydrodefluorination of a series of arenes with different functional groups, affording good to excellent yields (entries $17-26$ ). The wide applicability of complex $\mathbf{5 A}$ is also illustrated by the tolerance of different substituent groups, ranging from electro-withdrawing to electron-donating substituents.

If we compare the activity of different substituted fluorotoluenes, hindered substrates such as $o$-fluorotoluene (entry 21) displayed lower reaction rated compared to the $m$ (entry 22) and $p$ - (entry 7) isomers, indicating that steric factors seem to play an 
important role. Complex $\mathbf{5 A}$ was also able to quantitatively hydrodefluorinate polyfluorinated arenes, such as $p$ - and $o$-difluorobenzene, and 1,3,5-trifluorobenzene (entries 24, 25 and 26).

An important feature of this catalytic process is the small difference in activity shown by all aryl fluorides possessing electron-withdrawing or electron-donating substituents. In order to confirm this tendency we decided to carry out a competitive experiment, in which three different fluoroarenes with different electronic-properties were allowed to react together, as depicted in Scheme 1.28.<smiles>CC(C)c1ccc(N)c(N)c1</smiles>

Scheme 1.28 Competitive HDF of several aryl fluorides, catalyzed by $\mathbf{5 A}$

The evolution of the reaction was monitored by means of by ${ }^{19} \mathrm{~F}$ NMR, and indicated negligible differences in the hydrodefluorination of all three substrates used, as can be seen from the time-dependent reaction profile shown in Figure 1.12. This behavior suggests that the rate determining step of the process is not the oxidative addition of the aryl fluoride, as it has been previously observed in other studies regarding the dehalogenation of aryl chlorides. ${ }^{91-93}$

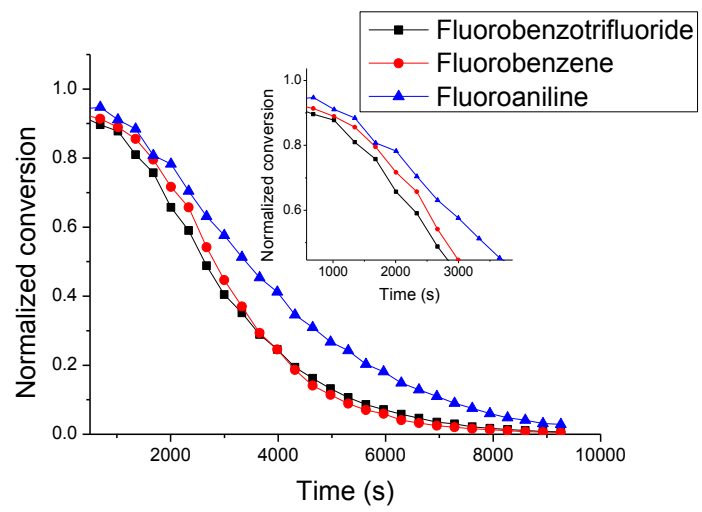

Figure 1.12 ${ }^{19} \mathrm{~F}$ NMR monitoring experiment. Reaction conditions: $0.3 \mathrm{mmol}$ of each fluoroarene, $\mathrm{NaO} t \mathrm{Bu}(0.9 \mathrm{mmol})$, catalyst ( $1 \mathrm{~mol} \%$ based on each fluoroarene), $1.5 \mathrm{~mL}$ of 2-propanol at $80^{\circ} \mathrm{C}$. 


\section{Hydrodefluorination of aliphatic C-F bonds}

Encouraged by the activity of the heterodimetallic complex $\mathbf{5 A}$ in the hydrodefluorination of fluoroarenes, we decided to test catalyst $\mathbf{5 A}$ in the hydrodefluorination of $\alpha, \alpha, \alpha-$ trifluorotoluenes. These substrates are known to lie among the most inert organofluorine compounds, and the reduction of benzylic C-F bonds has not been described until recently. ${ }^{94}$ Most of the active catalytic systems used so far, refer to early transition metals, ${ }^{82,95}$ and silylium compounds, ${ }^{63,64,94}$ although a few examples also describe the use of late transition metals such as $\mathrm{Ru}^{96}$ and $\mathrm{Ni}^{97}$

The reactions in the activation of aliphatic C-F bonds were performed using a catalyst loading of $5 \mathrm{~mol} \%$ and three equivalents of $\mathrm{NaO} t \mathrm{Bu}$, in 2-propanol at $80^{\circ} \mathrm{C}$. Yields and conversions were determined by gas chromatography using anisole as internal standard.

The results given in Table 1.8 , show that catalyst $\mathbf{5 A}$ is capable to HDF a series of $\alpha, \alpha, \alpha$ trifluorotoluenes, in good to excellent yields. The $\mathrm{Ru} / \mathrm{Pd}$ catalyst $\mathbf{5 A}$ was very active in the hydrodefluorination of $\alpha, \alpha, \alpha$-trifluoro-1-methylnaphthalene, $\alpha, \alpha, \alpha$-trifluoro-4-methylacetophenone and 4-trifluoro-methyl-pyridine (entries 3-6), but provided moderate yields in the hydrodefluorination of $\alpha, \alpha, \alpha$-trifluorotoluene, hexafluoro- $m$-xylene and hexafluorop-xylene.

In the case of the hexafluoro-xylenes, it is interesting that the reactions selectively yielded the hydrodefluorination of one of the trifluoromethyl groups, while the second group remained practically unaffected (only traces of the complete dehydrofluorinated compound were detected), regardless of the amount of base used (entries 9-10). In order to find a suitable explanation for the incomplete HDF of these two hexafluoro-xylenes, we also performed the reactions with trifluoro- $p$-xylene, and trifluoro- $m$-xylene, and observed that the catalyst was ineffective (entries 7 and 8). This suggests that the selective formation of the trifluoro-xylenes from the starting hexafluoro-xylenes is due to the inert character of the trifluorinated products, rather than to the deactivation of the catalyst during the reaction course. 
Table 1.8 Hydrodefluorination of $\alpha, \alpha, \alpha$-trifluorotoluenes with catalyst 5A

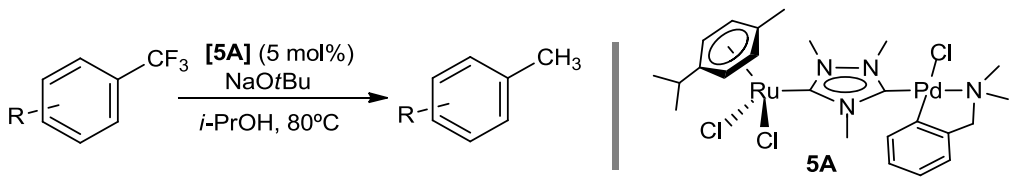

Entry

Reactions were carried out with $0.3 \mathrm{mmol}$ of trifluomethylarene, $\mathrm{NaOt} \mathrm{Bu}(0.9 \mathrm{mmol})$, catalyst (5 mol\%), 2 $\mathrm{mL}$ of 2-propanol at $80^{\circ} \mathrm{C}$. [a] Yields determined by $\mathrm{GC}$ analysis using anisole as internal standard. [b] Isolated yield in parenthesis. [c] $1.8 \mathrm{mmol}$ of $\mathrm{NaO} t \mathrm{Bu}$ 
Trifluorotoluenes with electron-withdrawing groups resulted to be more active than those having electron-donating functionalities (compare entries 3-6 and 7-10). The reduction of 4-fluoro-benzotrifluoride (entry 2), which was reduced to two different products in the presence of four equivalents of base, shows the higher selectivity of 5A towards the reduction of aromatic rather than aliphatic C-F bonds. In this case we can observe the first reduction of the aromatic $\mathrm{C}-\mathrm{F}$ bond, and then the slow activation of the $\mathrm{CF}_{3}$ group. As we previously observed, in the presence of one equivalent of base, the same substrate undergoes full conversion to trifluoro-toluene, therefore implying that only the aromatic $\mathrm{C}-\mathrm{F}$ bond was reduced while the $\mathrm{CF}_{3}$ group remained untouched (Table 1.7, entry 20). The selective activation of the aromatic C-F bond in this latter case is obviously due to the amount of base used, and illustrates how the outcome of the process may be selective to the reduction of aromatic C-F bonds if stoichiometric amounts of base are used.

\section{Kinetic studies}

All our results indicate that the $\mathrm{Ru} / \mathrm{Pd}$ heterodimetallic catalyst $\mathbf{5 A}$ behaves as an effective two-component activation system, which produces significant benefits in the hydrodefluorination of a wide set of fluorinated organic molecules. Although a mixture of two different homodimetallic complexes of palladium and ruthenium seems to partially facilitate the process, the reaction is clearly benefited when the two different metal fragments are linked to a single-frame ligand. From a mechanistic point of view, it is difficult to explain the intimate process, which should need a more detailed study, but we have enough evidences to justify that the most plausible mechanism implies the action of the two different metals to afford the full hydrodefluorination reaction. In this regard, the palladium center would be facilitating the $\mathrm{C}-\mathrm{F}$ bond activation, while the ruthenium fragment would complete the reaction by hydrogenating the substrate.

Aiming to shed some light on the reaction mechanism of the process, we decided to carry out some kinetic studies. Firstly, we decided to carry out the HDF of fluorobenzene and $p$-fluorotoluene using deuterium-labelled 2-propanol, $\left(\mathrm{CD}_{3}\right)_{2} \mathrm{CDOD}$. The reactions were carried out using $1 \mathrm{~mol} \%$ of complex $\mathbf{5 A}, \mathrm{NaO} t \mathrm{Bu}$ and $80^{\circ} \mathrm{C}$. The evolution of the reactions was monitored by ${ }^{19} \mathrm{~F}$ NMR, by measuring the disappearance of the ${ }^{19} \mathrm{~F}$ 
resonance of fluoroarene. Figure 1.13 displays the kinetic curves of the reactions carried out in 2-propanol (black), and 2-propanol- $d_{8}$ (red), for the HDF of $p$-fluorotoluene, and the plots of $\ln (\mathrm{I}) v s$. time ( $\mathrm{I}=$ Normalized conversion), with the linear regressions of the reactions in 2-propanol and 2-propanol- $d_{8}$ (top and bottom, respectively). The plots of $\ln (\mathrm{I}) v s$. time provided straight lines with negative slopes, thus revealing a $p s e u d o$-first order rate for the HDF process.

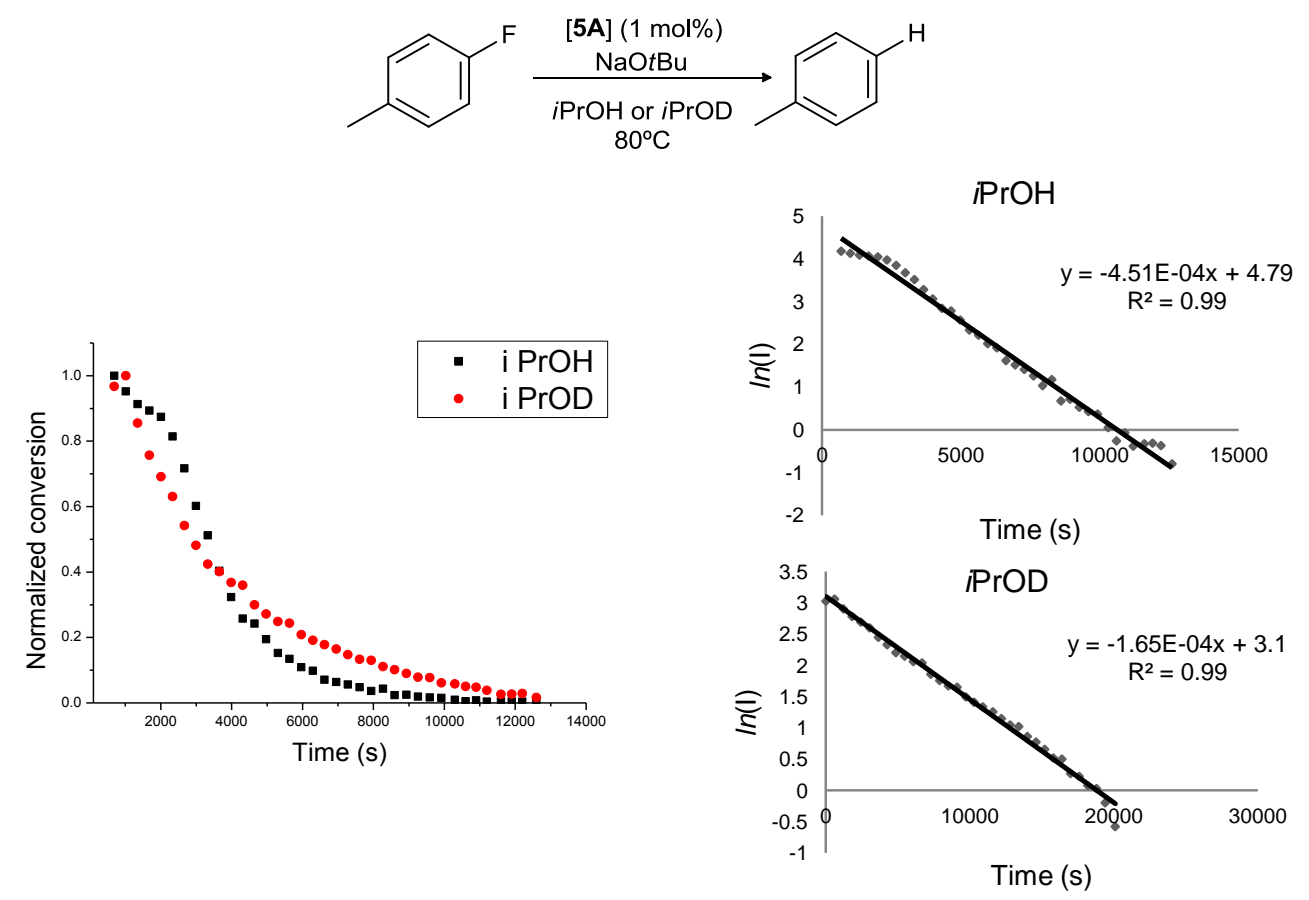

Figure 1.13 Kinetic curves for the HDF of fluorotoluene (right) in 2-propanol and 2-propanol- $d_{8}$, and the $\ln (\mathrm{I})$ vs. time plots with the linear regressions for the reaction in 2-propanol and 2propanol- $d_{8}$ (top and bottom, respectively). The reactions were performed using $0.3 \mathrm{mmol}$ of fluoroarene, $\mathrm{NaO} t \mathrm{Bu}(0.3 \mathrm{mmol})$, catalyst $(1 \mathrm{~mol} \%), 1.5 \mathrm{~mL}$ of 2-propanol at $80^{\circ} \mathrm{C}$. Evolution monitored by ${ }^{19} \mathrm{~F}$ NMR, by measuring the disappearance of the ${ }^{19} \mathrm{~F}$ resonance of fluoroarene

From these experiments we can extract two important mechanistic features of the process that should be considered: i) when the reactions were carried out with 2-propanol- $d_{8}$, the resulting arylated products contained deuterium, therefore confirming that 2-propanol is the hydrogen source of the process, and ii) these experiments allowed us to determine the 
kinetic isotopic effect (KIE) by calculating the kinetic constants of the hydrodefluorination of 4-fluorotoluene and fluorobenzene carried out in 2-propanol and 2-propanol- $d_{8}$ at $80^{\circ} \mathrm{C}$.

The determination of the kinetic isotopic effect (KIE) is a widely used technique for the study of reaction mechanisms. This parameter is the ratio between reaction rates shown by two different isotopically labelled molecules in a chemical reaction. A KIE involving hydrogen and deuterium is represented as $\mathrm{KIE}=\mathrm{k}_{H} / \mathrm{k}_{D}$ (where $\mathrm{k}_{H}$ and $\mathrm{k}_{D}$ are reaction rate constants).

The rate constants $\mathrm{k}_{H}$ and $\mathrm{k}_{D}$ were obtained from the kinetic curves of the HDF of fluorobenzene and $p$-fluorotoluene, both in 2-propanol and 2-propanol- $d_{8}$ under catalytic conditions: $1 \mathrm{~mol} \%$ of complex $\mathbf{5 A}, \mathrm{NaO} t \mathrm{Bu}$ and $80^{\circ} \mathrm{C}$ (Figure 1.13). $\mathrm{k}_{H}$ and $\mathrm{k}_{D}$ equal the slopes of the linear regressions of the plots of $\ln (\mathrm{I}) v s$. time ( $\mathrm{I}=$ Normalized conversion). The linear regression in all cases showed rather accurate regression-coefficient values (Table 1.9).

Table 1.9 Rate constants, regression coefficients and KIE values for the HDF of fluorobenzene and $p$-fluorotoluene.

\begin{tabular}{ccccc}
\hline Substrate & Solvent & $\boldsymbol{k}_{\boldsymbol{H}}\left(\mathbf{o r} \boldsymbol{k}_{\boldsymbol{D}}\right)\left(\mathbf{s}^{-1}\right)$ & $\mathbf{R}^{2}$ & KIE \\
\hline \multirow{2}{*}{ Fluorobenzene } & 2-propanol & $8.11 \cdot 10^{-4}$ & 0.973 & 2.4 \\
& 2-propanol- $d_{8}$ & $3.41 \cdot 10^{-4}$ & 0.939 & \\
\multirow{2}{*}{-Fluorotoluene } & 2-propanol & $4.51 \cdot 10^{-4}$ & 0.990 & 2.7 \\
& 2-propanol- $d_{8}$ & $1.65 \cdot 10^{-4}$ & 0.993 & \\
\hline
\end{tabular}

Table 1.9 also shows that both substrates present a positive KIE (Primary kinetic isotope effect). This primary KIE supports the idea that the rate determining step is not the oxidative addition of the aryl fluoride as stated before, therefore suggesting that the slow step of the overall process is associated with the reductive part of the reaction, rather than to $\mathrm{C}-\mathrm{F}$ bond activation.

As we saw from the analysis of the catalytic outcomes, only the combination of ruthenium and palladium results in an effective catalytic system for the HDF reaction. We decided to determine the reaction order in the catalyst, by using the heterodimetallic 
complex 5A. Assuming a pseudo-first order reaction with respect to fluorobenzene, the rate expression can be written as,

$$
v=k[\mathrm{cat}]^{n}[\mathrm{FBZ}]^{1}=k_{o b s}[\mathrm{FBZ}]
$$

(With FBZ = fluorobenzene)

where,

$$
k_{o b s}=k[c a t]^{n}
$$

The van't Hoff plot $\left(\ln \left(k_{\text {obs }}\right) v s\right.$. $\ln$ [cat] $)$ allows the determination of " $k$ " and "n". The $y$ intercept of the regression line equals $\ln (k)$. The slope of the regression line equals "n". 98,99

With these equations in mind, we performed a set of monitoring experiments, in which the evolution of the reactions was monitored by ${ }^{19} \mathrm{~F}$ NMR. We obtained the reaction profiles modifying the catalyst loading (from 0.25 to $2 \mathrm{~mol} \%$ ), while keeping the concentration of the substrates (fluorobenzene and $\mathrm{NaO} t \mathrm{Bu}$ ) fixed.

From the slope of the regression line of the van't Hoff plot (Figure 1.14) we concluded that the reaction is first order in the catalyst $\mathbf{5 A},(n=1.093)$.

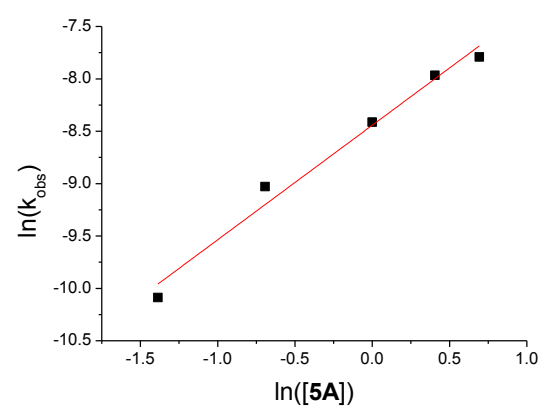

\begin{tabular}{ccc}
\hline $\begin{array}{c}\text { Equation } \\
\mathbf{R}^{\mathbf{2}}\end{array}$ & \multicolumn{2}{c}{$\mathrm{y}=\ln (k)+\mathrm{n} * \mathrm{x}$} \\
& & 0.977 \\
\hline & Value & Standard error \\
Intercept $\left(\mathbf{s}^{-1}\right)$ & -8.443 & 0.065 \\
Slope $\left(\mathbf{s}^{-1}\right)$ & 1.093 & 0.083 \\
\hline
\end{tabular}

Figure 1.14 Van’t Hoff plot for catalyst 5A

The first order reaction in catalyst $\mathbf{5} \mathbf{A}$ seems to discard the possibility of aggregation of metal initiators or growing polymer chains as have been seen in other reported catalytic systems. ${ }^{98,100}$ 
From the catalytic results, we have seen that the heterodimetallic complex $\mathbf{5 A}$ behaves as an effective two-component activation system, in which the ruthenium and the palladium active centers are in a 1:1 relation. We also observed that the mixture of two different homodimetallic complexes of ruthenium $(\mathbf{7 A})$ and palladium $(\mathbf{8 A})$ partially facilitate the process. Then, we were interested in determining the optimum ratio of the mixture of the homodimetallic complexes $([\mathbf{7 A}]:[\mathbf{8 A}])$, in order to quantify the participation of both metal centers in the catalytic cycle.

With this purpose, we planned a series of experiments using variable concentrations of palladium catalyst $\mathbf{8 A}$ (from $0.3 \mathrm{mM}$ to $2.62 \mathrm{mM}$ ), and maintaining constant the concentration of the diruthenium complex $([\mathbf{7 A}], 1.55 \mathrm{mM})$. The reactions were performed using fluorobenzene in the presence of $\mathrm{NaO} t \mathrm{Bu}$ (both in $1 \mathrm{M}$ concentration) in 2-propanol at $80^{\circ} \mathrm{C}$ for $30 \mathrm{~min}$. The yields were determined by $\mathrm{GC}$ analyses using anisole as internal standard. Figure 1.11 (left) shows the representation of the yield for each experiment $v$ s. the molar ratio of the homodimetallic complexes, $[\mathbf{8 A}] /[\mathbf{7 A}]$. From this representation we can observe a linear increase of the activity with respect to an increase of $[\mathbf{8 A}] /[\mathbf{7 A}]$ ratio, for $[\mathbf{8 A}] /[\mathbf{7 A}] \leq 1$. Then the process achieves the maximum yield when $[\mathbf{8 A}] /[\mathbf{7 A}]$ is 1 . We observed the same result using a constant loading of $\mathbf{8 A}$ and varying the concentration of the ruthenium complex 7A (Figure 1.15 right). These results are interesting, because they suggest an equal participation of both metal centers in the catalytic cycle.
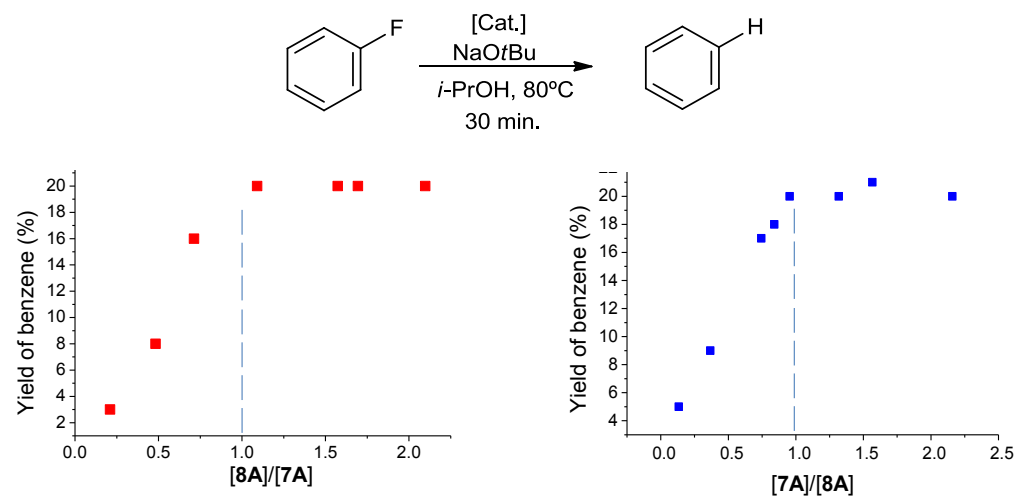

Figure 1.15 Yield of benzene $v s$. the molar ratio of the homodimetallic complexes, $[\mathbf{8 A}] /[\mathbf{7 A}]$ (left) and $[\mathbf{7 A}] /[\mathbf{8 A}]$ (right). The reactions were performed using fluorobenzene in the presence of $\mathrm{NaO} t \mathrm{Bu}$ (both in $1 \mathrm{M}$ concentration) in 2-propanol at $80^{\circ} \mathrm{C}$ for $30 \mathrm{~min}$. 
Once we determined the optimum $[\mathbf{7 A}]:[\mathbf{8 A}]$ ratio, we were interested in determining the reaction order with respect the sum of the homodimetallic complexes. With this purpose we performed a series of experiments similar to those used for the determination of the reaction order with respect to $\mathbf{5} \mathbf{A}$, but using the mixture of the related homodimetallic complexes. The van't Hoff plot (Figure 1.16) reveals that the reaction is first order with respect the sum of both homodimetallic complexes $(\mathrm{n}=1.199)$

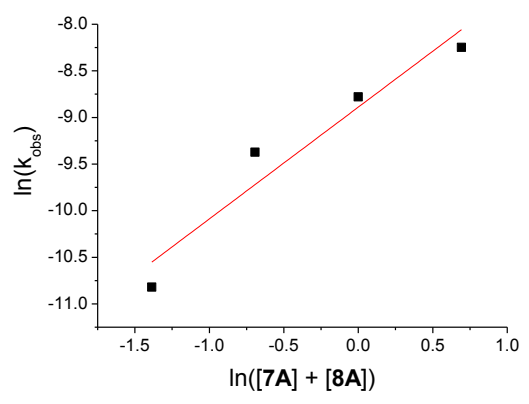

\begin{tabular}{ccc}
\hline $\begin{array}{c}\text { Equation } \\
\mathbf{R}^{\mathbf{2}}\end{array}$ & $\begin{array}{c}\mathrm{Y}=\ln (k)+\mathrm{n} * \mathrm{x} \\
0.9555\end{array}$ \\
\hline & Value & Standard error \\
Intercept $\left(\mathbf{s}^{-1}\right)$ & -8.889 & 0.190 \\
Slope $\left(\mathbf{s}^{-1}\right)$ & 1.199 & 0.223 \\
\hline
\end{tabular}

Figure 1.16 Reaction order in $([\mathbf{7 A}]+[\mathbf{8 A}])$

In summary, from kinetic and experimental results we can extract the following conclusions:

- 2-Propanol is the hydrogen source of the process by transfer hydrogenation.

- The rate determining step is the reduction step, rather than the C-F activation.

- Under the conditions used, the HDF is a homogeneously catalyzed process.

- The optimum $[\mathrm{Pd}]:[\mathrm{Ru}]$ ratio is 1 .

- The reaction is first order in catalysts, both when the heterodimetallic complex $\mathbf{5 A}$ and the combination of the homodimetallic complexes 7A and $\mathbf{8 A}$ are used.

- The heterodimetallic complex $\mathbf{5 A}$ is more effective than a mixture of the two homodimetallic complexes $\mathbf{7 A}$ and $\mathbf{8 A}$, thus illustrating a new example of catalytic cooperativity between the two metals comprised in the heterodimetallic unit.

With these conclusions in mind we may propose a tentative reaction mechanism (Scheme 1.29). 


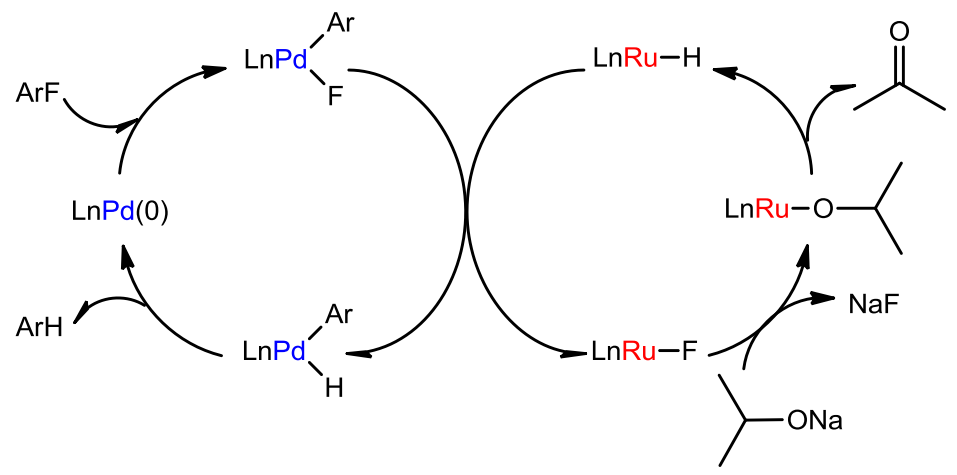

Scheme 1.29 Proposed mechanism for the HDF of fluoroarenes with a $\mathrm{Ru} / \mathrm{Pd}$ catalyst

This reaction pathway is also supported by previously published works. ${ }^{101}$ The mechanism basically implies that the aryl-fluoride oxidatively adds to Pd, to form an aryl-palladium-fluoride. In a parallel cycle, the ruthenium fragment produces a $\mathrm{Ru}-\mathrm{H}$ complex, by reaction with 2-propanol in basic conditions. The hydride is transferred from $\mathrm{Ru}$ to $\mathrm{Pd}$, while the fluoride goes to the ruthenium complex. We suggest that the rate determining step is the hydride transfer from the ruthenium to the palladium with the concomitant transfer of the fluoride from the palladium to the ruthenium center. A reductive elimination from palladium center leads to the formation of the defluorinated arene.

The hydride transfer from Pd to Ru has already been reported ${ }^{101}$ for the mechanism of olefin hydration. This reaction was based on a two-catalyst cooperative system involving an oxidation cycle $(\mathrm{Pd})$ followed by a reduction cycle $(\mathrm{Ru})$. The authors suggested that the two processes are combined through a hydride transfer from $\mathrm{Pd}$ to $\mathrm{Ru}$.

Attempts to get some more mechanistic insights form the isolation of intermediates were performed by means on NMR and ESI-MS studies. We observed the formation of Ruhydrides and Ru-alkoxydes. However, we were not able to detect any intermediates involving palladium hydrides or fluorides. 


\section{CONCLUSIONS}

A methodology process to synthesize heterodimetallic complexes has been developed. The metal centers are connected through a di-ylidine ligand (ditz), forming bis-carbene complexes. The analogous homodimetallic complexes have been obtained for comparative catalytic purposes. All complexes have been fully characterized by means of NMR spectroscopy, High Resolution Mass Spectroscopy (HRMS), elemental analysis and X-ray diffraction studies.

The electronic communication between the metal centers was evaluated by means of electrochemistry studies in the case of the heterodimetallic and homodimetallic Ru/Ir and $\mathrm{Ru} / \mathrm{Ru}$ complexes. The results show that there is a small electronic communication between the metal centers.

The heterodimetallic complexes have been used in designed multimetallic processes and the results show that:

- $\mathrm{Au} / \mathrm{Ir}$ is active in a dual cooperative process

- $\mathrm{Ru} / \mathrm{Ir}$ is active in a cascade tandem process

- $\mathrm{Ru} / \mathrm{Pd}$ is active in a synergistic cooperation between ruthenium and palladium capable of activating fluroarenes and trifluoromethylarenes.

We can state that the outcomes of complexes having two metal centers linked by one single ligand shows higher activities than the monometallic analogues as result of a cooperative behavior between the two metal centers. 


\section{REFERENCES}

(1) Lee, J. M.; Na, Y.; Han, H.; Chang, S. Chem. Soc. Rev. 2004, 33, 302-312.

(2) Kolb, H. C.; Finn, M. G.; Sharpless, K. B. Angew. Chem. Int. Edit. 2001, 40, 2004.

(3) Moses, J. E.; Moorhouse, A. D. Chem. Soc. Rev. 2007, 36, 1249-1262.

(4) Iha, R. K.; Wooley, K. L.; Nystrom, A. M.; Burke, D. J.; Kade, M. J.; Hawker, C. J. Chem. Rev. 2009, 109, 5620-5686.

(5) Ajamian, A.; Gleason, J. L. Angew. Chem. Int. Edit. 2004, 43, 3754-3760.

(6) Fogg, D. E.; dos Santos, E. N. Coord. Chem. Rev. 2004, 248, 2365-2379.

(7) Wasilke, J. C.; Obrey, S. J.; Baker, R. T.; Bazan, G. C. Chem. Rev. 2005, 105, 1001-1020.

(8) Yamada, Y.; Tsung, C.-K.; Huang, W.; Huo, Z.; Habas, S. E.; Soejima, T.; Aliaga, C. E.; Somorjai, G. A.; Yang, P. Nat. Chem. 2011, 3, 372-376.

(9) Vigato, P. A.; Tamburini, S.; Fenton, D. E. Coord. Chem. Rev. 1990, 106, 25170 .

(10) Bratko, I.; Gomez, M. Dalton Trans. 2013, 42, 10664-10681.

(11) Mata, J. A.; Hahn, F. E.; Peris, E. Chem. Sci. 2014, 5, 1723-1732.

(12) Perez-Temprano, M. H.; Casares, J. A.; Espinet, P. Chem. Eur. J. 2012, 18, 18641884.

(13) Multimetallic catalysts in organic synthesis; Shibasaki, Y. Y., Y., Ed.; WileyVCH: Weinheim, 2004.

(14) van den Beuken, E. K.; Feringa, B. L. Tetrahedron 1998, 54, 12985-13011.

(15) Hahn, F. E.; Jahnke, M. C. Angew. Chem. Int. Edit. 2008, 47, 3122-3172.

(16) Nelson, D. J.; Nolan, S. P. Chem. Soc. Rev. 2013, 42, 6723-6753.

(17) Hopkinson, M. N.; Richter, C.; Schedler, M.; Glorius, F. Nature 2014, 510, 485496.

(18) Poyatos, M.; Mata, J. A.; Peris, E. Chem. Rev. 2009, 109, 3677-3707.

(19) Curphey, T. J.; Prasad, K. S. J. Org. Chem. 1972, 37, 2259.

(20) Guerret, O.; Sole, S.; Gornitzka, H.; Teichert, M.; Trinquier, G.; Bertrand, G. J. Am. Chem. Soc. 1997, 119, 6668-6669. 
(21) Mas-Marza, E.; Mata, J. A.; Peris, E. Angew. Chem. Int. Edit. 2007, 46, 37293731 .

(22) Viciano, M.; Sanau, M.; Peris, E. Organometallics 2007, 26, 6050-6054.

(23) Zanardi, A.; Corberan, R.; Mata, J. A.; Peris, E. Organometallics 2008, 27, 35703576.

(24) Zanardi, A.; Mata, J. A.; Peris, E. J. Am. Chem. Soc. 2009, 131, 14531-14537.

(25) Zanardi, A.; Mata, J. A.; Peris, E. Organometallics 2009, 28, 4335-4339.

(26) Zanardi, A.; Mata, J. A.; Peris, E. Organometallics 2009, 28, 1480-1483.

(27) Zanardi, A.; Mata, J. A.; Peris, E. Chem. Eur. J. 2010, 16, 13109-13115.

(28) Zanardi, A.; Mata, J. A.; Peris, E. Chem. Eur. J. 2010, 16, 10502-10506.

(29) Guerret, O.; Sole, S.; Gornitzka, H.; Trinquier, G.; Bertrand, G. J. Organomet. Chem. 2000, 600, 112-117.

(30) Singh, A.; Ramanan, A.; Bandyopadhyay, D. Cryst. Growth Des. 2011, 11, 2743 2754.

(31) Laguna, A.; Lasanta, T.; Lopez-de-Luzuriaga, J. M.; Monge, M.; Naumov, P.; Elena Olmos, M. J. Am. Chem. Soc. 2010, 132, 456.

(32) Lopez-de-Luzuriaga, J. M.; Monge, M.; Olmos, M. E.; Rodriguez-Castillo, M.; Laguna, A.; Mendizabal, F. Theor. Chem. Acc. 2011, 129, 593-602.

(33) White-Morris, R. L.; Olmstead, M. M.; Jiang, F. L.; Tinti, D. S.; Balch, A. L. J. Am. Chem. Soc. 2002, 124, 2327-2336.

(34) Schmidbaur, H.; Schier, A. Chem. Soc. Rev. 2008, 37, 1931-1951.

(35) Ray, L.; Shaikh, M. M.; Ghosh, P. Inorg. Chem. 2008, 47, 230-240.

(36) Bratko, I.; Mallet-Ladeira, S.; Teuma, E.; Gomez, M. Organometallics 2014, 33, 1812-1819.

(37) Vuong, K. Q.; Wong, C. M.; Bhadbhade, M.; Messerle, B. A. Dalton Trans. 2014, 43, 7540-7553.

(38) Mercs, L.; Neels, A.; Albrecht, M. Dalton Trans. 2008, 5570-5576.

(39) Tennyson, A. G.; Rosen, E. L.; Collins, M. S.; Lynch, V. M.; Bielawski, C. W. Inorg. Chem. 2009, 48, 6924-6933.

(40) Nussbaum, M.; Schuster, O.; Albrecht, M. Chem. Eur. J. 2013, 19, 17517-17527.

(41) Gusev, D. G.; Peris, E. Dalton Trans. 2013, 42, 7359-7364.

(42) Wolf, S.; Plenio, H. J. Organomet. Chem. 2009, 694, 1487-1492. 
(43) Leuthaeusser, S.; Schwarz, D.; Plenio, H. Chem. Eur. J. 2007, 13, 7195-7203.

(44) Robin, M. B.; Day, P. Adv. Inorg. Chem. Radiochem 1967, 10, 247.

(45) Schuster, O.; Mercs, L.; Albrecht, M. Chimia 2010, 64, 184-187.

(46) Prades, A.; Peris, E.; Alcarazo, M. Organometallics 2012, 31, 4623-4626.

(47) Buchwalter, P.; Rosé, J.; Braunstein, P. Chem. Rev. 2014, DOI: $10.1021 /$ cr500208k.

(48) Gnanaprakasam, B.; Zhang, J.; Milstein, D. Angew. Chem. Int. Edit. 2010, 49, 1468-1471.

(49) Adams, J. P. J. Chem. Soc.-Perkin Trans. 1 2000, 125-139.

(50) Gawronski, J.; Wascinska, N.; Gajewy, J. Chem. Rev. 2008, 108, 5227-5252.

(51) Corma, A.; Gonzalez-Arellano, C.; Iglesias, M.; Sanchez, F. Appl. Catal. A-Gen. 2009, 356, 99-102.

(52) Grirrane, A.; Corma, A.; Garcia, H. Science 2008, 322, 1661-1664.

(53) Cui, W.; Xiao, Q.; Sarina, S.; Ao, W.; Xie, M.; Zhu, H.; Bao, Z. Catal. Today 2014, 235, 152-159.

(54) Wang, X.; Kawanami, H.; Dapurkar, S. E.; Venkataramanan, N. S.; Chatterjee, M.; Yokoyama, T.; Ikushima, Y. Appl. Catal. A-Gen. 2008, 349, 86-90.

(55) Oi, S.; Fukita, S.; Hirata, N.; Watanuki, N.; Miyano, S.; Inoue, Y. Org. Lett. 2001, 3, 2579-2581.

(56) Ackermann, L. Chem. Rev. 2011, 111, 1315-1345.

(57) Ackermann, L.; Vicente, R. C-H Activation 2010, 292, 211-229.

(58) Arockiam, P. B.; Bruneau, C.; Dixneuf, P. H. Chem. Rev. 2012, 112, 5879-5918.

(59) Prades, A.; Poyatos, M.; Peris, E. Adv. Synth. Catal. 2010, 352, 1155-1162.

(60) Wang, J.; Sanchez-Rosello, M.; Luis Acena, J.; del Pozo, C.; Sorochinsky, A. E.; Fustero, S.; Soloshonok, V. A.; Liu, H. Chem. Rev. 2014, 114, 2432-2506.

(61) O'Hagan, D. Chem. Soc. Rev. 2008, 37, 308-319.

(62) Amii, H.; Uneyama, K. Chem. Rev. 2009, 109, 2119-2183.

(63) Douvris, C.; Ozerov, O. V. Science 2008, 321, 1188-1190.

(64) Ahrens, M.; Scholz, G.; Braun, T.; Kemnitz, E. Angew. Chem. Int. Edit. 2013, 52, 5328-5332.

(65) Kuehnel, M. F.; Lentz, D.; Braun, T. Angew. Chem. Int. Edit. 2013, 52, 33283348 . 
(66) Han, W.; Li, Y.; Tang, H.; Liu, H. J Fluorine Chem 2012, 140, 7-16.

(67) Shine, K. P.; Sturges, W. T. Science 2007, 315, 1804-1805.

(68) Molina, M. J.; Rowland, F. S. Nature 1974, 249, 810-812.

(69) Rowland, F. S.; Molina, M. J. Rev. Geophys. 1975, 13, 1-35.

(70) Rowland, F. S.; Molina, M. J.; Chou, C. C. Nature 1975, 258, 775-776.

(71) Meier, G.; Braun, T. Angew. Chem. Int. Edit. 2009, 48, 1546-1548.

(72) Nova, A.; Mas-Balleste, R.; Lledos, A. Organometallics 2012, 31, 1245-1256.

(73) Aizenberg, M.; Milstein, D. Science 1994, 265, 359-361.

(74) Whittlesey, M. K.; Peris, E. ACS Catal. 2014, 4, 3152-3159.

(75) Chen, Z.; He, C.-Y.; Yin, Z.; Chen, L.; He, Y.; Zhang, X. Angew. Chem. Int. Edit. 2013, 52, 5813-5817.

(76) Reade, S. P.; Mahon, M. F.; Whittlesey, M. K. J. Am. Chem. Soc. 2009, 131, 1847-1861.

(77) Panetier, J. A.; Macgregor, S. A.; Whittlesey, M. K. Angew. Chem. Int. Edit. 2011, 50, 2783-2786.

(78) Vela, J.; Smith, J. M.; Yu, Y.; Ketterer, N. A.; Flaschenriem, C. J.; Lachicotte, R. J.; Holland, P. L. J. Am. Chem. Soc. 2005, 127, 7857-7870.

(79) Young, R. J.; Grushin, V. V. Organometallics 1999, 18, 294-296.

(80) Lv, H.; Zhan, J.-H.; Cai, Y.-B.; Yu, Y.; Wang, B.; Zhang, J.-L. J. Am. Chem. Soc. 2012, 134, 16216-16227.

(81) Lv, H.; Cai, Y.-B.; Zhang, J.-L. Angew. Chem. Int. Edit. 2013, 52, 3203-3207.

(82) Fuchibe, K.; Ohshima, Y.; Mitomi, K.; Akiyama, T. Org. Lett. 2007, 9, $1497-$ 1499.

(83) Davies, C. J. E.; Page, M. J.; Ellul, C. E.; Mahon, M. F.; Whittlesey, M. K. Chem. Commun. 2010, 46, 5151-5153.

(84) Desmarets, C.; Kuhl, S.; Schneider, R.; Fort, Y. Organometallics 2002, 21, $1554-$ 1559.

(85) Kuhl, S.; Schneider, R.; Fort, Y. Adv. Synth. Catal. 2003, 345, 341-344.

(86) Sawama, Y.; Yabe, Y.; Shigetsura, M.; Yamada, T.; Nagata, S.; Fujiwara, Y.; Maegawa, T.; Monguchi, Y.; Sajiki, H. Adv. Synth. Catal. 2012, 354, 777-782.

(87) Ukisu, Y.; Miyadera, T. J. Mol. Catal. A-Chem. 1997, 125, 135-142. 
(88) Cargill, M. R.; Sandford, G.; Tomlinson, D. J.; Hollfelder, N.; Pleis, F.; Nelles, G.; Kilickiran, P. J. Fluorine Chem. 2011, 132, 829-833.

(89) Foley, P.; Dicosimo, R.; Whitesides, G. M. J. Am. Chem. Soc. 1980, 102, 67136725.

(90) Crabtree, R. H. Chem. Rev. 2012, 112, 1536-54.

(91) Viciu, M. S.; Grasa, G. A.; Nolan, S. P. Organometallics 2001, 20, 3607-3612.

(92) Navarro, O.; Kaur, H.; Mahjoor, P.; Nolan, S. P. J. Org. Chem. 2004, 69, 31733180 .

(93) Navarro, O.; Marion, N.; Oonishi, Y.; Kelly, R. A.; Nolan, S. P. J. Org. Chem. 2006, 71, 685-692.

(94) Scott, V. J.; Celenligil-Cetin, R.; Ozerov, O. V. J. Am. Chem. Soc. 2005, 127, 2852-2853.

(95) Fuchibe, K.; Akiyama, T. J. Am. Chem. Soc. 2006, 128, 1434-1435.

(96) Stahl, T.; Klare, H. F. T.; Oestreich, M. J. Am. Chem. Soc. 2013, 135, 1248-1251.

(97) Wu, J.; Cao, S. ChemCatChem 2011, 3, 1582-1586.

(98) Chen, H.-Y.; Zhang, J.; Lin, C.-C.; Reibenspies, J. H.; Miller, S. A. Green Chem. 2007, 9, 1038-1040.

(99) Seo, S.; Marks, T. J. Chem. Eur. J. 2010, 16, 5148-5162.

(100) Chamberlain, B. M.; Cheng, M.; Moore, D. R.; Ovitt, T. M.; Lobkovsky, E. B.; Coates, G. W. J. Am. Chem. Soc. 2001, 123, 3229-3238.

(101) Dong, G.; Teo, P.; Wickens, Z. K.; Grubbs, R. H. Science 2011, 333, 1609-1612. 



\section{CHAPTER 2}

\section{ASYMMETRIC CATALYSIS WITH NHC COMPLEXES BEARING ENANTIOPURE ORTHO-PALLADATED AMINES}

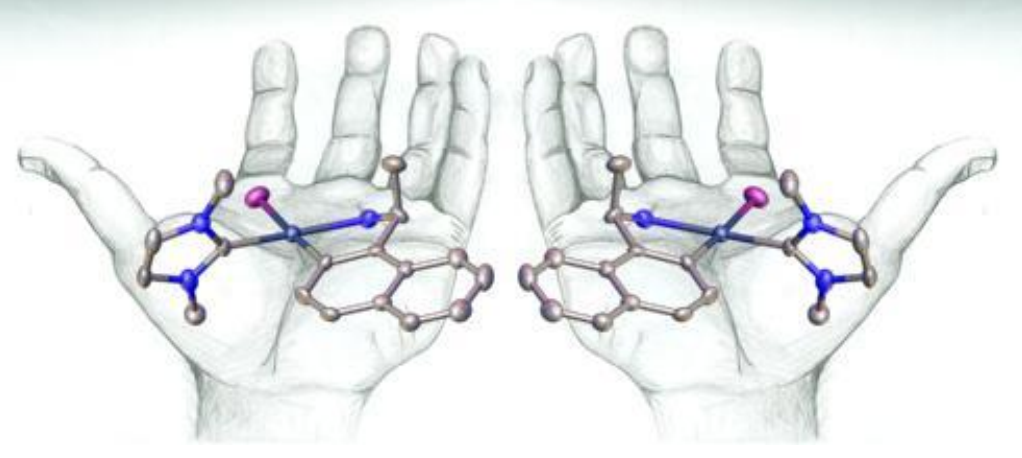





\section{INTRODUCTION}

The use of 1,2,4-trimethyltriazole-di-ylidene ligand (ditz) allowed to facilitate several homogeneous catalytic reactions, among which, sophisticated tandem processes probably constitute the most exciting application of this simple ligand. ${ }^{1}$ Although the benefits produced by ditz cover a wide variety of homogeneously catalyzed processes, we were concerned about applying the ligand for facilitating asymmetric catalyzed reactions. Due to the difficulties found for the modification of the ligand, we thought that the only way to synthesize a ditz-containing chiral metal complex was the coordination of ditz to metal fragments with chiral co-ligands.

Considering that our research group has a vast experience in the coordination of ditz to $\mathrm{Pd}(\mathrm{II})$, and palladium metallacycles are easily obtained in chiral forms, ${ }^{2,3}$ we decided to obtain ditz-based chiral palladacycles, and use them in asymmetric hydrophosphination, a reaction typically catalyzed by chiral palladium palladacycles. ${ }^{4,5}$ For comparative studies, together with the preparation of our chiral ditz-based iridium-palladium complexes, we also obtained several chiral imidazolylidene complexes of palladium, which were also tested in asymmetric hydrophosphination.

Palladacycles offer high flexibility in terms of synthetic procedures and modulation of the steric and electronic properties. This flexibility makes them useful for potential applications in organic synthesis, homogeneous catalysis, and medicinal chemistry. ${ }^{2,3,6-9}$ Some special features of palladacycles have been attributed to the reactivity of the labile Pd-C bond towards a wide range of nucleophilic and electrophilic reagents. This reactivity permits intriguing insertion reactions with small molecules, facilitating the formation of various nitrogen-containing heterocycles and carbocycles. ${ }^{3,10-15}$

Asymmetric hydrophosphination consists of a stereo-controlled addition of a P-H bond to unsaturated $\mathrm{C}-\mathrm{C}$ bonds and provides atom-efficient access to potentially useful chiral phosphines. The successful enantioselective preparation of chiral organophosphorous compounds via metal-catalyzed asymmetric addition of P-H bonds has recently gained great interest. ${ }^{4,5,16-32}$ Specifically, metal catalyzed hydrophosphination has attracted significant attention in the last decade ${ }^{33-35}$ due to its advantages over classical hydrophosphination, ${ }^{36-40}$ such as rate enhancement and regio- and stereo-selectivity 
control. In the last years, chiral cyclopalladated complexes, including pincer complexes, have been used for the asymmetric hydrophosphination of different unsaturated substrates such as enones, ${ }^{4,5}$ dienones, ${ }^{41}$ malonate esters, ${ }^{42}$ bisenones, ${ }^{16}$ and imines,,${ }^{18,43}$ facilitating the direct access to enantiopure tertiary phosphines (Scheme 2.1).

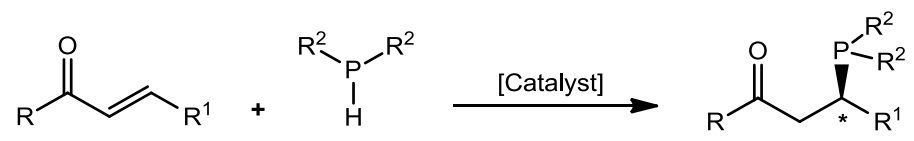

Scheme 2.1 Metal-catalyzed asymmetric hydrophosphination of enones 


\section{RESULTS AND DISCUSSION}

\subsection{Synthesis of ortho-metalated palladacycles}

The reaction of commercially available chiral tertiary amines (1-3) with palladium dichloride, and potassium carbonate, in acetonitrile at $80^{\circ} \mathrm{C}$, for $30 \mathrm{~min}$., afforded orthometalated chiral N,N-dimethylbenzylaminate palladacycles a - c (Scheme 2.2) in high yields $(85-95 \%)$. These complexes were described previously, although their synthesis involved the use of lithium tetrachloropalladate as palladium source. ${ }^{44,45}$ The ${ }^{1} \mathrm{H}$ and ${ }^{13} \mathrm{C}$ NMR spectra of palladacycles $\mathbf{a}-\mathbf{c}$ were in accordance with the published results, and showed cis/trans isomerism related to the relative orientation of the cyclometalated moieties in the dinuclear species. The thermodynamics of the activation for the cis/trans isomerization has elegantly been evaluated, and consists of a solvent-assisted associative process involving pentacoordinate palladium (II) transition states. ${ }^{46-48}$

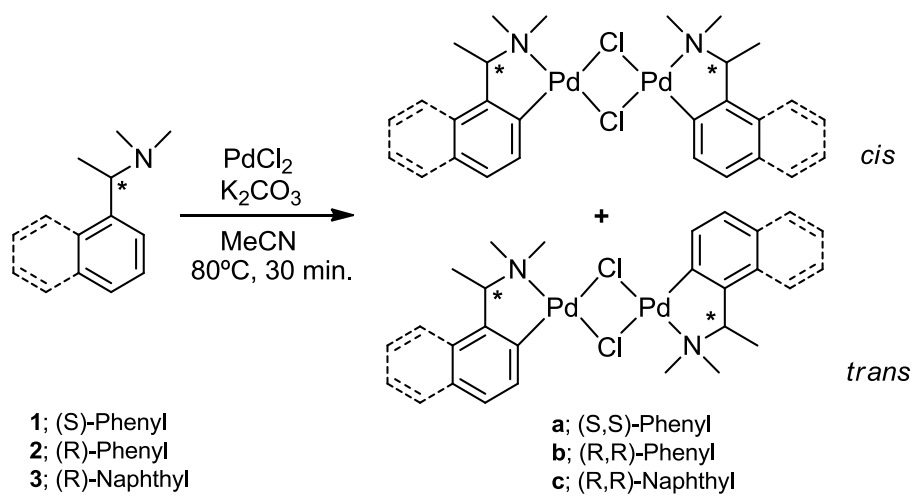

Scheme 2.2 Synthesis of palladacycles with tertiary amines

However, the reactivity of primary amines towards direct ortho-metalation is very different compared to tertiary amines. ${ }^{8}$ For example, under the same conditions, the reaction with primary amines affords monomeric diamine complexes, without the cyclometalation of the amine. The reaction of primary amine (S)-[1-(1naphthyl)ethyl]amine (5) with palladium dichloride, in the presence of potassium carbonate, in acetonitrile at $80^{\circ} \mathrm{C}$, for $30 \mathrm{~min}$., afforded the diamine dichloride palladium complex d, as a pale-yellow crystalline solid (Scheme 2.3). 


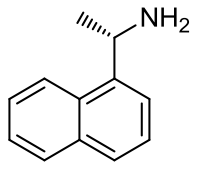

5; (S)-Naphthyl

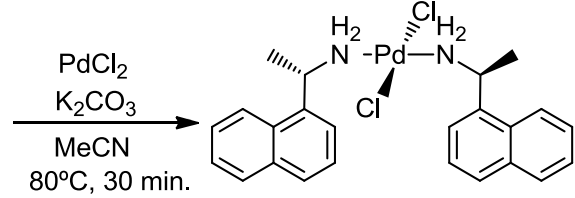

d; (S,S)-Naphthyl

Scheme 2.3 Synthesis of monomeric diamine complex d

The direct ortho-metalation using primary or secondary amines is a well studied field, mostly developed by Vicente and co-workers. ${ }^{25,27-30}$ In general, the preparation of palladacycles with primary amines is achieved using $\mathrm{Pd}(\mathrm{OAc})_{2}$, which is believed to play a significant role in the reaction mechanism. The acetate ligand enhances the electrophilicity of the palladium (II) center and also acts as an intramolecular base for the deprotonation of the ortho- $\mathrm{H}$ of the phenyl ring. ${ }^{49}$

The ortho-metalated chiral palladium complexes with primary amines described in this chapter were obtained following the above mentioned methodology. The reaction of chiral primary amines (4 and 5) with palladium acetate, in toluene at $50^{\circ} \mathrm{C}$ for $15 \mathrm{~h}$, afforded acetate-bridged palladacycles $\mathbf{e}$ and $\mathbf{f}$ (Scheme 2.4). The acetate-bridged dinuclear complexes $\mathbf{e}$ and $\mathbf{f}$ were obtained as yellow crystalline solids after recrystallization from dichloromethane/hexane mixtures in $90 \%$ and $85 \%$ yield, respectively. Both complexes were characterized by means of NMR spectroscopy, mass spectrometry and elemental analysis. All the details regarding the characterization of these complexes are described in the Experimental Section (Chapter 6).

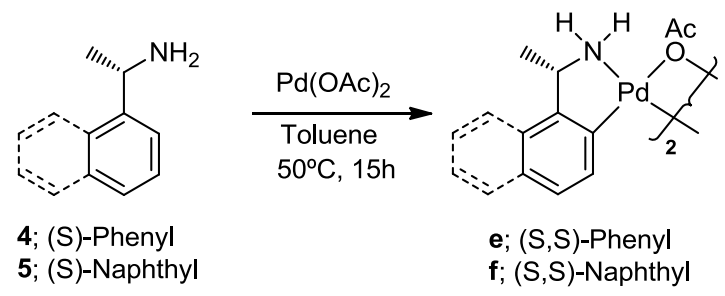

Scheme 2.4 Synthesis of palladacycles $\mathbf{e}$ and $\mathbf{f}$ with primary amines 


\section{Molecular structure of $f$}

Crystals of $\mathbf{f}$ suitable for X-ray diffraction studies were obtained by slow diffusion of hexane in a concentrated solution of the compound in dichloromethane. The molecular structure of $\mathbf{f}$ (Figure 2.1) consists of two palladium centers with two cis-bridging acetate groups and two cyclometalated (S)-[1-(1-naphthyl)ethyl]amine. The C-N ortho-metalated amine forms a five-membered ring in a puckered conformation, where the $\mathrm{N}$ atom is out of the plane by $0.48 \AA$. The methyl group attached at the benzylic carbon adopts an axial disposition enforced by the steric hindrance of the naphthyl group, as previously described for this ligand in a related palladium complex. ${ }^{47}$ The crystal structure corresponds to anti-exo disposition, which is the most thermodynamically stable stereoisomer in solid state. Table 2.1 shows the most representative bond lengths and angles of complex $\mathbf{f}$. The folded structure of the acetate bridge brings the palladium atoms into a relatively short Pd-Pd distance of $2.92 \AA$.

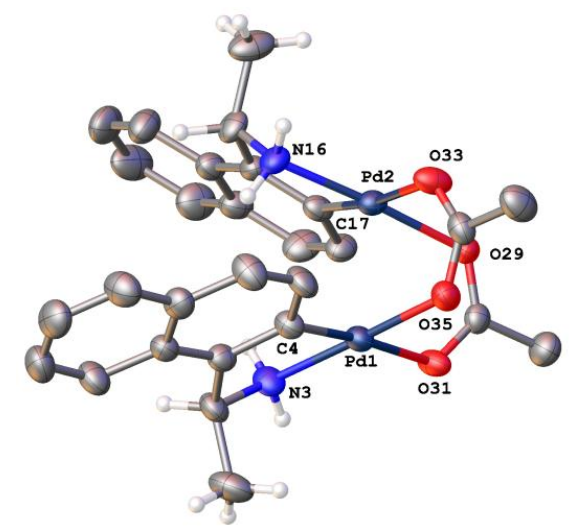

Figure 2.1 Molecular diagram of complex f. Ellipsoids are shown at 50\% probability level

Table 2.1 Selected bond lengths $(\AA)$ and angles $\left({ }^{\circ}\right)$ of complex $\mathbf{f}$

\begin{tabular}{|c|c|c|c|}
\hline Bonds & $(\AA)$ & Angles & $\left({ }^{\circ}\right)$ \\
\hline $\operatorname{Pd}(1)-\operatorname{Pd}(2)$ & $2.9159(5)$ & $\mathrm{O}(35)-\mathrm{Pd}(1)-\mathrm{O}(31)$ & $88.77(14)$ \\
\hline $\mathrm{Pd}(1)-\mathrm{O}(31)$ & $2.166(3)$ & $\mathrm{O}(29)-\mathrm{Pd}(2)-\mathrm{O}(33)$ & $87.98(14)$ \\
\hline $\mathrm{Pd}(1)-\mathrm{O}(35)$ & $2.049(3)$ & $C(4)-\operatorname{Pd}(1)-N(3)$ & $81.48(19)$ \\
\hline $\operatorname{Pd}(1)-N(3)$ & $2.026(4)$ & $C(17)-P d(2)-N(16)$ & $81.0(2)$ \\
\hline $\operatorname{Pd}(1)-C(4)$ & $1.962(5)$ & & \\
\hline
\end{tabular}




\subsection{Synthesis of chiral palladacycles with NHC ligands}

\subsubsection{Synthesis of NHC-palladacycles with tertiary amines}

The reaction of imidazolium salts $\mathbf{D}, \mathbf{E}$ and $\mathbf{F}$ with chiral N,N-dimethylbenzylaminate palladacycles, $\mathbf{a}$ and $\mathbf{c}$ in the presence of $\mathrm{Cs}_{2} \mathrm{CO}_{3}$, and an excess of $\mathrm{KI}$, afforded the NHCbased palladacycles 1D-1F and 2D-2F (Scheme 2.5). The reactions were performed in acetonitrile, at $100^{\circ} \mathrm{C}$ in a sealed Pyrex tube, for 60 minutes. The resulting suspensions were filtered through celite and the solvent was removed under reduced pressure. All complexes were prepared under similar conditions and yielded pale-yellow crystalline solids in moderate to high yields $(50-90 \%)$, after recrystallization from dichloromethane/hexane mixtures.

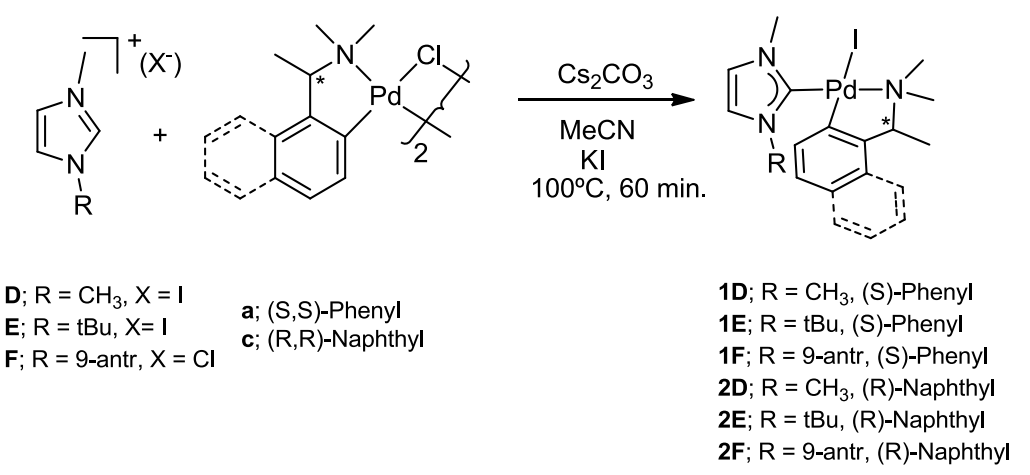

Scheme 2.5 Synthesis of NHC complexes with chiral palladacycles

The NHC-based palladacycles, with the general formula [PdI $\left.\left(\mathrm{C}-\mathrm{NMe}_{2}\right)(\mathrm{NHC})\right]$, were characterized by NMR spectroscopy, mass spectrometry and elemental analysis. In all cases, the N,N-dimethylamine ligands are mutually trans to the NHC ligand and the reactions proceed with retention of the configuration. As an example, a detailed description of the ${ }^{1} \mathrm{H}$ NMR and ${ }^{13} \mathrm{C}$ NMR of $\mathbf{1 D}$ (symmetrical NHC ligand) and 1E (unsymmetrical NHC ligand) is given. The characterization of the rest of complexes with symmetrically (2D) and asymmetrically (1F, 2E and 2F) NHC ligands are detailed in the Experimental Section (Chapter 6). 


\section{NMR Spectroscopic characterization of $\mathbf{1 D}$}

Figure 2.2 shows the ${ }^{1} \mathrm{H}$ NMR spectrum of 1D. The first evidence of the metalation of the carbene ligand is the absence of the characteristic signal attributed to the $\mathrm{NCHN}$ proton at $\delta$ 9.54. The resonance due to the $\mathrm{H}$ next to the ortho-metalated carbon is shown as a doublet at $5.84 \mathrm{ppm}\left({ }^{3} \mathrm{~J}_{\mathrm{H}, \mathrm{H}}=7.5 \mathrm{~Hz}\right)$. The spectrum shows two distinct signals due to the protons of the methyl groups at the azole ring at 3.93 and $3.82 \mathrm{ppm}$, indicating the loss of the two-fold symmetry of the ligand upon coordination to the palladium center. The resonances due to the protons of the two methyl groups of the N,N-dimethylbenzylamine ligand appear at 2.92 and $2.84 \mathrm{ppm}$. The signal due to the protons of the methyl group at the chiral carbon appears as a doublet at $1.62 \mathrm{ppm}\left({ }^{3} \mathrm{~J}_{\mathrm{H}, \mathrm{H}}=6.5 \mathrm{~Hz}\right)$.

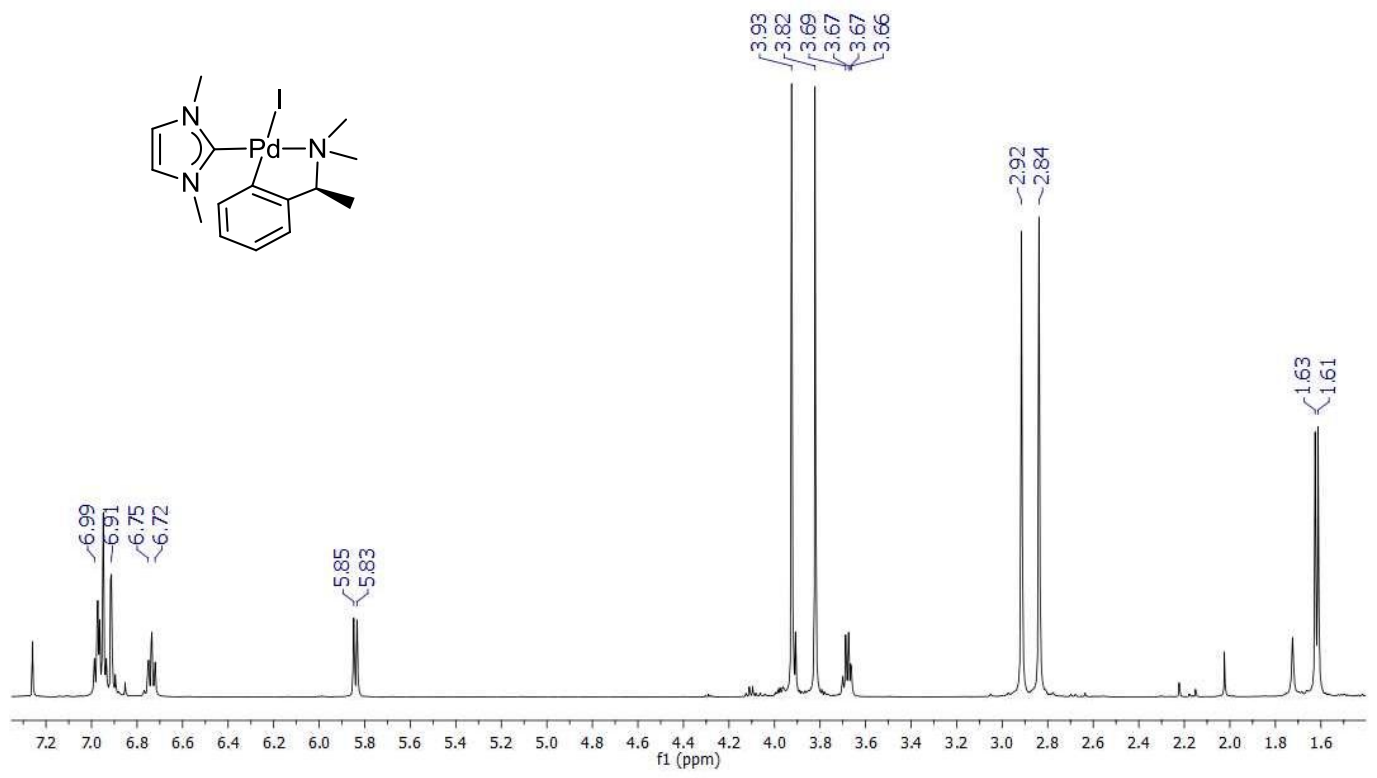

Figure 2.2 ${ }^{1} \mathrm{H}$ NMR spectrum of $\mathbf{1 D}$ in $\mathrm{CDCl}_{3}$

Figure 2.3 shows the ${ }^{13} \mathrm{C}\left\{{ }^{1} \mathrm{H}\right\}$ NMR spectrum of complex 1D. The most characteristic signal is the one attributed to the metalated carbene carbon at $173.1 \mathrm{ppm}$. The signal due to the ortho-metalated carbon of the phenyl ring appears at $154.8 \mathrm{ppm}$. The resonances due to the carbons of the two methyl groups of the N,N-dimethylbenzylamine appear as 
two singlets at 51.2 and $49.1 \mathrm{ppm}$. The signals due to the carbons of the methyl group of the imidazole ring are shown at 38.6 and $38.3 \mathrm{ppm}$.

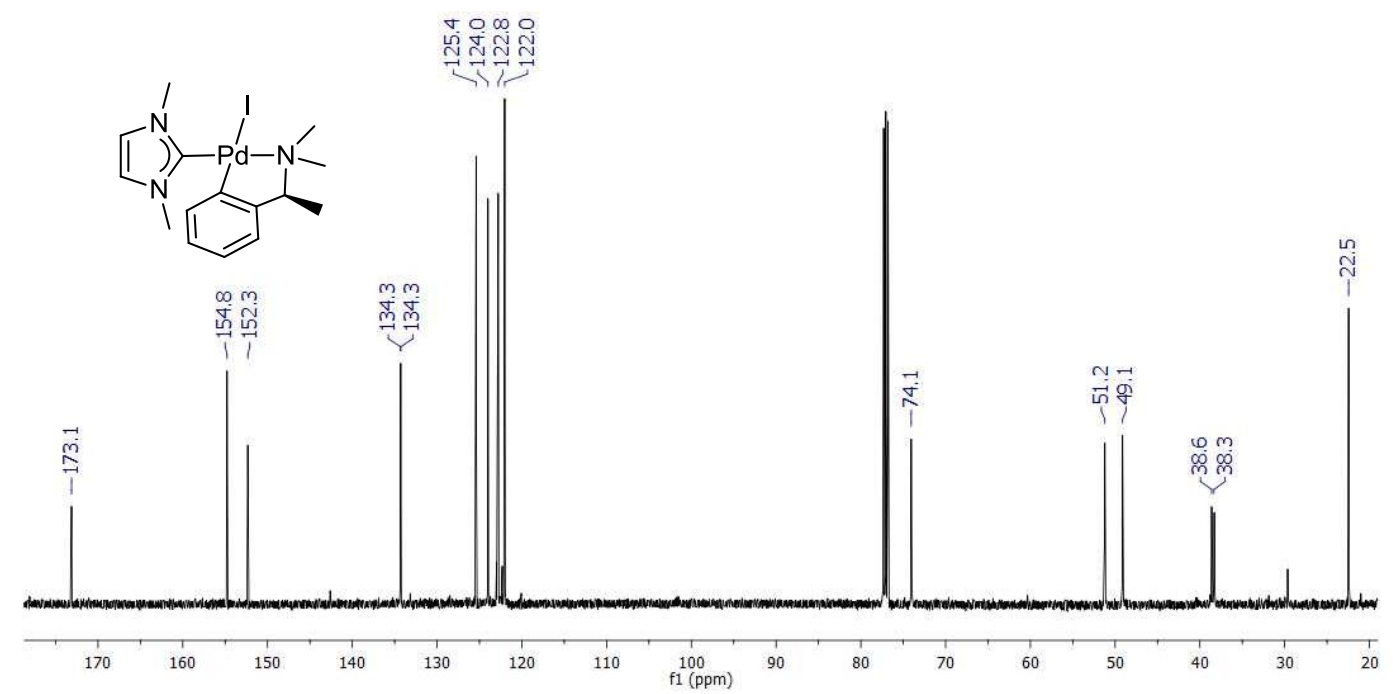

Figure $2.3{ }^{13} \mathrm{C}\left\{{ }^{1} \mathrm{H}\right\}$ NMR spectrum of $\mathbf{1 D}$ in $\mathrm{CDCl}_{3}$

\section{NMR Spectroscopic characterization of $\mathbf{1 E}$}

The ${ }^{1} \mathrm{H}$ NMR spectrum of $\mathbf{1 E}$ shows two distinct sets of signals due to the two Pd-NHC atropisomers in a 55/45 ratio, as determined from the integration of the representative signals for each isomer. This atropisomerism is consequence of the hindered rotation of the ligand about the $\mathrm{Pd}-\mathrm{C}_{\text {carbene }}$ bond (Scheme 2.6).

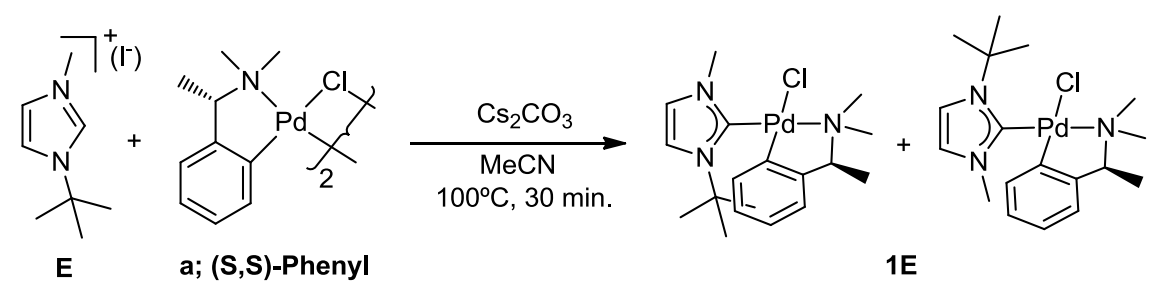

Scheme 2.6 Atropisomers formed during the preparation of unsymmetrical NHC ligands with palladacycles 
Figure 2.4 shows the ${ }^{1} \mathrm{H}$ NMR spectrum of 1E. The resonances due to the $\mathrm{H}$ next to the ortho-metalated carbon are shown as doublets at 5.88 and $5.77 \mathrm{ppm}\left({ }^{3} \mathrm{~J}_{\mathrm{H}, \mathrm{H}}=7.5 \mathrm{~Hz}\right)$. The most representative signals are the ones corresponding to the protons of the methyl group of the azole ring, at 4.07 and $3.98 \mathrm{ppm}$. The signals due to the benzylic $\mathrm{CH}$ proton of the $\mathrm{N}, \mathrm{N}$-dimethylbenzylamine ligand appear as quartets at 3.83 and $3.56 \mathrm{ppm}\left({ }^{3} \mathrm{~J}_{\mathrm{H}, \mathrm{H}}=6.6\right.$ $\mathrm{Hz}$ ). The singlets attributed to the protons of the two methyl groups of the N,Ndimethylbenzylamine ligand appear at 2.98, 2.97, 2.87 and $2.84 \mathrm{ppm}$. The protons of the methyl group at the chiral carbon appear as two doublets at $1.61 \mathrm{ppm}$ and $1.56 \mathrm{ppm}\left({ }^{3} \mathrm{~J}_{\mathrm{H}, \mathrm{H}}\right.$ $=6.5 \mathrm{~Hz})$.
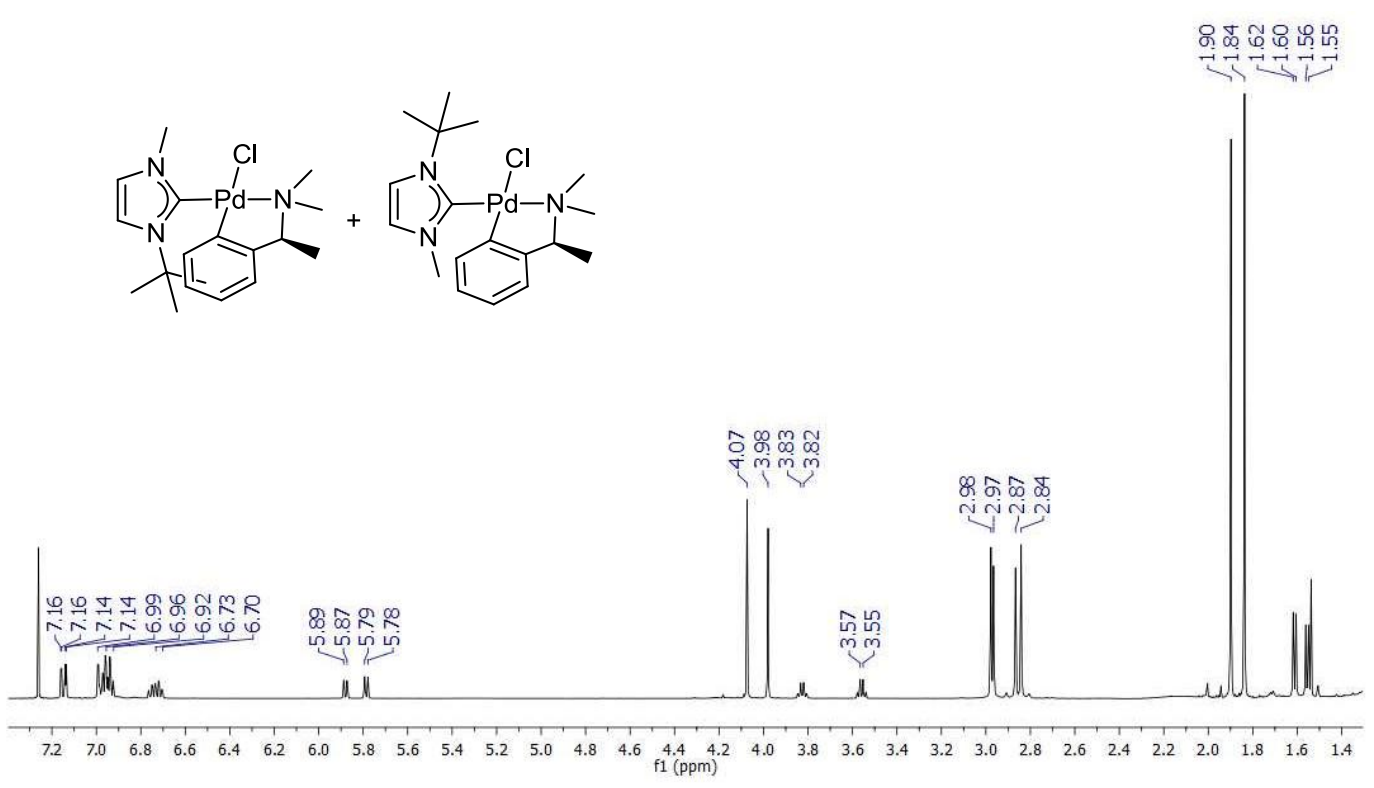

Figure 2.4 ${ }^{1} \mathrm{H}$ NMR spectrum of $\mathbf{1 E}$ in $\mathrm{CDCl}_{3}$

Figure 2.5 shows the ${ }^{13} \mathrm{C}\left\{{ }^{1} \mathrm{H}\right\}$ NMR spectrum of 1E. The spectrum also shows two distinct set of signals attributed to the two Pd-NHC atropisomers. The spectrum displays the representative signals due to the $\mathrm{Pd}-C_{\text {carbene }}$ of the two isomers at $170.3 \mathrm{ppm}$ and 169.7 ppm. The carbons of the two methyl groups of the N,N-dimethylbenzylamine appear as four different singlets at 52.2, 51.7, 50.42 and $48.02 \mathrm{ppm}$ respectively. The two signals attributed to the carbons of the methyl groups at the azole ring appear at 40.5 and 40.2 ppm. 


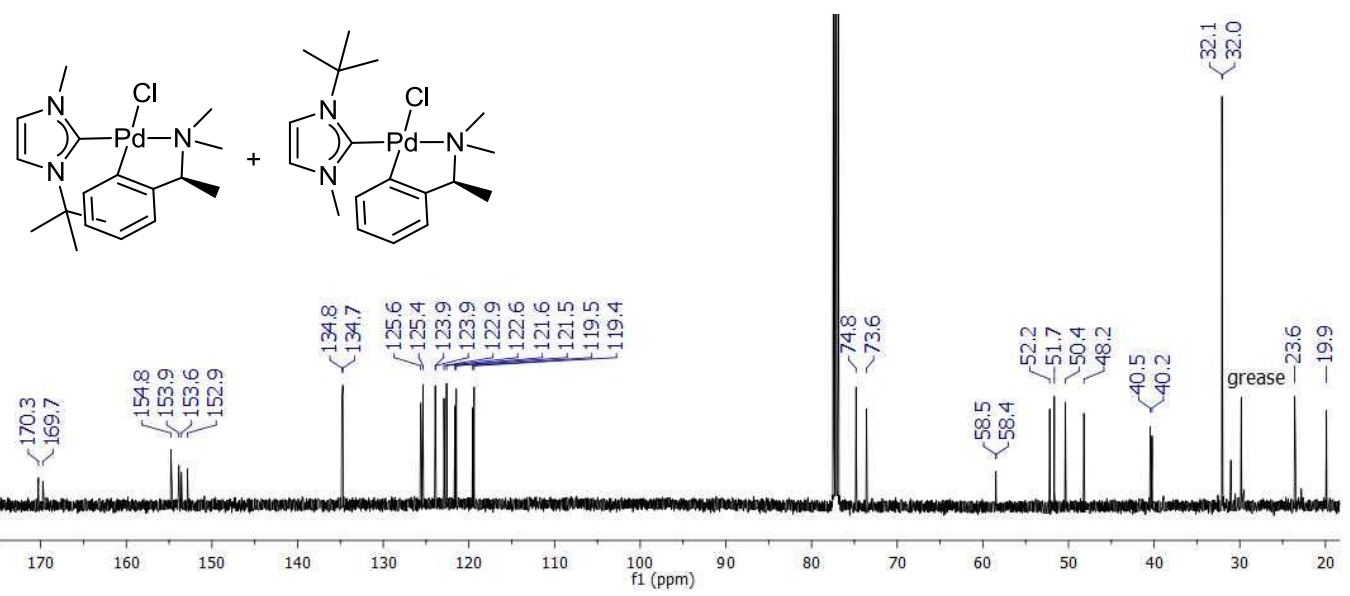

Figure $2.5{ }^{13} \mathrm{C}\left\{{ }^{1} \mathrm{H}\right\}$ NMR spectrum of complex $1 \mathrm{E}$ in $\mathrm{CDCl}_{3}$

In the case of complex $\mathbf{2 F}$, we were able to separate the two atropisomers by column chromatography, using a mixture of dichloromethane/ethyl acetate (9:1). The pure complexes 2Fa and 2Fb were characterized by ${ }^{1} \mathrm{H}$ NMR spectroscopy. Unfortunately, epimerization of both complexes was observed in solution overnight, as depicted in Scheme 2.7, rendering a 1:1 mixture of both atropisomers in solution. ${ }^{50,51}$ This made the accurate ${ }^{13} \mathrm{C}$ NMR characterization difficult to achieve.

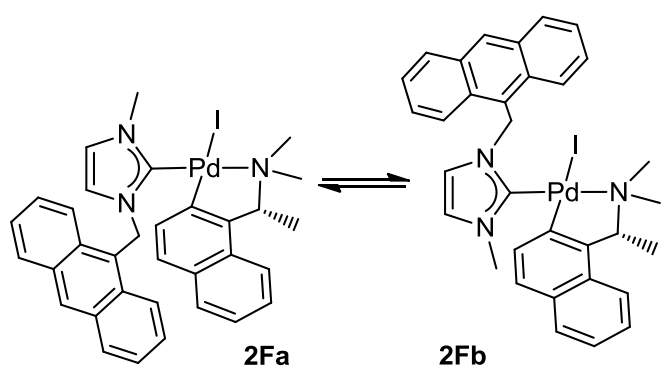

Scheme 2.7 Epimerization of both atropisomers of complex 2F

\section{Molecular structure of $\mathbf{1 D}$}

The molecular structure of compound 1D was confirmed by means of X-ray diffraction studies. The molecular structure consists of a palladium center with an iodide, an NHC ligand and a cyclometalated (S)-N,N-dimethylbenzylamine. The C-N ortho-metalated amine forms a five-membered ring in a puckered conformation. Two conformational 
isomers were found in the solid state (Figure 2.6, top). The conformational isomerism is due to two different positions that may adopt the methyl group attached to the benzylic carbon, axial or equatorial. At this point, it is worth mentioning that both isomers interconvert fast in solution, even at low temperatures (Figure 2.6, bottom). ${ }^{52-54}$ This situation clearly differs from that shown by complexes containing a naphthyl amine, for which the equatorial disposition is not affordable due to the steric hindrance of the naphthyl group, as was previously reported by Wild and co-workers. ${ }^{47}$

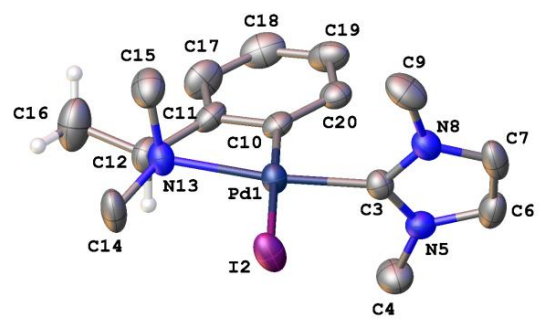

Equatorial

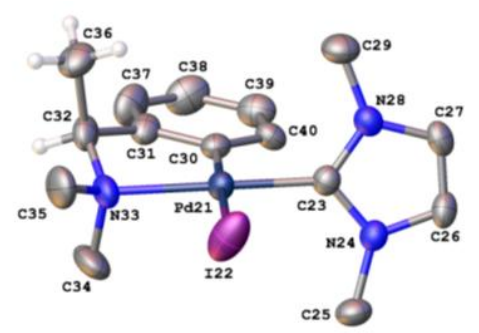

Axial
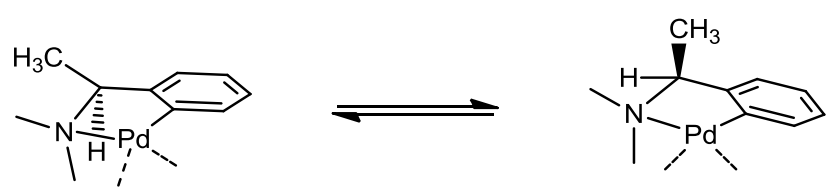

Figure 2.6 Top: Molecular diagram of complex 1D showing the two conformational isomers. Ellipsoids are shown at 50\% probability level. Hydrogens are omitted for clarity except at the benzylic position. Bottom: Schematic representation of the conformational isomerism equilibrium The most representative bond lengths and angles of the two isomers of 1D are shown in Table 2.2 (equatorial isomer), and Table 2.3 (axial isomer). The $\mathrm{Pd}-\mathrm{C}_{\text {carbene }}$ bond lengths are 1.979(5) and 1.961(5) $\AA$ for the equatorial and axial isomer, respectively.

Table 2.2 Selected bond lengths $(\AA)$ and angles $\left(^{\circ}\right)$ for the equatorial isomer of 1D

\begin{tabular}{|c|c|c|c|}
\hline Bonds & $(\AA ̊)$ & Angles & $\left({ }^{\circ}\right)$ \\
\hline $\operatorname{Pd}(1)-C(3)$ & $1.979(5)$ & $\mathrm{N}(13)-\mathrm{Pd}(1)-\mathrm{I}(2)$ & $97.71(11)$ \\
\hline $\operatorname{Pd}(1)-I(2)$ & $2.7195(5)$ & $N(13)-P d(1)-C(10)$ & $82.21(17)$ \\
\hline $\operatorname{Pd}(1)-C(10)$ & $2.166(3)$ & $\mathrm{C}(3)-\mathrm{Pd}(1)-\mathrm{I}(2)$ & $90.52(14)$ \\
\hline $\operatorname{Pd}(1)-\mathrm{N}(13)$ & $2.160(4)$ & $C(10)-C(11)-C(12)-C(16)$ & 160.3 \\
\hline
\end{tabular}


Table 2.3 Selected bond lengths $(\AA)$ and angles $\left(^{\circ}\right)$ for the axial isomer of 1D

\begin{tabular}{cccc}
\hline Bonds & $(\boldsymbol{\AA})$ & Angles & $\left.\mathbf{(}^{\mathbf{}}\right)$ \\
\hline $\mathrm{Pd}(21)-\mathrm{C}(23)$ & $1.961(5)$ & $\mathrm{N}(33)-\mathrm{Pd}(21)-\mathrm{I}(22)$ & $98.35(13)$ \\
$\mathrm{Pd}(21)-\mathrm{I}(22)$ & $2.6948(6)$ & $\mathrm{N}(33)-\mathrm{Pd}(21)-\mathrm{C}(30)$ & $82.19(19)$ \\
$\mathrm{Pd}(21)-\mathrm{C}(30)$ & $2.000(5)$ & $\mathrm{C}(23)-\mathrm{Pd}(21)-\mathrm{I}(22)$ & $88.91(14)$ \\
$\mathrm{Pd}(21)-\mathrm{N}(33)$ & $2.145(5)$ & $\mathrm{C}(30)-\mathrm{C}(31)-\mathrm{C}(32)-\mathrm{C}(36)$ & 91.3 \\
\hline
\end{tabular}

\subsubsection{Synthesis of NHC-palladacycles with primary amines}

For the preparation of NHC-based palladacycles with primary amines, when we followed the reaction conditions used for preparing palladacycles with tertiary amines, only decomposition products were observed. As an alternative, we used the alternative procedure shown in Scheme 2.8. The protocol consists of a stepwise coordination-orthometalation procedure. The reaction of imidazolium salts $\mathbf{D}$ and $\mathbf{F}$ with $\mathrm{PdCl}_{2}$, and chiral primary amine 5, in the presence of $\mathrm{Cs}_{2} \mathrm{CO}_{3}$ and $\mathrm{KI}$, in acetonitrile at $100^{\circ} \mathrm{C}$ for $2 \mathrm{~h}$, afforded NHC-palladium-amine complexes 3D and 3F. The complexes were washed with a saturated $\mathrm{NaCl}$ aqueous solution and were obtained as yellow solids after precipitation from a dichloromethane/hexane mixture, in good yields, 71\% (3D) and 79\% (3F). Subsequent reaction of $\mathbf{3 D}$ and $\mathbf{3 F}$ with silver acetate, in refluxing acetonitrile, facilitated the ortho-metalation of the amine, affording the ortho-metalated complexes $4 \mathbf{D}$ and $4 \mathbf{F}$. Complex 4D was washed with a saturated $\mathrm{NaCl}$ aqueous solution, and complex 4F was purified by column chromatography. Both complexes were precipitated from a dichloromethane/hexane mixture to give yellow solids in $80 \%$ (4D) and 56\% (4F) yield.

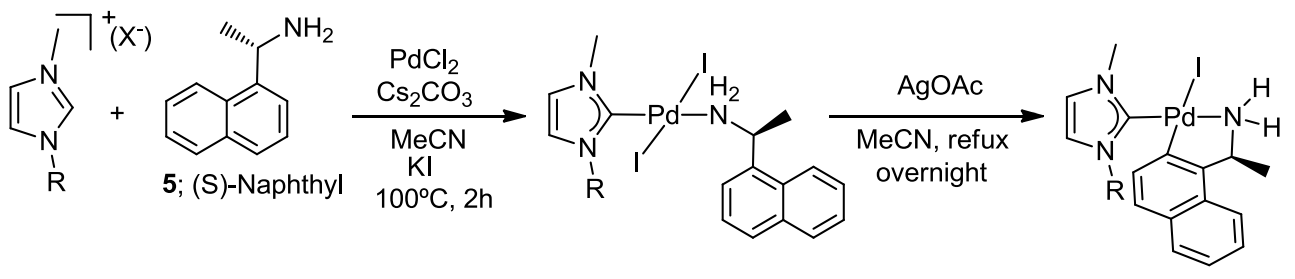

D; $\mathrm{R}=\mathrm{CH}_{3}, \mathrm{X}=\mathrm{I}$

F; $\mathrm{R}=9$-antr, $\mathrm{X}=\mathrm{Cl}$

3D; (S), $\mathrm{R}=\mathrm{CH}_{3}$

3F; (S), $\mathrm{R}=9$-antr
4D; (S), $\mathrm{R}=\mathrm{CH}_{3}$

4F; (S), $\mathrm{R}=9$-antr

Scheme 2.8 Synthesis of NHC-based palladacycles with primary amines 
All palladium complexes were characterized by means of NMR spectroscopy, mass spectrometry and elemental analysis. Due to the similarity of these complexes with the analogous bearing tertiary amines, which were described previously, only the ${ }^{1} \mathrm{H}$ NMR of complex 3D will be discussed in detail.

\section{${ }^{1} H$ NMR spectrum of complex $\mathbf{3 D}$}

Figure 2.7 shows the ${ }^{1} \mathrm{H}$ NMR spectrum of complex 3D. The most characteristic signals are those attributed to the $\mathrm{CH}$ and $\mathrm{CH}_{3}$ protons of the imidazolyl ligand, which appear as singlets at 6.88 and $3.84 \mathrm{ppm}$, indicating a two-fold symmetry of the complex. The resonances due to the aromatic protons of the naphthyl group appear between 8.46 and 7.44 ppm. The signals corresponding to the $\mathrm{CH}$ benzylic and $\mathrm{NH}_{2}$ protons of the naphthylethylamine are shown as multiplets between 5.63-5.39 ppm and 2.92-2.57 ppm, respectively. The protons of the methyl group at the chiral carbon appear as doublet at $1.99 \mathrm{ppm}\left({ }^{3} \mathrm{~J}_{\mathrm{H}, \mathrm{H}}=6.8 \mathrm{~Hz}\right)$.
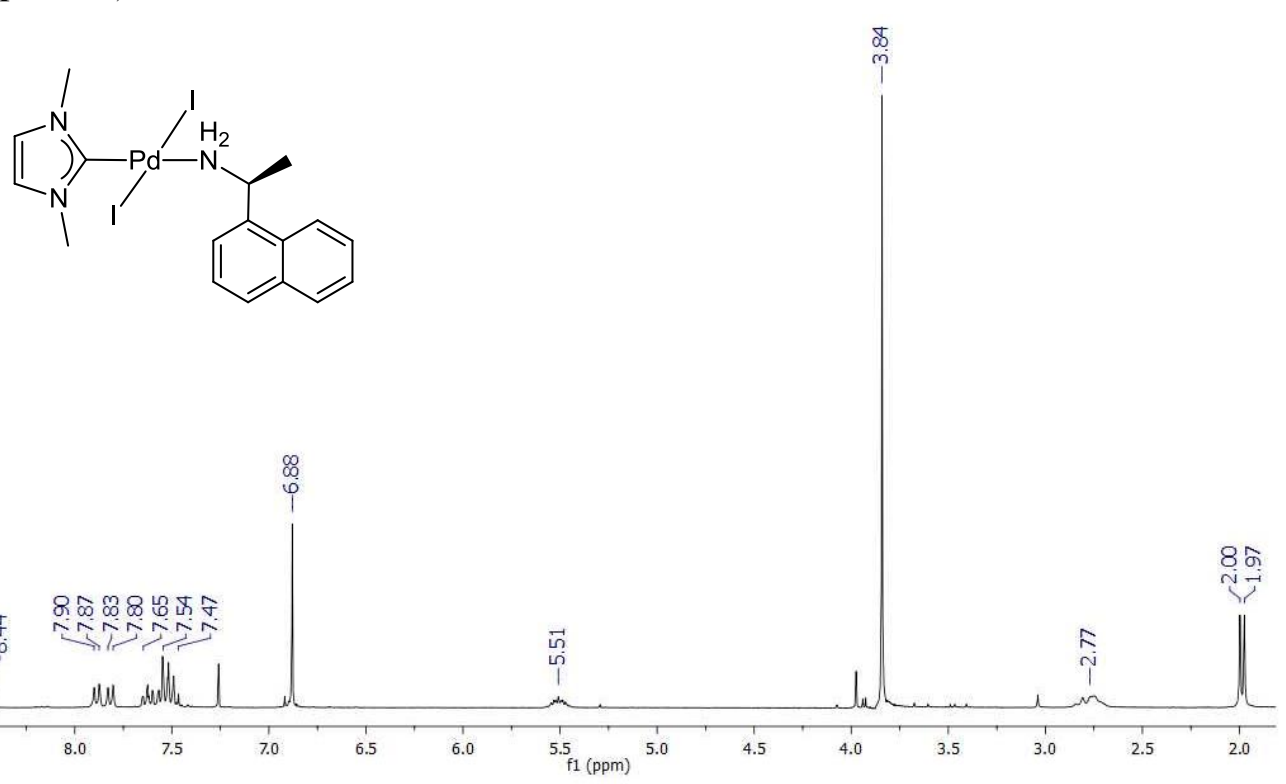

Figure 2.7 ${ }^{1} \mathrm{H}$ NMR spectrum of complex 3D in $\mathrm{CDCl}_{3}$

The molecular structures of compounds 3D and 4D were confirmed by means of X-ray diffraction studies. 


\section{Molecular structure of complex $\mathbf{4 D}$}

Crystals of 4D suitable for X-ray diffraction studies were obtained by slow diffusion of hexane in a concentrated solution of the complex in dichloromethane. The molecular structure of 4D shown in Figure 2.8 confirms that the ortho-metalation of the chiral primary amine has taken place. The C-N ortho-metalated amine forms a five-membered ring, with the methyl group attached at the chiral carbon in an axial disposition. In this case, the conformational isomerism observed in complex 1D is blocked due to the steric hindrance of the naphthyl group. Table 2.4 shows the most representative bond lengths and angles. The $\mathrm{Pd}-\mathrm{C}_{\text {carbene }}$ bond length is 1.975(7), similar to the related one shown in 1D (1.979(5), 1.961(5)).

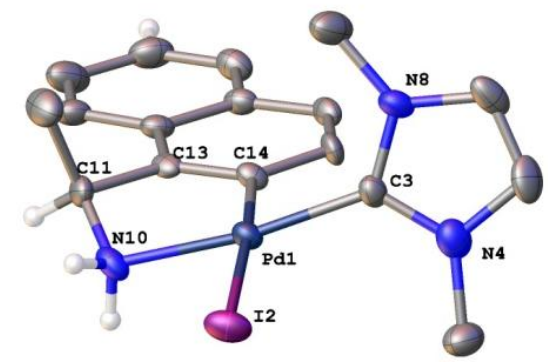

Figure 2.8 Molecular diagram of complex 4D. Ellipsoids are shown at 50\% probability level

Table 2.4 Selected bond lengths $(\AA)$ and angles $\left({ }^{\circ}\right)$ of complex 4D

\begin{tabular}{|c|c|c|c|}
\hline Bonds & $(\AA)$ & Angles & $\left({ }^{\circ}\right)$ \\
\hline $\operatorname{Pd}(1)-C(3)$ & $1.975(7)$ & $C(3)-\operatorname{Pd}(1)-I(2)$ & $93.30(19)$ \\
\hline $\operatorname{Pd}(1)-\mathrm{N}(10)$ & $2.095(5)$ & $C(14)-P d(1)-C(3)$ & $92.0(3)$ \\
\hline $\operatorname{Pd}(1)-I(2)$ & $2.6768(7)$ & $\mathrm{N}(10)-\operatorname{Pd}(1)-\mathrm{I}(2)$ & $93.85(15)$ \\
\hline $\operatorname{Pd}(1)-C(14)$ & $1.993(6)$ & $\mathrm{N}(10)-\mathrm{Pd}(1)-\mathrm{C}(14)$ & $81.1(2)$ \\
\hline
\end{tabular}




\subsection{Synthesis of heterodimetallic Ir-Pd* chiral complexes}

The reaction of $\left[\operatorname{IrCp}^{*} \mathrm{Cl}_{2}\right.$ (triazolium-ylidene) $] \mathrm{BF}_{4}, \quad \mathbf{1 A H}$, with chiral N,Ndimethylbenzylaminate palladacycles (b and $\mathbf{c}$ ), in the presence of $\mathrm{NaOAc}$ in acetone at $50^{\circ} \mathrm{C}$ for $15 \mathrm{~h}$, afforded $\mathrm{Ir}(\mathrm{III}) / \mathrm{Pd}(\mathrm{II})$ complexes $9 \mathrm{~A}$ and $\mathbf{1 0 A}$. Both complexes were washed with a saturated $\mathrm{NaCl}$ aqueous solution and precipitated from a dichloromethane/hexane mixture to give yellow solids in 98 and $97 \%$ yield, respectively.

Similar complexes with ortho-metalated primary amines (11A and 12A) were obtained after reaction of acetato-bridged palladacycles (e and $\mathbf{f}$ ) and complex $\mathbf{1 A H}$, in the presence of $\mathrm{NaCl}$, in acetone at $50^{\circ} \mathrm{C}$ for $15 \mathrm{~h}$. In this particular case, the addition of an external base was not necessary, as the acetate bridge acted as an internal base for the deprotonation of the azolium ring in $\mathbf{1 A H}$ (Scheme 2.9). Complexes 11A and 12A were obtained as pale-yellow crystalline solids after recrystallization in dichloromethane/hexane mixtures in $93 \%$ and $82 \%$ yield, respectively.

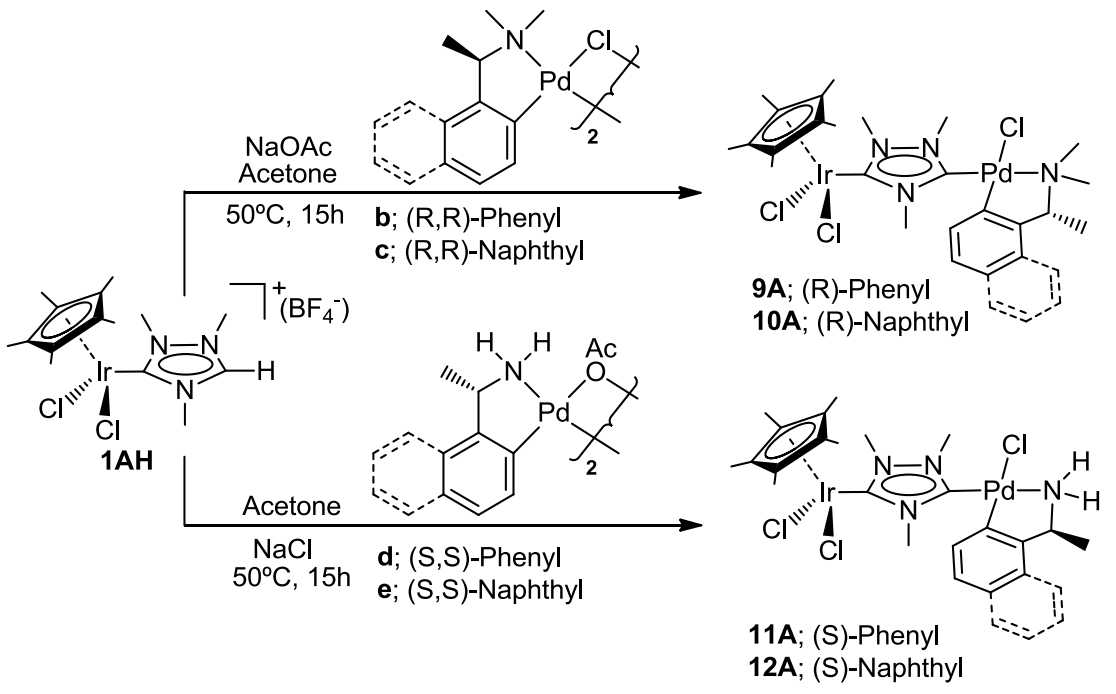

Scheme 2.9 Synthesis of heterodimetallic complexes 9A-12A

Complexes 9A - 12A were characterized by NMR spectroscopy, High Resolution Mass Spectrometry (HRMS) and elemental analysis. Due to the similarity of these compounds, 
only the spectroscopic characterization of $9 \mathbf{A}$ is discussed in detail. The characterization of the rest of complexes are detailed in the Experimental Section (Chapter 6).

\section{${ }^{1} H$ NMR spectrum of complex $9 \boldsymbol{A}$}

Figure 2.9 shows the ${ }^{1} \mathrm{H}$ NMR spectrum of complex 9A. The first evidence of coordination of the palladium center is the disappearance of the signal of the $\mathrm{NCHN}$ proton of the starting complex $\mathbf{1} \mathbf{A H}$. The signals attributed to the aromatic protons are shown between $\delta 7.02$ and $\delta 6.38$. The signals due to the protons of the methyl groups at the azole bridge are shown as multiplet between 4.44 and $4.24 \mathrm{ppm}$. The spectrum shows four different singlets at 2.86, 2.84, 2.70 and $2.68 \mathrm{ppm}$ corresponding to the protons of the two different methyl groups of the N,N-dimethylbenzylamine, indicating the presence in a 60:40 ratio of two atropisomers. Further evidences of the presence of two rotamers arises from the observation of the signals due to the benzylic $\mathrm{CH}$ protons, which appear as broad singlets at 3.87 and $374 \mathrm{ppm}$, and those attributed to the protons of the methyl groups at the $\mathrm{Cp}^{*}$ at 1.68 and $1.66 \mathrm{ppm}$. The signals due to the protons of the methyl group attached to the chiral carbon appear as doublets at 1.62 and $1.57 \mathrm{ppm}$. Variabletemperature ${ }^{1} \mathrm{H}$ NMR $\left(\mathrm{CDCl}_{3}, 60^{\circ} \mathrm{C}\right)$ studies of complex 9A confirmed that there is no rotation about the $\mathrm{M}-\mathrm{C}_{\text {carbene }}$ bond on the NMR timescale.
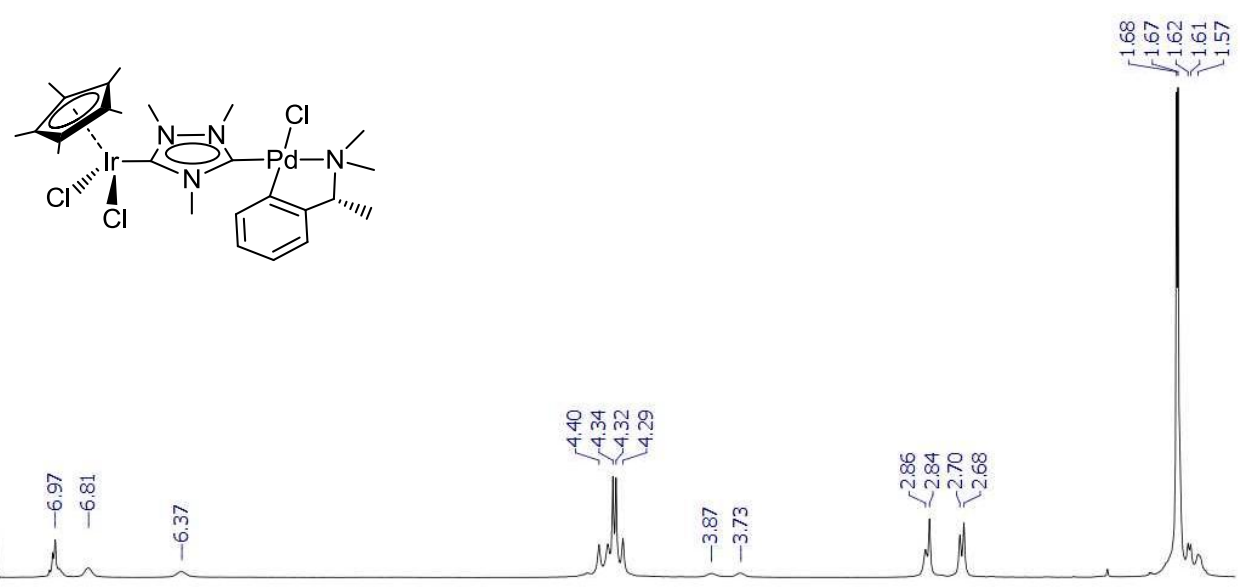

$\begin{array}{llllllllllllllllllllllllllllllllllllllll}7.4 & 7.2 & 7.0 & 6.8 & 6.6 & 6.4 & 6.2 & 6.0 & 5.8 & 5.6 & 5.4 & 5.2 & 5.0 & 4.8 & 4.6 & 4.4 & 4.2 & 4.0 & 3.8 & 3.6 & 3.4 & 3.2 & 3.0 & 2.8 & 2.6 & 2.4 & 2.2 & 2.0 & 1.8 & 1.6 & 1.4\end{array}$

Figure 2.9 ${ }^{1} \mathrm{H}$ NMR spectrum of $9 \mathrm{~A}$ at $60^{\circ} \mathrm{C}$ in $\mathrm{CDCl}_{3}$ 


\section{${ }^{13} \mathrm{C}\left\{{ }^{1} \mathrm{H}\right\}$ NMR spectrum of complex $9 \mathrm{~A}$}

Figure 2.10 shows the ${ }^{13} \mathrm{C}\left\{{ }^{1} \mathrm{H}\right\}$ NMR spectrum of complex 9A. The most relevant signals are the ones shown at 182.9 and $167.6 \mathrm{ppm}$, attributed to the carbene-carbons bound to $\mathrm{Pd}$ and $\mathrm{Ir}$, respectively. The signals due to the benzylic $\mathrm{CH}$ carbon are shown at 74.4 and $74.0 \mathrm{ppm}$. The singlets attributed to the carbons of the methyl groups of the amine appear at 50.7, 50.2, 46.1, $45.2 \mathrm{ppm}$. The signals due to the carbons of the methyl groups at the azole ring appear at 41.3, 38.4, 38.0 and $37.5 \mathrm{ppm}$. The signals corresponding to the carbon of the methyl group attached to the chiral carbon appear at 22.0 and $19.3 \mathrm{ppm}$.
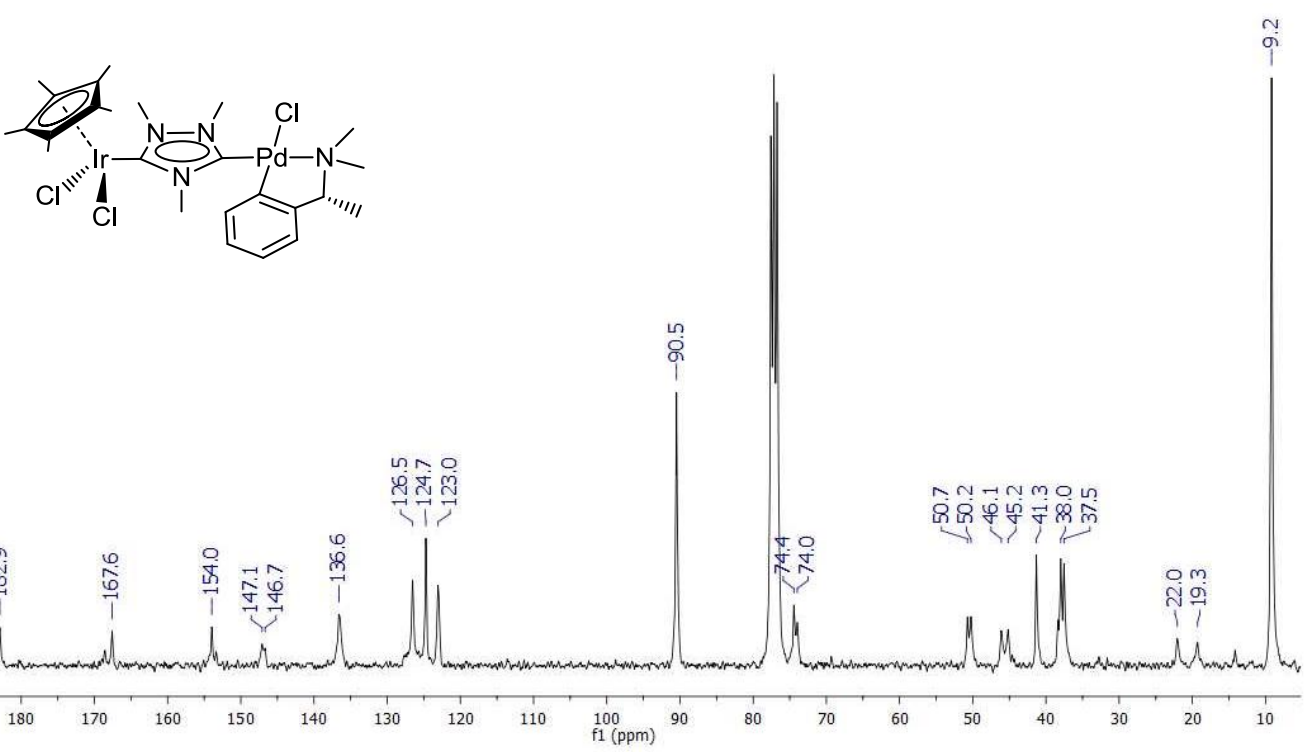

Figure 2.10 ${ }^{13} \mathrm{C}\left\{{ }^{1} \mathrm{H}\right\}$ NMR spectrum of $9 \mathrm{~A}$ in $\mathrm{CDCl}_{3}$

The presence of different isomers is consequence of the hindered rotation about the Ir$\mathrm{C}_{\text {carbene }}$ and $\mathrm{Pd}-\mathrm{C}_{\text {carbene }}$ bonds. The stereodynamics of other M-NHC complexes using monocarbene ligands with restricted $\mathrm{M}-\mathrm{C}_{\text {carbene }}$ rotation have been previously described, supporting the steric origin of the rotation barrier. ${ }^{50,51,55,56}$ The steric repulsions inhibit de $\mathrm{M}-\mathrm{C}_{\text {carbene }}$ rotation also in complexes $10 \mathrm{~A}-\mathbf{1 2 A}$. 
The bimetallic nature of the compounds was also confirmed by the data resulting from the electrospray mass spectrometry (ESI-MS), based on the mass/charge relation and the isotopic pattern of peaks. As an illustrative example, Figure 2.11 shows the positive ion electrospray mass spectrum of the bimetallic complex 9A in acetonitrile, displaying an intense peak for the $[\mathrm{M}-\mathrm{Cl}]^{+}$fragment at $763.1 \mathrm{~m} / \mathrm{z}$. The experimental isotopic pattern distribution fits with the theoretical simulation.

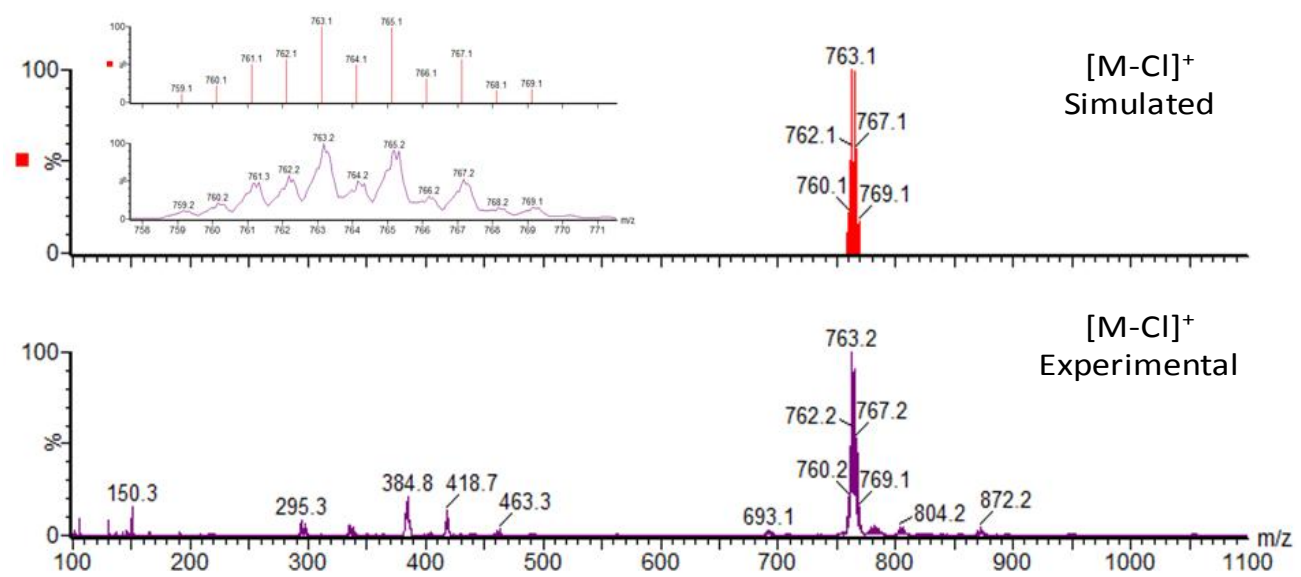

Figure 2.11 ESI-MS spectrum in acetonitrile solution of complex $\mathbf{9 A}$ and simulation of the molecular peak

Complexes bearing planar ligands with $C_{l}$-symmetry, such as triazole-ylidenes, in a perpendicular orientation relative to the coordination plane of the complex, present axial chirality. Monometallic square-planar palladium (II) complexes, containing two asymmetrically substituted NHC ligands, and therefore two axis of chirality, have been described previously. ${ }^{50,51,57}$

The $\mathrm{Ir} / \mathrm{Pd}^{*}$ heterodimetallic complexes $9 \mathrm{~A}-12 \mathrm{~A}$ contain two axes of chirality. One of the axes is defined by the $\mathrm{Ir}-\mathrm{C}_{\text {carbene }}$ bond and the other by the Pd- $\mathrm{C}_{\text {carbene }}$ bond (Scheme 2.10). 


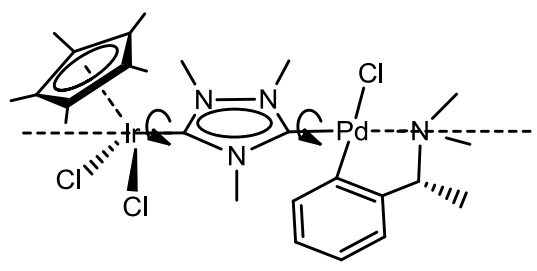

Scheme 2.10 Schematic representation of complex 9A showing the two axes of chirality

For the iridium center the substituents of the reference plane are the Ir atom and the $\mathrm{Cp}^{*}$ centroid, whereas the substituents of the second plane are the N-1and N-4 nitrogens next to the $\mathrm{C}_{\text {carbene. }}$ For the Palladium center the substituents in the reference plane are the chlorine atom and the phenyl ring trans to it, and in the second plane the N-2 and N-4 nitrogens next to the $\mathrm{C}_{\text {carbene }}$ (Scheme 2.11). The priorities have been assigned according to the Cahn-Ingold-Prelog (CIP) rules and termed as $\mathrm{R}_{\mathrm{aM}}$ or $\mathrm{S}_{\mathrm{aM}}(\mathrm{M}=\mathrm{Ir}$ or $\mathrm{Pd})$, depending of the chiral axis considered. ${ }^{58}$ According to these considerations all possible isomers are depicted in scheme 2.11 .
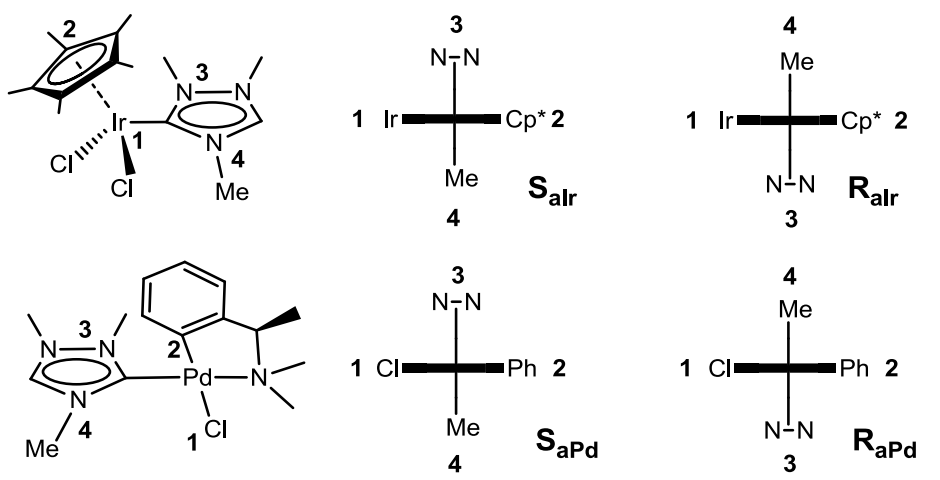

Scheme 2.11 Axes of chirality at iridium and palladium centers

Scheme 2.12 shows the lateral and front views of the four possible atropisomers that may be formed for the heterodimetallic Ir/Pd* complexes bearing a stereogenic chiral carbon at the cyclometalated ligand. 


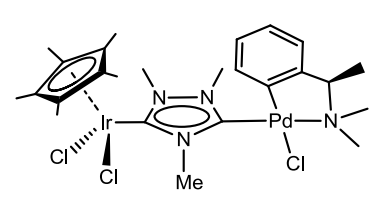

a) $S_{a \mid r}, S_{a P d}, R_{C}$

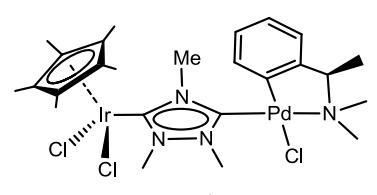

c) $R_{\mathrm{alr}}, \mathrm{R}_{\mathrm{aPd}}, \mathrm{R}_{\mathrm{C}}$
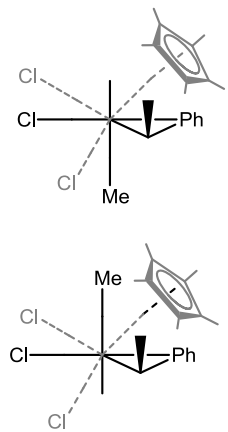
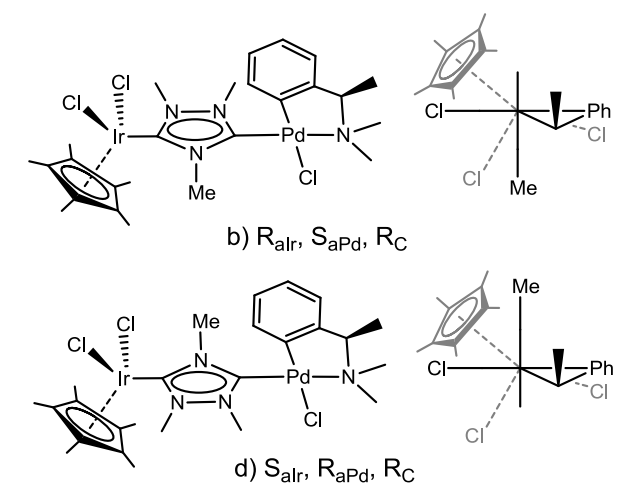

Scheme 2.12 Lateral and front views of the four possible atropisomers of complex 9A

\section{Molecular structure of complex $\mathbf{9 A}$}

The molecular structure of compound 9A was confirmed by means of X-ray diffraction studies. Single crystals were obtained by slow evaporation of dichloromethane/hexane mixtures. The crystal structure contains two of the four possible atropisomers (Figure 2.12), which expectedly are the ones that were detected by ${ }^{1} \mathrm{H}$ NMR spectroscopy.
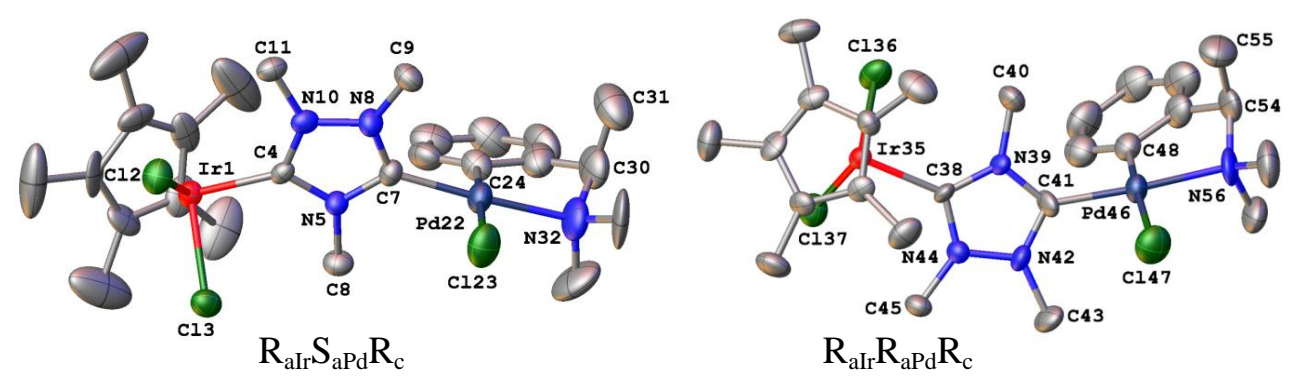

Figure 2.12 Molecular diagram of complex 9A. Ellipsoids are shown at 50\% probability level. Hydrogens omitted for clarity.

The absolute configuration of the atropisomers is $\mathrm{R}_{\mathrm{aIr}} \mathrm{S}_{\mathrm{aPd}} \mathrm{R}_{\mathrm{c}}$ (left) and $\mathrm{R}_{\mathrm{aII}} \mathrm{R}_{\mathrm{aPd}} \mathrm{R}_{\mathrm{c}}$ (right). The structure consists of a $\mathrm{Cp} * \mathrm{IrCl}_{2}$ connected, by a triazole-di-ylidene bridge to a palladium having a chloride, and a chelated (S)-N,N-dimethylbenzylamine. Both structures exhibit the expected square-planar coordination at the $\mathrm{Pd}(\mathrm{II})$ center, with the triazole ring nearly perpendicular to the coordination plane, and the expected pseudo- 
octahedral coordination at the $\operatorname{Ir}(\mathrm{III})$ center, as deduced form the torsional angle $\mathrm{Cl}(2)$ $\operatorname{Ir}(1)-\mathrm{Cl}(3) 85.14(8)^{\circ}$ and $\mathrm{Cl}(36)-\operatorname{Ir}(35)-\mathrm{Cl}(37) 84.98(9)^{\circ}$. The C-N ortho-metalated amine forms a five-membered ring that adopts an envelope conformation. The methyl group (C31 or C55) attached at the benzylic carbon adopts an axial disposition in both isomers. In the $\mathrm{R}_{\mathrm{aIr}} \mathrm{S}_{\mathrm{aPd}} \mathrm{R}_{\mathrm{c}}$ (left) isomer, the relative orientation of the Cp* and the phenyl ring is sin. The methyl group $(\mathrm{C} 31)$ is pointing towards the two methyls $(\mathrm{C} 9$ and $\mathrm{C} 11)$ of the triazole-di-ylidene ligand. For the $\mathrm{R}_{\mathrm{aIr}} \mathrm{R}_{\mathrm{aPd}} \mathrm{R}_{\mathrm{c}}$ (right) isomer, the relative orientation of the $\mathrm{Cp}^{*}$ and the phenyl ring is anti, and the methyl group attached at the benzylic carbon (C55) is pointing towards the side of the single methyl (C40) of the triazole-di-ylidene ligand. The bond lengths and angles in both isomers are very similar. The $\mathrm{Pd}-\mathrm{C}_{\text {carbene }}$ bond lengths are 1.970(8) $\AA$ and 1.963(8) $\AA$ for $\mathrm{R}_{\mathrm{aIr}} \mathrm{S}_{\mathrm{aPd}} \mathrm{R}_{\mathrm{c}}$ and $\mathrm{R}_{\mathrm{aIr}} \mathrm{S}_{\mathrm{aPd}} \mathrm{R}_{\mathrm{c}}$, respectively. The Ir$\mathrm{C}_{\text {carbene }}$ bond lengths are 2.027(7) $\AA$ and 2.039(7) $\AA$ for $\mathrm{R}_{\mathrm{aIr}} \mathrm{S}_{\mathrm{aPd}} \mathrm{R}_{\mathrm{c}}$ and $\mathrm{R}_{\mathrm{aIr}} \mathrm{S}_{\mathrm{aPd}} \mathrm{R}_{\mathrm{c}}$, respectively, and lie in the expected range for $\mathrm{Cp} * \operatorname{Ir}(\mathrm{NHC})$ complexes. ${ }^{59,60}$

\subsection{Catalytic studies}

\subsubsection{Asymmetric hydrophosphination using chiral palladacycles with NHC ligands}

The chiral palladacycles with NHC ligands, described in section 2.2, were tested as catalysts in hydrophosphination, using diphenylphosphine and 1,3-diphenyl-2(E)-propen1-one (trans-chalcone) as model substrates (Scheme 2.13).

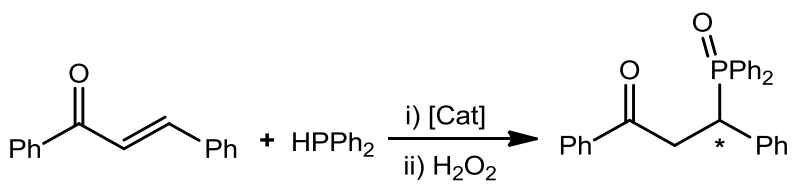

Scheme 2.13 Asymmetric hydrophosphination of trans-chalcone

The reactions were performed at room temperature with a catalyst loading of 2 mol\% under base-free conditions. The activation of the catalyst was performed by adding base ( $5 \mathrm{~mol} \%$ of $\mathrm{K}_{2} \mathrm{CO}_{3}$ ) and allowing the reaction with the precatalyst for two hours at room temperature. A slight excess of diphenylphosphine (1.2 equivalents), related to transchalcone, and nitrogen atmosphere were used in order to minimize the possible effects produced by the potential oxidation of the phosphine. After $15 \mathrm{~h}$ the reactions were 
quenched with hydrogen peroxide and ethyl acetate, and the products were purified by column chromatography as phosphine oxides, following the previous described methodologies. ${ }^{5}$ The $e e$ values were determined by HPLC chromatography using a Daicel Chiracel IC column.

The results in Table 2.5 show that all complexes tested performed high activity in terms of conversions and yields. The reaction produced moderate to good yields, even at low temperatures $\left(25^{\circ} \mathrm{C}\right.$ or lower). In all cases, the hydrophosphination proceeded with excellent regioselectivity, with the phosphorous addition occurring at the $\mathrm{C} 3$ position of the trans-chalcone.

Table 2.5 Selected data for trans-chalcone hydrophosphination

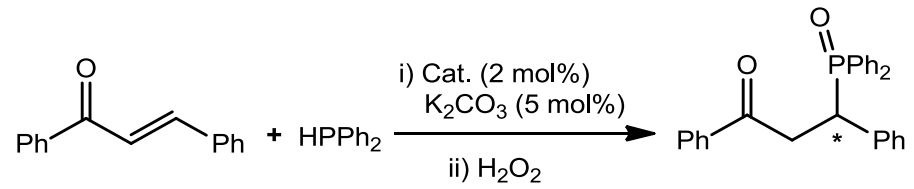

\begin{tabular}{cccccc}
\hline Entry & Catalyst & Solvent & Temp $\left({ }^{\mathbf{C}} \mathbf{C}\right)$ & ${\text { Yield }(\%)^{\mathbf{a}}}^{\text {a }}$ & ee (\%) $)^{\mathbf{b}}$ \\
\hline 1 & $\mathbf{f}$ & $\mathrm{CH}_{2} \mathrm{Cl}_{2}$ & 25 & 77 & 0 \\
2 & $\mathbf{2 D}$ & $\mathrm{CH}_{2} \mathrm{Cl}_{2}$ & 25 & 80 & 35 \\
3 & $\mathbf{2 E}$ & $\mathrm{CH}_{2} \mathrm{Cl}_{2}$ & 25 & 51 & 9 \\
4 & $\mathbf{2 F}$ & $\mathrm{CH}_{2} \mathrm{Cl}_{2}$ & 25 & 91 & 56 \\
5 & $\mathbf{2 F}$ & $\mathrm{CH}_{2} \mathrm{Cl}_{2}$ & -30 & 90 & 14 \\
6 & $\mathbf{2 F}$ & $\mathrm{Toluene}$ & 25 & 85 & 32 \\
7 & $\mathbf{2 F}$ & $\mathrm{THF}$ & 25 & 57 & 55 \\
8 & $\mathbf{4 D}$ & $\mathrm{CH}_{2} \mathrm{Cl}_{2}$ & 25 & 66 & 25 \\
\hline
\end{tabular}

Reactions carried out with $0.5 \mathrm{mmol}$ of trans-chalcone, $\mathrm{HPPh}_{2}(0.6 \mathrm{mmol}), 2 \mathrm{~mL}$ of solvent for 10 hours. [a] Isolated yields after column chromatography. [b] Determined using HPLC.

The chiral palladacycle $\mathbf{f}$ did not afford any enantiomeric excess (entry 1 ), while NHCpalladacycles 2D, 2E and 4D afforded modest enantioselectivities (entries 2, 3 and 8). Complex 2D, which contains a cyclometalated tertiary amine, yielded better outcomes in terms of activity and selectivity than complex $\mathbf{4 D}$, which contains the analogous primary amine (compare entries 2 and 8). The best results, albeit moderate, were obtained for catalyst 2F (entry 4), which contains the NHC ligand with the bulkier 9-methylantracyl 
group and a chelating N,N-dimethyl naphthyl benzylamine. This result compares well with previously published results by Leung and co-workers, in which a bis-acetonitrile palladium (II) complex with the same cyclometalated amine is used, although in their case catalyst loadings of $5 \mathrm{~mol} \%$ and temperatures of $-40^{\circ} \mathrm{C}$ were found to be optimum. ${ }^{4}$

In order to improve the catalytic outcomes, a solvent screening for the asymmetric hydrophosphination was performed. Dichloromethane was found to afford the best outcomes compared to toluene and tetrahydrofuran (compare entries 4, 6 and 7). For the reactions carried out in toluene, both yield and enantiomeric excess decreased. When the reactions were performed in tetrahydrofuran, the enantioselectivity did not change but the activity of the catalyst was reduced.

Interestingly, the enantiomeric excess decreased from $56 \%$ to $14 \%$ when lowering the temperature form $25^{\circ} \mathrm{C}$ to $-30^{\circ} \mathrm{C}$ (compare entries 4 and 5). Although we do not have a reasonable explanation for this behavior, there are some relevant examples in the literature in which a similar effect is observed. ${ }^{61}$ Some authors have attributed this abnormal effect of the temperature on the enantioselectivity to the dimerization of the precatalyst at low temperatures or differences in the solubility of the active catalytic species. $^{62}$

\subsubsection{Sequential isomerization of propargylic alcohols/hydrophosphination using Ir/Pd* chiral complexes}

The isomerization of propargyl alcohols provides straightforward synthetic routes to $\alpha-\beta$ unsaturated carbonyl compounds. The isomerization process may occur through three different reaction pathways: the Meyer-Schuster and Rupe rearrengements (Scheme 2.14, path a and b), in which a formal 1,3- or 1,2-shift of the hydroxyl group of the propargylic alcohol takes place, and the redox isomerization involving a simultaneous oxidation of the alcohol unit and the reduction of the $\mathrm{C} \equiv \mathrm{C}$ bond, i.e. not involving the transposition of the hydroxyl group (Scheme 2.14, path c).

The isomerization of propargyl alcohols ${ }^{63}$ is a reaction typically catalyzed by $\mathrm{Ru},{ }^{64,65}$ $\mathrm{Au},{ }^{66,67}$ and $\mathrm{Ag}^{68}$ complexes, which usually follow the Meyer-Schuster or Rupe 
rearrangement pathways. Otherwise, iridium complexes are known to facilitate the redox isomerization of internal propargylic alcohols. ${ }^{69,70}$

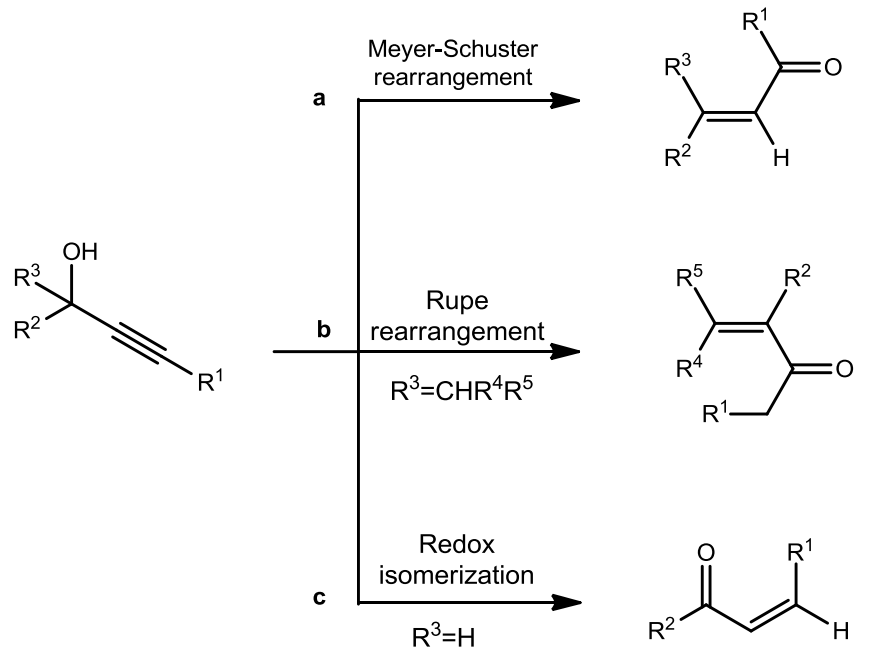

Scheme 2.14 Possible reaction pathways for the isomerisation of propargylic alcohols

The chiral heterodimetallic complexes 9A-12A were tested in a combination of two sequential catalytic reactions; the isomerization of 1,3-diphenylpropargyl alcohol and the asymmetric hydrophosphination of the resulting enone (Scheme 2.15). We wanted to explore the effect of the two metal centers in the bimetallic complexes in the final outcome of this sequential catalytic reaction, in which a stereocenter is created.

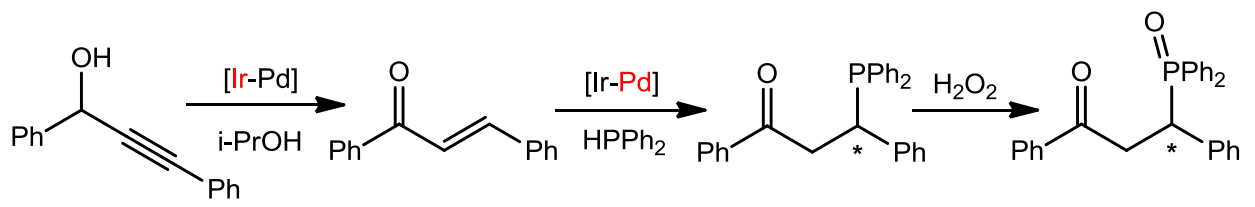

Scheme 2.15 Sequential isomerization of 1,3-diphenylpropargyl alcohol and hydrophosphination of the chalcone formed

Although both catalytic reactions have been studied deeply, there are no examples of such tandem reaction reported in the literature, so we believed that the study of this reaction may provide some interesting results. 
The sequential tandem reactions were performed in a stepwise manner, using a catalyst loading of $2 \mathrm{~mol} \%$ in the presence of $\mathrm{K}_{2} \mathrm{CO}_{3}$, under nitrogen atmosphere and 2-propanol. In a typical catalytic experiment, the isomerization was carried out for $5 \mathrm{~h}$ at $90^{\circ} \mathrm{C}$. The yield of trans-chalcone was determined by GC chromatography using anisole as internal standard. Then diphenylphosphine was added and the mixture was stirred for $10 \mathrm{~h}$ at room temperature. The final product was purified by column chromatography and isolated as phosphine oxide after being treated with hydrogen peroxide. The enantiomeric excess was determined by HPLC chromatography using an analogue procedure to the one described in Section 2.4.1: Asymmetric hydrophosphination with chiral palladacycles with NHC ligands.

The results shown in Table 2.6 indicate that catalysts $\mathbf{9 A - 1 2 A}$ are highly active in the isomerization process. Quantitative yields were obtained in short reaction times and mild reaction conditions (entries 1-4). Only small differences in activities were observed. This may seem rather logical if we take into account that analogous set of ligands are bound directly to the iridium centre in all catalysts.

Table 2.6 Sequential catalytic isomerization/hydrophosphination using Ir/Pd complexes

\begin{tabular}{cccc|ccc}
\hline & & \multicolumn{2}{c|}{ Isomerization } & \multicolumn{3}{c}{ Hydrophosphination } \\
\hline Entry & Catalyst & Time(h) & Yield(\%) & Time(h) & Yield(\%) $^{\mathbf{b}}$ & ee(\%) $)^{\mathbf{c}}$ \\
1 & $\mathbf{9 A}$ & 5 & 95 & 10 & 60 & 4 \\
2 & $\mathbf{1 0 A}$ & 5 & 97 & 10 & 75 & 8 \\
3 & $\mathbf{1 1 A}$ & 5 & 95 & 10 & 71 & 14 \\
4 & $\mathbf{1 2 A}$ & 5 & 90 & 10 & 56 & 17 \\
\hline
\end{tabular}

Reaction conditions: 1,3-diphenylpropargyl alcohol (100 $\mu \mathrm{L}, 0.47 \mathrm{mmol})$ catalyst (2 mol\%), $2 \mathrm{~mL}$ 2-propanol as solvent, $\mathrm{K}_{2} \mathrm{CO}_{3}(103 \mathrm{mg}, 0.7 \mathrm{mmol}), 90^{\circ} \mathrm{C}$, then $\mathrm{HPPh}_{2}(93 \mu \mathrm{L}, 0.52 \mathrm{mmol})$, room temperature. [a] Chalcone yield determined by GC (internal standard: anisole). [b] Isolated yield (related to 1,3diphenylpropargyl alcohol) after column chromatography using ${ }^{1} \mathrm{H}$ NMR.. [c] Determined using chiral HPLC.

The hydrophosphination proceeded from moderate to good yields at room temperature in 2-propanol (yields $56-75 \%$ ). In the previous study with monometalic chiral palladacycles-NHC complexes (Section 3.1) we observed that dichloromethane and 
tetrahydrofuran were convenient solvents for the asymmetric hydrophosphination. Unfortunately, for this process the isomerisation reaction did not proceed either in tetrahydrofuran or dichloromethane, thus 2-propanol was used as the optimal solvent for both catalytic processes.

In all cases, the hydrophosphination proceeded with excellent regioselectivity, with the phosphorous addition occurring at the $\mathrm{C} 3$ position. Unfortunately, the enantiomeric excesses were very low (ee $4-17 \%$ ). These results compare well with the other Pd-NHC complexes described in the last section, therefore confirming that palladium complexes with small R groups at the NHC ligands, such as methyls (complexes 2D and 4D), are not suitable for achieving high ee values. 


\section{CONCLUSIONS}

Novel chiral palladacycles with N-heterocyclic carbene ligands have been synthesized and characterized starting form commercially available enantiopure benzylamines. The complexes with asymmetric NHC ligands show the presence of two atropisomers, which were separated by column chromatography and allowed the performance of distinct ${ }^{1} \mathrm{H}$ NMR spectra. However, epimerization of the isolated complexes occurs slowly in solution, and this is why the ${ }^{13} \mathrm{C}$ NMR spectra of the complexes always showed the presence of mixtures of the two atropisomers.

The new complexes have been used as catalysts in the 1,4-addition of diarylphosphines to $\alpha, \beta$-unsaturated ketones. All the catalysts showed high activity in terms of conversion and yields obtained under mild reaction conditions. The hydrophosphination reaction proceeds with excellent regioselectivity (phosphorous addition occurs at the 3 carbon position) although modest enantioselectivities were achieved.

On the basis of previous results, we have described a new methodology that allowed us to obtain heterodimetallic complexes using ditz ligand. The method is based on the preparation of chiral palladium dimers that undergo facile bridge splitting leading to the formation of heterodimetallic $\operatorname{Ir}(\mathrm{III})-\mathrm{Pd}(\mathrm{II})^{*}$ complexes. The compounds obtained display two axes of chirality, one centered at the octahedral iridium center and the other at the square-planar palladium fragment.

The heterodimetallic complexes $\mathbf{9 A}-\mathbf{1 2} A$, have been tested in a new sequential tandem process involving the isomerization and chiral hydrophosphination of 1,3diphenylpropargyl alcohol. All the catalysts show high activity in the overall reaction process and excellent regioselectivity although poor enantioselectivities were obtained. 


\section{REFERENCES}

(1) Mata, J. A.; Hahn, F. E.; Peris, E. Chem. Sci. 2014, 5, 1723-1732.

(2) Albert, J.; Granell, J.; Muller, G. J. Organomet. Chem. 2006, 691, 2101-2106.

(3) Dupont, J.; Consorti, C. S.; Spencer, J. Chem. Rev. 2005, 105, 2527-2571.

(4) Huang, Y.; Pullarkat, S. A.; Li, Y.; Leung, P.-H. Chem. Commun. 2010, 46, 6950-6952.

(5) Feng, J.-J.; Chen, X.-F.; Shi, M.; Duan, W.-L. J. Am. Chem. Soc. 2010, 132, 5562.

(6) Beletskaya, I. P.; Cheprakov, A. V. J. Organomet. Chem. 2004, 689, 40554082.

(7) Elgazwy, A. S. S. H.; Ismail, N. S. M.; Atta-Allah, S. R.; Sarg, M. T.; Soliman, D. H. S.; Zaki, M. Y.; Elgamas, M. A. Curr. Med. Chem 2012, 19, 39673981.

(8) Vicente, J.; Saura-Llamas, I. Comments Inorg. Chem. 2007, 28, 39-72.

(9) Albert, J.; Bosque, R.; Crespo, M.; Garcia, G.; Granell, J.; Lopez, C.; Lovelle, M. V.; Qadir, R.; Gonzalez, A.; Jayaraman, A.; Mila, E.; Cortes, R.; Quirante, J.; Calvis, C.; Messeguer, R.; Badia, J.; Baldoma, L.; Cascante, M. Eur. J. Med. Chem. 2014, 84, 530-536.

(10) Vicente, J.; Teresa Chicote, M.; Jesus Martinez-Martinez, A.; Abellan-Lopez, A.; Bautista, D. Organometallics 2010, 29, 5693-5707.

(11) Gopi, K.; Thirupathi, N.; Nethaji, M. Organometallics 2011, 30, 572-583.

(12) Omae, I. J. Organomet. Chem. 2007, 692, 2608-2632.

(13) Omae, I. Coord. Chem. Rev. 2004, 248, 995-1023.

(14) Pfeffer, M. Pure Appl. Chem. 1992, 64, 335-342.

(15) Dupont, J.; Pfeffer, M. Palladacycles: Synthesis, Characterization and Applications; Wiley-VCH, 2008.

(16) Huang, Y.; Pullarkat, S. A.; Teong, S.; Chew, R. J.; Li, Y.; Leung, P.-H. Organometallics 2012, 31, 4871-4875.

(17) Huang, Y.; Pullarkat, S. A.; Li, Y.; Leung, P.-H. Inorg. Chem. 2012, 51, 2533-2540. 
(18) Huang, Y.; Chew, R. J.; Pullarkat, S. A.; Li, Y.; Leung, P.-H. J. Org. Chem. 2012, 77, 6849-6854.

(19) Du, D.; Duan, W.-L. Chem. Commun. 2011, 47, 11101-11103.

(20) Yuan, M.; Zhang, N.; Pullarkat, S. A.; Li, Y.; Liu, F.; Pham, P.-T.; Leung, P.H. Inorg. Chem. 2010, 49, 989-996.

(21) Yuan, M.; Pullarkat, S. A.; Li, Y.; Lee, Z.-Y.; Leung, P.-H. Organometallics 2010, 29, 3582-3588.

(22) Ibrahem, I.; Hammar, P.; Vesely, J.; Rios, R.; Eriksson, L.; Cordova, A. $A d v$. Synth. Catal. 2008, 350, 1875-1884.

(23) Butti, P.; Rochat, R.; Sadow, A. D.; Togni, A. Angew. Chem. Int. Edit. 2008, 47, 4878-4881.

(24) Barta, K.; Francio, G.; Leitner, W.; Lloyd-Jones, G. C.; Shepperson, I. R. Adv. Synth. Catal. 2008, 350, 2013-2023.

(25) Ibrahem, I.; Rios, R.; Vesely, J.; Hammar, P.; Eriksson, L.; Himo, F.; Cordova, A. Angew. Chem. Int. Edit. 2007, 46, 4507-4510.

(26) Carlone, A.; Bartoli, G.; Bosco, M.; Sambri, L.; Melchiorre, P. Angew. Chem. Int. Edit. 2007, 46, 4504-4506.

(27) Blank, N. F.; Moncarz, J. R.; Brunker, T. J.; Scriban, C.; Anderson, B. J.; Amir, O.; Glueck, D. S.; Zakharov, L. N.; Golen, J. A.; Incarvito, C. D.; Rheingold, A. L. J. Am. Chem. Soc. 2007, 129, 6847-6858.

(28) Xu, Q.; Han, L. B. Org. Lett. 2006, 8, 2099-2101.

(29) Shulyupin, M. O.; Francio, G.; Beletskaya, I. P.; Leitner, W. Adv. Synth. Catal. 2005, 347, 667-672.

(30) Scriban, C.; Kovacik, I.; Glueck, D. S. Organometallics 2005, 24, 4871-4874.

(31) Douglass, M. R.; Ogasawara, M.; Hong, S.; Metz, M. V.; Marks, T. J. Organometallics 2002, 21, 283-292.

(32) Kovacik, I.; Wicht, D. K.; Grewal, N. S.; Glueck, D. S.; Incarvito, C. D.; Guzei, I. A.; Rheingold, A. L. Organometallics 2000, 19, 950-953.

(33) Koshti, V.; Gaikwad, S.; Chikkali, S. H. Coord. Chem. Rev. 2014, 265, 52-73.

(34) Rosenberg, L. ACS Catal. 2013, 3, 2845-2855.

(35) Kolodiazhnyi, O. I.; Kukhar, V. P.; Kolodiazhna, A. O. Tetrahedron: Asymmetr. 2014, 25, 865-922. 
(36) Hoff, M. C.; Hill, P. J. Org. Chem. 1959, 24, 356-359.

(37) Dombek, B. D. J. Org. Chem. 1978, 43, 3408-3409.

(38) Rauhut, M. M.; Hechenbleikner, I.; Currier, H. A.; Schaefer, F. C.; Wystrach, V. P. J. Am. Chem. Soc. 1959, 81, 1103-1107.

(39) Bookham, J. L.; Smithies, D. M. J. Organomet. Chem. 1999, 577, 305-315.

(40) Bunlaksananusorn, T.; Knochel, P. Tetrahedron Lett. 2002, 43, 5817-5819.

(41) Huang, Y.; Chew, R. J.; Li, Y.; Pullarkat, S. A.; Leung, P.-H. Org. Lett. 2011, $13,5862-5865$.

(42) Xu, C.; Kennard, G. J. H.; Hennersdorf, F.; Li, Y.; Pullarkat, S. A.; Leung, P.H. Organometallics 2012, 31, 3022-3026.

(43) Huang, M.; Li, C.; Huang, J.; Duan, W.-L.; Xu, S. Chem. Commun. 2012, 48, 11148-11150.

(44) Tani, K.; Brown, L. D.; Ahmed, J.; Ibers, J. A.; Yokota, M.; Nakamura, A.; Otsuka, S. J. Am. Chem. Soc. 1977, 99, 7876-7886.

(45) Gul, N.; Nelson, J. H. J. Mol. Struct. 1999, 475, 121-130.

(46) Gul, N.; Nelson, J. H. Organometallics 2000, 19, 91-104.

(47) Hockless, D. C. R.; Gugger, P. A.; Leung, P. H.; Mayadunne, R. C.; Pabel, M.; Wild, S. B. Tetrahedron 1997, 53, 4083-4094.

(48) Ryabov, A. D.; Sakodinskaya, I. K.; Yatsimirsky, A. K. J. Chem. Soc.-Dalton Trans. 1985, 2629-2638.

(49) Davies, D. L.; Donald, S. M. A.; Macgregor, S. A. J. Am. Chem. Soc. 2005, $127,13754-13755$.

(50) Enders, D.; Gielen, H. J. Organomet. Chem. 2001, 617, 70-80.

(51) Enders, D.; Gielen, H.; Runsink, J.; Breuer, K.; Brode, S.; Boehn, K. Eur. J. Inorg. Chem. 1998, 913-919.

(52) Calmuschi, B.; Englert, U. Acta Crystallogr. C 2002, 58, m545-m548.

(53) Dunina, V. V.; Kuz'mina, L. G.; Kazakova, M. Y.; Golunova, O. N.; Grishin, Y. K.; Kazakova, E. I. Eur. J. Inorg. Chem. 1999, 1029-1039.

(54) Vicente, J.; Saurallamas, I.; Jones, P. G. J. Chem. Soc.-Dalton Trans. 1993, 3619-3624.

(55) Faller, J. W.; Fontaine, P. P. Organometallics 2006, 25, 5887-5893. 
(56) Ros, A.; Alcarazo, M.; Iglesias-Sigueenza, J.; Diez, E.; Alvarez, E.; Fernandez, R.; Lassaletta, J. M. Organometallics 2008, 27, 4555-4564.

(57) Enders, D.; Gielen, H.; Raabe, G.; Runsink, J.; Teles, J. H. Chem. Ber.-Recl. 1996, 129, 1483-1488.

(58) Cahn, R. S.; Ingold, C.; Prelog, V. Angew. Chem. Int. Edit. 1966, 5, 385.

(59) Corberan, R.; Lillo, V.; Mata, J. A.; Fernandez, E.; Peris, E. Organometallics 2007, 26, 4350-4353.

(60) Viciano, M.; Feliz, M.; Corberan, R.; Mata, J. A.; Clot, E.; Peris, E. Organometallics 2007, 26, 5304-5314.

(61) Xu, J. X.; Wei, T. Z.; Zhang, Q. H. J. Org. Chem. 2003, 68, 10146-10151.

(62) Xu, J. X.; Wei, T. Z.; Lin, S. S.; Zhang, Q. H. Helv. Chim. Acta 2005, 88, $180-186$.

(63) Cadierno, V.; Crochet, P.; Garcia-Garrido, S. E.; Gimeno, J. Dalton Trans. 2010, 39, 4015-4031.

(64) Cadierno, V.; Crochet, P.; Gimeno, J. Synlett 2008, 1105-1124.

(65) Trost, B. M.; Livingston, R. C. J. Am. Chem. Soc. 2008, 130, 11970-11978.

(66) Ramon, R. S.; Marion, N.; Nolan, S. P. Tetrahedron 2009, 65, 1767-1773.

(67) Wang, D.; Zhang, Y.; Harris, A.; Gautam, L. N. S.; Chen, Y.; Shi, X. Adv. Synth. Catal. 2011, 353, 2584-2588.

(68) Pennell, M. N.; Turner, P. G.; Sheppard, T. D. Chem. Eur. J. 2012, 18, 47484758.

(69) Ma, D. W.; Lu, X. Y. Tetrahedron Lett. 1989, 30, 2109-2112.

(70) Lu, X. Y.; Ma, D. W. Pure Appl. Chem. 1990, 62, 723-730. 



\section{CHAPTER 3}

\section{IRIDIUM COMPLEXES WITH CHIRAL ORTHO-METALATED AMINES: APPLICATIONS IN TRANSFER HYDROGENATION}

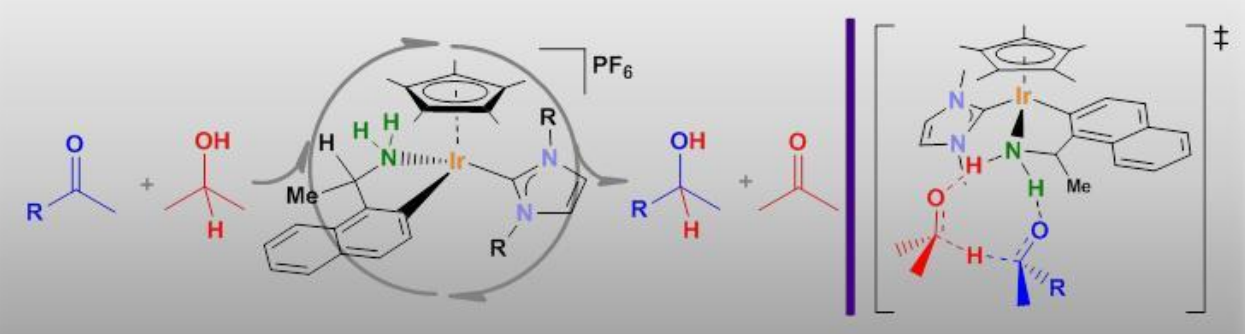





\section{INTRODUCTION}

\subsection{Metal catalyzed transfer hydrogenation reactions}

Catalytic transfer hydrogenation (TH) is a useful methodology for the reduction of unsaturated species. Formally, in TH processes the catalyst transfers hydrogen between two substrates, the hydrogen donor and the hydrogen acceptor without the need of hydrogen gas (Scheme 3.1). Since the first catalytic systems, reported by Mitchell and Henbest $^{1-3}$ and Mestroni, ${ }^{4}$ TH has attracted great interest because of its operational simplicity, mild conditions and the avoidance of using hydrogen gas. Today a plethora of efficient transition metal catalysts for $\mathrm{TH}$ based on $\mathrm{Ru}^{5-7} \mathrm{Rh}^{8-14}$ and $\mathrm{Ir}^{15-21}$ are known. Among them, the $\mathrm{IrCp}^{*}$ systems are emerging powerfully due to their stability and activity, ${ }^{18,22-25}$ and even their first application in industry has recently been reported. ${ }^{26}$

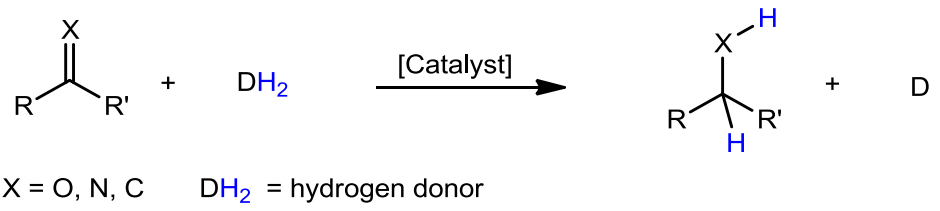

Scheme 3.1 Catalytic transfer hydrogenation

From a mechanistic point of view, two main pathways can be described either involving unsaturated metal species (Non-hydride mechanisms) or the participation of hydride derivatives (Hydride mechanisms) in the catalytic process. ${ }^{27,28}$

\section{Non-hydride mechanisms}

These mechanisms can be regarded as metal-templated concerted processes, in which the substrate and the hydrogen donor coordinate to the metal in one-step. The hydrogen is transferred directly from the donor to the unsaturated substrate via a six-membered transition state, and no participation of hydride intermediates is required. This pathway was proposed for the Meerwein-Ponndorf-Verley (MPV) reduction. In the original version, aluminum isopropoxide $\left[\mathrm{Al}(\mathrm{O} i \mathrm{Pr})_{3}\right]$ was used to promote hydrogen transfer from 2-propanol to a ketone. ${ }^{29-31}$ In this case, the metal acts as a Lewis acid and no participation of hydride intermediates is required. ${ }^{32}$ The process can be also run for the 
reverse cycle, known as Oppenauer oxidation, ${ }^{33}$ using acetone as hydrogen acceptor (Scheme 3.2). In the MPV reduction, 2-propanol is used in excess, while in the Oppenauer oxidation, acetone is used in excess.

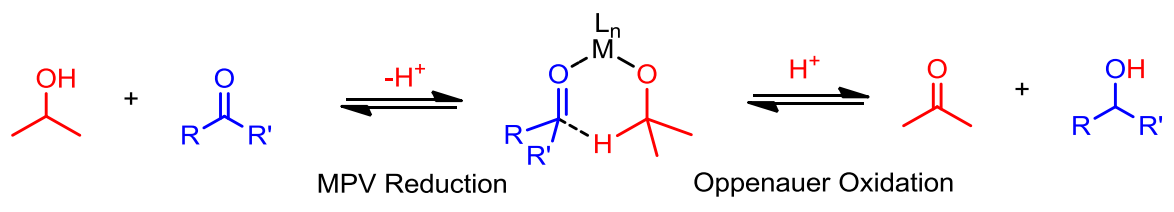

Scheme 3.2 MPV reduction and Oppenauer oxidation

\section{Hydride mechanisms}

In this class of mechanisms, a metal hydride is involved as key intermediate for hydrogen transfer, and represents the most common pathway in the case of transition metal catalyzed reactions. In general, the hydride mechanism consists of a stepwise process, where the donor and the acceptor interact separately with the metal at different stages of the reaction. First, the metal hydride is formed by the reaction of the catalyst with the hydrogen donor. Second, the hydride is transferred from the metal hydride to the substrate.

According to the hydride formation, it may involve the formation of a metal alkoxide followed by a $\beta-\mathrm{H}$ elimination to give the $\mathrm{M}-\mathrm{H}_{\mathrm{n}}$, (Scheme 3.3, a and b). Alternatively, the hydride generation can proceed without coordination of the alcohol to the metal (Scheme $3.3, \mathrm{c})$.

a)

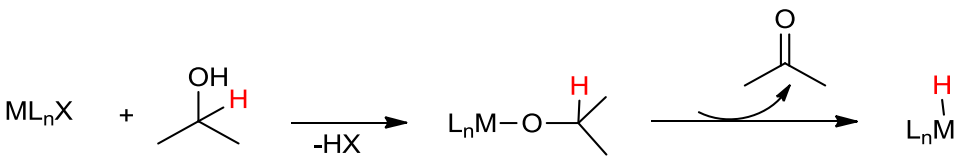

b)

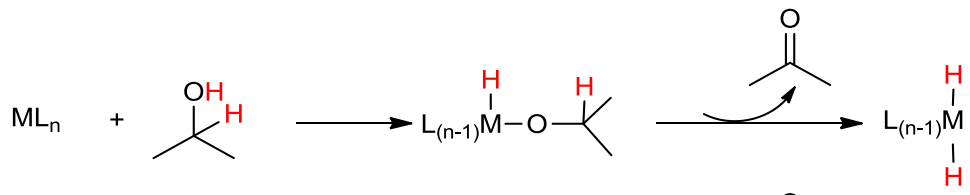

c)

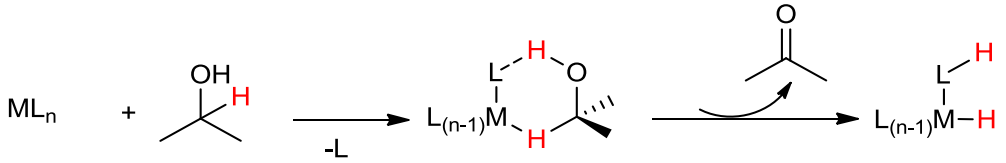

Scheme 3.3 Formation of monohydride and dihydride intermediates 
Considering the hydride transfer to the substrate, it has been shown to operate by innersphere and outer-sphere pathways. In both pathways the metal hydride migrates from the metal to the carbonyl carbon. ${ }^{34}$

\section{Inner-sphere mechanism}

In the inner-sphere mechanism, the unsaturated organic substrate binds directly to the metal. Then, the hydride transfer occurs in the inner sphere of the metal. The coordination of the substrate allows the electrophilic activation of the carbon of the unsaturated bond by the metal ion, so that a cis hydride can migrate to the carbon at the $\beta$ position to the metal (Scheme 3.4). Subsequent elimination of the hydrogenated substrate completes the catalytic cycle. ${ }^{35,36}$ As may be seen from the cycle depicted in Scheme 3.4, this mechanism requires that the metal has the possibility of generating, at least, two coordination vacant sites.

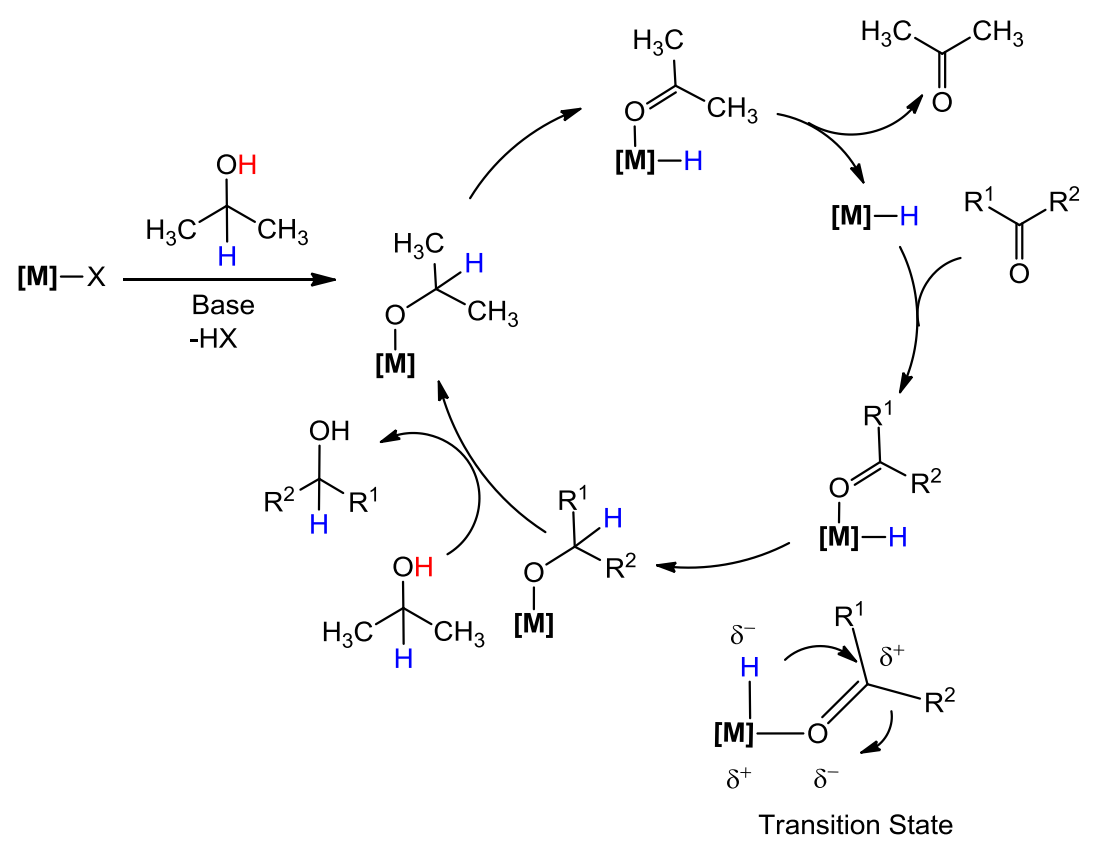

Scheme 3.4 Hydride transfer in the inner-sphere mechanism 


\section{Outer-sphere mechanism}

Similarly to the inner-sphere mechanism, a metal hydride intermediate is involved as key intermediate, although in this case the substrate does not bind to the metal. In this type of process, the $\mathrm{H}^{+}$and $\mathrm{H}^{-}$are transferred from the catalyst to the unbound substrate with a certain degree of synchronicity. ${ }^{37-39}$ In the outer-sphere mechanism, the hydrogen transfer may proceed either by the assistance of an external electrophile, or an ancillary ligand on the metal, which provide additional activation of the unsaturated substrate toward hydride attack (Scheme 3.5). As can be seen from the mechanism depicted in Scheme 3.5, this mechanism does require that the metal has one coordination vacant site.

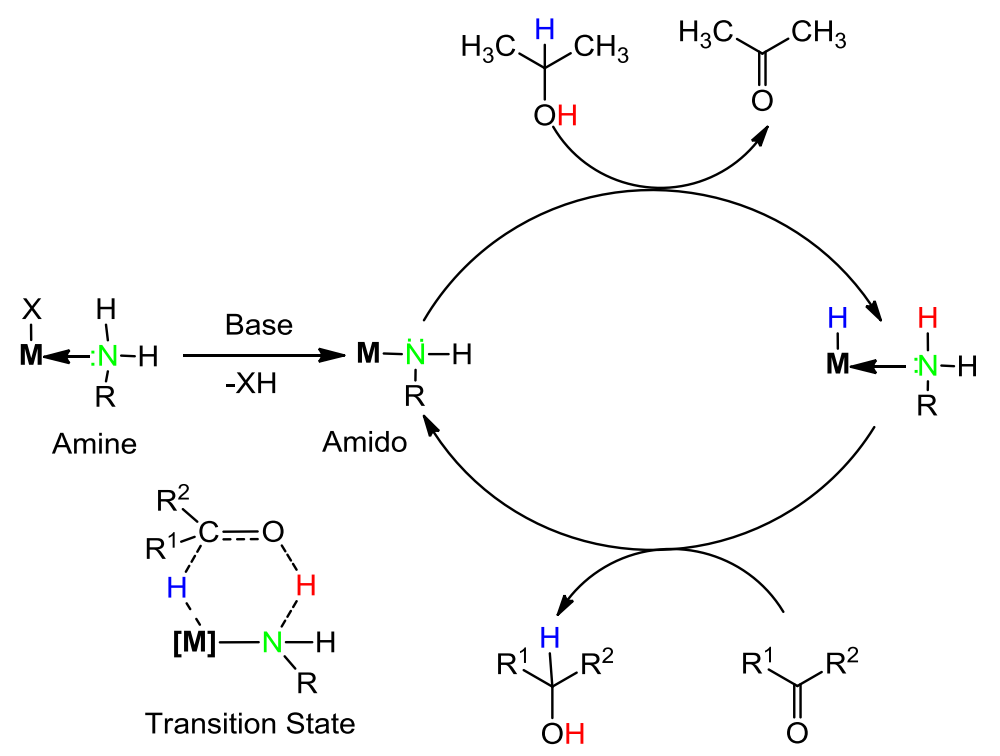

Scheme 3.5 Hydride transfer in the outer-sphere mechanism

Relevant examples of this kind of catalysts are metal-ligand bifunctional catalysts, based on the combination of two active sites working in concert. ${ }^{40-43}$ In this type of catalysts, the non-innocent ligands directly participate in the substrate activation, and in the bond formation. The first metal-ligand bifunctional catalyst for the reduction of carbonyl compounds was reported by Shvo in $1985 .^{44,45}$ In 1995, Noyori and Ikariya found a 
prototype of the bifunctional ruthenium catalysts based on the M/NH acid base synergetic effect. $^{46-48}$

As demonstrated in Chapter 2, the use of enantiomerically pure amines is an effective way of introducing chirality in organometallic complexes for the formation of enantiopure catalysts that can be applied in asymmetric catalysis. Ikariya, ${ }^{49-53}$ and Pfeffer, ${ }^{54-56}$ described the catalytic abilities of Ir-NH complexes in transfer hydrogenation processes. In light of the experience of our group in the use of $\mathrm{Ru}$ and $\mathrm{Ir}$ complexes in transfer hydrogenation processes, we thought that it would be interesting to develop chiral iridium-NH catalysts with NHC ligands for asymmetric transfer hydrogenation processes.

The main objectives of this chapter were the synthesis of enantiomerically pure $\operatorname{IrCp} *$ catalysts bearing ortho-metalated primary amines and the study of their catalytic performances in asymmetric transfer hydrogenation. ${ }^{57}$ We also studied the mechanistic insights based on experimental and theoretical investigations. At this point, it is important to mention that, whereas important efforts have been devoted to the determination of the mechanisms operating in Ru-catalyzed $\mathrm{TH}$ reactions by means of theoretical calculations, ${ }^{25,58-65}$ analogous studies on TH processes promoted by Ir-based systems are still scarce. ${ }^{6,17,66,67}$ 


\section{RESULTS AND DISCUSSION}

\subsection{Synthesis of Ir-NH-NHC complexes}

The reactions of $\left[\operatorname{IrCp} *(\mathrm{Cl})_{2}\left(\mathrm{Me}_{2} \operatorname{Im}\right)\right](5 \mathrm{D})$ or $\left[\operatorname{IrCp} *(\mathrm{Cl})_{2}\left(n \mathrm{Bu}_{2} \mathrm{Im}\right)\right](\mathbf{2 C})$ with the racemic primary amine [1-(1-naphthyl)ethyl]amine in the presence of two equivalents of AgOAc afforded the ortho-metalated iridium complexes $6 \mathbf{D}$ and $\mathbf{3 C}$, respectively. The reactions were performed in acetonitrile at room temperature for $4 \mathrm{~h}$ under nitrogen atmosphere. The enantiopure iridium complexes $6 \mathrm{D}^{*}$ and $\mathbf{3 C} *$ were obtained using the enantiomerically pure (S)-[1-(1-naphthyl)ethyl]amine (Scheme 3.6). All complexes were purified by column chromatography, using $\mathrm{KPF}_{6}$, and were precipitated from a mixture of acetonitrile/diethyl ether to afford yellow solids in 92\% (6D and 6D*) and 85\% (3C and 3C*) yield.

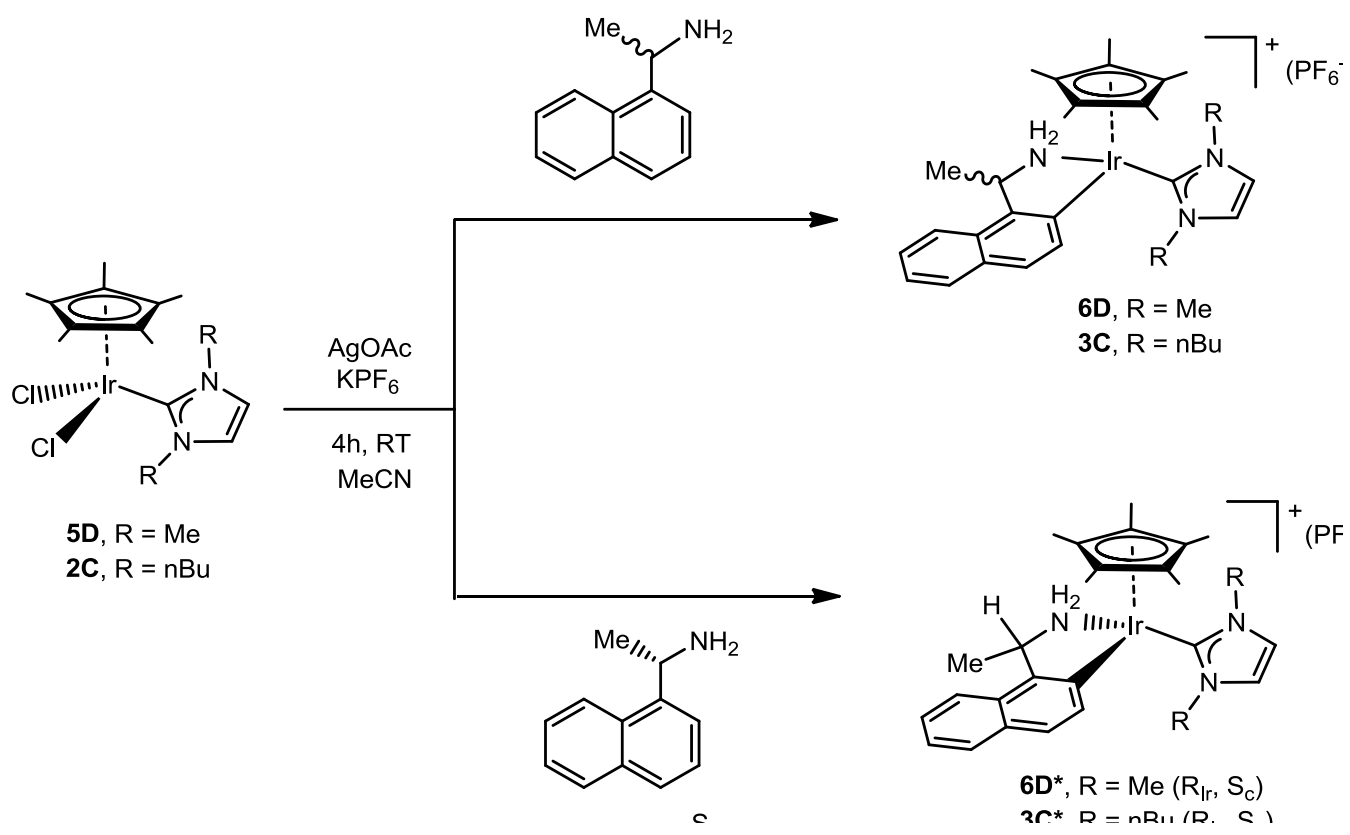

Scheme 3.6 Synthesis of Ir-NH-NHC complexes 6D, 3C, 6D* and 3C*

All compounds are stable under air atmosphere in the solid state and in solution, and were characterized by NMR spectroscopy, High Resolution Mass Spectrometry (HRMS) and 
elemental analysis. Due to the similarity of these compounds, only the spectroscopic characterization of $6 \mathrm{D}$ is discussed in detail.

\section{${ }^{1} H$ NMR spectrum of $\mathbf{6 D}$}

Figure 3.1 shows the ${ }^{1} \mathrm{H}$ NMR spectrum of $6 \mathbf{D}$. The most significant signal is the doublet due to the $\mathrm{CH}$ proton next to the ortho-metalated $\mathrm{Ir}-\mathrm{C}$ bond, at $\delta 7.96\left({ }^{3} \mathrm{~J}_{\mathrm{H}, \mathrm{H}}=8.4 \mathrm{~Hz}\right)$, which clearly indicates that the cyclometalation of the arylamine occurred. ${ }^{68}$ The rest of signals attributed to the naphthyl aromatic protons appear between 7.80 and $7.27 \mathrm{ppm}$. The resonances due to the protons of the methyl groups at the imidazole ring appear at 3.90 and $3.32 \mathrm{ppm}$, indicating the loss of the two-fold symmetry of the former complex (5D) upon coordination to the amine. The signals due to the protons at the $\mathrm{NH}_{2}$ group appear as broad singlets at 5.07 and $3.80 \mathrm{ppm}$. The resonance due to the $\mathrm{CH}$ benzylic proton of the amine is shown as multiplet at 5.01-4.87 ppm.

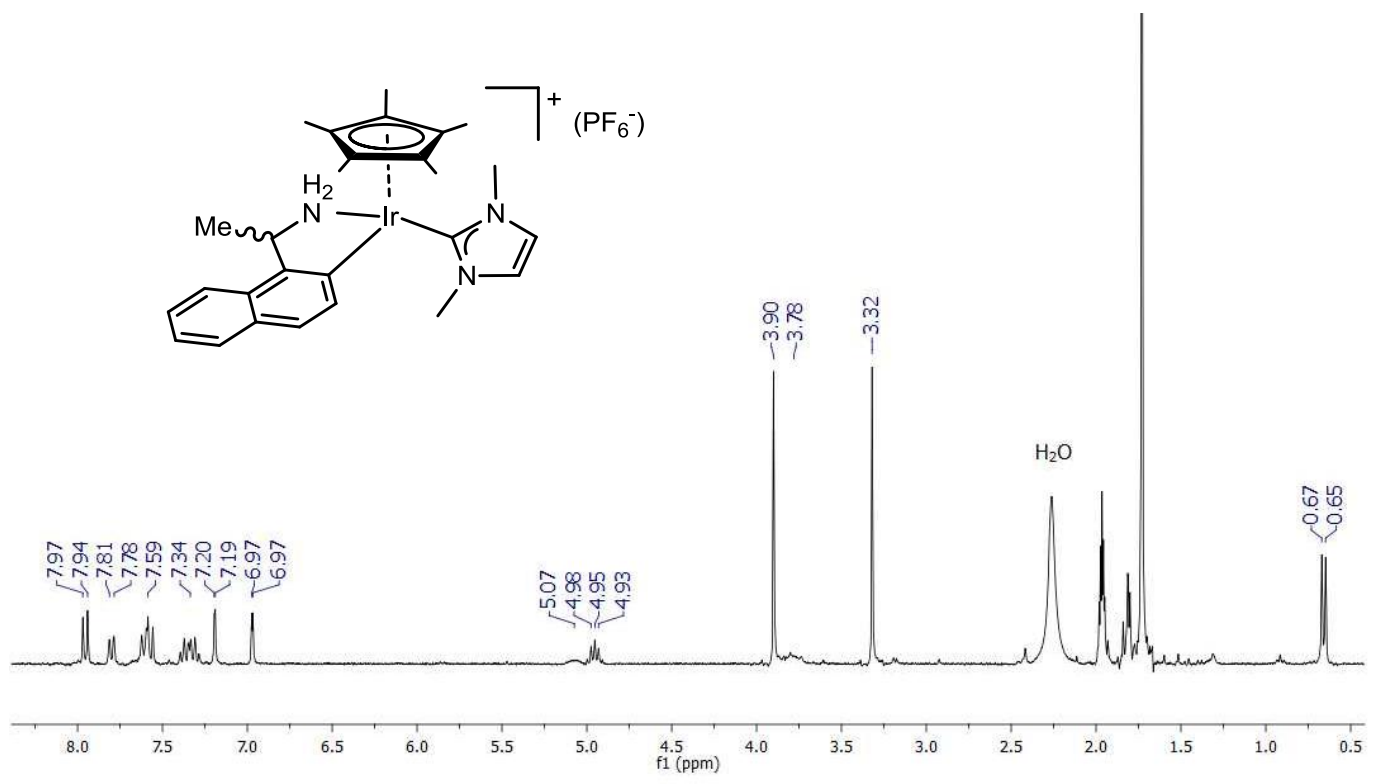

Figure 3.1 ${ }^{1} \mathrm{H}$ NMR spectra of complex $6 \mathrm{D}$ in $\mathrm{CD}_{3} \mathrm{CN}$ 


\section{${ }^{13} \mathrm{C}\left\{{ }^{1} \mathrm{H}\right\}$ NMR spectrum of $6 \mathrm{D}$}

Figure 3.2 shows the ${ }^{13} \mathrm{C}\left\{{ }^{1} \mathrm{H}\right\}$ NMR spectrum of complex 6D. The most characteristic signal is the one assigned to the metalated carbene carbon at $\delta$ 152.9. The signals corresponding to the $\mathrm{CH}$ carbons of the imidazole ring appear at 123.9 and $123.8 \mathrm{ppm}$. The resonance due to the benzylic $\mathrm{CH}$ of the amine is shown at $59.3 \mathrm{ppm}$. The signals attributed to the carbons of the methyl groups at the imidazole appear at 39.2 and 39.1 ppm.

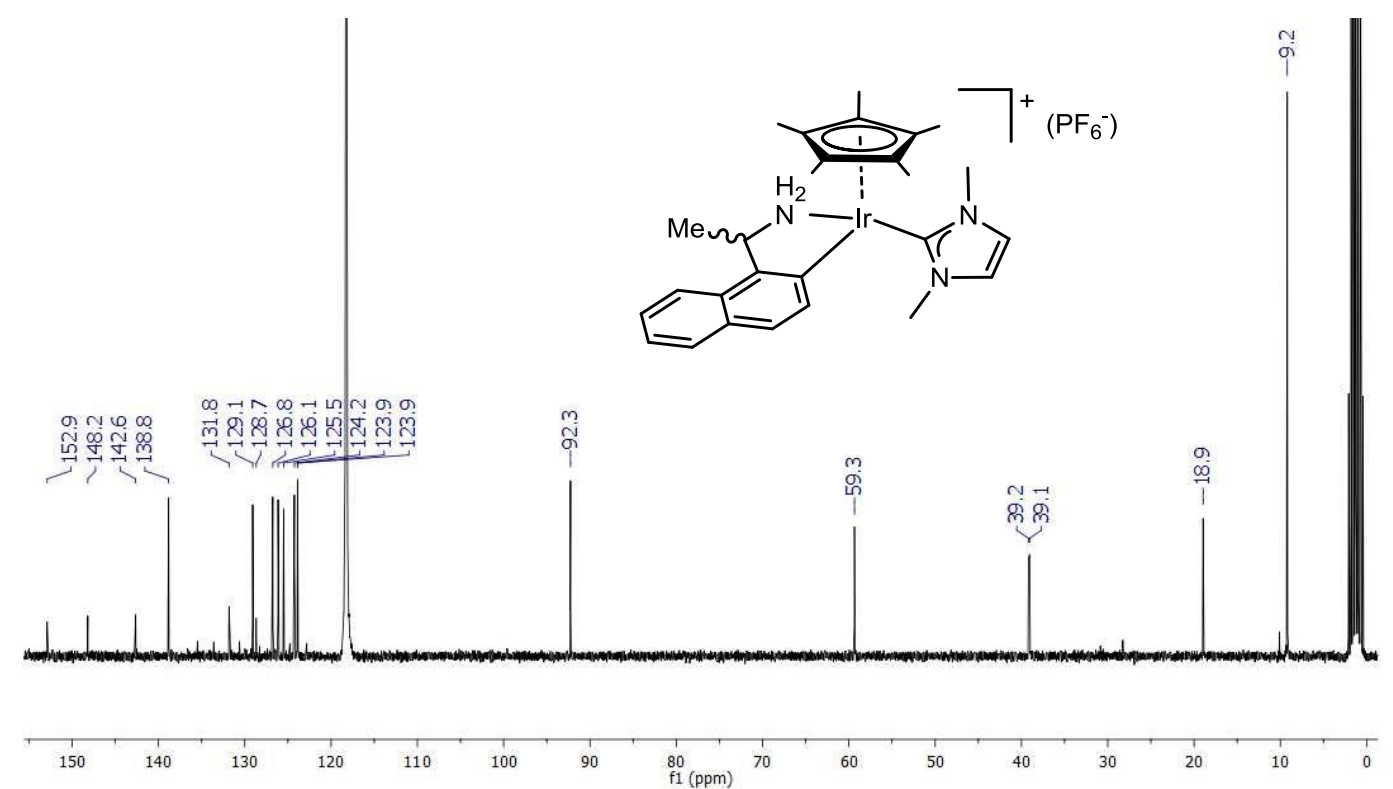

Figure 3.2 ${ }^{13} \mathrm{C}\left\{{ }^{1} \mathrm{H}\right\}$ NMR spectrum of $6 \mathrm{D}$ in $\mathrm{CD}_{3} \mathrm{CN}$

The ortho-metalated nature of the compounds was also confirmed by the data resulting from electrospray mass spectrometry (ESI-MS), based on the mass/charge relation and the isotopic pattern of peaks. Positive ion electrospray mass spectra analysis of complexes 6D and 3C in acetonitrile showed an intense positive ion peak for $[\mathrm{M}]^{+}$at $\mathrm{m} / \mathrm{z}$ $=594$ and 678 , respectively.

The enantiopure complexes $6 \mathrm{D}^{*}$ and $\mathbf{3 C} *$ were characterized by Circular Dichroism spectroscopy (CD). Figure 3.3 shows the $\mathrm{CD}$ spectra of $\mathbf{6 D}, \mathbf{6 D} *$ and $\mathbf{3 C}, \mathbf{3 C} *$. The $\mathrm{CD}$ spectrum of $6 \mathrm{D}^{*}$ shows three signals at $\lambda=254,281$ and $327 \mathrm{~nm}$ for $-10.2,5.3$ and 6.1 
mdeg, respectively. In the case of complex 6D, no bands are observed (Figure 3.3, left). The CD spectrum of $\mathbf{3 C} *$ shows three signals at $\lambda=251,283$ and $323 \mathrm{~nm}$ for $-7.6,2.6$ and 3.5 mdeg, while complex 3C does not show any bands (Figure 3.3, right).
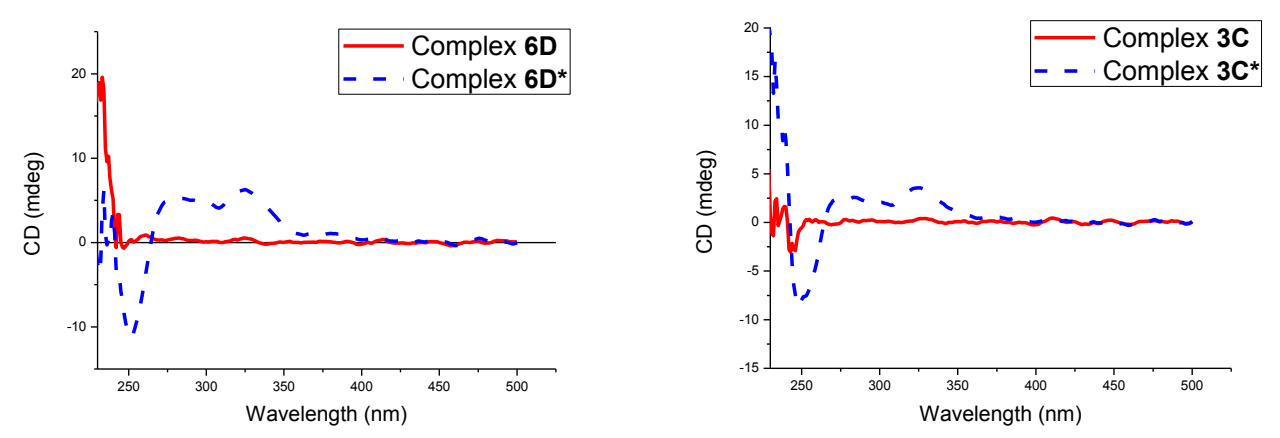

Figure 3.3 Circular dichroism spectra recorded in chloroform at $25^{\circ} \mathrm{C}$. Left: complex $6 \mathbf{D}(-)$ and complex 6D* (---); right: complex 3C (-) and complex 3C*(---).

The molecular structures of complexes $\mathbf{3 C}, \mathbf{6 D} *$ and $\mathbf{3 C} *$ were determined by $\mathrm{X}$-ray diffraction, and confirmed their absolute configuration. Due to their similarity, only the molecular structure of $\mathbf{3 C}$ and $\mathbf{6 D *}$ is discussed in detail.

\section{Molecular structure of $\mathbf{3 C}$}

The molecular structure of complex $\mathbf{3 C}$ was confirmed by means of $\mathrm{X}$-ray diffraction studies (Figure 3.4). Single crystals were obtained by slow diffusion of hexane into a concentrated solution of $\mathbf{3 C}$ in dichloromethane. Complex $\mathbf{3 C}$ crystallizes in the achiral space group P-1 and contains a single molecule in the asymmetric unit. The geometry of the complex can be regarded as three-legged piano-stool with dihedral angles close to $90^{\circ}$, except the $\mathrm{C}(6)-\operatorname{Ir}(1)-\mathrm{N}(2)$ angle of $77.51(14)^{\circ}$ due to the chelating coordination of the 1-(1-naphthyl)ethyl]amine. The C-N ortho-metalated amine forms a five-membered ring in an envelope conformation, where the $\mathrm{N}$ atom is out of the plane by $0.34 \AA$. The methyl group $\mathrm{C}(4)$ attached at the benzylic carbon adopts an axial disposition (exo regarding to the $\mathrm{Cp}^{*}$ ligand) enforced by the naphthyl group as previously described for this ligand in other complexes. ${ }^{69}$ Table 3.1 shows the most representative bond lengths and angles of complex 3C. The iridium distances are $\operatorname{Ir}(1)-\mathrm{C}_{\text {carbene }} 2.044(4) \AA, \operatorname{Ir}(1)$ - 
$\mathrm{C}(6) 2.061(4) \AA$ and $\operatorname{Ir}(1)-\mathrm{N}(2) 2.128(3) \AA$ and lie in the expected range for $\operatorname{IrCp}^{*}(\mathrm{NHC})$ complexes. $^{70}$
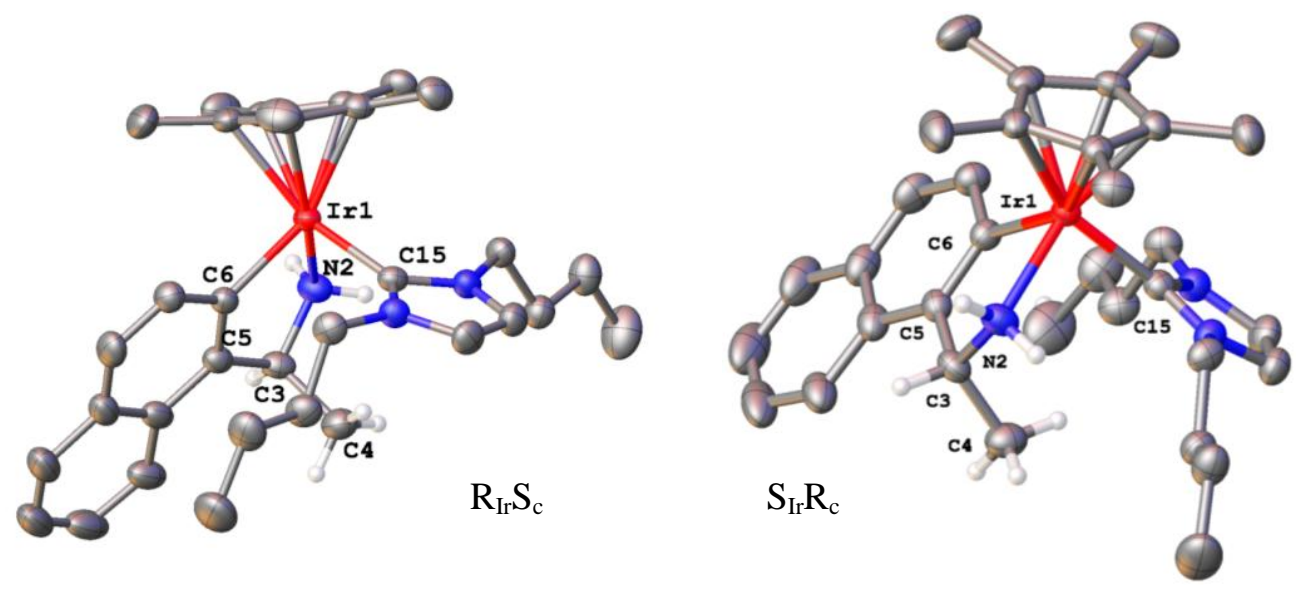

Figure 3.4 Molecular diagram of the pair of enantiomers of complex 3C. Ellipsoids are shown at $50 \%$ probability level. Only hydrogen atoms bonded to $\mathrm{N}(2), \mathrm{C}(3)$ and $\mathrm{C}(4)$ are shown. Counterions are omitted for clarity.

Table 3.1 Selected bond lengths $(\AA)$ and angles $\left(^{\circ}\right)$ of $\mathbf{3 C}$

\begin{tabular}{cccc}
\hline Bonds & $(\AA)$ & Angles & $\left.\mathbf{(}^{\circ}\right)$ \\
\hline $\operatorname{Ir}(1)-\mathrm{C}(15)$ & $2.044(4)$ & $\mathrm{C}(6)-\operatorname{Ir}(1)-\mathrm{N}(2)$ & $77.51(14)$ \\
$\operatorname{Ir}(1)-\mathrm{N}(2)$ & $2.128(3)$ & $\mathrm{C}(6)-\operatorname{Ir}(1)-\mathrm{C}(15)$ & $94.34(15)$ \\
$\operatorname{Ir}(1)-\mathrm{C}(6)$ & $2.061(4)$ & $\mathrm{C}(15)-\operatorname{Ir}(1)-\mathrm{N}(2)$ & $91.25(15)$ \\
$\operatorname{Ir}(1)-\mathrm{Cp}^{*}$ centroid & 1.861 & & \\
\hline
\end{tabular}

As depicted in figure 3.5, in the synthesis of $\mathrm{Cp} * \mathrm{Ir}$ complexes starting from the racemic primary amine [1-(1-naphthyl)ethyl]amine, four different isomers could be obtained. The ortho-metalated complexes $6 \mathrm{D}$ and $\mathbf{3 C}$ are chiral-at-metal and have a stereogenic carbon, so in principle two diastereomers could be expected. The ${ }^{1} \mathrm{H}$ NMR and ${ }^{13} \mathrm{C}$ NMR spectra of both complexes show only one set of signals, suggesting that the reaction is diastereoselective. Crystallographic studies of complex 3C confirm that only one diastereomer is obtained. The absolute configuration, determined by X-ray diffraction, 
according to the ligand priority sequence $\mathrm{C}_{5} \mathrm{Me}_{5}>\mathrm{NH}_{2}>\mathrm{C}_{\text {carbene }}>\mathrm{Ph},{ }^{71,72}$ is $\mathrm{R}_{\mathrm{Ir}} \mathrm{S}_{\mathrm{c}}$ and $\mathrm{S}_{\mathrm{Ir}} \mathrm{R}_{\mathrm{c}}$ (Figure 3.4).
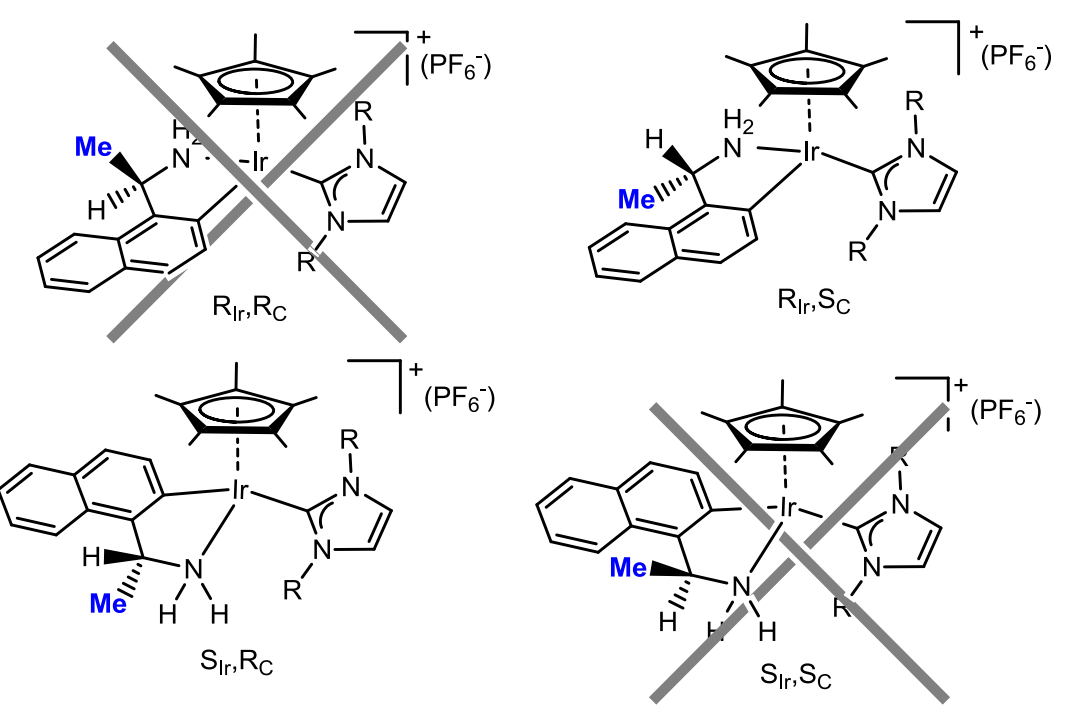

Figure 3.5 All possible isomers that can be obtained from the reaction of 5D (or 2C) with the racemic primary amine

\section{Molecular structure of $6 D^{*}$}

The absolute configuration of $6 \mathrm{D}^{*}$ was confirmed by X-ray diffraction studies (Figure 3.6). Complex 6D* crystallizes in the chiral space group $\mathrm{P} 2{ }_{1} 2_{1} 2_{1}$ and contains a single molecule in the asymmetric unit. This confirms that only one enantiomer is obtained. The absolute configuration is $\mathrm{R}_{\mathrm{Ir}} \mathrm{S}_{\mathrm{c}}$, according to the ligand priority sequence $\mathrm{C}_{5} \mathrm{Me}_{5}>\mathrm{NH}_{2}>$ $\mathrm{C}_{\text {carbene }}>\mathrm{Ph}$. Table 3.2 shows the most representative bond lengths and angles of complex 6D*.The geometry of complex $6 \mathrm{D}^{*}$, bond lengths and angles are similar to those described for complex 3C (Table 3.1). The five-membered ring C-N ortho-metalated amine is in an envelope conformation, and the $\mathrm{N}$ atom is out of the plane by $0.43 \AA$. The methyl group $\mathrm{C}(4)$ attached at the benzylic carbon adopts also an axial disposition. 


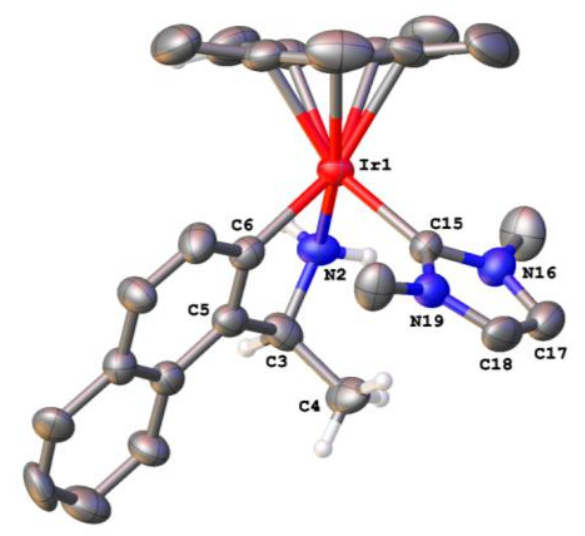

Figure 3.6 Molecular diagram of the cation of complex 6D*. Ellipsoids are shown at $50 \%$ probability level. Only hydrogen atoms bonded to $\mathrm{N}(2), \mathrm{C}(3)$ and $\mathrm{C}(4)$ are shown.

Table 3.2 Selected bond lengths $(\AA)$ and angles $\left(^{\circ}\right)$ of $6 D^{*}$

\begin{tabular}{|c|c|c|c|}
\hline Bonds & $(\AA)$ & Angles & $\left({ }^{\circ}\right)$ \\
\hline $\operatorname{Ir}(1)-C(15)$ & $2.043(7)$ & $C(6)-\operatorname{Ir}(1)-N(2)$ & $76.5(3)$ \\
\hline $\operatorname{Ir}(1)-N(2)$ & $2.138(6)$ & $C(6)-\operatorname{Ir}(1)-C(15)$ & $90.2(3)$ \\
\hline $\operatorname{Ir}(1)-C(6)$ & $2.063(8)$ & $C(15)-\operatorname{Ir}(1)-N(2)$ & $90.1(3)$ \\
\hline $\operatorname{Ir}(1)-\mathrm{Cp}_{\text {centroid }}$ & 1.870 & & \\
\hline
\end{tabular}

\subsection{Catalytic studies}

\subsubsection{Transfer hydrogenation of ketones}

The racemic complexes $\mathbf{6 D}$ and $\mathbf{3 C}$ were tested as catalyst precursors in transfer hydrogenation of ketones. The reactions were carried out with a $1 \mathrm{~mol} \%$ catalyst loading in the presence of $\mathrm{NaOt} \mathrm{Bu}(5 \mathrm{~mol} \%)$, in dry 2-propanol under nitrogen atmosphere. Both complexes required a catalytic amount of base in order to be active in transfer hydrogenation.

The results in Table 3.3 show that both complexes resulted highly active in the catalytic process. Aromatic and aliphatic carbonyls were reduced to the corresponding alcohols in quantitative yields in 2 hours at $70^{\circ} \mathrm{C}$. Although most of substrates required only $50^{\circ} \mathrm{C}$ to achieve yields over $95 \%$ in 2 hours. 
Table 3.3 Transfer hydrogenation of ketones using $6 \mathrm{D}$ and $3 \mathrm{C}$ as catalysts

\begin{tabular}{|c|c|c|c|}
\hline Entry & Catalyst & Substrate & Yield $^{\mathrm{a}}$ \\
\hline 1 & 6D & & 100 \\
\hline 2 & $3 \mathrm{C}$ & & $58(100)^{b}$ \\
\hline 3 & 6D & & 95 \\
\hline 4 & $3 \mathrm{C}$ & & $45(90)^{\mathrm{b}}$ \\
\hline 5 & 6D & & 97 \\
\hline 6 & $3 \mathrm{C}$ & & $55(100)^{\mathrm{b}}$ \\
\hline 7 & 6D & & 97 \\
\hline 8 & $3 \mathrm{C}$ & & 92 \\
\hline 9 & 6D & & 100 \\
\hline 10 & $3 \mathrm{C}$ & & 97 \\
\hline 11 & 6D & & 95 \\
\hline 12 & $3 \mathrm{C}$ & & $57(100)^{\mathrm{b}}$ \\
\hline 13 & 6D & & 100 \\
\hline 14 & $3 \mathrm{C}$ & & 100 \\
\hline
\end{tabular}

Reactions were carried out with $0.5 \mathrm{mmol}$ of substrate, $\mathrm{NaOtBu}(5 \mathrm{~mol} \%)$, catalyst $(1 \mathrm{~mol} \%), 2 \mathrm{~mL}$ of 2propanol for 2 hours at $50^{\circ} \mathrm{C}$. [a] Yields determined by GC analyses using anisole as internal standard. [b] In parenthesis yields after 2 hours at $70^{\circ} \mathrm{C}$.

In general, catalyst 6D showed higher activities than $\mathbf{3 C}$, for instance, acetophenone was quantitatively converted to 1-phenyl ethanol in 2 hours at $50^{\circ} \mathrm{C}$ using $6 \mathrm{D}$, whereas only $58 \%$ yield was obtained using catalyst $3 \mathrm{C}$ (entries 1 and 2). The differences in activity were also observed in the reduction of propiophenone (entries 3 and 4), 2-acetonaphthone 
(entries 5 and 6) and 2-butanone (entries 11 and 12). No significant differences were observed for $p$ - and $o$-bromoacetophenone (entries 7-10) and cyclohexanone (entries 13 and 14).

\subsubsection{Asymmetric transfer hydrogenation of ketones}

The enantiomerically pure complexes $\mathbf{6 D} *$ and $\mathbf{3 C} *$ were evaluated in asymmetric transfer hydrogenation of ketones. The reactions were performed under nitrogen atmosphere in 2-propanol at $30^{\circ} \mathrm{C}$ using a catalyst loading of $1 \mathrm{~mol} \%$ and a catalytic amount of $\mathrm{NaO} t \mathrm{Bu}(5 \mathrm{~mol} \%)$.

Table 3.4 Asymmetric transfer hydrogenation using complexes $6 \mathrm{D}^{*}$ and $3 \mathrm{C}^{*}$

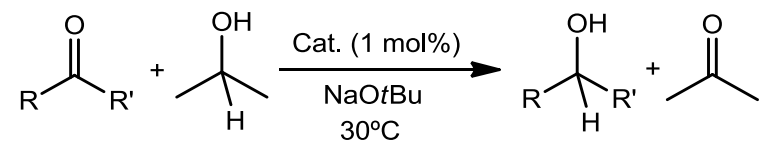

\begin{tabular}{|c|c|c|c|c|}
\hline Entry & Catalyst & Substrate & Yield $^{\mathrm{a}}$ & $e e(\%)^{\mathrm{b}}$ \\
\hline 1 & 6D* & & 100 & 20 \\
\hline 2 & $3 C^{*}$ & & 99 & 55 \\
\hline 3 & $6 D^{*}$ & & 95 & 12 \\
\hline 4 & $3 C^{*}$ & & $56^{\mathrm{c}}$ & 24 \\
\hline 5 & $6 D^{*}$ & & 58 & 16 \\
\hline 6 & $3 C^{*}$ & & 23 & 58 \\
\hline 7 & $6 D^{*}$ & & 63 & 2 \\
\hline 8 & $3 C^{*}$ & & 50 & 15 \\
\hline 9 & $6 D^{*}$ & & 92 & 19 \\
\hline 10 & $3 C^{*}$ & & 36 & 22 \\
\hline
\end{tabular}

Reactions were carried out with $0.5 \mathrm{mmol}$ of substrate, $\mathrm{NaO} t \mathrm{Bu}(5 \mathrm{~mol} \%)$, catalyst (1 mol\%), $2 \mathrm{~mL}$ of 2 propanol for 8 hours at $30^{\circ} \mathrm{C}$. [a] Yields determined by $\mathrm{GC}$ analyses using anisole as internal standard. [b] $e e$ 's determined using a chiral GC. 
The results in Table 3.4 show that both complexes were active in the asymmetric transfer hydrogenation of ketones, at $30^{\circ} \mathrm{C}$. In this case, longer times were needed ( 8 hours) if we compare the activity of the racemic counterparts 6D and 3C ( 2 hours). Acetophenone was quantitatively reduced to 1-phenyl ethanol (entries 1 and 2), while 2-acetonaphtone was only partially reduced (entries 7 and 8 ). In all substrates tested, the catalysts $\mathbf{6 D *}$ and $\mathbf{3 C} *$ presented enantioselectivity, albeit from low to moderate. Generally complex $3 \mathbf{C}^{*}$ lead to better results than $\mathbf{6} \mathbf{D}^{*}$, likely due to the presence of the bulkier $n \mathrm{Bu}$ instead of $\mathrm{Me}$ groups in the wingtips. The reduction of acetophenone and 2'-methylacetophenone with catalyst $3 \mathrm{C}^{*}$ gave the best $e e$ values, $55 \%$ and $58 \%$, respectively (entries 2 and 6).

\subsection{Mechanistic studies}

The above-presented experimental results indicate that all the iridium(III) catalytic systems are highly active in transfer hydrogenation at low temperatures $\left(30^{\circ} \mathrm{C}\right)$. It is worth pointing out that, despite of all complexes tested do not have coordination sites available on the Ir center, the compounds are efficient catalysts for the TH reduction of ketones. Thus, our aim was to assess a plausible reaction mechanism by means of DFT calculations. DFT studies were performed by Dr. Miguel Baya at University of Zaragoza, Spain.

From our preliminary studies by NMR spectroscopy and ESI-MS there are two important facts that should be taken into consideration:

- We did not observe decoordination of any of the ligands in the metal coordination sphere during the course of the experimental studies, although we are aware that catalyst activation by loss of $\mathrm{Cp}^{*}$ has been recently reported for $\mathrm{Cp} * \mathrm{Ir}$ complexes bearing two NHC ligands. ${ }^{22}$

- We did not find any evidence supporting either the formation of a hydride complex or the participation of such type of species in the catalytic cycle.

These observations, together with the imperative need for a strong base in the reaction media to activate the catalyst (5:1 base to catalyst molar ratio) suggest an outer-sphere mechanism involving an iridium-amido derivative as the active species in the catalytic cycle, with the iridium-amine precursor remaining as resting state. 
The mechanistic studies were carried out at the DFT/M06 level of theory and were based on the methyl-substituted NHC complex 6D*, using 2-propanol and acetophenone as hydrogen source and hydrogen acceptor, respectively. ${ }^{17,62,73} \mathrm{We}$ avoided the $n$-butylsubstituted complex $3 C^{*}$ in order to prevent collateral problems derived from the presence of different conformers. As a starting point, the relative energies of the two pairs of enantiomers were evaluated. The $\mathrm{R}_{\mathrm{Ir}} \mathrm{S}_{\mathrm{C}} / \mathrm{S}_{\mathrm{II}} \mathrm{R}_{\mathrm{C}}$ pair (6Da-DFT) was found to be 1.6 $\mathrm{kcal} / \mathrm{mol}$ more stable $\left(\Delta \mathrm{G}_{\mathrm{iPrOH}}\right)$ than the $\mathrm{R}_{\mathrm{Ir}} \mathrm{R}_{\mathrm{C}} / \mathrm{S}_{\mathrm{Ir}} \mathrm{S}_{\mathrm{C}}$ pair (6Db-DFT) (Scheme 3.7).

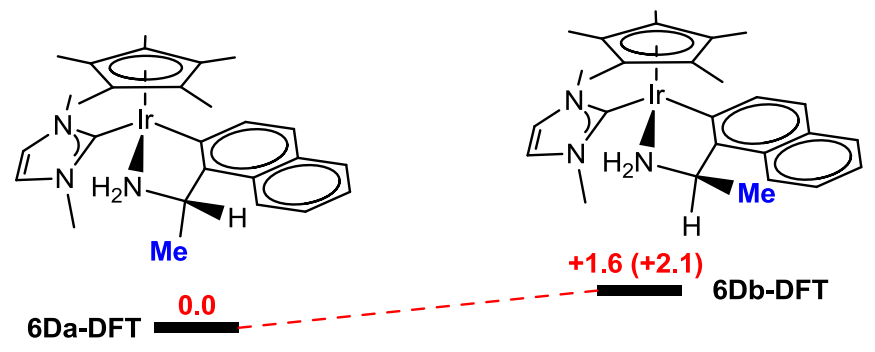

Scheme 3.7 Relative free energies in solution (2-propanol) of the two diastereomers $\mathrm{R}_{\mathrm{Ir}} \mathrm{S}_{\mathrm{C}}$ (or $S_{\text {Ir }} R_{C}$ ) (6Da-DFT) and $R_{\text {Ir }} R_{C}$ (or $S_{I r} S_{C}$ ) (6Db-DFT) in complex 6D* in Kcal $/$ mol. Relative free energies in the gas phase are shown in parentheses.

Although we are aware that small barriers should be taken with caution, this relative energy order is in agreement with the experimental results found in the diastereoselective synthesis of complexes $\mathbf{6 D}$ and $\mathbf{3 C}$, suggesting that the process is thermodynamically controlled. We only have observed the diastereomer in which the methyl group is pointing away from the $\mathrm{Cp}^{*}$ ring (Figure 3.6). The converged structure of 6Da-DFT compares well with that found for $\mathbf{6 D}^{*}$ by $\mathrm{X}$-ray diffraction (Figure 3.6), with the fivemember aza-irida-metalacycle ring showing an envelope conformation, in which the $\mathrm{N}$ atom is slightly out of the plane, by $0.46 \AA$. The Ir- $\mathrm{C}_{\text {carbene, }}$, Ir- $\mathrm{C}_{\text {metallacycle }}$ and Ir-N bond lengths of 6Da-DFT are 2.06, 2.08 and $2.19 \AA$, respectively.

Dealing with the interaction of the base with the catalyst precursor, it was considered the formation of an iridium-alkoxide complex via amine decoordination, and the interaction of the alkoxide ( $i$-propoxide) with any of the two hydrogen $\mathrm{NH}$ atoms to afford amidoalcohol species. Figure 3.7 shows the three most remarkable optimized adducts found, A- 
DFT, B-DFT and C-DFT. A-DFT represents the iridium-alkoxide adduct and B-DFT and C-DFT the amido-alcohol species.
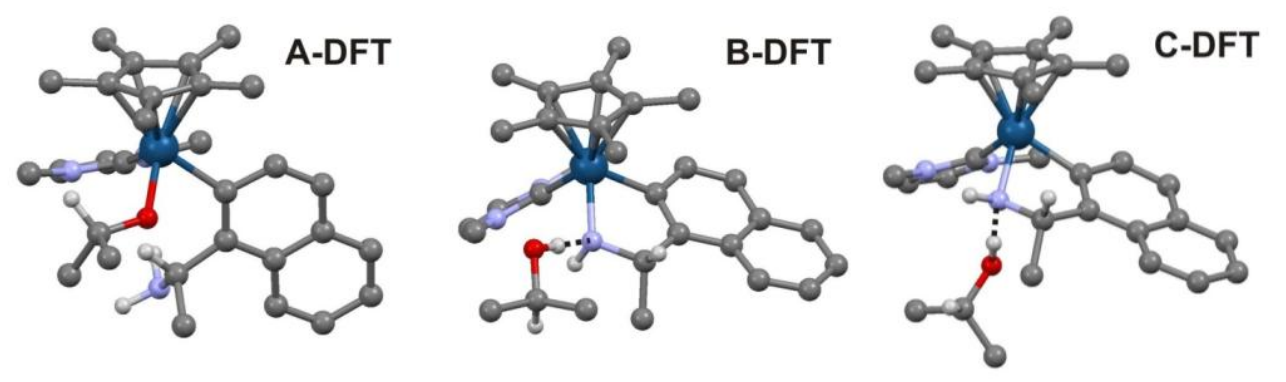

Figure 3.7 Molecular diagrams of the DFT optimized adducts A-DFT, B-DFT, C-DFT. Most of the hydrogen atoms have been omitted for clarity.

The relative energies of the optimized adducts are displayed in Scheme 3.8. The most favored alkoxide species (A-DFT) lays $22.5 \mathrm{kcal} / \mathrm{mol}$ higher in free energy in solution, related to the most favored amido-alcohol one (B-DFT), and $17.9 \mathrm{kcal} / \mathrm{mol}$ higher than a second amido-alcohol isomer (C-DFT). These relative energies suggest the unlikeness of the participation of an iridium-alkoxide species in the cycle.

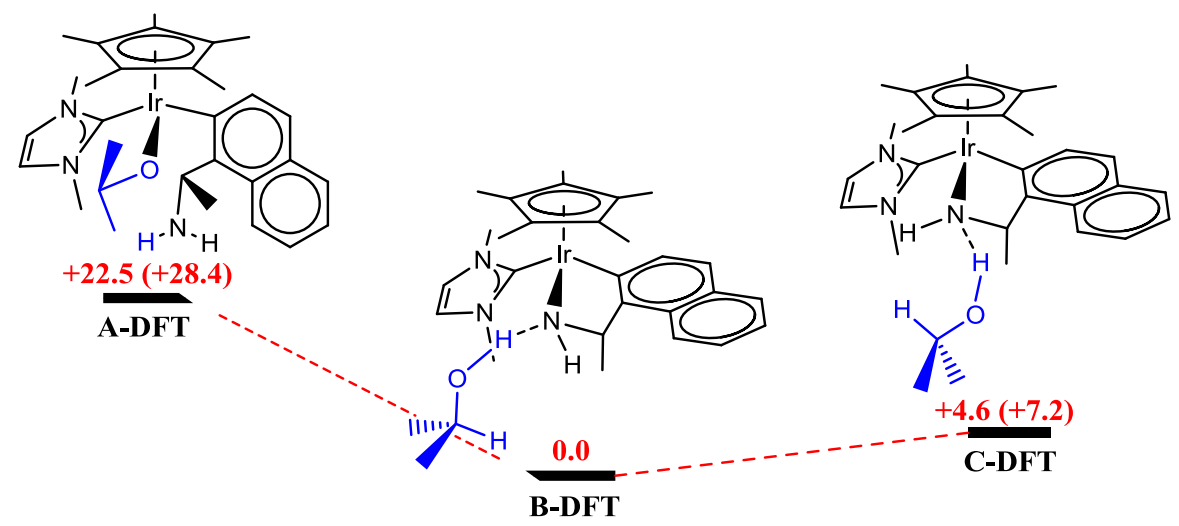

Scheme 3.8 Relative free energies in solution (2-propanol) of the iridium-alkoxide (A-DFT) and amido-alcohol species (B-DFT and C-DFT) in $\mathrm{kcal} / \mathrm{mol}$. Relative free energies in the gas phase are shown in parentheses. 
The structure of the putative iridium-amido complex resulting from the deprotonation of 6D* was also optimized (Figure 3.8, D-DFT). This species is most likely the catalyst of the studied TH process, as pointed out by the experimental and theoretical evidence.
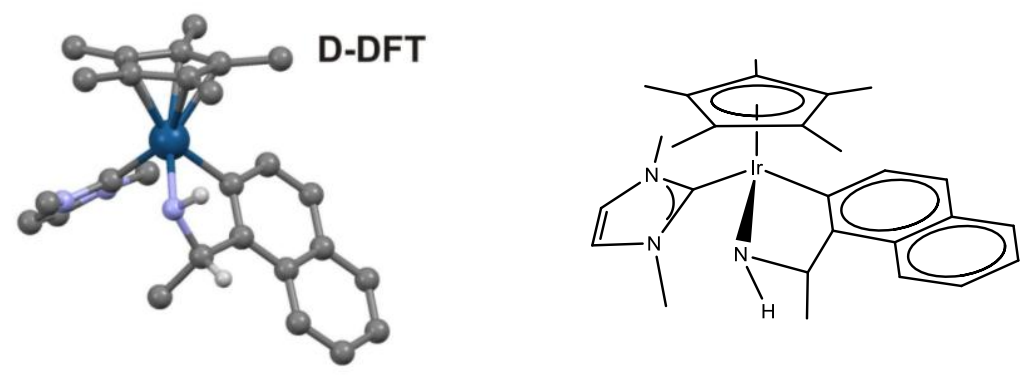

Figure 3.8 Molecular diagram (left) and schematic representation (right) of the DFT optimized complex D-DFT. Most hydrogen atoms have been omitted for clarity.

Taking all these considerations into account, two mechanisms consistent with the abovepresented discussion were evaluated.

Pathway 1: Concerted, two-step, outer-sphere process

This pathway is described as a concerted, two-step, outer-sphere process, involving the participation of the cyclopentadienyl ring and the amido group (Scheme 3.9) ${ }^{74}$ In the first step, the iridium-amido complex D-DFT dehydrogenates 2-propanol to afford an iridiumamine $\eta^{4}$-cyclopentadiene species (P1-03-DFT) that subsequently transfers $\mathrm{H}_{2}$ to the prochiral ketone in the second step. This latter transfer could happen with two possible substrate orientations, thus leading to the formation of the two alcohol enantiomers ( $R$ and $S$ ). 


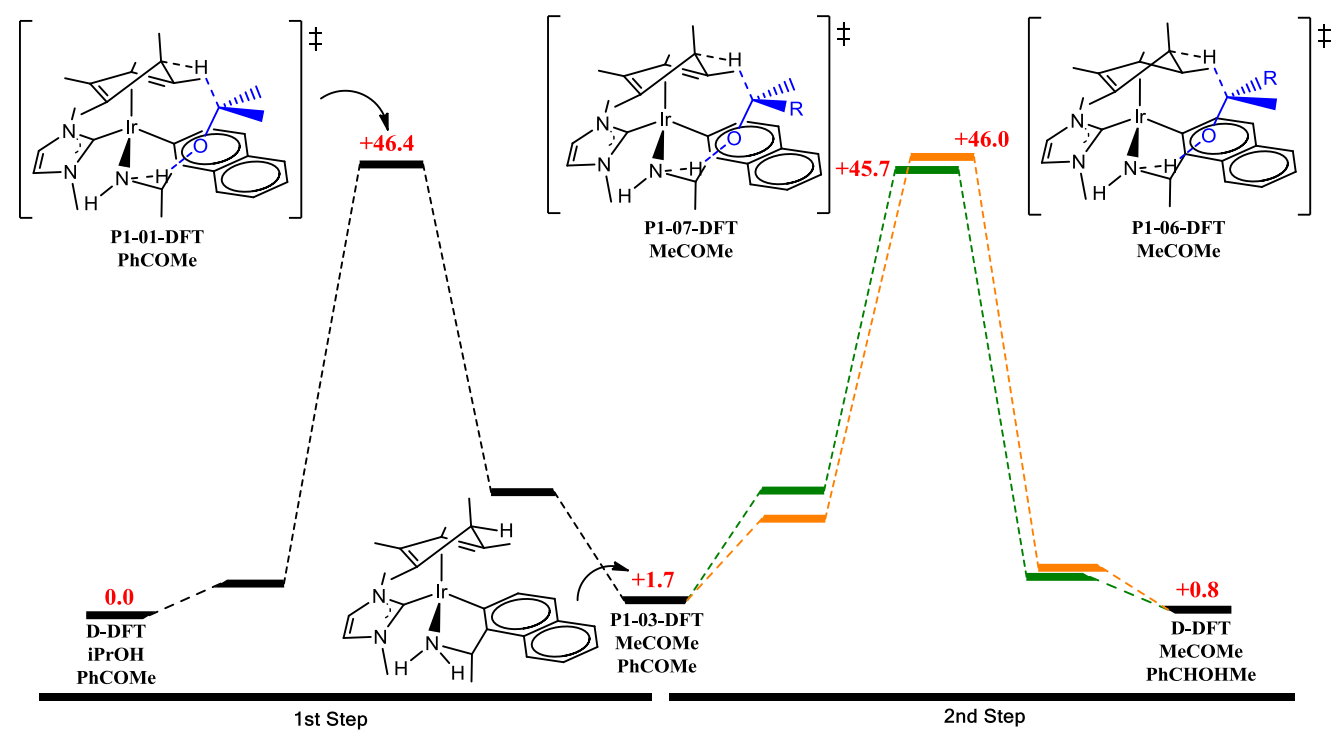

Scheme 3.9 DFT-calculated energy profile for the concerted, two-step, outer-sphere hydrogen transfer mechanism (Pathway 1). Relative free energies in 2-propanol solution in Kcal/mol. Only TS's and intermediate P1-03-DFT are shown for clarity.

The key species of this cycle is the iridium-amine $\eta^{4}$-cyclopentadiene P1-03-DFT, which results from the alcohol-to-catalyst transfer hydrogenation. Notably, the calculated transition state for the first step (P1-01-DFT) is $46.4 \mathrm{kcal} / \mathrm{mol}$ above in $\Delta \mathrm{G}_{i \mathrm{PrOH}}$, related to the free reactants. Consistent with this barrier, the subsequent transfer hydrogenation from P1-03-DFT to the prochiral ketone, in any of the two possible orientations to render (S) or (R)-1-phenylethanol, also require 46.0 and $45.7 \mathrm{kcal} / \mathrm{mol}$, respectively (transition states P1-06-DFT and P1-07-DFT). Thus, the high energy barriers calculated for these processes (dehydrogenation- transfer hydrogenation) clearly dismiss this reaction pathway.

\section{Pathway 2: Concerted, one-step, outer-sphere process}

Scheme 3.11 displays the second mechanism proposed. It consists of the hydrogen transfer from the iridium-amido-isopropyl alcohol adduct (either B-DFT or C-DFT, Figure 3.7) to the prochiral ketone, in a concerted, one-step, outer sphere process. Thus, depending on the relative orientation of the alcohol and the prochiral ketone with regard 
to the iridium center, four variants can be envisaged leading to the formation of one of the two enantiomers. Due to their similarity, only one of these pathways is displayed in Scheme 3.10 .

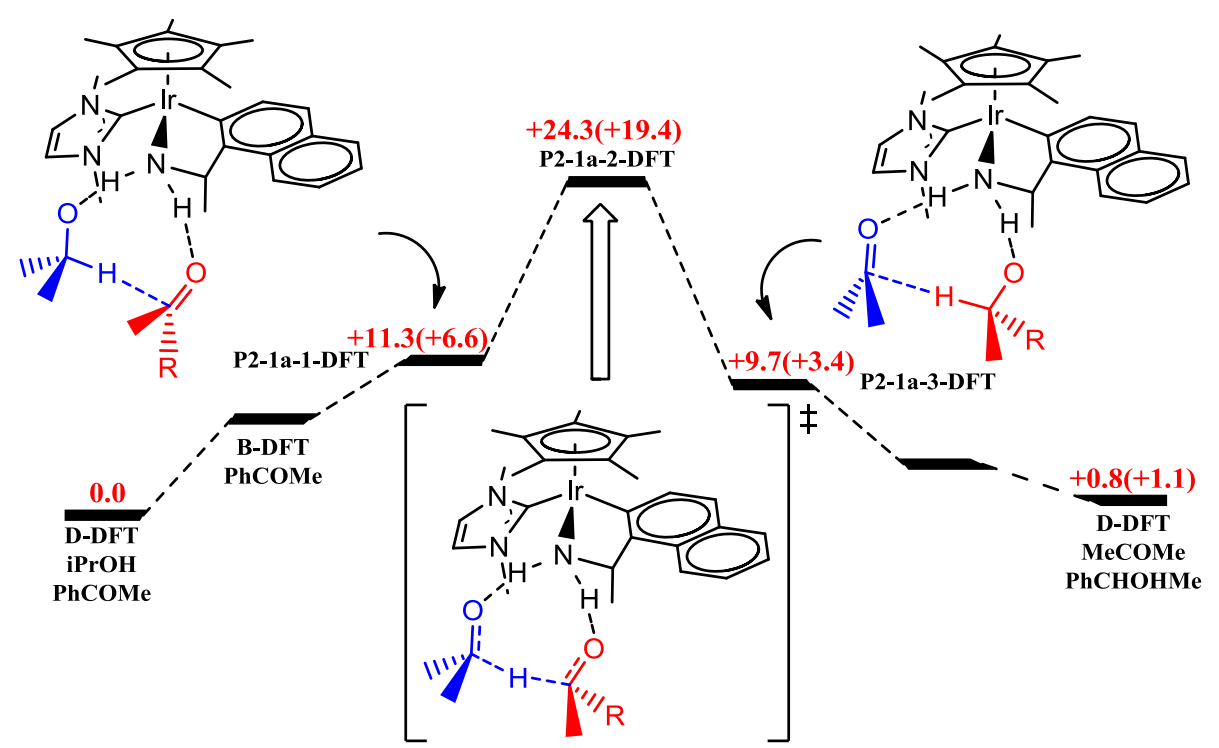

Scheme 3.10 DFT-calculated energy profile for one of the four possible concerted, one-step, outer sphere hydrogen mechanism (pathway 2) in kcal/mol. Relative free energies in 2-propanol solution and in the gas phase are shown in parentheses.

Four different transition states were found (Figure 3.9), two of them leading to (R)-1phenylethanol (P2-1a-2-DFT, P2-2b-2-DFT), and the other two leading to (S)-1phenylethanol (P2-1b-2-DFT, P2-2a-2-DFT). Interestingly, the calculated energy barriers $\left(\Delta \mathrm{G}_{\text {solv }}\right)$ of these concerted processes lay in the $24.3-25.7 \mathrm{kcal} / \mathrm{mol}$ range $(18.6-$ $21.2 \mathrm{Kcal} / \mathrm{mol}$ in $\Delta \mathrm{G}_{\mathrm{gas}}$ ). These data are consistent with the experimental results. Furthermore, the relative energies of the TS's found, leading to either R or S alcohol enantiomers, present very similar values with regard to each other. In other words, DFT calculations do not predict remarkable $e e$ 's in favor of any of the enantiomers. This result also is in good agreement with the poor enantiomeric excesses obtained experimentally. 

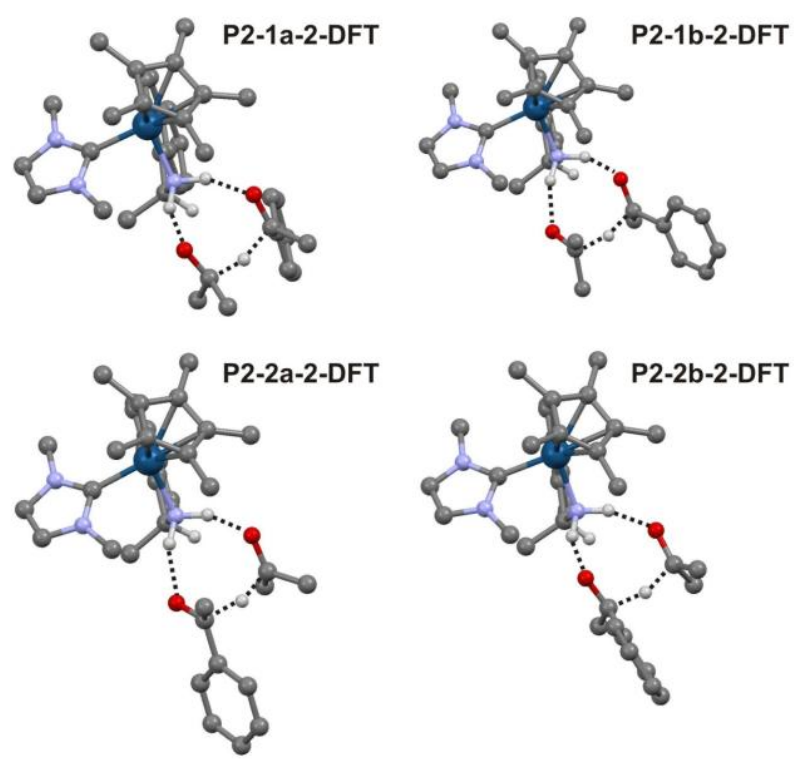

Figure 3.9 Molecular diagrams of the DFT optimized transition states P2-1a-2-DFT, P2-1b-2DFT, P2-2a-2-DFT, and P2-2b-2-DFT. Most hydrogen atoms have been omitted for clarity

The whole set of transition states found for this mechanism are "hydrogen-bonding organized" TS's. All of them are markedly synchronous thus, supporting concerted processes. Hydrogen-bonding organized transition states have been proposed previously in a few catalytic reactions, including the Ru-catalyzed enantioselective Michael addition of 1,3-dicarbonyl compounds to cyclic enones and the Ir-catalyzed asymmetric transfer hydrogenation of nitroalkenes. .7,75-77 $^{-1}$

In order to gain additional experimental evidences for the proposed mechanism replacement of the $\mathrm{NH}$ group by an $\mathrm{N}$-alkyl group was investigated. The introduction of this alkyl group should inhibit the formation of the hydrogen-bonding organized transition state and have an impact in the catalytic activity. The reaction of $\left[\mathrm{Cp} * \operatorname{Ir}(\mathrm{Cl})_{2}\left(\mathrm{Me}_{2} \mathrm{Im}\right)\right](5 \mathrm{D})$ or $\left[\mathrm{Cp} * \operatorname{Ir}(\mathrm{Cl})_{2}\left(\mathrm{nBu}_{2} \mathrm{Im}\right)\right](\mathbf{2 C})$ with the secondary amine $[(\mathrm{S})-$ $\mathrm{N}, \alpha$-dimethylbenzylamine] in the presence of two equivalents of AgOAc under the same conditions as described in Scheme 3.6 did not afford any ortho-metalated complex (Scheme 3.11). 


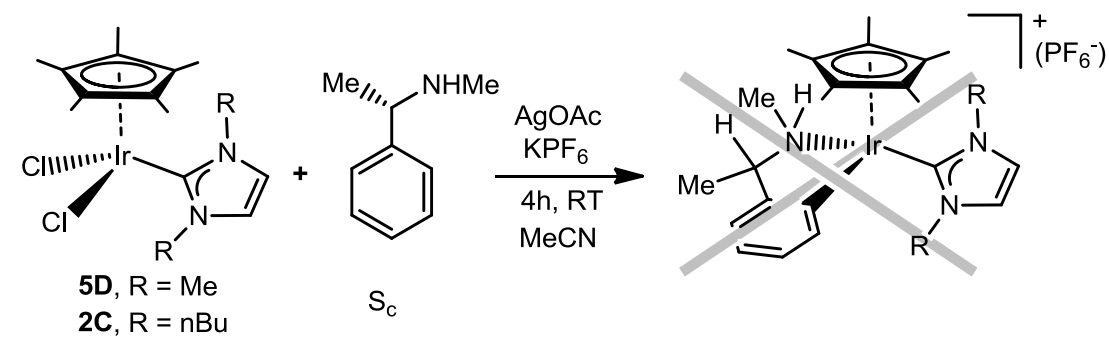

Scheme 3.11 


\section{CONCLUSIONS}

The synthesis on new IrCp* complexes bearing an $\mathrm{N}$-heterocyclic carbene ligand and a chelated primary benzylamine has been described. The complexes are chiral-at-metal and have a stereogenic carbon at the benzylamine ligand. The catalytic results reveal that cyclometalated iridium complexes based on chiral primary benzylamines are very active in transfer hydrogenation, for example, acetophenone is reduced to 1-phenylethanol in $2 \mathrm{~h}$ at $50^{\circ} \mathrm{C}$ using catalyst $6 \mathrm{D}$. More interestingly is that no base, apart from the required for catalyst activation, is needed in the process.

Density Functional Theory studies provided a mechanistic proposal which is consistent with the experimental observations and catalytic results. Interestingly, the transfer hydrogenation seems to proceed in a one-step process via a highly ordered transition state centered at the iridium-amido group, without the participation of any hydridic intermediate. According to the classification presented in the introduction of this chapter, this mechanism can be classified as a "non-hydride", albeit it implies some remarkable features. Contrary to the classical MPV reduction, in which the key feature is the Lewisacid character of the metal center, in the presented catalytic system, the Bronsted-acid character of the $\mathrm{NH}_{2}$ group bound to the iridium center is the key factor.

Substitutionally inert species, if catalytically active, may lead to robust catalysts therefore presenting higher turnover numbers. The catalysts presented in this chapter constitute a rare and remarkable example showing that inert and chiral-at-metal complexes can have successful applications in transfer hydrogenation processes. Although up to now our complexes only induce $e e$ 's from low to moderate, the synergy between $\mathrm{IrCp} *$ moieties, NHC ligands and primary amines holds great promise for the design of bulky systems showing improved performances in asymmetric transfer hydrogenation. 


\section{REFERENCES}

(1) Haddad, Y. M. Y.; Husbands, J.; Henbest, H. B.; Mitchell, T. R. P. Chem. Soc. London 1964, 361.

(2) Trochagr.J; Henbest, H. B. Chem. Commun. 1967, 544.

(3) Henbest, H. B.; Mitchell, T. R. J. Chem. Soc. C 1970, 785.

(4) Camus, A.; Mestroni, G.; Zassinovich, G. J. Mol. Catal. 1979, 6, 231-233.

(5) Baratta, W.; Chelucci, G.; Herdtweck, E.; Magnolia, S.; Siega, K.; Rigo, P. Angew. Chem. Int. Edit. 2007, 46, 7651-7654.

(6) O, W. W. N.; Lough, A. J.; Morris, R. H. Organometallics 2012, 31, 2137-2151.

(7) Ohara, H.; O, W. W. N.; Lough, A. J.; Morris, R. H. Dalton Trans. 2012, 41, 8797-8808.

(8) Mashima, K.; Abe, T.; Tani, K. Chem. Lett. 1998, 1199-1200.

(9) Murata, K.; Ikariya, T. J. Org. Chem. 1999, 64, 2186-2187.

(10) Annen, S.; Zweifel, T.; Ricatto, F.; Gruetzmacher, H. ChemCatChem 2010, 2, 1286-1295.

(11) Trincado, M.; Kuehlein, K.; Gruetzmacher, H. Chem. Eur. J. 2011, 17, 1190511913.

(12) Annen, S. P.; Gruetzmacher, H. Dalton Trans. 2012, 41, 14137-14145.

(13) Lang, F.; Breher, F.; Stein, D.; Grutzmacher, H. Organometallics 2005, 24, 2997 3007.

(14) Nova, A.; Taylor, D. J.; Blacker, A. J.; Duckett, S. B.; Perutz, R. N.; Eisenstein, O. Organometallics 2014, 33, 3433-3442.

(15) Bianchini, C. In Iridium Complexes in Organic Synthesis; Oro, L. A., Claver, C., Eds.; Wiley-VCH: Weinheim, 2008.

(16) Fujita, K.; Yamaguchi, R. In Iridium Complexes in Organic Synthesis; Oro, L. A., Claver, C., Eds.; Wiley-VCH: Weinheim, 2008.

(17) Wylie, W. N. O.; Lough, A. J.; Morris, R. H. Organometallics 2013, 32, 38083818.

(18) Dobereiner, G. E.; Nova, A.; Schley, N. D.; Hazari, N.; Miller, S. J.; Eisenstein, O.; Crabtree, R. H. J. Am. Chem. Soc. 2011, 133, 7547-7562. 
(19) Gnanamgari, D.; Sauer, E. L. O.; Schley, N. D.; Butler, C.; Incarvito, C. D.; Crabtree, R. H. Organometallics 2009, 28, 321-325.

(20) Gnanamgari, D.; Moores, A.; Rajaseelan, E.; Crabtree, R. H. Organometallics 2007, 26, 1226-1230.

(21) Chianese, A. R.; Crabtree, R. H. Organometallics 2005, 24, 4432-4436.

(22) Campos, J.; Hintermair, U.; Brewster, T. P.; Takase, M. K.; Crabtree, R. H. ACS Catal. 2014, 4, 973-985.

(23) Hintermair, U.; Campos, J.; Brewster, T. P.; Pratt, L. M.; Schley, N. D.; Crabtree, R. H. ACS Catal. 2014, 4, 99-108.

(24) Schley, N. D.; Halbert, S.; Raynaud, C.; Eisenstein, O.; Crabtree, R. H. Inorg. Chem. 2012, 51, 12313-12323.

(25) Eisenstein, O.; Crabtree, R. H. New J. Chem. 2013, 37, 21-27.

(26) Berliner, M. A.; Dubant, S. P. A.; Makowski, T.; Ng, K.; Sitter, B.; Wager, C.; Zhang, Y. Org. Process Res. Dev. 2011, 15, 1052-1062.

(27) Gladiali, S.; Alberico, E. Chem. Soc. Rev. 2006, 35, 226-236.

(28) Samec, J. S. M.; Backvall, J. E.; Andersson, P. G.; Brandt, P. Chem. Soc. Rev. 2006, 35, 237-248.

(29) Meerwein, H.; Schmidt, R. Liebigs Ann. Chem. 1925, 444, 221-238.

(30) Verley, A. Bull. Soc. Chim. Fr. 1925, 37, 537.

(31) Ponndorf, W. Angew. Chem. Int. Edit. 1926, 39, 138-143.

(32) Degraauw, C. F.; Peters, J. A.; Vanbekkum, H.; Huskens, J. Synthesis 1994, 1007-1017.

(33) Oppenauer, R. V. Recl. Trav. Chim. Pay-B 1937, 56, 137-144.

(34) Clapham, S. E.; Hadzovic, A.; Morris, R. H. Coord. Chem. Rev. 2004, 248, 22012237.

(35) Pamies, O.; Backvall, J. E. Chem. Eur. J. 2001, 7, 5052-5058.

(36) Bäckvall, J. E. J. Organomet. Chem. 2002, 652, 105-111.

(37) Haack, K. J.; Hashiguchi, S.; Fujii, A.; Ikariya, T.; Noyori, R. Angew. Chem. Int. Edit. 1997, 36, 285-288.

(38) Noyori, R.; Hashiguchi, S. Accounts Chem. Res. 1997, 30, 97-102.

(39) Noyori, R.; Yamakawa, M.; Hashiguchi, S. J. Org. Chem. 2001, 66, 7931-7944.

(40) Yamakawa, M.; Ito, H.; Noyori, R. J. Am. Chem. Soc. 2000, 122, 1466-1478. 
(41) Ikariya, T.; Murata, K.; Noyori, R. Org. Biomol. Chem. 2006, 4, 393-406.

(42) Ikariya, T.; Blacker, A. J. Accounts Chem. Res. 2007, 40, 1300-1308.

(43) Bifunctional Transition metal-based molecular catalysts for asymmetric synthesis; Ikariya, T., Ed.; Springer-Verlag Berlin Heidelberg, 2011; Vol. 37, pp 31-53.

(44) Blum, Y.; Czarkie, D.; Rahamim, Y.; Shvo, Y. Organometallics 1985, 4, 14591461.

(45) Shvo, Y.; Czarkie, D.; Rahamim, Y.; Chodosh, D. F. J. Am. Chem. Soc. 1986, 108, 7400-7402.

(46) Hashiguchi, S.; Fujii, A.; Takehara, J.; Ikariya, T.; Noyori, R. J. Am. Chem. Soc. 1995, 117, 7562-7563.

(47) Uematsu, N.; Fujii, A.; Hashiguchi, S.; Ikariya, T.; Noyori, R. J. Am. Chem. Soc. 1996, 118, 4916-4917.

(48) Fujii, A.; Hashiguchi, S.; Uematsu, N.; Ikariya, T.; Noyori, R. J. Am. Chem. Soc. 1996, 118, 2521-2522.

(49) Arita, S.; Koike, T.; Kayaki, Y.; Ikariya, T. Organometallics 2008, 27, 27952802.

(50) Arita, S.; Koike, T.; Kayaki, Y.; Ikariya, T. Chem.-Asian J. 2008, 3, 1479-1485.

(51) Watanabe, M.; Kashiwame, Y.; Kuwata, S.; Ikariya, T. Eur. J. Inorg. Chem. 2012, 504-511.

(52) Sato, Y.; Kayaki, Y.; Ikariya, T. Chem. Commun. 2012, 48, 3635-3637.

(53) Kashiwame, Y.; Kuwata, S.; Ikariya, T. Organometallics 2012, 31, 8444-8455.

(54) Barloy, L.; Issenhuth, J.-T.; Weaver, M. G.; Pannetier, N.; Sirlin, C.; Pfeffer, M. Organometallics 2011, 30, 1168-1174.

(55) Jerphagnon, T.; Haak, R.; Berthiol, F.; Gayet, A. J. A.; Ritleng, V.; Holuigue, A.; Pannetier, N.; Pfeffer, M.; Voelklin, A.; Lefort, L.; Verzijl, G.; Tarabiono, C.; Janssen, D. B.; Minnaard, A. J.; Feringa, B. L.; de Vries, J. G. Top. Catal. 2010, 53, 1002-1008.

(56) Pannetier, N.; Sortais, J.-B.; Issenhuth, J.-T.; Barloy, L.; Sirlin, C.; Holuigue, A.; Lefort, L.; Panella, L.; de Vries, J. G.; Pfeffer, M. Adv. Synth. Catal. 2011, 353, 2844-2852. 
(57) Cross, W. B.; Daly, C. G.; Boutadla, Y.; Singh, K. Dalton Trans. 2011, 40, 97229730.

(58) Comas-Vives, A.; Ujaque, G.; Lledós, A. J. Mol. Struct. -Theochem 2009, 903, 123-132.

(59) Comas-Vives, A.; Ujaque, G.; Lledós, A. In Advances in Inorganic Chemistry: Theoretical and Computational Inorganic Chemistry; VanEldik, R., Harvey, J., Eds., 2010; Vol. 62; pp 231-260.

(60) Alonso, D. A.; Brandt, P.; Nordin, S. J. M.; Andersson, P. G. J. Am. Chem. Soc. 1999, 121, 9580-9588.

(61) Di Tommaso, D.; French, S. A.; Zanotti-Gerosa, A.; Hancock, F.; Palin, E. J.; Catlow, C. R. A. Inorg. Chem. 2008, 47, 2674-2687.

(62) Dub, P. A.; Ikariya, T. J. Am. Chem. Soc. 2013, 135, 2604-2619.

(63) Nieto Faza, O.; Fernandez, I.; Silva Lopez, C. Chem. Commun. 2013, 49, $4277-$ 4279.

(64) Petra, D. G. I.; Reek, J. N. H.; Handgraaf, J. W.; Meijer, E. J.; Dierkes, P.; Kamer, P. C. J.; Brussee, J.; Schoemaker, H. E.; van Leeuwen, P. Chem. Eur. J. 2000, 6, 2818-2829.

(65) Zhang, X.; Guo, X.; Chen, Y.; Tang, Y.; Lei, M.; Fang, W. Phys. Chem. Chem. Phys. 2012, 14, 6003-6012.

(66) Bi, S.; Xie, Q.; Zhao, X.; Zhao, Y.; Kong, X. J. Organomet. Chem. 2008, 693, 633-638.

(67) Handgraaf, J. W.; Reek, J. N. H.; Meijer, E. J. Organometallics 2003, 22, 31503157.

(68) Davies, D. L.; Al-Duaij, O.; Fawcett, J.; Giardiello, M.; Hilton, S. T.; Russell, D. R. Dalton Trans. 2003, 4132-4138.

(69) Hockless, D. C. R.; Gugger, P. A.; Leung, P. H.; Mayadunne, R. C.; Pabel, M.; Wild, S. B. Tetrahedron 1997, 53, 4083-4094.

(70) Corberan, R.; Sanau, M.; Peris, E. J. Am. Chem. Soc. 2006, 128, 3974-3979.

(71) Cahn, R. S.; Ingold, C.; Prelog, V. Angew. Chem. Int. Edit. 1966, 5, 385-\&.

(72) Brunner, H. Enantiomer 1997, 2, 133-134.

(73) Comas-Vives, A.; Ujaque, G.; Lledós, A. Organometallics 2007, 26, 4135-4144.

(74) W. N. O, W.; Lough, A. J.; Morris, R. H. Organometallics 2012, 31, 2152-2165. 
(75) Gridnev, I. D.; Watanabe, M.; Wang, H.; Ikariya, T. J. Am. Chem. Soc. 2010, $132,16637-16650$.

(76) Chen, L.-A.; Xu, W.; Huang, B.; Ma, J.; Wang, L.; Xi, J.; Harms, K.; Gong, L.; Meggers, E. J. Am. Chem. Soc. 2013, 135, 10598-10601.

(77) Hounjet, L. J.; Ferguson, M. J.; Cowie, M. Organometallics 2011, 30, 41084114. 


\section{CHAPTER 4}

\section{RUTHENIUM COMPLEXES WITH FUNCTIONALIZED MIC LIGANDS: SYNTHESIS AND CATALYTIC APPLICATIONS}

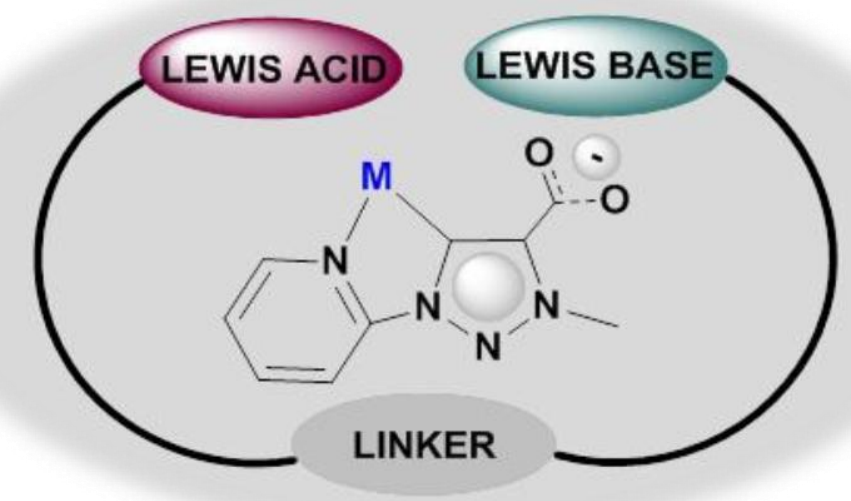





\section{INTRODUCTION}

The functionalization of key ligands for boosting their applications in catalytic reactions has been largely explored in coordination chemistry. ${ }^{1}$ Functionalized ligands have demonstrated to be non-innocent, and to potentially engage in reversible proton binding and release. Thus, functionalized ligands can play an important role in the activation of small molecules in a catalytic cycle, where both metal and ligand simultaneously participate in bond-forming and breaking processes. ${ }^{2-5}$

Mesoionic/abnormal carbenes have become attractive ligands in transition metal chemistry. ${ }^{6}$ Among them, functionalized 1,2,3-triazolylidene systems constitute a particularly versatile class of mesoionic carbenes (MICs), as they combine mesoionic properties with an extraordinary synthetic diversity. ${ }^{7}$ The ligand precursors are readily accessible either after alkylation of the corresponding 1,2,3-triazoles, obtained by [2+3] cycloaddition of alkynes and azides, as depicted in Scheme 4.1, or by cycloaddition between 1,2-diaza-2-azoniaallene salts and alkynes. ${ }^{8}$ These transformations stand for its exceptionally broad functional group tolerance. ${ }^{9}$
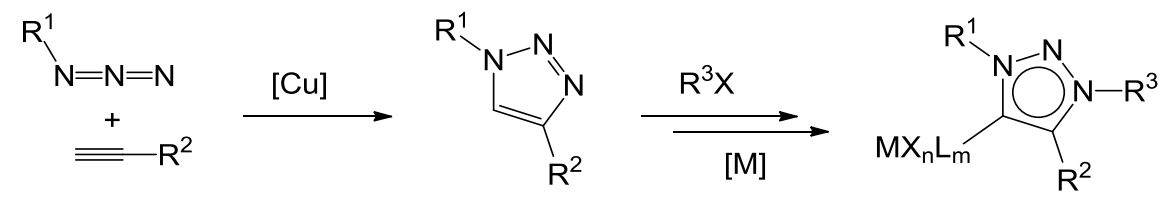

Scheme 4.1 Schematic route to 1,2,3-triazolylidene-based metal complexes

The synthetic flexibility of 1,2,3-triazolylidenes allows the introduction of functionalities at positions that are not accessible with other traditional N-heterocyclic carbenes. These functionalities, when introduced in close proximity to the metal center, may aid bond activation of substrates through ligand-metal cooperation (and eventually facilitate catalytic turnover). Moreover, the flexibility of MICs to adapt its donor strength and to stabilize low and high oxidation states, ${ }^{10}$ constitutes an exciting platform for performing challenging reactions that rely on synergistic ligand-metal interactions.

Frustrated Lewis pairs (FLPs), in which the combination of a Lewis acid and a Lewis base act cooperatively to activate small molecules, is at the forefront of chemistry 
research. ${ }^{11,12}$ The use of FLPs has already provided significant achievements in the heterolytic cleavage of $\mathrm{H}_{2}{ }^{13-15}$ FLP systems in which one of the Lewis acid/base components is a transition metal, may combine the well-established reactivity of transition metals with the inherent activity of the Lewis acid/base pairs. ${ }^{16}$ Transition metal complexes have long been exploited as Lewis acid catalysts for a variety of synthetic transformations, ${ }^{17}$ so marrying these properties with Lewis base functionalized MICs, opens a wide range of new possibilities for the design of bifunctional or cooperative metal-based FLP catalysts.

The main objective of this chapter is the synthesis and the catalytic applications of a new version of metal-based FLP systems. The ease to stereoelectronically modulate 1,2,3 triazolylidenes should facilitate the design of a new class of complexes able to hold a frustrated Lewis pair. With this aim, we decided to prepare a series of carboxylatefunctionalized Ru-triazolylidene complexes, in which the carboxylate functionality is introduced in close proximity to the metal center. The catalytic activity of the new complexes will be evaluated in transfer hydrogenation and dehydrogenative oxidation of alcohols processes. All the results presented in this chapter have been developed during a stay at the School of Chemistry \& Chemical Biology of the University College of Dublin under the supervision of Prof. Martin Albrecht. 


\section{RESULTS AND DISCUSSION}

\subsection{Synthesis and characterization of triazolium salts}

The syntheses of the triazolium salts $\mathbf{H}$ and $\mathbf{I}$ were carried out by a copper mediated [3+2] cycloaddition, followed by the $\mathrm{N}$-alkylation of the triazole formed (Scheme 4.2). The $[3+$ 2] cycloaddition of ethyl propiolate and pyridine-2-azide, in the presence of $\mathrm{CuI}$ and 2,6lutidine, in tetrahydrofuran/dimethyl sulfoxide mixtures, for $3 \mathrm{~h}$ at $100^{\circ} \mathrm{C}$, afforded the previously described triazole $\mathbf{h}^{18}$ The N-alkylation of $\mathbf{h}$ with methyl trifluoromethanesulfonate (MeOTf) in dichloromethane, afforded the triazolium salt $\mathbf{H}$, as a light brown solid in $89 \%$ yield. The hydrolysis of $\mathbf{h}$ with $\mathrm{LiOH} \cdot \mathrm{H}_{2} \mathrm{O}$ in a tetrahydrofuran/methanol/water $(1: 1: 1)$ mixture, at $70^{\circ} \mathrm{C}$ for 1.5 hours, yielded the triazole $\mathbf{i}$ as a yellow solid in $89 \%$ yield. The $\mathrm{N}$-alkylation of $\mathbf{i}$ with MeOTf in dichloromethane, afforded the triazolium salt $\mathbf{I}$ as a light brown solid in $94 \%$ yield.

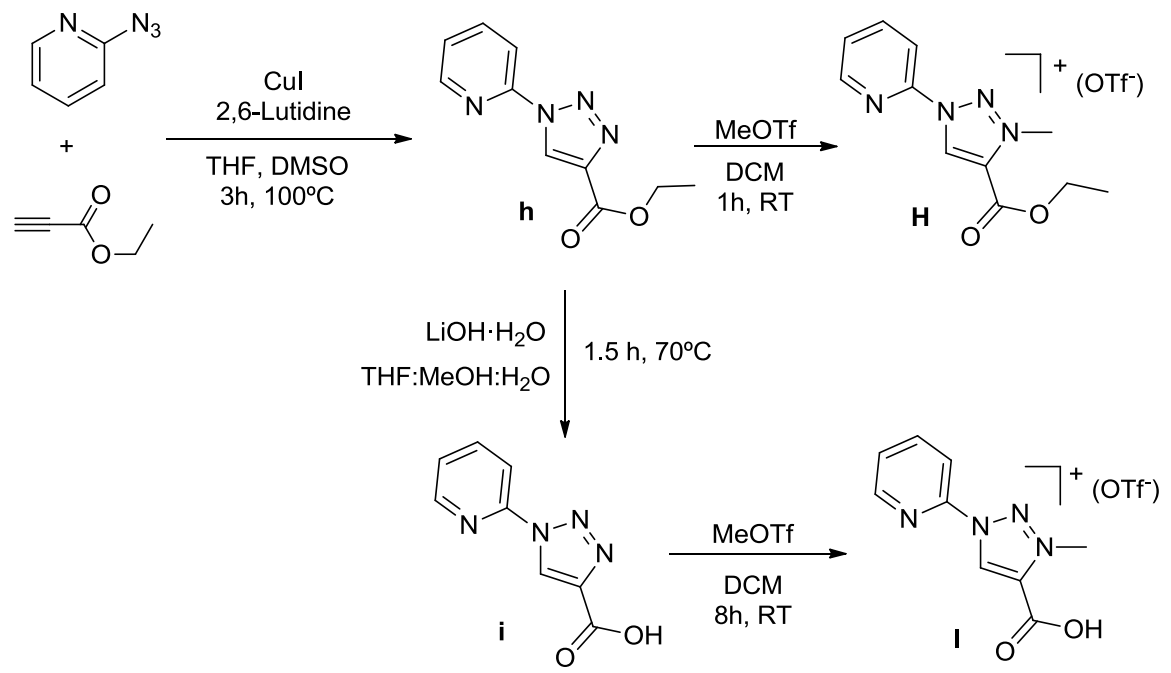

Scheme 4.2 Synthesis of triazolium salts $\mathbf{H}$ and $\mathbf{I}$

Both triazolium salts were characterized by NMR and IR spectroscopy, mass spectrometry, and elemental analysis. All the spectroscopic data for triazolium salts $\mathbf{H}$ and $\mathbf{I}$ can be found in the Experimental Section (Chapter 6). 


\subsection{Synthesis of Ru-triazolylidene complexes via decarboxylation}

The metalation of the triazolium salt I may mainly occur at the only available $\mathrm{C}-\mathrm{H}$ position of the triazole ring, due to the presence of the carboxylic acid group. The reaction of $\mathbf{I}$ in the presence of a base at room temperature, undergoes decarboxylation affording the previously described triazolium salt $\mathbf{J}$, which has two different triazolium C-H sites ${ }^{19}$ (Scheme 4.3). The lability of the carboxylic moiety in I may open new opportunities for the metalation of triazolium salts via decarboxylative methods. ${ }^{20}$ However, attempts to use the carboxylate group as a carbene transfer agent to the metal center by releasing $\mathrm{CO}_{2}$ were unsuccessful. The reaction of the triazolium salt $\mathbf{I}$ with $\left[\mathrm{RuCl}_{2}(p \text {-cymene })\right]_{2}$ in refluxing acetonitrile or toluene yielded the non-carboxylate triazolium salt J (Scheme 4.3).

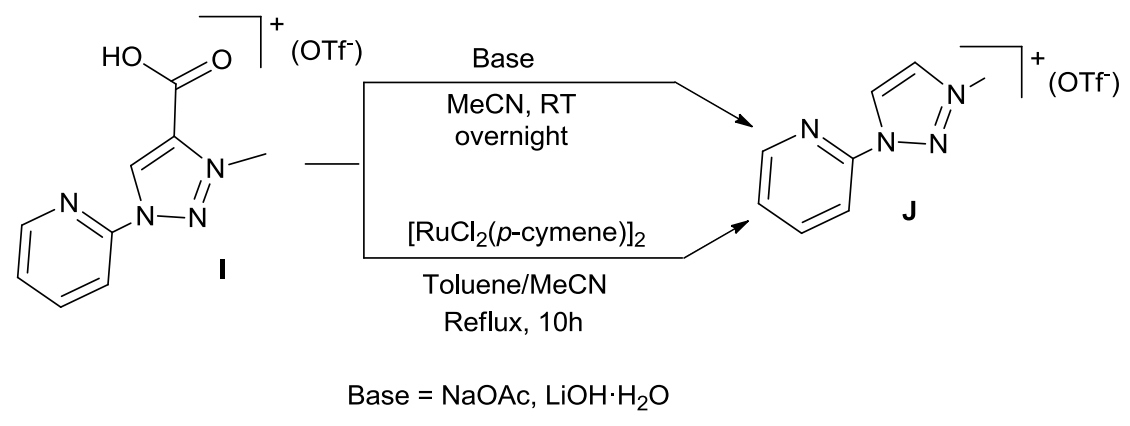

Scheme 4.3 Synthesis of $\mathbf{J}$ by decarboxylation of $\mathbf{I}$

The decarboxylation of $\mathbf{I}$ to afford $\mathbf{J}$ was confirmed by the absence of the carbonyl IR stretch vibration at $1746 \mathrm{~cm}^{-1}$ and by the presence of resonances due to two triazolium protons with equal intensity $\left(\delta_{\mathrm{H}} 9.14\right.$ and 8.72$)$ in the ${ }^{1} \mathrm{H}$ NMR spectrum. ${ }^{19}$

The syntheses of the triazolylidene-based complexes 1I, 2I and 3I were carried out by transmetalation from the corresponding pre-formed silver-NHC complexes, which were prepared by treatment of the triazolium salt I with $\operatorname{Ag}_{2} \mathrm{O}$, as depicted in Scheme 4.4. Complex 1 I was prepared by reaction of $\mathbf{I}$ with $\mathrm{Ag}_{2} \mathrm{O}$ in acetonitrile for two hours, and subsequent addition of $\left[\mathrm{RuCl}_{2}(p \text {-cymene })\right]_{2}$ at room temperature. Complex 2I was produced spontaneously when dissolving complex $\mathbf{1 I}$ in chloroform, which produced a mixture of 1I and 2I, as observed by ${ }^{1} \mathrm{H}$ NMR spectroscopy. Complex 2I was easily 
separated by column chromatography, as it is a neutral complex while $\mathbf{1 I}$ is cationic. The formation of $\mathbf{3 I}$ required 3 days of reaction of the triazolium salt $\mathbf{I}$ with $\mathrm{Ag}_{2} \mathrm{O}$ in acetonitrile, after which $\left[\mathrm{RuCl}_{2}(p \text {-cymene })\right]_{2}$ was added and the reaction mixture was allowed to stir at room temperature for $16 \mathrm{~h}$. After purification, compounds $\mathbf{1 I}$ and $\mathbf{3 I}$ were obtained as orange solids in $c a .30 \%$ yield.

The product selectivity occurs at the Ag-triazolylidene formation stage. The thermodynamically more stable chelate complex 3I is formed only upon prolonged reaction time of silver oxide in the presence of the triazolium carboxylate. Attempts to directly interconvert the ruthenium complexes $\mathbf{1 I}$ or 2 I to the supposedly more stable complex 3I did not meet any success. Prolonged heating of $\mathbf{1 I}$ or $\mathbf{2 I}$ in acetonitrile at elevated temperatures did not induce a shift of the metal center from the triazolium $\mathrm{C} 4$ position to C5 and instead, only gradual cymene loss and formation of the tetra(acetonitrile) species was observed.
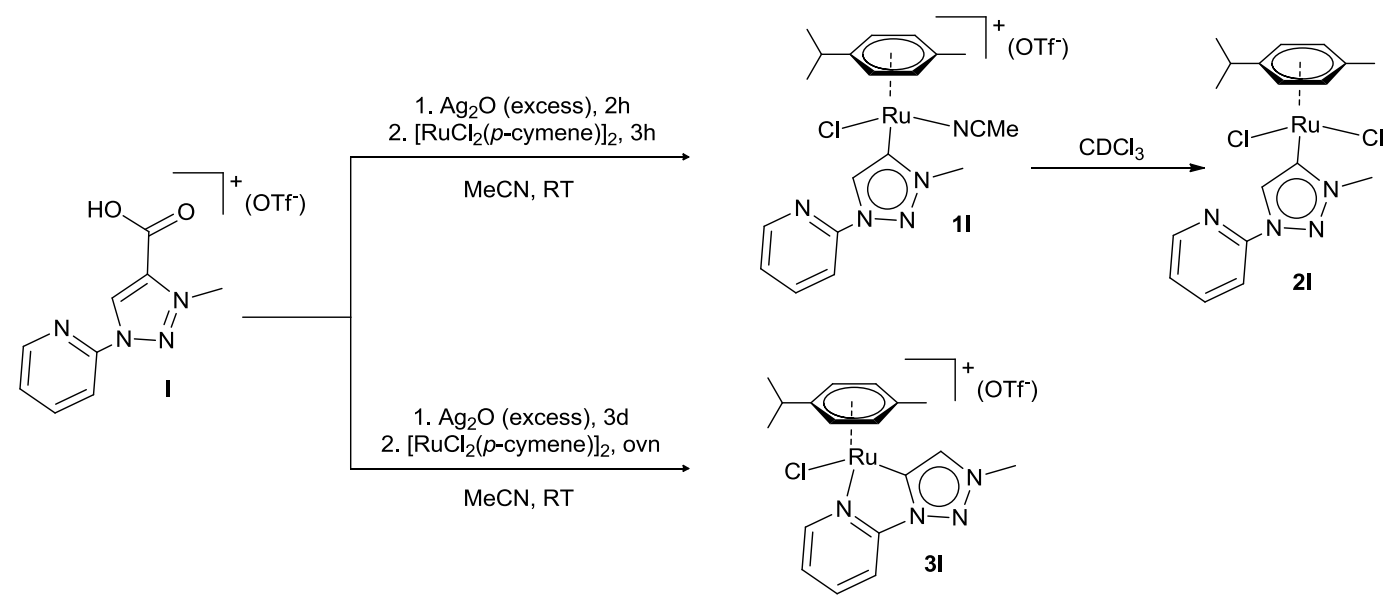

Scheme 4.4 Synthesis of complexes 1I, 2I and 3I

All complexes were characterized by NMR spectroscopy, mass spectrometry and elemental analysis. ${ }^{13} \mathrm{C}$ HSQC, ${ }^{13} \mathrm{C}$ HMBC, and NOE experiments were carried out in order to fully assign the signals displayed on the ${ }^{1} \mathrm{H}$ and ${ }^{13} \mathrm{C}$ NMR spectra of complexes 1I and 3I. 


\section{${ }^{1} H$ NMR spectrum of $\boldsymbol{1 I}$}

Figure 4.1 shows the ${ }^{1} \mathrm{H}$ NMR spectrum of 1I. The most characteristic signal is the one attributed to the $\mathrm{CH}$ proton of the triazole ring at $8.43 \mathrm{ppm}$, indicating that decarboxylation of the triazolylidene ligand has occurred. The upfield shift of the signal attributed to the ortho- $\mathrm{H}$ of the pyridyl group from $8.69 \mathrm{ppm}$ in the triazolium salt $\mathbf{I}$ to $8.63 \mathrm{ppm}$ in complex $1 \mathrm{I}$ suggests that the ruthenium center is not bound to the pyridine. NOE experiments suggest a through-space interaction of the $\mathrm{CH}$ group of the triazolylidene and the ortho- $\mathrm{H}$ of the pyridyl group, indicating metalation of the triazolium at the $\mathrm{C} 4$ position. The rest of the protons of the pyridyl group display their resonances as multiplets between 8.16 and $7.61 \mathrm{ppm}$. The asymmetric environment about the $\mathrm{Ru}$ center is supported by the desymmetrization of the aromatic protons of the $p$ cymene ligand, which are shown as four doublets at 5.75, 5.68, 5.62 and $5.48 \mathrm{ppm}$. The presence of the coordinated acetonitrile is confirmed by the signal at $2.26 \mathrm{ppm}$, due to the $\mathrm{CH}_{3} \mathrm{CN}$ protons. The signal due to the protons of the methyl group at the triazole ring appears at $4.36 \mathrm{ppm}$.

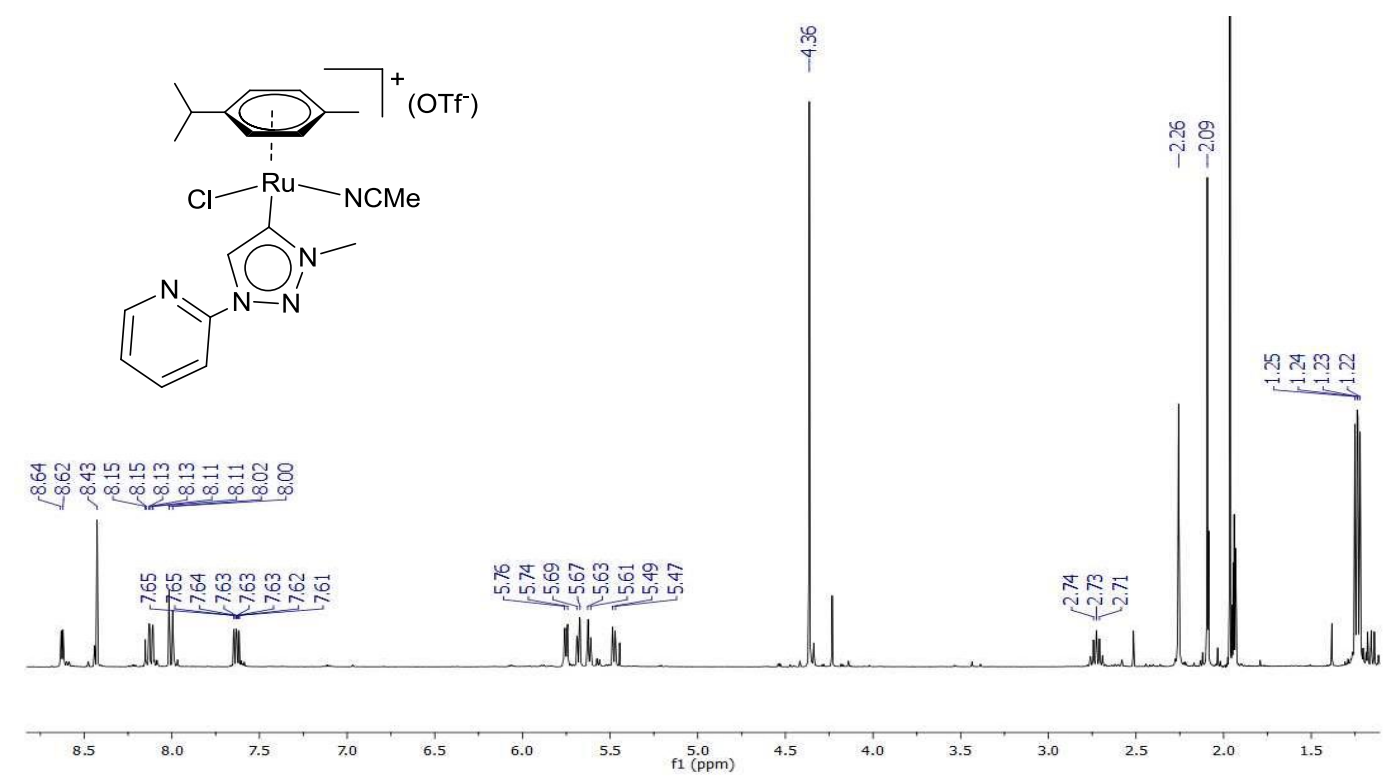

Figure 4.1 ${ }^{1} \mathrm{H}$ NMR spectrum of complex $1 \mathrm{I}$ in $\mathrm{CD}_{3} \mathrm{CN}$ 


\section{${ }^{I} H$ NMR spectrum of $\mathbf{3 I}$}

Figure 4.2 shows the ${ }^{1} \mathrm{H}$ NMR spectrum of 3I. The signal attributed to the $\mathrm{CH}$ proton of the triazole ring appears at $8.42 \mathrm{ppm}$, similar to $\mathrm{CH}_{\text {trz }}$ of $\mathbf{1 I}$, and constitutes a diagnostic indication of the decarboxylation of the triazolylidene ligand. The signal attributed to the ortho-H of the pyridyl group is shown as a doublet of doublet at $9.25 \mathrm{ppm}\left({ }^{3} \mathrm{~J}_{\mathrm{H}, \mathrm{H}}=5.7\right.$, $0.8 \mathrm{~Hz}$ ), which is significantly downfield-shifted compared to the resonance of the same proton in $\mathbf{I}(8.69 \mathrm{ppm})$. NOE experiments suggest a through-space interaction of the proton of the $\mathrm{CH}$ and the protons of the $\mathrm{N}-\mathrm{CH}_{3}$ of the triazolylidene, indicating metalation of the triazolium at the $\mathrm{C} 5$ position. The rest of the protons of the pyridyl group display their resonances as multiplets between 8.29 and $7.59 \mathrm{ppm}$. The bidentate (chelate) coordination is supported by the desymmetrization of the aromatic protons of the $p$-cymene ligand, which are shown as four doublets at 6.06, 5.88, 5.70 and $5.50 \mathrm{ppm}$. The signal due to the protons of the methyl group at the triazole ring appears at $4.33 \mathrm{ppm}$.

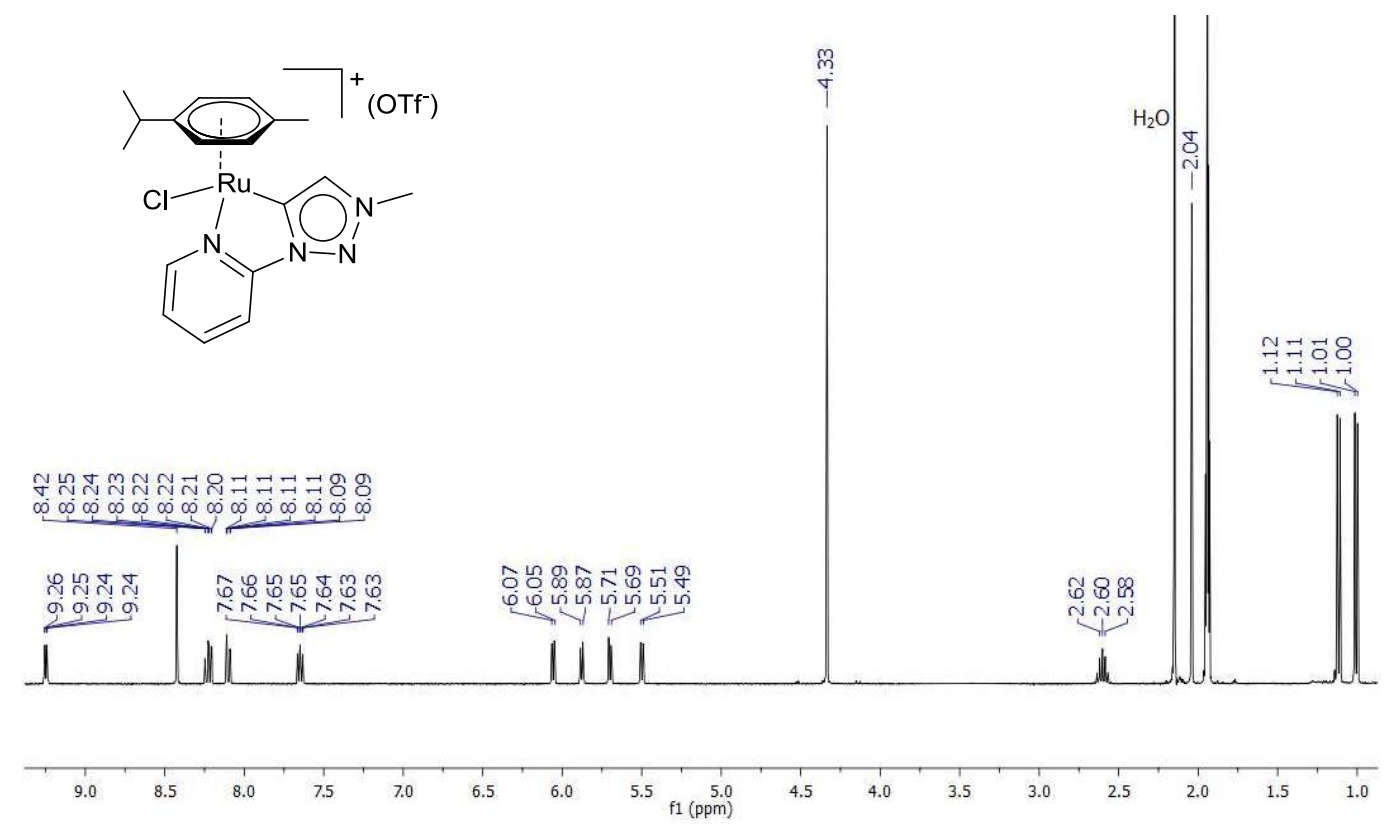

Figure 4.2 ${ }^{1} \mathrm{H}$ NMR spectrum of complex $3 \mathrm{I}$ in $\mathrm{CD}_{3} \mathrm{CN}$ 
The ${ }^{13} \mathrm{C}$ NMR spectra of 1I, 2I and 3I show the signal due to the metalated carbene carbon at $161.4,167.8$ and $171.5 \mathrm{ppm}$, respectively. The signals attributed to the $\mathrm{CH}$ at the triazole ring appear at $130.7(\mathbf{1 I}), 130.9(\mathbf{2 I})$, and $136.9(\mathbf{3 I}) \mathrm{ppm}$.

The mass spectra of complexes 1I, 2I and 3I showed peaks at $\mathrm{m} / \mathrm{z}=472.47[\mathrm{M}]^{+}, 431.48$ $[\mathrm{M}-\mathrm{Cl}]^{+}$and $431.43[\mathrm{M}]^{+}$, respectively, confirming the proposed molecular composition of the complexes based on the mass/charge relation and the isotopic pattern.

\section{Molecular structure of complex 2 I}

Crystals of 2I suitable for X-ray diffraction analysis were obtained by slow diffusion of pentane in a concentrated solution of the compound in chloroform. The molecular structure of 2I confirms the monodentate coordination at the $\mathrm{C} 4$ position of the triazolylidene ligand ( $\mathrm{C} 1$ in Figure 4.3). The structure can be regarded as a three-legged piano stool. Along with the triazolylidene ligand, two chlorides and a $p$-cymene ligand complete the coordination sphere about the ruthenium center. Table 4.1 shows the most representative bond lengths and angles of complex $2 \mathrm{I}$. The $\mathrm{Ru}-\mathrm{C}_{\text {carbene }}$ bond length is 2.052(5) $\AA$, in the range of other ' $\mathrm{Ru}\left(p\right.$-cymene)MIC' complexes. ${ }^{21} \mathrm{The} \mathrm{Ru}-\mathrm{Cl}$ and $\mathrm{Ru}-$ $\mathrm{C}_{\text {centroid }}$ bond lengths and the $\mathrm{C}_{\text {carbene }}-\mathrm{Ru}-\mathrm{Cl}$ angles lie in the expected range.

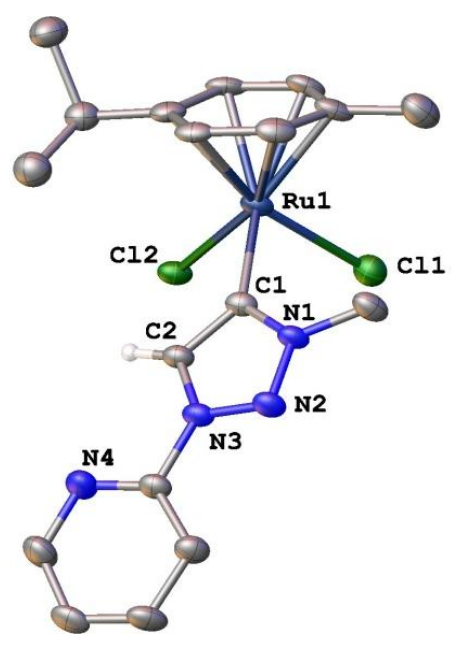

Figure 4.3 Molecular diagram of complex 2I. Ellipsoids are shown at 50\% probability level. All hydrogen atoms except $\mathrm{H}-\mathrm{C} 2$ are omitted for clarity. 
Table 4.1 Selected bond lengths $(\AA)$ and angles $\left(^{\circ}\right)$ of complex $2 \mathbf{I}$.

\begin{tabular}{cccc}
\hline Bonds & $\mathbf{( \AA )}$ & Angles & $\left.\mathbf{(}^{\circ}\right)$ \\
\hline $\mathrm{Ru}(1)-\mathrm{C}(1)$ & $2.052(5)$ & $\mathrm{C}(1)-\mathrm{Ru}(1)-\mathrm{Cl}(2)$ & $84.29(15)$ \\
$\mathrm{Ru}(1)-\mathrm{Cl}(2)$ & $2.4277(12)$ & $\mathrm{C}(1)-\mathrm{Ru}(1)-\mathrm{Cl}(1)$ & $87.14(15)$ \\
$\mathrm{Ru}(1)-\mathrm{Cl}(1)$ & $2.4313(14)$ & $\mathrm{Cl}(2)-\mathrm{Ru}(1)-\mathrm{Cl}(1)$ & $87.25(5)$ \\
$\mathrm{Ru}(1)-\mathrm{C}_{\text {centroid }}$ & 1.689 & & \\
\hline
\end{tabular}

\subsection{Synthesis of 'Ru(p-cymene)' triazolylidene-based complexes}

The synthesis of the triazolylidene-based complex $\mathbf{1 H}$ was carried out by transmetalation from the pre-formed silver-NHC complex, which was obtained by reaction of $\mathbf{H}$ with $\mathrm{Ag}_{2} \mathrm{O}$ in dichloromethane (Scheme 4.5). Then $\left[\mathrm{RuCl}_{2}(p \text {-cymene) }]_{2}\right.$ was added, and the reaction mixture was stirred at room temperature overnight. The crude mixture was filtered through celite, and the solvent was removed under reduced pressure. Compound 1H was purified by column chromatography, and the pure complex was precipitated from a mixture of acetonitrile/diethyl ether, as an orange solid in $77 \%$ yield.

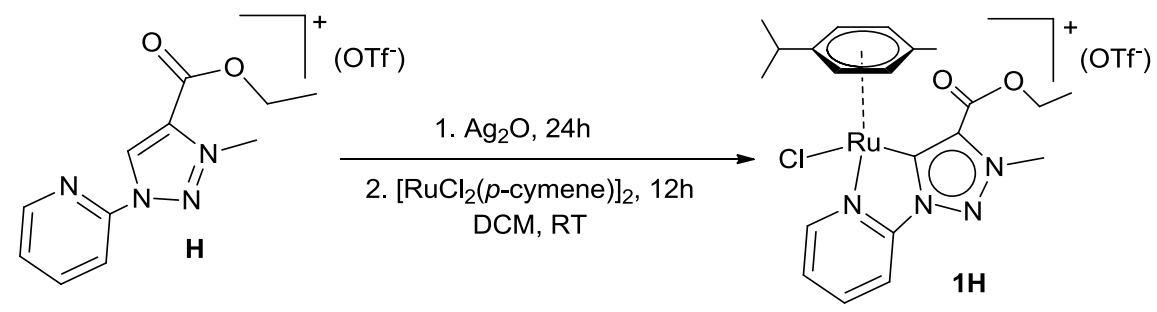

Scheme 4.5 Synthesis of complex $1 \mathrm{H}$

Scheme 4.6 shows the synthetic protocol used for the basic hydrolysis of the ester functionality in complex $\mathbf{1 H}$ to afford the carboxylate $(\mathbf{2 H})$ and the carboxylic acid $(\mathbf{3 H})$ derivatives. The zwitterionic ${ }^{22}$ complex $\mathbf{2 H}$ was obtained by reaction of $\mathbf{1 H}$ with two equivalents of $\mathrm{LiOH} \cdot \mathrm{H}_{2} \mathrm{O}$ in a methanol/water (1:1) mixture. After purification, $\mathbf{2 H}$ was obtained as yellow solid, in $83 \%$ yield. Complex $\mathbf{3 H}$ was obtained following a similar protocol, but acidifying with an aqueous $\mathrm{HCl}$ solution $(1 \mathrm{M})$ after the hydrolysis. Complex 3H was purified and obtained as a yellow solid in $92 \%$ yield. 


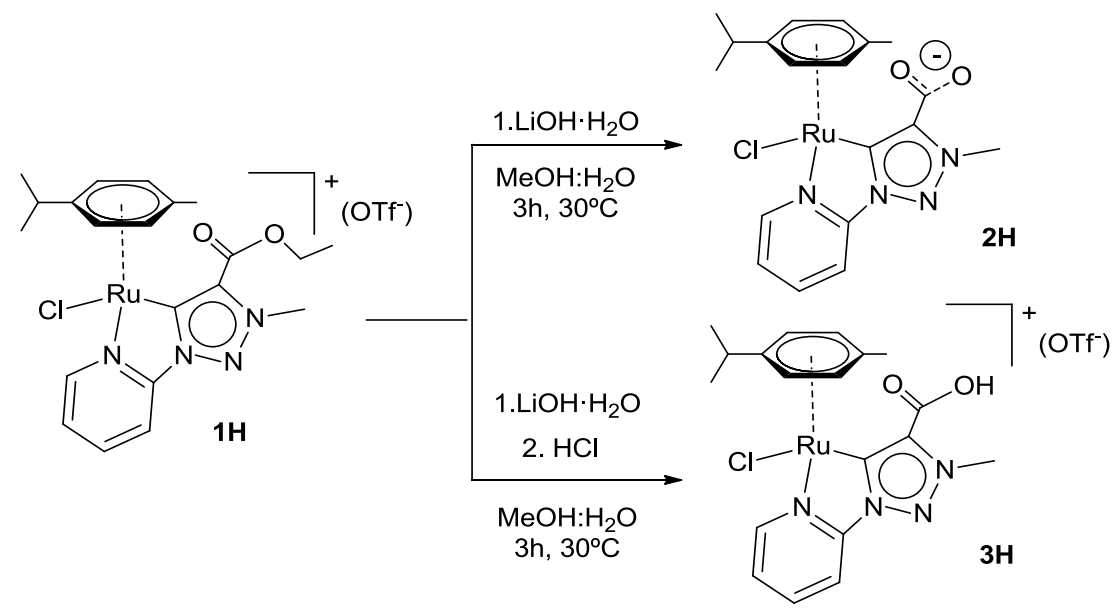

Scheme 4.6 Synthesis of complexes $2 \mathrm{H}$ and $3 \mathrm{H}$

Complexes $\mathbf{1 H}$ and $\mathbf{3 H}$ are air- and moisture-stable in the solid state and in solution, while complex $\mathbf{2 H}$ is air-stable but highly hygroscopic. All three complexes were characterized by NMR and IR spectroscopy, mass spectrometry and elemental analysis.

\section{${ }^{1}$ H NMR spectrum of complex $1 \boldsymbol{H}$}

Figure 4.4 shows the ${ }^{1} \mathrm{H}$ NMR spectrum of complex $\mathbf{1 H}$. The first indication that the metalation has taken place is the disappearance of the signal attributed to the NCHC proton of the triazolium salt $\mathbf{H}$. The signal due to the ortho-H of the pyridyl group is shown as a doublet at $9.27 \mathrm{ppm}\left({ }^{3} \mathrm{~J}_{\mathrm{H}, \mathrm{H}}=5.7 \mathrm{~Hz}\right)$, providing evidence of metal bonding to the pyridine, as the signal is significantly downfield shifted compared to the one shown in the ligand precursor $\mathbf{H}$. The remaining protons of the pyridyl group display their resonances as multiplets between 8.30 and $7.65 \mathrm{ppm}$. The bidentate coordination was also supported by the desymmetrization of the aromatic protons of the $p$-cymene ligand, which appear as four doublets at 6.23, 6.15, 5.82 and $5.43 \mathrm{ppm}$. The signal due to the protons of the methyl group at the triazole ring appears at $4.53 \mathrm{ppm}$. 


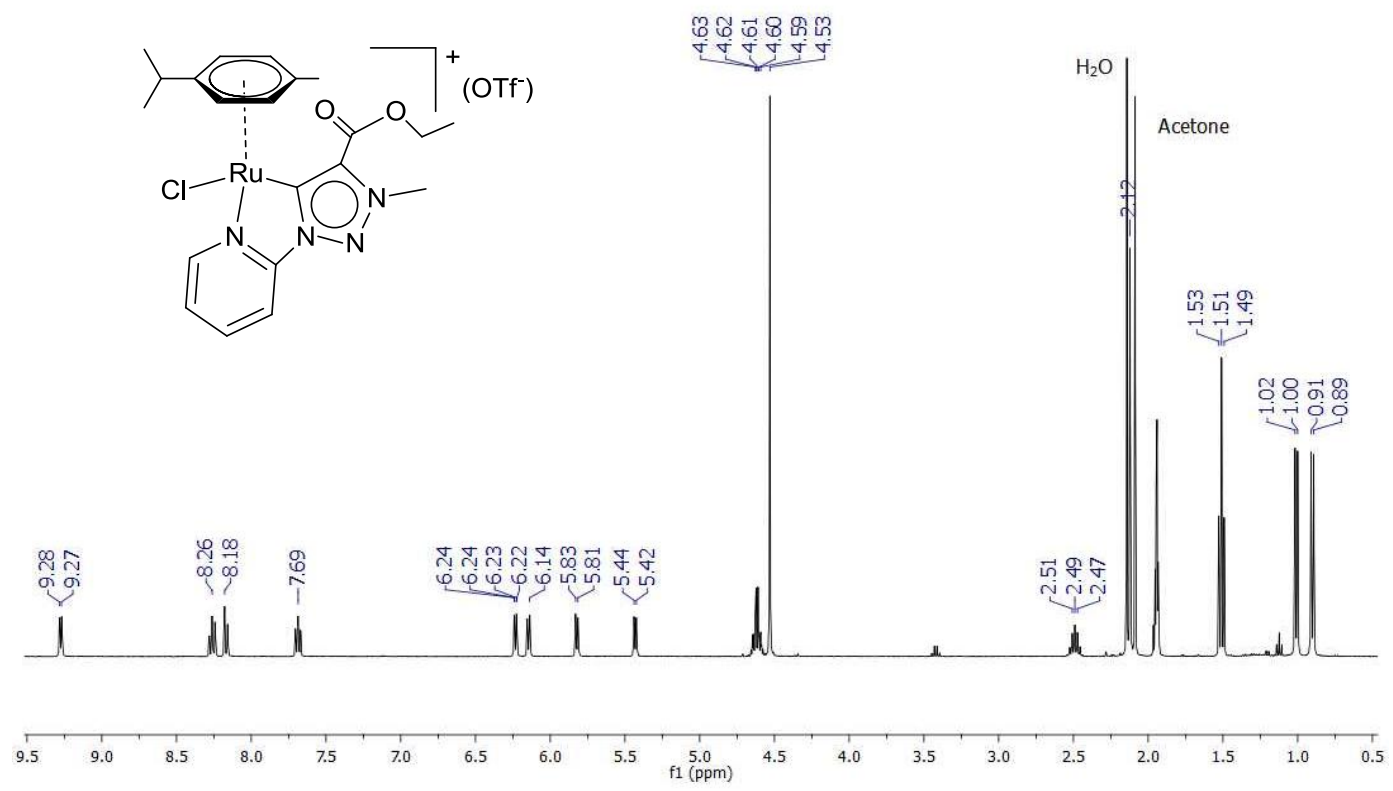

Figure 4.4 ${ }^{1} \mathrm{H}$ NMR spectrum of complex $1 \mathrm{H}$ in $\mathrm{CD}_{3} \mathrm{CN}$

\section{${ }^{13} \mathrm{C}\left\{{ }^{1} \mathrm{H}\right\}$ NMR spectrum of complex $1 \mathrm{H}$}

Figure 4.5 shows the ${ }^{13} \mathrm{C}\left\{{ }^{1} \mathrm{H}\right\}$ NMR spectrum of $\mathbf{1 H}$, with a clearly distinguishable signal due to the metalated carbene carbon at $177.1 \mathrm{ppm}$. The signal attributed to the carbonyl carbon is seen at $159.2 \mathrm{ppm}$. The quaternary carbons of the pyridyl group and the triazole ring resonate at 150.5 and $139.8 \mathrm{ppm}$, respectively. The signal attributed to the $\mathrm{N}-\mathrm{CH}_{3}$ carbon is displayed at $43.3 \mathrm{ppm}$. All other signals including those attributed to the carbons of the pyridyl group, $p$-cymene ligand and ethyl group are well-resolved in the NMR spectrum shown in Figure 4.5. ${ }^{13} \mathrm{C}$ HSQC NMR experiments were carried out in order to confirm the assignation of the signals displayed on the ${ }^{1} \mathrm{H}$ and ${ }^{13} \mathrm{C}$ NMR spectra of $\mathbf{1 H}$ (see Experimental Section). 


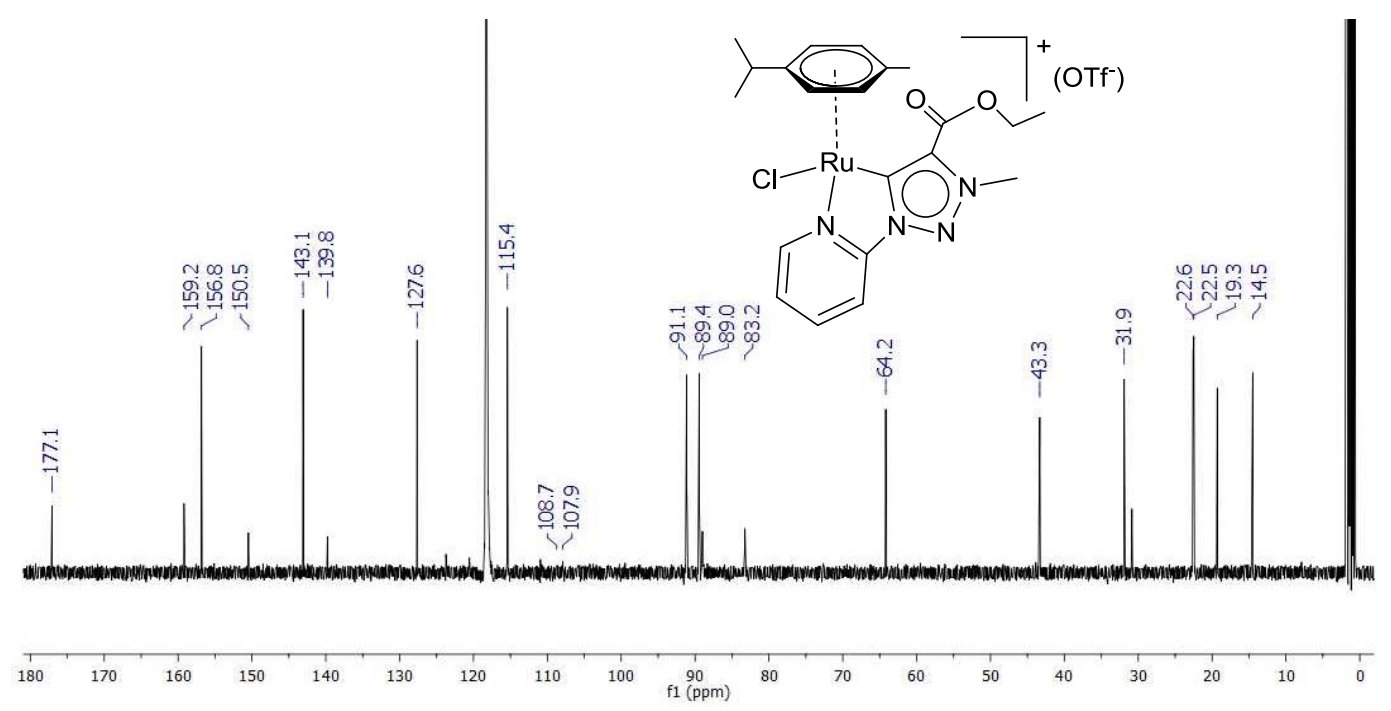

Figure 4.5 ${ }^{13} \mathrm{C}\left\{{ }^{1} \mathrm{H}\right\}$ NMR spectrum of complex $1 \mathrm{H}$ in $\mathrm{CD}_{3} \mathrm{CN}$

\section{${ }^{1} \mathrm{H}$ NMR spectrum of complex $\mathbf{3} \boldsymbol{H}$}

Figure 4.6 shows the ${ }^{1} \mathrm{H}$ NMR spectrum of $\mathbf{3 H}$. The most significant feature of the NMR spectrum of $\mathbf{3 H}$ is the absence of the signals corresponding to the protons of the ethyl group (shown in $\mathbf{1 H}$ at $\delta 4.61$ and 1.51 for $\mathrm{CH}_{2}$ and $\mathrm{CH}_{3}$, respectively), thus confirming that the hydrolysis of the ester moiety has taken place. The signal attributed to the protons of the methyl group at the triazole ring appears as a singlet at $4.57 \mathrm{ppm}$. The signal due to the acidic $\mathrm{COOH}$ proton appears as broad singlet at $4.25 \mathrm{ppm}$. All other signals attributed to the protons of the pyridyl group and $p$-cymene ligand appear at similar chemical shifts as those shown for $\mathbf{1 H}$ (Figure 4.4). 


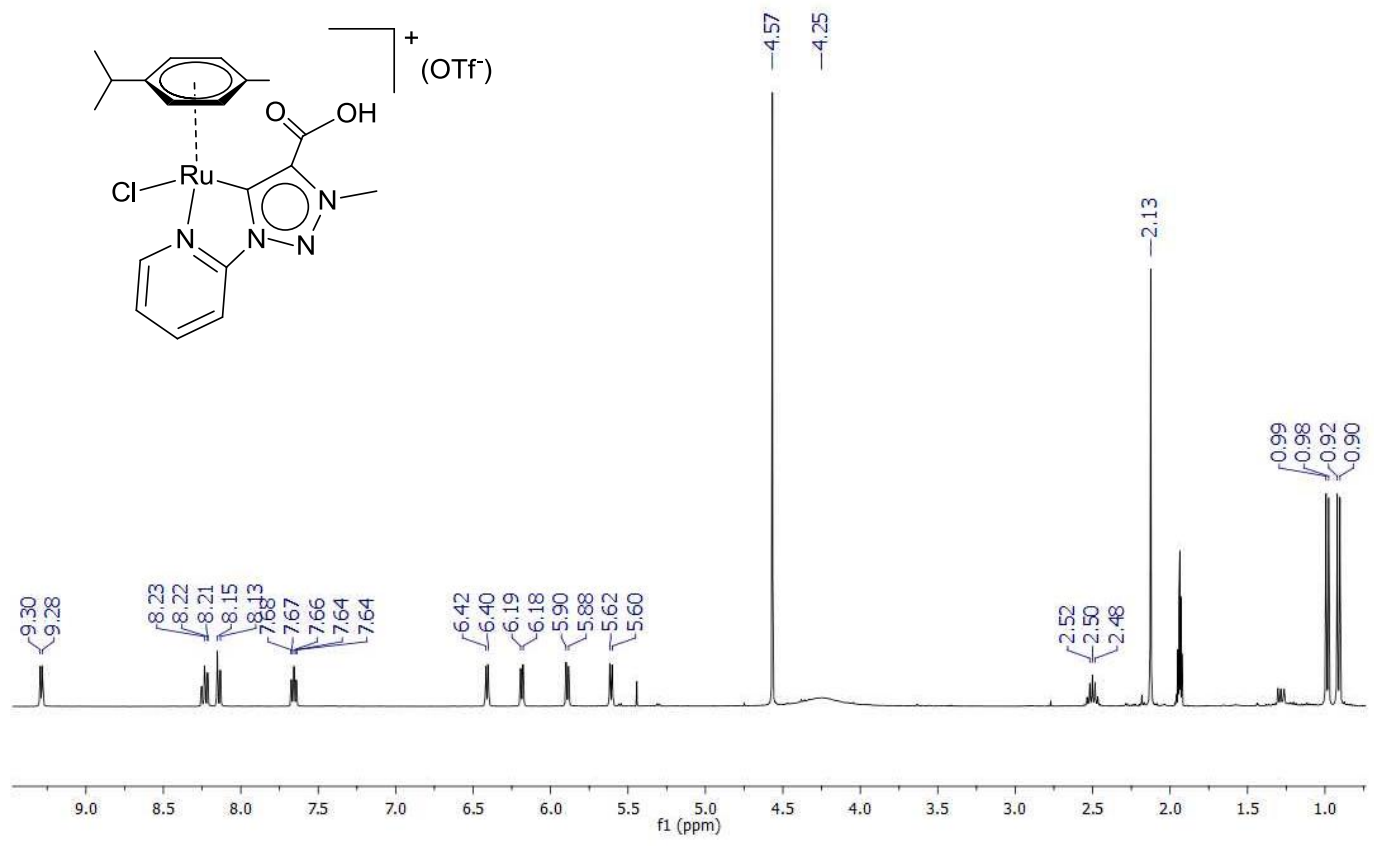

Figure 4.6 ${ }^{1} \mathrm{H}$ NMR spectrum of complex $3 \mathrm{H}$ in $\mathrm{CD}_{3} \mathrm{CN}$

Complex 2H shows a qualitatively similar ${ }^{1} \mathrm{H}$ NMR spectrum as the one displayed in Figure 4.3 , for $\mathbf{3 H}$. The ${ }^{13} \mathrm{C}$ NMR spectra of $\mathbf{2 H}$ and $\mathbf{3 H}$ show the signal due to the metalated carbene carbon at 172.3 and $174.9 \mathrm{ppm}$, respectively. The signal attributed to the carbonyl carbon of the carboxylate in $\mathbf{2 H}$ and carboxylic acid in $\mathbf{3 H}$ is seen at 156.7 and $161.4 \mathrm{ppm}$.

The mass spectra of complexes $\mathbf{1 H}, \mathbf{2 H}$ and $\mathbf{3 H}$ showed an intense positive ion peak at $\mathrm{m} / \mathrm{z}=502.3[\mathrm{M}]^{+}, 439[\mathrm{M}-\mathrm{Cl}]^{+}$and $475.7[\mathrm{M}]^{+}$, respectively. These peaks confirm the proposed molecular composition of the complexes, based on the mass/charge relation and the isotopic patterns.

The IR spectra of complexes $\mathbf{1 H}-\mathbf{3 H}$ display the characteristic stretching vibration modes of the $\mathrm{C}=\mathrm{O}$ at 1729,1618 and $1735 \mathrm{~cm}^{-1}$, respectively. These values are in accordance with the standard stretching $v(\mathrm{C}=\mathrm{O})$ frequencies for an ester, a carboxylate and a carboxylic acid group. ${ }^{23}$ 


\section{Molecular structure of complex $\mathbf{1 H}$}

The molecular structure of $\mathbf{1 H}$ (Figure 4.7) was confirmed by X-ray diffraction studies. Crystals of $\mathbf{1 H}$ suitable for X-ray diffraction analysis were obtained by slow diffusion of pentane into a solution of the compound in dichloromethane. The geometry of $\mathbf{1 H}$ can be regarded as a three-legged piano stool, with a $p$-cymene ligand, one chloride and a chelating triazolium-ylidene bound to the $\mathrm{Ru}$ (II) center. Table 4.2 shows the most representative bond and angles of complex $\mathbf{1 H}$. The $\mathrm{Ru}-\mathrm{C}_{\text {carbene }}$ bond length is 2.049(2) $\AA$, and lies in the expected range, when compared to similar complexes reported for the same metal fragment containing triazolylidene ligands. ${ }^{21}$ The molecular structure confirms the chelation of the pyridyl group, which produces a five-membered metallacycle with an acute bite angle $\mathrm{C}(1)-\mathrm{Ru}-\mathrm{N}(4)$ of $77.92(8)^{\circ}$. The cationic nature of the complex is confirmed by the presence of a $\mathrm{OTf}^{-}$counterion.

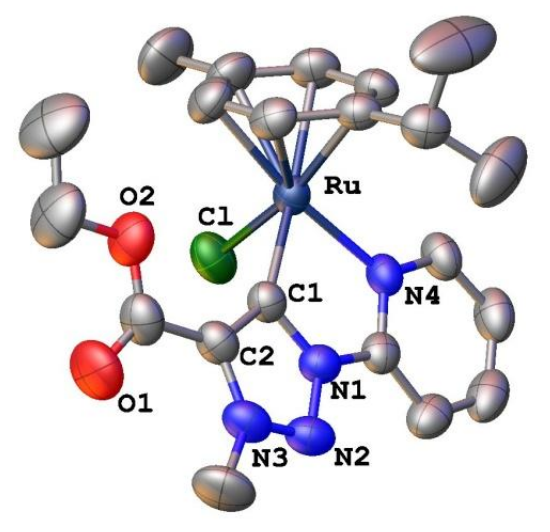

Figure 4.7 Molecular diagram of complex 1H. Ellipsoids are shown at 50\% probability level. Counteranion (OTf) and hydrogen atoms of the $p$-cymene ligand and pyridyl group are omitted for clarity.

Table 4.2 Selected bond lengths (Á) and angles ( $\left(^{\circ}\right)$ of complex $\mathbf{1 H}$

\begin{tabular}{cccc}
\hline Bonds & $(\AA)$ & Angles & $\left.\mathbf{(}^{\circ}\right)$ \\
\hline $\mathrm{Ru}-\mathrm{C}(1)$ & $2.049(2)$ & $\mathrm{C}(1)-\mathrm{Ru}-\mathrm{Cl}$ & $84.96(6)$ \\
$\mathrm{Ru}-\mathrm{N}(4)$ & $2.0983(18)$ & $\mathrm{C}(1)-\mathrm{Ru}-\mathrm{N}(4)$ & $77.92(8)$ \\
$\mathrm{Ru}-\mathrm{Cl}$ & $2.4004(6)$ & $\mathrm{N}(4)-\mathrm{Ru}-\mathrm{Cl}$ & $82.34(5)$ \\
$\mathrm{Ru}-\mathrm{C}_{\text {centroid }}$ & 1.706 & & \\
\hline
\end{tabular}




\subsection{Synthesis of solvento Ru-triazolylidene complexes}

The substitution of the ancillary $p$-cymene ligand in $\mathbf{1 H}$ was achieved by thermal replacement according to established procedures. ${ }^{21,24-27}$ Scheme 4.5 shows the synthetic protocol used for the preparation of complexes $\mathbf{4 H}$ and $\mathbf{5 H}$. The reaction of complex $\mathbf{1 H}$ with AgOTf in refluxing acetonitrile for $24 \mathrm{~h}$ allowed the formation of the solvento complex $4 \mathbf{H}$. The reaction mixture was filtered over celite and the solvent was removed under reduced pressure. Complex $\mathbf{4 H}$ was obtained as a yellow solid in $75 \%$ yield after precipitation from acetonitrile/diethyl ether mixture. The reaction of $\mathbf{4 H}$ with 2.5 equivalents of $\mathrm{PPh}_{3}$, in $o$-dichlorobenzene $\left(o\right.$-DCB) at $110^{\circ} \mathrm{C}$ for 3 days, afforded complex $\mathbf{5 H}$, which was obtained by precipitation with diethyl ether as a yellow solid in $95 \%$ yield.

The basic hydrolysis of the ester functionality in $\mathbf{5 H}$ afforded the carboxylate $(\mathbf{6 H})$ and carboxylic acid (7H) derivatives. The protocol used was similar as the one described for the preparation of $\mathbf{2 H}$ and $\mathbf{3 H}$ but using a mixture of acetonitrile/water as solvent in order to avoid solvent exchange processes (Scheme 4.7). After purification, complex $6 \mathbf{H}$ was obtained as a yellow solid in $64 \%$ yield. Complex $7 \mathbf{H}$ was obtained as a yellow solid in $52 \%$ yield. All complexes were characterized by NMR and IR spectroscopy, mass spectrometry and elemental analysis.

Complexes $\mathbf{5 H - 7 H}$ were air- and moisture- stable in solution and in the solid state. The solvento complex $\mathbf{4 H}$ is sensitive to air, and its gradual degradation was indicated by a color change from yellow to green over several days. The successful replacement of the $p$-cymene ligand in $\mathbf{4 H}$ was deduced from the loss of the resonances due to the $p$-cymene ligand and to the appearance of the signals due to the coordinated $\mathrm{MeCN}$ ligands by ${ }^{1} \mathrm{H}$ NMR spectroscopy. Only two sets of $\mathrm{CH}_{3} \mathrm{CN}$ resonances are observed in 1:2 ratio $(\delta 2.50$ and 2.08, respectively); this pattern suggests octahedral coordination geometry about the ruthenium center, with two symmetry-related $\mathrm{MeCN}$ ligands in mutual trans position, and one MeCN trans to either the carbene or the pyridine. In a octahedral complex a 1:1:2 ratio of MeCN signals would be expected, the other $\mathrm{MeCN}$, trans to the carbene or pyridine, rapidly exchanges with $\mathrm{CD}_{3} \mathrm{CN}$, thus becomes part of the solvent signal (see Experimental section for further details). 

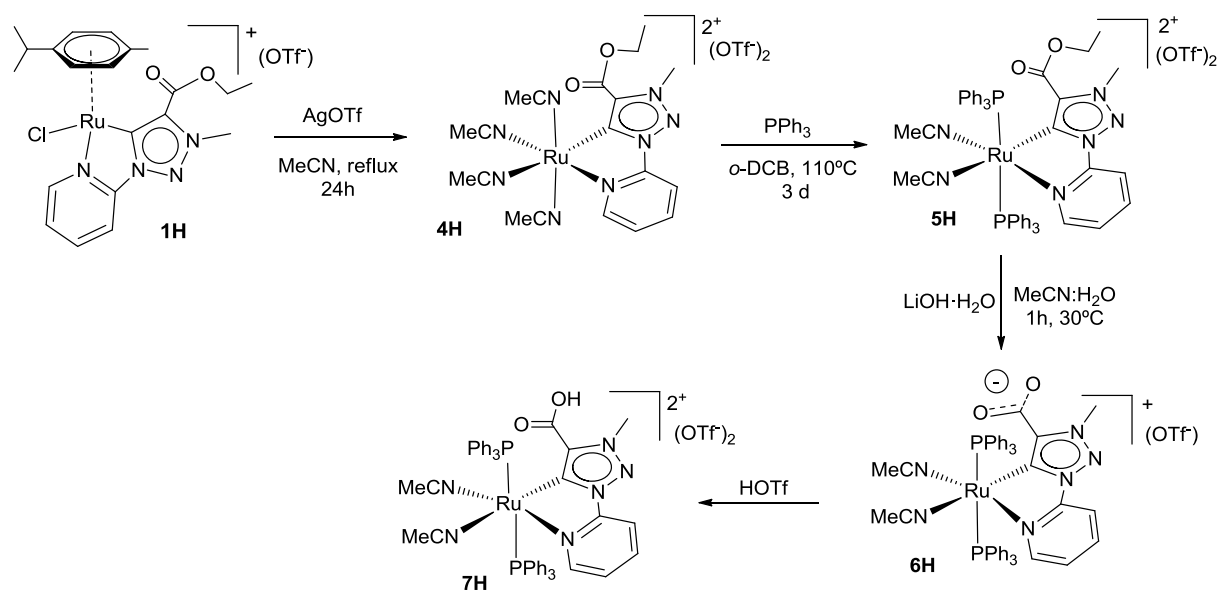

Scheme 4.7 Synthesis of complexes $4 \mathbf{H}$ - 7H

\section{${ }^{1} H$ NMR spectrum of complex $\mathbf{5} \boldsymbol{H}$}

Figure 4.8 shows the ${ }^{1} \mathrm{H}$ NMR spectrum of complex 5H. The coordination of two $\mathrm{PPh}_{3}$ ligands is evidenced by the emergence of a multiplet in the aromatic region, between 7.47 and $7.15 \mathrm{ppm}$, due to 30 new protons, and the disappearance of the signal attributed to two coordinated $\mathrm{MeCN}$ ligands ( $\delta 2.50$ in $\mathbf{4 H}$ ). The spectrum shows only one set of signals, indicating the presence of only one of the two possible diastereomers. The presence of one singlet in the ${ }^{31} \mathrm{P}$ NMR spectrum of $\mathbf{5 H}$ at $29.5 \mathrm{ppm}$ is indicative of the presence of symmetry-related phosphines, thus indicating that the $\mathrm{PPh}_{3}$ ligands are in mutual trans orientation. 


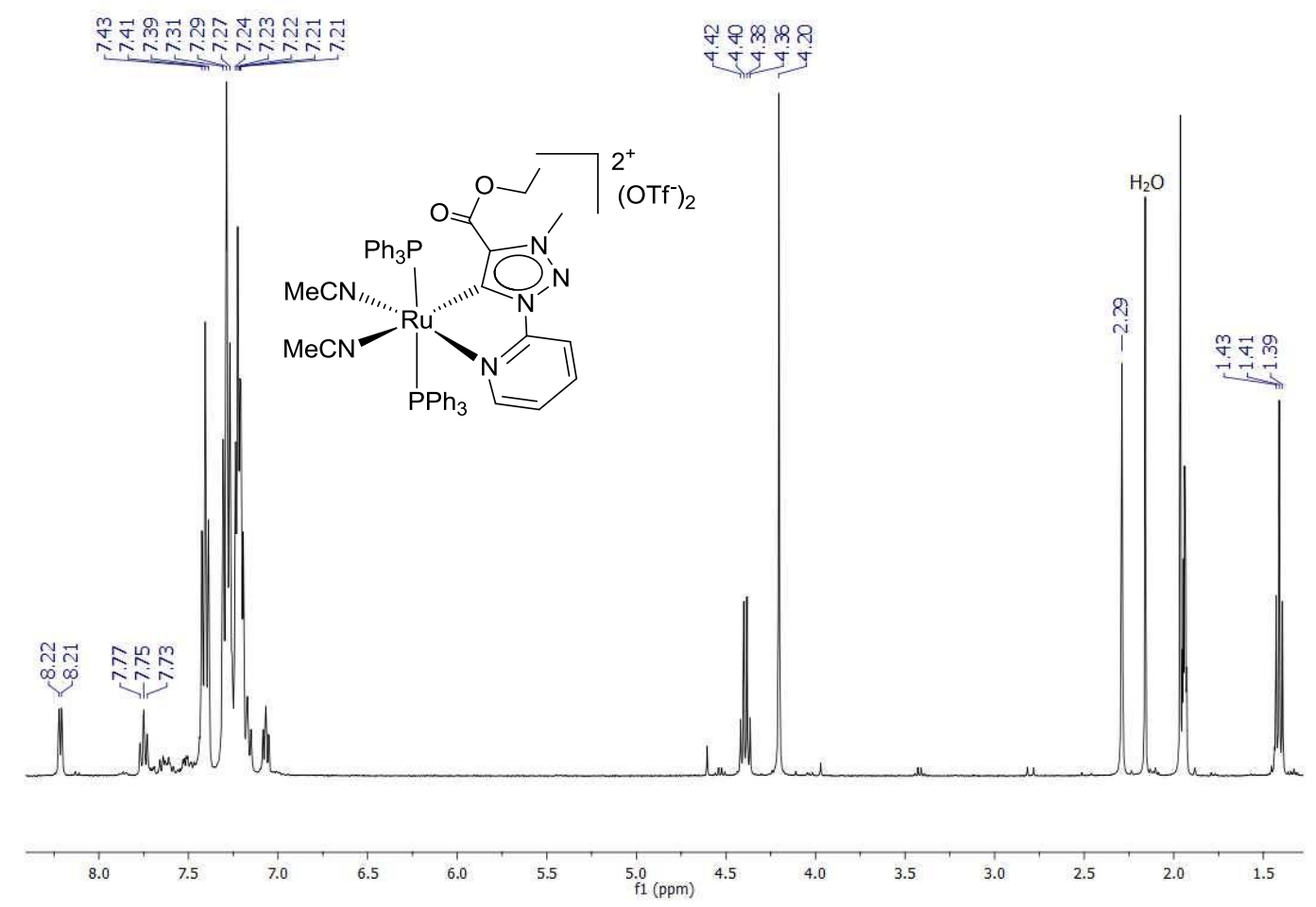

Figure 4.8 ${ }^{1} \mathrm{H}$ NMR spectrum of complex $5 \mathrm{H}$ in $\mathrm{CD}_{3} \mathrm{CN}$

\section{${ }^{13} \mathrm{C}\left\{{ }^{1} \mathrm{H}\right\}$ NMR spectrum of complex $\mathbf{5} \boldsymbol{H}$}

Figure 4.9 shows the ${ }^{13} \mathrm{C}\left\{{ }^{1} \mathrm{H}\right\}$ NMR spectrum of $\mathbf{5 H}$. The signal attributed to the metalated carbene carbon is displayed as a triplet at $183.4 \mathrm{ppm}\left({ }^{2} \mathrm{~J}_{\mathrm{C}-\mathrm{P}}=11.5 \mathrm{~Hz}\right)$, and constitutes an indication that the two $\mathrm{PPh}_{3}$ ligands are in mutual trans disposition. ${ }^{28}$ The signal attributed to the carbonyl carbon of the ester appears at $158.2 \mathrm{ppm}$. The resonances due to the quaternary carbons of the pyridyl group and triazole ring are shown at 150.7 and $141.0 \mathrm{ppm}$. The signal due to the $\mathrm{N}-\mathrm{CH}_{3}$ carbon is displayed at $43.9 \mathrm{ppm}$. 

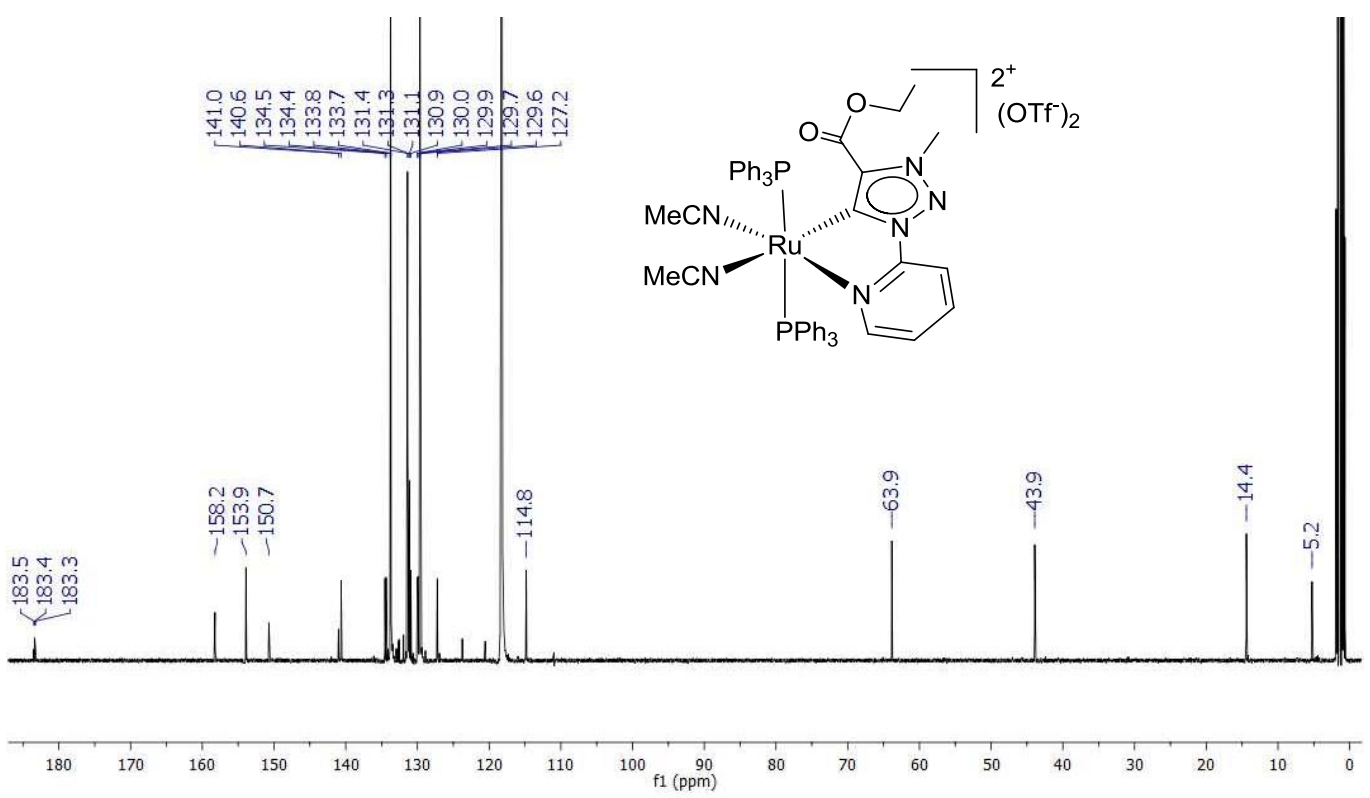

Figure 4.9 ${ }^{13} \mathrm{C}\left\{{ }^{1} \mathrm{H}\right\}$ NMR spectrum of $\mathbf{5} \mathbf{H}$ in $\mathrm{CD}_{3} \mathrm{CN}$

The NMR spectra of complexes $\mathbf{6 H}$ and $\mathbf{7 H}$ are similar to the one described above for 5H. The absence of the signals attributed to the protons $\left({ }^{1} \mathrm{H}\right.$ NMR) and carbons $\left({ }^{13} \mathrm{C}\right.$ NMR) of the ethyl group indicates that the hydrolysis has taken place.

The mass spectra of complexes $\mathbf{4 H}-\mathbf{7 H}$ displayed intense peaks at $\mathrm{m} / \mathrm{z}=647.2[\mathrm{M}+$ $\left.\mathrm{CF}_{3} \mathrm{SO}_{3}\right]^{+}(\mathbf{4 H}), 1088.8\left[\mathrm{M}+\mathrm{CF}_{3} \mathrm{SO}_{3}\right]^{+}(\mathbf{5 H}), 869.9[\mathrm{M}-\mathrm{MeCN}]^{+}(\mathbf{6 H})$ and $415.6[\mathrm{M}-$ $2(\mathrm{MeCN})]^{2+}(\mathbf{7 H})$, confirming the proposed molecular composition of the complexes based on the mass/charge relation and the isotopic pattern.

The IR spectra of complex $\mathbf{5 H}$ displays the characteristic stretching vibration mode of the $(\mathrm{C}=\mathrm{O})$ at1728 $\mathrm{cm}^{-1}$, very similar to complex $\mathbf{1 H}$. However, $\mathbf{6 H}$ and $\mathbf{7 H}$ afforded a strong absorption at 1645 and $1646 \mathrm{~cm}^{-1}$, respectively, which is lower than the expected for an acid, yet higher than typical carboxylate (see $\mathbf{1 H}-\mathbf{3 H}$, above). Probably a dynamic equilibrium operates between the carboxylate and carboxylic acid derivatives. 


\subsection{Catalytic studies}

In the previous sections, a series of functionalized Ru-triazolylidene complexes were described. As stated in the introduction, the objective of introducing functionalities into the triazolium skeleton is to explore the benefits that synergistic ligand-metal interactions may provide. With this target in mind, the effect of the carboxylate functionality in $\mathbf{1 H}$ 7H was evaluated in transfer hydrogenation and in dehydrogenative oxidation of alcohols. The same benchmark processes were used in order to evaluate the influence of the pyridyl group in complexes 1I-3I.

\subsubsection{Transfer hydrogenation}

The new ruthenium complexes were investigated as catalysts for the ketone reduction via transfer hydrogenation. The reactions were carried out using acetophenone as benchmark substrate, 2-propanol as a formal dihydrogen donor, and a catalyst loading of $1 \mathrm{~mol} \%$, in the presence of $\mathrm{KOH}(10 \mathrm{~mol} \%)$. The experiments were performed at $82^{\circ} \mathrm{C}$.

All triazolylidene-based $\mathrm{Ru}(\mathrm{II})$ complexes tested were active in the transfer hydrogenation of acetophenone. Table 4.3 summarizes the catalytic results for this process. The data revealed the highest activity of the ' $\mathrm{Ru}(p$-cymene)' derivatives containing an ester $(\mathbf{1 H})$ and carboxylic acid $(\mathbf{3 H})$ functionalities, which achieved quantitative yields within $1 \mathrm{~h}$ of reaction (entries 1 and 3). The carboxylate derivative $\mathbf{2 H}$ was less effective than $\mathbf{1 H}$ and $\mathbf{3 H}$, yielding $75 \%$ of 1-phenyl ethanol under the same reaction time (compare entries 1, 2 and 3). This result is likely due to a faster decarboxylation (vide infra) of $\mathbf{2 H}$ compared to $\mathbf{1 H}$ and $\mathbf{3 H}$. The transfer hydrogenation under base-free conditions using complex $\mathbf{3 H}$ resulted ineffective (entry 3, yield in parenthesis), indicating that the process requires the presence of the base. The octahedral carboxylate derivative $\mathbf{6 H}$ did not provide any enhancement of the catalytic activity compared to $\mathbf{2 H}$ (compare entries 2 and 4), indicating that modification of the chemical environment about the ruthenium center does not have clear impact in the catalytic process. Complex $\mathbf{3 I}$ (similar to $\mathbf{1 H}-\mathbf{3 H}$ but without the carboxylate functionality at the triazole ligand) was less active than $\mathbf{1 H - 3 H}$ (compare entries 1, 2, 3, and 9). 
Table 4.3 Catalytic transfer hydrogenation of acetophenone

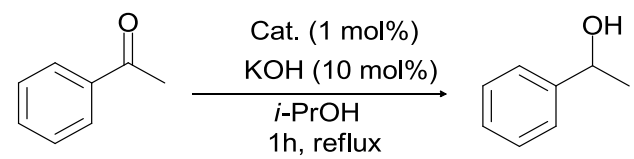

\begin{tabular}{cccc}
\hline Entry & Catalyst & Time $(\mathbf{h})$ & Yield $^{(\%)^{\mathbf{a}}}$ \\
\hline 1 & $\mathbf{1 H}$ & 1 & 97 \\
2 & $\mathbf{2 H}$ & 1 & 75 \\
3 & $\mathbf{3 H}$ & 1 & $95(0)$ \\
4 & $\mathbf{6 H}$ & 1 & 16 \\
\hline 5 & $\mathbf{1 I}$ & 1 & 88 \\
6 & & 4 & 100 \\
\hline 7 & $\mathbf{2 I}$ & 1 & 48 \\
8 & & 4 & 92 \\
\hline 9 & $\mathbf{3 I}$ & 1 & 29 \\
10 & & 4 & 58 \\
\hline
\end{tabular}

General reaction conditions: acetophenone $(0.6 \mathrm{mmol}), \mathrm{KOH}(0.06 \mathrm{mmol}),[\mathrm{Ru}](0.006 \mathrm{mmol}, 1 \mathrm{~mol} \%), 2-$ propanol (2 mL), reflux temperature. [a] Determined by ${ }^{1} \mathrm{H}$ NMR spectroscopy (anisole as internal standard). Yields in parenthesis: under base-free conditions.

If we compare the catalytic outcomes of complexes 1I-3I, we may conclude that 1I provided the best catalytic outcomes, achieving quasi-quantitative yield within $1 \mathrm{~h}$ of reaction (entry 5). The lower catalytic activity of the pyridine-chelated ruthenium complex 3I (entry 9) compared to 1I or 2I (entries 5 and 7), may be ascribed to the chelate coordination of the phenyl-pyridine ligand, which blocks two coordination sites at the metal center.

As a tentative explanation of the similarities in the catalytic activities shown by complexes $\mathbf{1 H}$ and $\mathbf{3 H}$, we believe that both complexes may undergo the in situ hydrolysis of the ester moiety, since the catalytic experiments were performed under basic conditions and in protic media. These conditions are identical to the ones used for the hydrolysis of $\mathbf{1 H}$ to afford $\mathbf{3 H}$ (Scheme 4.4). The catalytic transfer hydrogenation was monitored using complexes $\mathbf{1 H}$ and $\mathbf{3 H}$ in order to evaluate if the differences in the 
catalytic activity reflect the ester $v s$. carboxylic acid functionalization. Time-resolved monitoring of the catalytic reaction (Figure 4.10) showed indeed a slightly higher activity of complex $\mathbf{3 H}$, suggesting comparably slow initial preactivation of $\mathbf{1 H}$ via basic hydrolysis.

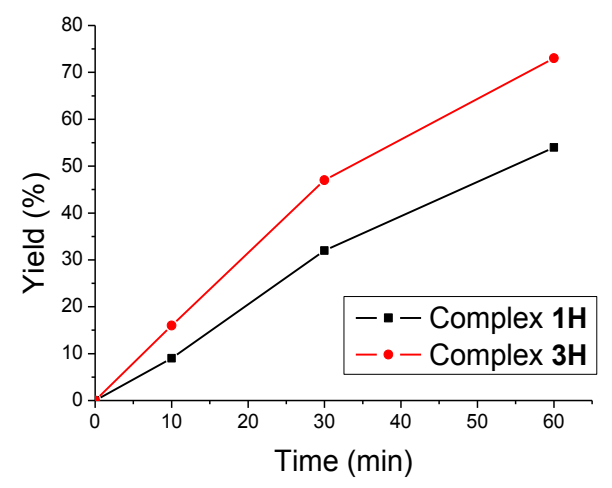

Figure 4.10 Monitoring of the transfer hydrogenation of acetophenone using $\mathbf{1 H}$ and $\mathbf{3 H}$ as catalyst precursors. Reaction conditions: acetophenone $(0.6 \mathrm{mmol}), \mathrm{KOH}(0.06 \mathrm{mmol}),[\mathrm{Ru}]$ (0.006 mmol, $1 \mathrm{~mol} \%)$, 2-propanol $(2 \mathrm{~mL})$, reflux temperature. Yields were determined by ${ }^{1} \mathrm{H}$ NMR spectroscopy (anisole as internal standard).

\subsubsection{Base-free dehydrogenation of benzyl alcohol}

The triazolylidene-based ruthenium complexes $\mathbf{1 H}-\mathbf{7 H}$ and $\mathbf{1 I - 3 I}$ were evaluated as catalysts for the oxidant- and base-free oxidation of benzyl alcohol. The catalytic experiments were performed in $o$-dichlorobenzene with a catalyst loading of $5 \mathrm{~mol} \%$, at $150^{\circ} \mathrm{C}$ in an open system.

The results shown in Table 4.4 indicate that all ruthenium complexes were active in the dehydrogenative oxidation of benzyl alcohol. The data reveal the highest activity of complex 1H, which achieved full conversion to benzaldehyde within $16 \mathrm{~h}$ (entry 1 ). The carboxylic acid derivative $\mathbf{3 H}$ was less effective than the carboxylates $\mathbf{2 H}$ and $\mathbf{6 H}$ (compare entries 2, 3 and 4). Interestingly, complex 3H showed also lower catalytic performance than the analogous 3 I without functional group at $\mathrm{C} 4$ (compare entries 3 and 7). The different catalytic activity is probably due to the difference in solubility or 
stability in $o$-dichlorobenzene at $150^{\circ} \mathrm{C}$, and not necessarily due to the presence/absence of functional groups at the ligand. Thermal decarboxylation has been observed (vide infra), so complexes $\mathbf{2 H}$ and $\mathbf{3 H}$ should transform to the same species (3I), and hence display largely similar activities.

Table 4.4 Oxidation of benzyl alcohol with triazolylidene $\mathrm{Ru}(\mathrm{II})$ complexes

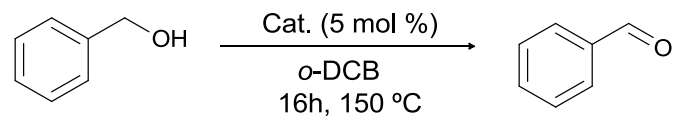

\begin{tabular}{ccc}
\hline Entry & Catalyst & ${\text { Yield }(\boldsymbol{\%})^{\mathbf{a}}}^{\text {a }}$ \\
\hline 1 & $\mathbf{1 H}$ & 95 \\
2 & $\mathbf{2 H}$ & 73 \\
3 & $\mathbf{3 H}$ & 54 \\
4 & $\mathbf{6 H}$ & 86 \\
5 & $\mathbf{1 I}$ & 75 \\
6 & $\mathbf{2 I}$ & 50 \\
7 & $\mathbf{3 I}$ & 83 \\
\hline
\end{tabular}

General reaction conditions: benzyl alcohol $(0.2 \mathrm{mmol}),[\mathrm{Ru}](0.01 \mathrm{mmol}, 5 \mathrm{~mol} \%), o$-dichlorobenzene $(2$ $\mathrm{mL}$ ), $150{ }^{\circ} \mathrm{C}$. [a] Yields determined by ${ }^{1} \mathrm{H}$ NMR integration (anisole as a standard).

When comparing the catalytic outcomes induced by complexes 1I-3I, slight differences in activity were observed (entries 5-7). The catalytic activity of the pyridine-chelated complex 3I was higher than the ones shown by the monodentate complexes $\mathbf{1 I}$ and $\mathbf{2 I}$. Recent studies revealed that chelation of a pyridine consistently induces lower activity than monodentate triazolylidenes, ${ }^{21}$ therefore the different catalytic activity imparted by complexes 1I-3I is presumably attributed again to the difference of solubility of the complexes in $o$-dichlorobenzene, or to the hemilability of the ligand at high temperatures.

The stability of complex $\mathbf{3 H}$ towards decarboxylation was evaluated in order to gain a better understanding of the differences in activity. Complex $\mathbf{3 H}$ undergoes decarboxylation under neutral and basic conditions in acetonitrile at $80^{\circ} \mathrm{C}$. The reaction of 3H with 2 equivalents of $\mathrm{KOH}$ in acetonitrile at $80^{\circ} \mathrm{C}$ for $30 \mathrm{~min}$., afforded the formation of complex 3I in 30\% NMR yield. After 5 hours of reaction the product partially lost the 
p-cymene ligand and decarboxylation of complex $\mathbf{3 H}$ reached $90 \%$. The same result was obtained under base-free conditions in acetonitrile at $80^{\circ} \mathrm{C}$ where a $95 \%$ decarboxylation of $\mathbf{3 H}$ was detected (Scheme 4.8).

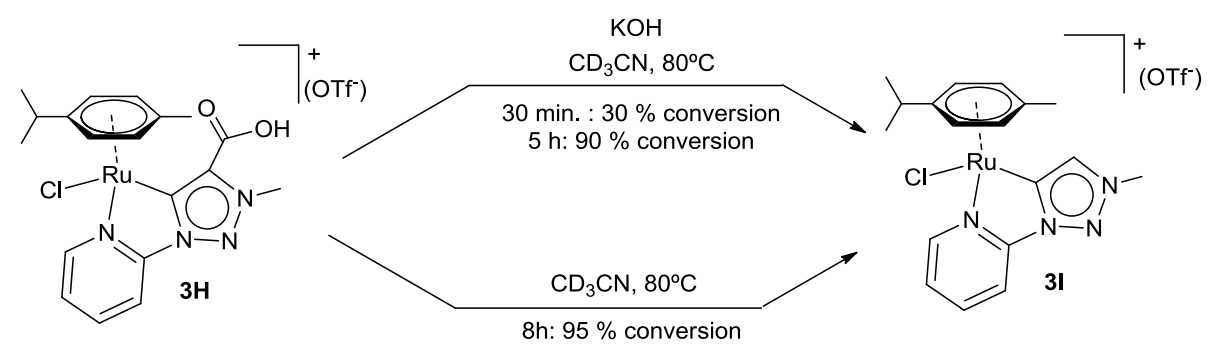

Scheme 4.8 Decarboxylation of $\mathbf{3 H}$

This reactivity of $\mathbf{3 H}$ towards decarboxylation should explain the differences in activity of the complexes in the catalytic transformations, as the decarboxylation of $\mathbf{3 H}$ occurs within $5 \mathrm{~h}$. The transfer hydrogenation processes were achieved within $1 \mathrm{~h}$ of reaction, thus the carboxylate moiety presumably was still present and playing an active role as demonstrated by the catalytic results. In the oxidative dehydrogenation of benzyl alcohol longer reaction times were needed, therefore the differences in the catalytic activity of the complexes are not attributed to the presence/absence of functional groups at the triazole ligand. 


\section{CONCLUSIONS}

A straightforward synthetic route to ruthenium complexes with mesoionic triazolylidenes functionalized with carboxylates was disclosed. The introduction of the carboxylate functionality was evaluated first starting from a carboxylic acid-functionalized triazolium salt (I). In this case, the carboxylic acid easily underwent decarboxylation opening new opportunities for the metalation of triazolium salts via decarboxylative methods. However, all the attempts of using the carboxylate functionality as carbene transfer agent were unsuccessful, evidencing the different reactivity of triazolylidenes compared to 2imidazolylidenes. The formation of the donor-functionalized Ru-triazolylidene complexes was achieved by an alternative route, starting from the triazolium salt which contains an ester functionality at its skeleton $(\mathbf{H})$.

All new complexes were evaluated as catalyst precursors in the dehydrogenative oxidation of alcohols and transfer hydrogenation processes. The results revealed the positive influence in transfer hydrogenation of the donor pyridyl group when is not bound to the ruthenium center, and an enhancement of the activity when the carboxylate functionality is present. These results, which suggest an active ligand-metal cooperation, together with the ease of the functionalization of the ligand consist a promising start point in order to obtain a wide range of bifunctional or cooperative metal-based FLP systems. 


\section{REFERENCES}

(1) Hartwig, J. Organotransition metal chemistry : from bonding to catalysis; University Science Books, cop., 2010.

(2) Noyori, R.; Yamakawa, M.; Hashiguchi, S. J. Org. Chem. 2001, 66, 7931-7944.

(3) Ikariya, T.; Murata, K.; Noyori, R. Org. Biomol. Chem. 2006, 4, 393-406.

(4) Grützmacher, H. Angew. Chem. Int. Edit. 2008, 47, 1814-1818.

(5) Askevold, B.; Roesky, H. W.; Schneider, S. ChemCatChem 2012, 4, 307-320.

(6) Crabtree, R. H. Coord. Chem. Rev. 2013, 257, 755-766.

(7) Donnelly, K. F.; Petronilho, A.; Albrecht, M. Chem. Commun. 2013, 49, 1145 1159.

(8) Bouffard, J.; Keitz, B. K.; Tonner, R.; Guisado-Barrios, G.; Frenking, G.; Grubbs, R. H.; Bertrand, G. Organometallics 2011, 30, 2617-2627.

(9) Kolb, H. C.; Finn, M. G.; Sharpless, K. B. Angew. Chem. Int. Edit. 2001, 40, 2004.

(10) Heckenroth, M.; Neels, A.; Garnier, M. G.; Aebi, P.; Ehlers, A. W.; Albrecht, M. Chem. Eur. J. 2009, 15, 9375-9386.

(11) Power, P. P. Nature 2010, 463, 171-177.

(12) Stephan, D. W.; Erker, G. Angew. Chem. Int. Edit. 2010, 49, 46-76.

(13) Hounjet, L. J.; Stephan, D. W. Org. Process Res. Dev. 2014, 18, 385-391.

(14) Paradies, J. Angew. Chem. Int. Edit. 2014, 53, 3552-3557.

(15) Eisenberger, P.; Bestvater, B. P.; Keske, E. C.; Crudden, C. M. Angew. Chem. Int. Edit. 2015, 54, 2467-2471.

(16) Flynn, S. R.; Wass, D. F. ACS Catal. 2013, 3, 2574-2581.

(17) Yamamoto, H. Lewis Acids in Organic Synthesis; Wiley-VHC: New York, 2000.

(18) Chattopadhyay, B.; Vera, C. I. R.; Chuprakov, S.; Gevorgyan, V. Org. Lett. 2010, 12, 2166-2169.

(19) Petronilho, A.; Woods, J. A.; Mueller-Bunz, H.; Bernhard, S.; Albrecht, M. Chem. Eur. J. 2014, 20, 15775-15784.

(20) Voutchkova, A. M.; Appelhans, L. N.; Chianese, A. R.; Crabtree, R. H. J. Am. Chem. Soc. 2005, 127, 17624-17625. 
(21) Delgado-Rebollo, M.; Canseco-Gonzalez, D.; Hollering, M.; Mueller-Bunz, H.; Albrecht, M. Dalton Trans. 2014, 43, 4462-4473.

(22) Kirkwood, J. G. J. Chem. Phys. 1934, 2.

(23) Oomens, J.; Steill, J. D. J. Phys. Chem. A 2008, 112, 3281-3283.

(24) Le Lagadec, R.; Rubio, L.; Alexandrova, L.; Toscano, R. A.; Ivanova, E. V.; Meskys, R.; Laurinavicius, V.; Pfeffer, M.; Ryabov, A. D. J. Organomet. Chem. 2004, 689, 4820-4832.

(25) Ghattas, W.; Bunz, H. M.; Albrecht, M. Organometallics 2010, 29, 6782-6789.

(26) Leigh, V.; Ghattas, W.; Lalrempuia, R.; Mueller-Bunz, H.; Pryce, M. T.; Albrecht, M. Inorg. Chem. 2013, 52, 5395-5402.

(27) Nussbaum, M.; Schuster, O.; Albrecht, M. Chem. Eur. J. 2013, 19, 17517-17527.

(28) Burling, S.; Mahon, M. F.; Paine, B. M.; Whittlesey, M. K.; Williams, J. M. J. Organometallics 2004, 23, 4537-4539. 


\section{CHAPTER 5}

\section{IMMOBILIZATION OF PYRENE-TAGGED CATALYSTS ONTO GRAPHENE-TYPE SURFACES}

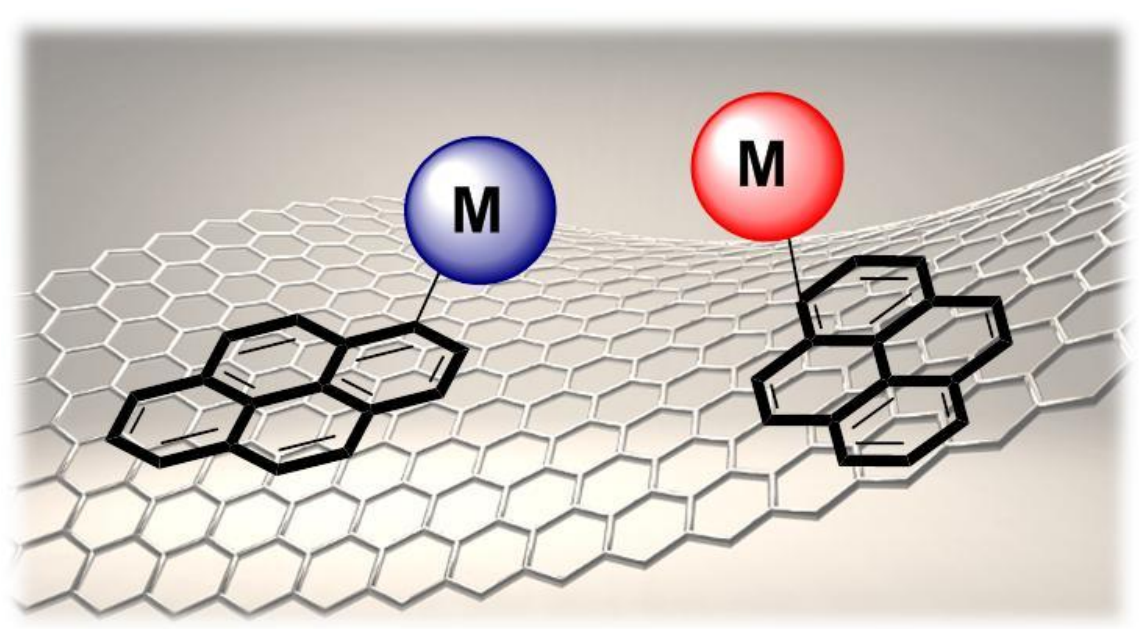





\section{INTRODUCTION}

One of the major challenges in catalysis is to transform a successful homogeneous catalyst into a recyclable catalytic system. ${ }^{1}$ Homogeneous catalysts have many attractive properties in terms of activity and selectivity, but they suffer from difficulties associated with separating the products from the catalyst and from catalyst recycling. ${ }^{2}$ These drawbacks make industries to be relatively reluctant to use them for the large scale production of materials (over $90 \%$ of all commercial chemical processes make use of heterogeneous catalysts). ${ }^{3}$ To tackle these problems, the simplest strategy arises from the immobilization of known catalysts onto solid supports. ${ }^{4-9}$ The most widely used method for the immobilization of a homogeneous catalyst is the formation of covalent bonds between a solid support and a ligand. By doing so, the supported catalyst is expected to be robust enough to withstand the harsh reaction conditions needed for some catalytic processes. However, this immobilization method has two important drawbacks, first arising from the need for an additional functionalization of the catalyst ligand and solid surface (which, in turn implies an increase in the catalyst preparation cost) and second, arising from the possible changes in chemical reactivity (and therefore in catalytic activity) derived from the formation of the covalent bond between the support and the catalyst. As an alternative, non-covalent interactions are emerging as a powerful tool that gives rise to supported catalysts without chemical modification of the homogeneous catalyst, although the stability against leaching may become a problem that needs to be avoided by the careful selection of the reaction conditions, immobilization method, and the nature of the support and the catalyst. ${ }^{7}$

Graphene and its derivatives have attracted increasing attention due to their unique physical and chemical properties, ${ }^{10,11}$ which make them particularly interesting in the fields of nanochemistry and catalysis. ${ }^{12-15}$ Chemically derived graphenes (CDGs) such as graphene oxide $(\mathrm{GO})$ and reduced graphene oxide ( $\mathrm{rGO}$ ), are excellent candidates for catalyst immobilization due to its inertness, large surface area, stability and availability. The superior electron mobility of graphene may facilitate electron transfer during catalytic reactions, which can lead to the enhancement of the catalytic activity of the immobilized complex. Together with their inherent properties, graphene and derivatives 
offer a unique opportunity for non-covalent modifications by $\pi$-stacking interactions with molecules containing polycyclic aromatic hydrocarbons, ${ }^{16-21}$ from which pyrenefunctionalized systems constitute promising examples. ${ }^{22-25}$ Thus, it becomes rather obvious that one potentially effective form for catalyst-heterogenization should be the preparation of pyrene-containing catalysts, which could be non-covalently supported onto a graphene surface. The non-covalent interaction between the pyrene and rGO is based on van der Waals forces ( $\pi$-stacking) and is thermodynamically controlled. ${ }^{26,27}$ This type of rGO-functionalization is particularly attractive, because it offers the possibility of attaching a metal complex without affecting the electronic network. For the choice of the suitable catalysts, N-heterocyclic carbene-based complexes seem to be good candidates, due to the $\sigma$-donor capacity of NHC ligands and the stability of the metal complexes derived that make them suitable to operate under harsh reaction conditions. ${ }^{28-30}$ On the basis of all these arguments, we now describe the synthesis of an imidazolium salt with a pyrene-tag that allows the preparation of palladium and ruthenium complexes that have been immobilized onto a rGO surface by $\pi$-stacking interactions.

With these ideas in mind, the main objective in this chapter is the synthesis of an imidazolium salt with a pyrene-tag, its coordination to metal fragments, and their noncovalent immobilization onto rGO surfaces. The catalytic properties of these new hybrid materials will be first tested in the ruthenium catalyzed oxidation of alcohols, and in the palladium-mediated hydrogenation of organic molecules. Then, the mixed hybrid materials (containing both, palladium and ruthenium complexes) will be tested in the hydrodefluorination (HDF) of fluoro-organic molecules, a reaction for which we already demonstrated in Chapter 1 that the combination of these two metals affords important benefits. $^{31}$ 


\section{RESULTS AND DISCUSSION}

\subsection{Synthesis of metal complexes bearing a pyrene-tagged-NHC ligand}

In order to prepare metal complexes capable to be anchored onto a graphene surface, the pyrene-tagged imidazolium salt $\mathbf{G}$ was synthesized. The preparation of $\mathbf{G}$ was carried out by reaction of 1-methyimidazole with 1-(bromomethyl)pyrene in refluxing tetrahydrofuran for $16 \mathrm{~h}$ (Scheme 5.1). The resulting suspension was filtered and washed thoroughly with diethyl ether to yield the imidazolium salt $\mathbf{G}$ as a white crystalline solid in $96 \%$ yield. G was characterized by NMR spectroscopy, High Resolution Mass Spectrometry (HRMS) and elemental analysis.

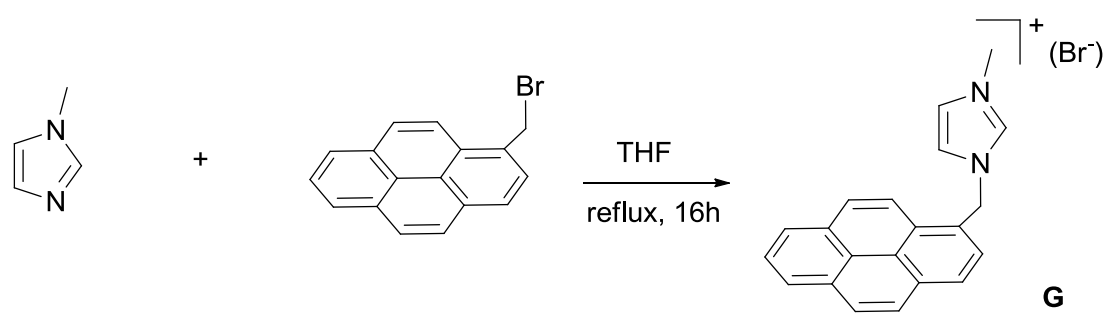

Scheme 5.1 Synthesis of imidazolium salt $\mathbf{G}$

A series of metal complexes of Pd (1G), Ru (2G), Au (3G) and Ir (4G) were prepared starting from the imidazolium salt G. Different metalation strategies were used depending on the metal precursor (Scheme 5.2).

Complex $1 \mathbf{G}$ was obtained by reaction of the imidazolium salt $\mathbf{G}$ and $[\operatorname{Pd}(\mathrm{OAc})(\mathrm{dmba})]_{2}$ $(\mathrm{dmba}=$ dimethylbenzylamine $)$ in the presence of $\mathrm{KBr}$ in refluxing acetonitrile for $16 \mathrm{~h}$. The resulting suspension was filtered through celite and the solvent was evaporated under reduced pressure. The crude solid was purified by column chromatography. Precipitation from a dichloromethane/hexanes mixture afforded $\mathbf{1 G}$ as a yellow solid in $60 \%$ yield. 


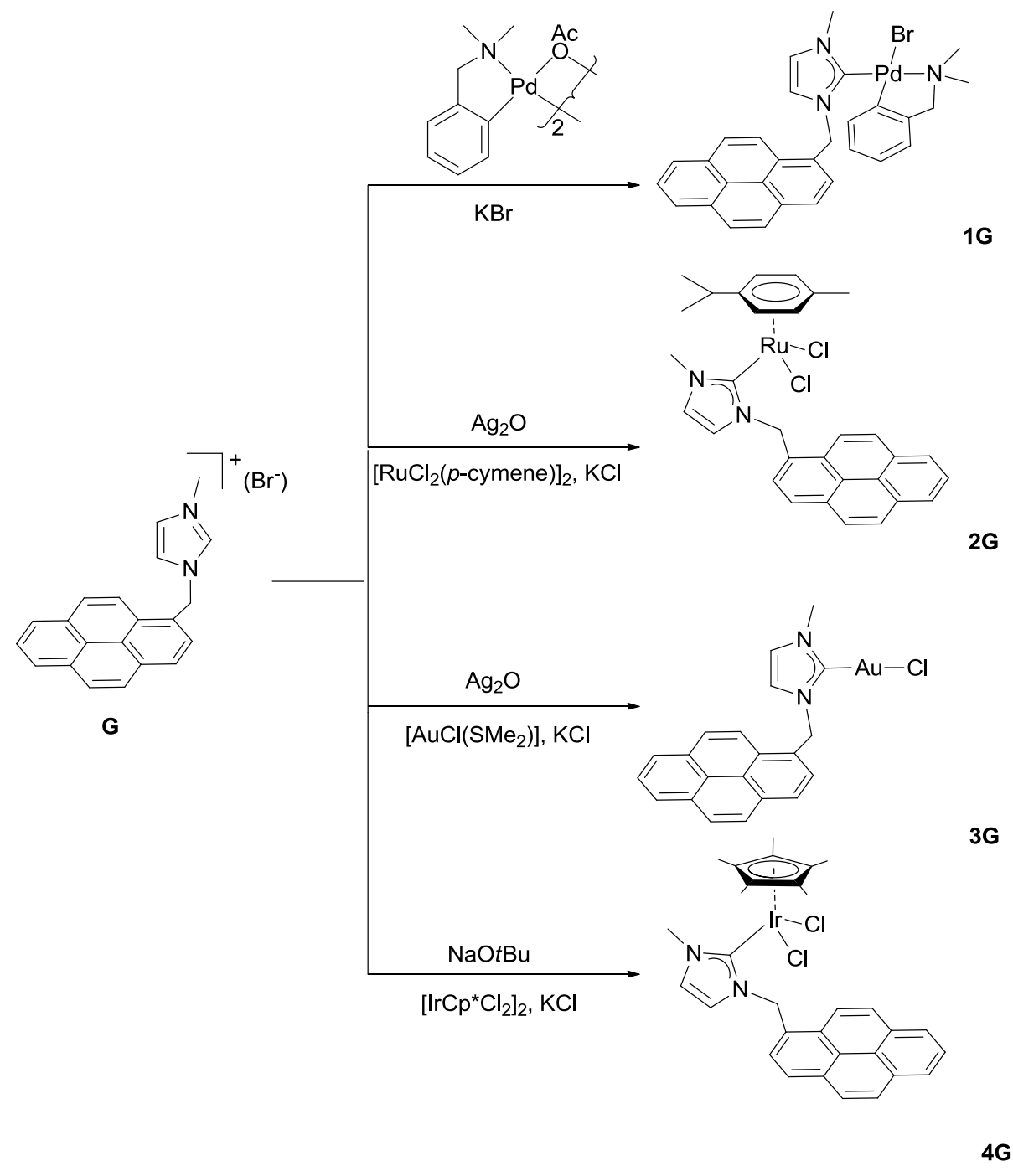

Scheme 5.2 Synthesis of complexes 1G - 4G

The synthesis of complexes $\mathbf{2 G}$ and $\mathbf{3 G}$ was performed by transmetallation from the preformed silver-NHC complex, prepared by treatment of $\mathbf{G}$ with $\mathrm{Ag}_{2} \mathrm{O}$ in refluxing acetonitrile for $5 \mathrm{~h}$, under the exclusion of light. $\left[\mathrm{RuCl}_{2}(p \text {-cymene) }]_{2}\right.$ or $\left[\mathrm{AuCl}\left(\mathrm{SMe}_{2}\right)\right]$ (for 2G and 3G, respectively) were added and the reaction mixtures were stirred at room temperature overnight in the presence of $\mathrm{KCl}$. Complex $\mathbf{2 G}$ was purified by column 
chromatography and precipitated from a mixture of dichloromethane/hexane to yield an orange solid in $61 \%$ yield. Complex 3G was purified by crystallization from a dichloromethane/hexane mixture and was obtained as white solid in $81 \%$ yield.

The iridium complex $\mathbf{4 G}$ was synthesized starting from the pre-formed free carbene, prepared by treatment of $\mathbf{G}$ with $\mathrm{NaO} t \mathrm{Bu}$ in tetrahydrofuran for $30 \mathrm{~min}$. at $0^{\circ} \mathrm{C}$. Then $\left[\mathrm{IrCp} * \mathrm{Cl}_{2}\right]_{2}$ and $\mathrm{KCl}$ were added, and the reaction mixture was stirred for 5 hours at room temperature. Complex 4G was purified by column chromatography and precipitated from a mixture of dichloromethane/hexane to yield an orange solid in $46 \%$ yield.

All metal complexes 1G - 4G were characterized by NMR spectroscopy, High Resolution Mass Spectrometry (HRMS) and elemental analysis. In general, the first evidence that metalation had occurred came from the ${ }^{1} \mathrm{H}$ NMR spectra, by observing the disappearance of the signal attributed to the $\mathrm{NCHN}$ proton of the imidazolium salt $\mathbf{G}$. More concluding evidences were provided by the ${ }^{13} \mathrm{C}$ NMR spectra, which showed the signals assigned to the metalated carbene carbons. These signals appeared at 172.9 (1G), 174.8 (2G), 171.3 (3G), and 157.1 (4G) ppm. As an example of the characterization of these complexes, the ${ }^{1} \mathrm{H}$ NMR and ${ }^{13} \mathrm{C}$ NMR spectra of complex $2 \mathrm{G}$ will be described. All the details regarding the NMR spectroscopic characterization of the rest of the complexes are given in the Experimental Section (Chapter 6).

\section{${ }^{1} H$ NMR spectrum of $\mathbf{2 G}$}

Figure 5.1 shows the ${ }^{1} \mathrm{H}$ NMR spectrum of complex 2G. The signals attributed to the pyrene aromatic protons appear between 8.38 and $7.70 \mathrm{ppm}$. The signal due to the benzylic protons of the imidazolylidene appears as broad singlet between 6.75 and 6.33 ppm. The signals due to the aromatic protons of the $p$-cymene ligand appear as a multiplet at $5.25 \mathrm{ppm}$ and a doublet at $4.89 \mathrm{ppm}$. The signal assigned to the protons of the $\mathrm{N}-\mathrm{CH}_{3}$ group appears as a singlet at $4.11 \mathrm{ppm}$. 


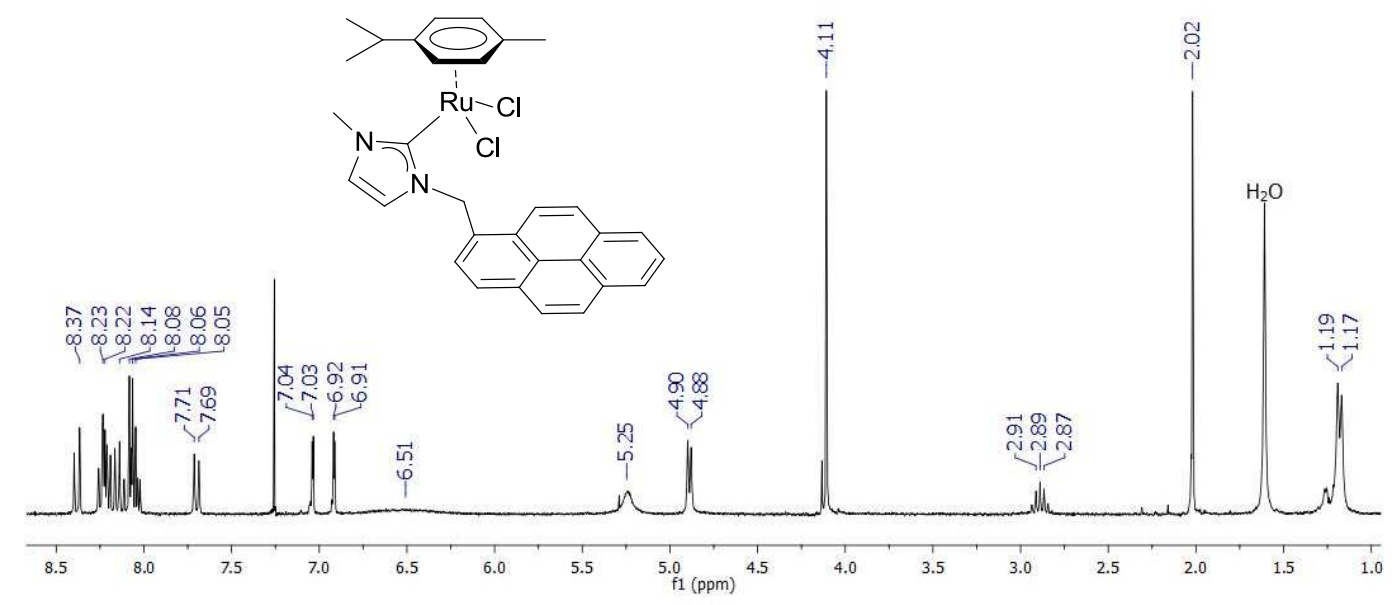

Figure 5.1 ${ }^{1} \mathrm{H}$ NMR spectrum of complex $2 \mathrm{G}$ in $\mathrm{CDCl}_{3}$

\section{${ }^{13} C\left\{{ }^{1} H\right\}$ NMR spectrum of complex $\mathbf{2} \boldsymbol{G}$}

Figure 5.2 shows the ${ }^{13} \mathrm{C}\left\{{ }^{1} \mathrm{H}\right\}$ NMR spectrum of complex 2 G. The most characteristic signal is the one attributed to the metalated carbon at $174.8 \mathrm{ppm}$. The resonance due to the benzylic carbon of the imidazolylidene ligand appears at $52.8 \mathrm{ppm}$. The signal attributed to the carbon of the $\mathrm{N}-\mathrm{CH}_{3}$ group is displayed at $39.9 \mathrm{ppm}$.

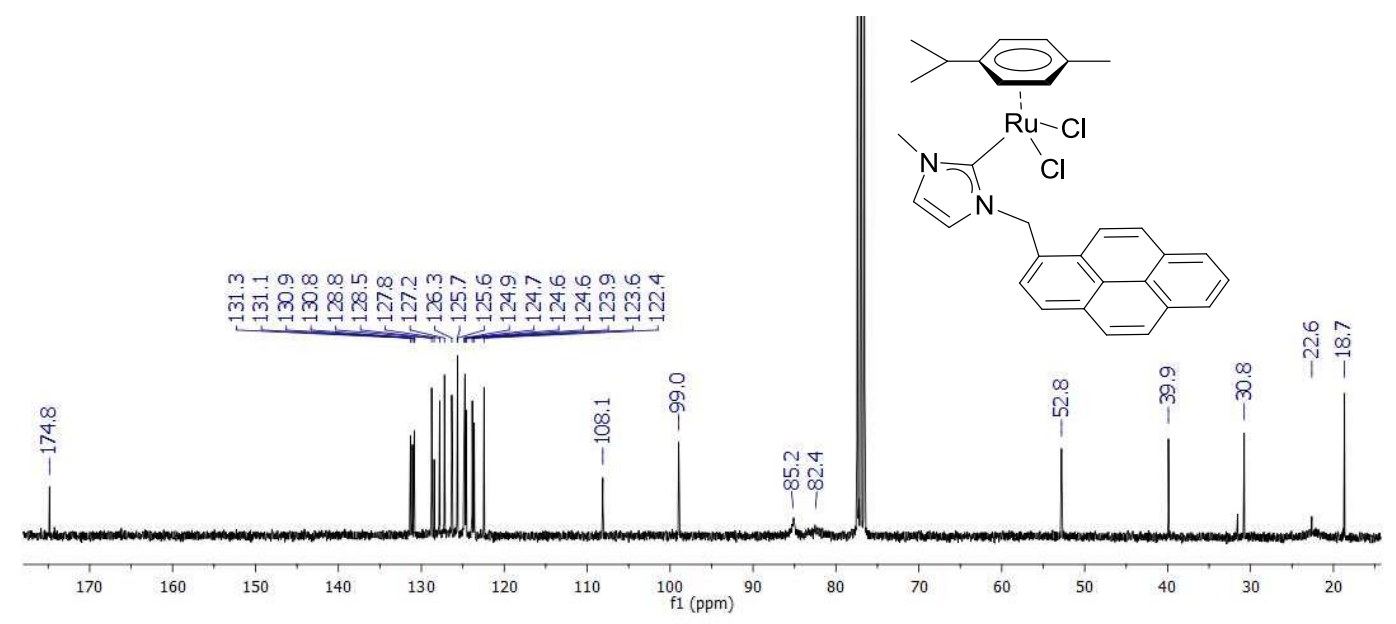

Figure 5.2 ${ }^{13} \mathrm{C}\left\{{ }^{1} \mathrm{H}\right\}$ NMR spectrum of complex 2G 
The HRMS spectra for complexes $\mathbf{1 G}-\mathbf{4 G}$ showed an intense positive ion peak at $\mathrm{m} / \mathrm{z}=$ $616.8912\left([\mathrm{M}-\mathrm{Br}]^{+}, \mathbf{1 G}\right), 567.1144\left([\mathrm{M}-\mathrm{Cl}]^{+}, \mathbf{2 G}\right), 567.0309\left([\mathrm{M}+\mathrm{K}]^{+}, \mathbf{3 G}\right)$ and $659.1803\left([\mathrm{M}-\mathrm{Cl}]^{+}, \mathbf{4 G}\right)$, which confirm the proposed molecular composition of the complexes, based on the mass/charge relation and the isotopic pattern.

The molecular structures of compounds 1G, 2G and 3G were confirmed by means of Xray diffraction. In the next section, the molecular structure of $\mathbf{1 G}$ will be described. The details about the molecular structures of $\mathbf{2 G}$ and $\mathbf{3 G}$ are given in the Experimental Section (Chapter 6).

\section{Molecular structure of $\mathbf{1 G}$}

Crystals of $1 G$ suitable for X-ray diffraction analysis were obtained by slow diffusion of hexane in a concentrated solution of the compound in dichloromethane. The structure analysis reveals a palladium center with a bromide, the NHC ligand and the N,Cchelating N,N-dimethylbenzylamine in a pseudo-square-planar coordination (Figure 5.3, left).The $\mathrm{C}-\mathrm{N}$ ortho-metalated amine forms a five-membered ring that adopts an envelope conformation. The Pd- $\mathrm{C}_{\text {carbene }}$ bond length measures 1.970(8) $\AA$. The packing diagram of complex 1G (Figure 5.3, right) shows an intermolecular slipped $\pi$-stacking between pairs of molecules (interplanar distance: $3.6 \AA$ ) indicating the tendency of the pyrene moiety to display this type of non-covalent interaction. ${ }^{32}$ Interestingly, the packing diagram of complexes 2G and 3G (see Figure 6.24, Experimental Section) reveals the same intermolecular slipped $\pi$-stacking between pairs of molecules with the same interplanar distance of 3.6 $\AA$, confirming the general tendency of the pyrene moiety to present noncovalent interactions of $\pi$-stacking type. See Experimental Section for further details. 

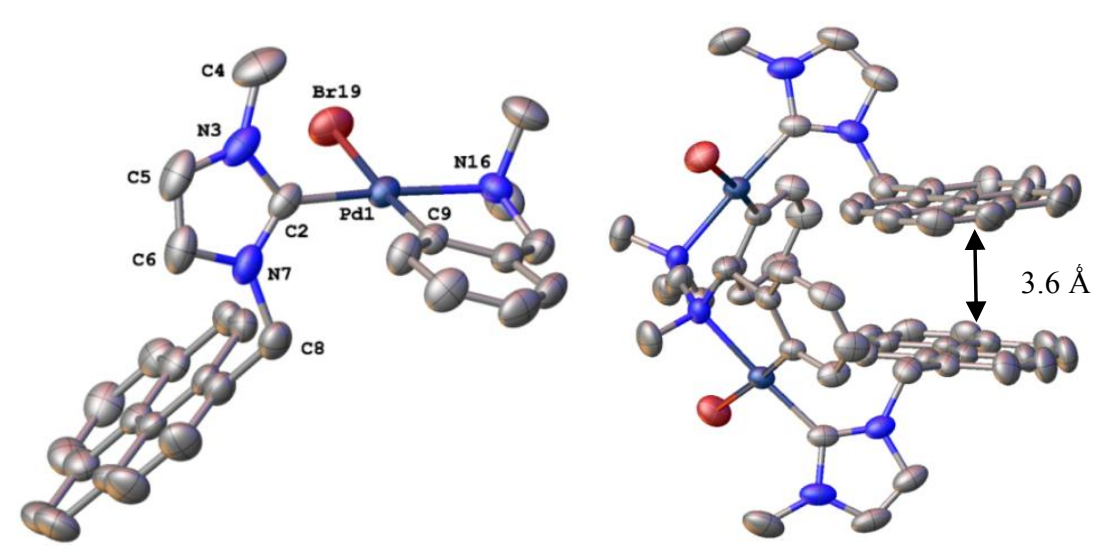

Figure 5.3 Molecular (left) and packing (right) diagrams showing the $\pi$-stacking of complex 1G. Ellipsoids are shown at 50\% probability level. Hydrogens are omitted for clarity. Selected bond lengths $(\AA)$ and angles $\left({ }^{\circ}\right) \operatorname{Pd}(1)-\mathrm{C}(2) 1.978(3), \operatorname{Pd}(1)-\mathrm{C}(9)$ 2.002(3), $\operatorname{Pd}(1)-\operatorname{Br}(19)$ 2.5435(7), $\mathrm{Pd}(1)-\mathrm{N}(16)$ 2.138(3), C(2) - N(7) 1.343(5), N(7) - C(8) 1.471(4), Br(19) - Pd(1) - C(2) 92.99(8), $\operatorname{Br}(19)-\operatorname{Pd}(1)-\mathrm{N}(16)$ 93.25(7), N(16) - Pd(1) - C(9) 83.00(11), C(9) - Pd(1) - C(2) 90.77(12), $\operatorname{Br}(19)-\operatorname{Pd}(1)-\mathrm{C}(2)-\mathrm{N}(3)$ 67.5.The $\pi$-stacking interplanar distance measures $3.6 \AA$

\subsection{Synthesis and characterization of hybrid M - rGO materials}

Complexes 1G - 4G were supported onto reduced graphene oxide (rGO) to afford the hybrid materials 1G-rGO, 2G-rGO, 3G-rGO and 4G-rGO, which contained Pd, Ru, $\mathrm{Au}$ and Ir, respectively (Scheme 5.3). In general, for the preparation of the hybrid materials, rGO was suspended in dichloromethane and the suspension was sonicated for 30 minutes. Then, the corresponding molecular complex $(\mathbf{1 G}-\mathbf{4 G})$ was added and the suspension was stirred at room temperature for 10 hours until the solution became colorless. The resulting solid was filtrated and washed thoroughly with dichloromethane affording the resulting product as a black solid.

The first evidence that the molecular complexes were anchored over the surface of rGO arises from the disappearance of the color of the solutions. The molecular complexes 1G - 4G give yellow-orangish colors in dichloromethane solutions. The disappearance of the color is attributed to the anchoring in the surface of rGO. The ${ }^{1} \mathrm{H}$ NMR spectroscopic analyses of the solid residues resulting from the elimination of the solvent of the filtered 
dichloromethane solutions, revealed the absence of any trace of molecular complexes, suggesting the quantitative deposition of the metal compounds onto rGO. The exact metallic content on the hybrid materials was determined by ICP-MS analysis after digestion of the samples in a refluxing mixture of concentrated nitric and hydrochloric acids (3:1) for 12h. The results obtained accounted for a $9.3 \mathrm{wt} \%$ of 1G in 1G-rGO, a 9.0 wt $\%$ of 2 G in 2G-rGO, a 9.7 wt $\%$ of 3G in 3G-rGO and a 10.72 wt\% of 4G in 4GrGO.
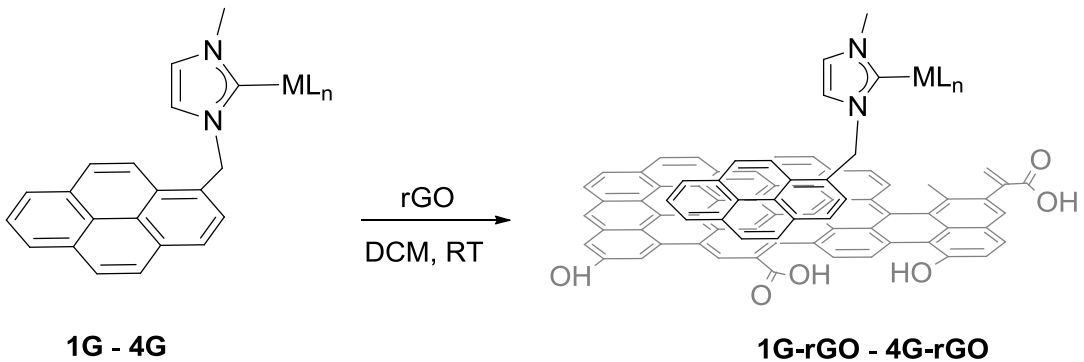

Scheme 5.3

The characterization of the hybrid materials 1G-rGO - 4G-rGO was performed using UV/Vis, FTIR and Raman spectroscopy, Scanning Electron Microscopy (SEM), High Resolution Transmission Electron Microscopy (HRTEM) and Brunauer-Emmett-Teller (BET) Surface Area Analysis. In the following sections, as an example, the description of the characterization of 1G-rGO is given. A detailed description of all the characterization techniques applied to 2G-rGO - 4G-rGO is given in the Experimental section (Chapter $6)$.

\section{UV/Vis spectroscopy}

Figure 5.4 shows the UV/Vis spectra of 1G, rGO and 1G-rGO. The UV/Vis measurements revealed that the spectrum of the hybrid material 1G-rGO (red curve) contains the UV/Vis bands due to $\mathbf{1 G}$, thus confirming the presence of the complex on the rGO surface. Both the spectra of 1G and 1G-rGO show the pyrene UV/Vis characteristic signals assigned to $\pi-\pi^{*}$ transitions. ${ }^{33-36}$ 


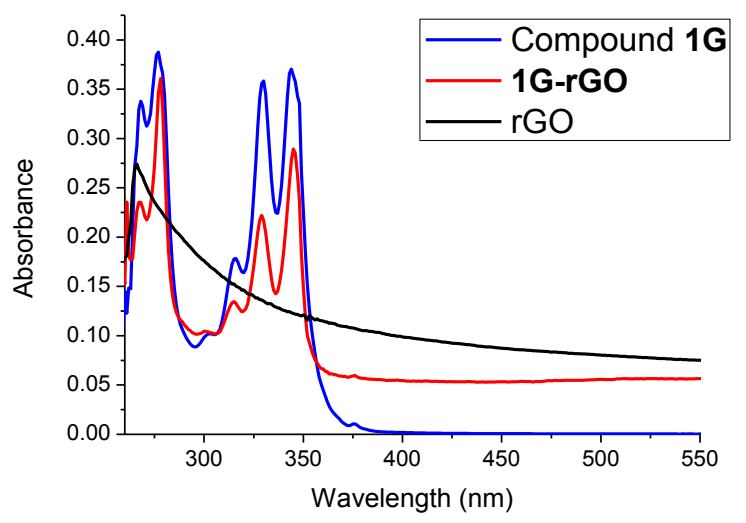

Figure 5.4 UV/Vis spectra of 1G, 1G-rGO and rGO. The solid samples were suspended in N,Ndimethylformamide and sonicated for 5 minutes before the measurements. The spectrum of $\mathbf{1 G}$ was taken from $\mathrm{N}, \mathrm{N}$-dimethylformamide solutions $\left(10^{-6} \mathrm{M}\right)$

\section{Scanning Electron Microscopy (SEM) Images}

The morphological properties of 1G-rGO were examined by means of Scanning Electron Microscopy (SEM). Figure 5.5 shows the SEM images of 1G-rGO at different magnifications using the secondary electrons (SE). The images show the typical rGO morphology consisting of sheets randomly aggregated, thin and crumpled (with wrinkles and folded edges) and forming a disordered solid.
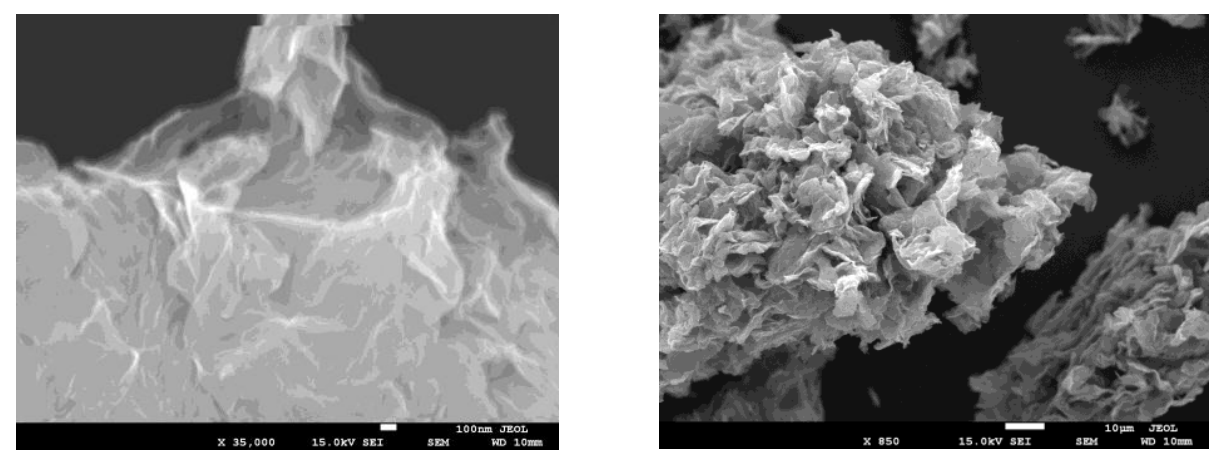

Figure 5.5 SEM images of 1G-rGO using secondary electrons (SE). The samples were covered with a Pt film. Scale bar: $100 \mathrm{~nm}$ (left), $10 \mu \mathrm{m}$ (right) 


\section{High Resolution Transmission Electron Microscopy (HRTEM) images}

HRTEM was used to characterize the microstructure and qualitative composition of the hybrid solids. Figure 5.6 shows the HRTEM (a, b), the STEM (c), and Energy-Dispersive X-ray spectroscopy (EDS) elemental mapping (d) images of 1G-rGO. The HRTEM images $(a, b)$ show typical rGO sheets at different magnifications. The elemental mapping by EDS analysis performed by means of HRTEM of 1G-rGO, confirmed the homogeneous distribution of palladium in the hybrid material.
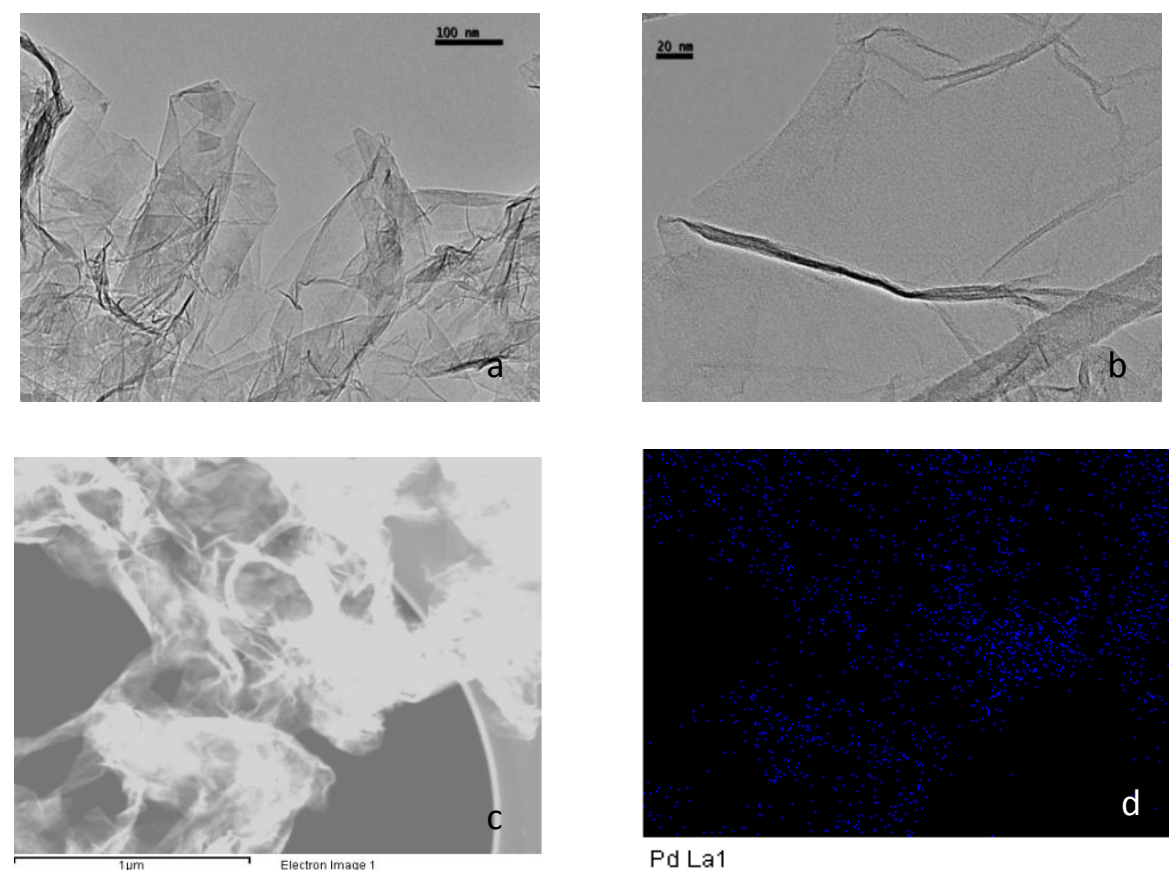

Figure 5.6 HRTEM (a, b) and STEM (c) images of 1G-rGO. The EDS elemental mapping image (d) shows the homogeneous distribution of palladium. Scale bar: $100 \mathrm{~nm}$ (a), $20 \mathrm{~nm}$ (b), $1 \mu \mathrm{m}(\mathrm{c})$

\section{Brunauer-Emmett-Teller (BET) Surface Area Analysis}

The analysis of the surface area was performed by argon sorption analysis at $77 \mathrm{~K}$. The BET area for 1G-rGO was $263 \mathrm{~m}^{2} \mathrm{~g}^{-1}$, significantly lower than that shown by the rGO raw material $\left(456 \mathrm{~m}^{2} \mathrm{~g}^{-1}\right)$, probably due to the agglomeration of the rGO sheets upon functionalization with $\mathbf{1 G}$. 


\subsection{Synthesis and characterization of hybrid bimetallic Ru-Pd-rGO material 5rGO}

In the last section we demonstrated the applicability of the non-covalent anchoring method for the preparation of four different hybrid materials based on reduced graphene oxide (rGO). Following the same procedure described for the immobilization of only one molecular complex, the hybrid bimetallic material 5rGO, which contained $\mathrm{Pd}$ and $\mathrm{Ru}$, was prepared. An equimolecular mixture of complexes 1G $(\mathrm{Pd})$ and 2G $(\mathrm{Ru})$ were supported onto rGO by mixing the molecular complexes and $\mathrm{rGO}$ in dichloromethane in ultrasound bath for $30 \mathrm{~min}$. and stirring for $10 \mathrm{~h}$ (Scheme 5.4).

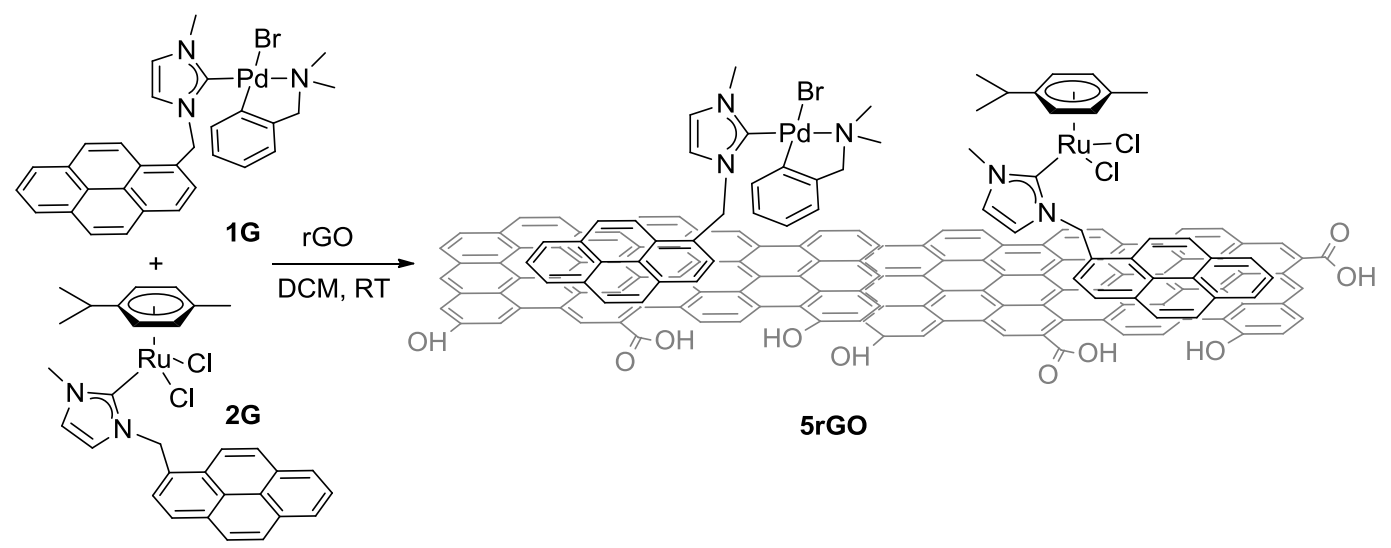

Scheme 5.4 Synthesis of hybrid bimetallic material 5rGO

As stated for the hybrid materials described in the previous section, the first indication that the immobilization had taken place was the loss of the color of the solution. The resulting black solid was filtered and washed thoroughly with dichloromethane. The ${ }^{1} \mathrm{H}$ NMR spectrum of the filtrate revealed the absence of signals due to $\mathbf{1 G}$ or $\mathbf{2 G}$, and therefore constituted indirect evidence that the two complexes had been effectively grafted on the solid surface. The exact palladium and ruthenium content in the final solid 5rGO was determined by ICP-MS analysis after digestion of the solid in a refluxing mixture of concentrated nitric and hydrochloric acids $(3: 1)$ for $12 \mathrm{~h}$. The results indicated 5.19 and $4.97 \mathrm{wt} \%$ for palladium and ruthenium, respectively. The characterization of 5rGO was carried out by using UV/Vis and FTIR spectroscopy, Ar-sorption experiments, 


\section{Atomic Force Microscopy (AFM) and High Resolution Transmission Electron Microscopy (HRTEM).}

The analysis of the surface area was performed by argon sorption analysis at $77 \mathrm{~K}$. The BET area for 5 rGO was $165 \mathrm{~m}^{2} \mathrm{~g}^{-1}$, significantly lower than that shown by the rGO raw material $\left(373 \mathrm{~m}^{2} \mathrm{~g}^{-1}\right)$.

Atomic Force Microscopy (AFM) analyses were performed in order to establish the thickness and surface roughness of $\mathbf{5 r G O}$ sheets (Figure 5.7). The height profile indicated an approximate $2 \mathrm{~nm}$ step from the surface of mica to the rGO sheet. The deviation from the theoretical value about $0.3 \mathrm{~nm}$ for a single graphene sheet is attributed to the presence of residual oxygen-containing groups on the sheet surface. The height of the rGO sheets of about $2 \mathrm{~nm}$ is within the accepted range of few-layer graphenes. ${ }^{37}$
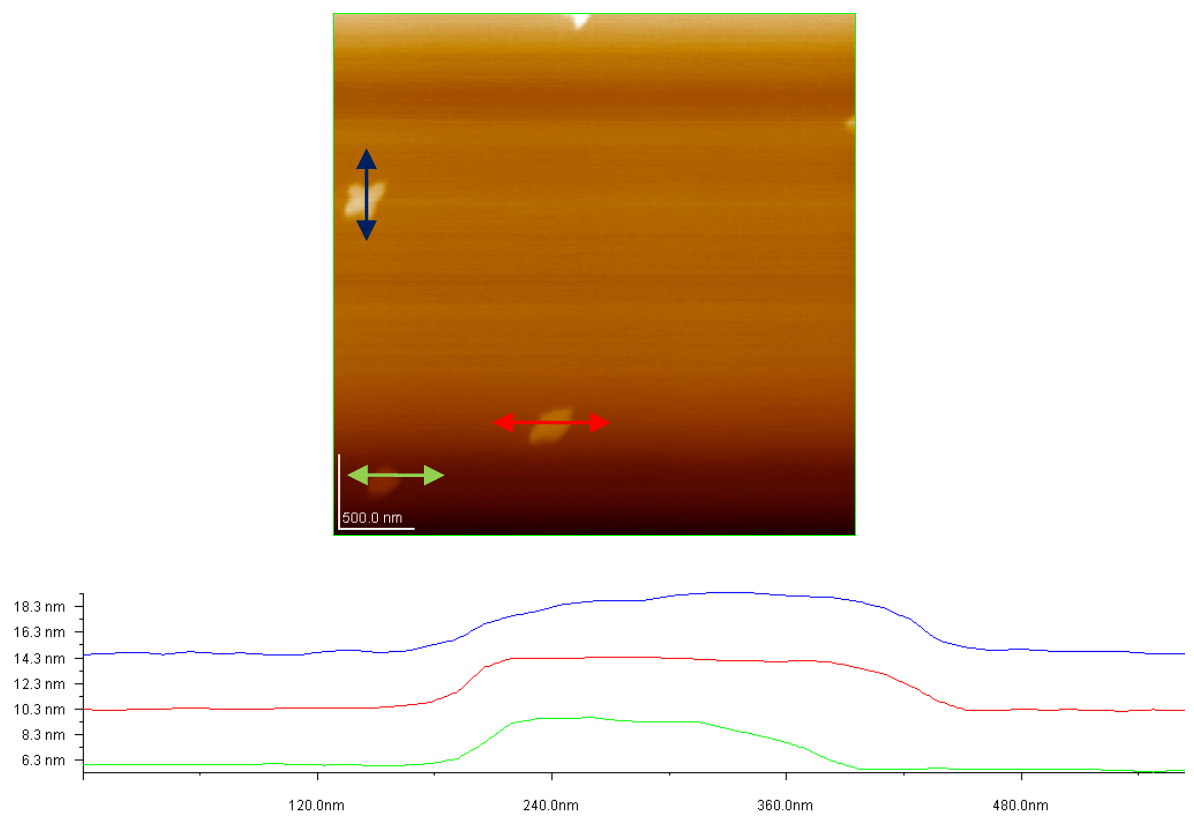

Figure 5.7 AFM image using a contact mode of 5rGO and its height profiles for the indicated lines across the sheets. The solid sample was dispersed in methanol, deposited on mica by drop casting and dried at room temperature. Scale bar: $500 \mathrm{~nm}$ 
The elemental mapping by energy-dispersive X-ray spectroscopic analysis (EDS) performed by means of HRTEM of 5rGO, confirmed the homogeneous distribution of palladium and ruthenium in the hybrid material (Figure 5.8).
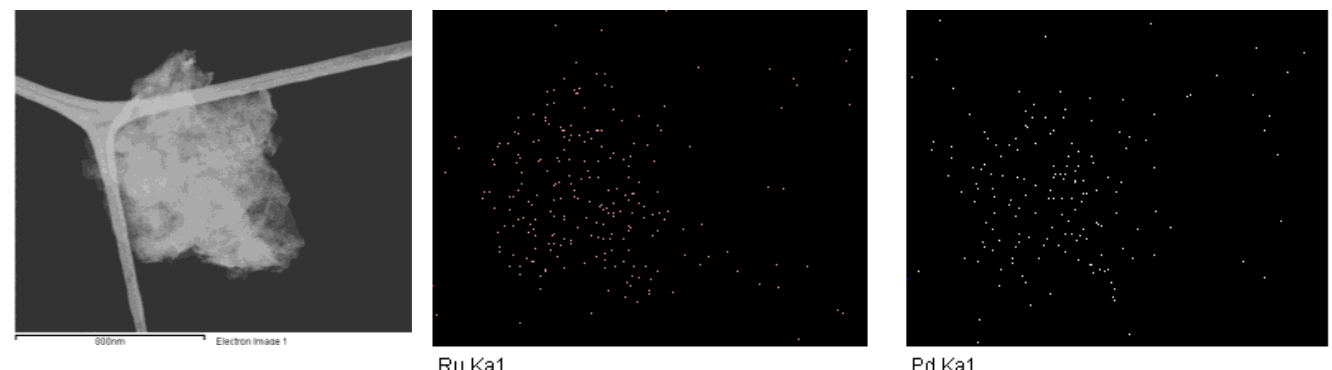

Figure 5.8 STEM image of 5rGO (left), and EDS elemental mapping images showing the homogeneous distribution of ruthenium (middle) and palladium (right). Scale bar: $600 \mathrm{~nm}$

\subsection{Catalytic Studies}

The catalytic activity of the hybrid materials 1G-rGO and 2G-rGO was tested in two benchmark reactions typically catalyzed by $\mathrm{Pd}$ and $\mathrm{Ru}$. The palladium-containing material (1G-rGO) was tested in the hydrogenation of unsaturated organic substrates (alkenes and nitrobenzenes) using molecular hydrogen. ${ }^{38,39}$ The ruthenium containing material (2G-rGO) was tested in the dehydrogenation of alcohols. ${ }^{40,41}$ The catalytic activity of the bimetallic Pd-Ru-rGO material (5rGO) was tested in the hydrodefluorination (HDF) of a series of fluoroarenes.

\subsubsection{Hydrogenation of unsaturated organic substrates using molecular hydrogen}

\section{$\underline{\text { Hydrogenation of alkenes }}$}

The palladium-containing material 1G-rGO was tested in the reduction of alkenes. The reactions were performed in toluene in the presence of $\mathrm{Cs}_{2} \mathrm{CO}_{3}$ at $100^{\circ} \mathrm{C}$ using $\mathrm{H}_{2}$ (1 atm) for $15 \mathrm{~min}$. with a catalyst loading of $1 \mathrm{~mol} \%$, based on the amount of metal (ICP-MS analysis). The yields were determined by GC analyses using anisole as internal standard. 
The results shown in Table 5.1 indicate that the palladium hybrid material 1G-rGO is highly effective in the reduction of a wide variety of substrates. The inherent catalytic properties of rGO have been studied in different processes, ${ }^{12,42,43}$ thus control experiments using the rGO under exactly the same reaction conditions were carried out, showing that the palladium-free material was completely inactive (entry 1 ).

Table 5.1 Alkene hydrogenation using molecular hydrogen

\begin{tabular}{|c|c|c|c|}
\hline \multicolumn{4}{|c|}{ Cat. (1 mol \%) } \\
\hline \multirow[b]{3}{*}{ Entry } & \multirow[b]{3}{*}{ Catalyst } & \multirow[b]{3}{*}{ Substrate } & \multirow[b]{3}{*}{ Yield $(\%)^{a}$} \\
\hline & & & \\
\hline & & & \\
\hline 1 & rGO & & 0 \\
\hline 2 & $1 G$ & & 48 \\
\hline $3^{c}$ & $1 G$ & & 10 \\
\hline 4 & 1G-rGO & & 100 \\
\hline 5 & 1G-rGO & & 88 \\
\hline 6 & 1G-rGO & & 100 \\
\hline 7 & 1G-rGO & & $100^{\mathrm{b}}$ \\
\hline 8 & 1G-rGO & & 100 \\
\hline 9 & 1G-rGO & & 100 \\
\hline
\end{tabular}

Reactions were carried out with $0.3 \mathrm{mmol}$ of alkene, $\mathrm{Cs}_{2} \mathrm{CO}_{3}(0.3 \mathrm{mmol}), \mathrm{H}_{2}(1 \mathrm{~atm})$, catalyst $(1 \mathrm{~mol} \%), 5$ $\mathrm{mL}$ of toluene for $15 \mathrm{~min}$. at $100^{\circ} \mathrm{C}$. [a] Yields determined by GC analyses using anisole as internal standard. [b] Isolated yield. [c] The mercury-drop experiment under the same reaction conditions resulted in the deactivation of the catalyst. An excess of $\mathrm{Hg}(0)$ was added to the reaction mixture with efficient stirring after the catalyst was activated ( $5 \mathrm{~min}$. of reaction). 
For the reaction carried out using complex $\mathbf{1 G}$ in solution, we obtained a moderate yield of ethyl benzene, while 1G-rGO produced a quantitative yield under the same reaction conditions (compare entries 2 and 4). These results suggest that the support provides a significant benefit in the catalytic hydrogenation reaction, most probably due to the surface area of rGO, which may facilitate the interaction between the substrates and the catalyst. ${ }^{44}$ This 'abnormal' enhancement in the activity of the immobilized catalyst onto the graphene material has already been observed before in the case of the hydrogenation of cyclohexene facilitated by a rhodium complex covalently supported onto graphene oxide. $^{45}$

In order to determine if the reaction with $\mathbf{1 G}$ is homogeneously catalyzed, we performed the mercury-drop experiment, and observed that the reaction rate significantly decreased (entry 3). This result suggests that the reaction may be heterogeneously rather than homogeneously catalyzed, and should imply that palladium-nanoparticles are produced along the reaction course. ${ }^{46}$ We thought that the formation of nanoparticles should also account for the reactions catalyzed by 1G-rGO, and for that reason we decided to analyze the solid by HTREM after being used in the reduction of styrene. Figure 5.9 shows the HRTEM image of 1G-rGO, after being used in the reduction of styrene (Table 5.1 , entry 4). The image shows the formation of small palladium nanoparticles. This result is in agreement with the results shown by the mercury drop experiment carried out at the molecular level using the molecular complex 1G. Very recent examples of NHCbased complexes covalently-immobilized onto graphene-oxide (GO) also described the decomposition of the parent original complexes onto palladium nanoparticles. ${ }^{47,48}$ Although the molecular complexes generate palladium nanoparticles, the catalytic performances are maintained and good recyclability could be achieved, as will be described below. 


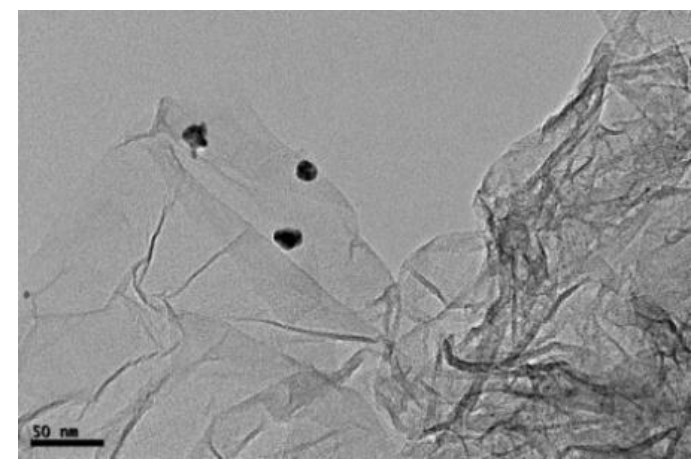

Figure 5.9 HRTEM of 1G-rGO after 1 catalytic cycle showing the formation of small amounts of palladium nanoparticles. Scale bar: $50 \mathrm{~nm}$

\section{$\underline{\text { Reduction of nitroarenes }}$}

Catalyst 1G-rGO was also tested in the reduction of nitroarenes, a very interesting process due to the applications of functionalized anilines in the synthesis of pharmaceuticals and fine chemicals. ${ }^{49,50}$ The reactions were carried out using a catalyst loading of $2 \mathrm{~mol} \%$, based on the amount of metal (ICP-MS analysis), in the presence of $\mathrm{Cs}_{2} \mathrm{CO}_{3}$, and $\mathrm{H}_{2}(1 \mathrm{~atm})$, in toluene for 2 hours at $100^{\circ} \mathrm{C}$. The yields were determined by GC analyses using anisole as internal standard.

Table 5.2 Nitro reduction using molecular hydrogen

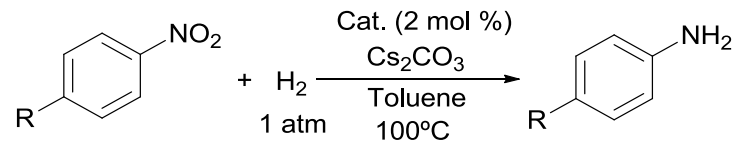

\begin{tabular}{cccc}
\hline Entry & Catalyst & R & Yield(\%) $^{\mathbf{a}}$ \\
\hline 1 & rGO & $\mathrm{H}$ & 0 \\
2 & $\mathbf{1 G}$ & $\mathrm{H}$ & 50 \\
3 & $\mathbf{1 G - r G O}$ & $\mathrm{H}$ & 100 \\
4 & $\mathbf{1 G - r G O}$ & $\mathrm{CH}_{3}$ & 100 \\
5 & $\mathbf{1 G - r G O}$ & $\mathrm{CH}_{3} \mathrm{O}$ & $100^{\mathrm{b}}$ \\
\hline
\end{tabular}

Reactions were carried out with $0.3 \mathrm{mmol}$ of nitro compound, $\mathrm{Cs}_{2} \mathrm{CO}_{3}(0.3 \mathrm{mmol}), \mathrm{H}_{2}(1 \mathrm{~atm})$, catalyst (2 $\mathrm{mol} \%$ ), $5 \mathrm{~mL}$ of toluene for 2 hours at $100^{\circ} \mathrm{C}$. [a] Yields determined by GC analyses using anisole as internal standard. [b] Isolated yield. 
The results in Table 5.2 show that 1G-rGO is also an efficient catalyst in the reduction of nitroarenes in short times under mild reaction conditions $\left(1 \mathrm{~atm}\right.$ of $\mathrm{H}_{2}$ ). For this reaction we also observed that rGO is completely inactive (entry 1), and that the molecular complex 1G is less active than the supported counterpart (compare entries 2 and 3), therefore suggesting that the $\mathrm{rGO}$ is playing a positive role in the overall catalytic cycle, as we observed before in the hydrogenation of alkenes.

\section{$\underline{\text { Recycling and large scale experiments }}$}

In view of the good results obtained with the hybrid material 1G-rGO in the hydrogenation of organic substrates, we decided to explore the catalyst stability by exploring their recyclability, and activity at large scale experiments. Recycling experiments of the palladium-containing catalyst 1G-rGO were carried out using styrene as model substrate (Figure 5.10). The reaction conditions used in these experiments were the same as those described in the hydrogenation reactions, and the reaction progress was monitored by GC. After completion of each run (15 min.), the reaction mixture was allowed to reach room temperature, and the solid catalyst was separated by centrifugation, washed with dichloromethane, dried and reused in the subsequent run. By following this methodology the palladium-containing catalyst 1G-rGO was reused up to ten times without any decrease in activity, affording ethyl benzene in quantitative yield for all ten runs.

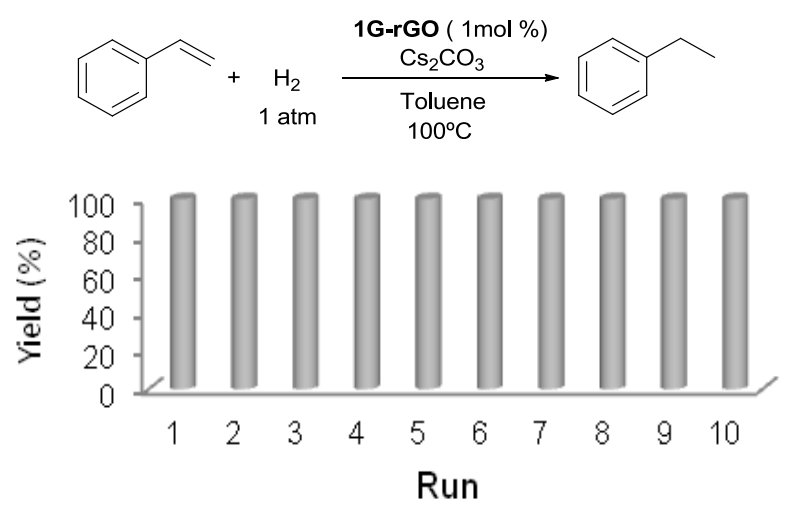

Figure 5.10 Recycling experiment of styrene using 1G-rGO 
Interestingly, the base was only needed for the first run. This observation may be due to the transformation of the complex into the active catalytic species during the first run, or simply to the adsorption of residual $\mathrm{Cs}_{2} \mathrm{CO}_{3}$ to the $\mathrm{rGO}$ surface, which avoided the need of further addition in the subsequent experiments. EDS elemental mapping of 1G-rGO after the tenth run, revealed the presence of homogenously distributed Cs, which may suggest that some base remains attached to surface of the solid (Figure 5.11).
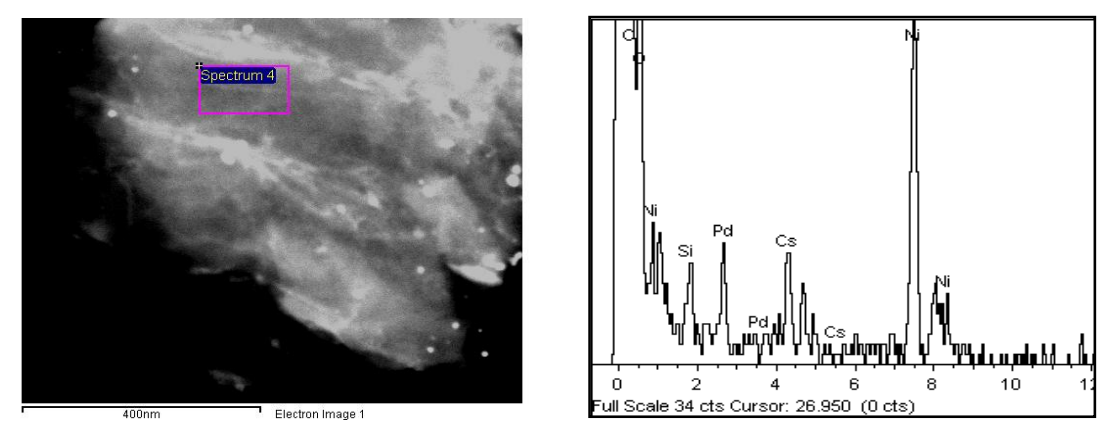

Figure 5.11 STEM image and EDS spectrum of 1G-rGO after 10 catalytic cycles showing the homogeneous distribution of Cs on the solid surface. Scale bar: $400 \mathrm{~nm}$

The ICP-MS analysis after the recycling experiments accounts for $8.5 \mathrm{wt} \%$ of palladium, indicating a total loss of $0.8 \mathrm{wt} \%$ with respect to the initial palladium content, and thus $8.6 \%$ of the palladium is lost after ten catalytic cycles. After the tenth experiment, a SEM image of the 1G-rGO revealed that the morphology of rGO is maintained (Figure 5.12).
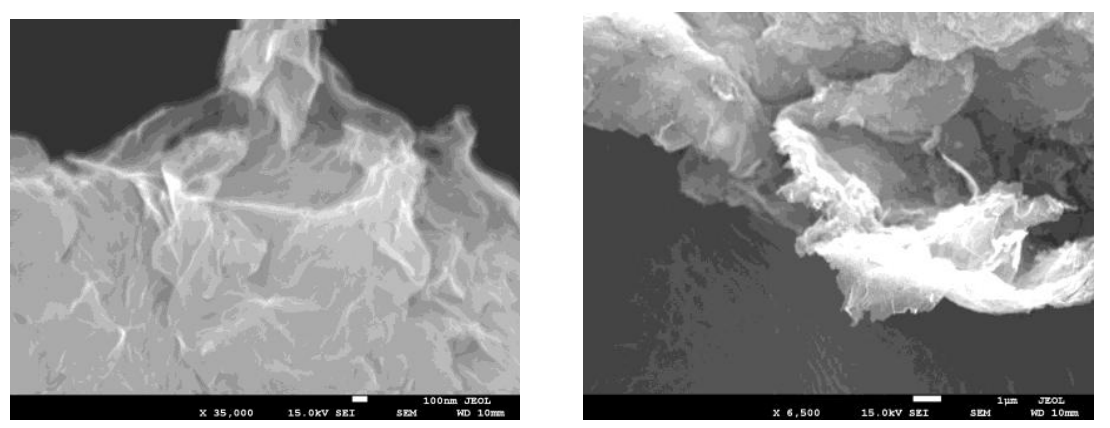

Figure 5.12 SEM images of 1G-rGO before (left) and after (right) 10 catalytic cycles. Scale bar: $100 \mathrm{~nm}$ (left), $1 \mu \mathrm{m}$ (right) 
A deeper analysis by HRTEM showed that there is a well-dispersed palladium distribution, although the presence of some small $(<8 \mathrm{~nm}$ diameter $)$ palladium nanoparticles (Figure 5.13) was detected.
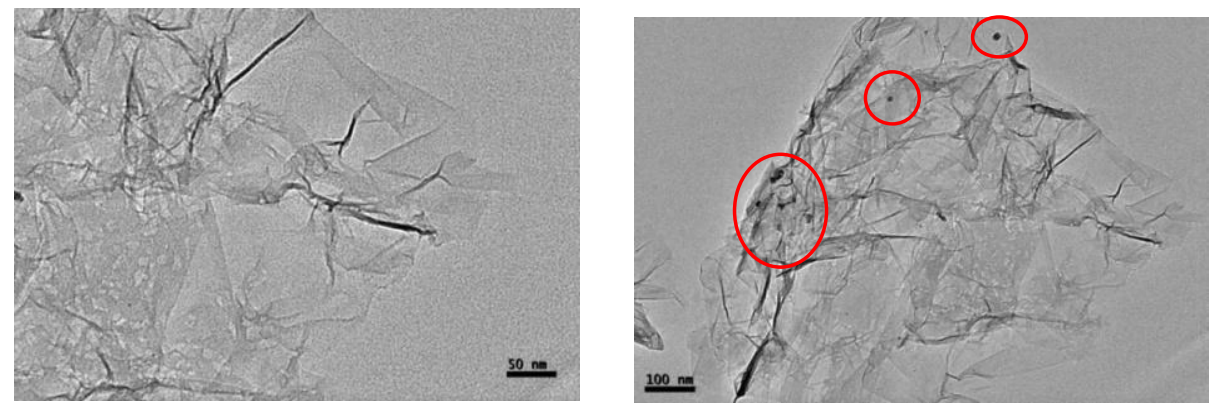

Figure 5.13 HRTEM images of 1G-rGO before (left) and after (right) 10 catalytic cycles indicating the formation of palladium nanoparticles. Scale bar: $50 \mathrm{~nm}$ (left), $100 \mathrm{~nm}$ (right)

The high activity of catalyst 1G-rGO was further analyzed in a larger scale experiment. The reaction was carried out using $2 \mathrm{~mL}$ of styrene $(17.3 \mathrm{mmol})$ and a catalyst loading of $0.01 \mathrm{~mol} \%$ based on palladium $\left(1.74 \times 10^{-3} \mathrm{mmol}\right)$. The reaction achieved quantitative yield after 20h, therefore achieving a maximum TON of 10.000. A recyclability study using these conditions reveals that the system is active at least for three runs without decrease in activity (Figure 5.14). The monitoring of the time-dependent reaction progress by GC indicates that the activity of catalyst 1G-rGO is maintained along the three consecutive runs (the accumulated TON is 30.000), therefore revealing that there is no trace of catalyst deactivation. An interesting feature that is worth mentioning is the zero order dependence of the rate on the concentration of the substrate (styrene), as can be observed from the time-dependent reaction profiles shown in Figure 5.14. Zero-order dependence on the concentration of the substrate is usually observed in heterogeneous hydrogenations of olefins and is interpreted to indicate that the surface of the catalysts saturated with the substrate, ${ }^{51-54}$ and therefore suggests that in our case there is probably some interaction between the substrate and the support. 


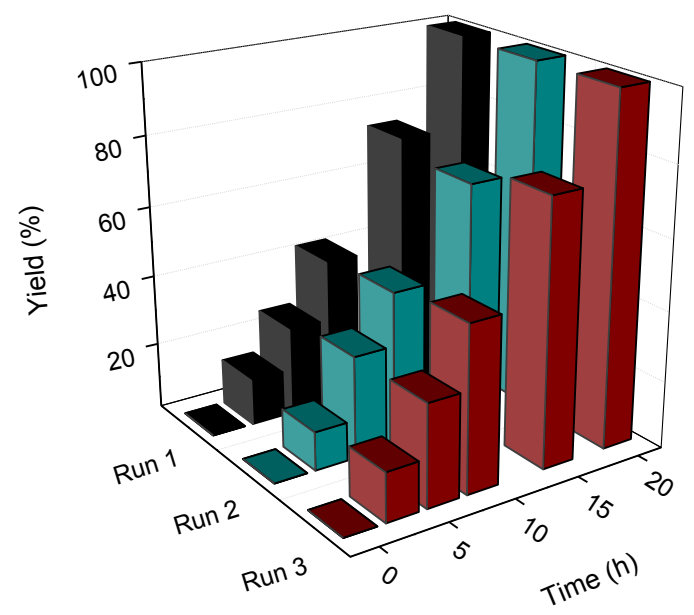

Figure 5.14 Recycling experiment, and time-dependent reaction profiles of styrene hydrogenation using 1G-rGO at a catalyst loading of $0.01 \mathrm{~mol} \%$

\subsubsection{Dehydrogenative oxidation of alcohols}

The ruthenium-containing material 2G-rGO was tested in the dehydrogenative oxidation of alcohols, which is a highly attractive process for the synthesis of carbonyl species and for the generation of molecular hydrogen from readily available alcohols. ${ }^{55}$ Metal-based ruthenium catalysts have been widely used for this process. ${ }^{56-60}$

The reactions were carried out in toluene for $12 \mathrm{~h}$ at $100^{\circ} \mathrm{C}$, under aerobic conditions, in the presence of $\mathrm{Cs}_{2} \mathrm{CO}_{3}$, with a catalyst loading of $2 \mathrm{~mol} \%$, based on the metal amount (ICP-MS analysis). Yields were determined by GC analyses using anisole as internal standard.

Table 5.3 shows that 2G-rGO was active in the oxidation of a wide variety of substrates. The optimization of the reaction was carried out using 1-phenyl ethanol (entries 1 - 3) and benzyl alcohol (entries $4-6$ ) as model substrates. Blank experiments on both substrates using rGO under the same reaction conditions, revealed that the oxidation does not occur (entries 1 and 4). The oxidation of 1-phenyl ethanol and benzyl alcohol under homogeneous conditions using the molecular catalyst 2G afforded low yields of acetophenone (26\%) and moderate yields of benzaldehyde (47\%) (entries 2 and 5, 
respectively). When the oxidation was carried out using the hybrid material 2G-rGO, the yields increased up to $60 \%$, and $>99 \%$ for the cases of acetophenone and benzaldehyde, respectively (entries 3 and 7). These results suggest that immobilization of the molecular catalyst $2 \mathrm{G}$ on $\mathrm{rGO}$ provides a benefit in the catalytic process, as previously observed in the case of the hydrogenation processes. Catalyst 2G-rGO was very effective in the oxidation of a wide set of benzyl alcohols, affording quantitative yields, regardless the presence of electron-accepting or -donating substituents in the substrate (entries 8-11). We performed the mercury-drop experiment, which provided similar results as those without the mercury drop, thus suggesting that the reaction is homogeneously catalyzed (entry 6).

Table 5.3 Oxidant-free dehydrogenation of alcohols

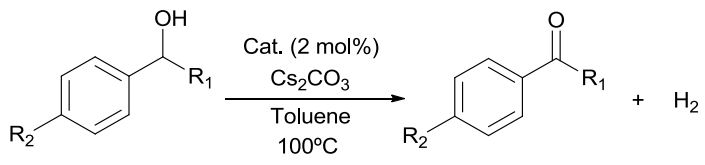

\begin{tabular}{ccccc}
\hline Entry & Catalyst & $\mathbf{R}_{\mathbf{1}}$ & $\mathbf{R}_{\mathbf{2}}$ & Yield(\%) $^{\mathbf{a}}$ \\
\hline 1 & rGO & $\mathrm{CH}_{3}$ & $\mathrm{H}$ & 0 \\
2 & $\mathbf{2 G}$ & $\mathrm{CH}_{3}$ & $\mathrm{H}$ & 26 \\
3 & $\mathbf{2 G - r G O}$ & $\mathrm{CH}_{3}$ & $\mathrm{H}$ & 60 \\
\hline 4 & rGO & $\mathrm{H}$ & $\mathrm{H}$ & 0 \\
5 & $\mathbf{2 G}$ & $\mathrm{H}$ & $\mathrm{H}$ & 47 \\
$6^{\mathrm{b}}$ & $\mathbf{2 G}$ & $\mathrm{H}$ & $\mathrm{H}$ & 47 \\
7 & $\mathbf{2 G - r G O}$ & $\mathrm{H}$ & $\mathrm{H}$ & 100 \\
\hline 8 & $\mathbf{2 G}-\mathbf{H G O}$ & $\mathrm{H}$ & $\mathrm{Cl}$ & 100 \\
9 & $\mathbf{2 G - r G O}$ & $\mathrm{H}$ & $\mathrm{NO}_{2}$ & 100 \\
10 & $\mathbf{2 G - r G O}$ & $\mathrm{H}$ & $\mathrm{OMe}$ & 100 \\
11 & $\mathbf{2 G - r G O}$ & $\mathrm{H}$ & $\mathrm{CH}_{3}$ & 100 \\
\hline
\end{tabular}

Reactions were carried out with $0.3 \mathrm{mmol}$ of alcohol, $\mathrm{Cs}_{2} \mathrm{CO}_{3}(0.3 \mathrm{mmol})$, catalyst $(2 \mathrm{~mol} \%), 5 \mathrm{~mL}$ of toluene for 12 hours at $100^{\circ} \mathrm{C}$. [a] Yields determined by GC analyses using anisole as internal standard. [b] An excess of $\operatorname{Hg}(0)$ was added to the reaction mixture with efficient stirring after the catalyst was activated (1h of reaction). 
The HRTEM analysis of the catalyst 2G-rGO, after being used in the oxidation of benzyl-alcohol (Figure 5.15), revealed the absence of Ru-based nanoparticles in the solid, therefore suggesting that the nature of the catalytic species for this system should be molecular.
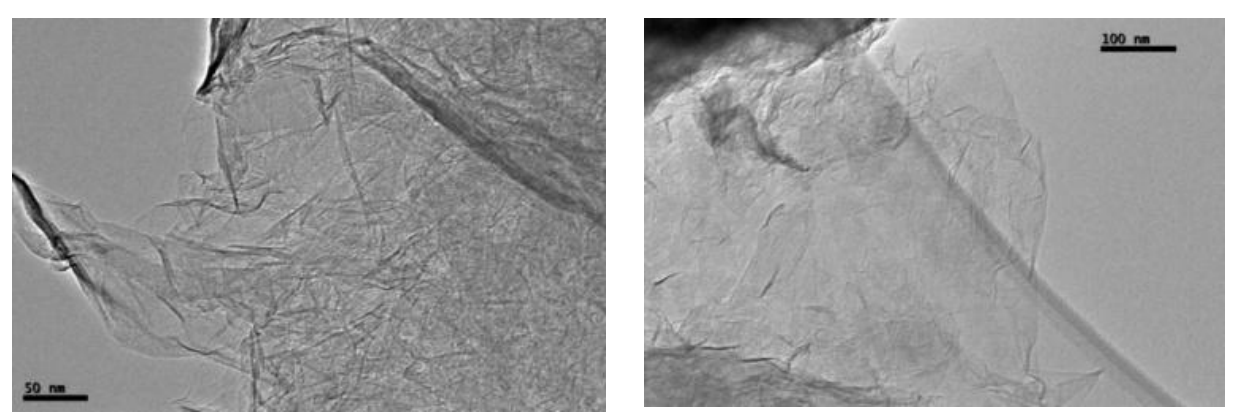

Figure 5.15 HRTEM images of 2G-rGO after being used in the dehydrogenation of benzyl alcohol. Scale bar: $50 \mathrm{~nm}$ (left), $100 \mathrm{~nm}$ (right)

\section{$\underline{\text { Recycling and large scale experiments }}$}

Recycling experiments for the ruthenium-containing catalyst 2G-rGO, were performed using benzyl alcohol as model substrate (Figure 5.16). The reactions were carried out using a catalyst load of $2 \mathrm{~mol} \%$, at $100^{\circ} \mathrm{C}$ in the presence of $\mathrm{Cs}_{2} \mathrm{CO}_{3}$ (only in the first run). The mixture was allowed to react for $12 \mathrm{~h}$ for each run. Then the catalyst was separated from the reaction mixture by centrifugation, washed, dried and replaced in the reaction vessel to be used in the subsequent run. As stated above, in the hydrogenation of alkenes using 1G-rGO, the fact that the base was only needed in the first cycle may indicate either that once the pre-catalyst is transformed into its active form, the active species is stable for recyclability during the subsequent runs, or that the base is adsorbed onto the solid support, and therefore there is no need for subsequent additions. In this case, similarly to 1G-rGO, the EDS elemental mapping of the solid after the first run shows a homogeneous distribution of Cs, thus suggesting that the base is adsorbed onto the solid. Catalyst 2G-rGO was reused up to ten times without any decrease in activity, affording quantitative yields of benzaldehyde (Figure 5.16). 

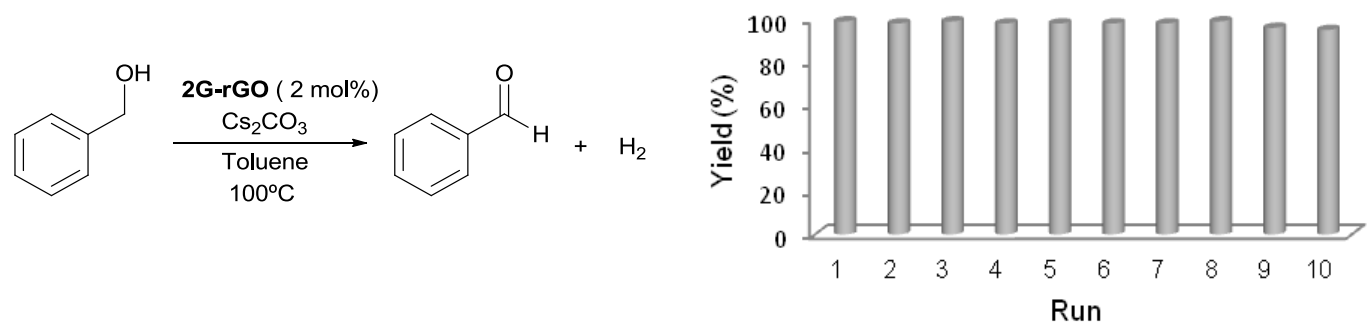

Figure 5.16 Recycling experiment of benzyl alcohol oxidation using 2G-rGO

The ICP-MS analysis after the recycling experiments accounts for $7.7 \mathrm{wt} \%$ of ruthenium (2G), indicating that a total loss of $1.3 \mathrm{wt} \%$, with respect the original content on $\mathrm{Ru}$, and thus $14.4 \%$ of all original ruthenium complex is lost. The comparison of the SEM and HRTEM analysis of 2G-rGO before and after the recycling experiments revealed the same well-dispersed ruthenium distribution, without modification of the support morphology and the absence of ruthenium nanoparticles (Figure 5.17).
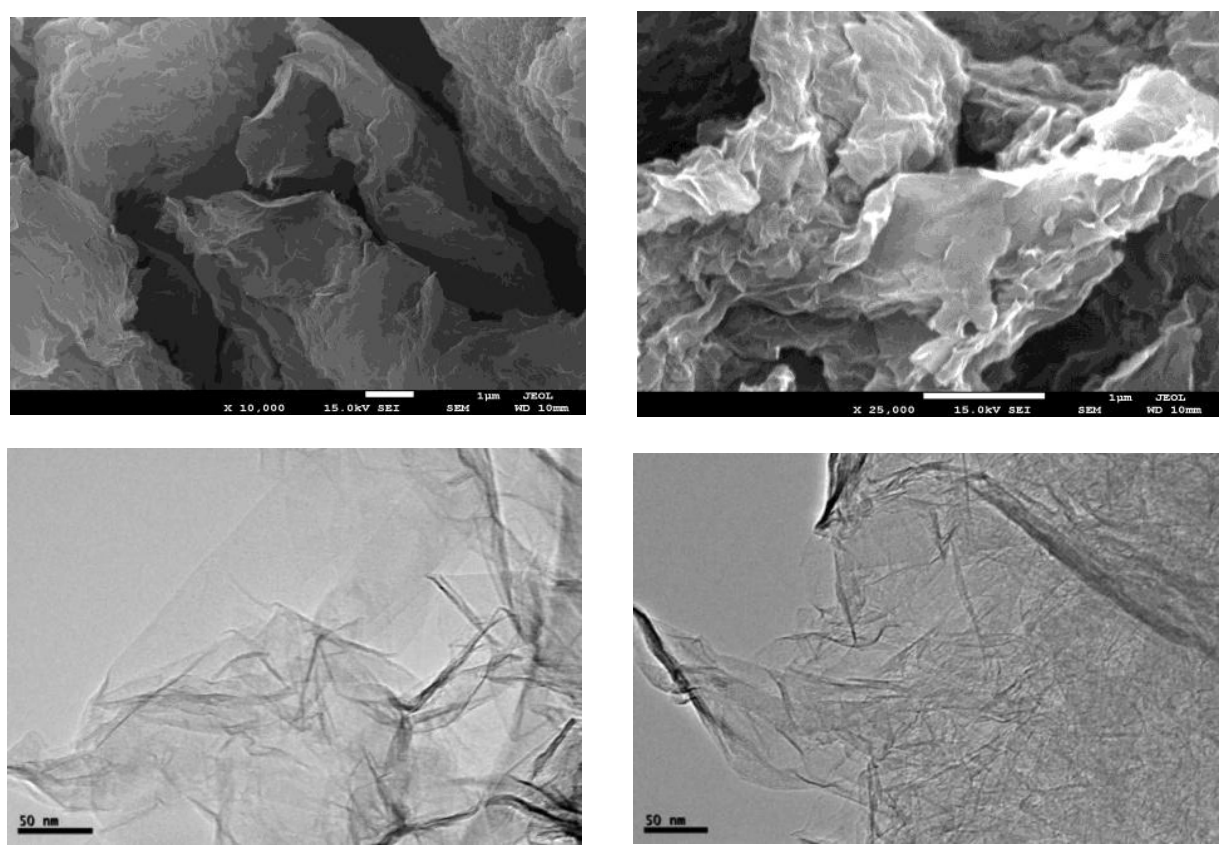

Figure 5.17 SEM (top) and HRTEM (bottom) images of 2G-rGO before (left) and after (right) 10 catalytic runs. Scale bar: $1 \mu \mathrm{m}$ (top), $50 \mathrm{~nm}$ (bottom) 
The large scale experiments were carried out using $1 \mathrm{~mL}$ of benzyl alcohol $(9.7 \mathrm{mmol})$ and a catalyst loading of $0.03 \mathrm{~mol} \%$ based on ruthenium $\left(3.0 \times 10^{-3} \mathrm{mmol}\right)$. The reaction yielded $75 \%$ of benzaldehyde after $24 \mathrm{~h}$. Figure 5.18 shows that 2G-rGO could be reused up to 3 times, and the reaction profiles where identical in the two first cycles, therefore suggesting that the deactivation of the catalyst is negligible. In the third cycle a slight decrease in catalyst activity is observed, and the reaction afforded $65 \%$ yield after $24 \mathrm{~h}$. This experiment is very interesting, because the reaction conditions used for this catalytic experiment show that the catalyst is using its full potential to achieve a $75 \%$ yield in the first cycle, therefore any minimum loss of catalyst, or any deactivation should have measurable negative consequences in the recycling of the catalyst. Again, the reaction shows quasi-zero order dependence of the rate on the concentration of the substrate (benzyl alcohol), thus suggesting surface saturation due to the interaction between the substrate and the support.

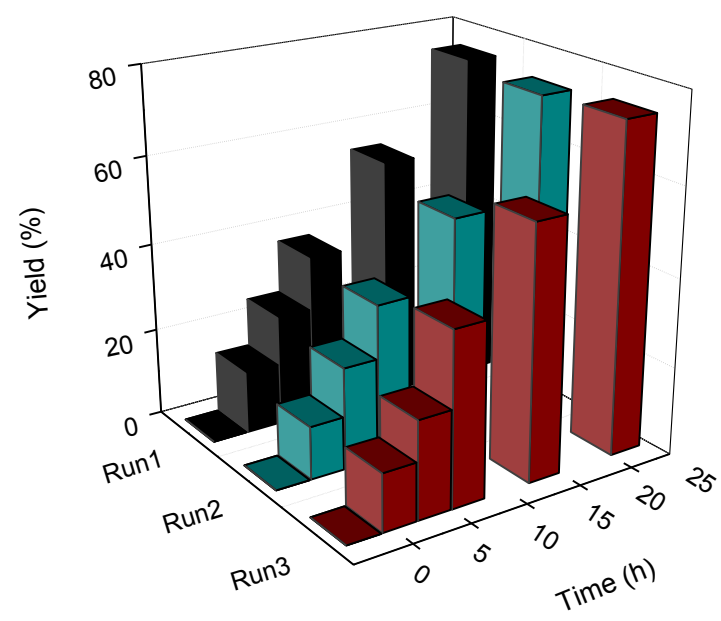

Figure 5.18 Recycling experiment and time-dependent reaction profiles of benzyl alcohol oxidation using 2G-rGO at a catalyst loading of $0.03 \mathrm{~mol} \%$

At this stage, in order to prove that the reaction was heterogeneously catalyzed, we performed a hot filtration experiment. This experiment was carried out using the general conditions described in Table 5.3 using benzyl alcohol as substrate. After 6h (GC yield $48 \%$ ) the catalyst was filtered off at $100^{\circ} \mathrm{C}$. The filtrate was allowed to stir for $16 \mathrm{~h}$ under 
identical conditions, but GC analysis indicated that no further oxidation occurred (GC final yield is maintained in $48 \%$ ). This experiment confirmed the absence of catalytic active species in solution due to ruthenium leaching or desorption of the molecular catalyst by the "boomerang effect". ${ }^{61-63}$ The boomerang effect consists of a release-andreturn of the molecular complex from the support, therefore implying that the catalytic active species may be homogeneous in nature. ${ }^{64}$ This observation contrasts with previously published results regarding the $\pi$-stacking between pyrene and carbon nanotubes, which are affected by the polarity of the solvent and by temperature. ${ }^{33,65,66} \mathrm{In}$ our case, we did not observe desorption of the molecular complexes 1G or $2 \mathbf{G}$ in polar or non-polar solvents such us, dichloromethane, acetone, toluene or 2-propanol.

\subsubsection{Hydrodefluorination of fluoroarenes}

In the last two sections, we proved that, not only the catalytic efficiencies of the molecular complexes $\mathbf{1 G}$ and $\mathbf{2 G}$ were enhanced upon immobilization, but also that the catalysts could be recycled ten times without apparent loss of activity. On the other hand, the combination of these two metals into a single-frame heterodimetallic complex, allowed us to obtain a highly efficient catalyst for the hydrodefluorination (HDF) of a wide set of fluoro-organic molecules, as was described in Chapter 1 (section 2.3.3. Carbon-Fluor bond activation via hydrodefluorination). ${ }^{31}$ Based on these previous findings, we decided to test the hybrid material 5rGO, which contains two molecular complexes of Pd (1G) and Ru (2G), in the HDF of fluoroarenes.

In order to evaluate the activity of the palladium-ruthenium hybrid material 5rGO in HDF, we first tested its activity toward a series of fluoroarenes. For comparative purposes, we also tested the activity of $\mathrm{rGO}$, and the previously described materials PdrGO (1G-rGO) and Ru-rGO (2G-rGO), which contain only one of the two metal complexes grafted onto the solid surface. The reactions were carried out in 2-propanol at $80^{\circ} \mathrm{C}$, during 3 hours in the presence of $\mathrm{NaO} t \mathrm{Bu}$ with a catalyst loading of $1 \mathrm{~mol} \%$, based on the amount of each metal. We noticed that the supported catalysts needed to be activated prior to their use in the catalytic reactions (vide infra). The activation of the catalysts was carried out by refluxing the solid in 2-propanol in the presence of $\mathrm{NaO} t \mathrm{Bu}$, for $24 \mathrm{~h}$. 
The results shown in Table 5.4 indicate that in the HDF of fluorobenzene, rGO and RurGO (2G-rGO) were completely inactive, while the palladium containing solid (1GrGO) produced moderate amounts (50\%) of benzene (compare entries 1, 2 and 3). Interestingly, when the molecular complexes $\mathbf{1 G}$ and $\mathbf{2 G}$ were mixed together and used for the homogeneously catalyzed HDF of fluorobenzene, we did not observe the formation of benzene. This result is rather surprising, especially if we compare it with the result obtained when the heterogeneized catalyst 5rGO was used, which afforded full conversion to benzene (compare entries 4 and 5). This unexpected enhancement in the activity of the immobilized catalyst onto the graphene material has already been observed before for 1G and 2G supported onto reduced graphene oxide (1G-rGO and 2G-rGO), but this new example that we report here is far more striking, because it shows that the catalyst is only active when it is immobilized onto the graphene surface.

Catalyst 5rGO was also very active in the HDF of 4-fluoroaniline and 4fluorobenzotrifluoride (entries 7 and 8), and showed moderate activity for the C-F activation of 4-fluorotoluene, 1,4-difluorobenzene and 1,3,5-trifluorobenzene, although the conversions increased upon increasing the reaction times (entries 6,9 and 10). Despite the fact that the palladium-containing material (1G-rGO) is active in the HDF of fluorobenzene, its activity is much lower than that shown by the Ru-Pd material (5rGO) which afforded better results even using a lower loading of palladium (1G-rGO contains 2 mol\% of palladium, while 5rGO contains 1 mol\% of palladium). This result verifies our initial hypothesis that the synergistic role of the two metals is playing an important effect in the catalytic performance of our hybrid catalyst 5rGO.

In order to test if the activity of the catalysts could be maintained upon catalyst recovery, we performed a second run by recycling the catalyst from the reaction mixture. The activity of the palladium-supported catalyst (1G-rGO) improved with respect to the first cycle (entry 3, first run $50 \%$, and second run $75 \%$ ). This result is probably due to further activation of the catalyst along the reaction course, and may be ascribed to the slow formation of palladium nanoparticles (vide infra). In the case of the bimetallic solid material (5rGO) we also observed that the activity was maintained in the case of the HDF of fluorobenzene (entry 5). For the HDF of the rest of the substrates that we tested, 
we observed a slight enhancement of the catalytic activity, again probably as a consequence of the slow formation of active palladium nanoparticles.

Table 5.4 Hydrodefluorination of fluoroarenes<smiles>Fc1cc[R]cc1</smiles>

\begin{tabular}{|c|c|c|c|c|c|}
\hline Entry & Catalyst & Substrate & Product & $\begin{array}{c}\text { Yield (\%) } \\
1^{\text {st }} \text { run }\end{array}$ & $\begin{array}{c}\text { Yield (\%) } \\
2^{\text {nd }} \text { run }\end{array}$ \\
\hline $1^{\mathrm{a}}$ & $\mathrm{rGO}$ & & & 0 & 0 \\
\hline $2^{a, b}$ & 2G-rGO & & & 0 & 0 \\
\hline $3^{\mathrm{b}}$ & 1G-rGO & & & 51 & 75 \\
\hline $4^{\mathrm{a}}$ & $1 G+2 G$ & & & 3 & -- \\
\hline 5 & 5rGO & & & 100 & 100 \\
\hline 6 & 5rGO & & & 52 & 60 \\
\hline 7 & 5rGO & & & 85 & 95 \\
\hline 8 & 5rGO & & & 63 & 65 \\
\hline $9^{\mathrm{c}, \mathrm{d}}$ & 5rGO & & & 50 & $50(90)$ \\
\hline $10^{\mathrm{d}, \mathrm{e}}$ & 5rGO & & & 43 & $45(63)$ \\
\hline
\end{tabular}

Reactions were carried out with $0.3 \mathrm{mmol}$ of fluoroarene, $\mathrm{NaO} t \mathrm{Bu}(0.3 \mathrm{mmol})$, preactivated catalyst $(1 \mathrm{~mol} \%$ base on each metal), $2 \mathrm{~mL}$ of 2-propanol for 3 hours at $80^{\circ} \mathrm{C}$. Yields determined by $\mathrm{GC}$ analyses using anisole as internal standard. [a] GC Yields after 20h. [b] catalyst loading 2 mol\%. [c] 2 Equivalents of $\mathrm{NaO} t \mathrm{Bu}(0.6$ mmol). [d] Yields in parenthesis are after 6 hours. [e] 3 Equivalents of $\mathrm{NaO} t \mathrm{Bu}(0.9 \mathrm{mmol})$.

As already mentioned, the supported catalysts needed to be activated prior to their use in the catalytic reactions. We were interested in observing the activation process of the 
catalyst, and thus we performed an analysis of the reaction profiles of the HDF of fluorobenzene using non-activated catalyst 5rGO. The time-course reaction profiles are shown in Figure 5.19, and are displayed for eight successive cycles. The reactions were monitored by ${ }^{19}$ F NMR spectroscopy. As can be seen from the profiles shown in Figure 5.19, the curve corresponding to the first run has a sigmoidal form, indicating that the rate of formation of benzene is slowly increased with the reaction time, as a consequence of the gradual activation of the catalyst. Run 2 needs 12 hours to completion, and runs 3 to 8 display quasi-identical profiles, achieving completion in about 3 hours. The similarities of the reaction profiles displayed for runs 3-8 are a very good indication that the catalyst deactivation is negligible.

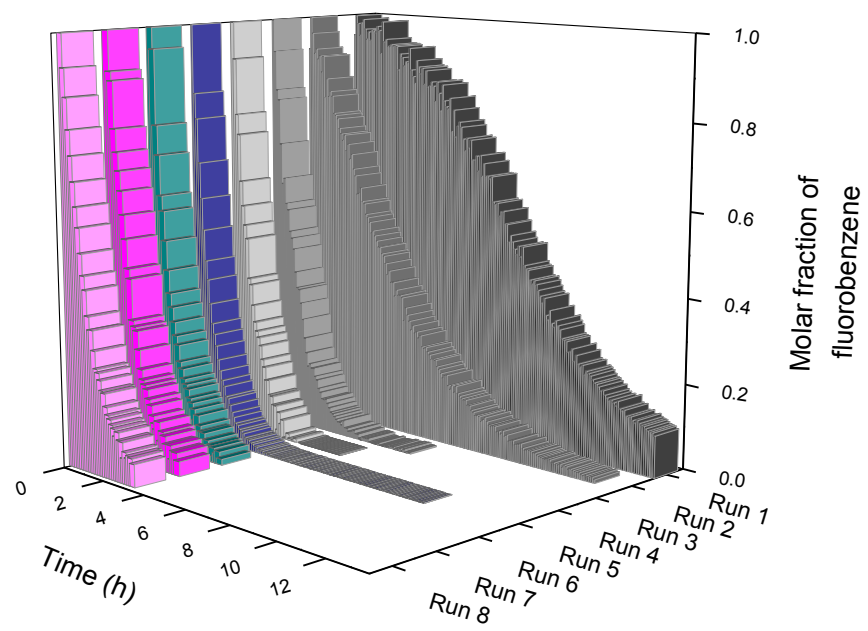

Figure 5.19 Reaction profiles for eight successive cycles of the HDF of fluorobenzene with 5rGO. The reactions were carried out in 2-propanol in the presence of $\mathrm{NaO} t \mathrm{Bu}$, at $80^{\circ} \mathrm{C}$. After each run, the catalyst was filtered, washed with dichloromethane and dried. The monitoring of the reactions was carried out by ${ }^{19} \mathrm{~F}$ NMR spectroscopy

We were also interested in determining if we could recycle catalyst 5rGO for several catalytic cycles without loss of its catalytic activity. The recycling experiments were performed using fluorobenzene as model substrate. The catalyst was activated in 2propanol in the presence of $\mathrm{NaO} t \mathrm{Bu}$, for $24 \mathrm{~h}$. The reactions were carried out in 2propanol at $80^{\circ} \mathrm{C}$, during 3 hours in the presence of $\mathrm{NaO} t \mathrm{Bu}$ with a catalyst loading of 2 
mol\%, based on the amount of metal ( $1 \mathrm{~mol} \%$ of $\mathrm{Ru}$, and $1 \mathrm{~mol} \%$ of $\mathrm{Pd})$. The yields were determined by GC analyses using anisole as internal standard. After completion of each run (3 hours), the reaction mixture was allowed to reach room temperature, and the solid catalyst was separated by centrifugation, washed with dichloromethane, dried and reused in the subsequent cycle. Each cycle required the addition of one equivalent of $\mathrm{NaO} t \mathrm{Bu}$ with respect to fluorobenzene. By following this procedure, catalyst 5rGO could be recycled for up to 12 runs, without any observable decrease of activity (Figure 5.20). Only during the $13^{\text {th }}$ run, we observed a decrease of the activity to $85 \%$, but we believe that this result may be due to the accumulated loss of catalyst during the successive filtrations.

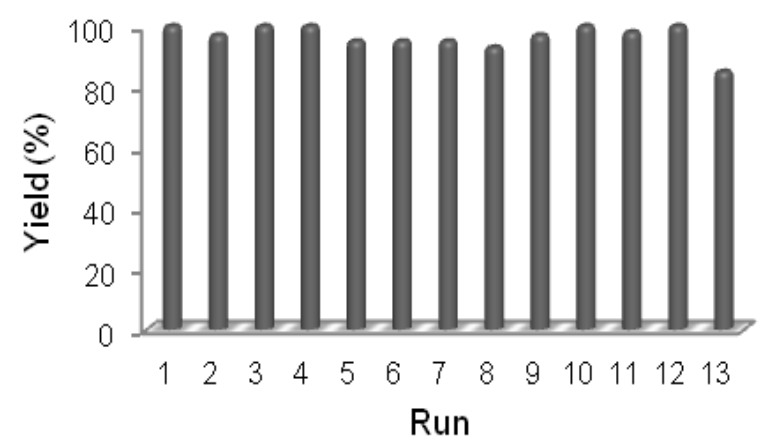

Figure 5.20 Recycling experiments of hydrodefluorination of fluorobenzene in the presence of 5rGO. The reactions were carried out in 2-propanol in the presence of $\mathrm{NaO} t \mathrm{Bu}$, at $80^{\circ} \mathrm{C}$. After each run, the catalyst was filtered, washed with dichloromethane and dried. Run number 13 was carried out under air and using technical grade 2-propanol

The ICP-MS analysis after the first run accounted for $4.87 \mathrm{wt} \%$ of ruthenium and 4.99 wt $\%$ of palladium, indicating a negligible loss of metals. When the ICP-MS analysis was carried out after the final 13 runs, we did observe that the total amount of metals found was $3.94 \mathrm{wt} \%$ for ruthenium and $3.85 \mathrm{wt} \%$ for palladium, indicating an approximate $20 \%$ loss of palladium and ruthenium respect to the initial metal content. However, this loss of percent of metal content may be due to the mass gain during the subsequent cycles due to the adsorption of substances from the catalyst reactions (we believe that a mass gain due to $\mathrm{NaO} t \mathrm{Bu}$ adsorption may be produced), so it may not be ascribed to desorption or leaching of the metal complex. 
In order to determine the nature of the active catalytic species, we performed HRTEM analyses of 1G-rGO, 2G-rGO and 5 rGO after being activated at $80^{\circ} \mathrm{C}$ with $\mathrm{NaO} t \mathrm{Bu}$ in 2-propanol for 24h. Figure 5.21 (top) shows the HRTEM images of 5rGO after being activated and it shows the formation of well-dispersed small nanoparticles $(<5 \mathrm{~nm}$ diameter). The analysis by Energy-Dispersive X-ray spectroscopy (EDS) performed by means of HRTEM of 5rGO, indicated the homogeneously distributed presence of ruthenium and palladium in the areas where the nanoparticles were present (Figure 5.21, bottom), so the question remains about what is the nature of the nanoparticles formed along the reaction course.
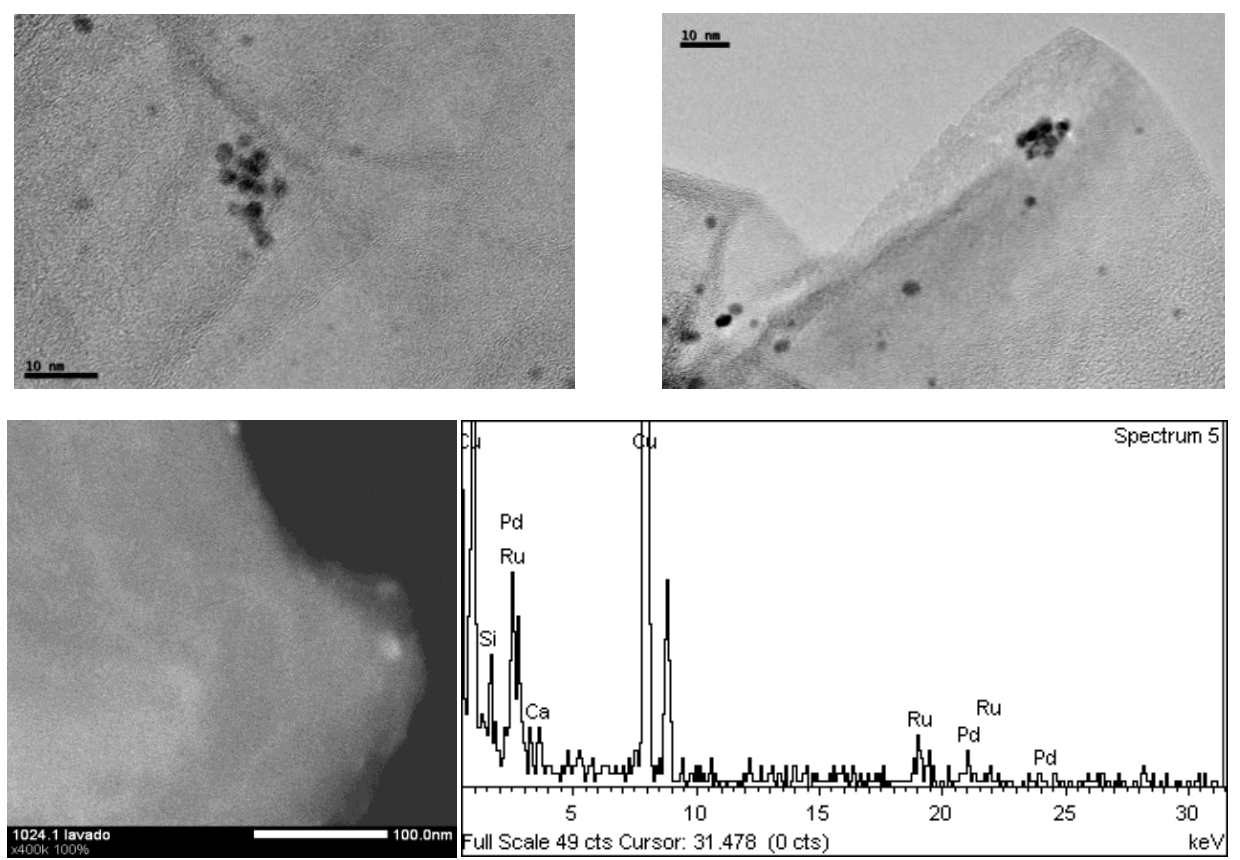

Figure 5.21 HRTEM (top), STEM images and EDS spectrum (bottom) of 5rGO after being activated at $80^{\circ} \mathrm{C}$ with $\mathrm{NaOt} \mathrm{Bu}$ in 2-propanol for $24 \mathrm{~h}$. Scale bar: $10 \mathrm{~nm}$ (top). $100 \mathrm{~nm}$ (bottom)

In order to clarify this point, we performed separate experiments in which the palladium and ruthenium containing solids, 1G-rGO and 2G-rGO were heated in 2-propanol in the presence of $\mathrm{NaO} t \mathrm{Bu}$ for $24 \mathrm{~h}$. The HRTEM analyses for 1G-rGO after being activated showed the formation of small nanoparticles $(<5 \mathrm{~nm}$ diameter) (Figure 5.22, top). The 
analysis by Energy-Dispersive X-ray spectroscopy (EDS) performed by means of HRTEM of 1G-rGO, indicated the homogeneously distributed presence of palladium in the areas where the nanoparticles were present (Figure 5.22, bottom).
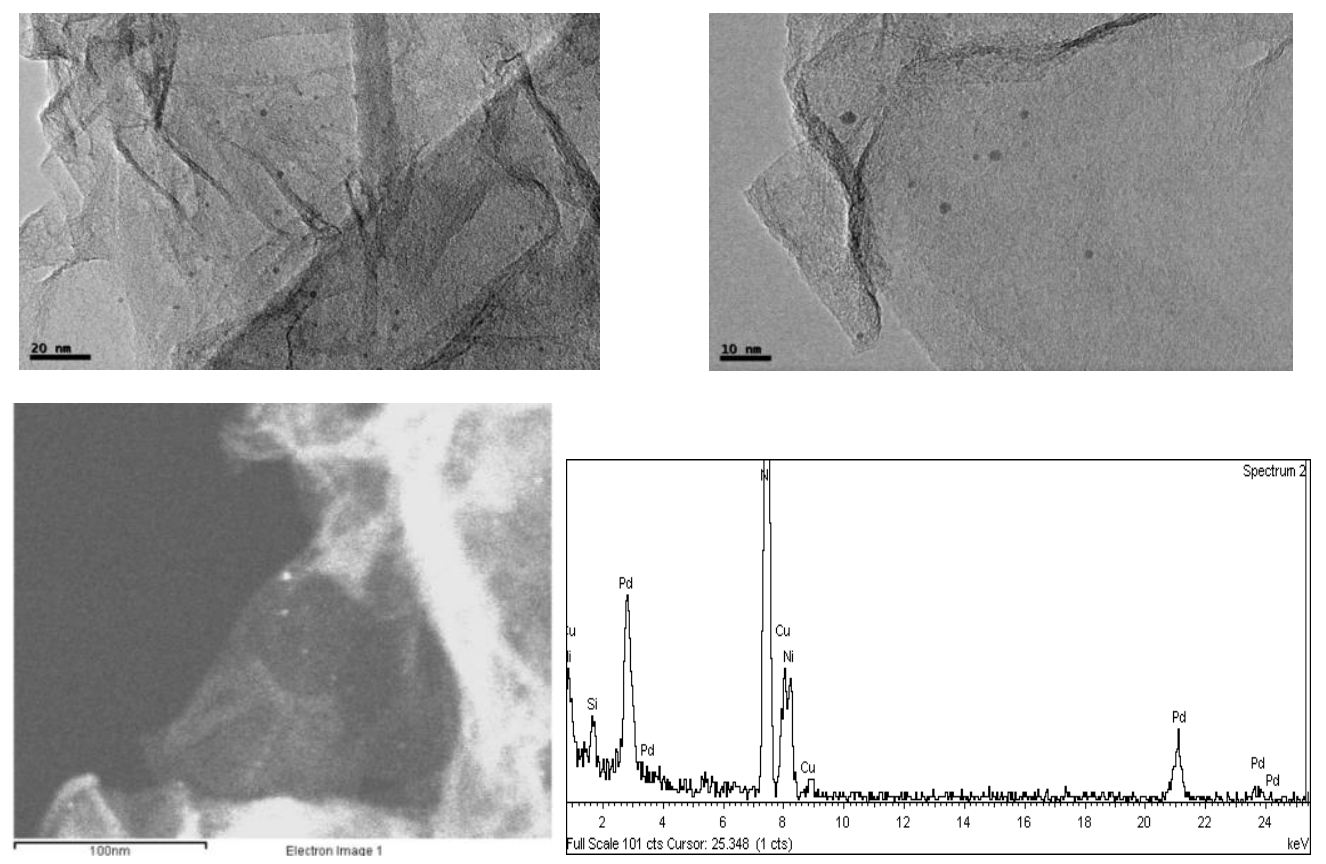

Figure 5.22 HRTEM (top), STEM images and EDS spectrum (bottom) of 1G-rGO after being activated at $80^{\circ} \mathrm{C}$ with $\mathrm{NaO} t \mathrm{Bu}$ in 2-propanol for $24 \mathrm{~h}$. Scale bar: $10 \mathrm{~nm}$ (top), $100 \mathrm{~nm}$ (bottom)

The HRTEM analyses for 2G-rGO, after being activated, did not show visible formation of nanoparticles (Figure 5.23, top). The elemental mapping by energy-dispersive X-ray spectroscopic analysis (EDS) performed by means of HRTEM of 2G-rGO, confirmed the homogeneous distribution of ruthenium in the activated material (Figure 5.23, bottom). 

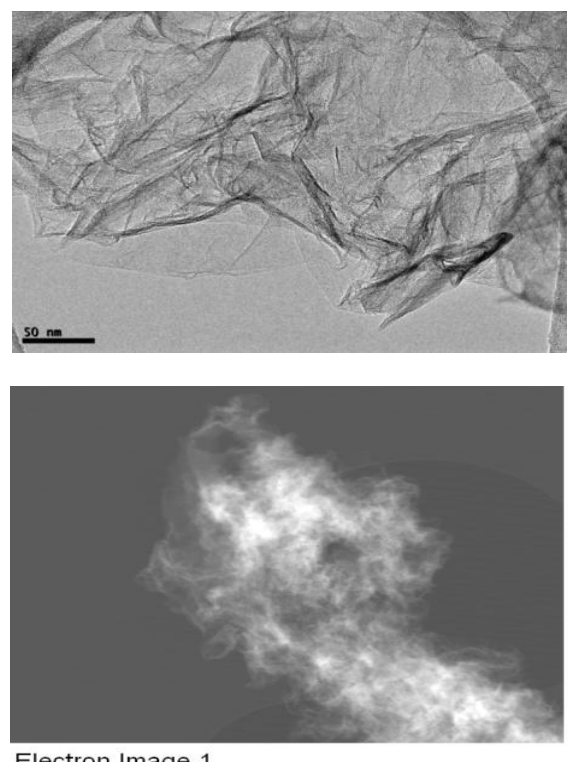

Electron Image 1
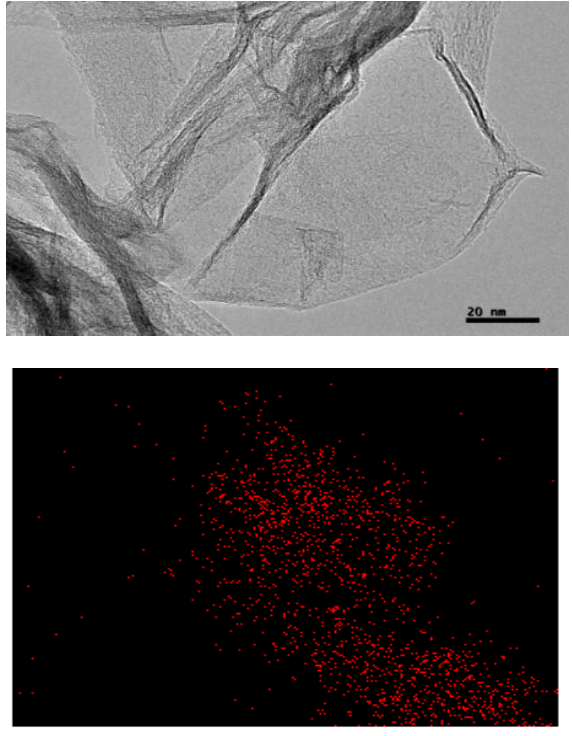

Ru Ka1

Figure 5.23 HRTEM (top), STEM and EDS elemental mapping (bottom) images of 2G-rGO after being activated at $80^{\circ} \mathrm{C}$ with $\mathrm{NaO} t \mathrm{Bu}$ in 2-propanol for $24 \mathrm{~h}$

The HRTEM analyses of 1G-rGO, 2G-rGO and 5rGO after being activated, suggest that the small nanoparticles should be mainly formed by palladium, which has a higher tendency to aggregate than ruthenium under the reaction conditions used. This finding should explain the differences found in the catalytic activity of the solid material 5rGO, compared to the inactive catalytic system formed by the mixture of the two homogeneous catalysts $\mathbf{1 G}$ and $\mathbf{2 G}$. Also, the mechanism for this process is different to the mechanism for our previously described homogeneous $\mathrm{Ru}-\mathrm{Pd}$ catalyst. In this case, we believe that the C-F activation should be promoted by the palladium nanoparticles, while the introduction of the hydrogen by transfer hydrogenation should be facilitated by the ruthenium heterogeneized complex, and therefore the synergistic action between the two metals is crucial for explaining the superior activity of the catalyst.

The Atomic Force Microscopy (AFM) analyses of 5rGO before and after its activation, revealed that the height of the rGO sheets is of about 2 and $1.5 \mathrm{~nm}$, respectively, both within the accepted range of few-layer graphenes (see Experimental Section). ${ }^{37}$ Interestingly, the BET surface area of $\mathbf{5 r G O}$ is 164 and $221 \mathrm{~m}^{2} / \mathrm{g}$ for the unactivated and 
activated materials, respectively. These data are in agreement with the data coming from the AFM analysis, which suggests a partial exfoliation of the catalyst upon activation. 


\section{CONCLUSIONS}

The preparation of molecular complexes containing an N-heterocyclic carbene ligand with a pyrene-tag was described. The molecular complexes were anchored on the surface of rGO by non-covalent interactions under mild reaction conditions affording the hybrid materials of type M-rGO and M-M'-rGO. This modular methodology allows incorporation of well-defined molecular complexes onto chemical derived graphenes (CDGs) where the properties of the complex are not altered by the effect of the immobilization.

The catalytic properties of the molecular complexes and hybrid materials were studied in the hydrogenation of alkenes, alcohol oxidation, and hydrodefluorination. We proved that, not only the catalytic efficiencies of the catalysts were enhanced upon immobilization, but also that the catalysts could be recycled several times without apparent loss of activity, even in larger scale experiments, with catalysts loadings of 0.01 and $0.03 \mathrm{~mol} \%$.

We strongly believe that the simplicity of the design of these heterogeneous catalytic systems, in combination with its great catalytic performance and recyclability, may inspire future researches in the field of catalyst heterogeneization. To the best of our knowledge, the three examples that we described in this chapter, constitute the first evidences of supported NHC-molecular catalysts onto a graphene derivative, and we demonstrated its great potential practical applications. 


\section{REFERENCES}

(1) Corma, A.; Garcia, H. Adv. Synth. Catal. 2006, 348, 1391-1412.

(2) Cole-Hamilton, D. J. Science 2003, 299, 1702-1706.

(3) Wegener, S. L.; Marks, T. J.; Stair, P. C. Accounts Chem. Res. 2012, 45, 206214.

(4) Jones, C. W. Top. Catal. 2010, 53, 942-952.

(5) Wang, Z.; Chen, G.; Ding, K. L. Chem. Rev. 2009, 109, 322-359.

(6) Gladysz, J. A. Chem. Rev. 2002, 102, 3215-3216.

(7) Fraile, J. M.; Garcia, J. I.; Mayoral, J. A. Chem. Rev. 2009, 109, 360-417.

(8) Coperet, C.; Chabanas, M.; Saint-Arroman, R. P.; Basset, J. M. Angew. Chem. Int. Edit. 2003, 42, 156-181.

(9) Bond, G. C. Chem. Soc. Rev. 1991, 20, 441-475.

(10) Edwards, R. S.; Coleman, K. S. Accounts Chem. Res. 2013, 46, 23-30.

(11) Castro Neto, A. H.; Guinea, F.; Peres, N. M. R.; Novoselov, K. S.; Geim, A. K. Rev. Mod. Phys. 2009, 81, 109-162.

(12) Machado, B. F.; Serp, P. Catal. Sci. Technol. 2012, 2, 54-75.

(13) Pyun, J. Angew. Chem. Int. Edit. 2011, 50, 46-48.

(14) Su, C. L.; Loh, K. P. Accounts Chem. Res. 2013, 46, 2275-2285.

(15) Luo, B.; Liu, S. M.; Zhi, L. J. Small 2012, 8, 630-646.

(16) Eda, G.; Chhowalla, M. Adv. Mater. 2010, 22, 2392-2415.

(17) Podeszwa, R. J. Chem. Phys. 2010, 132.

(18) Pan, B.; Xing, B. S. Environ. Sci. Technol. 2008, 42, 9005-9013.

(19) Chen, J. Y.; Chen, W.; Zhu, D. Environ. Sci. Technol. 2008, 42, 7225-7230.

(20) Muller, S.; Totsche, K. U.; Kogel-Knabner, I. Eur. J. Soil Sci. 2007, 58, 918-931.

(21) Balapanuru, J.; Yang, J.-X.; Xiao, S.; Bao, Q.; Jahan, M.; Polavarapu, L.; Wei, J.; Xu, Q.-H.; Loh, K. P. Angew. Chem. Int. Edit. 2010, 49, 6549-6553.

(22) Sun, Y. B.; Yang, S. B.; Zhao, G. X.; Wang, Q.; Wang, X. K. Chem.-Asian J. 2013, 8, 2755-2761.

(23) Yang, K.; Wang, X. L.; Zhu, L. Z.; Xing, B. S. Environ. Sci. Technol. 2006, 40, 5804-5810. 
(24) Chefetz, B.; Deshmukh, A. P.; Hatcher, P. G.; Guthrie, E. A. Environ. Sci. Technol. 2000, 34, 2925-2930.

(25) Mao, X.; Su, H.; Tian, D.; Li, H.; Yang, R. ACS Appl. Mater. Interfaces 2013, 5, $592-597$.

(26) Georgakilas, V.; Otyepka, M.; Bourlinos, A. B.; Chandra, V.; Kim, N.; Kemp, K. C.; Hobza, P.; Zboril, R.; Kim, K. S. Chem. Rev. 2012, 112, 6156-6214.

(27) Rodriguez-Perez, L.; Herranz, M. A.; Martin, N. Chem. Commun. 2013, 49, 3721-3735.

(28) Herrmann, W. A.; Elison, M.; Fischer, J.; Kocher, C.; Artus, G. R. J. Angew. Chem. Int. Edit. 1995, 34, 2371-2374.

(29) Bourissou, D.; Guerret, O.; Gabbai, F. P.; Bertrand, G. Chem. Rev. 2000, 100, $39-91$.

(30) Diez-Gonzalez, S.; Marion, N.; Nolan, S. P. Chem. Rev. 2009, 109, 3612-3676.

(31) Sabater, S.; Mata, J. A.; Peris, E. Nat. Commun. 2013, 4.

(32) Janiak, C. J. Chem. Soc.-Dalton Trans. 2000, 3885-3896.

(33) Wittmann, S.; Schaetz, A.; Grass, R. N.; Stark, W. J.; Reiser, O. Angew. Chem. Int. Edit. 2010, 49, 1867-1870.

(34) Herranz, M. A.; Ehli, C.; Campidelli, S.; Gutierrez, M.; Hug, G. L.; Ohkubo, K.; Fukuzumi, S.; Prato, M.; Martin, N.; Guldi, D. M. J. Am. Chem. Soc. 2008, 130, 66-73.

(35) Ehli, C.; Guldi, D. M.; Herranz, M. A.; Martin, N.; Campidelli, S.; Prato, M. J. Mater. Chem. 2008, 18, 1498-1503.

(36) Kavakka, J. S.; Heikkinen, S.; Kilpelainen, I.; Mattila, M.; Lipsanen, H.; Helaja, J. Chem. Commun. 2007, 519-521.

(37) Geim, A. K.; Novoselov, K. S. Nat. Mater. 2007, 6, 183-191.

(38) Clapham, S. E.; Hadzovic, A.; Morris, R. H. Coord. Chem. Rev. 2004, 248, 22012237.

(39) Cui, X. H.; Burgess, K. Chem. Rev. 2005, 105, 3272-3296.

(40) Bertoli, M.; Choualeb, A.; Lough, A. J.; Moore, B.; Spasyuk, D.; Gusev, D. G. Organometallics 2011, 30, 3479-3482.

(41) Nielsen, M.; Kammer, A.; Cozzula, D.; Junge, H.; Gladiali, S.; Beller, M. Angew. Chem. Int. Edit. 2011, 50, 9593-9597. 
(42) Dreyer, D. R.; Jia, H.-P.; Bielawski, C. W. Angew. Chem. Int. Edit. 2010, 49, 6813-6816.

(43) Huang, C.; Li, C.; Shi, G. Energy Environ. Sci. 2012, 5, 8848-8868.

(44) Gao, Y.; Ma, D.; Wang, C.; Guan, J.; Bao, X. Chem. Commun. 2011, 47, 24322434.

(45) Zhao, Q.; Chen, D.; Li, Y.; Zhang, G.; Zhang, F.; Fan, X. Nanoscale 2013, 5, 882-885.

(46) Crabtree, R. H. Chem. Rev. 2012, 112, 1536-54.

(47) Shang, N.; Gao, S.; Feng, C.; Zhang, H.; Wang, C.; Wang, Z. RSC Adv. 2013, 3, 21863-21868.

(48) Movahed, S. K.; Esmatpoursalmani, R.; Bazgir, A. RSC Adv. 2014, 4, 1458614591.

(49) Blaser, H.-U. Science 2006, 313, 312-313.

(50) Siegrist, U.; Baumeister, P.; Blaser, H. U.; Studer, M. The selective hydrogenation of functionalized nitroarenes: New catalytic systems, 1998; Vol. 75 .

(51) Zaera, F. Prog. Surf. Sci. 2001, 69, 1-98.

(52) Lee, T. R.; Whitesides, G. M. Accounts Chem. Res. 1992, 25, 266-272.

(53) Kung, H. H.; Pellet, R. J.; Burwell, R. L. J. Am. Chem. Soc. 1976, 98, 5603-5611.

(54) Pomogailo, A. D. Catalysis by Polymer-Immobilized Metal Complexes; Gordon and Breach Science Publishers: Amsterdam, 1998.

(55) Adair, G. R. A.; Williams, J. M. J. Tetrahedron Lett. 2005, 46, 8233-8235.

(56) Blum, Y.; Czarkie, D.; Rahamim, Y.; Shvo, Y. Organometallics 1985, 4, 14591461.

(57) Shvo, Y.; Czarkie, D.; Rahamim, Y.; Chodosh, D. F. J. Am. Chem. Soc. 1986, 108, 7400-7402.

(58) Spasyuk, D.; Gusev, D. G. Organometallics 2012, 31, 5239-5242.

(59) Hollmann, D.; Baehn, S.; Tillack, A.; Beller, M. Angew. Chem. Int. Edit. 2007, 46, 8291-8294.

(60) Prades, A.; Peris, E.; Albrecht, M. Organometallics 2011, 30, 1162-1167.

(61) Clavier, H.; Caijo, F.; Borre, E.; Rix, D.; Boeda, F.; Nolan, S. P.; Mauduit, M. Eur. J. Org. Chem. 2009, 4254-4265. 
(62) Clavier, H.; Nolan, S. P.; Mauduit, M. Organometallics 2008, 27, 2287-2292.

(63) Kingsbury, J. S.; Harrity, J. P. A.; Bonitatebus, P. J.; Hoveyda, A. H. J. Am. Chem. Soc. 1999, 121, 791-799.

(64) Vriamont, C.; Devillers, M.; Riant, O.; Hermans, S. Chem. Eur. J. 2013, 19, 12009-12017.

(65) Liu, G.; Wu, B.; Zhang, J.; Wang, X.; Shao, M.; Wang, J. Inorg. Chem. 2009, 48, 2383-2390.

(66) Schaetz, A.; Reiser, O.; Stark, W. J. Chem. Eur. J. 2010, 16, 8950-8967. 

CHAPTER 6

\section{EXPERIMENTAL SECTION}

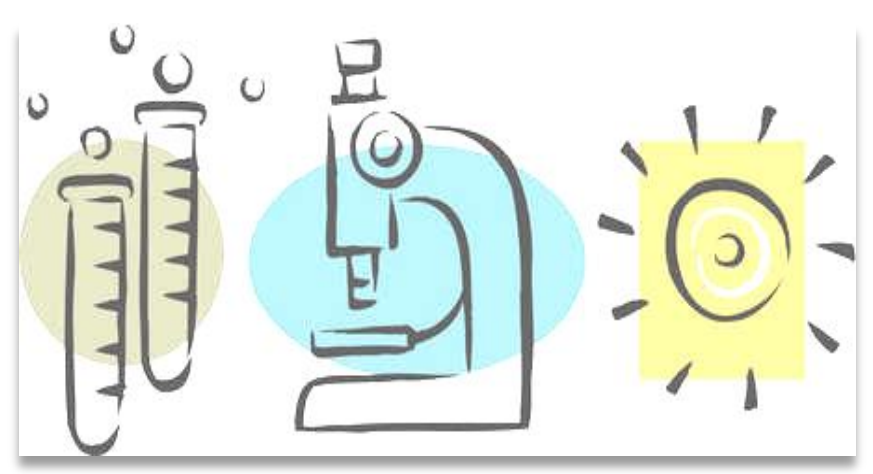





\section{ANALYTICAL TECHNIQUES}

\section{Nuclear Magnetic Resonance (NMR)}

${ }^{1} \mathrm{H},{ }^{1} \mathrm{H}-{ }^{1} \mathrm{H}$ gNOESY, ${ }^{13} \mathrm{C},{ }^{1} \mathrm{H}-{ }^{13} \mathrm{C}$ gHSQC and ${ }^{1} \mathrm{H}-{ }^{13} \mathrm{C}$ gHMBC NMR spectra were recorded on the following spectrometers at $298 \mathrm{~K}$ :

- Varian Innova $300 \mathrm{MHz}\left({ }^{1} \mathrm{H} 300 \mathrm{MHz},{ }^{13} \mathrm{C} 75 \mathrm{MHz}\right)$

- Varian Innova $400 \mathrm{MHz}\left({ }^{1} \mathrm{H} 400 \mathrm{MHz},{ }^{13} \mathrm{C} 100 \mathrm{MHz}\right)$

- Varian Innova $500 \mathrm{MHz}\left({ }^{1} \mathrm{H} 500 \mathrm{MHz},{ }^{13} \mathrm{C} 125 \mathrm{MHz}\right)$

Chemical shifts are given in ppm $(\delta)$, referred to the residual peak of the deuterated solvent $\left(\mathrm{CDCl}_{3}, \mathrm{CD}_{2} \mathrm{Cl}_{2}, \mathrm{CD}_{3} \mathrm{CN}, \mathrm{CD}_{3} \mathrm{OD}\right.$ and DMSO- $\left.d_{6}\right)$ and reported downfield of $\mathrm{SiMe}_{4}$.

\section{Electrospray Mass Spectra (ESI-MS) and High Resolution Mass Spectroscopy (HRMS)}

Electrospray Mass Spectra (ESI-MS) were recorded on a Micromass Quatro LC instrument; $\mathrm{CH}_{3} \mathrm{OH}$ or $\mathrm{CH}_{3} \mathrm{CN}$ were used as mobile phase and nitrogen was employed as drying and nebulizing gas. High Resolution Mass Spectra (HRMS) were recorded on a QTOF I (quadrupole-hexapole-TOF) mass spectrometer with an orthogonal Z-sprayelectrospray interface (Micromass, Manchester, UK). The drying gas as well as nebulizing gas was nitrogen at a flow of $400 \mathrm{~L} / \mathrm{h}$ and $80 \mathrm{~L} / \mathrm{h}$ respectively. The temperature of the source block was set to $120^{\circ} \mathrm{C}$ and the desolvation temperature to $150^{\circ} \mathrm{C}$. A capillary voltage of $3.5 \mathrm{kV}$ was used in the positive scan mode and the cone voltage was set to $30 \mathrm{~V}$.

\section{Elemental Analysis (EA)}

Elemental analyses were carried out on a EuroEA3000 Eurovector Analyzer.

\section{Gas Chromatography: GC and GC/MS}

GC analyses were obtained on a Shimadzu GC-2010 apparatus equipped with a FID and a Technokroma (TRB-5MS, $30 \mathrm{~m} \times 0.25 \mathrm{~mm} \times 0.25 \mu \mathrm{m}$ ) column and on a Shimadzu 
GCMS-QP2010 apparatus equipped with a Technokroma (TRB-5MS, $30 \mathrm{~m}$ x $0.25 \mathrm{~mm}$ x $0.25 \mu \mathrm{m}$ ) column were used.

\section{High Performance Liquid Chromatography (HPLC)}

High Performance Liquid Chromatography (HPLC) analyses were carried out in a Merck HITACHI LaChrom chromatograph UV detector at $250 \mathrm{~nm}$ using a using a Daicel CHIRALPACK IC column $(25 \mathrm{~cm} \times 4.6 \mathrm{~mm}$ I.D. $)$ at $30^{\circ} \mathrm{C}$ with hexane/2-propanol $=$ $70 / 30$, flow $=0.6 \mathrm{~mL} / \mathrm{min}, \lambda=250 \mathrm{~nm}$.

\section{Infrared Spectroscopy (IR)}

Infrared spectra (FTIR) were performed on a JASCO FT/IR-6200spectrometer with a spectral window of $4000-500 \mathrm{~cm}^{-1}$. The samples were prepared as $\mathrm{KBr}$ disks.

\section{Circular Dichroism (CD)}

Circular Dichroism (CD) measurements were recorded on a JASCO J-810 spectropolarimeter in chloroform solutions.

\section{UV/Vis Spectroscopy}

UV/Vis spectra were recorded between 250 and $600 \mathrm{~nm}$ by a Cary 300 Bio UV-Vis Varian spectrophotometer. The samples were suspended in DMF and sonicated for 5 minutes before the measurements. Molecular complexes were dissolved in DMF $\left(10^{-6} \mathrm{M}\right)$.

\section{Raman Spectroscopy}

Raman spectra were acquired on a JASCO NRS-3100 dispersive spectrometer under the following conditions: $632.80 \mathrm{~nm}$ laser with a single monochromator, grating 600 lines $\mathrm{mm}^{-1}$, slit $0.1 \times 6 \mathrm{~mm}$, resolution $9.36 \mathrm{~cm}^{-1}$, with a center wavenumber of $2000.03 \mathrm{~cm}^{-1}$, a laser power of $80.1 \mathrm{~mW}$, and 10 accumulations of $20 \mathrm{~s}$ each.

\section{Scanning Electron Microscopy (SEM)}

Scanning Electron Micrographs of the samples were taken with a field emission gun scanning electron microscope (FEG-SEM) model JEOL 7001F, equipped with a 
spectrometer of energy dispersion of X-ray (EDX) from Oxford instruments. The samples for microstructural and microanalysis determinations were covered with a Pt film.

\section{High Resolution Transmission Electron Microscopy (HRTEM)}

High-Resolution images of Transmission Electron Microscopy HRTEM and High-Angle Annular Dark-Field HAADF-STEM images of the samples were obtained using a Jem2100 LaB6 (JEOL) transmission electron microscope coupled with an INCA Energy TEM 200 (Oxford) Energy Dispersive X-ray spectrometer (EDX) operating at $200 \mathrm{kV}$. Samples were prepared by drying a droplet of a methanolic dispersion on a carbon-coated copper grid.

\section{Brunauer-Emmett-Teller (BET) Surface Area Analysis}

Argon adsorption-desorption isotherms were collected on a Micromeritics Gemini V gas adsorption analyzer at $77 \mathrm{~K}$, after degassing the samples overnight at $100^{\circ} \mathrm{C}$ in a Micromeritics Flow prep 060 system with argon flux gas. The BET surface areas were calculated from the adsorption branch of the isotherm by the BJH method.

\section{Atomic Force Microscopy (AFM) Images}

Atomic Force Microscopy measurements (AFM) were carried out with a JSPM-5200 JEOL Scanning Probe Microscope using a contact mode. The solid samples were dispersed in methanol, deposited on mica by drop casting and dried at room temperature.

\section{Electrochemical Measurements}

The measurements were carried out using a GPES equipped PGSTAT-30 potentiostat from Autolab at room temperature. A three-electrode configuration was used, where two Pt microelectrodes were connected to the working electrode and counter electrode and a $\mathrm{Ag}$ wire was used as the pseudo-reference electrode. Redox potential of $\mathrm{Fe}\left(\mathrm{C}_{2} \mathrm{H}_{5}\right)_{2}{ }^{+} / \mathrm{Fe}\left(\mathrm{C}_{2} \mathrm{H}_{5}\right)_{2}(445 \mathrm{mV})$ was used to calibrate the potential scale. Measurements were performed on $10 \mathrm{mM}$ analyte in dichloromethane with $0.1 \mathrm{mM}\left[\mathrm{NBu}_{4}\right] \mathrm{PF}_{6}$ as supporting electrolyte, at a $100 \mathrm{mV} \mathrm{s}^{-1}$ scan rate. 


\section{SYNTHESIS AND CHARACTERIZATION}

All manipulations were carried out by using standard Schlenk techniques under nitrogen atmosphere unless otherwise stated. Anhydrous solvents were dried using a solvent purification system (SPS M BRAUN) or purchased from Aldrich and degassed prior to use by purging with dry nitrogen and kept over molecular sieves. All other reagents were used as received from commercial suppliers. Metal precursors: $\left[\mathrm{RuCl}_{2}(p \text {-cymene })\right]_{2}{ }^{1}$ $\left[\mathrm{IrCp} * \mathrm{Cl}_{2}\right]_{2},{ }^{2} \quad\left[\mathrm{AuCl}\left(\mathrm{SMe}_{2}\right)\right],{ }^{3}$ palladacycles: $[\mathrm{Pd}(\mathrm{OAc})(\mathrm{dmba})]_{2},{ }^{4}$ and $e^{5}$ ligand precursors: 1,2,4-trimethyltriazolium tetrafluoroborate $\left.\mathbf{( A H}_{2}\right){ }^{6}$ 2-ethyl-1,4-dimethyl triazolium tetrafluoroborate $\left(\mathbf{B H}_{2}\right){ }^{6}{ }^{6}$ 1,3-dibuthylimidazolium iodide $(\mathbf{C}){ }^{7} \quad$ 1,3dimethylimidazolium iodide (D), ${ }^{7}$ 1-tert-buthyl-3-methylimidazolium iodide (E), ${ }^{8}$ 1-(9methylantracene)-3-methylimidazolium chloride (F), ${ }^{9}$ and ethyl-1-(2-pyridyl)-1H-1,2,3triazole-4-carboxylate $(\mathbf{h}),{ }^{10}$ and organometallic complexes $\mathbf{1 A H},{ }^{11} \mathbf{6 A},{ }^{11} \mathbf{7 A},{ }^{\mathbf{1 2}} \mathbf{1 C},{ }^{13}$ $\mathbf{2 C},{ }^{14}$ and $\mathbf{5 D}{ }^{15}$ were prepared according to literature procedures. Graphitic Oxide (GO) was prepared from graphite powder (natural, universal grade, 200 mesh, 99.9995\%) by the Hummers' method ${ }^{16}$ and reduced graphene oxide (rGO) was prepared by reduction of a colloidal suspension of exfoliated GO sheets in water with hydrazine hydrate. ${ }^{17}$

\subsection{Synthesis and characterization of azolium salts}

\section{Synthesis of G}

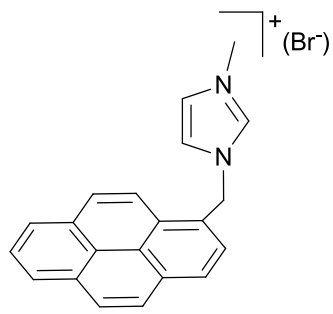

1-(bromomethyl)pyrene (1 g, $3.39 \mathrm{mmol})$ and 1-methylimidazole $(0.28 \mathrm{ml}, 3.39 \mathrm{mmol})$ were refluxed under nitrogen in dry tetrahydrofuran $(10 \mathrm{~mL})$ for 16 hours. The resulting suspension was filtered yielding a white precipitate which was washed with diethyl ether (2x10 mL). Yield: $1.2 \mathrm{~g}, 96 \%$. ${ }^{1} \mathrm{H} \mathrm{NMR}(300 \mathrm{MHz}$, DMSO): $\delta 9.21(\mathrm{~s}, 1 \mathrm{H}, \mathrm{NCHN}), 8.51-8.08\left(\mathrm{~m}, 9 \mathrm{H}, \mathrm{CH}_{\text {pyrene }}\right)$, $7.87\left(\mathrm{~d},{ }^{3} \mathrm{~J}_{\mathrm{H}, \mathrm{H}}=1.7 \mathrm{~Hz}, 1 \mathrm{H}, \mathrm{C} H_{\text {imidazole }}\right), 7.73\left(\mathrm{~d},{ }^{3} \mathrm{~J}_{\mathrm{H}, \mathrm{H}}=1.7 \mathrm{~Hz}\right.$, $1 \mathrm{H}, \mathrm{CH}_{\text {imidazole }}$ ), 6.23 (s, 2H, $\left.\mathrm{NCH}_{2^{-}}\right), 3.82\left(\mathrm{~s}, 3 \mathrm{H}, \mathrm{NCH} \mathrm{H}_{3}\right) \cdot{ }^{13} \mathrm{C}\left\{{ }^{1} \mathrm{H}\right\} \mathrm{NMR}(75 \mathrm{MHz}$, DMSO): $\delta$ 137.1(NCHN), [131.9, 131.1, 130.6, 129.2, 129.1, 128.6, 128.4, 127.8, 127.7, $127.1,126.4,126.2,125.6,124.5,124.3,124.1,123.1,122.8]\left(C_{\text {pyrene, }} C \mathrm{H}_{\text {imidazole }}\right), 50.4$ $\left(\mathrm{NCH}_{2}-\right)$, $36.3\left(\mathrm{NCH}_{3}\right)$. Anal. Calcd. for $\mathrm{C}_{21} \mathrm{H}_{17} \mathrm{~N}_{2} \mathrm{Br} \cdot \mathrm{H}_{2} \mathrm{O}(395.29 \mathrm{~g} / \mathrm{mol}): \mathrm{C}, 63.80 ; \mathrm{H}$, 4.84; N, 7.08. Found: C, 64.23; H, 5.21; N, 6.94. Electrospray Ms. (Cone 20V) (m/z, 
fragment): $297.4[\mathrm{M}]^{+}$. HRMS ESI-TOF-MS (positive mode): $[\mathrm{M}]^{+}$monoisotopic peak 297.1389; calc. 297.1392, $\varepsilon_{\mathrm{r}}: 1 \mathrm{ppm}$.

\section{Synthesis of $\mathbf{H}$}

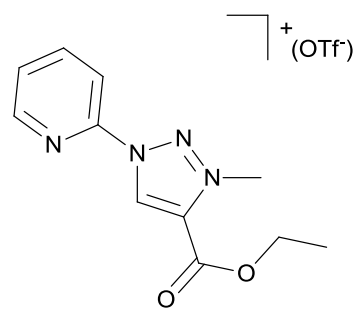

In a Schlenk, a solution of ethyl-1-(2-pyridyl)-1H-1,2,3-triazole4-carboxylate (h) $(500 \mathrm{mg}, 2.29 \mathrm{mmol})$ in freshly distilled dichloromethane, was cooled to $0^{\circ} \mathrm{C}$, in an ice bath. Then, MeOTf $(317.6 \mu \mathrm{L}, 2.75 \mathrm{mmol})$ was added, and the mixture was stirred for $1 \mathrm{~h}$ at room temperature. All volatiles were removed under reduced pressure to afford an analytically pure light brown solid. Yield: $779 \mathrm{mg}, 89 \% .{ }^{1} \mathrm{H}$ NMR $\left(400 \mathrm{MHz}, \mathrm{CD}_{3} \mathrm{CN}\right): \delta 9.54(\mathrm{~s}, 1 \mathrm{H}, \mathrm{NCHC}$ ), $8.69\left(\mathrm{~d},{ }^{3} \mathrm{~J}_{\mathrm{H}, \mathrm{H}}=4.8, \mathrm{~Hz}, 1 \mathrm{H}, \mathrm{CH}_{\mathrm{py}}\right), 8.28-8.19\left(\mathrm{~m}, 1 \mathrm{H}, \mathrm{CH}_{\mathrm{py}}\right), 8.19-8.07(\mathrm{~m}, 1 \mathrm{H}$, $\left.\mathrm{CH}_{\mathrm{py}}\right), 7.83-7.68\left(\mathrm{~m}, 1 \mathrm{H}, \mathrm{CH}_{\mathrm{py}}\right), 4.60\left(\mathrm{~s}, 3 \mathrm{H}, \mathrm{NCH}_{3}\right), 4.53\left(\mathrm{q},{ }^{3} \mathrm{~J}_{\mathrm{H}, \mathrm{H}}=7.1 \mathrm{~Hz}, 2 \mathrm{H}, \mathrm{O}-\right.$ $\left.\mathrm{CH}_{2^{-}}\right), 1.44\left(\mathrm{t},{ }^{3} \mathrm{~J}_{\mathrm{H}, \mathrm{H}}=7.1 \mathrm{~Hz}, 3 \mathrm{H},-\mathrm{CH}_{3}\right) \cdot{ }^{13} \mathrm{C}\left\{{ }^{1} \mathrm{H}\right\} \mathrm{NMR}\left(101 \mathrm{MHz}, \mathrm{CD}_{3} \mathrm{CN}\right): \delta 155.1$ $(C O O-), 149.7\left(\mathrm{CH}_{\mathrm{py}}\right), 146.3\left(C_{\mathrm{q}(\mathrm{py})}\right), 141.1\left(\mathrm{CH}_{\mathrm{py}}\right), 134.3\left(C_{\mathrm{q}(\mathrm{tz})}\right), 129.4(\mathrm{NCHC}), 127.8$ $\left(\mathrm{CH}_{\mathrm{py}}\right)$, $115.1\left(\mathrm{CH}_{\mathrm{py}}\right)$, 64.1(- $\left.\mathrm{CH}_{2}-\right), 41.5\left(\mathrm{~N}-\mathrm{CH}_{3}\right), 13.2\left(-\mathrm{CH}_{3}\right)$. Anal. Calcd. for $\mathrm{C}_{11} \mathrm{H}_{13} \mathrm{~N}_{4} \mathrm{O}_{2} \mathrm{CF}_{3} \mathrm{SO}_{3}$ (382.31 g/mol): C, 37.70; H, 3.42; N, 14.65. Found: C, 37.68; H, 2.98; N, 14.67. Electrospray Ms. (Cone 15V) (m/z, fragment): $233.4[\mathrm{M}]^{+}$.

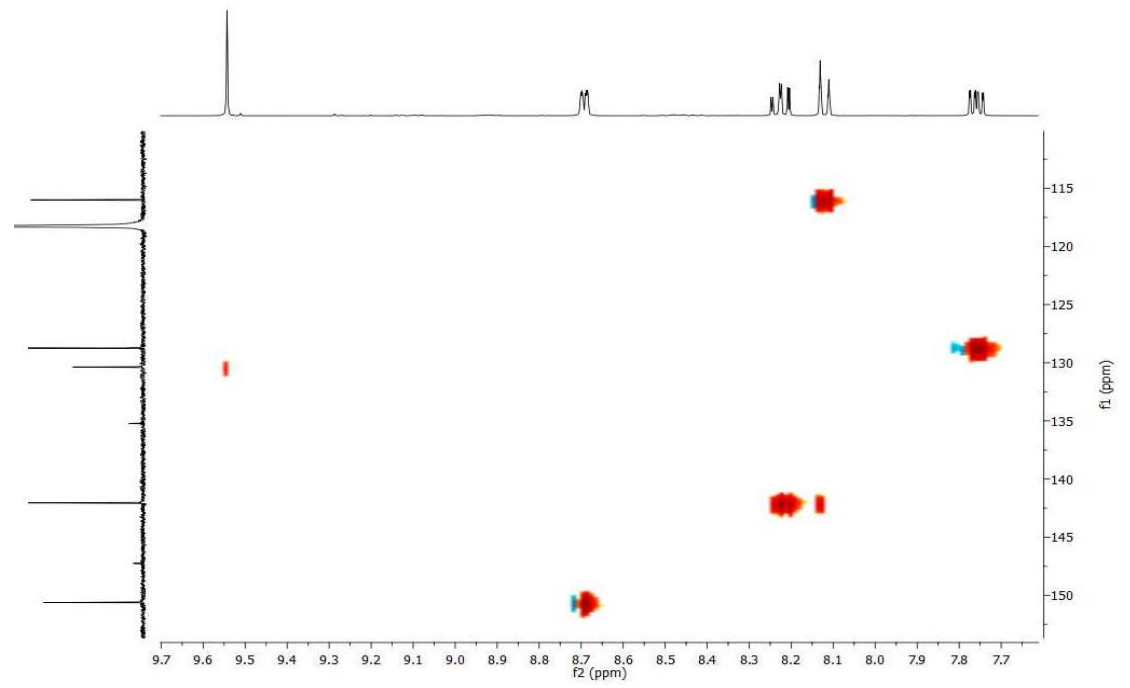

Figure 6.1 ${ }^{13} \mathrm{C}$ HSQC NMR spectrum $\left({ }^{1} \mathrm{H}, 400 \mathrm{MHz}\right.$ and $\left.{ }^{13} \mathrm{C}, 100 \mathrm{MHz}\right)$ of $\mathbf{H}$ in $\mathrm{CD}_{3} \mathrm{CN}$ 


\section{Synthesis of $i$}

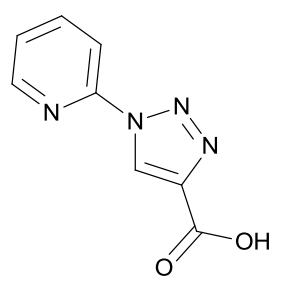

In a round-bottom flask were mixed, ethyl-1-(2-pyridyl)-1H-1,2,3triazole-4-carboxylate $(\mathbf{h})(500 \mathrm{mg}, 2.29 \mathrm{mmol})$ and $\mathrm{LiOH} \cdot \mathrm{H}_{2} \mathrm{O}(289$ $\mathrm{mg}, 6.88 \mathrm{mmol})$ in $15 \mathrm{~mL}$ of a tetrahydrofuran/methanol/water $(1: 1: 1)$ mixture and the solution was stirred for 1.5 hours at $70^{\circ} \mathrm{C}$. The organic solvents were concentrated under reduced pressure and the mixture was extracted using ethyl acetate/water mixtures. The combined aqueous layers were acidified with an $\mathrm{HCl}$ solution (4M). The product precipitated and the mixture was extracted with ethyl acetate. The organic layers were combined and the solvent was removed under reduced pressure to afford an analytically pure light brown solid. Yield: $387 \mathrm{mg}, 89 \% .{ }^{1} \mathrm{H} \mathrm{NMR}\left(300 \mathrm{MHz}, \mathrm{CDCl}_{3}\right): \delta 9.22(\mathrm{~s}, 1 \mathrm{H}$, $\mathrm{NCHC}), 8.61-8.53\left(\mathrm{~m}, 1 \mathrm{H}, \mathrm{CH}_{\mathrm{py}}\right), 8.34-8.24\left(\mathrm{~m}, 1 \mathrm{H}, \mathrm{CH}_{\mathrm{py}}\right), 8.05-7.95(\mathrm{~m}, 1 \mathrm{H}$, $\left.\mathrm{CH}_{\mathrm{py}}\right), 7.51-7.41\left(\mathrm{~m}, 1 \mathrm{H}, \mathrm{CH}_{\mathrm{py}}\right)$.

\section{Synthesis of I}

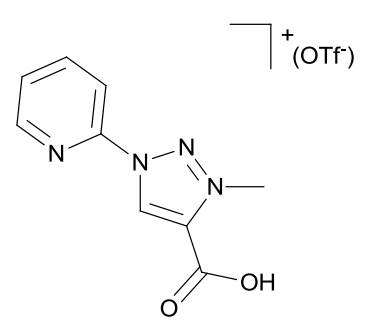

In a Schlenk, a suspension of 1-(2-pyridyl)-1H-1,2,3-triazole-4carboxylic acid (i) $(300 \mathrm{mg}, 1.57 \mathrm{mmol})$ in freshly distilled dichloromethane, was cooled to $0^{\circ} \mathrm{C}$, in an ice bath. Then, MeOTf $(219 \mu \mathrm{L}, 1.89 \mathrm{mmol})$ was added, and the mixture was stirred for $8 \mathrm{~h}$ at room temperature. All volatiles were removed under reduced pressure to afford an analytically pure light brown solid. Yield: $523 \mathrm{mg}, 94 \% .{ }^{1} \mathrm{H}$ NMR (300 MHz, $\mathrm{CD}_{3} \mathrm{CN}$ ): $\delta 9.53$ (s, 1H, NCHC), 8.69 (d, $\left.{ }^{3} \mathrm{~J}_{\mathrm{H}, \mathrm{H}}=5.1 \mathrm{~Hz}, 1 \mathrm{H}, \mathrm{CH}_{\mathrm{py}}\right), 8.26-8.18\left(\mathrm{~m}, 1 \mathrm{H}, \mathrm{CH}_{\mathrm{py}}\right), 8.14-8.08\left(\mathrm{~m}, 1 \mathrm{H}, \mathrm{CH}_{\mathrm{py}}\right), 7.79-$ $7.72\left(\mathrm{~m}, 1 \mathrm{H}, \mathrm{CH}_{\mathrm{py}}\right), 6.53$ (broad s, $\left.-\mathrm{OH}\right) 4.59\left(\mathrm{~s}, 3 \mathrm{H}, \mathrm{NCH}_{3}\right) .{ }^{13} \mathrm{C}\left\{{ }^{1} \mathrm{H}\right\} \mathrm{NMR}(101 \mathrm{MHz}$, $\left.\mathrm{CD}_{3} \mathrm{CN}\right): \delta 156.2(C O O-), 150.6\left(\mathrm{CH}_{\mathrm{py}}\right), 147.3\left(C_{\mathrm{q}(\mathrm{py})}\right), 142.0\left(\mathrm{CH}_{\mathrm{py}}\right), 135.2\left(C_{\mathrm{q}(\mathrm{tz})}\right), 130.5$ ( $\mathrm{NCHC}), 128.7\left(\mathrm{CH}_{\mathrm{py}}\right), 116.0\left(\mathrm{CH}_{\mathrm{py}}\right), 42.4\left(\mathrm{~N}-\mathrm{CH}_{3}\right)$. Anal. Calcd. for $\mathrm{C}_{9} \mathrm{H}_{9} \mathrm{~N}_{4} \mathrm{O}_{2} \mathrm{CF}_{3} \mathrm{SO}_{3}$ (354.26 g/mol): C, 33.90; H, 2.56; N, 15.81. Found: C, 34.30; H, 2.07; N, 16.27. Electrospray Ms. (Cone 15V) (m/z, fragment): $205.0[\mathrm{M}]^{+}$. 


\subsection{Synthesis and characterization of bimetallic complexes}

\section{Synthesis of $2 \mathrm{AH}$}

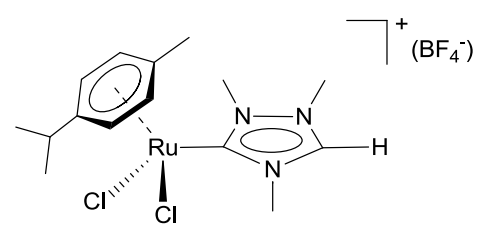

A suspension of sodium hydride $(60 \%$ in mineral oil, $15.7 \mathrm{mg}, 0.39 \mathrm{mmol})$ in dry methanol was stirred at $0^{\circ} \mathrm{C}$ under a nitrogen atmosphere until a clear solution of sodium methoxide was formed. 1,2,4trimethyltriazolium tetrafluoroborate $(93.6 \mathrm{mg}, 0.32$ mmol) was added, and the mixture was stirred at room temperature for $1 \mathrm{~h}$. After addition of $\left[\mathrm{RuCl}_{2}(p \text {-cymene })\right]_{2}(100 \mathrm{mg}, 0.16 \mathrm{mmol})$ the mixture was refluxed for $2 \mathrm{~h}$. The suspension was filtered and the solution concentrated under reduced pressure. Compound 2AH was obtained as an orange solid after crystallization from acetonitrile/methanol. Yield: $129 \mathrm{mg}, 80 \% .{ }^{1} \mathrm{H}$ NMR (300 MHz, $\left.\mathrm{CD}_{3} \mathrm{CN}\right): \delta 9.54(\mathrm{~s}, 1 \mathrm{H}, \mathrm{NCHN}), 5.66\left(\mathrm{~d},{ }^{3} \mathrm{~J}_{\mathrm{H}, \mathrm{H}}\right.$ $\left.=6 \mathrm{~Hz}, 1 \mathrm{H}, \mathrm{CH}_{p \text {-cym }}\right), 5.43\left(\mathrm{~d},{ }^{3} \mathrm{~J}_{\mathrm{H}, \mathrm{H}}=6 \mathrm{~Hz}, 1 \mathrm{H}, \mathrm{CH}_{p \text {-cym }}\right), 4.38\left(\mathrm{~s}, 3 \mathrm{H}, \mathrm{NCH}_{3}\right), 4.21(\mathrm{~s}, 3 \mathrm{H}$, $\left.\mathrm{NCH}_{3}\right), 4.17\left(\mathrm{~s}, 3 \mathrm{H}, \mathrm{NCH}_{3}\right), 2.94\left(\mathrm{sept},{ }^{3} \mathrm{~J}_{\mathrm{H}, \mathrm{H}}=7.5 \mathrm{~Hz}, 1 \mathrm{H}, \mathrm{CH}_{\text {isop }(p \text {-cym })}\right), 2.10(\mathrm{~s}, 3 \mathrm{H}$, $\left.\mathrm{CH}_{3(p-c y m)}\right), 1.33\left(\mathrm{~d},{ }^{3} \mathrm{~J}_{\mathrm{H}, \mathrm{H}}=6 \mathrm{~Hz}, 6 \mathrm{H}, \mathrm{CH}_{3(\text { isop }(p \text {-cym) })}\right) .{ }^{13} \mathrm{C}\left\{{ }^{1} \mathrm{H}\right\} . \mathrm{NMR}\left(75 \mathrm{MHz}, \mathrm{CD}_{3} \mathrm{CN}\right): \delta$ $192.3\left(C_{\text {carbene }}-\mathrm{Ru}\right), 145.7(\mathrm{NCHN}),[112.6,99.8]\left(\mathrm{Cq}_{p \text {-cym }}\right),[88.2,85.3]\left(C \mathrm{H}_{p \text {-cym }}\right)$, [40.1, 39.6, 38.1] $\left(\mathrm{NCH}_{3}\right), 31.6\left(\mathrm{CH}_{\text {isop }(p-c y m)}\right), 22.4\left(\mathrm{CH}_{3(\operatorname{sisop}(p-\mathrm{cym}))}\right), 18.7\left(\mathrm{CH}_{3(p-c y m)}\right)$. Anal. Calcd. for $\mathrm{C}_{15} \mathrm{H}_{24} \mathrm{~N}_{3} \mathrm{BCl}_{2} \mathrm{~F}_{4} \mathrm{Ru}(505.15 \mathrm{~g} / \mathrm{mol})$ : C, 35.66; H, 4.79; N, 8.32. Found: $\mathrm{C}$, 35.50; H, 5.05; N, 7.99. Electrospray Ms. (Cone 15V) (m/z, fragment): $418.2[\mathrm{M}]^{+}$. HRMS ESI-TOF-MS (positive mode): $[\mathrm{M}-\mathrm{Cl}]^{+}$monoisotopic peak 418.0385; calc. $418.0389, \varepsilon_{\mathrm{r}}: 0.9 \mathrm{ppm}$.

\section{Synthesis of 3A}

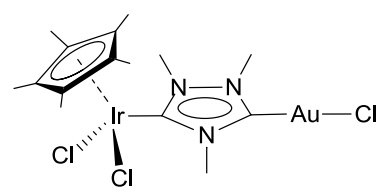

Compound 1AH (100 mg, $0.17 \mathrm{mmol})$ and $\mathrm{Ag}_{2} \mathrm{O}$ (20mg, 0.085 $\mathrm{mmol})$ were stirred under nitrogen in dry tetrahydrofuran $(10$ $\mathrm{mL}$ ) at room temperature for 8 hours, under the exclusion of light. Then $20 \mathrm{~mL}$ of dry acetonitrile, $\left[\mathrm{AuCl}\left(\mathrm{SMe}_{2}\right)\right](50 \mathrm{mg}$, $0.17 \mathrm{mmol})$ and $\mathrm{KCl}(18 \mathrm{mg}, 0.24 \mathrm{mmol})$ were added and stirred at room temperature overnight. The final suspension was filtered over celite and the solvents were concentrated under reduced pressure. Crystallization from acetonitrile/diethyl ether gave 3A as a yellow crystalline solid. Yield: $88 \mathrm{mg}, 70 \%$. ${ }^{1} \mathrm{H}$ NMR $\left(500 \mathrm{MHz}, \mathrm{CD}_{3} \mathrm{CN}\right): \delta$ 
$4.23\left(\mathrm{~s}, 3 \mathrm{H}, \mathrm{NCH}_{3}\right), 4.14$ (s, 3H, NCH$\left.H_{3}\right), 4.09$ (s, 3H, NCH$), 1.61\left(\mathrm{~s}, 15 \mathrm{H}, \mathrm{C}_{5}\left(\mathrm{CH}_{3}\right)_{5}\right)$. ${ }^{13} \mathrm{C}\left\{{ }^{1} \mathrm{H}\right\}$ NMR $\left(125 \mathrm{MHz}, \mathrm{CD}_{3} \mathrm{CN}\right): \delta 175.0\left(C_{\text {carbene }}-\mathrm{Au}\right), 164.9\left(C_{\text {carbene }}-\mathrm{Ir}\right), 94.1$ $\left(C_{5}\left(\mathrm{CH}_{3}\right)_{5}\right), \quad\left[\begin{array}{llllll}41.1, & 39.2, & 38.8\end{array} \quad\left(\mathrm{NCH}_{3}\right), \quad 9.2 \quad\left(\mathrm{C}_{5}\left(\mathrm{CH}_{3}\right)_{5}\right)\right.$. Anal. Calcd. for $\mathrm{C}_{15} \mathrm{H}_{24} \mathrm{~N}_{3} \mathrm{AuIrCl}_{3} \cdot \mathrm{H}_{2} \mathrm{O} \cdot \mathrm{CH}_{3} \mathrm{CN}$ (800.98 g/mol): C, 25.49; H, 3.65; N, 6.99. Found: $\mathrm{C}$, 25.95; H, 3.88; N, 6.97. Electrospray Ms. (Cone 15V) (m/z, fragment): $706[\mathrm{M}-\mathrm{Cl}]^{+}$. HRMS ESI-TOF-MS (positive mode): $[\mathrm{M}-\mathrm{Cl}]^{+}$monoisotopic peak 706.0634; calc. 706.0628, $\varepsilon_{\mathrm{r}}: 0.8 \mathrm{ppm}$.

\section{Synthesis of 4A}

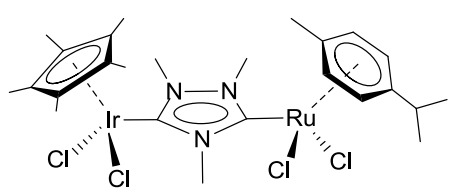

Method A: Compound 2AH (100 mg, $0.198 \mathrm{mmol}$ ), $\mathrm{NaOAc}(17 \mathrm{mg}, 0.207 \mathrm{mmol})$ and $\left[\mathrm{IrCp}^{*} \mathrm{Cl}_{2}\right]_{2}(79 \mathrm{mg}$, $0.099 \mathrm{mmol})$ were stirred under nitrogen in deoxygenated acetone $(10 \mathrm{~mL})$ at $50^{\circ} \mathrm{C}$ for 16 hours. The final suspension was filtered giving an orange solid which was

extracted with dichloromethane $\left(\begin{array}{lllll}3 & \mathrm{x} & 10 & \mathrm{~mL}\end{array}\right)$. Crystallization from dichloromethane/diethyl ether gave compound $\mathbf{4 A}$ as an orange crystalline solid. Yield: $130 \mathrm{mg}$ (81\%). Method B: Compound 1AH (150 mg, $0.247 \mathrm{mmol}), \mathrm{NaOAc}$ (20.6 mg, $0.247 \mathrm{mmol})$ and $\left[\mathrm{RuCl}_{2}(p \text {-cymene })\right]_{2}(75.3 \mathrm{mg}, 0.123 \mathrm{mmol})$ were stirred under nitrogen in deoxygenated acetone $(10 \mathrm{~mL})$ at $50^{\circ} \mathrm{C}$ for 16 hours. The final suspension was filtered giving an orange solid which was extracted with dichloromethane (3 x $10 \mathrm{~mL})$. Crystallization from dichloromethane/diethyl ether gave compound $\mathbf{4 A}$ as an orange crystalline solid. Yield: $141 \mathrm{mg}, 70 \% .{ }^{1} \mathrm{H}$ NMR (300 MHz, $\left.\mathrm{CD}_{3} \mathrm{Cl}\right): \delta 5.53\left(\mathrm{~d},{ }^{3} \mathrm{~J}_{\mathrm{H}, \mathrm{H}}=6\right.$ $\left.\mathrm{Hz}, 1 \mathrm{H}, \mathrm{CH}_{p \text {-cym }}\right), 5.49\left(\mathrm{~d},{ }^{3} \mathrm{~J}_{\mathrm{H}, \mathrm{H}}=6 \mathrm{~Hz}, 1 \mathrm{H}, \mathrm{CH}_{p \text {-cym }}\right), 5.42\left(\mathrm{~d},{ }^{3} \mathrm{~J}_{\mathrm{H}, \mathrm{H}}=6 \mathrm{~Hz}, 1 \mathrm{H}, \mathrm{CH}_{p \text {-cym }}\right)$, $5.25\left(\mathrm{~d},{ }^{3} \mathrm{~J}_{\mathrm{H}, \mathrm{H}}=6 \mathrm{~Hz}, 1 \mathrm{H}, \mathrm{CH}_{p-\mathrm{cym}}\right), 4.37\left(\mathrm{~s}, 3 \mathrm{H}, \mathrm{NCH}_{3}\right), 4.32\left(\mathrm{~s}, 3 \mathrm{H}, \mathrm{NCH}_{3}\right), 4.27(\mathrm{~s}, 3 \mathrm{H}$, $\left.\mathrm{NCH}_{3}\right), 2.93\left(\mathrm{~m}, 1 \mathrm{H}, \mathrm{CH}_{\text {isop(p-cym })}\right), 2.18\left(\mathrm{~s}, 3 \mathrm{H}, \mathrm{CH}_{3(p-c y m)}\right), 1.63\left(\mathrm{~s}, 15 \mathrm{H}, \mathrm{C}_{5}\left(\mathrm{CH}_{3}\right)_{5}\right), 1.32$ $\left(\mathrm{d},{ }^{3} \mathrm{~J}_{\mathrm{H}, \mathrm{H}}=6 \mathrm{~Hz}, 6 \mathrm{H}, \mathrm{CH}_{3(\text { isop }(p-\mathrm{cym}))}\right) \cdot{ }^{13} \mathrm{C}\left\{{ }^{1} \mathrm{H}\right\} \mathrm{NMR}\left(75 \mathrm{MHz}, \mathrm{CD}_{2} \mathrm{Cl}_{2}\right): \delta 186.4\left(C_{\text {carbene }^{-}}\right.$ $\mathrm{Ru}), 168.1\left(C_{\text {carbene }}-\mathrm{Ir}\right),[109.6,98.5]\left(C \mathrm{q}_{p \text {-cym }}\right), 90.2\left(C_{5}\left(\mathrm{CH}_{3}\right)_{5}\right),[86.6,85.4,84.9,83.9]$ $\left(\mathrm{CH}_{p \text {-cym }}\right),[42.1,39.2,38.1]\left(\mathrm{NCH}_{3}\right), 30.8\left(\mathrm{CH}_{\mathrm{isop}(p-c y m)}\right),[22.3,21.9]\left(\mathrm{CH}_{3 \text { isop }(p-c y m)}\right), 18.6$ $\left(\mathrm{CH}_{3(p-c y m)}\right)$, $8.8\left(\mathrm{C}_{5}\left(\mathrm{CH}_{3}\right)_{5}\right)$. Anal. Calcd. for $\mathrm{C}_{25} \mathrm{H}_{38} \mathrm{~N}_{3} \mathrm{Cl}_{4} \mathrm{RuIr}(815.69 \mathrm{~g} / \mathrm{mol})$ : C, 36.81; H, 4.69; N, 5.15. Found: C, 36.76; H, 4.73; N, 4.75. Electrospray Ms. (Cone 15V) (m/z, fragment): $780.3[\mathrm{M}-\mathrm{Cl}]^{+}, 372.5[\mathrm{M}-2 \mathrm{Cl}]^{2+}$. HRMS ESI-TOF-MS (positive mode): [M $-\mathrm{Cl}]^{+}$monoisotopic peak 780.0796; calc. 780.0789, $\varepsilon_{\mathrm{r}}: 0.9 \mathrm{ppm}$. 


\section{Synthesis of $5 \mathrm{~A}$}

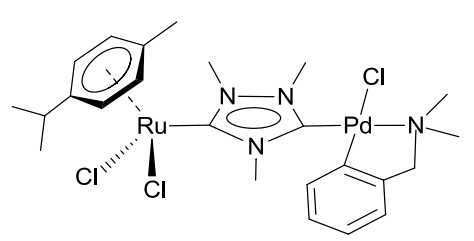

Compound $\mathbf{2 A H} \quad(100 \quad \mathrm{mg}, \quad 0.198 \mathrm{mmol})$, $[\mathrm{Pd}(\mathrm{OAc})(\mathrm{dmba})]_{2}(59 \mathrm{mg}, 0,099 \mathrm{mmol})$ and $\mathrm{NaCl}(79$ $\mathrm{mg}, \quad 1.35 \mathrm{mmol})$ were stirred under nitrogen in deoxygenated acetone $(10 \mathrm{~mL})$ at $50^{\circ} \mathrm{C}$ for 15 hours. The final suspension was filtered through celite and the solvent was removed under reduced pressure. The crude solid was purified by column chromatography. Elution with a mixture of dichloromethane/acetone (4:6) afforded an orange band. Crystallization from dichloromethane/hexane gave compound $\mathbf{5 A}$ as an orange crystalline solid. Yield: $100 \mathrm{mg}, 73 \% .{ }^{1} \mathrm{H} \mathrm{NMR}\left(300 \mathrm{MHz}, \mathrm{CDCl}_{3}\right): \delta 6.96(\mathrm{~m}$, $\left.2 \mathrm{H}, \mathrm{CH}_{A r}\right), 6.78\left(\mathrm{~m}, 1 \mathrm{H}, \mathrm{CH}_{A r}\right), 6.24\left(\mathrm{~d},{ }^{3} \mathrm{~J}_{\mathrm{H}, \mathrm{H}}=7.5 \mathrm{~Hz}, 1 \mathrm{H}, \mathrm{CH}_{A r}\right), 5.56\left(\mathrm{~d},{ }^{3} \mathrm{~J}_{\mathrm{H}, \mathrm{H}}=6.0 \mathrm{~Hz}\right.$, $\left.1 \mathrm{H}, \mathrm{CH}_{p \text {-cym }}\right), 5.53\left(\mathrm{~d},{ }^{3} \mathrm{~J}_{\mathrm{H}, \mathrm{H}}=5.8 \mathrm{~Hz}, 1 \mathrm{H}, \mathrm{CH}_{p \text {-cym }}\right), 5.31\left(\mathrm{~d},{ }^{3} \mathrm{~J}_{\mathrm{H}, \mathrm{H}}=5.9 \mathrm{~Hz}, 1 \mathrm{H}, \mathrm{CH}_{p \text {-cym }}\right)$, $5.18\left(\mathrm{~d},{ }^{3} \mathrm{~J}_{\mathrm{H}, \mathrm{H}}=5.9 \mathrm{~Hz}, 1 \mathrm{H}, \mathrm{CH}_{p \text {-cym }}\right), 4.41\left(\mathrm{~s}, 3 \mathrm{H}, \mathrm{N}_{\text {triazole }} \mathrm{CH}_{3}\right), 4.35\left(\mathrm{~s}, 3 \mathrm{H}, \mathrm{N}_{\text {triazole }} \mathrm{CH}_{3}\right)$, $4.30\left(\mathrm{~s}, 3 \mathrm{H}, \mathrm{N}_{\text {triazole }} \mathrm{CH}_{3}\right), 4.01,3.85\left(\mathrm{AB},{ }^{3} \mathrm{~J}_{\mathrm{AB}}=13.5 \mathrm{~Hz}, 2 \mathrm{H}, \mathrm{N}_{\text {amine }} \mathrm{CH}_{2}\right), 2.99$ (sept, $\left.{ }^{3} \mathrm{~J}_{\mathrm{H}, \mathrm{H}}=6.9 \mathrm{~Hz}, 1 \mathrm{H}, \mathrm{CH}_{\text {isop(p-cym) }}\right), 2.85\left(\mathrm{~s}, 3 \mathrm{H}, \mathrm{N}_{\text {amine }} \mathrm{CH}_{3}\right), 2.83$ (s, 3H, $\mathrm{N}_{\text {amine }} \mathrm{CH}_{3}$ ), 2.13 (s, $\left.3 \mathrm{H}, \mathrm{CH}_{3(p-c y m)}\right), 1.33\left(\mathrm{~d},{ }^{3} \mathrm{~J}_{\mathrm{H}, \mathrm{H}}=4.1 \mathrm{~Hz}, 3 \mathrm{H}, \mathrm{CH}_{3 \mathrm{isop}(p-c y m)}\right), 1.31\left(\mathrm{~d},{ }^{3} \mathrm{~J}_{\mathrm{H}, \mathrm{H}}=4.1 \mathrm{~Hz}, 3 \mathrm{H}\right.$, $\left.\mathrm{CH}_{3 \text { (isop(p-cym) }))}\right) \cdot{ }^{13} \mathrm{C}\left\{{ }^{1} \mathrm{H}\right\}$ NMR $\left(75 \mathrm{MHz}, \mathrm{CDCl}_{3}\right): \delta 186.4\left(C_{\text {carbene }}-\mathrm{Ru}\right), 182.4\left(C_{\text {carbene }}-\right.$ $\mathrm{Pd}),\left[147.8,146.9,136.1,126.5,124.6,122.4\left(C_{A r}\right)\right],\left[110.2,99.4\left(C \mathrm{q}_{p-\text { cym }}\right)\right],[87.3,86.3$, 84.2, $\left.82.9\left(\mathrm{CH}_{p \text {-cym }}\right)\right], 72.3\left(\mathrm{~N}_{\text {amine }} C \mathrm{H}_{2}\right)$, [50.7, $\left.50.4\left(\mathrm{~N}_{\text {amine }} C_{3}\right)\right]$, [42.2, 38.5, 37.9 $\left(\mathrm{N}_{\text {triazole }} \mathrm{CH}_{3}\right)$ ], $30.8\left(\mathrm{CH}_{\text {isop }(p \text {-cym })}\right)$, [22.6, $22.3\left(\mathrm{CH}_{3 \text { (isop(p-cym) }))}\right)$, $18.8\left(\mathrm{CH}_{3 p \text {-cym }}\right)$. Anal. Calcd. for $\mathrm{C}_{24} \mathrm{H}_{35} \mathrm{~N}_{4} \mathrm{Cl}_{3} \mathrm{RuPd} \cdot 2 \mathrm{H}_{2} \mathrm{O}(729.44 \mathrm{~g} / \mathrm{mol}): \mathrm{C}, 39.51 ; \mathrm{H}, 5.38 ; \mathrm{N}, 7.68$. Found: $\mathrm{C}$, 40.0; H, 5.77; N, 7.75. Electrospray Ms. (Cone 20V) (m/z, fragment): $700.1[\mathrm{M}-\mathrm{Cl}+$ $\mathrm{MeCN}]^{+}$.HRMS ESI-TOF-MS (positive mode): $[\mathrm{M}-\mathrm{Cl}]^{+}$monoisotopic peak 659.0305; calc. $659.0319, \varepsilon_{\mathrm{r}}: 2.1 \mathrm{ppm}$.

\section{Synthesis of 8A}

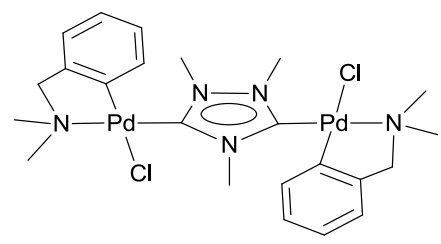

Compound $\mathbf{A H}_{\mathbf{2}}$ (48 mg, $\left.0.167 \mathrm{mmol}\right),[\mathrm{Pd}(\mathrm{OAc})(\mathrm{dmba})]_{2}$ (100 mg, $0.167 \mathrm{mmol})$ and $\mathrm{NaCl}(50 \mathrm{mg}, 0.85 \mathrm{mmol})$ were refluxed under nitrogen in acetonitrile $(10 \mathrm{~mL})$ for 15 hours. The final suspension was filtered through celite and the solvent was evaporated under reduced pressure.

Crystallization from dichloromethane/hexane gave pure compound $\mathbf{8 A}$ as an off-white 
crystalline solid. Yield: $90 \mathrm{mg}, 82 \% .{ }^{1} \mathrm{H} \mathrm{NMR}\left(300 \mathrm{MHz}, \mathrm{CDCl}_{3}\right): \delta 7.10-6.91(\mathrm{~m}, 8 \mathrm{H}$, $\left.\mathrm{CH}_{A r}\right), 6.90-6.75\left(\mathrm{~m}, 4 \mathrm{H}, \mathrm{CH}_{A r}\right), 6.28\left(\mathrm{~d},{ }^{3} \mathrm{~J}_{\mathrm{H}, \mathrm{H}}=7.0 \mathrm{~Hz}, 2 \mathrm{H}, \mathrm{CH}_{A r}\right), 6.19\left(\mathrm{~d},{ }^{3} \mathrm{~J}_{\mathrm{H}, \mathrm{H}}=7.3\right.$ $\left.\mathrm{Hz}, 2 \mathrm{H}, \mathrm{CH}_{A r}\right), 4.36\left(\mathrm{~s}, 6 \mathrm{H}, \mathrm{N}_{\text {triazole }} \mathrm{CH}_{3}\right), 4.33\left(\mathrm{~s}, 6 \mathrm{H}, \mathrm{N}_{\text {triazole }} \mathrm{CH}_{3}\right), 4.26(\mathrm{~s}, 3 \mathrm{H}$, $\left.\mathrm{N}_{\text {triazole }} \mathrm{CH}_{3}\right), 4.23\left(\mathrm{~s}, 3 \mathrm{H}, \mathrm{N}_{\text {triazole }} \mathrm{CH}_{3}\right), 4.05-3.88\left(\mathrm{~m}, 8 \mathrm{H}, \mathrm{N}_{\text {amine }} \mathrm{CH}_{2}\right), 2.90-2.79$ (m, $\left.24 \mathrm{H}, \mathrm{N}_{\text {amine }} \mathrm{CH}_{3}\right) .{ }^{13} \mathrm{C}\left\{{ }^{1} \mathrm{H}\right\} \mathrm{NMR}\left(75 \mathrm{MHz}, \mathrm{CDCl}_{3}, \delta 182.6\left(C_{\text {carbene }}-\mathrm{Pd}\right), 182.4\left(C_{\text {carbene }^{-}}\right.\right.$ $\mathrm{Pd}),\left[148.4,147.9,147.2,135.8,134.7,126.6,125.9,124.6,122.8,122.5\left(C_{A r}\right)\right],[72.5$, $72.4\left(\mathrm{~N}_{\text {amine }} C \mathrm{H}_{2}\right)$ ], [50.6, 50.5, $50.4\left(\mathrm{~N}_{\text {amine }} \mathrm{CH}_{3}\right)$ ], [41.4, 41.3, 37.7, $37.6\left(\mathrm{~N}_{\text {triazole }} C \mathrm{H}_{3}\right)$ ]. Anal. Calcd. for $\mathrm{C}_{23} \mathrm{H}_{33} \mathrm{~N}_{5} \mathrm{Cl}_{2} \mathrm{Pd}_{2} \cdot 3 \mathrm{H}_{2} \mathrm{O}$ (717,34 g/mol): C, 38,51; H, 5.48; N, 9.76. Found: C, 38.6; H, 5.86; N, 9.67. Electrospray Ms. (Cone 20V) (m/z, fragment): 669.0 $[\mathrm{M}-\mathrm{Cl}+\mathrm{MeCN}]^{+}, 337.7[\mathrm{M}-2 \mathrm{Cl}+2 \mathrm{MeCN}]^{2+}$. HRMS ESI-TOF-MS (positive mode): $[\mathrm{M}-\mathrm{Cl}+\mathrm{MeCN}]^{+}$monoisotopic peak 669.0758; calc. 669.0769, $\varepsilon_{\mathrm{r}}: 1.5 \mathrm{ppm}$

\section{Synthesis of 1B}

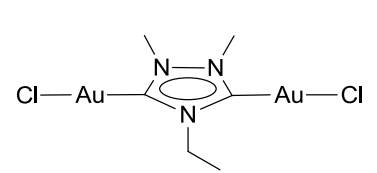

2-ethyl-1,4-dimethyltriazolium tetrafluoroborate $\left(\mathbf{B H}_{\mathbf{2}}\right)$ (93 $\mathrm{mg}$, $0.31 \mathrm{mmol})$ and 2 equivalents of silver acetate $(104 \mathrm{mg}, 0.62$ $\mathrm{mmol})$ were refluxed under nitrogen in dry tetrahydrofuran (10 $\mathrm{mL}$ ) for $3 \mathrm{~h}$, under the exclusion of light. The mixture was cooled at room temperature and $20 \mathrm{ml}$ of dry acetonitrile, [ $\left.\mathrm{AuCl}\left(\mathrm{SMe}_{2}\right)\right](183 \mathrm{mg}, 0.62$ mmol) and $\mathrm{KCl}(70 \mathrm{mg}, 0.93 \mathrm{mmol})$ were added and stirred at room temperature overnight. The suspension was filtered over celite and the solvents were concentrated under reduced pressure. Crystallization from acetonitrile/diethyl ether gave $\mathbf{1 B}$ as a white crystalline solid. Yield: $147 \mathrm{mg}, 80 \%$. ${ }^{1} \mathrm{H}$ NMR (300 MHz, DMSO-d 6 ): $\delta 4.32$ (q, ${ }^{3} \mathrm{~J}_{\mathrm{H}-\mathrm{H}}=$ $\left.7.5 \mathrm{~Hz}, 2 \mathrm{H}, \mathrm{NCH}_{2} \mathrm{CH}_{3}\right), 4.10\left(\mathrm{~s}, 6 \mathrm{H}, \mathrm{NCH}_{3}\right), 1.50\left(\mathrm{t},{ }^{3} \mathrm{~J}_{\mathrm{H}-\mathrm{H}}=7.2 \mathrm{~Hz}, 3 \mathrm{H}, \mathrm{NCH}_{2} \mathrm{CH}_{3}\right)$. ${ }^{13} \mathrm{C}\left\{{ }^{1} \mathrm{H}\right\}$ NMR (75 MHz, DMSO- $\left.d_{6}\right): \delta 174.9\left(C_{\text {carbene }}-\mathrm{Au}\right), 48.7\left(\mathrm{NCH}_{2} \mathrm{CH}_{3}\right), 38.7$ $\left(\mathrm{NCH}_{3}\right), 16.9\left(\mathrm{NCH}_{2} \mathrm{CH}_{3}\right)$. Anal. Calcd. for $\mathrm{C}_{6} \mathrm{H}_{11} \mathrm{~N}_{3} \mathrm{Au}_{2} \mathrm{Cl}_{2} \cdot 2 \mathrm{H}_{2} \mathrm{O}(626.04 \mathrm{~g} / \mathrm{mol}): \mathrm{C}$, $11.51 ; \mathrm{H}, 2.42$; N, 6.71. Found: C, 11.25; H, 2.78; N, 6.62. Electrospray Ms. (Cone 20V) (m/z, fragment): $595\left[\mathrm{M}-\mathrm{Cl}+\mathrm{CH}_{3} \mathrm{CN}\right]^{+}$. HRMS ESI-TOF-MS (positive mode): $[\mathrm{M}-\mathrm{Cl}$ $\left.+\mathrm{CH}_{3} \mathrm{CN}\right]^{+}$monoisotopic peak 595.0235; calc. 595.0238, $\varepsilon_{\mathrm{r}}: 0.5$ ppm. 


\subsubsection{Synthesis and characterization of heterodimetallic Ir-Pd chiral complexes}

General procedure for the synthesis of chiral palladacycles with tertiary amines: In a Pyrex tube $0.56 \mathrm{mmol}$ of finely powered $\mathrm{PdCl}_{2}$ and $0.59 \mathrm{mmol}$ of the corresponding tertiary amine were suspended in acetonitrile. The suspension was heated at $80^{\circ}$ until an orange solution was formed. Then, $1.4 \mathrm{mmol}$ of $\mathrm{K}_{2} \mathrm{CO}_{3}$ was added and the reaction was continued at $80^{\circ}$ until a yellow solution was formed. The mixture was filtered over celite and the solvent was concentrated under reduced pressure. The crude was redisolved in dichloromethane and washed with a saturated $\mathrm{NaCl}$ solution $(3 \times 10 \mathrm{~mL})$. The organic phase was dried with anhydrous $\mathrm{MgSO}_{4}$. Crystallization from dichloromethane/hexanes afforded the products as yellow crystalline solids. Complexes $\mathbf{a}-\mathbf{c}$ have been described previously starting from lithiumtetrachloropalladate. ${ }^{18,19}$

General procedure for the synthesis of chiral palladacycles with primary amines: In a Schlenk were added $0.45 \mathrm{mmol}$ of finely powered $\mathrm{Pd}(\mathrm{OAc})_{2}$ and $0.446 \mathrm{mmol}$ of corresponding amine in $10 \mathrm{~mL}$ of deoxygenated toluene and the suspension was heated at $50^{\circ} \mathrm{C}$ for $15 \mathrm{~h}$. The solvent was removed under reduced pressure. Recrystallization from dichloromethane/hexanes afforded the products as pale-yellow crystalline solids.

\section{Compound $\mathbf{f}$}

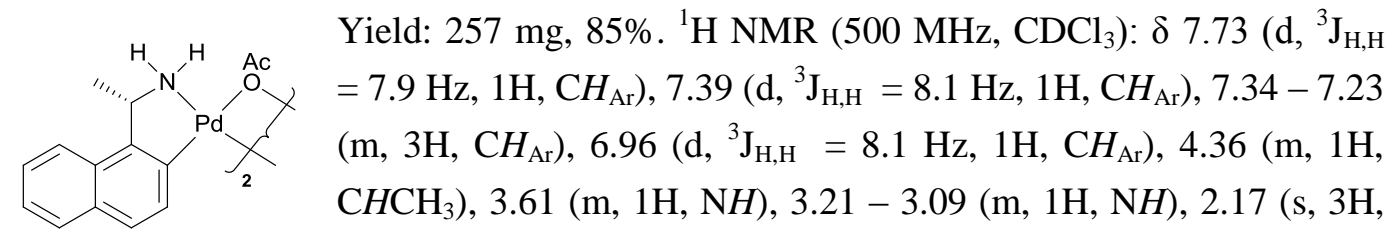
$\left.\mathrm{CH}_{3} \mathrm{OO}\right), 1.74-1.65\left(\mathrm{~m}, 3 \mathrm{H}, \mathrm{CHCH}_{3}\right) .{ }^{13} \mathrm{C}\left\{{ }^{1} \mathrm{H}\right\} \mathrm{NMR}\left(126 \mathrm{MHz}, \mathrm{CDCl}_{3}\right): \delta 181.4$ $\left(\mathrm{CH}_{3} \mathrm{COO}\right),[149.3,141.3,131.6,129.6,128.8,127.8,125.9,125.6,123.9,123.2]\left(C_{\mathrm{Ar}}\right)$, $58.3\left(\mathrm{CHCH}_{3}\right), 24.6\left(\mathrm{CHCH}_{3}, \mathrm{CH}_{3} \mathrm{COO}\right)$. Anal. Calcd. for $\mathrm{C}_{28} \mathrm{H}_{30} \mathrm{~N}_{2} \mathrm{Pd}_{2} \mathrm{O}_{4}(671.39$ g/mol): C, 50.09; H, 4.50; N, 4.17. Found: C, 50.06; H, 4.75; N, 4.47. Electrospray Ms. (Cone $15 \mathrm{~V})\left(\mathrm{m} / \mathrm{z}\right.$, fragment): $358.1\left[\mathrm{Pd}(\mathrm{L})(\mathrm{MeCN})_{2}\right]^{+}$. HRMS ESI-TOF-MS (positive mode): $[\mathrm{Pd}(\mathrm{L})+\mathrm{MeCN}]^{+}$monoisotopic peak 317.0275; calc. 317.0276, $\varepsilon_{\mathrm{r}}: 0.3 \mathrm{ppm}$. 
General synthesis of heterodimetallic complexes bearing tertiary amines: Compound 1AH $(0.167 \mathrm{mmol}), 0.084 \mathrm{mmol}$ of the corresponding palladacycle (b or c), $0.167 \mathrm{mmol}$ of sodium acetate and $10 \mathrm{~mL}$ of deoxygenated acetone were stirred at $50^{\circ} \mathrm{C}$ for $20 \mathrm{~h}$ under inert atmosphere. The resulting mixture was filtered over celite and the solvent was removed under reduced pressure. The crude was redisolved in dichloromethane and washed with a saturated $\mathrm{NaCl}$ solution $(3 \times 10 \mathrm{~mL})$. The organic phase was dried with anhydrous $\mathrm{MgSO}_{4}$. Crystallization from dichloromethane/hexanes afforded the products as yellow crystalline solids.

\section{Compound 9A}

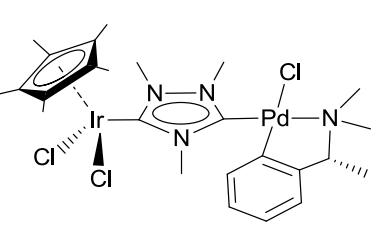

Yield: $130 \mathrm{mg}, 98 \% .{ }^{1} \mathrm{H}$ NMR $\left(500 \mathrm{MHz}, \mathrm{CDCl}_{3}\right): \delta 7.02-$ $6.91\left(\mathrm{~m}, 4 \mathrm{H}, \mathrm{CH} H_{\mathrm{Ar}}\right), 6.82\left(\mathrm{~m}, 2 \mathrm{H}, \mathrm{CH}_{\mathrm{Ar}}\right), 6.38\left(\mathrm{~m}, 2 \mathrm{H}, \mathrm{CH} H_{\mathrm{Ar}}\right)$, $4.44-4.24\left(\mathrm{~m}, 18 \mathrm{H}, \mathrm{N}_{\text {triazole }} \mathrm{CH}_{3}\right), 3.87\left(\mathrm{~m}, 1 \mathrm{H}, \mathrm{CHCH}_{3}\right), 3.74$ $\left(\mathrm{m}, 1 \mathrm{H}, \mathrm{CHCH}_{3}\right), 2.86\left(\mathrm{~s}, 3 \mathrm{H}, \mathrm{N}_{\text {amine }} \mathrm{CH}_{3}\right), 2.84(\mathrm{~s}, 3 \mathrm{H}$, $\left.\mathrm{N}_{\text {amine }} \mathrm{CH}_{3}\right), 2.70\left(\mathrm{~s}, 3 \mathrm{H}, \mathrm{N}_{\text {amine }} \mathrm{CH}_{3}\right), 2.68\left(\mathrm{~s}, 3 \mathrm{H}, \mathrm{N}_{\text {amine }} \mathrm{CH}_{3}\right.$ ), $1.68\left(\mathrm{~s}, 15 \mathrm{H},\left(\mathrm{C}_{5}\left(\mathrm{CH}_{3}\right)_{5}\right)\right), 1.66\left(\mathrm{~s}, 15 \mathrm{H},\left(\mathrm{C}_{5}\left(\mathrm{CH}_{3}\right)_{5}\right)\right), 1.62\left(\mathrm{~d},{ }^{3} \mathrm{~J}_{\mathrm{H}, \mathrm{H}}=6.2 \mathrm{~Hz}, 3 \mathrm{H}, \mathrm{CHCH}_{3}\right)$, $1.57\left(\mathrm{~d},{ }^{3} \mathrm{~J}_{\mathrm{H}, \mathrm{H}}=6.2 \mathrm{~Hz}, 3 \mathrm{H}, \mathrm{CHCH}_{3}\right) .{ }^{13} \mathrm{C}\left\{{ }^{1} \mathrm{H}\right\} \mathrm{NMR}\left(125 \mathrm{MHz}, \mathrm{CDCl}_{3}\right): \delta 183.1\left(C_{\text {carbene }}\right.$ - Pd), 167.6 ( $\left.C_{\text {carbene }}-\mathrm{Ir}\right),[153.9,136.5,126.5,124.6,122.9]\left(\mathrm{CH}_{\mathrm{Ar}}\right), 90.4\left(C_{5}\left(\mathrm{CH}_{3}\right)_{5}\right)$, [74.3, 73.8] $\left(\mathrm{CHCH}_{3}\right),[50.6,50.1,46.0,45.1]\left(\mathrm{N}_{\text {amine }} \mathrm{CH}_{3}\right),[41.2,37.8,37.3]$ $\left(\mathrm{N}_{\text {triazole }} \mathrm{CH}_{3}\right)$, [21.9, 19.1] $\quad\left(\mathrm{CHCH}_{3}\right), \quad 9.1 \quad\left(\mathrm{C}_{5}\left(\mathrm{CH}_{3}\right)_{5}\right)$. Anal. Calcd. for $\mathrm{C}_{25} \mathrm{H}_{38} \mathrm{~N}_{4} \mathrm{PdIrCl}_{3} \cdot 9 \mathrm{H}_{2} \mathrm{O}$ (961.73 g/mol): C, 31.22; H, 5.86; N, 5.82. Found: C, 31.61; H, 5.65; N, 5.83. Electrospray Ms. (Cone 20V) (m/z, fragment): $763.2[\mathrm{M}-\mathrm{Cl}]^{+}$. HRMS ESI-TOF-MS (positive mode): [M - Cl $]^{+}$monoisotopic peak 763.1128; calc. 763.1128, $\varepsilon_{\mathrm{r}}$ : $0.01 \mathrm{ppm}$.

\section{Compound 10A}

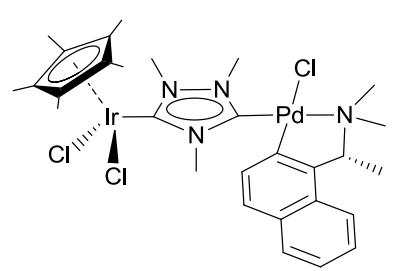

Yield $137 \mathrm{mg}, 97 \% .{ }^{1} \mathrm{H}$ NMR (300 $\left.\mathrm{MHz}, \mathrm{CDCl}_{3}\right): \delta 7.76-$ $6.70\left(\mathrm{~m}, 1 \mathrm{H}, \mathrm{C} H_{\mathrm{Ar}}\right), 7.69-7.62\left(\mathrm{~m}, 1 \mathrm{H}, \mathrm{CH}_{\mathrm{Ar}}\right), 7.44-7.28$ $\left(\mathrm{m}, 3 \mathrm{H}, \mathrm{C} H_{\mathrm{Ar}}\right), 6.55\left(\mathrm{~m}, 1 \mathrm{H}, \mathrm{C} H_{\mathrm{Ar}}\right), 4.52-4.21(\mathrm{~m}, 10 \mathrm{H}$, $\left.\mathrm{N}_{\text {triazole }} \mathrm{CH}_{3}, \mathrm{CHCH}_{3}\right), 2.88-2.78\left(\mathrm{~m}, 6 \mathrm{H}, \mathrm{N}_{\text {amine }} \mathrm{CH}_{3}\right), 1.86-$ $1.80\left(\mathrm{~m}, 3 \mathrm{H}, \mathrm{CHCH}_{3}\right),\left[1.69,1.66\left(\mathrm{~s}, 15 \mathrm{H},\left(\mathrm{C}_{5}\left(\mathrm{CH}_{3}\right)_{5}\right)\right)\right]$.

${ }^{13} \mathrm{C}\left\{{ }^{1} \mathrm{H}\right\} \mathrm{NMR}\left(75 \mathrm{MHz}, \mathrm{CDCl}_{3}\right): \delta 183.1\left(C_{\text {carbene }}-\mathrm{Pd}\right), 167.7\left(C_{\text {carbene }}-\mathrm{Ir}\right)$, [153.7,146.1, 
131.6, 129.1, 129.0, 128.9, $126.2125 .8,124.1,122.9]\left(\mathrm{CH}_{\mathrm{Ar}}\right),[90.5,90.4]\left(C_{5}\left(\mathrm{CH}_{3}\right)_{5}\right)$, [72.5, 72.4] $\left(\mathrm{CHCH}_{3}\right),[51.8,47.6]\left(\mathrm{N}_{\text {amine }} \mathrm{CH}_{3}\right)$, [41.3, 41.1, 38.3, 37.9 37.7, 37.4] $\left(\mathrm{N}_{\text {triazole }} \mathrm{CH}_{3}\right)$, [29.6, 23.7] $\left(\mathrm{CHCH}_{3}\right), \quad$ [9.1, 9.0] $\left(\mathrm{C}_{5}\left(\mathrm{CH}_{3}\right)_{5}\right)$. Anal. Calcd. for $\mathrm{C}_{29} \mathrm{H}_{40} \mathrm{~N}_{4} \mathrm{PdIrCl} 3 \cdot 2 \mathrm{H}_{2} \mathrm{O}$ (885.68 g/mol): C, 39.33; H, 5.00; N, 6.32. Found: C, 39.90; H, 5.45; N, 5.82. Electrospray Ms. (Cone $15 \mathrm{~V})\left(\mathrm{m} / \mathrm{z}\right.$, fragment): $813.1[\mathrm{M}-\mathrm{Cl}]^{+}$. HRMS ESI-TOF-MS (positive mode): $[\mathrm{M}-\mathrm{Cl}]^{+}$monoisotopic peak 813.1285; calc. 813.1285, $\varepsilon_{\mathrm{r}}$ : $0.01 \mathrm{ppm}$.

General synthesis of heterodimetallic complexes bearing tertiary amines: Compound 1AH $(0.167 \mathrm{mmol}), 0.084 \mathrm{mmol}$ of the corresponding palladacycle (e or $\mathbf{f}), 0.5 \mathrm{mmol}$ of sodium chloride and $10 \mathrm{~mL}$ of deoxygenated acetone were stirred at $50^{\circ} \mathrm{C}$ for $20 \mathrm{~h}$ under inert atmosphere. The resulting mixture was filtered over celite and the solvent was removed under reduced pressure. The crude was redisolved in dichloromethane and washed with a saturated $\mathrm{NaCl}$ solution $(3 \times 10 \mathrm{~mL})$. The organic phase was dried with anhydrous $\mathrm{MgSO}_{4}$. Crystallization from dichloromethane/hexanes afforded the products as yellow crystalline solids.

\section{Compound 11A}

Yield: $120 \mathrm{mg}, 93 \% .{ }^{1} \mathrm{H}$ NMR $\left(500 \mathrm{MHz}, \mathrm{CDCl}_{3}\right) \delta 6.94-$

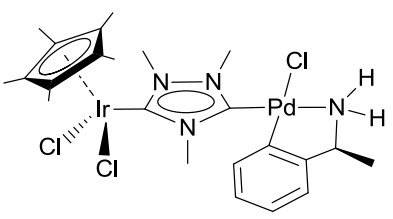
$6.88\left(\mathrm{~m}, 1 \mathrm{H}, \mathrm{CH}_{\mathrm{Ar}}\right), 6.85\left(\mathrm{~d},{ }^{3} \mathrm{~J}_{\mathrm{H}, \mathrm{H}}=7.1 \mathrm{~Hz}, 1 \mathrm{H}, \mathrm{CH} H_{\mathrm{Ar}}\right), 6.76$ $\left(\mathrm{m}, 1 \mathrm{H}, \mathrm{CH}_{\mathrm{Ar}}\right), 6.36\left(\mathrm{~m}, 1 \mathrm{H}, \mathrm{CH}_{\mathrm{Ar}}\right), 4.42-4.31(\mathrm{~m}, 1 \mathrm{H}$, $\left.\mathrm{CHCH}_{3}\right), 4.30-4.18\left(\mathrm{~m}, 9 \mathrm{H}, \mathrm{N}_{\text {triazole }} \mathrm{CH}_{3}\right), 4.03(\mathrm{~m}, 1 \mathrm{H}$, $\left.\mathrm{N} H_{2}\right), 3.00\left(\mathrm{~m}, 1 \mathrm{H}, \mathrm{N} H_{2}\right), 1.60\left(\mathrm{~s}, 15 \mathrm{H},\left(\mathrm{C}_{5}\left(\mathrm{CH}_{3}\right)_{5}\right)\right), 1.55(\mathrm{~m}$, $\left.3 \mathrm{H}, \mathrm{CHCH}_{3}\right) .{ }^{13} \mathrm{C}\left\{{ }^{1} \mathrm{H}\right\}$ NMR $\left(125 \mathrm{MHz}, \mathrm{CDCl}_{3}\right): \delta 183.5\left(C_{\text {carbene }}-\mathrm{Pd}\right), 167.6\left(C_{\text {carbene }}-\right.$ Ir), [156.0,146.3, 137.1, 127.0, 124.8, 122.6] $\left(\mathrm{CH}_{\mathrm{Ar}}\right), 90.5\left(\mathrm{C}_{5}\left(\mathrm{CH}_{3}\right)_{5}\right), 58.9\left(\mathrm{CHCH}_{3}\right)$, [41.3, 38.1, 37.8, 37.6] $\left(\mathrm{N}_{\text {triazole }} \mathrm{CH}_{3}\right)$, [26.3, 25.7] $\left(\mathrm{CHCH}_{3}\right), 9.2\left(\mathrm{C}_{5}\left(\mathrm{CH}_{3}\right)_{5}\right)$. Anal. Calcd. for $\mathrm{C}_{23} \mathrm{H}_{34} \mathrm{~N}_{4} \mathrm{PdIrCl} 3 \cdot 2 \mathrm{H}_{2} \mathrm{O}$ (807.57 g/mol): C, 34.20; H, 4.74; N, 6.93. Found: C, 34.47; $\mathrm{H}, 5.09$; N, 6.87. Electrospray Ms. (Cone 15V) (m/z, fragment): $735.3[\mathrm{M}-\mathrm{Cl}]^{+}$. HRMS ESI-TOF-MS (positive mode): $[\mathrm{M}-\mathrm{Cl}]^{+}$monoisotopic peak 735.0814; calc. 735.0812, $\varepsilon_{\mathrm{r}}$ : $0.3 \mathrm{ppm}$. 


\section{Compound 12A}

Yield: $112 \mathrm{mg}, 82 \% .{ }^{1} \mathrm{H}$ NMR $\left(300 \mathrm{MHz}, \mathrm{CDCl}_{3}\right): \delta 7.66-$

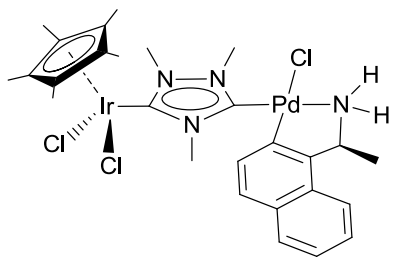
$7.46\left(\mathrm{~m}, 2 \mathrm{H}, \mathrm{CH}_{\mathrm{Ar}}\right), 7.37-7.18\left(\mathrm{~m}, 3 \mathrm{H}, \mathrm{C} H_{\mathrm{Ar}}\right), 6.54(\mathrm{~m}$, $\left.1 \mathrm{H}, \mathrm{CH}_{\mathrm{Ar}}\right), 4.34-3.97\left(\mathrm{~m}, 11 \mathrm{H}, \mathrm{N}_{\text {triazole }} \mathrm{CH}_{3}, \mathrm{CHCH}_{3}, \mathrm{NH}_{2}\right)$, $3.09\left(\mathrm{~m}, 1 \mathrm{H}, \mathrm{NH} \mathrm{H}_{2}\right), \quad 1.76-1.67\left(\mathrm{~m}, 3 \mathrm{H}, \mathrm{CHCH}_{3}\right), 1.64-$ $\left.1.55\left(\mathrm{~m}, 15 \mathrm{H},\left(\mathrm{C}_{5}\left(\mathrm{CH}_{3}\right)_{5}\right)\right)\right] .{ }^{13} \mathrm{C}\left\{{ }^{1} \mathrm{H}\right\} \quad \mathrm{NMR}(75 \mathrm{MHz}$, $\left.\mathrm{CDCl}_{3}\right): \delta 183.3\left(C_{\text {carbene }}-\mathrm{Pd}\right), 167.6\left(C_{\text {carbene }}-\mathrm{Ir}\right)$, [151.1,145.5, 135.2, 131.5, 131.3 128.7, 128.3 126.5, 126.0, 125.5, 124,3, 123.7, 123.4] $\left(\mathrm{CH}_{\mathrm{Ar}}\right)$, [90.4, 90.3] $\left(\mathrm{C}_{5}\left(\mathrm{CH}_{3}\right)_{5}\right)$, [56.8, 56.7] $\left(\mathrm{CHCH}_{3}\right)$, [41.1, 38.4, 38.1, 37.6] $\left(\mathrm{N}_{\text {triazole }} \mathrm{CH}_{3}\right), \quad 25.7 \quad\left(\mathrm{CHCH}_{3}\right), \quad[9.1, \quad 9.0] \quad\left(\mathrm{C}_{5}\left(\mathrm{CH}_{3}\right)_{5}\right)$. Anal. Calcd. for $\mathrm{C}_{27} \mathrm{H}_{36} \mathrm{~N}_{4} \mathrm{PdIrCl} 3 \cdot \mathrm{H}_{2} \mathrm{O}(839.71 \mathrm{~g} / \mathrm{mol}): \mathrm{C}, 38.62 ; \mathrm{H}, 4.56 ; \mathrm{N}, 6.67$. Found: $\mathrm{C}, 38.73 ; \mathrm{H}$, 4.89; N, 6.14. Electrospray Ms. (Cone 15V) (m/z, fragment): $785.1[\mathrm{M}-\mathrm{Cl}]^{+}$. HRMS ESI-TOF-MS (positive mode): [M - Cl $]^{+}$monoisotopic peak 785.0977; calc. 785.0972, $\varepsilon_{\mathrm{r}}$ : $0.6 \mathrm{ppm}$.

\subsection{Synthesis and characterization of chiral palladacycles with NHC ligands}

General procedure for the synthesis of NHC palladacycles with tertiary amines: In a Pyrex tube $0.15 \mathrm{mmol}$ of chiral palladacycle (a-c), $0.30 \mathrm{mmol}$ of imidazolium salt (D-F), $0.3 \mathrm{mmol}$ of $\mathrm{KI}$ and $0.43 \mathrm{mmol}$ of $\mathrm{Cs}_{2} \mathrm{CO}_{3}$ were heated at $100^{\circ}$ and stirred for $1 \mathrm{~h}$. The resulting mixture was filtered over celite and the solvent was removed under reduced pressure. The crude was redisolved in dichloromethane and washed with a saturated $\mathrm{NaCl}$ solution (3 $\mathrm{x} 10 \mathrm{~mL}$ ). The organic phase was dried with anhydrous $\mathrm{MgSO}_{4}$. Crystallization from dichloromethane/hexanes afforded the products as pale yellow crystalline solids.

\section{Compound 1D}

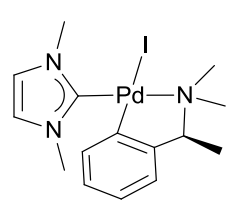

Yield: $128 \mathrm{mg}, 90 \% .{ }^{1} \mathrm{H}$ NMR $\left(300 \mathrm{MHz}, \mathrm{CDCl}_{3}\right): \delta 7.02-6.90(\mathrm{~m}, 4 \mathrm{H}$, $\left.\mathrm{CH}_{\mathrm{Ar}}, \mathrm{CH}_{\text {imidazole }}\right), 6.78-6.71\left(\mathrm{~m}, 1 \mathrm{H}, \mathrm{C} H_{\mathrm{Ar}}\right), 5.84\left(\mathrm{~d},{ }^{3} \mathrm{~J}_{\mathrm{H}, \mathrm{H}}=7.5 \mathrm{~Hz}, 1 \mathrm{H}\right.$, $\mathrm{CH}_{\mathrm{Ar}}$ ), 3.93 (s, 3H, $\mathrm{N}_{\text {imidazole }} \mathrm{CH}_{3}$ ), 3.83 (s, 3H, $\mathrm{N}_{\text {imidazole }} \mathrm{CH}_{3}$ ), 3.69 (q, $\left.{ }^{3} \mathrm{~J}_{\mathrm{H}, \mathrm{H}}=6.4 \mathrm{~Hz}, 1 \mathrm{H}, \mathrm{CHCH}_{3}\right), 2.92\left(\mathrm{~s}, 3 \mathrm{H}, \mathrm{N}_{\text {amine }} \mathrm{CH}_{3}\right), 2.85(\mathrm{~s}, 3 \mathrm{H}$, $\left.\mathrm{N}_{\text {amine }} \mathrm{CH}_{3}\right), 1.63\left(\mathrm{~d},{ }^{3} \mathrm{~J}_{\mathrm{H}, \mathrm{H}}=6.5 \mathrm{~Hz}, 3 \mathrm{H}, \mathrm{CHCH}_{3}\right) .{ }^{13} \mathrm{C}\left\{{ }^{1} \mathrm{H}\right\} \quad \mathrm{NMR}\left(75 \mathrm{MHz}, \mathrm{CDCl}_{3}\right): \delta$ $173.4\left(C_{\text {carbene }}-\mathrm{Pd}\right),[154.9,152.5,134.4,125.5,124.1,122.9,122.1]\left(C_{\mathrm{Ar},} C_{\text {imidazole }}\right), 74.2$ 
$\left(\mathrm{CHCH}_{3}\right), 51.3\left(\mathrm{~N}_{\text {amine }} C \mathrm{H}_{3}\right), 49.2\left(\mathrm{~N}_{\text {amine }} C \mathrm{H}_{3}\right), 38.7\left(\mathrm{~N}_{\text {imidazole }} C \mathrm{H}_{3}\right), 38.5\left(\mathrm{~N}_{\text {imidazole }} C \mathrm{H}_{3}\right)$, $22.6\left(\mathrm{CHCH}_{3}\right)$. Anal. Calcd. for $\mathrm{C}_{15} \mathrm{H}_{22} \mathrm{~N}_{3} \mathrm{PdI}(477.68 \mathrm{~g} / \mathrm{mol})$ : C, 37.71; H, 4.64; N, 8.79. Found: C, 37.43; H, 4.96; N, 8.89. Electrospray Ms. (Cone 15V) (m/z, fragment): 350.1 $[\mathrm{M}-\mathrm{I}]^{+}$. HRMS ESI-TOF-MS (positive mode): $[\mathrm{M}-\mathrm{I}]^{+}$monoisotopic peak 350.0852; calc. $350.0855, \varepsilon_{\mathrm{r}}: 0.9 \mathrm{ppm}$.

\section{Compound 1E}

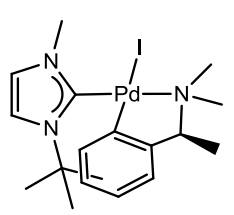

Purification by silica gel chromatography with dichloromethane/ethyl acetate $=1 / 1$ afforded the product as a yellow solid. Yield: $93 \mathrm{mg}, 60 \%$. Isomer 1: ${ }^{1} \mathrm{H}$ NMR $\left(500 \mathrm{MHz}, \mathrm{CDCl}_{3}\right): \delta 7.16\left(\mathrm{~d},{ }^{3} \mathrm{~J}_{\mathrm{H}, \mathrm{H}}=2.1 \mathrm{~Hz}, 1 \mathrm{H}\right.$, $\left.\mathrm{CH}_{\mathrm{Ar}}\right), 7.00-6.92\left(\mathrm{~m}, 3 \mathrm{H}, \mathrm{CH}_{\mathrm{Ar}}, \mathrm{CH}_{\text {imidazole }}\right), 6.73\left(\mathrm{~m}, 1 \mathrm{H}, \mathrm{C} H_{\mathrm{Ar}}\right), 5.88$

$\left(\mathrm{d},{ }^{3} \mathrm{~J}_{\mathrm{H}, \mathrm{H}}=7.5 \mathrm{~Hz}, 1 \mathrm{H}, \mathrm{CH}_{\mathrm{Ar}}\right), 3.98\left(\mathrm{~s}, 3 \mathrm{H}, \mathrm{N}_{\text {imidazole }} \mathrm{CH}_{3}\right), 3.83\left(\mathrm{q},{ }^{3} \mathrm{~J}_{\mathrm{H}, \mathrm{H}}=6.6 \mathrm{~Hz}, 1 \mathrm{H}\right.$, $\mathrm{CHCH}_{3}$ ), 2.97 (s, 3H, N amine $\left.\mathrm{CH}_{3}\right), 2.87$ (s, 3H, N amine $\left.\mathrm{CH}_{3}\right), 1.90$ (s, 9H, C(CH $\left.\mathrm{CH}_{3}\right), 1.56$ (d, $\left.{ }^{3} \mathrm{~J}_{\mathrm{H}, \mathrm{H}}=6.5 \mathrm{~Hz}, 3 \mathrm{H}, \mathrm{CHCH}_{3}\right)$. Isomer 2: ${ }^{1} \mathrm{H} \mathrm{NMR}\left(500 \mathrm{MHz}, \mathrm{CDCl}_{3}\right): \delta 7.14\left(\mathrm{~d},{ }^{3} \mathrm{~J}_{\mathrm{H}, \mathrm{H}}=2.1\right.$ $\left.\mathrm{Hz}, 1 \mathrm{H}, \mathrm{C} H_{\mathrm{Ar}}\right), 7.00-6.92\left(\mathrm{~m}, 3 \mathrm{H}, \mathrm{CH}_{\mathrm{Ar}}, \mathrm{CH}_{\text {imidazole }}\right), 6.73\left(\mathrm{~m}, 1 \mathrm{H}, \mathrm{C} H_{\mathrm{Ar}}\right), 5.79\left(\mathrm{~d},{ }^{3} \mathrm{~J}_{\mathrm{H}, \mathrm{H}}=\right.$ $\left.7.5 \mathrm{~Hz}, 1 \mathrm{H}, \mathrm{CH}_{\mathrm{Ar}}\right), 4.07\left(\mathrm{~s}, 3 \mathrm{H}, \mathrm{N}_{\text {imidazole }} \mathrm{CH}_{3}\right), 3.56\left(\mathrm{q},{ }^{3} \mathrm{~J}_{\mathrm{H}, \mathrm{H}}=6.5 \mathrm{~Hz}, 1 \mathrm{H}, \mathrm{CHCH}_{3}\right), 2.98$ (s, 3H, $\left.\mathrm{N}_{\text {amine }} \mathrm{CH}_{3}\right), 2.84\left(\mathrm{~s}, 3 \mathrm{H}, \mathrm{N}_{\mathrm{amine}} \mathrm{CH}_{3}\right), 1.84\left(\mathrm{~s}, 9 \mathrm{H}, \mathrm{C}\left(\mathrm{CH}_{3}\right)_{3}\right), 1.61\left(\mathrm{~d},{ }^{3} \mathrm{~J}_{\mathrm{H}, \mathrm{H}}=6.5 \mathrm{~Hz}\right.$, $\left.3 \mathrm{H}, \mathrm{CHCH}_{3}\right)$. Assignments for isomers are arbitrary. Diastereomeric ratio: 55/45. ${ }^{13} \mathrm{C}\left\{{ }^{1} \mathrm{H}\right\}$ NMR $\left(126 \mathrm{MHz}, \mathrm{CDCl}_{3}\right): \delta[170.0,169.5]\left(C_{\text {carbene }}-\mathrm{Pd}\right),[154.6,153.6,153.3$, 152.6, 134.6, 134.5, 125.4, 125.1, 123.7, 123.7, 122.7, 122.4, 121.4, 121.2, 119.3, 119.2] $\left(C_{\mathrm{Ar}}, C_{\text {imidazole }}\right),[74.6,73.4]\left(\mathrm{CHCH}_{3}\right),[58.3,58.3]\left(C\left(\mathrm{CH}_{3}\right)_{3}\right),[52.0,51.5,50.2,48.0]$ $\left(\mathrm{N}_{\text {amine }} \mathrm{CH}_{3}\right)$, [40.3, 40.0] $\left(\mathrm{N}_{\text {imidazole }} \mathrm{CH}_{3}\right)$, [31.9, 31.9] $\left(\mathrm{C}\left(\mathrm{CH}_{3}\right)_{3}\right)$, [23.4, 19.7] $\left(\mathrm{CHCH}_{3}\right)$. Anal. Calcd. for $\mathrm{C}_{18} \mathrm{H}_{28} \mathrm{~N}_{3} \mathrm{PdI}(519.76 \mathrm{~g} / \mathrm{mol})$ : C, 41.59; H, 5.43; N, 8.08. Found: C, 41.92; H, 5.67; N, 8.39. Electrospray Ms. (Cone 15V) (m/z, fragment): $392.1[\mathrm{M}-\mathrm{I}]^{+}$. HRMS ESI-TOF-MS (positive mode): $[\mathrm{M}-\mathrm{I}]^{+}$monoisotopic peak 392.1330; calc. $392.1325, \varepsilon_{\mathrm{r}}: 1.3 \mathrm{ppm}$. 


\section{Compound 1F}

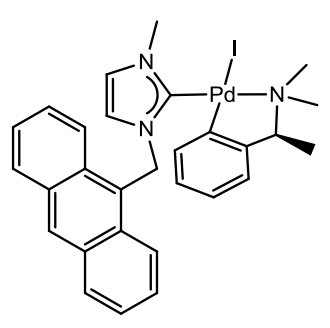

Yield: $150 \mathrm{mg}, 76 \% .{ }^{1} \mathrm{H}$ NMR $\left(500 \mathrm{MHz}, \mathrm{CDCl}_{3}\right): \delta 8.53(\mathrm{~d}$, $\left.{ }^{3} \mathrm{~J}_{\mathrm{H}, \mathrm{H}}=7.9 \mathrm{~Hz}, 2 \mathrm{H}, \mathrm{CH} H_{\mathrm{Ar}}\right), 8.45\left(\mathrm{~d},{ }^{3} \mathrm{~J}_{\mathrm{H}, \mathrm{H}}=8.8 \mathrm{~Hz}, 2 \mathrm{H}, \mathrm{CH}_{\mathrm{Ar}}\right), 8.34$ $\left(\mathrm{d},{ }^{3} \mathrm{~J}_{\mathrm{H}, \mathrm{H}}=8.8 \mathrm{~Hz}, 2 \mathrm{H}, \mathrm{C} H_{\mathrm{Ar}}\right), 8.02\left(\mathrm{t},{ }^{3} \mathrm{~J}_{\mathrm{H}, \mathrm{H}}=8.4 \mathrm{~Hz}, 4 \mathrm{H}, \mathrm{C} H_{\mathrm{Ar}}\right)$, $7.46\left(\mathrm{~m}, 4 \mathrm{H}, \mathrm{CH}_{\mathrm{Ar}}\right), 7.42-7.37\left(\mathrm{~m}, 2 \mathrm{H}, \mathrm{CH}_{\mathrm{Ar}}\right), 7.35-7.30(\mathrm{~m}$, $\left.2 \mathrm{H}, \mathrm{C} H_{\mathrm{Ar}}\right), 7.12-7.03\left(\mathrm{~m}, 4 \mathrm{H}, \mathrm{CH}_{\mathrm{Ar}}\right), 6.86\left(\mathrm{~m}, 2 \mathrm{H}, \mathrm{C} H_{\mathrm{Ar}}\right), 6.74-$ $6.69\left(\mathrm{~m}, 2 \mathrm{H}, \mathrm{CH}_{\mathrm{Ar}}\right), 6.55\left(\mathrm{~m}, 4 \mathrm{H}, \mathrm{CH}_{\mathrm{Ar}}\right), 6.29-6.22(\mathrm{~m}, 4 \mathrm{H},-$ $\mathrm{CH}_{2}$ ), 4.07 (s, 3H, $\mathrm{N}_{\text {imidazole }} \mathrm{CH}_{3}$ ), 3.94 (s, 3H, $\mathrm{N}_{\text {imidazole }} \mathrm{CH}_{3}$ ), 3.89 (q, ${ }^{3} \mathrm{~J}_{\mathrm{H}, \mathrm{H}}=6.8 \mathrm{~Hz}, 1 \mathrm{H}$, $\left.\mathrm{CHCH}_{3}\right), 3.82\left(\mathrm{q},{ }^{3} \mathrm{~J}_{\mathrm{H}, \mathrm{H}}=6.3 \mathrm{~Hz}, 1 \mathrm{H}, \mathrm{CHCH}_{3}\right), 2.97\left(\mathrm{~s}, 3 \mathrm{H}, \mathrm{N}_{\text {amine }} \mathrm{CH}_{3}\right), 2.93(\mathrm{~s}, 3 \mathrm{H}$, $\mathrm{N}_{\text {amine }} \mathrm{CH}_{3}$ ), $2.82\left(\mathrm{~s}, 3 \mathrm{H}, \mathrm{N}_{\text {amine }} \mathrm{CH}_{3}\right), 2.79\left(\mathrm{~s}, 3 \mathrm{H}, \mathrm{N}_{\text {amine }} \mathrm{CH}_{3}\right), 1.72\left(\mathrm{~d},{ }^{3} \mathrm{~J}_{\mathrm{H}, \mathrm{H}}=6.5 \mathrm{~Hz}, 3 \mathrm{H}\right.$, $\left.\mathrm{CHCH}_{3}\right), 1.69\left(\mathrm{~d},{ }^{3} \mathrm{~J}_{\mathrm{H}, \mathrm{H}}=6.5 \mathrm{~Hz}, 3 \mathrm{H}, \mathrm{CHCH}_{3}\right) .{ }^{13} \mathrm{C}\left\{{ }^{1} \mathrm{H}\right\} \quad \mathrm{NMR}\left(75 \mathrm{MHz}, \mathrm{CDCl}_{3}\right): \delta$ $[173.5,173.3]\left(C_{\text {carbene }}-\mathrm{Pd}\right),[154.9,154.6,148.5,148.2,136.2,136.0,131.4,131.3$, 131.2, 131.1, 129.2, 129.1, 129.0, 127.1, 125.6, 125.6, 125.4, 125.3, 124.4, 124.3, 124.0, $123.9,122.7,121.6,121.5,120.1,120.0]\left(C_{\mathrm{Ar}}, C_{\text {imidazole }}\right)[74.3,73.9]\left(\mathrm{CHCH}_{3}\right),[50.3$, 50.3, 47.7, 47.6] $\left(\mathrm{N}_{\text {amine }} C \mathrm{H}_{3}\right),[45.8,45.3]\left(\mathrm{N}_{\text {imidazole }} C \mathrm{H}_{2^{-}}\right)$, [38.6, 38.5] $\left(\mathrm{N}_{\text {imidazole }} C \mathrm{CH}_{3}\right)$, [21.9, 20.8] $\left(\mathrm{CHCH}_{3}\right)$. Anal. Calcd. for $\mathrm{C}_{19} \mathrm{H}_{30} \mathrm{~N}_{3} \mathrm{PdI}(653.89 \mathrm{~g} / \mathrm{mol}): \mathrm{C}, 53.26 ; \mathrm{H}, 4.62$; $\mathrm{N}, 6.42$. Found: C, 56.33; H, 4.70; N, 6.83. Electrospray Ms. (Cone 15V) (m/z, fragment): $526.3[\mathrm{M}-\mathrm{I}]^{+}$. HRMS ESI-TOF-MS (positive mode): $[\mathrm{M}-\mathrm{I}]^{+}$monoisotopic peak 526.1488; calc. 526.1486, $\varepsilon_{\mathrm{r}}: 0.4 \mathrm{ppm}$.

\section{Compound 2D}

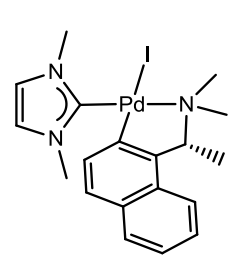

Yield: $135 \mathrm{mg}, 85 \% .{ }^{1} \mathrm{H}$ NMR (300 MHz, $\left.\mathrm{CDCl}_{3}\right): \delta 7.72\left(\mathrm{~d},{ }^{3} \mathrm{~J}_{\mathrm{H}, \mathrm{H}}=8.3\right.$ $\left.\mathrm{Hz}, 2 \mathrm{H}, \mathrm{CH}_{\mathrm{Ar}}\right), 7.44-7.25\left(\mathrm{~m}, 3 \mathrm{H}, \mathrm{CH}_{\mathrm{Ar}}\right), 6.98\left(\mathrm{~m}, 2 \mathrm{H}, \mathrm{CH}_{\mathrm{Ar}}\right), 6.07(\mathrm{~d}$, $\left.{ }^{3} \mathrm{~J}_{\mathrm{H}, \mathrm{H}}=8.3 \mathrm{~Hz}, 1 \mathrm{H}, \mathrm{CH}_{\mathrm{Ar}}\right), 4.37\left(\mathrm{q},{ }^{3} \mathrm{~J}_{\mathrm{H}, \mathrm{H}}=6.3 \mathrm{~Hz}, 1 \mathrm{H},\left(\mathrm{CHCH}_{3}\right), 4.06(\mathrm{~s}\right.$, $3 \mathrm{H},\left(\mathrm{N}_{\text {imidazole }} \mathrm{CH}_{3}\right), 3.79\left(\mathrm{~s}, 3 \mathrm{H},\left(\mathrm{N}_{\text {imidazole }} \mathrm{CH}_{3}\right), 3.16\left(\mathrm{~s}, 3 \mathrm{H},\left(\mathrm{N}_{\text {amine }} \mathrm{CH}_{3}\right)\right.\right.$, $2.80\left(\mathrm{~s}, 3 \mathrm{H},\left(\mathrm{N}_{\text {amine }} \mathrm{CH}_{3}\right)\right), 1.85\left(\mathrm{~d},{ }^{3} \mathrm{~J}_{\mathrm{H}, \mathrm{H}}=6.4 \mathrm{~Hz}, 3 \mathrm{H},\left(\mathrm{CHCH}_{3}\right) .{ }^{13} \mathrm{C}\left\{{ }^{1} \mathrm{H}\right\}\right.$ NMR (75 MHz, $\left.\mathrm{CDCl}_{3}\right): \delta 173.5\left(C_{\text {carbene }}-\mathrm{Pd}\right),[151.7,149.2,133.3,131.3,129.2,128.7$, 125.7, 125.1, 123.8, 123.1, 122.1, 122.1] $\left(C_{\mathrm{Ar}}, C_{\text {imidazole }}\right), 71.9\left(\mathrm{CHCH}_{3}\right), 51.6\left(\mathrm{~N}_{\text {amine }} C_{3}\right)$, $51.4\left(\mathrm{~N}_{\text {amine }} \mathrm{CH}_{3}\right), 38.8\left(\mathrm{~N}_{\text {imidazole }} \mathrm{CH}_{3}\right), 38.2\left(\mathrm{~N}_{\text {imidazole }} \mathrm{CH}_{3}\right), 23.9\left(\mathrm{CHCH}_{3}\right)$. Anal. Calcd. for $\mathrm{C}_{19} \mathrm{H}_{24} \mathrm{~N}_{3} \mathrm{PdI}(527.74 \mathrm{~g} / \mathrm{mol}): \mathrm{C}, 43.24 ; \mathrm{H}, 4.58 ; \mathrm{N}, 7.96$. Found: $\mathrm{C}, 43.37 ; \mathrm{H}, 4.56 ; \mathrm{N}$, 8.26. Electrospray Ms. (Cone 15V) (m/z, fragment): 400.1 [M - I] $]^{+}$. HRMS ESI-TOF-MS (positive mode): $[\mathrm{M}-\mathrm{I}]^{+}$monoisotopic peak 400.1006; calc. 400.1013, $\varepsilon_{\mathrm{r}}: 1.7$ ppm. 


\section{Compound 2E}

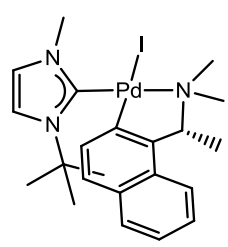

Purification by silica gel chromatography with dichloromethane afforded the product as a yellow solid. Yield: $85 \mathrm{mg}, 50 \%$. Isomer $1:{ }^{1} \mathrm{H}$ NMR $\left(300 \mathrm{MHz}, \mathrm{CDCl}_{3}\right) \delta 7.71\left(\mathrm{~d},{ }^{3} \mathrm{~J}_{\mathrm{H}, \mathrm{H}}=8.2 \mathrm{~Hz}, 2 \mathrm{H}, \mathrm{CH}_{\mathrm{Ar}}\right), 7.43-$ $7.23\left(\mathrm{~m}, 3 \mathrm{H}, \mathrm{C} H_{\mathrm{Ar}}\right), 7.22\left(\mathrm{~d},{ }^{3} \mathrm{~J}_{\mathrm{H}, \mathrm{H}}=2.1 \mathrm{~Hz}, 1 \mathrm{H}, \mathrm{C} H_{\mathrm{Ar}}\right), 6.96\left(\mathrm{~d},{ }^{3} \mathrm{~J}_{\mathrm{H}, \mathrm{H}}=\right.$ $\left.2.1 \mathrm{~Hz}, 1 \mathrm{H}, \mathrm{C} H_{\mathrm{Ar}}\right), 6.14\left(\mathrm{~d},{ }^{3} \mathrm{~J}_{\mathrm{H}, \mathrm{H}}=8.3 \mathrm{~Hz}, 1 \mathrm{H}, \mathrm{C} H_{\mathrm{Ar}}\right), 4.34\left(\mathrm{q},{ }^{3} \mathrm{~J}_{\mathrm{H}, \mathrm{H}}=\right.$ $\left.6.3 \mathrm{~Hz}, 1 \mathrm{H}, \mathrm{CHCH}_{3}\right), 3.89$ (s, $3 \mathrm{H}, \mathrm{N}_{\text {imidazole }} \mathrm{CH}_{3}$ ), 3.17 (s, $\left.3 \mathrm{H}, \mathrm{N}_{\text {amine }} \mathrm{CH}_{3}\right), 2.82$ (s, $3 \mathrm{H}$, $\left.\mathrm{N}_{\text {amine }} \mathrm{CH}_{3}\right), 2.00\left(\mathrm{~s}, 9 \mathrm{H}, \mathrm{C}\left(\mathrm{CH}_{3}\right)_{3}\right), 1.78\left(\mathrm{~d},{ }^{3} \mathrm{~J}_{\mathrm{H}, \mathrm{H}}=6.3 \mathrm{~Hz}, 3 \mathrm{H}, \mathrm{CHCH}_{3}\right)$. Isomer 2: ${ }^{1} \mathrm{H}$ $\operatorname{NMR}\left(300 \mathrm{MHz}, \mathrm{CDCl}_{3}\right) \delta 7.71\left(\mathrm{~d},{ }^{3} \mathrm{~J}_{\mathrm{H}, \mathrm{H}}=8.2 \mathrm{~Hz}, 2 \mathrm{H}, \mathrm{CH}_{\mathrm{Ar}}\right), 7.43-7.23\left(\mathrm{~m}, 3 \mathrm{H}, \mathrm{CH}_{\mathrm{Ar}}\right)$, $7.16\left(\mathrm{~d},{ }^{3} \mathrm{~J}_{\mathrm{H}, \mathrm{H}}=2.1 \mathrm{~Hz}, 1 \mathrm{H}, \mathrm{CH}_{\mathrm{Ar}}\right), 7.04\left(\mathrm{~d},{ }^{3} \mathrm{~J}_{\mathrm{H}, \mathrm{H}}=2.1 \mathrm{~Hz}, 1 \mathrm{H}, \mathrm{CH}_{\mathrm{Ar}}\right), 5.98\left(\mathrm{~d},{ }^{3} \mathrm{~J}_{\mathrm{H}, \mathrm{H}}=8.3\right.$ $\left.\mathrm{Hz}, 1 \mathrm{H}, \mathrm{CH}_{\mathrm{Ar}}\right), 4.34\left(\mathrm{q},{ }^{3} \mathrm{~J}_{\mathrm{H}, \mathrm{H}}=6.3 \mathrm{~Hz}, 1 \mathrm{H}, \mathrm{CHCH}_{3}\right), 4.15\left(\mathrm{~s}, 3 \mathrm{H}, \mathrm{N}_{\text {imidazole }} \mathrm{CH}_{3}\right), 3.13$ (s, $\left.3 \mathrm{H}, \mathrm{N}_{\text {amine }} \mathrm{CH}_{3}\right), 2.84\left(\mathrm{~s}, 3 \mathrm{H}, \mathrm{N}_{\text {amine }} \mathrm{CH}_{3}\right), 1.80\left(\mathrm{~s}, 9 \mathrm{H}, \mathrm{C}\left(\mathrm{CH}_{3}\right)_{3}\right), 1.78\left(\mathrm{~d},{ }^{3} \mathrm{~J}_{\mathrm{H}, \mathrm{H}}=6.3 \mathrm{~Hz}\right.$, $\left.3 \mathrm{H}, \mathrm{CHCH}_{3}\right)$. Assignments for isomers are arbitrary. Diastereomeric ratio: 70/30. ${ }^{13} \mathrm{C}\left\{{ }^{1} \mathrm{H}\right\} \mathrm{NMR}\left(126 \mathrm{MHz}, \mathrm{CDCl}_{3}\right): \delta[170.6,169.5]\left(C_{\text {carbene }}-\mathrm{Pd}\right),[152.3,152.1,148.7$, $148.4,134.0,133.5,131.3,131.3,129.2$, 129.1, 129.0, 128.7, 128.2, 125.7, 125.6, 125.1, $125.0,123.7,123.6,123.2,121.7,121.3,119.6,119.2]\left(C_{\mathrm{Ar}}, C_{\text {imidazole }}\right),[71.9,71.8]$ $\left(\mathrm{CHCH}_{3}\right),[58.4,58.3]\left(C\left(\mathrm{CH}_{3}\right)_{3}\right),[52.6,52.3,51.7,51.4]\left(\mathrm{N}_{\text {amine }} \mathrm{CH}_{3}\right),[40.4,39.9]$ $\left(\mathrm{N}_{\text {imidazole }} \mathrm{CH}_{3}\right)$, [31.9, 31.8] $\left(\mathrm{C}\left(\mathrm{CH}_{3}\right)_{3}\right)$, [23.6, 22.8] $\left(\mathrm{CHCH}_{3}\right)$. Anal. Calcd. for $\mathrm{C}_{22} \mathrm{H}_{30} \mathrm{~N}_{3} \mathrm{PdI}(569.82 \mathrm{~g} / \mathrm{mol}): \mathrm{C}, 46.37 ; \mathrm{H}, 5.30 ; \mathrm{N}, 7.37$. Found: $\mathrm{C}, 46.81 ; \mathrm{H}, 5.66 ; \mathrm{N}$, 7.74. Electrospray Ms. (Cone 15V) (m/z, fragment): 442.3 [M - I] ${ }^{+}$. HRMS ESI-TOF-MS (positive mode): [M - I $]^{+}$monoisotopic peak 442.1480; calc. 442.1483, $\varepsilon_{\mathrm{r}}: 0.7 \mathrm{ppm}$.

\section{Compound 2F}

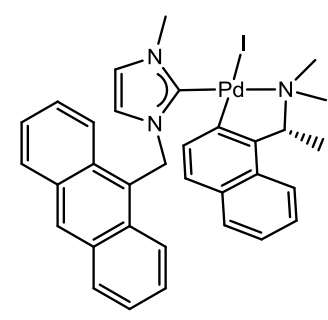

Yield: $148 \mathrm{mg}, 70 \% .{ }^{1} \mathrm{H}$ NMR (300 MHz, $\left.\mathrm{CDCl}_{3}\right): \delta 8.63(\mathrm{~s}, 1 \mathrm{H}$, $\left.\mathrm{CH}_{\mathrm{Ar}}\right), 8.58\left(\mathrm{~d},{ }^{3} \mathrm{~J}_{\mathrm{H}, \mathrm{H}}=11.7 \mathrm{~Hz}, 2 \mathrm{H}, \mathrm{CH}_{\mathrm{Ar}}\right), 8.49\left(\mathrm{~s}, 1 \mathrm{H}, \mathrm{CH}_{\mathrm{Ar}}\right)$, $8.24\left(\mathrm{~d},{ }^{3} \mathrm{~J}_{\mathrm{H}, \mathrm{H}} J=8.6 \mathrm{~Hz}, 2 \mathrm{H}, \mathrm{CH}_{\mathrm{Ar}}\right), 8.06\left(\mathrm{~d},{ }^{3} \mathrm{~J}_{\mathrm{H}, \mathrm{H}}=8.9 \mathrm{~Hz}, 2 \mathrm{H}\right.$, $\left.\mathrm{CH}_{\mathrm{Ar}}\right), 7.99\left(\mathrm{~d},{ }^{3} \mathrm{~J}_{\mathrm{H}, \mathrm{H}} J=8.3 \mathrm{~Hz}, 2 \mathrm{H}, \mathrm{CH}_{\mathrm{Ar}}\right), 7.85-7.75(\mathrm{~m}, 4 \mathrm{H}$, $\left.\mathrm{CH}_{\mathrm{Ar}}\right), 7.52-7.28\left(\mathrm{~m}, 12 \mathrm{H}, \mathrm{C} H_{\mathrm{Ar}}\right), 7.21\left(\mathrm{~d},{ }^{3} \mathrm{~J}_{\mathrm{H}, \mathrm{H}}=7.5 \mathrm{~Hz}, 2 \mathrm{H}\right.$, $\left.\mathrm{CH}_{\mathrm{Ar}}\right), 6.99\left(\mathrm{~d},{ }^{3} \mathrm{~J}_{\mathrm{H}, \mathrm{H}}=15.5 \mathrm{~Hz}, 1 \mathrm{H}, \mathrm{CH}_{\mathrm{Ar}}\right), 6.75\left(\mathrm{~m}, 2 \mathrm{H}, \mathrm{CH}_{\mathrm{Ar}}\right)$, $6.63\left(\mathrm{~d},{ }^{3} \mathrm{~J}_{\mathrm{H}, \mathrm{H}}=15.2 \mathrm{~Hz}, 1 \mathrm{H}, \mathrm{C} H_{\mathrm{Ar}}\right), 6.52-6.39\left(\mathrm{~m}, 4 \mathrm{H},-\mathrm{CH}_{2}\right), 6.33\left(\mathrm{~m}, 2 \mathrm{H}, \mathrm{CH}_{\mathrm{Ar}}\right), 4.44$ $\left(\mathrm{m}, 2 \mathrm{H}, \mathrm{CHCH}_{3}\right), 4.20\left(\mathrm{~s}, 3 \mathrm{H}, \mathrm{N}_{\text {imidazole }} \mathrm{CH}_{3}\right), 3.80\left(\mathrm{~s}, 3 \mathrm{H}, \mathrm{N}_{\text {imidazole }} \mathrm{CH}_{3}\right), 3.00-2.90(\mathrm{~m}$, $\left.12 \mathrm{H}, \mathrm{N}_{\text {amine }} \mathrm{CH}_{3}\right), 1.98-1.91\left(\mathrm{~m}, 6 \mathrm{H}, \mathrm{CHCH}_{3}\right)$. Diastereomeric ratio: 50/50. ${ }^{13} \mathrm{C}\left\{{ }^{1} \mathrm{H}\right\}$ 
NMR (75 MHz, $\left.\mathrm{CDCl}_{3}\right): \delta[173.9,173.2]\left(C_{\text {carbene }}-\mathrm{Pd}\right),[149.5,149.3,147.7,147.4$, 135.3, 134.8, 131.5, 131.4, 131.2, 131.2, 131.0, 129.2, 129.2, 129.2, 129.1, 129.1, 129.0, $128.8,127.2,127.1,125.7,125.7,125.6,125.30,125.2,125.2,125.2,125.2,125.1,124.3$, $124.1,123.8,123.7,123.1,123.1,123.0,121.7,121.5,120.2,120.0]\left(C_{\mathrm{Ar}}, C_{\text {imidazole }}\right),[72.1$, 72.0] $\left(\mathrm{CHCH}_{3}\right)$, [51.6, 51.4] $\left(\mathrm{N}_{\text {imidazole }} C \mathrm{H}_{2^{-}}\right)$, [47.9, 47.5, 47.4] $\left(\mathrm{N}_{\text {amine }} \mathrm{CH}_{3}\right),[38.6,38.5]$ $\left(\mathrm{N}_{\text {imidazole }} \mathrm{CH}_{3}\right)$, [23.8, 23.7] $\left(\mathrm{CHCH}_{3}\right)$. Anal. Calcd. for $\mathrm{C}_{33} \mathrm{H}_{32} \mathrm{~N}_{3} \mathrm{PdI}(703.95 \mathrm{~g} / \mathrm{mol})$ : $\mathrm{C}$, 56.30; H, 4.58; N, 5.97. Found: C, 56.47; H, 4.63; N, 6.23. Electrospray Ms. (Cone 15V) (m/z, fragment): $576.1[\mathrm{M}-\mathrm{I}]^{+}$. HRMS ESI-TOF-MS (positive mode): $[\mathrm{M}-\mathrm{I}]^{+}$ monoisotopic peak 576.1644; calc. 576.1644, $\varepsilon_{\mathrm{r}}: 0.01 \mathrm{ppm}$.

General procedure for the synthesis of NHC-palladium-amine complexes: In a Pyrex tube $0.3 \mathrm{mmol}$ of finely powered $\mathrm{PdCl}_{2}, 0.3 \mathrm{mmol}$ of imidazolium salt (D or F), 0.35 mmol of chiral primary amine (5), $0.6 \mathrm{mmol}$ of $\mathrm{KI}$ and $0.6 \mathrm{mmol}$ of $\mathrm{Cs}_{2} \mathrm{CO}_{3}$ were suspended in acetonitrile. The suspension was heated at $100^{\circ}$ for $2 \mathrm{~h}$. The mixture was filtered over celite and the solvent was concentrated under reduced pressure. The crude was redisolved in dichloromethane and washed with a saturated $\mathrm{NaCl}$ solution $(3 \mathrm{x} 10$ $\mathrm{mL}$ ). The organic phase was dried with anhydrous $\mathrm{MgSO}_{4}$. Crystallization from dichloromethane/hexanes afforded the products as yellow crystalline solids.

\section{Compound 3D}

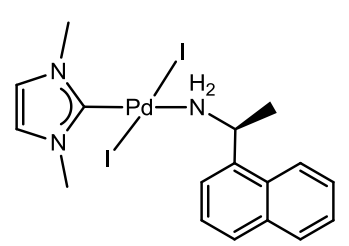

Yield: $143 \mathrm{mg}, 71 \%$. ${ }^{1} \mathrm{H}$ NMR $\left(300 \mathrm{MHz}, \mathrm{CDCl}_{3}\right): \delta 8.45(\mathrm{~d}$, $\left.{ }^{3} \mathrm{~J}_{\mathrm{H}, \mathrm{H}}=8.6 \mathrm{~Hz}, 1 \mathrm{H}, \mathrm{C} H_{\mathrm{Ar}}\right), 7.89\left(\mathrm{~d},{ }^{3} \mathrm{~J}_{\mathrm{H}, \mathrm{H}}=8.3 \mathrm{~Hz}, 1 \mathrm{H}, \mathrm{CH}_{\mathrm{Ar}}\right)$, $7.82\left(\mathrm{~d},{ }^{3} \mathrm{~J}_{\mathrm{H}, \mathrm{H}}=7.9 \mathrm{~Hz}, 1 \mathrm{H}, \mathrm{CH}_{\mathrm{Ar}}\right), 7.67-7.44\left(\mathrm{~m}, 4 \mathrm{H}, \mathrm{CH}_{\mathrm{Ar}}\right)$, $6.88\left(\mathrm{~s}, 2 \mathrm{H}, \mathrm{CH}_{\text {imidazole }}\right), 5.63-5.39\left(\mathrm{~m}, 1 \mathrm{H}, \mathrm{C} \mathrm{CHCH}_{3}\right), 3.85(\mathrm{~s}$, $\left.6 \mathrm{H}, \mathrm{N}_{\text {imidazole }} \mathrm{CH}_{3}\right), 2.92-2.57\left(\mathrm{~m}, 2 \mathrm{H}, \mathrm{NH}_{2}\right), 1.99\left(\mathrm{~d},{ }^{3} \mathrm{~J}_{\mathrm{H}, \mathrm{H}}=6.8\right.$ $\left.\mathrm{Hz}, 3 \mathrm{H}, \mathrm{CHCH}_{3}\right) .{ }^{13} \mathrm{C}\left\{{ }^{1} \mathrm{H}\right\} \quad \mathrm{NMR}\left(75 \mathrm{MHz}, \mathrm{CDCl}_{3}\right): \delta 148.9\left(C_{\text {carbene }}-\mathrm{Pd}\right),[140.1$, $134.0,130.3,129.0,128.5,126.8,125.9,125.4,122.9,122.4]\left(C_{\mathrm{Ar}}, C_{\text {imidazole }}\right), 49.8$ $\left(\mathrm{CHCH}_{3}\right), 38.7\left(\mathrm{~N}_{\text {imidazole }} \mathrm{CH}_{3}\right), 23.3\left(\mathrm{CHCH}_{3}\right)$. Anal. Calcd. for $\mathrm{C}_{17} \mathrm{H}_{21} \mathrm{~N}_{3} \mathrm{PdI}_{2}(627.60$ g/mol): C, 32.53; H, 3.37; N, 6.69. Found: C, 32.65; H, 3.56; N, 6.71. Electrospray Ms. (Cone $15 \mathrm{~V}$ ) (m/z, fragment): 500.0 [M - I] $]^{+}$. HRMS ESI-TOF-MS (positive mode): [M $\mathrm{I}]^{+}$monoisotopic peak 499.9818; calc. 499.9822, $\varepsilon_{\mathrm{r}}: 0.8 \mathrm{ppm}$. 


\section{Compound 3F}

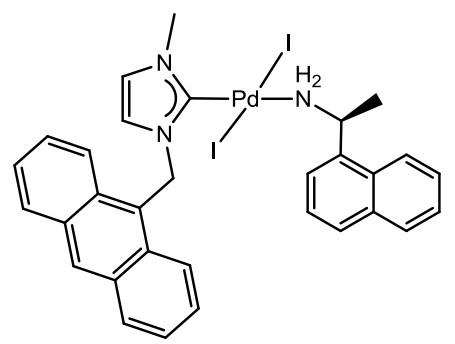

Yield: $190 \mathrm{mg}, 79 \% .{ }^{1} \mathrm{H}$ NMR $\left(300 \mathrm{MHz}, \mathrm{CDCl}_{3}\right): \delta 8.57$ $\left(\mathrm{s}, 1 \mathrm{H}, \mathrm{C} H_{\mathrm{Ar}}\right), 8.42\left(\mathrm{~d},{ }^{3} \mathrm{~J}_{\mathrm{H}, \mathrm{H}}=8.9 \mathrm{~Hz}, 3 \mathrm{H}, \mathrm{CH}_{\mathrm{Ar}}\right), 8.06(\mathrm{~d}$, $\left.{ }^{3} \mathrm{~J}_{\mathrm{H}, \mathrm{H}}=7.8 \mathrm{~Hz}, 2 \mathrm{H}, \mathrm{CH}_{\mathrm{Ar}}\right), 7.83-7.73\left(\mathrm{~m}, 2 \mathrm{H}, \mathrm{CH} \mathrm{Ar}_{\mathrm{r}}\right), 7.64$ - $7.40\left(\mathrm{~m}, 9 \mathrm{H}, \mathrm{CH}_{\mathrm{Ar}}\right), 6.60\left(\mathrm{~d},{ }^{3} \mathrm{~J}_{\mathrm{H}, \mathrm{H}}=2.0 \mathrm{~Hz}, 1 \mathrm{H}\right.$, $\left.\mathrm{CH}_{\text {imidazole }}\right), 6.58\left(\mathrm{~s}, 1 \mathrm{H}, \mathrm{CH}_{\mathrm{Ar}}\right), 6.09\left(\mathrm{~d},{ }^{3} \mathrm{~J}_{\mathrm{H}, \mathrm{H}}=2.0 \mathrm{~Hz}, 1 \mathrm{H}\right.$, $\left.\mathrm{CH}_{\text {imidazole }}\right), \quad 5.47 \quad\left(\mathrm{~m}, 1 \mathrm{H}, \mathrm{CHCH}_{3}\right), 4.10 \quad(\mathrm{~s}, 3 \mathrm{H}$, $\left.\mathrm{N}_{\text {imidazole }} \mathrm{CH}_{3}\right), 2.91(\mathrm{~m}, 1 \mathrm{H}, \mathrm{NH}), 2.75(\mathrm{~m}, 1 \mathrm{H}, \mathrm{NH}), 2.10$ $\left(\mathrm{d},{ }^{3} \mathrm{~J}_{\mathrm{H}, \mathrm{H}}=6.7 \mathrm{~Hz}, 3 \mathrm{H}, \mathrm{CHCH}_{3}\right) .{ }^{13} \mathrm{C}\left\{{ }^{1} \mathrm{H}\right\} \quad \mathrm{NMR}\left(75 \mathrm{MHz}, \mathrm{CDCl}_{3}\right): \delta, 151.6\left(C_{\text {carbene }}-\right.$ Pd), [139.6, 133.9, 131.3, 131.2, 130.5, 129.5, 129.1, 128.9, 128.4, 127.4, 126.9, 125.9, $125.4,125.4,124.3,124.0,122.9,122.4,122.3,120.4]\left(C_{\mathrm{Ar}}, C_{\text {imidazole }}\right), 47.7\left(\mathrm{CHCH}_{3}\right)$, $46.9\left(\mathrm{~N}_{\text {imidazole }} \mathrm{CH}_{2}-\right), 37.8\left(\mathrm{~N}_{\text {imidazole }} \mathrm{CH}_{3}\right), 23.1\left(\mathrm{CHCH}_{3}\right)$. Anal. Calcd. for $\mathrm{C}_{31} \mathrm{H}_{29} \mathrm{~N}_{3} \mathrm{PdI}_{2}$ (803.81 g/mol): C, 46.32; H, 3.63; N, 5.22. Found: C, 46.53; H, 3.87; N, 5.67. Electrospray Ms. (Cone 15V) (m/z, fragment): $676.9[\mathrm{M}-\mathrm{I}]^{+}$.

\section{General procedure for the synthesis of chiral palladacycles bearing primary amines} with NHC ligands: In a round bottom flask were placed together $0.3 \mathrm{mmol}$ of NHCpalladium-amine compound (3D or 3F) and $0.72 \mathrm{mmol}$ of $\mathrm{AgOAc}$ in $10 \mathrm{~mL}$ of acetonitrile. The mixture was refluxed overnight. The resulting mixture was filtered over celite and the solvent was removed under reduced pressure. The crude was redisolved in dichloromethane and washed with a saturated $\mathrm{NaCl}$ solution $(3 \times 10 \mathrm{~mL})$. The organic phase was dried with anhydrous $\mathrm{MgSO}_{4}$. Crystallization from dichloromethane/hexanes afforded the products as pale yellow crystalline solids.

\section{Compound 4D}

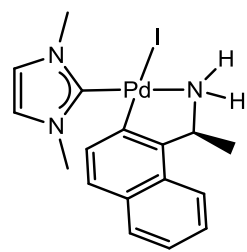

Yield: $120 \mathrm{mg}, 80 \% .{ }^{1} \mathrm{H}$ NMR $\left(300 \mathrm{MHz}, \mathrm{CDCl}_{3}\right): \delta 7.70\left(\mathrm{~d},{ }^{3} \mathrm{~J}_{\mathrm{H}, \mathrm{H}}=\right.$ $\left.8.9 \mathrm{~Hz}, 1 \mathrm{H}, \mathrm{CH}_{\mathrm{Ar}}\right), 7.64\left(\mathrm{~d},{ }^{3} \mathrm{~J}_{\mathrm{H}, \mathrm{H}}=8.0 \mathrm{~Hz}, 1 \mathrm{H}, \mathrm{CH}_{\mathrm{Ar}}\right), 7.42-7.26(\mathrm{~m}$, $\left.3 \mathrm{H}, \mathrm{CH}_{\mathrm{Ar}}\right), 6.95\left(\mathrm{dd},{ }^{3} \mathrm{~J}_{\mathrm{H}, \mathrm{H}}=11.3,1.7 \mathrm{~Hz}, 2 \mathrm{H}, \mathrm{CH}_{\mathrm{Ar}}\right), 6.18\left(\mathrm{~d},{ }^{3} \mathrm{~J}_{\mathrm{H}, \mathrm{H}}=\right.$ $\left.8.3 \mathrm{~Hz}, 1 \mathrm{H}, \mathrm{CH}_{\mathrm{Ar}}\right), 5.21\left(\mathrm{~m}, 1 \mathrm{H}, \mathrm{CHCH}_{3}\right), 3.94\left(\mathrm{~s}, 3 \mathrm{H}, \mathrm{N}_{\text {imidazole }} \mathrm{CH}_{3}\right)$, $3.81\left(\mathrm{~s}, 3 \mathrm{H}, \mathrm{N}_{\text {imidazole }} \mathrm{CH}_{3}\right), 3.76(\mathrm{~m}, 1 \mathrm{H}, \mathrm{NH}), 3.12(\mathrm{~m}, 1 \mathrm{H}, \mathrm{NH}), 1.80$ $\left(\mathrm{d},{ }^{3} \mathrm{~J}_{\mathrm{H}, \mathrm{H}}=6.4 \mathrm{~Hz}, 3 \mathrm{H}, \mathrm{CHCH}_{3}\right) \cdot{ }^{13} \mathrm{C}\left\{{ }^{1} \mathrm{H}\right\} \quad \mathrm{NMR}\left(75 \mathrm{MHz}, \mathrm{CDCl}_{3}\right): \delta 174.9\left(C_{\text {carbene }}-\right.$ $\mathrm{Pd}),[152.1,150.1,133.7,131.2,128.5,128.3,125.9,125.8,123.9,123.5,122.1,122.0]$ $\left(C_{\mathrm{Ar}}\right), 58.4\left(\mathrm{CHCH}_{3}\right), 38.7\left(\mathrm{~N}_{\text {imidazole }} C \mathrm{H}_{3}\right), 38.3\left(\mathrm{~N}_{\text {imidazole }} \mathrm{CH}_{3}\right), 25.8\left(\mathrm{CHCH}_{3}\right)$. Anal. 
Calcd. for $\mathrm{C}_{17} \mathrm{H}_{20} \mathrm{~N}_{3} \mathrm{PdI}$ (499.68 g/mol): C, 40.86; H, 4.03; N, 8.41. Found: C, 40.57; H, 3.76; N, 8.21. Electrospray Ms. (Cone 20V) (m/z, fragment): $413.0[\mathrm{M}-\mathrm{I}+\mathrm{MeCN}]^{+}$. HRMS ESI-TOF-MS (positive mode): $[\mathrm{M}-\mathrm{I}]^{+}$monoisotopic peak 372.0705; calc. $372.0699, \varepsilon_{\mathrm{r}}: 1.6 \mathrm{ppm}$.

\section{Compound 4F}

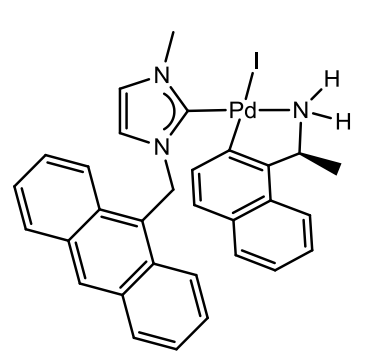

Purification by silica gel chromatography with dichloromethane/ethyl acetate $=7 / 3$ afforded the product as a pale yellow solid. Yield: $113 \mathrm{mg}, 56 \% .{ }^{1} \mathrm{H}$ NMR $(300 \mathrm{MHz}$, $\left.\mathrm{CDCl}_{3}\right): \delta 8.52-8.41\left(\mathrm{~m}, 4 \mathrm{H}, \mathrm{CH}_{\mathrm{Ar}}\right), 8.28\left(\mathrm{~d},{ }^{3} \mathrm{~J}_{\mathrm{H}, \mathrm{H}}=8.7 \mathrm{~Hz}\right.$, $\left.2 \mathrm{H}, \mathrm{CH}_{\mathrm{Ar}}\right), 8.06-7.95\left(\mathrm{~m}, 4 \mathrm{H}, \mathrm{CH}_{\mathrm{Ar}}\right), 7.79-7.65(\mathrm{~m}, 4 \mathrm{H}$, $\left.\mathrm{CH}_{\mathrm{Ar}}\right), 7.48-7.14\left(\mathrm{~m}, 16 \mathrm{H}, \mathrm{CH}_{\mathrm{Ar}}\right), 6.71\left(\mathrm{~m}, 2 \mathrm{H}, \mathrm{CH}_{\mathrm{Ar}}\right), 6.56-$ $6.44\left(\mathrm{~m}, 4 \mathrm{H}, \mathrm{CH}_{\mathrm{Ar}}\right), 6.26\left(\mathrm{~m}, 2 \mathrm{H},-\mathrm{CH}_{2}\right), 5.33\left(\mathrm{~m}, 2 \mathrm{H}, \mathrm{CHCH}_{3}\right)$, $4.31(\mathrm{~m}, 2 \mathrm{H}, \mathrm{NH}), 4.03\left(\mathrm{~s}, 3 \mathrm{H}, \mathrm{N}_{\text {imidazole }} \mathrm{CH}_{3}\right), 3.85$ (s, $\left.3 \mathrm{H}, \mathrm{N}_{\text {imidazole }} \mathrm{CH}_{3}\right), 3.29(\mathrm{~m}, 2 \mathrm{H}$, $\mathrm{NH}), 1.97-1.86\left(\mathrm{~m}, 6 \mathrm{H}, \mathrm{CHCH}_{3}\right)$. Diastereomeric ratio: 60/40 ${ }^{13} \mathrm{C}\left\{{ }^{1} \mathrm{H}\right\} \mathrm{NMR}(75 \mathrm{MHz}$, $\left.\left.\mathrm{CDCl}_{3}\right):\right) \delta[174.8,174.4]\left(C_{\text {carbene }}-\mathrm{Pd}\right),[151.5,135.3,134.9,134.0,131.3,131.3,131.2$, 131.2, 131.0, 129.2, 129.1, 129.0, 128.9, 128.6, 128.6, 128.5, 128.5, 127.1, 127.1, 125.8, 125.6, 125.3, 125.2, 125.2, 125.1, 124.2, 124.1, 123.9, 123.8, 123.4, 123.3, 121.7, 121.4] $\left(C_{\mathrm{Ar}}, C_{\text {imidazole }}\right), 56.5\left(\mathrm{CHCH}_{3}\right),[47.5,47.4]\left(\mathrm{N}_{\text {imidazole }} \mathrm{CH}_{2}-\right),[38.6,38.2]\left(\mathrm{N}_{\text {imidazole }} C \mathrm{H}_{3}\right)$, [29.7, 25.8] $\left(\mathrm{CHCH}_{3}\right)$. Anal. Calcd. for $\mathrm{C}_{31} \mathrm{H}_{28} \mathrm{~N}_{3} \mathrm{PdI}(675.90 \mathrm{~g} / \mathrm{mol})$ : C, 55.08; H, 4.17; $\mathrm{N}$, 6.22. Found: C, 55.27; H, 4.46; N, 6.35. Electrospray Ms. (Cone 20V) (m/z, fragment): $548.2[\mathrm{M}-\mathrm{I}]^{+}$. HRMS ESI-TOF-MS (positive mode): $[\mathrm{M}-\mathrm{I}]^{+}$monoisotopic peak 548.1332; calc. 548.1330, $\varepsilon_{\mathrm{r}}: 0.4 \mathrm{ppm}$.

\subsection{Synthesis and characterization of iridium complexes with ortho-metalated primary amines}

In a Schlenk was placed $0.17 \mathrm{mmol}$ of the corresponding iridium compound (5D or $\mathbf{2 C}$ ), $0.34 \mathrm{mmol}$ of silver acetate and $10 \mathrm{~mL}$ of deoxygenated acetonitrile. The mixture was stirred at room temperature for 15 minutes. To the suspension, $0.17 \mathrm{mmol}$ of 1-(1Naphthyl)ethylamine or (S)-(-)-1-(1-Naphthyl)ethylamine was added and stirred at room temperature for $4 \mathrm{~h}$. The resulting mixture was filtered over celite and the solvent was removed under reduced pressure. The crude was purified by column chromatography. 
Elution with acetone- $\mathrm{KPF}_{6}$ solution afforded the separation of a yellow band that contained the title compound. Crystallization from dichloromethane/hexane gave the corresponding compound as a yellow crystalline solid.

\section{Compound 3C}

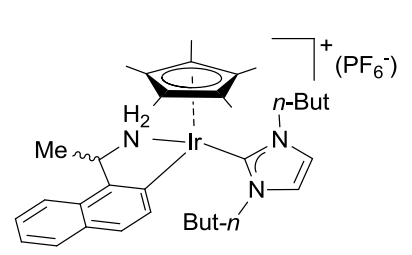

Yield: $120 \mathrm{mg}, 85 \% .{ }^{1} \mathrm{H}$ NMR $\left(300 \mathrm{MHz}, \mathrm{CD}_{2} \mathrm{Cl}_{2}\right) \delta 7.82-$ $7.71\left(\mathrm{~m}, 2 \mathrm{H}, \mathrm{CH}_{\mathrm{Ar}}\right), 7.61-7.48\left(\mathrm{~m}, 2 \mathrm{H}, \mathrm{CH}_{\mathrm{Ar}}\right), 7.39-7.23(\mathrm{~m}$, $\left.2 \mathrm{H}, \mathrm{CH}_{\mathrm{Ar}}\right), 7.17\left(\mathrm{~d},{ }^{3} \mathrm{~J}_{\mathrm{H}, \mathrm{H}}=2.1 \mathrm{~Hz}, 1 \mathrm{H}, \mathrm{CH}_{\text {imidazole }}\right), 6.93(\mathrm{~d}$, $\left.{ }^{3} \mathrm{~J}_{\mathrm{H}, \mathrm{H}}=2.1 \mathrm{~Hz}, 1 \mathrm{H}, \mathrm{CH}_{\text {imidazole }}\right), 5.06-4.91\left(\mathrm{~m}, 1 \mathrm{H}, \mathrm{CHCH}_{3}\right)$, $4.72\left(\right.$ broad s, $\left.1 \mathrm{H}, \mathrm{NH}_{2}\right), 4.30-4.13\left(\mathrm{~m}, 1 \mathrm{H}, \mathrm{NCH}_{2}-\right), 4.13-$ $3.94\left(\mathrm{~m}, 2 \mathrm{H}, \mathrm{NCH}_{2^{-}}\right), 3.85$ (broad s, $\left.1 \mathrm{H}, \mathrm{NH}_{2}\right), 3.71-3.55$ (m, $1 \mathrm{H}, \mathrm{NCH}_{2^{-}}$), $2.16-1.98$ $\left(\mathrm{m}, 1 \mathrm{H},-\mathrm{CH}_{2^{-}}\right), 1.98-1.79\left(\mathrm{~m}, 1 \mathrm{H},-\mathrm{CH}_{2^{-}}\right), 1.74-1.58\left(\mathrm{~m}, 17 \mathrm{H}, \mathrm{C}_{5}\left(\mathrm{CH}_{3}\right)_{5},-\mathrm{CH}_{2^{-}}\right), 1.14$ $-0.98\left(\mathrm{~m}, 7 \mathrm{H},-\mathrm{CH}_{2^{-}},-\mathrm{CH}_{3}\right), 0.66\left(\mathrm{~d},{ }^{3} \mathrm{~J}_{\mathrm{H}, \mathrm{H}}=6.8 \mathrm{~Hz}, 3 \mathrm{H}, \mathrm{CHCH}_{3}\right), 0.58\left(\mathrm{t},{ }^{3} \mathrm{~J}_{\mathrm{H}, \mathrm{H}}=7.1 \mathrm{~Hz}\right.$, $\left.3 \mathrm{H},-\mathrm{CH}_{3}\right) .{ }^{13} \mathrm{C}\left\{{ }^{1} \mathrm{H}\right\} \mathrm{NMR}\left(75 \mathrm{MHz}, \mathrm{CD}_{3} \mathrm{CN}\right): \delta 152.2\left(C_{\text {carbene }}-\mathrm{Ir}\right),[148.1,142.7,137.9$, $131.7,129.1,128.4,127.0,126.8,124.3]\left(C_{\mathrm{Ar}}, C_{\text {imidazole }}\right),[123.9,123.7]\left(C \mathrm{H}_{\text {imidazole }}\right), 122.0$ $\left(C_{\mathrm{Ar}}\right), 92.3\left(C_{5}\left(\mathrm{CH}_{3}\right)_{5}\right), 59.5\left(\mathrm{CHCH}_{3}\right),[51.5,50.4]\left(\mathrm{N}-\mathrm{CH}_{2}-n-\mathrm{Bu}\right),[33.6,33.5]\left(-\mathrm{CH}_{2}-\right.$ $\left.\mathrm{CH}_{2} n-\mathrm{Bu}\right),[21.0,20.4]\left(-\mathrm{CH}_{2}-\mathrm{CH}_{3} n-\mathrm{Bu}\right), 19.3\left(\mathrm{CHCH}_{3}\right),[14.2,13.8]\left(-\mathrm{CH}_{3} n-\mathrm{Bu}\right), 9.2$ $\left(\mathrm{C}_{5}\left(\mathrm{CH}_{3}\right)_{5}\right)$. Anal. Calcd. for $\mathrm{C}_{33} \mathrm{H}_{47} \mathrm{~N}_{3} \mathrm{IrPF}_{6}(822.93 \mathrm{~g} / \mathrm{mol}): \mathrm{C}, 48.16 ; \mathrm{H}, 5.75 ; \mathrm{N}, 5.10$. Found: C, 48.46; H, 5.97; N, 5.33. Electrospray Ms. (Cone 20V) (m/z, fragment): 678.5 $[\mathrm{M}]^{+}$. HRMS ESI-TOF-MS (positive mode): $[\mathrm{M}]^{+}$monoisotopic peak 678.3411; calc. $678.3401, \varepsilon_{\mathrm{r}}: 1.5 \mathrm{ppm}$.

\section{Compound 6D}

Yield $117 \mathrm{mg}, 92 \% .{ }^{1} \mathrm{H}$ NMR (300 MHz, $\left.\mathrm{CD}_{3} \mathrm{CN}\right): \delta 7.96(\mathrm{~d}$,

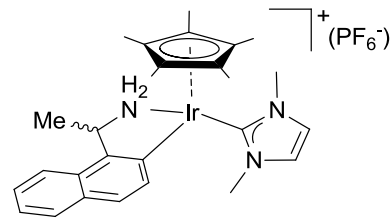

$\left.{ }^{3} \mathrm{~J}_{\mathrm{H}, \mathrm{H}}=8.4 \mathrm{~Hz}, 1 \mathrm{H}, \mathrm{C} H_{\mathrm{Ar}}\right), 7.80\left(\mathrm{~d},{ }^{3} \mathrm{~J}_{\mathrm{H}, \mathrm{H}}=8.5 \mathrm{~Hz}, 1 \mathrm{H}, \mathrm{C} H_{\mathrm{Ar}}\right)$, $7.65-7.53\left(\mathrm{~m}, 2 \mathrm{H}, \mathrm{C} H_{\mathrm{Ar}}\right), 7.41-7.27\left(\mathrm{~m}, 2 \mathrm{H}, \mathrm{C} H_{\mathrm{Ar}}\right), 7.19$ $\left(\mathrm{d},{ }^{3} \mathrm{~J}_{\mathrm{H}, \mathrm{H}}=1.9 \mathrm{~Hz}, 1 \mathrm{H}, \mathrm{C} H_{\text {imidazole }}\right), 6.97\left(\mathrm{~d},{ }^{3} \mathrm{~J}_{\mathrm{H}, \mathrm{H}}=1.9 \mathrm{~Hz}, 1 \mathrm{H}\right.$, $\mathrm{CH}_{\text {imidazole }}$ ), 5.07 (broad s, $\left.1 \mathrm{H}, \mathrm{NH}_{2}\right), 5.01-4.87(\mathrm{~m}, 1 \mathrm{H}$, $\left.\mathrm{CHCH}_{3}\right), 3.90\left(\mathrm{~s}, 3 \mathrm{H}, \mathrm{NCH}_{3}\right), 3.80$ (broad s, $\left.1 \mathrm{H}, \mathrm{NH}_{2}\right), 3.32\left(\mathrm{~s}, 3 \mathrm{H}, \mathrm{NCH}_{3}\right), 1.72(\mathrm{~s}, 15 \mathrm{H}$, $\left.\mathrm{C}_{5}\left(\mathrm{CH}_{3}\right)_{5}\right), 0.66\left(\mathrm{~d},{ }^{3} \mathrm{~J}_{\mathrm{H}, \mathrm{H}}=6.8 \mathrm{~Hz}, 3 \mathrm{H}, \mathrm{CHCH}_{3}\right) .{ }^{13} \mathrm{C}\left\{{ }^{1} \mathrm{H}\right\} \mathrm{NMR}\left(75 \mathrm{MHz}, \mathrm{CD}_{3} \mathrm{CN}\right): \delta$ 152.9 ( $\left.C_{\text {carbene }}-\mathrm{Ir}\right)$, [148.2, 142.6, 138.8, 131.8, 129.1, 128.7, 126.7, 126.1, 125.5, 124.2] $\left(C_{\mathrm{Ar},} C_{\text {imidazole }}\right),[123.9,123.8]\left(\mathrm{CH}_{\text {imidazole }}\right), 92.2\left(C_{5}\left(\mathrm{CH}_{3}\right)_{5}\right), 59.3\left(\mathrm{CHCH}_{3}\right),[39.2,39.0]$ $\left(\mathrm{NCH}_{3}\right), 18.9\left(\mathrm{CHCH}_{3}\right), 9.2\left(\mathrm{C}_{5}\left(\mathrm{CH}_{3}\right)_{5}\right)$. Anal. Calcd. for $\mathrm{C}_{27} \mathrm{H}_{35} \mathrm{~N}_{3} \mathrm{IrPF}_{6}(738.77 \mathrm{~g} / \mathrm{mol})$ : 
C, 43.89; H, 4.77; N, 5.68. Found: C, 44.25; H, 4.26; N, 5.74. Electrospray Ms. (Cone $20 \mathrm{~V})\left(\mathrm{m} / \mathrm{z}\right.$, fragment): $594.2[\mathrm{M}]^{+}$. HRMS ESI-TOF-MS (positive mode): $[\mathrm{M}]^{+}$ monoisotopic peak 594.2464; calc. 594.2462, $\varepsilon_{\mathrm{r}}: 0.3 \mathrm{ppm}$.
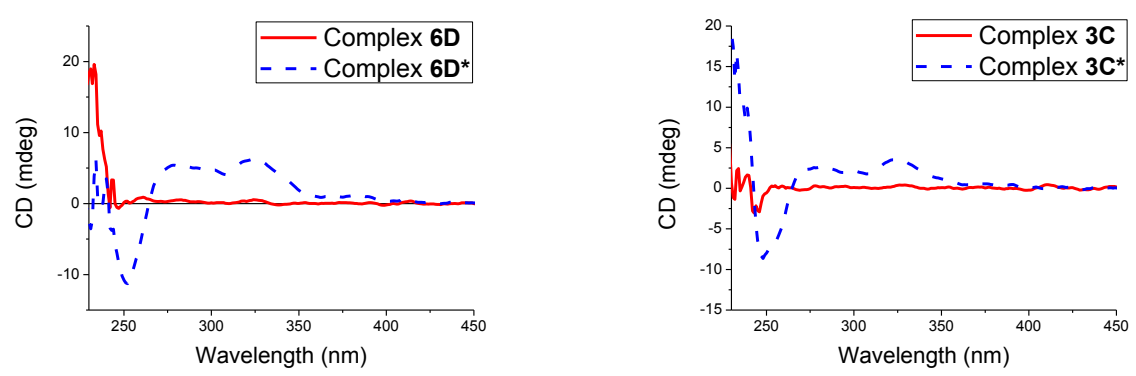

Figure 6.2 Circular dichroism spectra recorded in chloroform at $25^{\circ} \mathrm{C}$. Left: complex $6 \mathrm{D}(-)$ and complex 6D* (---). Right: complex 3C (-)and complex 3C* (---).

\subsection{Synthesis and characterization of metal complexes bearing a pyrene-tagged- NHC ligand}

Synthesis of $1 G$

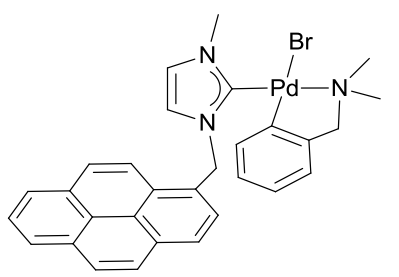

Imidazolium salt $\quad$ G $\quad(127 \quad \mathrm{mg}, \quad 0.334 \quad \mathrm{mmol})$, $[\mathrm{Pd}(\mathrm{OAc})(\mathrm{dmba})]_{2}(100 \mathrm{mg}, 0.167 \mathrm{mmol})$, and $\mathrm{KBr}(40 \mathrm{mg}$, $0.334 \mathrm{mmol}$ ), were refluxed under nitrogen in $10 \mathrm{~mL}$ of acetonitrile for 16 hours. The resulting suspension was filtered through celite and the solvent was evaporated under reduced pressure. The crude solid was purified by column chromatography. Elution with a mixture dichloromethane/Acetone 9:1 afforded the separation of a yellowish band containing the desired product. Precipitation with dichloromethane/Hexanes afforded an analytically pure yellow solid. Yield: $128 \mathrm{mg}, 60 \% .{ }^{1} \mathrm{H} \mathrm{NMR}\left(300 \mathrm{MHz}, \mathrm{CDCl}_{3}\right) \delta 8.38$ $\left(\mathrm{d},{ }^{3} \mathrm{~J}_{\mathrm{H}, \mathrm{H}}=9.3 \mathrm{~Hz}, 1 \mathrm{H}, \mathrm{C} H_{\text {pyrene }}\right), 8.19-7.89\left(\mathrm{~m}, 8 \mathrm{H}, \mathrm{CH}_{\text {pyrene }}\right), 7.09-7.01(\mathrm{~m}, 2 \mathrm{H}$, $\left.\mathrm{CH}_{\text {phenyl }}\right), 6.87\left(\mathrm{td},{ }^{3} \mathrm{~J}_{\mathrm{H}, \mathrm{H}}=7.4,2.8 \mathrm{~Hz}, 1 \mathrm{H}, \mathrm{CH}_{\text {phenyl }}\right), 6.77\left(\mathrm{~d},{ }^{3} \mathrm{~J}_{\mathrm{H}, \mathrm{H}}=1.9 \mathrm{~Hz}, 1 \mathrm{H}\right.$, $\left.\mathrm{CH}_{\text {imidazole }}\right), 6.43\left(\mathrm{~d},{ }^{3} \mathrm{~J}_{\mathrm{H}, \mathrm{H}}=1.9 \mathrm{~Hz}, 1 \mathrm{H}, \mathrm{CH}_{\text {imidazole }}\right), 6.31,6.18\left(\mathrm{AB},{ }^{3} \mathrm{~J}_{\mathrm{AB}}=14.5 \mathrm{~Hz}, 2 \mathrm{H}\right.$, $\left.\mathrm{N}_{\text {imidazole }} \mathrm{CH}_{2^{-}}\right), 6.15\left(\mathrm{~d},{ }^{3} \mathrm{~J}_{\mathrm{H}, \mathrm{H}}=6,9 \mathrm{~Hz}, 1 \mathrm{H}, \mathrm{C} H_{\text {Arphenyl }}\right), 4.01,3.87\left(\mathrm{AB},{ }^{3} \mathrm{~J}_{\mathrm{AB}}=14.0 \mathrm{~Hz}\right.$, $2 \mathrm{H}, \mathrm{N}_{\text {amine }} \mathrm{CH}_{2^{-}}$), 3.99 (s, 3H, N $\left.\mathrm{N}_{\text {imidazole }} \mathrm{CH}_{3}\right), 2.95$ (s, 3H, $\left.\mathrm{N}_{\text {amine }} \mathrm{CH}_{3}\right), 2.92$ (s, 3H, $\left.\mathrm{N}_{\text {amine }} \mathrm{CH}_{3}\right) .{ }^{13} \mathrm{C}\left\{{ }^{1} \mathrm{H}\right\} \mathrm{NMR}\left(75 \mathrm{MHz}, \mathrm{CDCl}_{3}\right): \delta 172.9\left(C_{\text {carbene }}-\mathrm{Pd}\right),[150.3,148.7,135.6$, 
$131.8,131.1,130.7,129.7,128.8,128.6,128.1,127.9,127.2,126.1,125.7,125.6,125.5$, $124.9,124.7,124.5,124.1,123.7,122.5,122.1,120.3]\left(C_{\text {pyrene, }} C \mathrm{H}_{\text {imidazole }}\right), \quad 72.2$ $\left(\mathrm{N}_{\text {amine }} C \mathrm{H}_{2}-\right), 53.7\left(\mathrm{~N}_{\text {imidazole }} C \mathrm{H}_{2}-\right)$, [51.2, 50.6] $\left(\mathrm{N}_{\text {amine }} C \mathrm{H}_{3}\right), 38.7\left(\mathrm{NCH}_{3}\right)$. Anal. Calcd. for $\mathrm{C}_{30} \mathrm{H}_{28} \mathrm{~N}_{3} \mathrm{PdBr} \cdot \mathrm{H}_{2} \mathrm{O}$ (634.90 g/mol): C, 56.75; H, 4.76; N, 6.62. Found: C, 56.93; H, 5.09; $\mathrm{N}$, 6.35. Electrospray Ms. (Cone 20V) (m/z, fragment): $536.3[\mathrm{M}-\mathrm{Br}]^{+}$. HRMS ESITOF-MS (positive mode): $[\mathrm{M}-\mathrm{Br}]^{+}$monoisotopic peak 536.1333; calc. 536.1330, $\varepsilon_{\mathrm{r}}$ : 0.6 ppm.

\section{Synthesis of 2G}

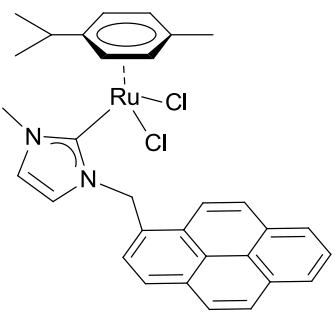

In a round-bottom flask were mixed, under exclusion of light, the imidazolium salt $\mathbf{G}(124.2 \mathrm{mg}, 0.326 \mathrm{mmol})$ and $\mathrm{Ag}_{2} \mathrm{O}(75.5 \mathrm{mg}$, $0.326 \mathrm{mmol})$ in $10 \mathrm{~mL}$ of acetonitrile and the suspension was refluxed for 5 hours. Then $\left[\mathrm{RuCl}_{2}(p \text {-cymene })\right]_{2}$ (100 mg, 0,163 $\mathrm{mmol})$ and $\mathrm{KCl}(243 \mathrm{mg}, 3,25 \mathrm{mmol})$ were added and the reaction mixture was stirred at room temperature for 15 hours.

The resulting suspension was filtered through celite and the solvent was evaporated under reduced pressure. The crude solid was purified by column chromatography. Elution with a mixture of dichloromethane/Acetone (9:2) afforded the separation of an orange band containing the desired product. Precipitation with dichloromethane/ diethyl ether afforded an analytically pure orange solid. Yield: $120 \mathrm{mg}, 61 \% .{ }^{1} \mathrm{H} \mathrm{NMR}\left(300 \mathrm{MHz}, \mathrm{CDCl}_{3}\right): \delta$ $8.38\left(\mathrm{~d},{ }^{3} \mathbf{J}_{\mathrm{H}, \mathrm{H}}=9.3 \mathrm{~Hz}, 1 \mathrm{H}, \mathrm{CH}_{\text {pyrene }}\right), 8.27-8.00\left(\mathrm{~m}, 7 \mathrm{H}, \mathrm{C} H_{\text {pyrene }}\right), 7.70\left(\mathrm{~d},{ }^{3} \mathbf{J}_{\mathrm{H}, \mathrm{H}}=8.0\right.$ $\left.\mathrm{Hz}, 1 \mathrm{H}, \mathrm{CH}_{\text {pyrene }}\right), 7.04\left(\mathrm{~d},{ }^{3} \mathrm{~J}_{\mathrm{H}, \mathrm{H}}=1.9 \mathrm{~Hz}, 1 \mathrm{H}, \mathrm{C} H_{\text {imidazole }}\right), 6.92\left(\mathrm{~d},{ }^{3} \mathrm{~J}_{\mathrm{H}, \mathrm{H}}=1.8 \mathrm{~Hz}, 1 \mathrm{H}\right.$, $\left.\mathrm{CH}_{\text {imidazole }}\right), 6.75-6.33\left(\right.$ broad s, $\left.2 \mathrm{H}, \mathrm{NCH}_{2^{-}}\right), 5.26\left(\operatorname{broad~s}, 2 \mathrm{H}, \mathrm{CH}_{p \text {-cym }}\right), 4.89\left(\mathrm{~d},{ }^{3} \mathrm{~J}_{\mathrm{H}, \mathrm{H}}=\right.$ $\left.5.9 \mathrm{~Hz}, 2 \mathrm{H} . \mathrm{CH}_{p \text {-cym }}\right), 4.11\left(\mathrm{~s}, 3 \mathrm{H}, \mathrm{NCH}_{3}\right), 2.98-2.76\left(\mathrm{~m}, 1 \mathrm{H}, \mathrm{CH}_{\text {isop }(p-\mathrm{cym})}\right), 2.02(\mathrm{~s}, 3 \mathrm{H}$, $\left.\mathrm{CH}_{3(p-c y m)}\right), 1.18\left(\mathrm{~d},{ }^{3} \mathrm{~J}_{\mathrm{H}, \mathrm{H}}=6.7 \mathrm{~Hz}, 6 \mathrm{H}, \mathrm{CH}_{3 \mathrm{isop}(p-\mathrm{cym})}\right) .{ }^{13} \mathrm{C}\left\{{ }^{1} \mathrm{H}\right\} \mathrm{NMR}\left(75 \mathrm{MHz}, \mathrm{CDCl}_{3}\right): \delta$ $174.8\left(C_{\text {carbene }}-\mathrm{Ru}\right),[131.3,131.1,130.8,130.8,128.7,128.4,127.8,127.2,126.3,125.6$, $125.6,124.9,124.7,124.6,124.6,123.8,123.6,122.4]\left(C_{\text {pyrene, }} C H_{\text {imidazole }}\right),[108.1,98.9]$ $\left(C \mathrm{q}_{p \text {-cym }}\right),[85.1,82.4]\left(\mathrm{CH}_{p \text {-cym }}\right), 52.8\left(\mathrm{NCH}_{2}\right), 39.9\left(\mathrm{NCH}_{3}\right), 30.7\left(\mathrm{CH}_{\text {isop }(p-\mathrm{cym})}\right)$,

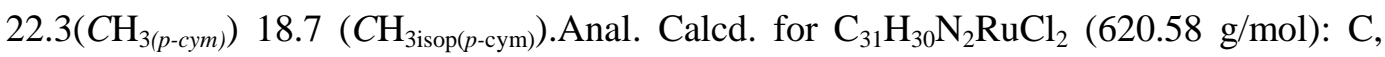
59.99; H, 5.19; N, 4.51. Found: C, 60.38; H, 5.43; N, 4.62. Electrospray Ms. (Cone 20V) (m/z, fragment): $567.2[\mathrm{M}-\mathrm{Cl}]^{+}$. HRMS ESI-TOF-MS (positive mode): $[\mathrm{M}-\mathrm{Cl}]^{+}$ monoisotopic peak 567.1144; calc. 567.1146, $\varepsilon_{\mathrm{r}}: 0.4 \mathrm{ppm}$. 


\section{Synthesis of 3G}

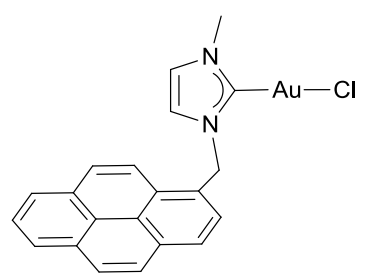

In a round-bottom flask were mixed, under exclusion of light, the imidazolium salt $\mathbf{G}(128.3 \mathrm{mg}, 0.339 \mathrm{mmol})$ and $\mathrm{Ag}_{2} \mathrm{O}(78$ $\mathrm{mg}, 0.339 \mathrm{mmol})$ in $10 \mathrm{~mL}$ of acetonitrile and the suspension was refluxed for 5 hours. Then $\left[\mathrm{AuCl}\left(\mathrm{SMe}_{2}\right)\right](100 \mathrm{mg}, 0,339$ $\mathrm{mmol})$ and $\mathrm{KCl}(243 \mathrm{mg}, 3,25 \mathrm{mmol})$ were added and the reaction mixture was stirred at room temperature for 15 hours. The resulting suspension was filtered through celite and the solvent was evaporated under reduced pressure. Precipitation with dichloromethane/ hexane afforded an analytically pure white solid. Yield: $145 \mathrm{mg}, 81 \% .{ }^{1} \mathrm{H}$ NMR (300 MHz, $\left.\mathrm{CDCl}_{3}\right): \delta 8.20-7.91\left(\mathrm{~m}, 8 \mathrm{H}, \mathrm{CH}_{\text {pyrene }}\right), 7.79$ $\left(\mathrm{d},{ }^{3} \mathrm{~J}_{\mathrm{H}, \mathrm{H}}=7.8 \mathrm{~Hz}, 1 \mathrm{H}, \mathrm{C} H_{\text {pyrene }}\right), 6.71\left(\mathrm{~d},{ }^{3} \mathrm{~J}_{\mathrm{H}, \mathrm{H}}=1.9 \mathrm{~Hz}, 1 \mathrm{H}, \mathrm{C} H_{\text {imidazole }}\right), 6.54\left(\mathrm{~d},{ }^{3} \mathrm{~J}_{\mathrm{H}, \mathrm{H}}=\right.$ $\left.1.9 \mathrm{~Hz}, 1 \mathrm{H}, \mathrm{CH}_{\text {imidazole }}\right), 5.84\left(\mathrm{~s}, 2 \mathrm{H}, \mathrm{CH}_{2}\right), 3.78\left(\mathrm{~s}, 3 \mathrm{H}, \mathrm{NCH}_{3}\right) .{ }^{13} \mathrm{C}\left\{{ }^{1} \mathrm{H}\right\} \mathrm{NMR}(75 \mathrm{MHz}$, $\left.\mathrm{CDCl}_{3}\right): \delta 171.3\left(C_{\text {carbene }}-\mathrm{Au}\right),[131.8,131.0,130.4,128.9,128.1,127.5,127.1,126.9$, $126.3,125.8,125.7,124.8,124.3]\left(C_{\text {pyrene }}, C \mathrm{H}_{\text {imidazole }}\right), 52.9\left(\mathrm{CH}_{2}\right), 38.3\left(\mathrm{NCH}_{3}\right)$. Anal. Calcd. for $\mathrm{C}_{21} \mathrm{H}_{16} \mathrm{~N}_{2} \mathrm{AuCl}(528.78 \mathrm{~g} / \mathrm{mol}): \mathrm{C}, 47.70 ; \mathrm{H}, 3.05 ; \mathrm{N}, 5.30$. Found: C, 47.87; H, 3.27; N, 5.39. Electrospray Ms. (Cone 20V) (m/z, fragment): $567.2[\mathrm{M}+\mathrm{K}]^{+}$. HRMS ESI-TOF-MS (positive mode): $[\mathrm{M}+\mathrm{K}]^{+}$monoisotopic peak 567.0309; calc. 567.0305, $\varepsilon_{\mathrm{r}}$ : $0.7 \mathrm{ppm}$.

\section{Synthesis of 4G}

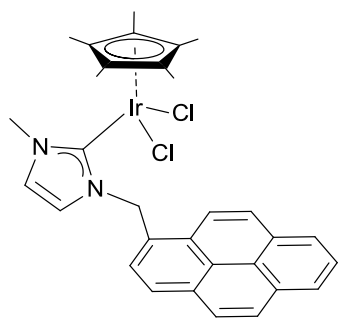

In a Schlenk, a mixture of the imidazolium salt $\mathbf{G}(95 \mathrm{mg}, 0.250$ mmol) and potassium tert-butoxide (33 $\mathrm{mg}, 0.280 \mathrm{mmol}$ ) was cooled to $0^{\circ} \mathrm{C}$, in an ice bath. Freshly distilled tetrahydrofuran $(5$ $\mathrm{mL}$ ) was added, and the mixture was stirred for $10 \mathrm{~min}$. and allowed to reach room temperature. Then, $\left[\mathrm{IrCp}^{*} \mathrm{Cl}_{2}\right]_{2}(100 \mathrm{mg}$, $0.125 \mathrm{mmol})$ and $\mathrm{KCl}(243 \mathrm{mg}, 3.25 \mathrm{mmol})$ were added and the reaction mixture was stirred for $4 \mathrm{~h}$ at room temperature. The resulting suspension was filtered through celite and the solvent was evaporated under reduced pressure. The crude solid was purified by column chromatography. Elution with a mixture of dichloromethane/Acetone (9:1) afforded the separation of a yellow band containing the desired product. Precipitation with dichloromethane/ hexane afforded an analytically pure yellow solid. Yield: $111 \mathrm{mg}, 64 \% .{ }^{1} \mathrm{H}$ NMR $\left(300 \mathrm{MHz}, \mathrm{CDCl}_{3}\right): \delta 8.40-8.35(\mathrm{~m}, 1 \mathrm{H}$, 
$\left.\mathrm{CH}_{\text {pyrene }}\right), 8.26-8.00\left(\mathrm{~m}, 7 \mathrm{H}, \mathrm{CH}_{\text {pyrene }}\right), 7.87-7.82\left(\mathrm{~m}, 1 \mathrm{H}, \mathrm{CH}_{\text {pyrene }}\right), 6.90\left(\mathrm{~d},{ }^{3} \mathrm{~J}_{\mathrm{H}, \mathrm{H}}=2.0\right.$ $\left.\mathrm{Hz}, 1 \mathrm{H}, \mathrm{CH}_{\text {imidazole }}\right), 6.72-6.61\left(\mathrm{~m}, 2 \mathrm{H}, \mathrm{CH}_{2}\right), 6.28\left(\mathrm{~d},{ }^{3} \mathrm{~J}_{\mathrm{H}, \mathrm{H}}=2.0 \mathrm{~Hz}, 1 \mathrm{H}, \mathrm{CH}_{\text {imidazole }}\right)$, $4.07\left(\mathrm{~s}, 3 \mathrm{H}, \mathrm{NCH}_{3}\right), 1.62\left(\mathrm{~s}, 15 \mathrm{H}, \mathrm{C}\left(\mathrm{CH}_{3}\right)_{5}\right) \cdot{ }^{13} \mathrm{C}\left\{{ }^{1} \mathrm{H}\right\} \mathrm{NMR}\left(75 \mathrm{MHz}, \mathrm{CDCl}_{3}\right): \delta 157.1$ $\left(C_{\text {carbene- }}\right.$ Ir), [131.2, 131.1, 130.7, 129.8, 129.0, 128.6, 127.7, 127.2, 126.2, 125.9, 125.6, 125.5, 124.7, 123.1, 122.5, 122.3] $\left(C_{\text {pyrene, }} C \mathrm{H}_{\text {imidazole }}\right), 88.9\left(C\left(\mathrm{CH}_{3}\right)_{5}\right), 52.1\left(\mathrm{CH}_{2}\right), 38.9$ $\left(\mathrm{NCH}_{3}\right), 9.2\left(\mathrm{C}\left(\mathrm{CH}_{3}\right)_{5}\right)$. Anal. Calcd. for $\mathrm{C}_{31} \mathrm{H}_{31} \mathrm{~N}_{2} \mathrm{IrCl}_{2}(694.71 \mathrm{~g} / \mathrm{mol}): \mathrm{C}, 53.59 ; \mathrm{H}, 4.49$; $\mathrm{N}$, 4.03. Found: C, 53.67; H, 4.27; N, 4.37. Electrospray Ms. (Cone 20V) $(\mathrm{m} / \mathrm{z}$, fragment): $659.3[\mathrm{M}-\mathrm{Cl}]^{+}$. HRMS ESI-TOF-MS (positive mode): $[\mathrm{M}-\mathrm{Cl}]^{+}$ monoisotopic peak 659.1803 ; calc. $659.1798, \varepsilon_{\mathrm{r}}: 0.8 \mathrm{ppm}$.

\subsection{Synthesis and characterization of functionalized Ru-triazolylidene complexes}

\section{Synthesis of $1 \mathrm{H}$}

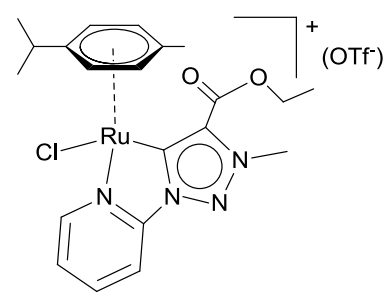

In a round-bottom flask were mixed, under exclusion of light, the triazolium salt $\mathbf{H}(300 \mathrm{mg}, 0.784 \mathrm{mmol})$ and $\mathrm{Ag}_{2} \mathrm{O}(272.3$ $\mathrm{mg}, 1.176 \mathrm{mmol}$ ) in $10 \mathrm{~mL}$ of dichloromethane and the suspension was stirred for 24 hours. Then $\left[\mathrm{RuCl}_{2}(p \text {-cymene })\right]_{2}$ (235 $\mathrm{mg}, 0,384 \mathrm{mmol}$ ) was added and the reaction mixture was stirred at room temperature for 12 hours. The resulting suspension was filtered through celite and the solvent was evaporated under reduced pressure. The crude solid was purified by column chromatography. Elution with a mixture of dichloromethane/methanol (40:1) afforded the separation of an orange band containing the desired product. Precipitation with acetonitrile/ diethyl ether afforded an analytically pure orange solid. Yield: $385 \mathrm{mg}, 77 \%$. ${ }^{1} \mathrm{H} \mathrm{NMR}\left(400 \mathrm{MHz}, \mathrm{CD}_{3} \mathrm{CN}\right.$ ): $\delta 9.27$ $\left(\mathrm{d},{ }^{3} \mathrm{~J}_{\mathrm{H}, \mathrm{H}}=5.7 \mathrm{~Hz}, 1 \mathrm{H}, \mathrm{C} H_{\mathrm{py}}\right), 8.30-8.23\left(\mathrm{~m}, 1 \mathrm{H}, \mathrm{C} H_{\mathrm{py}}\right), 8.19-8.14\left(\mathrm{~d}, 1 \mathrm{H}, \mathrm{C} H_{\mathrm{py}}\right), 7.73-$ $7.65\left(\mathrm{~m}, 1 \mathrm{H}, \mathrm{CH}_{\mathrm{py}}\right), 6.23\left(\mathrm{~d},{ }^{3} \mathrm{~J}_{\mathrm{H}, \mathrm{H}}=6.1 \mathrm{~Hz}, 1 \mathrm{H}, \mathrm{CH}_{p \text {-cym }}\right), 6.15\left(\mathrm{~d},{ }^{3} \mathrm{~J}_{\mathrm{H}, \mathrm{H}}=6.2 \mathrm{~Hz}, 1 \mathrm{H}\right.$, $\left.\mathrm{C}_{p \text {-cym }}\right), 5.82\left(\mathrm{~d},{ }^{3} \mathrm{~J}_{\mathrm{H}, \mathrm{H}}=6.2 \mathrm{~Hz}, 1 \mathrm{H}, \mathrm{CH}_{p \text {-cym }}\right), 5.43\left(\mathrm{~d},{ }^{3} \mathrm{~J}_{\mathrm{H}, \mathrm{H}}=6.1 \mathrm{~Hz}, 1 \mathrm{H}, \mathrm{CH}_{p \text {-cym }}\right), 4.66$

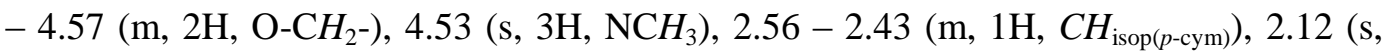
$\left.3 \mathrm{H}, \mathrm{CH}_{3(p-\mathrm{cym})}\right), 1.51\left(\mathrm{t},{ }^{3} \mathrm{~J}_{\mathrm{H}, \mathrm{H}}=7.1 \mathrm{~Hz}, 3 \mathrm{H},-\mathrm{CH}_{3}\right), 1.01\left(\mathrm{~d},{ }^{3} \mathrm{~J}_{\mathrm{H}, \mathrm{H}}=6.9 \mathrm{~Hz}, 3 \mathrm{H}, \mathrm{CH}_{3 \text { (isop }(p-}\right.$ cym)) $), 0.90\left(\mathrm{~d},{ }^{3} \mathrm{~J}_{\mathrm{H}, \mathrm{H}}=6.9 \mathrm{~Hz}, 3 \mathrm{H}, \mathrm{CH}_{3(\text { isop }(p \text {-cym) })}\right) .{ }^{13} \mathrm{C}\left\{{ }^{1} \mathrm{H}\right\} \mathrm{NMR}\left(101 \mathrm{MHz}, \mathrm{CD}_{3} \mathrm{CN}\right): \delta$ $177.1\left(C_{\text {carbene }}-\mathrm{Ru}\right), 159.2(C \mathrm{OO}-), 156.8\left(C_{\mathrm{py}}\right), 150.5\left(C_{\mathrm{q}(\mathrm{py})}\right), 143.1\left(\mathrm{CH}_{\mathrm{py}}\right), 139.8$ $\left(C_{\mathrm{q}(\mathrm{tz})}\right), 127.6\left(\mathrm{CH}_{\mathrm{py}}\right), 115.4\left(\mathrm{CH}_{\mathrm{py}}\right),[108.7,107.9]\left(C_{\mathrm{q}(p-\mathrm{cym}}\right),[91.1,89.4,89.0,83.2]$ 
$\left(\mathrm{CH}_{p \text {-cym }}\right), 64.2\left(\mathrm{O}-\mathrm{CH}_{2}-\right), 43.3\left(\mathrm{~N}-\mathrm{CH}_{3}\right), 31.9\left(\mathrm{CH}_{\text {isop }(p \text {-cym })}\right)$, [22.6, 22.5] $\left(\mathrm{CH}_{3 \text { (isop }(p \text {-cym }))}\right)$, $19.3\left(\mathrm{CH}_{3(p-\text { cym })}\right), 14.5\left(-\mathrm{CH}_{3}\right)$. Anal. Calcd. for $\mathrm{C}_{21} \mathrm{H}_{26} \mathrm{~N}_{4} \mathrm{O}_{2} \mathrm{RuClCF}_{3} \mathrm{SO}_{3}(652.05 \mathrm{~g} / \mathrm{mol})$ : C, 40.51; H, 4.02; N, 8.59. Found: C, 39.99; H, 3.79; N, 8.62. Electrospray Ms. (Cone $20 \mathrm{~V})\left(\mathrm{m} / \mathrm{z}\right.$, fragment): $502.3[\mathrm{M}]^{+}$. IR $\left(\mathrm{CH}_{3} \mathrm{CN}\right)=1729.1 \mathrm{~cm}^{-1},(\mathrm{C}=\mathrm{O})$.

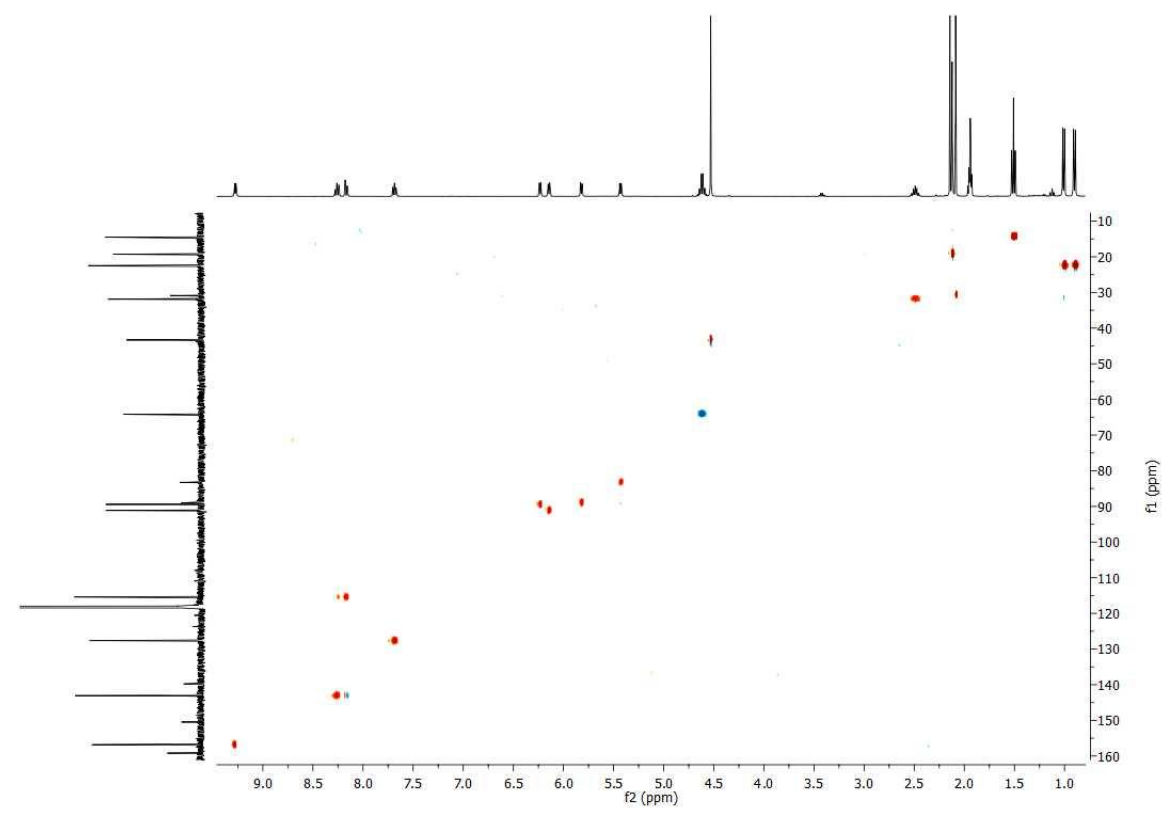

Figure 6.3 ${ }^{13} \mathrm{C}$ HSQC NMR spectrum $\left({ }^{1} \mathrm{H}, 400 \mathrm{MHz}\right.$ and $\left.{ }^{13} \mathrm{C}, 100 \mathrm{MHz}\right)$ of $\mathbf{1 H}$ in $\mathrm{CD}_{3} \mathrm{CN}$

\section{Synthesis of $2 \mathrm{H}$}

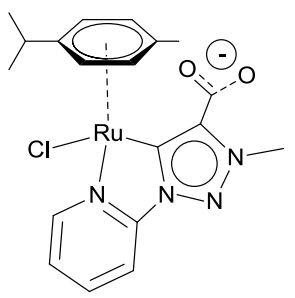

In a round-bottom flask were mixed, the complex $\mathbf{1 H}(100 \mathrm{mg}$, $0.153 \mathrm{mmol})$ and $\mathrm{LiOH} \cdot \mathrm{H}_{2} \mathrm{O}(14 \mathrm{mg}, 0.306 \mathrm{mmol})$ in $5 \mathrm{~mL}$ of a methanol/water (1:1) mixture and the suspension was stirred for 3 hours at $30^{\circ} \mathrm{C}$. The reaction mixture was extracted with dichloromethane/water mixtures. The aqueous layers were combined and the solvent was removed under reduced pressure. The

crude was redisolved in acetonitrile and filtered through celite. Precipitation with acetonitrile/ diethyl ether afforded an analytically pure orange solid. Yield: $60 \mathrm{mg}, 83 \%$. ${ }^{1} \mathrm{H}$ NMR $\left(300 \mathrm{MHz}, \mathrm{CD}_{3} \mathrm{CN}\right): \delta 9.28\left(\mathrm{~d},{ }^{3} \mathrm{~J}_{\mathrm{H}, \mathrm{H}}=5.3 \mathrm{~Hz}, 1 \mathrm{H}, \mathrm{CH}_{\mathrm{py}}\right), 8.25-8.19(\mathrm{~m}, 1 \mathrm{H}$, $\left.\mathrm{CH}_{\mathrm{py}}\right), 8.14-8.09\left(\mathrm{~m}, 1 \mathrm{H}, \mathrm{CH}_{\mathrm{py}}\right), 7.66-7.61\left(\mathrm{~m}, 1 \mathrm{H}, \mathrm{CH}_{\mathrm{py}}\right), 6.53\left(\mathrm{~d},{ }^{3} \mathrm{~J}_{\mathrm{H}, \mathrm{H}}=5.8 \mathrm{~Hz}, 1 \mathrm{H}\right.$, 
$\left.\mathrm{CH}_{p \text {-cym }}\right), 6.25\left(\mathrm{~d},{ }^{3} \mathrm{~J}_{\mathrm{H}, \mathrm{H}}=6.1 \mathrm{~Hz}, 1 \mathrm{H}, \mathrm{C} H_{p \text {-cym }}\right), 5.78\left(\mathrm{~d},{ }^{3} \mathrm{~J}_{\mathrm{H}, \mathrm{H}}=6.2 \mathrm{~Hz}, 1 \mathrm{H}, \mathrm{CH}_{p \text {-cym }}\right), 5.54$ $\left(\mathrm{d},{ }^{3} \mathrm{~J}_{\mathrm{H}, \mathrm{H}}=5.6 \mathrm{~Hz}, 1 \mathrm{H}, \mathrm{CH}_{p \text {-cym }}\right), 4.59\left(\mathrm{~s}, 3 \mathrm{H}, \mathrm{NCH}_{3}\right), 2.54-2.42\left(\mathrm{~m}, 1 \mathrm{H}, \mathrm{CH}_{\text {isop }(p-\mathrm{cym})}\right)$, $2.09\left(\mathrm{~s}, 3 \mathrm{H}, \mathrm{CH}_{3(p-\mathrm{cym})}\right), 0.98\left(\mathrm{~d},{ }^{3} \mathrm{~J}_{\mathrm{H}, \mathrm{H}}=6.9 \mathrm{~Hz}, 3 \mathrm{H}, \mathrm{CH}_{3 \text { (isop(p-cym) })}\right), 0.85\left(\mathrm{~d},{ }^{3} \mathrm{~J}_{\mathrm{H}, \mathrm{H}}=6.9 \mathrm{~Hz}\right.$, $\left.3 \mathrm{H}, \mathrm{CH}_{3 \text { (isop }(p \text {-cym) })}\right) .{ }^{13} \mathrm{C}\left\{{ }^{1} \mathrm{H}\right\} \mathrm{NMR}\left(101 \mathrm{MHz}, \mathrm{CD}_{3} \mathrm{CN}\right): \delta 172.3\left(C_{\text {carbene }}-\mathrm{Ru}\right), 162.6$ (COO-), $156.7\left(\mathrm{CH}_{\mathrm{py}}\right), 151.1\left(\mathrm{CH}_{\mathrm{py}}\right), 150.5\left(C_{\mathrm{q}(\mathrm{py})}\right), 146.0\left(C_{\mathrm{q}(\mathrm{tz})}\right), 142.6\left(C_{\mathrm{py}}\right), 126.9$ $\left(C \mathrm{H}_{\mathrm{py}}\right), 114.9\left(\mathrm{CH}_{\mathrm{py}}\right),[108.0,106.4]\left(C_{\mathrm{q}(p-\mathrm{cym})}\right),[90.9,90.3,88.5,82.5]\left(C_{p-\text { cym }}\right), 41.9(\mathrm{~N}-$ $\left.\mathrm{CH}_{3}\right), 31.9\left(\mathrm{CH}_{\text {isop }(p \text {-cym })}\right), 22.7\left(\mathrm{CH}_{3 \text { (isop }(p \text {-cym }))}\right), 19.5\left(\mathrm{CH}_{3(p-\text { cym })}\right)$. Attempts to obtain satisfactory elemental analysis of $\mathbf{2 H}$ failed, probably because this species is highly hygroscopic. Electrospray Ms. (Cone 20V) (m/z, fragment): $439.0[\mathrm{M}-\mathrm{Cl}]^{+}$. IR $\left(\mathrm{CH}_{3} \mathrm{CN}\right)=1618.2 \mathrm{~cm}^{-1},(\mathrm{C}=\mathrm{O})$.

\section{Synthesis of $3 \mathbf{H}$}

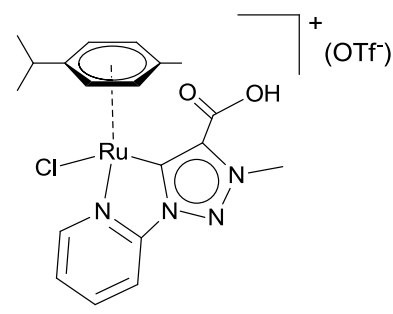

In a round-bottom flask were mixed, the complex $\mathbf{1 H}$ (100 $\mathrm{mg}, 0.153 \mathrm{mmol})$ and $\mathrm{LiOH} \cdot \mathrm{H}_{2} \mathrm{O}(14 \mathrm{mg}, 0.306 \mathrm{mmol})$ in 5 $\mathrm{mL}$ of a methanol/water $(1: 1)$ mixture and the suspension was stirred for 3 hours at $30^{\circ} \mathrm{C}$. The reaction mixture was acidified with $\mathrm{HCl}$ aqueous solution (1M) and extracted with dichloromethane/water mixtures. The organic layers were combined and the solvent was removed under reduced pressure. Precipitation with acetonitrile/ diethyl ether afforded an analytically pure orange solid. Yield: $88 \mathrm{mg}, 92 \%$. ${ }^{1} \mathrm{H}$ NMR $\left(400 \mathrm{MHz}, \mathrm{CD}_{3} \mathrm{CN}\right): \delta 9.29\left(\mathrm{~d},{ }^{3} \mathrm{~J}_{\mathrm{H}, \mathrm{H}}=5.6 \mathrm{~Hz}, 1 \mathrm{H}, \mathrm{CH}_{\mathrm{py}}\right), 8.26-8.21(\mathrm{~m}, 1 \mathrm{H}$, $\left.\mathrm{CH}_{\mathrm{py}}\right), 8.16-8.12\left(\mathrm{~m}, 1 \mathrm{H}, \mathrm{CH}_{\mathrm{py}}\right), 7.68-7.63\left(\mathrm{~m}, 1 \mathrm{H}, \mathrm{CH}_{\mathrm{py}}\right), 6.41\left(\mathrm{~d},{ }^{3} \mathrm{~J}_{\mathrm{H}, \mathrm{H}}=6.0 \mathrm{~Hz}, 1 \mathrm{H}\right.$, $\left.\mathrm{CH}_{p \text {-cym }}\right), 6.19\left(\mathrm{~d},{ }^{3} \mathrm{~J}_{\mathrm{H}, \mathrm{H}}=5.4 \mathrm{~Hz}, 1 \mathrm{H}, \mathrm{CH}_{p \text {-cym }}\right), 5.89\left(\mathrm{~d},{ }^{3} \mathrm{~J}_{\mathrm{H}, \mathrm{H}}=6.2 \mathrm{~Hz}, 1 \mathrm{H}, \mathrm{CH}_{p \text {-cym }}\right), 5.61$ $\left(\mathrm{d},{ }^{3} \mathrm{~J}_{\mathrm{H}, \mathrm{H}}=6.0 \mathrm{~Hz}, 1 \mathrm{H}, \mathrm{CH}_{p \text {-cym }}\right), 4.57\left(\mathrm{~s}, 3 \mathrm{H}, \mathrm{NCH}_{3}\right), 4.20($ broad s, $1 \mathrm{H},-\mathrm{OH}) 2.57-2.43$ $\left(\mathrm{m}, 1 \mathrm{H}, \mathrm{CH}_{\text {isop }(p \text {-cym })}\right), 2.13\left(\mathrm{~s}, 3 \mathrm{H}, \mathrm{CH}_{3(p-\text { cym })}\right), 0.98\left(\mathrm{~d},{ }^{3} \mathrm{~J}_{\mathrm{H}, \mathrm{H}}=6.9 \mathrm{~Hz}, 3 \mathrm{H}, \mathrm{CH}_{3 \text { (isop }(p \text {-cym) })}\right)$, $0.91\left(\mathrm{~d},{ }^{3} \mathrm{~J}_{\mathrm{H}, \mathrm{H}}=6.9 \mathrm{~Hz}, 3 \mathrm{H}, \mathrm{CH}_{3(\mathrm{isop}(p \text {-cym) }))}\right){ }^{13} \mathrm{C}\left\{{ }^{1} \mathrm{H}\right\} \mathrm{NMR}\left(101 \mathrm{MHz}, \mathrm{CD}_{3} \mathrm{CN}\right): \delta 174.9$ $\left(C_{\text {carbene }}-\mathrm{Ru}\right), 161.4(C \mathrm{OO}-), 156.8\left(\mathrm{CH}_{\mathrm{py}}\right), 150.8\left(C_{\mathrm{q}(\mathrm{py})}\right), 142.8\left(\mathrm{CH}_{\mathrm{py}}\right), 142.5\left(C_{\mathrm{q}(\mathrm{tz})}\right)$, $127.3\left(\mathrm{CH}_{\mathrm{py}}\right), 115.2\left(\mathrm{CH}_{\mathrm{py}}\right),[108.1,106.9]\left(C_{\mathrm{q}(p-\mathrm{cym})}\right),[91.2,89.7,89.2,83.6]\left(\mathrm{CH}_{p-\text { cym }}\right)$, $42.3\left(\mathrm{~N}-\mathrm{CH}_{3}\right), 31.9$, [22.6, 22.6] $\left(\mathrm{CH}_{3(\mathrm{isop}(p-\mathrm{cym}))}\right), 19.3\left(\mathrm{CH}_{3(p-\mathrm{cym})}\right)$. Anal. Calcd. for $\mathrm{C}_{19} \mathrm{H}_{22} \mathrm{~N}_{4} \mathrm{O}_{2} \mathrm{RuClCF}_{3} \mathrm{SO}_{3}(623.99 \mathrm{~g} / \mathrm{mol}): \mathrm{C}, 38.50 ; \mathrm{H}, 3.55 ; \mathrm{N}, 8.98$. Found: $\mathrm{C}, 38.49 ; \mathrm{H}$, 3.41; N, 8.92. Electrospray Ms. (Cone $20 \mathrm{~V})\left(\mathrm{m} / \mathrm{z}\right.$, fragment): $475.7[\mathrm{M}]^{+}$. IR $\left(\mathrm{CH}_{3} \mathrm{CN}\right)=$ $1735.5 \mathrm{~cm}^{-1},(\mathrm{C}=\mathrm{O})$. 


\section{Synthesis of $4 \mathbf{H}$}

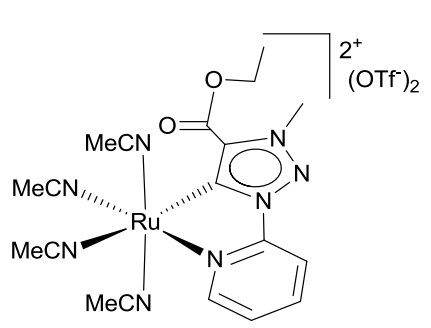

In a round-bottom flask were mixed, the complex $\mathbf{1 H}$ (256 $\mathrm{mg}, 0.402 \mathrm{mmol})$ and AgOTf (156.7 $\mathrm{mg}, 0.603 \mathrm{mmol})$ in 10 $\mathrm{mL}$ of acetonitrile, and the suspension was stirred at reflux for 24 hours. The reaction mixture was filtered through celite and the solvent was removed under reduced pressure. Precipitation with acetonitrile/ diethyl ether afforded an analytically pure yellow solid. Yield: $240 \mathrm{mg}, 75 \% .{ }^{1} \mathrm{H}$ NMR $\left(400 \mathrm{MHz}, \mathrm{CD}_{3} \mathrm{CN}\right): \delta$ $9.00-8.97\left(\mathrm{~m}, 1 \mathrm{H}, \mathrm{C} H_{\mathrm{py}}\right), 8.24-8.21\left(\mathrm{~m}, 2 \mathrm{H}, \mathrm{CH}_{\mathrm{py}}\right), 7.70-7.65\left(\mathrm{~m}, 1 \mathrm{H}, \mathrm{C} H_{\mathrm{py}}\right), 4.57-$ $4.49\left(\mathrm{~m}, 5 \mathrm{H}, \mathrm{NCH}_{3}, \mathrm{O}_{-} \mathrm{CH}_{2}-\right), 2.50\left(\mathrm{~s}, 3 \mathrm{H}, \mathrm{CH}_{3} \mathrm{CN}\right), 2.08\left(\mathrm{~s}, 6 \mathrm{H}, \mathrm{CH}_{3} \mathrm{CN}\right), 1.45\left(\mathrm{t},{ }^{3} \mathrm{~J}_{\mathrm{H}, \mathrm{H}}=\right.$ $\left.7.1 \mathrm{~Hz}, 3 \mathrm{H},-\mathrm{CH}_{3}\right) .{ }^{13} \mathrm{C}\left\{{ }^{1} \mathrm{H}\right\}$ NMR $\left(101 \mathrm{MHz}, \mathrm{CD}_{3} \mathrm{CN}\right): \delta 181.6\left(C_{\text {carbene }}-\mathrm{Ru}\right), 159.2$ $(C O O-), 154.5\left(C_{\mathrm{py}}\right), 153.7\left(C_{\mathrm{q}(\mathrm{py})}\right), 141.9\left(\mathrm{CH}_{\mathrm{py}}\right), 141.8\left(C_{\mathrm{q}(\mathrm{tz})}\right), 126.7\left(\mathrm{CH}_{\mathrm{py}}\right)$, [125.1, 124.8] $\left(\mathrm{CH}_{3} \mathrm{CN}\right), 114.7\left(\mathrm{CH}_{\mathrm{py}}\right), 63.5\left(\mathrm{O}-\mathrm{CH}_{2}-\right), 42.6\left(\mathrm{~N}-\mathrm{CH}_{3}\right), 14.5\left(-\mathrm{CH}_{3}\right),[4.3,3.9]$ $\left(\mathrm{CH}_{3} \mathrm{CN}\right)$. Electrospray Ms. (Cone $\left.20 \mathrm{~V}\right)\left(\mathrm{m} / \mathrm{z}\right.$, fragment): $647.2\left[\mathrm{M}+\mathrm{CF}_{3} \mathrm{SO}_{3}\right]^{+}$.

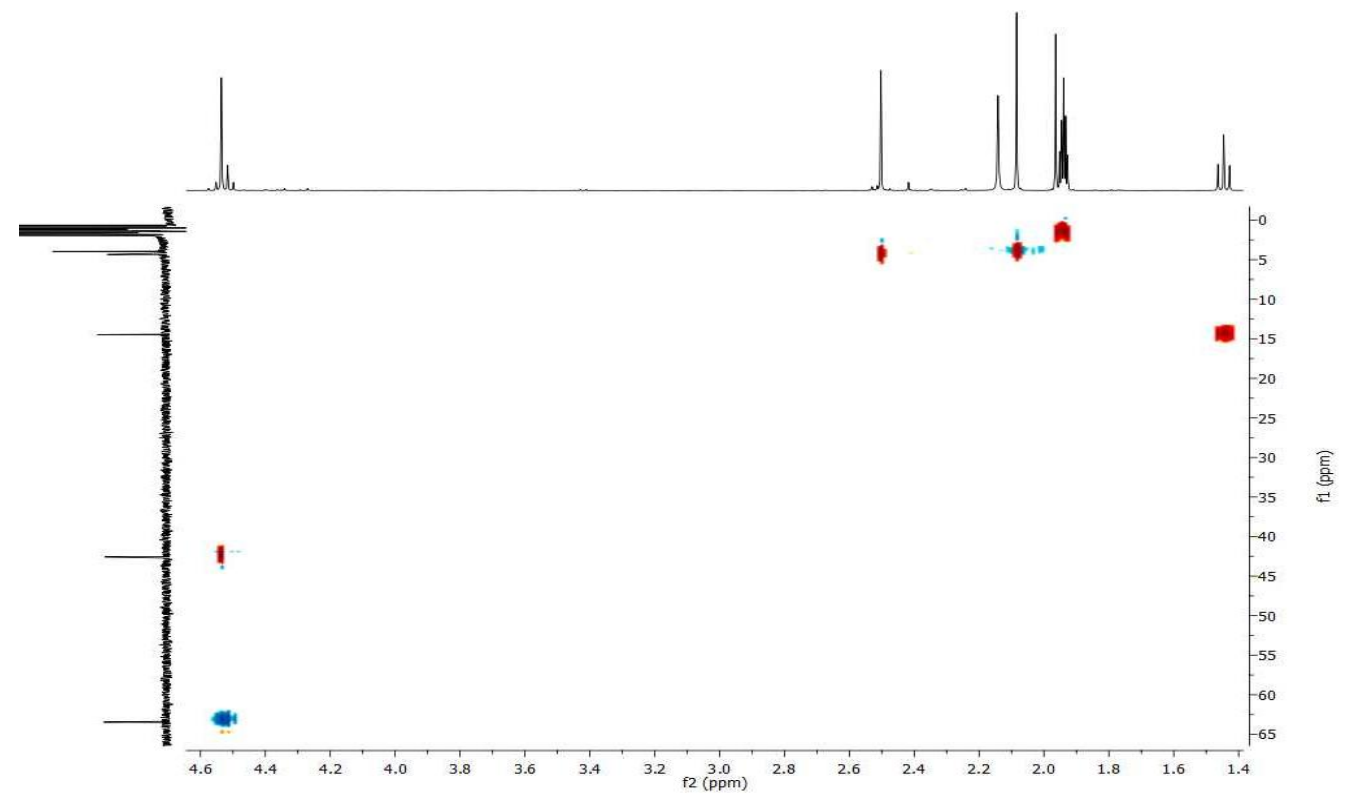

Figure 6.4 ${ }^{13} \mathrm{C}$ HSQC NMR spectrum $\left({ }^{1} \mathrm{H}, 400 \mathrm{MHz}\right.$ and $\left.{ }^{13} \mathrm{C}, 100 \mathrm{MHz}\right)$ of $\mathbf{4 H}$ in $\mathrm{CD}_{3} \mathrm{CN}$ 


\section{Synthesis of $5 \mathrm{H}$}

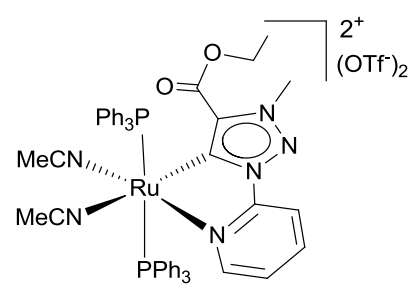

In a Schlenk were mixed the complex $\mathbf{4 H}(100 \mathrm{mg}, 0.125$ mmol) and $\mathrm{PPh}_{3}(84 \mathrm{mf}, 0.314 \mathrm{mmol})$ in $5 \mathrm{~mL}$ of $o$ dichlorobenzene, and the suspension was stirred at $110^{\circ} \mathrm{C}$ for 3 days, under nitrogen atmosphere. After cooling, the reaction mixture was poured into diethyl ether $(20 \mathrm{~mL})$ and the formed precipitate was filtered off, washed thoroughly with diethyl ether, and dried to afford an analytically pure yellow solid. Yield: $155 \mathrm{mg}$, 95\%. ${ }^{1} \mathrm{H}$ NMR (400 MHz, $\left.\mathrm{CD}_{3} \mathrm{CN}\right): \delta 8.21\left(\mathrm{~d},{ }^{3} \mathrm{~J}_{\mathrm{H}, \mathrm{H}}=5.5 \mathrm{~Hz}, 1 \mathrm{H}, \mathrm{CH}_{\mathrm{py}}\right), 7.78-7.72(\mathrm{~m}$, $\left.1 \mathrm{H}, \mathrm{CH}_{\mathrm{py}}\right), 7.47-7.36\left(\mathrm{~m}, 7 \mathrm{H}, \mathrm{CH}_{\mathrm{py}}, \mathrm{PPh}_{3}\right), 7.32-7.15\left(\mathrm{~m},{ }^{3} \mathrm{~J}_{\mathrm{H}, \mathrm{H}}=22.7,17.5,8.0 \mathrm{~Hz}\right.$, $\left.24 \mathrm{H}, \mathrm{PPh}_{3}\right), 7.09-7.04\left(\mathrm{~m}, 1 \mathrm{H}, \mathrm{CH}_{\mathrm{py}}\right), 4.39\left(\mathrm{q},{ }^{3} \mathrm{~J}_{\mathrm{H}, \mathrm{H}}=7.1 \mathrm{~Hz}, 1 \mathrm{H}, \mathrm{O}-\mathrm{CH}_{2^{-}}\right), 4.20(\mathrm{~s}, 3 \mathrm{H}$, $\left.\mathrm{NCH}_{3}\right), 2.29\left(\mathrm{~s}, 3 \mathrm{H}, \mathrm{CH}_{3} \mathrm{CN}\right), 1.41\left(\mathrm{t},{ }^{3} \mathrm{~J}_{\mathrm{H}, \mathrm{H}}=7.1 \mathrm{~Hz}, 3 \mathrm{H},-\mathrm{CH}_{3}\right) \cdot{ }^{13} \mathrm{C}\left\{{ }^{1} \mathrm{H}\right\} \mathrm{NMR}(101$ $\left.\mathrm{MHz}, \mathrm{CD}_{3} \mathrm{CN}\right): \delta 183.4\left(\mathrm{t},{ }^{2} \mathrm{~J}_{\mathrm{C}, \mathrm{P}}=11.5 \mathrm{~Hz},\left(C_{\text {carbene }}-\mathrm{Ru}\right), 158.2(C \mathrm{OO}-), 153.9\left(\mathrm{CH}_{\mathrm{py}}\right)\right.$, $150.7\left(C_{\mathrm{q}(\mathrm{py})}\right), 141.0\left(C_{\mathrm{q}(\mathrm{tz})}\right), 140.6\left(\mathrm{CH}_{\mathrm{py}}\right),[134.5,134.4]\left(\mathrm{s}, \mathrm{PPh}_{3}\right), 133.7\left(\mathrm{t},{ }^{2} \mathrm{~J}_{\mathrm{C}, \mathrm{P}}=5.5\right.$ $\left.\mathrm{Hz}, \mathrm{PPh}_{3}\right), 131.4\left(\mathrm{CH}_{3} \mathrm{CN}\right), 131.1\left(\mathrm{t},{ }^{2} \mathrm{~J}_{\mathrm{C}, \mathrm{P}}=21.3 \mathrm{~Hz}, \mathrm{PPh}_{3}\right),[130.0,129.92]\left(\mathrm{s}, \mathrm{PPh}_{3}\right)$, $129.64\left(\mathrm{t},{ }^{2} \mathrm{~J}_{\mathrm{C}, \mathrm{P}}=4.7 \mathrm{~Hz}, \mathrm{PPh}_{3}\right), 127.2\left(\mathrm{CH}_{\mathrm{py}}\right), 114.8\left(\mathrm{CH}_{\mathrm{py}}\right), 63.8\left(\mathrm{O}_{-} \mathrm{CH}_{2}-\right), 43.9(\mathrm{~N}-$ $\left.\mathrm{CH}_{3}\right), 14.4\left(-\mathrm{CH}_{3}\right), 5.2\left(\mathrm{CH}_{3} \mathrm{CN}\right) .{ }^{31} \mathrm{P}\left\{{ }^{1} \mathrm{H}\right\} \mathrm{NMR}\left(121 \mathrm{MHz}, \mathrm{CD}_{3} \mathrm{CN}\right) \delta 29.5\left(\mathrm{~s}, \mathrm{PPh}_{3}\right)$. Anal. Calcd. for $\mathrm{C}_{51} \mathrm{H}_{48} \mathrm{~N}_{6} \mathrm{P}_{2} \mathrm{O}_{2} \mathrm{Ru}\left(\mathrm{CF}_{3} \mathrm{SO}_{3}\right)_{2}(1238.12 \mathrm{~g} / \mathrm{mol}): \mathrm{C}, 51.81 ; \mathrm{H}, 4.35 ; \mathrm{N}, 6.47$. Found: C, 51.90; H, 3.58; N, 5.98. Electrospray Ms. (Cone 20V) (m/z, fragment): 1088.8 $\left[\mathrm{M}+\mathrm{CF}_{3} \mathrm{SO}_{3}\right]^{+}$. IR $\left(\mathrm{CH}_{3} \mathrm{CN}\right)=1728.1 \mathrm{~cm}^{-1},(\mathrm{C}=\mathrm{O})$.

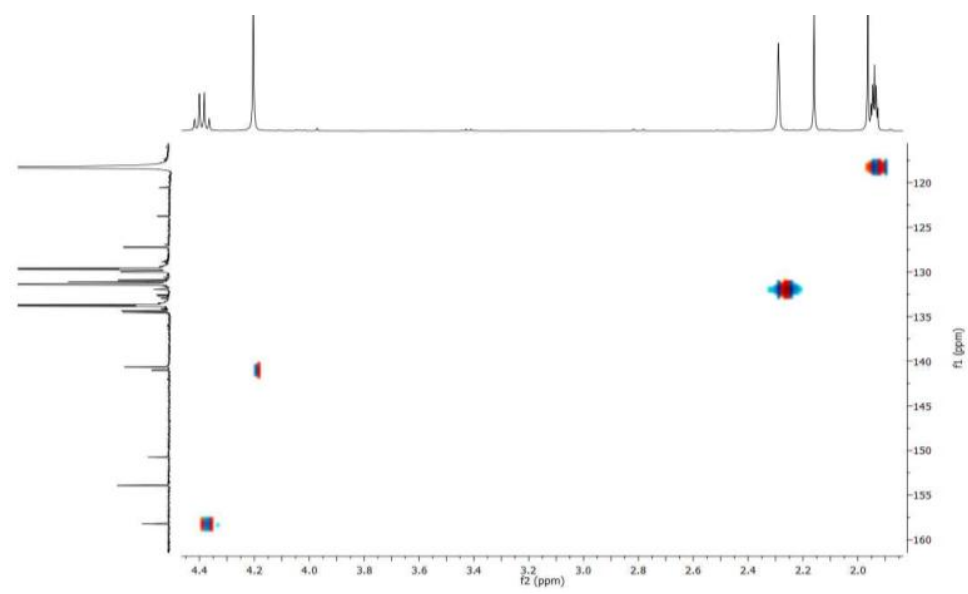

Figure 6.5 ${ }^{13} \mathrm{C}$ HMBC NMR spectrum $\left({ }^{1} \mathrm{H}, 400 \mathrm{MHz}\right.$ and $\left.{ }^{13} \mathrm{C}, 100 \mathrm{MHz}\right)$ of $\mathbf{5} \mathbf{H}$ in $\mathrm{CD}_{3} \mathrm{CN}$ 


\section{Synthesis of $6 \mathrm{H}$}

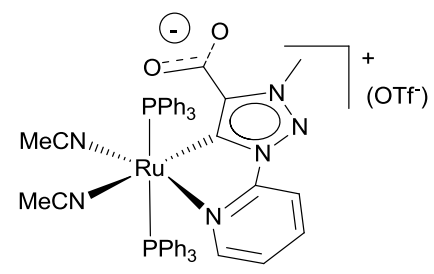

In a round-bottom flask were mixed, the complex $\mathbf{5 H}(78.5$ $\mathrm{mg}, 0.064 \mathrm{mmol})$ and $\mathrm{LiOH} \cdot \mathrm{H}_{2} \mathrm{O}(5.4 \mathrm{mg}, 0.127 \mathrm{mmol})$ in $3 \mathrm{~mL}$ of a acetonitrile/water (2:1) mixture and the suspension was stirred for 1 hours at $30^{\circ} \mathrm{C}$. The reaction mixture was extracted with dichloromethane/water mixtures. The organic layers were combined and the solvent was removed under reduced pressure. Precipitation with acetonitrile/ diethyl ether afforded an analytically pure yellow solid. Yield: $46 \mathrm{mg}, 64 \% .{ }^{1} \mathrm{H}$ NMR (500 MHz, $\left.\mathrm{CD}_{3} \mathrm{CN}\right): \delta 7.99\left(\mathrm{~d},{ }^{3} \mathrm{~J}_{\mathrm{H}, \mathrm{H}}=5.2 \mathrm{~Hz}, 1 \mathrm{H}, \mathrm{CH}_{\mathrm{py}}\right), 7.72-7.67\left(\mathrm{~m}, 1 \mathrm{H}, \mathrm{CH}_{\mathrm{py}}\right), 7.45-7.22(\mathrm{~m}$, $\left.30 \mathrm{H}, \mathrm{PPh}_{3}\right), 7.18-7.15\left(\mathrm{~m}, 1 \mathrm{H}, \mathrm{C} H_{\mathrm{py}}\right), 7.07-7.03\left(\mathrm{~m}, 1 \mathrm{H}, \mathrm{CH} H_{\mathrm{py}}\right), 4.21\left(\mathrm{~s}, 3 \mathrm{H}, \mathrm{NCH}_{3}\right)$, $2.16\left(\mathrm{~s}, 3 \mathrm{H}, \mathrm{CH}_{3} \mathrm{CN}\right) .{ }^{13} \mathrm{C}\left\{{ }^{1} \mathrm{H}\right\} \mathrm{NMR}\left(126 \mathrm{MHz}, \mathrm{CD}_{3} \mathrm{CN}\right): \delta 174.1-173.7\left(\mathrm{~m}, C_{\text {carbene }}{ }^{-}\right.$ $\mathrm{Ru}), 161.5-161.2(\mathrm{~m}, C O O-), 153.0\left(\mathrm{CH}_{\mathrm{py}}\right), 152.0\left(C_{\mathrm{q}(\mathrm{py})}\right), 150.0-149.9\left(\mathrm{~m}, C_{\mathrm{q}(\mathrm{tz})}\right)$, $139.9\left(\mathrm{CH}_{\mathrm{py}}\right),[134.7,134.5]\left(\mathrm{s}, \mathrm{PPh}_{3}\right), 134.2\left(\mathrm{t},{ }^{2} \mathrm{~J}_{\mathrm{C}, \mathrm{P}}=5.5 \mathrm{~Hz}, \mathrm{PPh}_{3}\right), 131.0\left(\mathrm{t},{ }^{2} \mathrm{~J}_{\mathrm{C}, \mathrm{P}}=20.9\right.$ $\left.\mathrm{Hz}, \mathrm{PPh}_{3}\right), 130.9\left(\mathrm{CH}_{3} \mathrm{CN}\right),[130.0,129.9]\left(\mathrm{s}, \mathrm{PPh}_{3}\right), 129.4\left(\mathrm{t},{ }^{2} \mathrm{~J}_{\mathrm{C}, \mathrm{P}}=4.7 \mathrm{~Hz}, \mathrm{PPh}_{3}\right), 126.3$ $\left(\mathrm{CH}_{\mathrm{py}}\right), 114.5\left(\mathrm{CH}_{\mathrm{py}}\right), 41.2\left(\mathrm{~N}-\mathrm{CH}_{3}\right), 5.3\left(\mathrm{CH}_{3} \mathrm{CN}\right) .{ }^{31} \mathrm{P}\left\{{ }^{1} \mathrm{H}\right\} \quad \mathrm{NMR}\left(121 \mathrm{MHz}, \mathrm{CD}_{3} \mathrm{CN}\right)$ : $\delta 32.0\left(\mathrm{~s}, \mathrm{PPh}_{3}\right)$. Anal. Calcd. for $\mathrm{C}_{49} \mathrm{H}_{43} \mathrm{~N}_{6} \mathrm{P}_{2} \mathrm{O}_{2} \mathrm{Ru}\left(\mathrm{CF}_{3} \mathrm{SO}_{3}\right) \cdot 4 \mathrm{H}_{2} \mathrm{O}(1132.05 \mathrm{~g} / \mathrm{mol})$ : $\mathrm{C}$, 53.05; H, 4.54; N, 7.42. Found: C, 53.03; H, 4.76; N, 6.93. Electrospray Ms. (Cone 20V) $\left(\mathrm{m} / \mathrm{z}\right.$, fragment): $869.9[\mathrm{M}-\mathrm{MeCN}]^{+}$. IR $\left(\mathrm{CH}_{3} \mathrm{CN}\right)=1645.2 \mathrm{~cm}^{-1},(\mathrm{C}=\mathrm{O})$.

\section{Synthesis of $7 \mathbf{H}$}

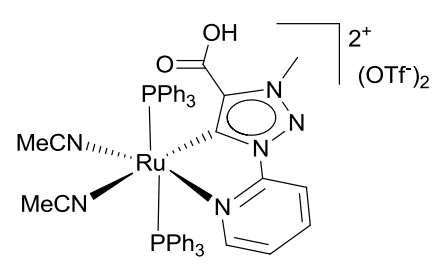

In a round-bottom flask were mixed, the complex $\mathbf{5 H}$ (78.5 $\mathrm{mg}, 0.064 \mathrm{mmol})$ and $\mathrm{LiOH} \cdot \mathrm{H}_{2} \mathrm{O}(5.4 \mathrm{mg}, 0.127 \mathrm{mmol})$ in $3 \mathrm{~mL}$ of a acetonitrile/water (2:1) mixture and the suspension was stirred for 1 hours at $30^{\circ} \mathrm{C}$. The reaction mixture was acidified with triflic acid aqueous solution (1M) and extracted with dichloromethane/water mixtures. The organic layers were combined and the solvent was removed under reduced pressure. Precipitation with acetonitrile/ diethyl ether afforded an analytically pure yellow solid. Yield: $40 \mathrm{mg}, 52 \%$. ${ }^{1} \mathrm{H}$ NMR (400 MHz, CD $\left.\mathrm{CD}_{3} \mathrm{CN}\right) \delta 8.03\left(\mathrm{~d},{ }^{3} \mathrm{~J}_{\mathrm{H}, \mathrm{H}}=5.6 \mathrm{~Hz}, 1 \mathrm{H}, \mathrm{CH}_{\mathrm{py}}\right), 7.75-7.67(\mathrm{~m}, 1 \mathrm{H}$, $\left.\mathrm{C} H_{\text {py }}\right), 7.45-7.21\left(\mathrm{~m}, 31 \mathrm{H}, \mathrm{PPh}_{3}, \mathrm{CH}_{\mathrm{py}}\right), 7.08-7.00\left(\mathrm{~m}, 1 \mathrm{H}, \mathrm{C} H_{\mathrm{py}}\right), 4.24\left(\mathrm{~s}, 3 \mathrm{H}, \mathrm{NCH}_{3}\right)$. ${ }^{13} \mathrm{C}\left\{{ }^{1} \mathrm{H}\right\}$ NMR (101 MHz, $\left.\mathrm{CD}_{3} \mathrm{CN}\right): \delta 173.6$ - 173.0 (m, $\left.C_{\text {carbene }}-\mathrm{Ru}\right), 160.4$ (COO-), 
$152.2\left(\mathrm{CH}_{\mathrm{py}}\right), 151.0\left(C_{\mathrm{q}(\mathrm{py})}\right), 148.7-148.0\left(\mathrm{~m}, C_{\mathrm{q}(\mathrm{tz})}\right), 139.1\left(\mathrm{CH}_{\mathrm{py}}\right),[133.7,133.6](\mathrm{s}$, $\left.\mathrm{PPh}_{3}\right), 133.2\left(\mathrm{t},{ }^{2} \mathrm{~J}_{\mathrm{C}, \mathrm{P}}=5.5 \mathrm{~Hz}, \mathrm{PPh}_{3}\right), 130.1\left(\mathrm{t},{ }^{2} \mathrm{~J}_{\mathrm{C}, \mathrm{P}}=20.9 \mathrm{~Hz}, \mathrm{PPh}_{3}\right), 130.0\left(\mathrm{CH}_{3} \mathrm{CN}\right)$, [129.0, 128.9] $\left(\mathrm{s}, \mathrm{PPh}_{3}\right), 128.5\left(\mathrm{t},{ }^{2} \mathrm{~J}_{\mathrm{C}, \mathrm{P}}=4.7 \mathrm{~Hz}, \mathrm{PPh}_{3}\right), 125.4\left(\mathrm{CH}_{\mathrm{py}}\right), 113.6\left(\mathrm{CH}_{\mathrm{py}}\right), 40.4$ $\left(\mathrm{N}-\mathrm{CH}_{3}\right), 4.4\left(\mathrm{CH}_{3} \mathrm{CN}\right) .{ }^{31} \mathrm{P}\left\{{ }^{1} \mathrm{H}\right\} \quad \mathrm{NMR}\left(162 \mathrm{MHz}, \mathrm{CD}_{3} \mathrm{CN}\right): \delta 36.7\left(\mathrm{~s}, \mathrm{PPh}_{3}\right)$. Electrospray Ms. (Cone 20V) (m/z, fragment): $415.6[\mathrm{M}-2(\mathrm{MeCN})]^{2+}$.

\section{Synthesis of $1 \mathrm{I}$}

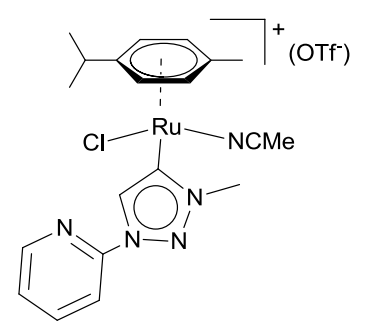

In a round-bottom flask were mixed, under exclusion of light, the triazolium salt $\mathbf{I}(174 \mathrm{mg}, 0.491 \mathrm{mmol})$ and $\mathrm{Ag}_{2} \mathrm{O}(228 \mathrm{mg}$, $0.982 \mathrm{mmol}$ ) in $10 \mathrm{~mL}$ of acetonitrile and the suspension was stirred for 2 hours. Then $\left[\mathrm{RuCl}_{2}(p \text {-cymene })\right]_{2}$ (150 mg, 0,245 mmol) was added and the reaction mixture was stirred at room temperature for 3 hours. The resulting suspension was filtered through celite and the solvent was evaporated under reduced pressure. The crude solid was purified by column chromatography. Elution with a mixture of dichloromethane/Acetone (1:1) afforded the separation of an orange band containing the desired product. Precipitation with acetonitrile/ diethyl ether afforded an analytically pure orange solid. Yield: $85 \mathrm{mg}, 28 \%$. ${ }^{1} \mathrm{H}$ NMR $\left(400 \mathrm{MHz}, \mathrm{CD}_{3} \mathrm{CN}\right): \delta 8.63\left(\mathrm{ddd},{ }^{3} \mathrm{~J}_{\mathrm{H}, \mathrm{H}}=4.8\right.$, $\left.1.8,0.8 \mathrm{~Hz}, 1 \mathrm{H}, \mathrm{CH}_{\mathrm{py}}\right), 8.43\left(\mathrm{~s}, 1 \mathrm{H}, \mathrm{CH}_{\mathrm{tz}}\right), 8.16-8.10\left(\mathrm{~m}, 1 \mathrm{H}, \mathrm{CH}_{\mathrm{py}}\right), 8.02-7.98(\mathrm{~m}, 1 \mathrm{H}$, $\left.\mathrm{C} H_{\mathrm{py}}\right), 7.66-7.61\left(\mathrm{~m}, 1 \mathrm{H}, \mathrm{C} H_{\mathrm{py}}\right), 5.75\left(\mathrm{~d},{ }^{3} \mathrm{~J}_{\mathrm{H}, \mathrm{H}}=6.1 \mathrm{~Hz}, 1 \mathrm{H}, \mathrm{CH}_{p-\mathrm{cym}}\right), 5.68\left(\mathrm{~d},{ }^{3} \mathrm{~J}_{\mathrm{H}, \mathrm{H}}=\right.$ $\left.6.1 \mathrm{~Hz}, 1 \mathrm{H}, \mathrm{C} H_{p \text {-cym }}\right), 5.62\left(\mathrm{~d},{ }^{3} \mathrm{~J}_{\mathrm{H}, \mathrm{H}}=6.1 \mathrm{~Hz}, 1 \mathrm{H}, \mathrm{C} H_{p \text {-cym }}\right), 5.48\left(\mathrm{~d},{ }^{3} \mathrm{~J}_{\mathrm{H}, \mathrm{H}}=6.0 \mathrm{~Hz}, 1 \mathrm{H}\right.$, $\left.\mathrm{CH}_{p \text {-cym }}\right), 4.36\left(\mathrm{~s}, 3 \mathrm{H}, \mathrm{NCH}_{3}\right), 2.78-2.66\left(\mathrm{~m}, 1 \mathrm{H}, \mathrm{CH}_{\text {isop }(p \text {-cym })}\right), 2.26\left(\mathrm{~s}, 3 \mathrm{H}, \mathrm{CH}_{3} \mathrm{CN}\right)$, 2.09 (s, 3H, CH $\left.H_{3(p-c y m)}\right), 1.24\left(\mathrm{~d},{ }^{3} \mathrm{~J}_{\mathrm{H}, \mathrm{H}}=5.1 \mathrm{~Hz}, 3 \mathrm{H}, \mathrm{CH}_{3(\text { (isop }(p \text {-cym) })}\right), 1.23\left(\mathrm{~d},{ }^{3} \mathrm{~J}_{\mathrm{H}, \mathrm{H}}=5.1 \mathrm{~Hz}\right.$, $\left.3 \mathrm{H}, \mathrm{CH}_{3 \text { (isop }(p \text {-cym) }))}\right){ }^{13} \mathrm{C}\left\{{ }^{1} \mathrm{H}\right\} \mathrm{NMR}\left(101 \mathrm{MHz}, \mathrm{CD}_{3} \mathrm{CN}\right): \delta 161.4\left(C_{\text {carbene }}-\mathrm{Ru}\right), 149.9$ $\left(C \mathrm{H}_{\mathrm{py}}\right), 148.0\left(C_{\mathrm{q}(\mathrm{py})}\right), 141.1\left(\mathrm{CH}_{\mathrm{py}}\right), 130.7\left(\mathrm{CH}_{\mathrm{tz}}\right), 126.9\left(\mathrm{CH}_{\mathrm{py}}\right), 115.4\left(\mathrm{CH}_{\mathrm{py}}\right)$, [108.9, 102.6] $\left(C_{\mathrm{q}(p-\mathrm{cym})}\right),[88.0,87.8,87.2,85.0]\left(\mathrm{CH}_{p \text {-cym }}\right), 41.8\left(\mathrm{NCH}_{3}\right), 31.3\left(\mathrm{CH}_{\mathrm{isop}(p-\mathrm{cym})}\right)$, [22.2, 22.0] $\left(\mathrm{CH}_{3(\operatorname{sisp}(p-\text { cym }))}\right), 18.2\left(\mathrm{CH}_{3(p-\mathrm{cym})}\right)$. Anal. Calcd. for $\mathrm{C}_{20} \mathrm{H}_{25} \mathrm{~N}_{5} \mathrm{RuClCF}_{3} \mathrm{SO}_{3}$ (621.04 g/mol): C, 40.61; H, 4.06; N, 11.27. Found: C, 40.34; H, 4.42; N, 10.87. Electrospray Ms. (Cone 20V) (m/z, fragment): $472.47[\mathrm{M}]^{+}$. 


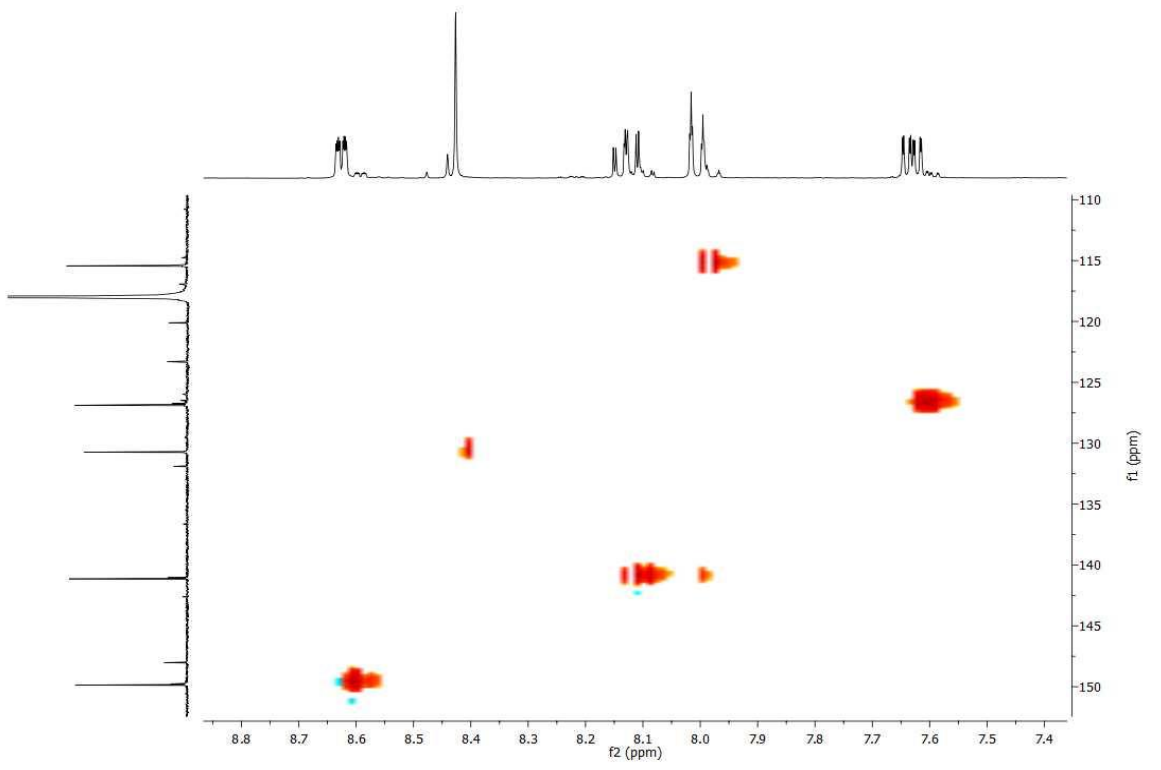

Figure 6.6 ${ }^{13} \mathrm{C}$ HSQC NMR spectrum $\left({ }^{1} \mathrm{H}, 400 \mathrm{MHz}\right.$ and $\left.{ }^{13} \mathrm{C}, 100 \mathrm{MHz}\right)$ of $\mathbf{1 I}$ in $\mathrm{CD}_{3} \mathrm{CN}$

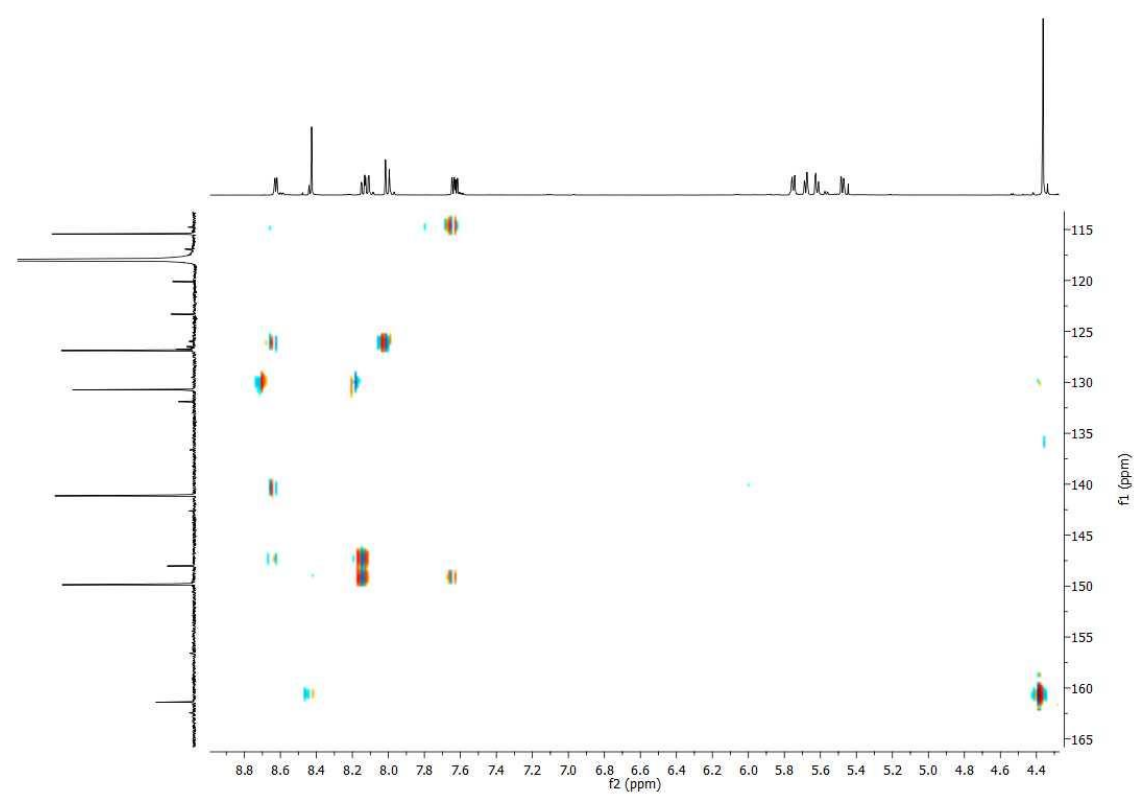

Figure 6.7 ${ }^{13} \mathrm{C}$ HMBC NMR spectrum $\left({ }^{1} \mathrm{H}, 400 \mathrm{MHz}\right.$ and $\left.{ }^{13} \mathrm{C}, 100 \mathrm{MHz}\right)$ of $\mathbf{1 I}$ in $\mathrm{CD}_{3} \mathrm{CN}$ 


\section{Synthesis of 2I}

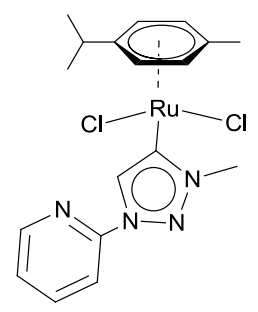

Complex 2I was produced by the evolution of a solution of $\mathbf{1 I}$ in chloroform, where a mixture of $\mathbf{1 I}$ and $\mathbf{2 I}$ was observed by ${ }^{1} \mathrm{H}$ NMR spectroscopy Complex 2I was easily separated by column chromatography as it is a neutral complex while $\mathbf{1 I}$ is cationic. Elution with a mixture of dichloromethane/Acetone (8:2) afforded the separation of an orange band containing the desired product. Crystallization by slow diffusion of pentane in a concentrated solution of $\mathbf{2 I}$ in chloroform gave analytically pure sample and crystals suitable for X-ray diffraction analysis. ${ }^{1} \mathrm{H}$ NMR (400 MHz, $\left.\mathrm{CD}_{3} \mathrm{CN}\right): \delta 8.63-8.60\left(\mathrm{~m}, 1 \mathrm{H}, \mathrm{CH}_{\mathrm{py}}\right), 8.44\left(\mathrm{~s}, 1 \mathrm{H}, \mathrm{CH}_{\mathrm{tz}}\right)$, $8.12-8.07\left(\mathrm{~m}, 1 \mathrm{H}, \mathrm{CH}_{\mathrm{py}}\right), 7.99-7.95\left(\mathrm{~m}, 1 \mathrm{H}, \mathrm{CH}_{\mathrm{py}}\right), 7.62-7.57\left(\mathrm{~m}, 1 \mathrm{H}, \mathrm{CH}_{\mathrm{py}}\right), 5.42(\mathrm{~d}$, $\left.{ }^{3} \mathrm{~J}_{\mathrm{H}, \mathrm{H}}=6.0 \mathrm{~Hz}, 2 \mathrm{H}, \mathrm{CH}_{p \text {-cym }}\right), 5.28\left(\mathrm{~d},{ }^{3} \mathrm{~J}_{\mathrm{H}, \mathrm{H}}=5.9 \mathrm{~Hz}, 2 \mathrm{H}, \mathrm{CH}_{p \text {-cym }}\right), 4.45\left(\mathrm{~s}, 3 \mathrm{H}, \mathrm{NCH}_{3}\right)$, $2.79-2.65\left(\mathrm{~m}, 1 \mathrm{H}, \mathrm{CH}_{\text {isop }(p \text {-cym })}\right), 2.10\left(\mathrm{~s}, 3 \mathrm{H}, \mathrm{CH}_{3(p-\mathrm{cym})}\right), 1.22\left(\mathrm{~d},{ }^{3} \mathrm{~J}_{\mathrm{H}, \mathrm{H}}=7.2 \mathrm{~Hz}, 6 \mathrm{H}\right.$, $\mathrm{CH}_{3 \text { (isop(p-cym)) }) .}{ }^{13} \mathrm{C}\left\{{ }^{1} \mathrm{H}\right\} \mathrm{NMR}\left(101 \mathrm{MHz}, \mathrm{CD}_{3} \mathrm{CN}\right): \delta 167.8\left(\mathrm{C}_{\text {carbene }}-\mathrm{Ru}\right), 150.0\left(\mathrm{CH}_{\mathrm{py}}\right)$, $148.5\left(C_{\mathrm{q}(\mathrm{py})}\right), 141.2\left(\mathrm{CH}_{\mathrm{py}}\right), 130.9\left(\mathrm{CH}_{\mathrm{tz}}\right), 126.7\left(\mathrm{CH}_{\mathrm{py}}\right), 115.4\left(\mathrm{CH}_{\mathrm{py}}\right),[104.1,100.4]$ $\left(C_{\mathrm{q}(p \text {-cym })}\right),[85.5,84.9]\left(\mathrm{CH}_{p \text {-cym }}\right), 41.9\left(\mathrm{NCH}_{3}\right), 31.4\left(\mathrm{CH}_{\text {isop }(p \text {-cym })}\right), 22.5\left(\mathrm{CH}_{3(\text { isop }(p \text {-cym }))}\right)$, $18.5\left(\mathrm{CH}_{3(p-c y m)}\right)$. Anal. Calcd. for $\mathrm{C}_{18} \mathrm{H}_{22} \mathrm{~N}_{4} \mathrm{RuCl}_{2}(466.37 \mathrm{~g} / \mathrm{mol}): \mathrm{C}, 46.35 ; \mathrm{H}, 4.75 ; \mathrm{N}$, 12.01. Found: C, 46.85; H, 4.42; N, 11.94. Electrospray Ms. (Cone 20V) (m/z, fragment): $431.48[\mathrm{M}-\mathrm{Cl}]^{+}$.

\section{Synthesis of 3I}

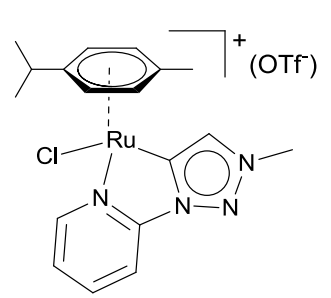

In a round-bottom flask were mixed, under exclusion of light, the triazolium salt I (174 mg, $0.491 \mathrm{mmol}$ ) and $\mathrm{Ag}_{2} \mathrm{O}$ (342 mg, 1.47 $\mathrm{mmol})$ in $10 \mathrm{~mL}$ of acetonitrile and the suspension was stirred for 3 days. Then $\left[\operatorname{RuCl}_{2}(p \text {-cymene })\right]_{2}(150 \mathrm{mg}, 0,245 \mathrm{mmol})$ was added and the reaction mixture was stirred at room temperature for 15 hours. The resulting suspension was filtered through celite and the solvent was evaporated under reduced pressure. The crude solid was purified by column chromatography. Elution with a mixture of dichloromethane/Acetone (1:1) afforded the separation of an orange band containing the desired product. Precipitation with acetonitrile/ diethyl ether afforded an analytically pure orange solid. Yield: $80 \mathrm{mg}$, 28\%. ${ }^{1} \mathrm{H}$ NMR (400 MHz, $\left.\mathrm{CD}_{3} \mathrm{CN}\right): \delta 9.25\left(\mathrm{dd},{ }^{3} \mathrm{~J}_{\mathrm{H}, \mathrm{H}}=5.7,0.8 \mathrm{~Hz}, 1 \mathrm{H}, \mathrm{CH}_{\mathrm{py}}\right), 8.42(\mathrm{~s}$, 
$\left.1 \mathrm{H}, \mathrm{CH}_{\mathrm{tz}}\right), 8.29-8.17\left(\mathrm{~m}, 1 \mathrm{H}, \mathrm{CH}_{\mathrm{py}}\right), 8.15-8.07\left(\mathrm{~m}, 1 \mathrm{H}, \mathrm{CH}_{\mathrm{py}}\right), 7.72-7.59(\mathrm{~m}, 1 \mathrm{H}$, $\left.\mathrm{CH}_{\mathrm{py}}\right), 6.06\left(\mathrm{~d},{ }^{3} \mathrm{~J}_{\mathrm{H}, \mathrm{H}}=6.1 \mathrm{~Hz}, 1 \mathrm{H}, \mathrm{CH}_{p-\mathrm{cym}}\right), 5.88\left(\mathrm{~d},{ }^{3} \mathrm{~J}_{\mathrm{H}, \mathrm{H}}=6.1 \mathrm{~Hz}, 1 \mathrm{H}, \mathrm{CH}_{p \text {-cym }}\right), 5.70(\mathrm{~d}$, $\left.{ }^{3} \mathrm{~J}_{\mathrm{H}, \mathrm{H}}=6.1 \mathrm{~Hz}, 1 \mathrm{H}, \mathrm{C} H_{p \text {-cym }}\right), 5.50\left(\mathrm{~d},{ }^{3} \mathrm{~J}_{\mathrm{H}, \mathrm{H}}=6.1 \mathrm{~Hz}, 1 \mathrm{H}, \mathrm{CH}_{p \text {-cym }}\right), 4.33\left(\mathrm{~s}, 3 \mathrm{H}, \mathrm{NCH}_{3}\right)$, $2.69-2.52\left(\mathrm{~m}, 1 \mathrm{H}, \mathrm{CH}_{\text {isop }(p \text {-cym })}\right), 2.04\left(\mathrm{~s}, 3 \mathrm{H}, \mathrm{CH}_{3(p \text {-cym })}\right), 1.12\left(\mathrm{~d},{ }^{3} \mathrm{~J}_{\mathrm{H}, \mathrm{H}}=6.9 \mathrm{~Hz}, 3 \mathrm{H}\right.$, $\left.\mathrm{CH}_{3 \text { (isop(p-cym)) }}\right), 1.01\left(\mathrm{~d},{ }^{3} \mathrm{~J}_{\mathrm{H}, \mathrm{H}}=6.9 \mathrm{~Hz}, 3 \mathrm{H}, \mathrm{CH}_{3(\text { isop }(p \text {-cym) })}\right) .{ }^{13} \mathrm{C}\left\{{ }^{1} \mathrm{H}\right\} \mathrm{NMR}(101 \mathrm{MHz}$, $\left.\mathrm{CD}_{3} \mathrm{CN}\right): \delta 171.5\left(C_{\text {carbene }}-\mathrm{Ru}\right), 156.8\left(\mathrm{CH}_{\mathrm{py}}\right), 150.8\left(C_{\mathrm{q}(\mathrm{py})}\right), 142.9\left(\mathrm{CH}_{\mathrm{py}}\right), 136.9\left(C_{\mathrm{tz}}\right)$, $127.2\left(C_{\mathrm{py}}\right), 115.1\left(\mathrm{CH}_{\mathrm{py}}\right),[108.0,104.2]\left(C_{\mathrm{q}(p-\mathrm{cym})}\right),[90.7,89.5,85.9,85.3]\left(\mathrm{CH}_{p-\mathrm{cym}}\right)$, $40.6\left(\mathrm{NCH}_{3}\right), 31.8\left(\mathrm{CH}_{\text {isop }(p \text {-cym })}\right)$, [22.6, 22.00] $\left(\mathrm{CH}_{3 \text { (isop(p-cym) }))}\right), 18.8\left(\mathrm{CH}_{3(p \text {-cym })}\right)$. Anal. Calcd. for $\mathrm{C}_{18} \mathrm{H}_{22} \mathrm{~N}_{4} \mathrm{RuClCF}_{3} \mathrm{SO}_{3}(579.98 \mathrm{~g} / \mathrm{mol})$ : C, 39.34; H, 3.82; N, 9.66. Found: $\mathrm{C}$, 39.22; H, 3.70; N, 9.41. Electrospray Ms. (Cone 20V) (m/z, fragment): $431.43[\mathrm{M}]^{+}$.

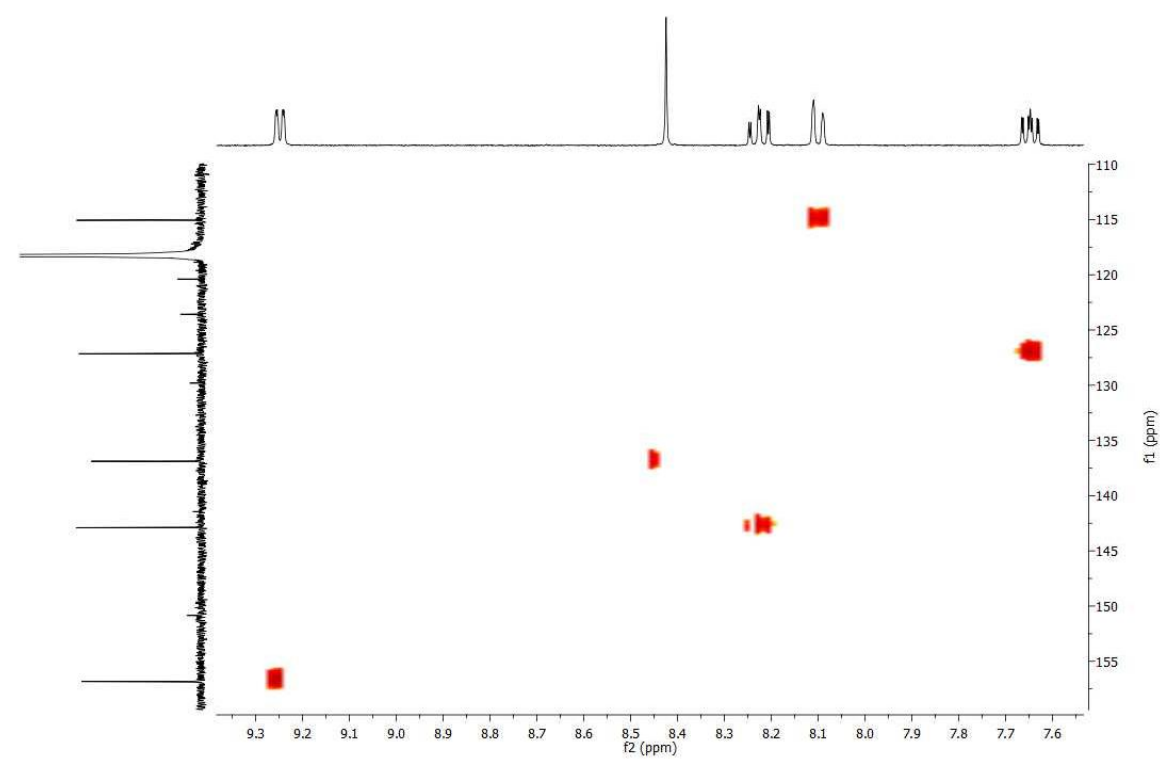

Figure 6.8 ${ }^{13} \mathrm{C}$ HSQC NMR spectrum $\left({ }^{1} \mathrm{H}, 400 \mathrm{MHz}\right.$ and $\left.{ }^{13} \mathrm{C}, 100 \mathrm{MHz}\right)$ of $\mathbf{3 I}$ in $\mathrm{CD}_{3} \mathrm{CN}$ 


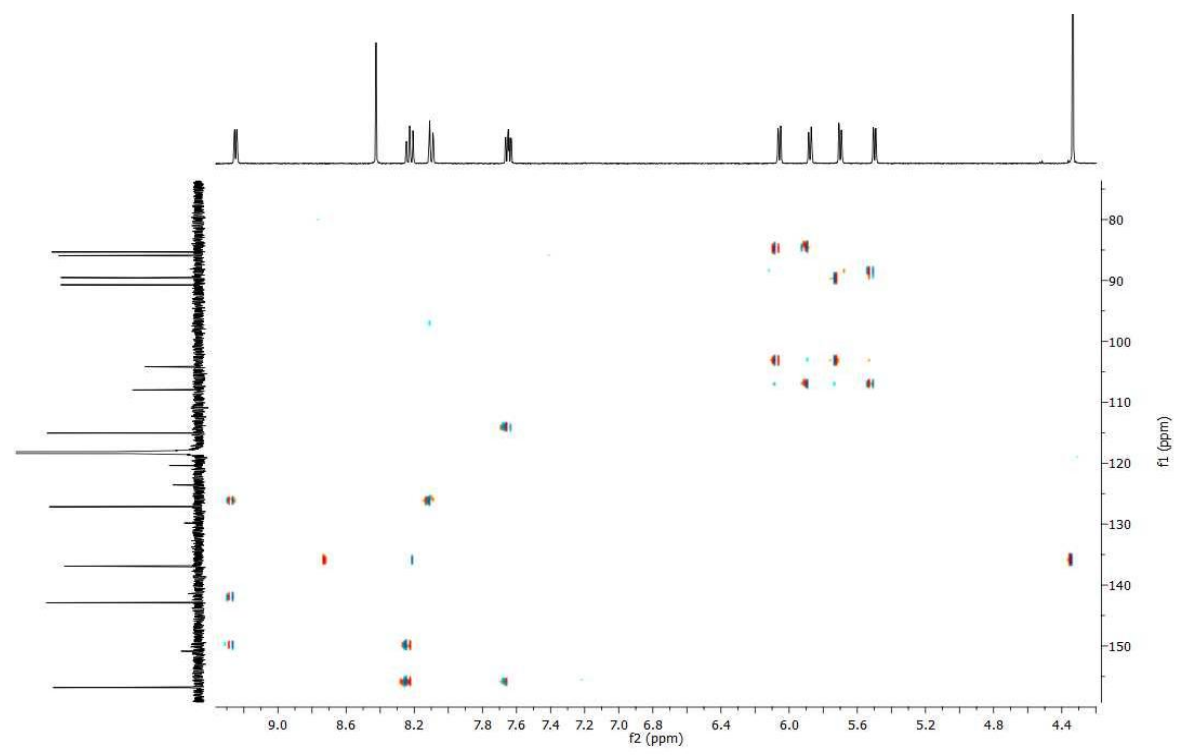

Figure 6.9 ${ }^{13} \mathrm{C}$ HMBC NMR spectrum $\left({ }^{1} \mathrm{H}, 400 \mathrm{MHz}\right.$ and $\left.{ }^{13} \mathrm{C}, 100 \mathrm{MHz}\right)$ of $\mathbf{3 I}$ in $\mathrm{CD}_{3} \mathrm{CN}$

\subsection{Synthesis and characterization of hybrid M-rGO materials}

General procedure: In a round-bottom flask were introduced $90 \mathrm{mg}$ of $\mathrm{rGO}$ and $10 \mathrm{~mL}$ of dichloromethane. The suspension was sonicated for 30 minutes. Then, $10 \mathrm{mg}$ of the corresponding metal complex 1G-4G was added. The suspension was stirred at room temperature for 10 hours until the solution become clear. The black solid was filtrated and washed with $2 \times 15 \mathrm{~mL}$ of dichloromethane affording the resulting product as a black solid. The filtrates were combined and evaporated to dryness under reduced pressure. Unsupported complex 1G-4G was analyzed by ${ }^{1} \mathrm{H}$ NMR using anisole as internal standard. Integration of the characteristic signal of anisole (-OMe) vs. ( $\mathrm{NCH}_{2}$-Pyr) reveals the amount complex that has been deposited on the rGO. The exact amount of complex supported was determined by ICP-MS analysis. Digestion of the materials was performed in a refluxing mixture of nitric and hydrochloric acids (3:1) for $12 \mathrm{~h}$.

Preparation of 5rGO: In a round-bottom flask were introduced $270 \mathrm{mg}$ of rGO and 10 $\mathrm{mL}$ of dichloromethane. The suspension was sonicated for 30 minutes. Then, $30 \mathrm{mg}$ of an equimolecular mixture of $\mathbf{1 G}$ and $\mathbf{2} \mathbf{G}$ was added. The suspension was stirred at room temperature for $10 \mathrm{~h}$. The black solid was filtrated and washed with $2 \times 15 \mathrm{~mL}$ of 
dichloromethane affording the resulting product as a black solid. The exact amount of complex supported was determined by ICP-MS analysis.

\section{UV/Vis spectroscopy}
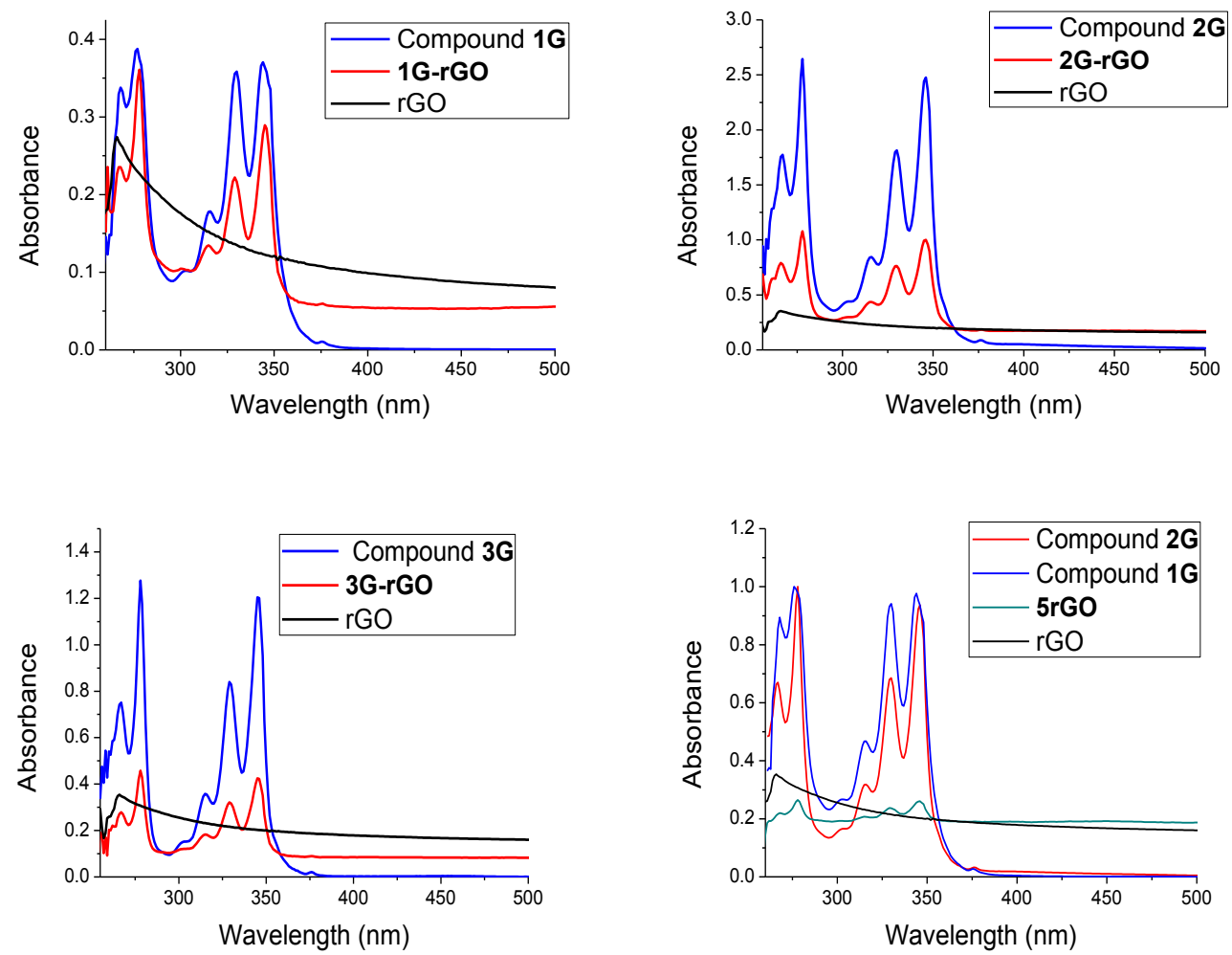

Figure 6.10 UV/Vis spectra of hybrid materials, molecular complexes and rGO. The samples were suspended in DMF and sonicated for 5 minutes before the measurements. The molecular complexes were dissolved in DMF $\left(10^{-6} \mathrm{M}\right)$

\section{FTIR spectroscopy}

The samples were prepared as $\mathrm{KBr}$ disks. Due to the weak signal and overlapping of absorption by other groups, hardly evident additional peaks or shifts can be observed in the spectrum of the hybrid materials. 

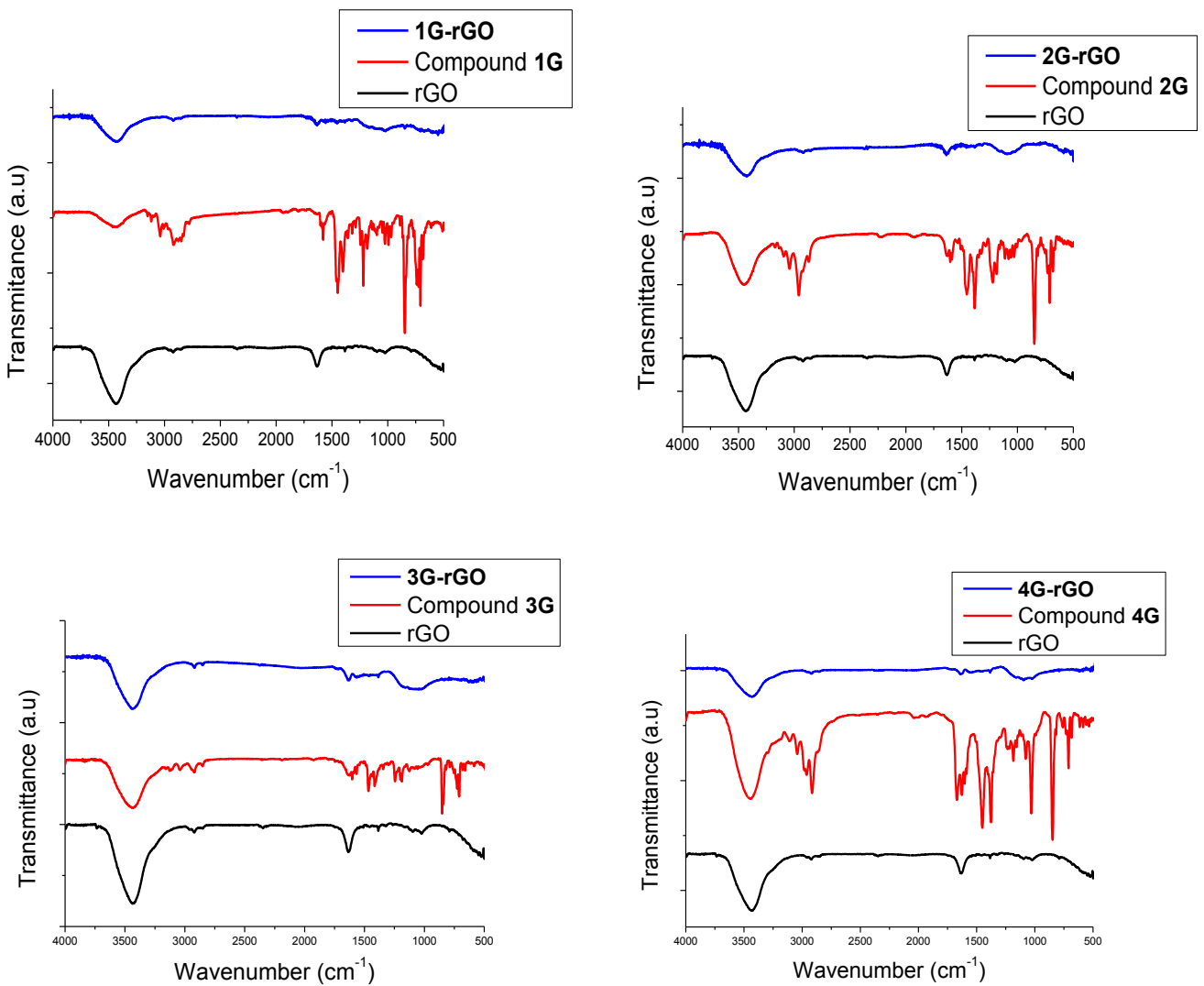

Figure 6.11 FTIR spectra of hybrid materials, molecular complexes and rGO

\section{Raman spectroscopy}

The Raman spectra exhibit a typical graphene-like pattern with characteristic D and G bands, attributed to $s p^{3}$ bonds (or the disorder in $s p^{2}$-hybridized bonds) and $s p^{2}$ bonds, respectively. The relative intensity of both bands $\left(\mathrm{I}_{\mathrm{D}} / \mathrm{I}_{\mathrm{G}}\right)$ is often applied as an indicator for the extent of defects occurring on graphene materials. The $D / G$ intensity ratios of the hybrid materials are slightly larger than rGO (1.44). This suggests the appearance of more defects over the rGO surface, and reflects the degree of functionalization. 


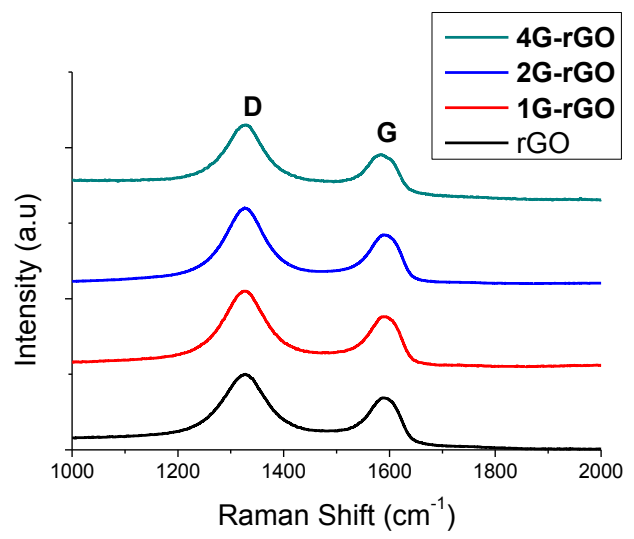

Figure 6.12 Raman spectra recorded using a $633 \mathrm{~nm}$ laser excitation

Table 6.1 D/G intensity ratios of $\mathrm{rGO}$ and the hybrid materials

\begin{tabular}{cc}
\hline Material & $\mathbf{I}_{\mathbf{D}} / \mathbf{I}_{\mathbf{G}}$ \\
\hline rGO & 1.44 \\
1G-rGO & 1.49 \\
2G-rGO & 1.56 \\
4G-rGO & 1.67 \\
\hline
\end{tabular}

\section{Brunauer-Emmett-Teller (BET) surface area analysis}

The BET area of the hybrid materials were significantly lower than that shown by the rGO raw material $\left(456 \mathrm{~m}^{2} \mathrm{~g}^{-1}\right)$, probably due to the agglomeration of the rGO sheets upon functionalization with the molecular complexes.

Table 6.2 BET Surface Area analysis of rGO and hybrid materials

\begin{tabular}{cc}
\hline Material & BET surface $\left(\mathbf{m}^{2} / \mathbf{g}\right)$ \\
\hline rGO & 455.94 \\
1G-rGO & 262.44 \\
2G-rGO & 237.80 \\
3G-rGO & 223.60 \\
5rGO & 165.34 \\
5rGO (Activated) & 221.34 \\
\hline
\end{tabular}




\section{XPS Analysis of 4G-rGO}

The hybrid material of iridium (4G-rGO) was also characterized by means of X-ray Photoelectron Spectroscopy (XPS). The Figure 6.13 shows the XPS spectra for the Ir $4 \mathrm{f}$ core level of the molecular complex 4G and the hybrid material 4G-rGO. The spectra show two characteristic peaks, the $\operatorname{Ir}_{4} \mathrm{f}_{7 / 2}$ peak centered at $62.5 \mathrm{eV}$ and the $\operatorname{Ir}_{4} \mathrm{f}_{5 / 2}$ peak

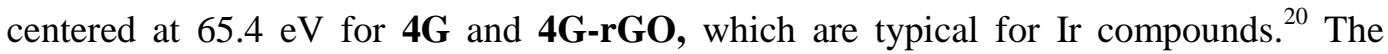
presence of the characteristic Ir4f peaks in the hybrid solid 4G-rGO confirms the presence of the molecular complex $\mathbf{4 G}$ over the surface of the reduced graphene oxide.

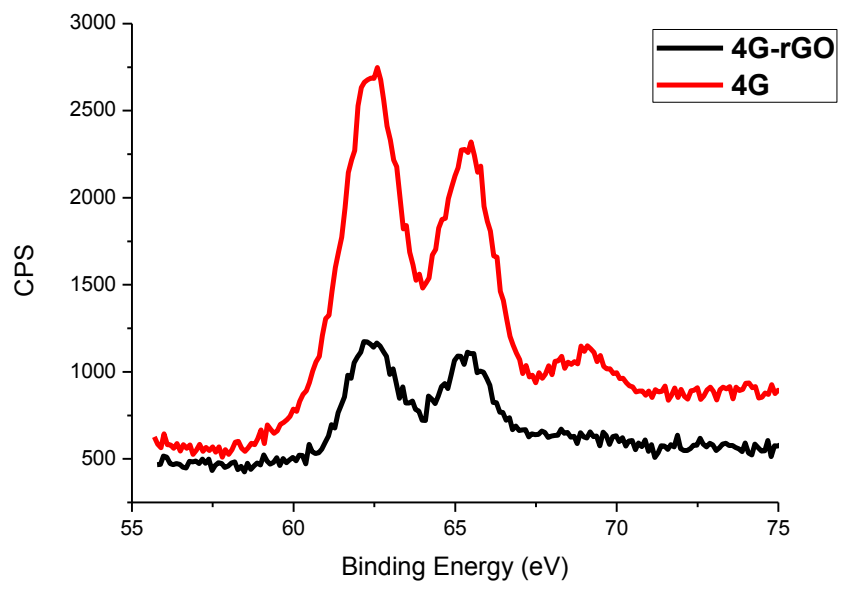

Figure 6.13 XPS spectra for the Ir4f core level of $\mathbf{4 G}$ and $\mathbf{4 G - r G O}$

\section{Scanning Electron Microscopy (SEM) Images}

The samples for microstructural and microanalysis determinations were covered with a $\mathrm{Pt}$ film. 

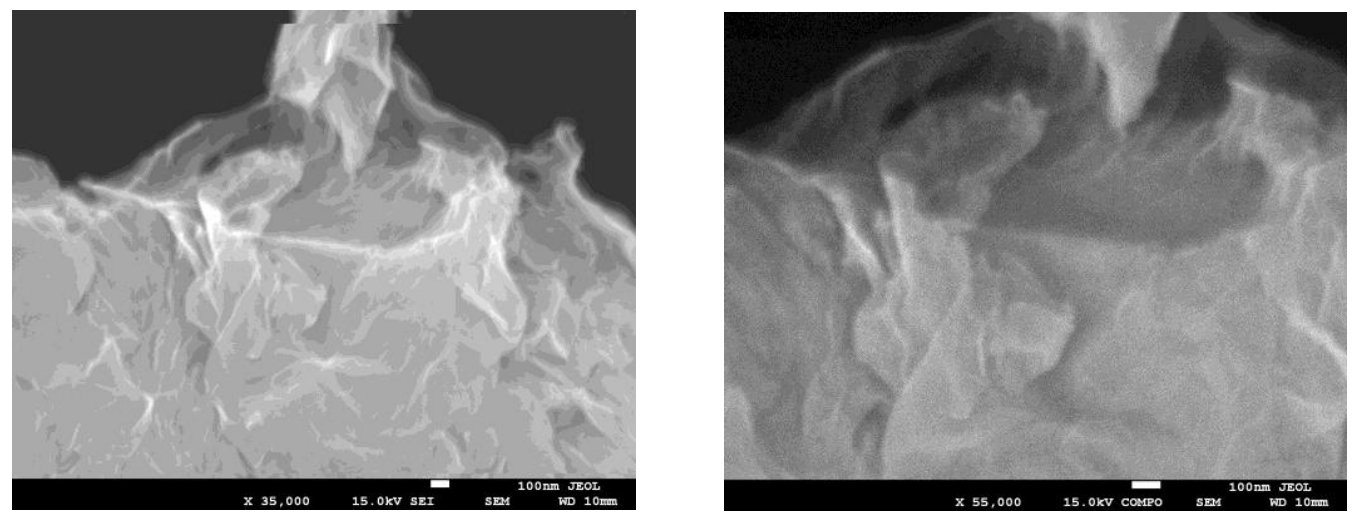

Figure 6.14 SEM images of 1G-rGO hybrid using the secondary electrons (SE) (left) and the back-scattered electrons (BSE) (right) signals of the SEM. Scale bar: $100 \mathrm{~nm}$
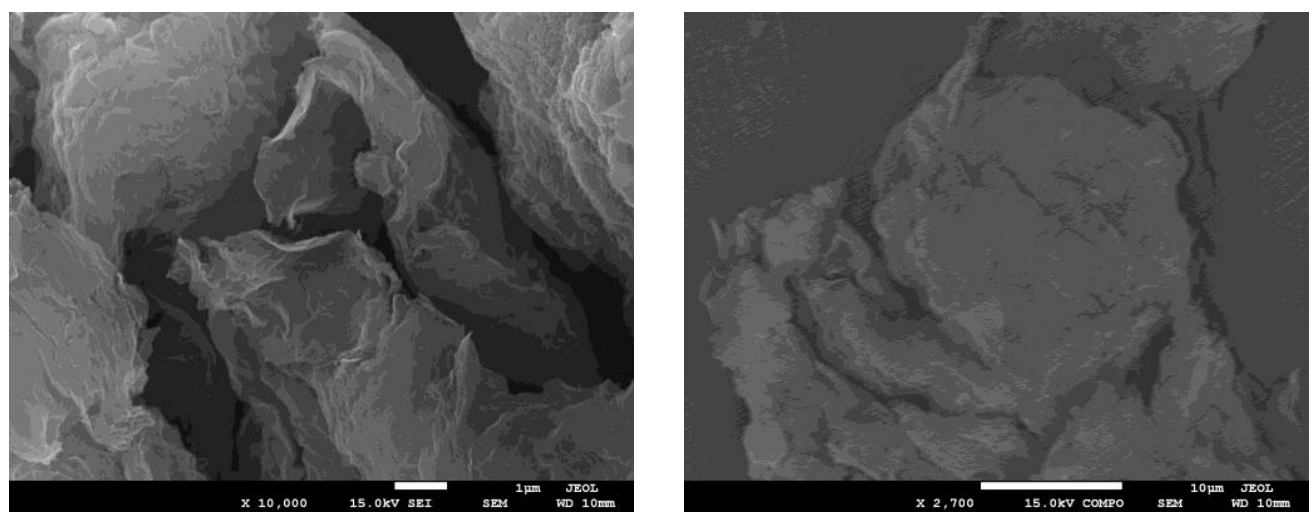

Figure 6.15 SEM images of 2G-rGO hybrid using the secondary electrons (SE) (left) and the back-scattered electrons (BSE) (right) signals of the SEM. Scale bar: $1 \mu \mathrm{m}$ (left), $10 \mu \mathrm{m}$ (right)

\section{High Resolution Transmission Electron Microscopy (HRTEM) images}

The samples were prepared by drying a droplet of a methanolic dispersion on a carboncoated copper grid. 


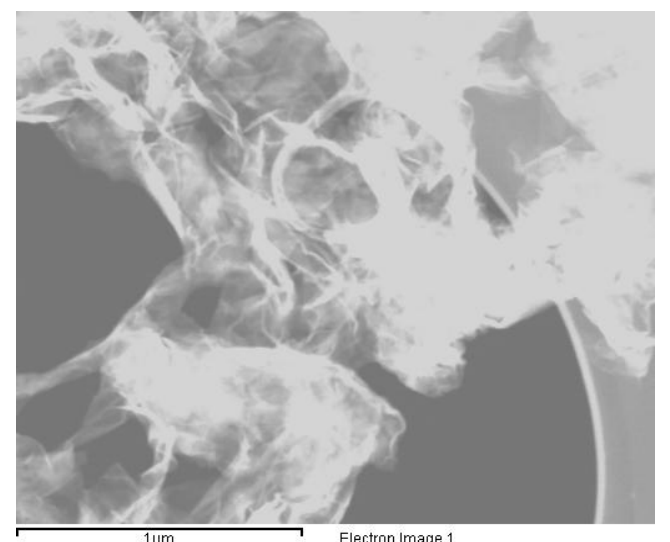

Electron Imaqe 1

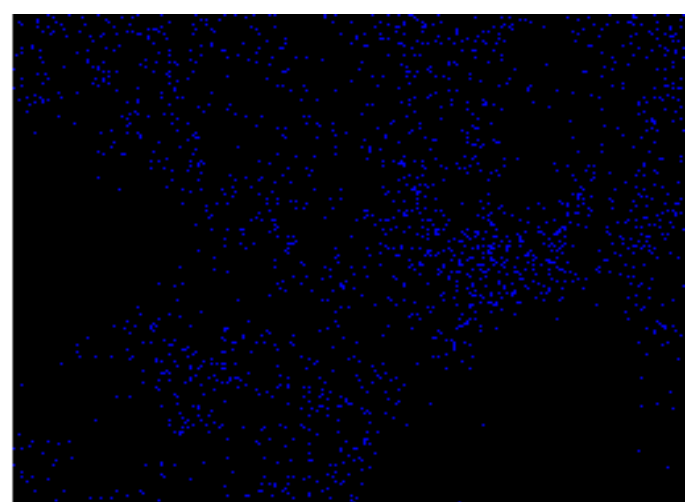

Pd La1

Figure 6.16 STEM image (left) of 1G-rGO. The EDS elemental mapping image (right) shows the homogeneous distribution of palladium. Scale bar: $1 \mu \mathrm{m}$

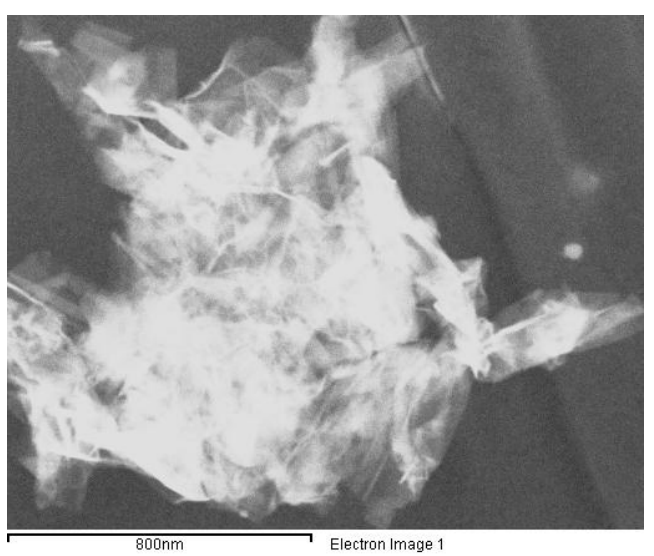

Electron Image 1

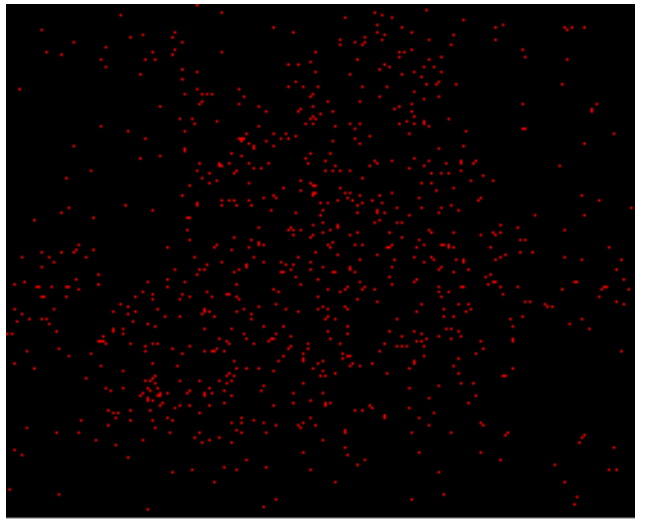

Ru La1

Figure 6.17 STEM image (left) of 2G-rGO. The EDS elemental mapping image (right) shows the homogeneous distribution of ruthenium. Scale bar: $600 \mathrm{~nm}$ 

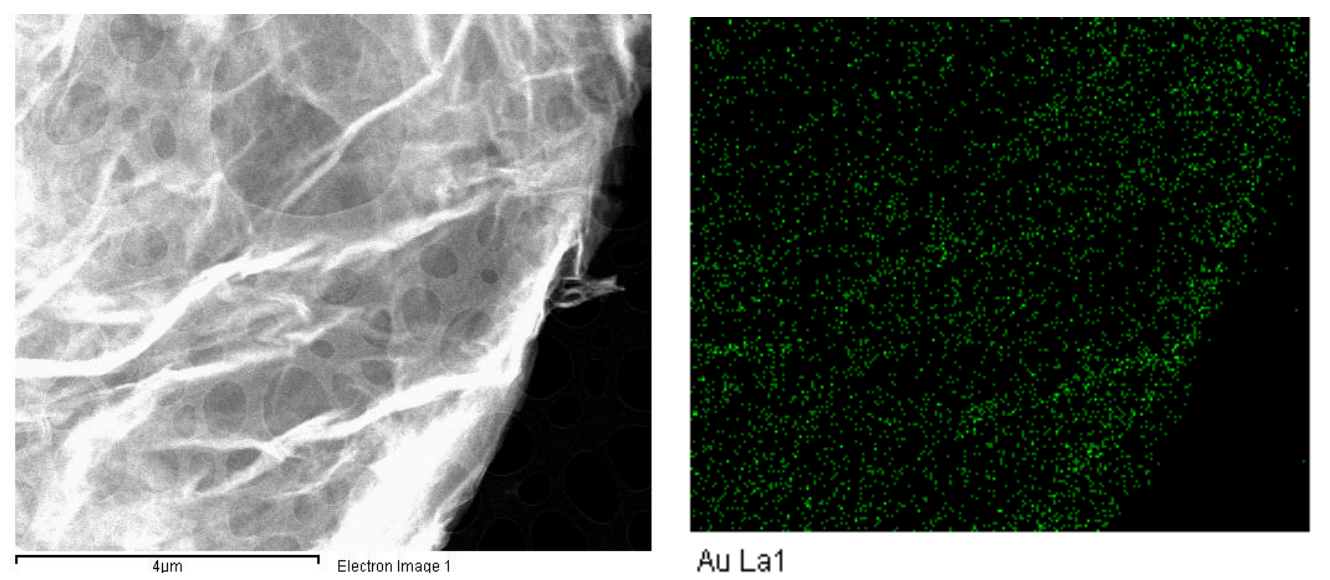

Au La1

Figure 6.18 STEM image (left) of 3G-rGO. The EDS elemental mapping image (right) shows the homogeneous distribution of gold. Scale bar: $4 \mu \mathrm{m}$

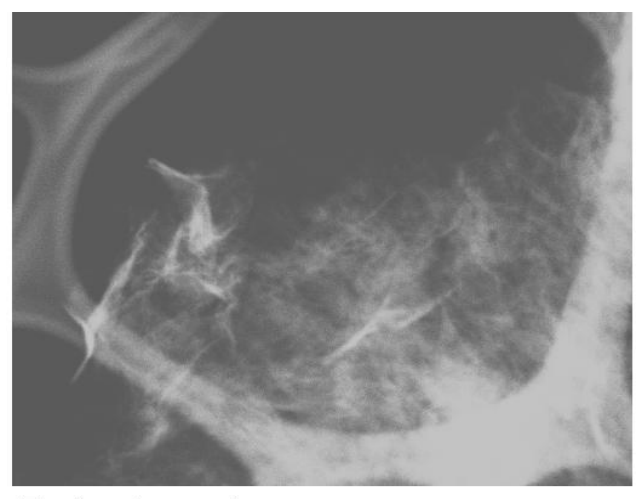

Electron Image 1

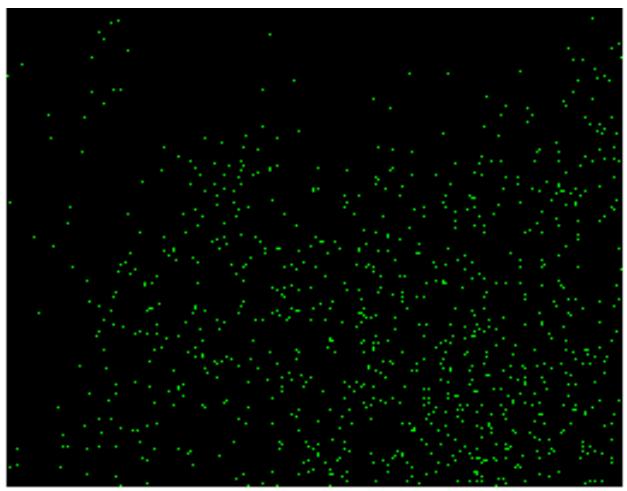

Ir Lb1

Figure 6.19 STEM image (left) of 4G-rGO. The EDS elemental mapping image (right) shows the homogeneous distribution of iridium 


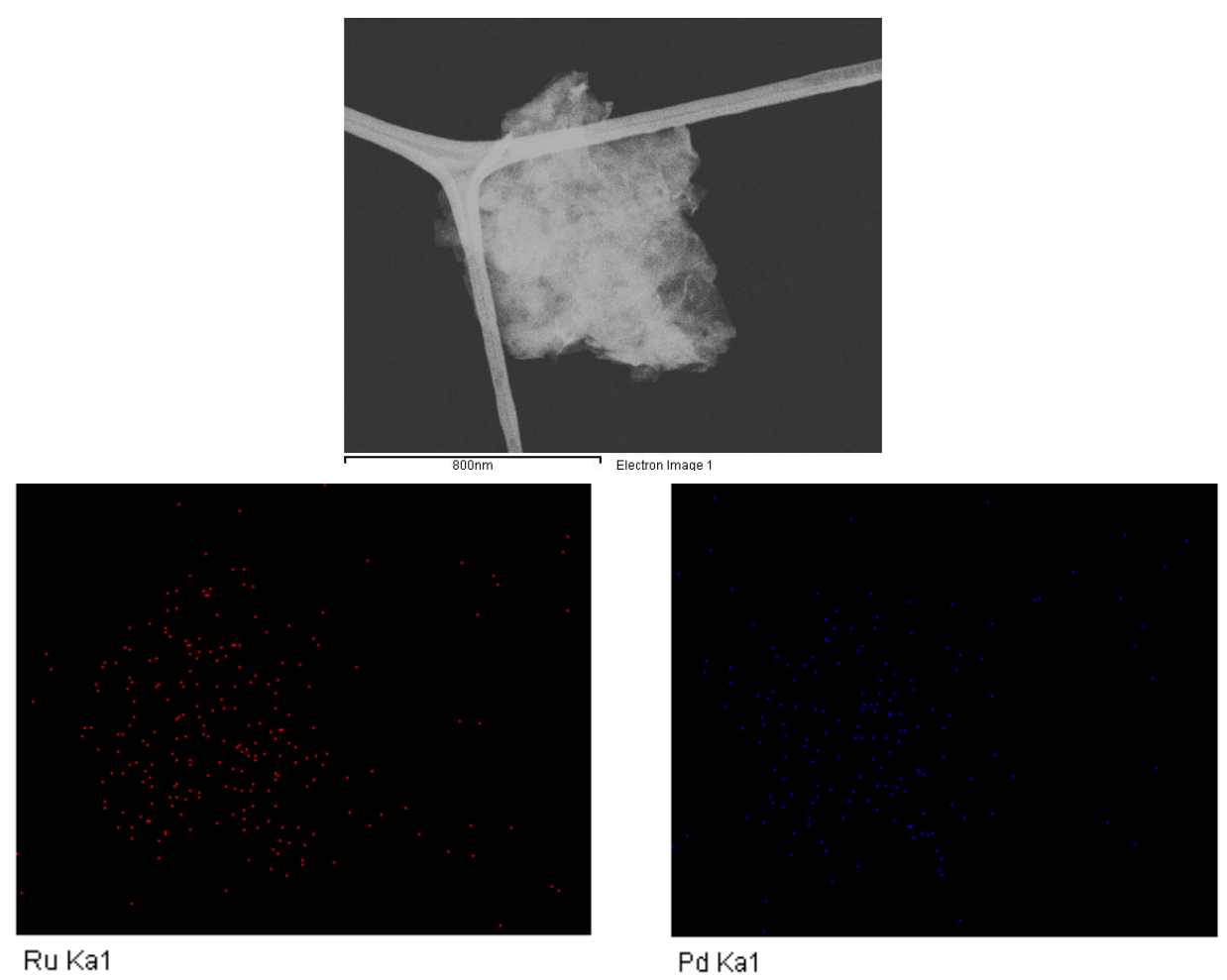

Figure 6.20 STEM image of 5rGO (top). The EDS elemental mapping images show the homogeneous distribution of ruthenium (left) and palladium (right). Scale bar: $600 \mathrm{~nm}$

\section{Atomic Force Microscopy (AFM) images}

The solid samples were dispersed in methanol, deposited on mica by drop casting and dried at room temperature. 

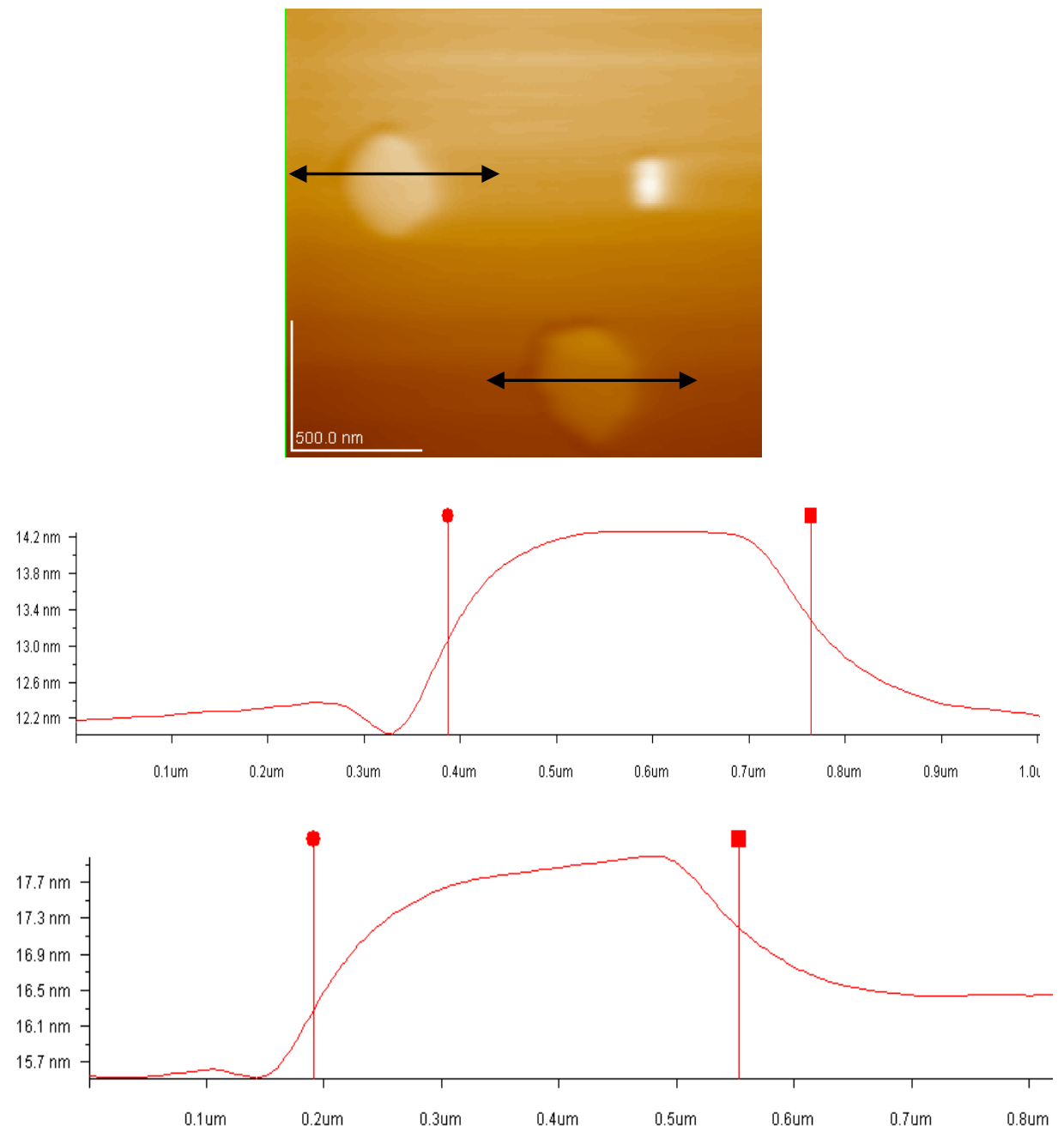

Figure 6.21 AFM image of rGO and its height profiles for the indicated lines across the sheets 

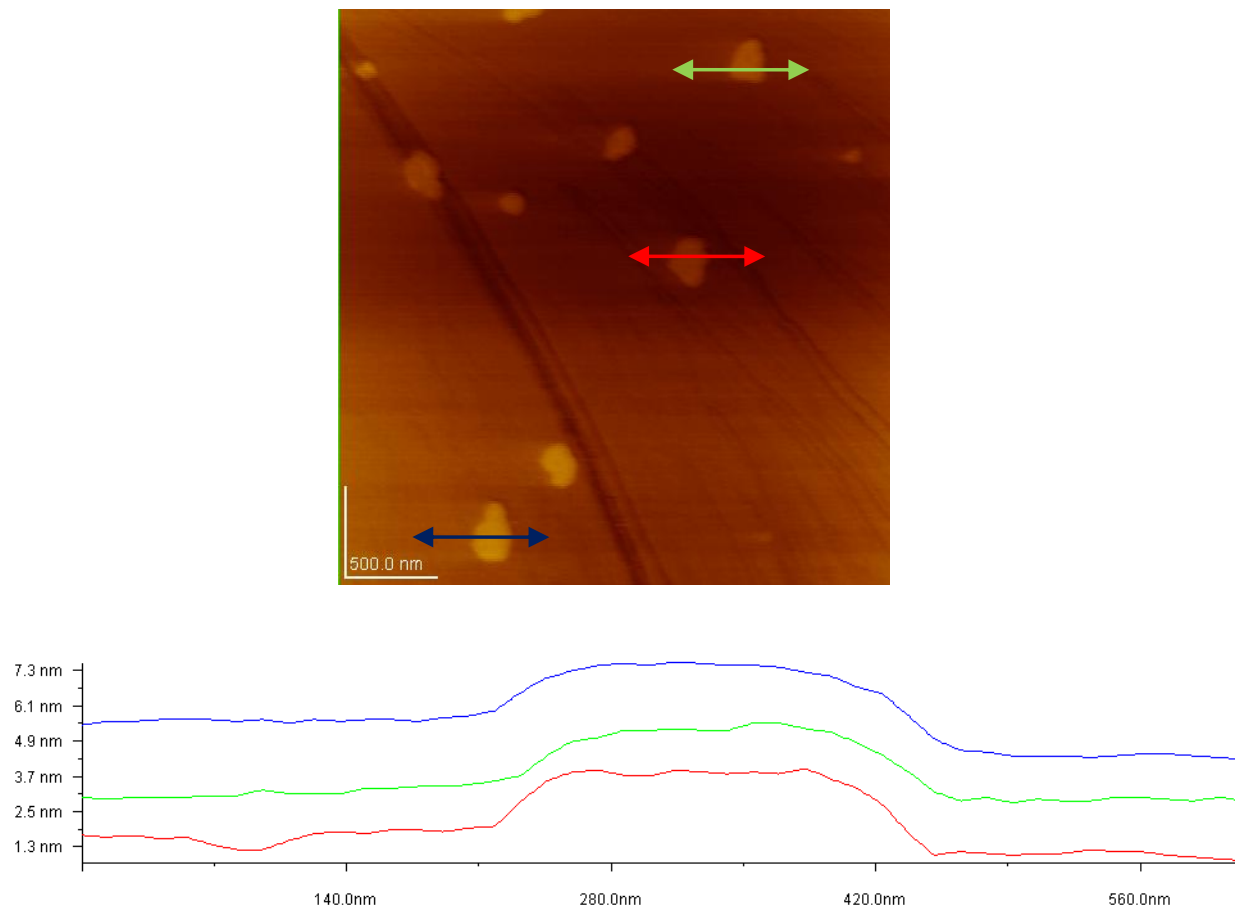

Figure 6.22 AFM image of activated 5rGO and its height profiles for the indicated lines across the sheets

\section{CATALYTIC EXPERIMENTS}

\subsection{Reduction of nitroarenes by transfer hydrogenation using primary alcohols using bimetallic Au/Ir complexes}

In a capped vessel containing a stirrer bar, nitroarene $(0.3 \mathrm{mmol})$, benzylalcohol (10 mmol), catalyst $(1 \mathrm{~mol} \%$ metal $), \mathrm{Cs}_{2} \mathrm{CO}_{3}(0.3 \mathrm{mmol})$ and anisole $(0.3 \mathrm{mmol})$ as the internal reference, were vigorously stirred at $100^{\circ} \mathrm{C}$ under aerobic conditions for the given reaction time. The yields, conversion and product selectivity were determined GC chromatography using anisole as internal standard. The products were identified by GCMS, and the spectra obtained were compared to the spectroscopic data of the commercially available compounds. 


\subsection{Chelation assisted C-H activation of arylpyridines and Oppenauer oxidation using bimetallic $\mathrm{Ru} / \mathrm{Ir}$ complexes}

\section{Oppenauer oxidation}

The catalyst $(1 \mathrm{~mol} \%)$, 1-phenylethanol $(1 \mathrm{mmol}), \mathrm{K}_{2} \mathrm{CO}_{3}(1 \mathrm{mmol})$ and deoxygenated acetone $(2 \mathrm{~mL})$ were placed together in a thick-walled glass tube. The mixture was stirred at $100^{\circ} \mathrm{C}$ for the desired time. Yields were determined by GC chromatography using anisole $(1 \mathrm{mmol})$ as internal standard. According to the spectroscopic data of the commercially available compounds, products were identified.

\section{Chelation assisted C-H activation of arylpyridines and Oppenauer oxidation}

The ruthenium complex $(5 \mathrm{~mol} \%)$ and KOAc $(10 \mathrm{~mol} \%)$ were placed together in a thickwalled Schlenk tube with a Teflon cap. The tube was then evacuated and filled with nitrogen three times. NMP $(1 \mathrm{~mL})$ and acetone $(1 \mathrm{~mL})$ were added and the mixture stirred at room temperature for $1 \mathrm{~h}$. Then arylpyridine $(0.5 \mathrm{mmol})$, $\mathrm{Ar}-\mathrm{X}(1.25 \mathrm{mmol})$ and $\mathrm{K}_{2} \mathrm{CO}_{3}$ $(2.5 \mathrm{mmol})$ were added. The resulting mixture was stirred at $120^{\circ} \mathrm{C}$ for the desired time. $\mathrm{H}_{2} \mathrm{O}$ and ethyl acetate were added to the cold reaction mixture. The organic phase was dried with $\mathrm{Na}_{2} \mathrm{SO}_{4}$ and concentrated under vacuum. The remaining residue was purified by column chromatography on silica gel (hexane/diethyl ether mixture) to yield the corresponding ortho-arylated products. Yields and ratios were determined by ${ }^{1} \mathrm{H}$ NMR spectroscopy and by GC analyses using anisole $(0.5 \mathrm{mmol})$ as internal standard. According to previously reported spectroscopic data, products were identified..$^{21,22}$

\subsection{Carbon-fluor activation via hydrodefluorination using bimetallic $\mathbf{R u} / \mathbf{P d}$ complexes}

\section{Hydrodefluorination of fluoroarene compounds}

The catalyst $(1 \mathrm{~mol} \%)$ and $\mathrm{NaOt} \mathrm{Bu}(0.3 \mathrm{mmol})$ were placed together in a thick-walled Schlenk tube with a Teflon cap. The tube was then evacuated and filled with nitrogen three times. 2-propanol $(2 \mathrm{~mL})$ and fluoroarene $(0.3 \mathrm{mmol})$ were added and the mixture was stirred at $80^{\circ} \mathrm{C}$ for the desired time. Yields were determined by GC analyses using anisole $(0.3 \mathrm{mmol})$ as internal standard. Isolated products were characterized by ${ }^{1} \mathrm{H}$ NMR 
and ${ }^{13} \mathrm{C}$ NMR after extraction with dichloromethane and a saturated $\mathrm{NaCl}$ solution $(3 \times 5$ $\mathrm{mL})$.

\section{Hydrodefluorination of fluoroalkyl compounds}

The heterodimetallic complex $\mathbf{5 A}(5 \mathrm{~mol} \%)$ and $\mathrm{NaO} t \mathrm{Bu}(0.9 \mathrm{mmol})$ were placed together in a thick-walled Schlenk tube with a Teflon cap. The tube was then evacuated and filled with nitrogen three times. 2-propanol $(2 \mathrm{~mL})$ and fluoroalkyl $(0.3 \mathrm{mmol})$ were added and the mixture was stirred at $80^{\circ} \mathrm{C}$ for the desired time. Yields were determined by GC analyses using anisole $(0.3 \mathrm{mmol})$ as internal standard. Isolated products were characterized by ${ }^{1} \mathrm{H}$ and ${ }^{13} \mathrm{C}$ NMR after extraction with dichloromethane and a saturated $\mathrm{NaCl}$ solution $(3 \times 5 \mathrm{~mL})$.

\section{Kinetic measurements}

General procedure for ${ }^{19} \mathrm{~F}$ NMR monitoring experiments: A J. Young tube was loaded with catalyst $(1 \mathrm{~mol} \%)$ and $\mathrm{NaO} t \mathrm{Bu}(0.3 \mathrm{mmol})$. The tube was then evacuated and filled with nitrogen three times. 2-propanol $(1.5 \mathrm{~mL})$ and fluoroarene $(0.3 \mathrm{mmol})$ were added and the mixture was stirred at room temperature until homogeneous solution was formed. The tube was the introduced in an NMR probe preheated at $80^{\circ} \mathrm{C}$. The conversion of fluoroaromatic compound was monitored by ${ }^{19} \mathrm{~F}$ NMR spectroscopy at intervals over the course of the desired time. The corresponding deuterated products were identified by GCMS-QP2010, ${ }^{1} \mathrm{H}$ NMR and ${ }^{13} \mathrm{C}$ NMR.

Determination of the KIE for the HDF of fluorobenzene and p-fluorotoluene: The reactions were carried out using complex 5A $(1 \mathrm{~mol} \%)$, fluoroarene $(0.3 \mathrm{mmol}), \mathrm{NaO} t \mathrm{Bu}$ $(0.3 \mathrm{mmol})$, at $80^{\circ} \mathrm{C}$ in $i \mathrm{PrOH}$ or deuterium-labeled isopropyl alcohol, $\left(\mathrm{CD}_{3}\right)_{2} \mathrm{CDOD}(1.5$ $\mathrm{mL}$ ). The evolution of the reactions was monitored by ${ }^{19} \mathrm{~F}$ NMR, by measuring the disappearance of the ${ }^{19} \mathrm{~F}$ resonance of fluoroarene. 
$\underset{\substack{i \mathrm{PrOH} \text { or } i \mathrm{PrOD} \\ 80^{\circ} \mathrm{C}}}{\stackrel{[5 \mathrm{~A}](1 \mathrm{~mol} \%)}{\mathrm{NaOtBu}}}$
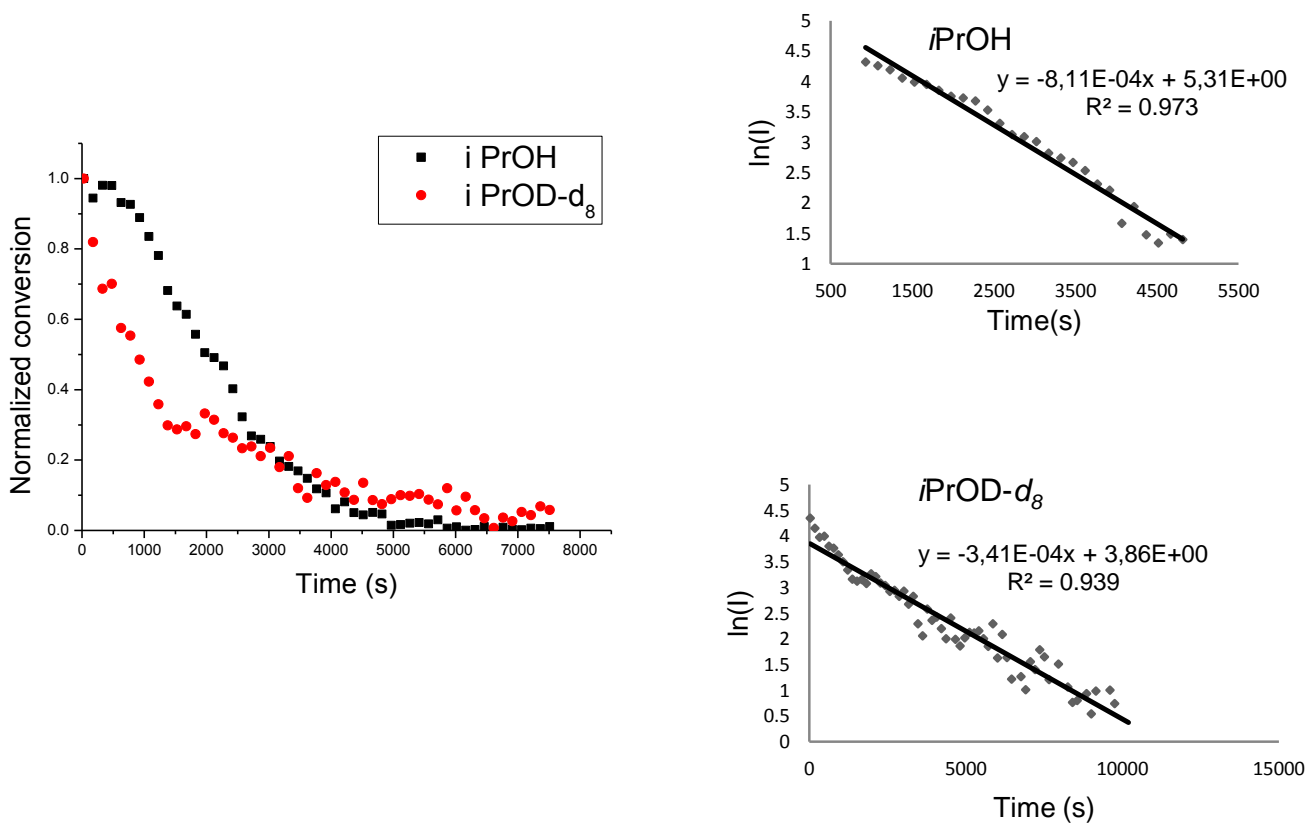

Figure 6.23 Kinetic curves for the HDF of fluorobenzene (right) in $i \mathrm{PrOH}$ and $i \mathrm{PrOD} \mathrm{d}_{8}$, and the $\ln (\mathrm{I})$ vs. time plots with the linear regressions for the reaction in $i \mathrm{PrOH}$ and $i \operatorname{PrOD} \mathrm{d}_{8}$ (top and bottom, respectively).

Table 6.3 Rate constants, regression coefficients and KIE values for the HDF of fluorobenzene and $p$-fluorotoluene.

\begin{tabular}{ccccc}
\hline Substrate & Solvent & $\boldsymbol{k}_{\boldsymbol{H}}\left(\mathbf{o r} \boldsymbol{k}_{\boldsymbol{D}}\right)\left(\mathbf{s}^{-1}\right)$ & $\mathbf{R}^{2}$ & KIE \\
\hline \multirow{2}{*}{ Fluorobenzene } & $i \operatorname{PrOH}$ & $8.11 \cdot 10^{-4}$ & 0.973 & 2.4 \\
& $i \operatorname{PrOD~d} \mathrm{d}_{8}$ & $3.41 \cdot 10^{-4}$ & 0.939 & \\
\multirow{3}{*}{-fluorotoluene } & $i \operatorname{PrOH}$ & $4.51 \cdot 10^{-4}$ & 0.990 & 2.7 \\
& $i \operatorname{PrOD~d}_{8}$ & $1.65 \cdot 10^{-4}$ & 0.993 & \\
\hline
\end{tabular}


Determination of the reaction order in the catalyst: A set of experiments were performed modifying the catalyst loading (from 0.25 to $2 \mathrm{~mol} \%$ ), while keeping the concentration of the substrates fluorobenzene $(0.3 \mathrm{mmol})$ and $\mathrm{NaO} t \mathrm{Bu}(0.3 \mathrm{mmol})$ fixed, at $80^{\circ} \mathrm{C}$ in $i \mathrm{PrOH}$ $(1.5 \mathrm{~mL})$. The evolution of the reactions was monitored by ${ }^{19} \mathrm{~F}$ NMR, by measuring the disappearance of the ${ }^{19} \mathrm{~F}$ resonance of fluorobenzene.

Determination of the optimum ratio of the mixture of the homodimetallic complexes ([7A]:[8A]): A set of experiments were carried out using variable concentrations of palladium catalyst $8 \mathrm{~A}$ (from $0.3 \mathrm{mM}$ to $2.62 \mathrm{mM}$ ) and maintaining constant the ruthenium concentration $([7 \mathrm{~A}], 1.55 \mathrm{mM})$. The reactions were performed using fluorobenzene in the presence of $\mathrm{NaO} t \mathrm{Bu}$ (both in $1 \mathrm{M}$ concentration) in $i \mathrm{PrOH}$ at $80^{\circ} \mathrm{C}$ for $30 \mathrm{~min}$. A set of parallel experiments were carried out using a constant loading of $\mathbf{8 A}$ and varying the concentration of the ruthenium complex 7A. The yields were determined by GC analyses using anisole as internal standard.

\subsection{Asymmetric hydrophosphination using chiral palladacycles with NHC ligands}

The palladium complex $(2 \mathrm{~mol} \%)$ and $\mathrm{K}_{2} \mathrm{CO}_{3}(5 \mathrm{~mol} \%)$ were placed together in Schlenk with a Teflon cap. The tube was purged with nitrogen three times. The solvent $(2 \mathrm{~mL})$ was added and the mixture was stirred at room temperature for $2 \mathrm{~h}$. Then diphenylphosphine $(106.6 \mu \mathrm{L}, 0.6 \mathrm{mmol})$ was added, the resulting yellow solution was stirred for 5 minutes. After addition of 1,3-diphenyl-2-propen-1-one (106 mg, $0.5 \mathrm{mmol}$ ) the resulting mixture was stirred for $15 \mathrm{~h}$ at room temperature, then quenched with $\mathrm{H}_{2} \mathrm{O}_{2}$ aqueous solution $(30 \%, 200 \mu \mathrm{L})$ and EtOAc $(3 \mathrm{~mL})$. The mixture was stirred for $2 \mathrm{~h}$ and was added $0.4 \mathrm{~mL}$ of a saturated $\mathrm{Na}_{2} \mathrm{~S}_{2} \mathrm{O}_{3}$ aqueous solution. The mixture was extracted with dichloromethane three times. The organic phase was dried with $\mathrm{MgSO}_{4}$ and concentrated under vacuum. The residue was purified by silica gel chromatography with dichloromethane/methanol $=70 / 1$ to afford the product as a white solid. The ee was determined on a Daicel Chiracel IC column at $30^{\circ} \mathrm{C}$ with hexane/2-propanol $=70 / 30$, flow $=0.6 \mathrm{~mL} / \mathrm{min}, \lambda=250 \mathrm{~nm}$. Retention times: $13.2 \mathrm{~min}$ [(S)-enantiomer], $17.2 \mathrm{~min}$ [(R)-enantiomer]. 


\subsection{Sequential isomerization of propargylic alcohols/hydrophosphination using Ir/Pd* complexes}

The heterodimetallic complex $(2 \mathrm{~mol} \%)$ and $\mathrm{K}_{2} \mathrm{CO}_{3}(103 \mathrm{mg}, 0.7 \mathrm{mmol})$ were placed together in a Schlenk tube with a Teflon cap. The tube was then evacuated and filled with nitrogen three times. 1,3-Diphenyl-2-propyn-1-ol (100 $\mu \mathrm{L}, 0.47 \mathrm{mmol})$ and solvent (2 $\mathrm{mL})$ was added and the mixture stirred at $90^{\circ} \mathrm{C}$ for $5 \mathrm{~h}$. Then diphenylphosphine $(93 \mu \mathrm{L}$, $0.52 \mathrm{mmol}$ ) was added, the resulting mixture was stirred for $10 \mathrm{~h}$ at room temperature, then quenched with $\mathrm{H}_{2} \mathrm{O}_{2}$ aqueous solution $(30 \%, 200 \mu \mathrm{L})$ and EtOAc $(3 \mathrm{~mL})$. The mixture was stirred for $2 \mathrm{~h}$ and was added $0.4 \mathrm{~mL}$ of a saturated $\mathrm{Na}_{2} \mathrm{~S}_{2} \mathrm{O}_{3}$ aqueous solution. The mixture was extracted with dichloromethane three times. The organic phase was dried with $\mathrm{Na}_{2} \mathrm{SO}_{4}$ and concentrated under vacuum. The residue was purified by silica gel chromatography with dichloromethane/methanol $=70 / 1$ to afford product as a white solid. According to previously reported spectroscopic data, products were identified. The $e e$ was determined on a Daicel Chiracel IC column at $30^{\circ} \mathrm{C}$ with hexane/2propanol $=70 / 30$, flow $=0.6 \mathrm{~mL} / \mathrm{min}, \lambda=250 \mathrm{~nm}$. Retention times: $13.2 \mathrm{~min}[(\mathrm{~S})-$ enantiomer], $17.2 \mathrm{~min}[(\mathrm{R})$-enantiomer].

\subsection{Transfer hydrogenation using Ir-NH-NHC complexes}

General procedure: The iridium complex $(1 \mathrm{~mol} \%)$ and $\mathrm{NaO} t \mathrm{Bu}(5 \mathrm{~mol} \%)$ were placed together in Schlenk tube with a Teflon cap. The tube was then evacuated and filled with nitrogen three times. 2-propanol $(2 \mathrm{~mL})$ and the corresponding ketone $(0.5 \mathrm{mmol})$ were added and the mixture was stirred at $50^{\circ} \mathrm{C}$ for $2 \mathrm{~h}$. Yields were determined by GC analyses using anisole $(0.5 \mathrm{mmol})$ as internal standard.

General procedure for asymmetric transfer hydrogenation: The iridium complex (1 $\mathrm{mol} \%)$ and $\mathrm{NaO} t \mathrm{Bu}(5 \mathrm{~mol} \%)$ were placed together in Schlenk tube with a Teflon cap. The tube was then evacuated and filled with nitrogen three times. 2-propanol (2 mL) and the corresponding ketone $(0.5 \mathrm{mmol})$ were added and the mixture was stirred at $30^{\circ} \mathrm{C}$ for $8 \mathrm{~h}$. Yields were determined by GC analyses using anisole $(0.5 \mathrm{mmol})$ as internal standard. The ee was determined using Chiral GC. 


\subsection{Catalytic experiments using Ru-MIC complexes}

\section{Transfer hydrogenation}

In a one-neck round bottom flask, a mixture of acetophenone $(0.6 \mathrm{mmol})$, the ruthenium complex $(0.006 \mathrm{~mol})$, a solution of $\mathrm{KOH}(2 \mathrm{M}, 0.06 \mathrm{mmol})$ and anisole $(0.6 \mathrm{mmol})$ as internal standard was refluxed in 2-propanol $(2 \mathrm{~mL})$ for the desired time. The reaction mixture was analyzed by ${ }^{1} \mathrm{H}$ NMR spectroscopy, by introducing aliquots $(0.1 \mathrm{~mL})$ of the reacting solution in a NMR tube with $0.5 \mathrm{~mL}$ of $\mathrm{CDCl}_{3}$. Anisole was used in all cases as internal standard in order to determine conversions and yields.

\section{Base-free dehydrogenation of alcohols}

In a one-neck round bottom flask, a mixture of benzyl alcohol $(0.2 \mathrm{mmol})$, the ruthenium complex $(0.01 \mathrm{~mol})$, and anisole $(0.2 \mathrm{mmol})$ as internal standard was heated in $o$-DCB (2 $\mathrm{mL}$ ) at $150^{\circ} \mathrm{C}$ for $16 \mathrm{~h}$. The reaction mixture was analyzed by ${ }^{1} \mathrm{H}$ NMR spectroscopy, by introducing aliquots $(0.1 \mathrm{~mL})$ of the reacting solution in a NMR tube with $0.5 \mathrm{~mL}$ of $\mathrm{CDCl}_{3}$. Anisole was used in all cases as internal standard in order to determine conversions and yields.

\subsection{Catalytic experiments and recyclability studies using hybrid materials}

\section{Alkene reductions using 1G-rGO}

General Procedure: Molecular hydrogen was added with a balloon filled with 1 atm of $\mathrm{H}_{2}$ to a mixture of alkene $(0.3 \mathrm{mmol}), \mathrm{Cs}_{2} \mathrm{CO}_{3}(0.3 \mathrm{mmol})$, and 1G-rGO $\left(3 \cdot 10^{-3} \mathrm{mmol}\right.$, based on metal) in toluene $(5 \mathrm{~mL})$. The system was then evacuated and backfilled with $\mathrm{H}_{2}$ in cycles for three times before putting the reaction vessel in an oil bath at $100{ }^{\circ} \mathrm{C}$ for 15 minutes. Yields were determined by GC analyses using anisole $(0.3 \mathrm{mmol})$ as internal standard. Products were identified according to spectroscopic data of the commercially available compounds.

Recycling experiments: The hydrogenation reaction was carried out under identical reaction conditions as described in the alkene reduction procedure. After completion of each run $(15 \mathrm{~min}$.$) , the reaction mixture was allowed to reach room temperature and$ 
centrifuged. The remaining solid was washed thoroughly with dichloromethane $(5 \times 10$ $\mathrm{mL}$ ), dried and reused in the following run. The base was only added in the first run.

Large-scale recycling experiments: Molecular hydrogen was added with a balloon filled with $1 \mathrm{~atm}$ of $\mathrm{H}_{2}$ to a mixture of styrene $(2 \mathrm{~mL}, 17.3 \mathrm{mmol}), \mathrm{Cs}_{2} \mathrm{CO}_{3}(2.8 \mathrm{~g}, 8.65 \mathrm{mmol})$, anisole as internal standard $(1.9 \mathrm{~mL}, 17.3 \mathrm{mmol})$ and 1G-rGO $\left(1.74 \cdot 10^{-3} \mathrm{mmol}\right.$, based on metal) in toluene $(20 \mathrm{~mL})$. The system was then evacuated and backfilled with $\mathrm{H}_{2}$ in cycles for three times before putting the reaction vessel in an oil bath at $100{ }^{\circ} \mathrm{C}$. Reaction monitoring, yields and conversions were determined by GC analyses. After completion of each run $(20 \mathrm{~h})$, the reaction mixture was allowed to reach room temperature and centrifuged. The remaining solid was washed thoroughly with dichloromethane $(5 \times 10$ $\mathrm{mL}$ ), dried and reused in the following run. The base was only added in the first run.

\section{Nitroarene reductions using 1G-rGO}

Molecular hydrogen was added with a balloon filled with 1 atm of $\mathrm{H}_{2}$ to a mixture of nitroarene $(0.3 \mathrm{mmol}), \mathrm{Cs}_{2} \mathrm{CO}_{3}(0.3 \mathrm{mmol})$, anisole as internal standard $(0.3 \mathrm{mmol})$ and $1 \mathrm{G}-\mathrm{rGO}\left(6 \cdot 10^{-3} \mathrm{mmol}\right.$, based on metal) in toluene $(5 \mathrm{~mL})$. The system was then evacuated and backfilled with $\mathrm{H}_{2}$ in cycles for three times before putting the reaction vessel in an oil bath at $100{ }^{\circ} \mathrm{C}$ for $2 \mathrm{~h}$. Yields were determined by $\mathrm{GC}$ analyses using anisole $(0.3 \mathrm{mmol})$ as internal standard. Products were identified according to spectroscopic data of the commercially available compounds.

\section{Dehydrogenative alcohol oxidations using 2G-rGO}

General procedure: In a round-bottom flask a mixture of the alcohol $(0.3 \mathrm{mmol}), \mathrm{Cs}_{2} \mathrm{CO}_{3}$ $(0.3 \mathrm{mmol})$ and 2G-rGO $\left(6 \cdot 10^{-3} \mathrm{mmol}\right.$, based on metal) was refluxed in toluene $(4 \mathrm{~mL})$ for 12 hours. Yields were determined by GC analyses using anisole $(0.3 \mathrm{mmol})$ as internal standard. Products were identified according to spectroscopic data of the commercially available compounds.

Recycling experiments: The recycling of benzyl alcohol oxidation was carried out under identical reaction conditions as described before. After completion of each run (12 h.), the reaction mixture was allowed to reach room temperature and centrifuged. The remaining 
solid was washed thoroughly with dichloromethane $(5 \times 10 \mathrm{~mL})$, dried and reused in the following run. The base was only added in the first run.

Large-scale recycling experiments: In a round-bottom flask a mixture of benzyl alcohol $(1 \mathrm{~mL}, 9.66 \mathrm{mmol}), \mathrm{Cs}_{2} \mathrm{CO}_{3}(1.5 \mathrm{~g}, 4.83 \mathrm{mmol})$, anisole as internal standard (1 mL, 9.66 $\mathrm{mmol})$ and the 2G-rGO $\left(3 \cdot 10^{-3} \mathrm{mmol}\right.$, based on metal) was refluxed in toluene $(10 \mathrm{~mL})$ for 24 hours. Reaction monitoring, yields and conversions were determined by GC analyses. After completion of each run $(24 \mathrm{~h})$, the reaction mixture was allowed to reach room temperature and centrifuged. The remaining solid was washed thoroughly with dichloromethane $(5 \times 10 \mathrm{~mL})$, dried and reused in the following run. The base was only added in the first run.

Hot filtration test: This experiment was carried out under identical reaction conditions as earlier described. After 6h (yield 48\%) the catalyst was filtered off at the reaction temperature $\left(100{ }^{\circ} \mathrm{C}\right)$, and the solid free filtrate was allowed to stir for $16 \mathrm{~h}$ under identical reaction conditions. GC analysis monitoring indicated that no further oxidation of benzyl alcohol occurred (GC final yield 48\%).

\section{Catalytic HDF using bimetallic Ru/Pd hybrid 5rGo}

General procedure: In a typical experiment the catalyst, ( $1 \mathrm{~mol} \%$ based on each metal) and $\mathrm{NaO} t \mathrm{Bu}(0.3 \mathrm{mmol})$ were placed together in a thick walled Schlenk tube with a Teflon cap. The tube was then evacuated and filled with nitrogen three times. 2-propanol $(2 \mathrm{~mL})$ was added and the mixture was stirred at $80^{\circ} \mathrm{C}$ for $24 \mathrm{~h}$. Then fluoroarene $(0.3$ mmol) and anisole $(0.3 \mathrm{mmol})$ were added and the mixture was stirred at $80^{\circ} \mathrm{C}$ for 3 hours. Yields were determined by GC analyses using anisole as internal standard.

Recycling experiments: The recycling experiments were carried out under identical reaction conditions as described in the HDF procedure. After completion of each run, the reaction mixture was allowed to reach room temperature and centrifuged. The remaining solid was washed thoroughly with dichloromethane $(5 \times 10 \mathrm{~mL})$, dried and reused in the following run. 
${ }^{19}$ F NMR monitoring experiments: A J. Young tube was loaded with catalyst (1 mol\% based on each metal) and $\mathrm{NaO} t \mathrm{Bu}(0.3 \mathrm{mmol})$. The tube was then evacuated and filled with nitrogen three times. 2-propanol $(2 \mathrm{~mL})$ and fluoroarene $(0.3 \mathrm{mmol})$ were added. The tube was the introduced in an NMR probe preheated at $80^{\circ} \mathrm{C}$. The conversion of fluorobenzene was monitored by ${ }^{19} \mathrm{~F}$ NMR spectroscopy at intervals over the course of the desired time. After completion of each run, the reaction mixture was allowed to reach room temperature and centrifuged. The remaining solid was washed thoroughly with dichloromethane $(5 \times 10 \mathrm{~mL})$, dried and reused in the following run.

\section{DFT STUDIES}

Quantum mechanical calculations were performed with the Gaussian09 package $^{23}$ at the DFT/M06 level of theory. ${ }^{24}$ SDD basis set and its corresponding effective core potentials (ECPs) were used to describe the iridium atom. ${ }^{25}$ An additional set of f-type functions was also added. ${ }^{26}$ Carbon, nitrogen, oxygen and hydrogen atoms were described with a 6$31 \mathrm{G}^{* *}$ basis set. ${ }^{27,28}$ Frequency calculations have been performed in order to determine the nature of the stationary points found (no imaginary frequencies for minima, only one imaginary frequency for transition states). Free Gibbs energies in $i \mathrm{PrOH}$ solution $(\varepsilon=$ 19.264) were calculated by computing the energy in the solvent by means of single-point calculations on the gas-phase optimized geometries with the SMD continuum solvation model, ${ }^{29}$ and subsequently applying the following expression:

$$
\mathrm{G}_{\mathrm{iPrOH}}=\mathrm{E}_{\mathrm{iPrOH}}+\left(\mathrm{G}_{\text {gas phase }}-\mathrm{E}_{\text {gas phase }}\right)
$$

The TS's connecting two minima were investigated by using a freeze-scan strategy. $\mathrm{C} \cdots \mathrm{H}$ distances involved in the $\mathrm{C}-\mathrm{H}$ bond forming processes have been frozen in subsequent $0.05 \AA$ steps and the structures have been optimized with the referred restriction. The optimized geometry for the first point has been used as a starting one for the following point in the profile, and the process has been repeated for subsequent points. A first approximation for the $\mathrm{C}-\mathrm{H} \cdots \mathrm{C}$ to $\mathrm{C} \cdots \mathrm{H}-\mathrm{C}$ reaction energy profile is therefore obtained. The highest energy structure in each studied profile is then used as a starting geometry in order to find the corresponding TS. In all cases the shape of the transition vector provided by the frequency calculation in each of the TS's is consistent 
with the proposed mechanism. IRC computations have been additionally carried out in order to connect minima and TS's and further strengthen the mechanism proposal.

Table 6.4 Relative energies and representative geometric parameters of the four transition states found for the concerted, one-step, outer-sphere hydrogen mechanism (pathway 2). Energies are expressed in $\mathrm{kcal} / \mathrm{mol}$, and distances in $\AA$.

\begin{tabular}{|c|c|c|c|c|c|}
\hline \multirow[b]{4}{*}{ enantiomer } & \multicolumn{5}{|c|}{$\mathrm{R}_{2 \mathrm{a}}$} \\
\hline & & P2-1a-2-DFT & P2-1b-2-DFT & P2-2a-2-DFT & P2-2b-2-DFT \\
\hline & & $\mathrm{R} 1 \mathrm{a}=\mathrm{Ph}$ & $\mathrm{R} 1 \mathrm{~b}=\mathrm{Ph}$ & $\mathrm{R} 2 \mathrm{a}=\mathrm{Ph}$ & $\mathrm{R} 2 \mathrm{~b}=\mathrm{Ph}$ \\
\hline & & $\mathrm{R}$ & S & S & $\mathrm{R}$ \\
\hline$\Delta \mathrm{G}(\mathrm{iPrOH})$ & & 24.3 & 24.6 & 24.7 & 25.7 \\
\hline$\Delta \mathbf{G}($ gas) & & 19.4 & 18.6 & 18.8 & 21.2 \\
\hline N-H & (a) & 1.04 & 1.04 & 1.04 & 1.04 \\
\hline N-H & (b) & 1.04 & 1.04 & 1.04 & 1.04 \\
\hline $\mathbf{H} \cdots \mathrm{O}$ & (c) & 1.85 & 1.85 & 1.81 & 1.79 \\
\hline $\mathbf{H} \cdots \mathrm{O}$ & (d) & 1.77 & 1.89 & 1.93 & 1.81 \\
\hline $\mathrm{O}=\mathrm{C}$ & (e) & 1.27 & 1.28 & 1.29 & 1.28 \\
\hline $\mathrm{O}=\mathrm{C}$ & (f) & 1.28 & 1.28 & 1.28 & 1.28 \\
\hline $\mathbf{C} \cdots \mathbf{H}$ & (g) & 1.39 & 1.34 & 1.33 & 1.33 \\
\hline $\mathbf{C} \cdots \mathbf{H}$ & (h) & 1.34 & 1.35 & 1.36 & 1.35 \\
\hline
\end{tabular}

\section{X-RAY DIFFRACTION}

Single crystals suitable for X-ray diffraction were mainly obtained by slow diffusion of hexane or diethyl ether into a concentrated solution of the compound in different solvents or slow evaporation of a concentrated solution of the compound in appropriated solvent. Diffraction data was collected on a Agilent SuperNova diffractometer equipped with an Atlas CCD detector using Mo-K $\alpha$ radiation $(\lambda=0.71073 \AA)$. Single crystals were mounted on a MicroMount ${ }^{\circledR}$ polymer tip (MiteGen) in a random orientation. Absorption 
corrections based on the multiscan method were applied. ${ }^{30}$ Structures were solved by direct methods in SHELXS-97 and refined by the full-matrix method based on $\mathrm{F}^{2}$ with the program SHELXL-97 using the OLEX software package. ${ }^{31,32}$

Key details of the crystal and structure refinement data are summarized in the following tables.

Table 6.5 Structural parameters, register conditions and refinement of $\mathbf{2 A H}$

\begin{tabular}{|c|c|}
\hline & 2AH \\
\hline Empirical formula & $\mathrm{C}_{15} \mathrm{H}_{24} \mathrm{BCl}_{2} \mathrm{~F}_{4} \mathrm{~N}_{3} \mathrm{Ru}$ \\
\hline Formula weight & 505.15 \\
\hline Temperature/K & $150.05(10)$ \\
\hline Crystal system & Monoclinic \\
\hline Space group & $\mathrm{P} 2{ }_{1} / \mathrm{c}$ \\
\hline $\mathrm{a} / \AA$ & $8.7587(10)$ \\
\hline $\mathrm{b} / \mathrm{A}$ & $20.7199(19)$ \\
\hline $\mathrm{c} / \AA ̊$ & $11.3341(15)$ \\
\hline$\alpha /^{\circ}$ & 90.00 \\
\hline$\beta /{ }^{\circ}$ & $106.939(13)$ \\
\hline$\gamma /{ }^{\circ}$ & 90.00 \\
\hline Volume $/ \AA^{3}$ & $1967.7(4)$ \\
\hline $\mathrm{Z}$ & 4 \\
\hline$\rho_{\text {calc }} \mathrm{mg} / \mathrm{mm}^{3}$ & 1.705 \\
\hline$\mu / \mathrm{mm}^{-1}$ & 1.109 \\
\hline $\mathrm{F}(000)$ & 1016.0 \\
\hline Crystal size $/ \mathrm{mm}^{3}$ & $0.18 \times 0.13 \times 0.11$ \\
\hline $2 \Theta$ range for data collection & 6.26 to $58.06^{\circ}$ \\
\hline Index ranges & $-11 \leq \mathrm{h} \leq 11,-26 \leq \mathrm{k} \leq 27,-15 \leq 1 \leq 14$ \\
\hline Reflections collected & 22343 \\
\hline Independent reflections & $4909[\mathrm{R}(\mathrm{int})=0.0429]$ \\
\hline Data/restraints/parameters & $4909 / 64 / 241$ \\
\hline Goodness-of-fit on $\mathrm{F}^{2}$ & 1.064 \\
\hline Final $R$ indexes $[\mathrm{I}>=2 \sigma(\mathrm{I})]$ & $\mathrm{R}_{1}=0.0412, \mathrm{wR}_{2}=0.0873$ \\
\hline Final R indexes [all data] & $\mathrm{R}_{1}=0.0616, \mathrm{wR}_{2}=0.0986$ \\
\hline Largest diff. peak/hole / e $\AA^{-3}$ & $1.30 /-1.05$ \\
\hline
\end{tabular}


Table 6.6 Structural parameters, register conditions and refinement of $\mathbf{3 A}, \mathbf{1 B}$ and $\mathbf{9 A}$

\begin{tabular}{|c|c|c|c|}
\hline & $3 \mathbf{A}$ & $1 B$ & 9A \\
\hline Empirical formula & $\mathrm{C}_{15} \mathrm{H}_{24} \mathrm{AuIr} \mathrm{Cl}_{3} \mathrm{~N}_{3}$ & $\mathrm{C}_{6} \mathrm{H}_{11} \mathrm{Au}_{2} \mathrm{~N}_{3} \mathrm{Cl}_{2}$ & $\mathrm{C}_{57} \mathrm{H}_{91} \mathrm{Cl}_{8} \mathrm{Ir}_{2} \mathrm{~N}_{8} \mathrm{Pd}_{2}$ \\
\hline Formula weight & 741.89 & 590.01 & 1769.18 \\
\hline Temperature/K & 293(2) & 293(2) & $293(2)$ \\
\hline Crystal system & Monoclinic & Monoclinic & Monoclinic \\
\hline Space group & $\mathrm{P} 2(1) / \mathrm{c}$ & $\mathrm{C} 2 / \mathrm{c}$ & $\mathrm{P} 2_{1}$ \\
\hline $\mathrm{a} / \AA$ & $8.0096(5)$ & $20.342(5)$ & $12.7019(3)$ \\
\hline $\mathrm{b} / \AA$ & $22.4094(14)$ & $11.088(2)$ & $14.4194(3)$ \\
\hline $\mathrm{c} / \AA$ & $11.6630(8)$ & $13.711(3)$ & $19.5497(5)$ \\
\hline$\alpha /^{\circ}$ & 90 & 90 & 90.00 \\
\hline$\beta /{ }^{\circ}$ & $109.725(7)$ & $129.089(4)$ & $103.615(2)$ \\
\hline$\gamma /{ }^{\circ}$ & 90 & 90 & 90.00 \\
\hline Volume $/ \AA^{3}$ & $1970.6(2)$ & 2400.3(9) & $3479.98(14)$ \\
\hline $\mathrm{Z}$ & 4 & 8 & 2 \\
\hline$\rho_{\text {calc }} \mathrm{mg} / \mathrm{mm}^{3}$ & 2.501 & 3.265 & 1.688 \\
\hline$\mu / \mathrm{mm}^{-1}$ & 14.591 & 24.827 & 4.667 \\
\hline $\mathrm{F}(000)$ & 1368.0 & 2080.0 & 1742.0 \\
\hline Crystal size $/ \mathrm{mm}^{3}$ & $0.16 \times 0.11 \times 0.10$ & $\begin{array}{c}0.14 \times 0.12 \times \\
0.09\end{array}$ & $0.13 \times 0.10 \times 0.08$ \\
\hline $\begin{array}{l}2 \Theta \text { range for data } \\
\text { collection }\end{array}$ & 5.7 to $55^{\circ}$ & 4.48 to $60^{\circ}$ & 5.66 to $58.88^{\circ}$ \\
\hline Index ranges & $\begin{array}{c}-10 \leq \mathrm{h} \leq 10,-29 \\
\leq \mathrm{k} \leq 29,-14 \leq 1 \leq \\
14\end{array}$ & $\begin{array}{c}-28 \leq \mathrm{h} \leq 11,- \\
15 \leq \mathrm{k} \leq 15,-5 \leq \\
1 \leq 19\end{array}$ & $\begin{array}{l}-16 \leq \mathrm{h} \leq 17,-18 \leq \\
\mathrm{k} \leq 18,-26 \leq 1 \leq 24\end{array}$ \\
\hline Reflections collected & 18903 & 4522 & 40990 \\
\hline Independent reflections & $\begin{array}{c}4465\left[\mathrm{R}_{\text {int }}=\right. \\
0.0895, \mathrm{R}_{\text {sigma }}= \\
0.0802]\end{array}$ & $\begin{array}{c}2891\left[\mathrm{R}_{\text {int }}=\right. \\
0.0310, \mathrm{R}_{\text {sigma }}= \\
0.0568]\end{array}$ & $\begin{array}{c}16650[\mathrm{R}(\mathrm{int})= \\
0.0519]\end{array}$ \\
\hline Data/restraints/parameters & $4465 / 517 / 216$ & $2891 / 0 / 121$ & $16650 / 111 / 723$ \\
\hline Goodness-of-fit on $\mathrm{F}^{2}$ & 1.049 & 0.990 & 1.028 \\
\hline Final $R$ indexes $[\mathrm{I}>=2 \sigma(\mathrm{I})]$ & $\begin{aligned} \mathrm{R}_{1}= & 0.0621, \mathrm{wR} \mathrm{R}_{2} \\
= & 0.1465\end{aligned}$ & $\begin{array}{c}\mathrm{R}_{1}=0.0467 \\
\mathrm{wR}_{2}=0.1056\end{array}$ & $\begin{array}{c}\mathrm{R}_{1}=0.0453, \mathrm{wR}_{2}= \\
0.0824\end{array}$ \\
\hline Final $\mathrm{R}$ indexes [all data] & $\begin{aligned} \mathrm{R}_{1}= & 0.0892, \mathrm{wR}_{2} \\
= & 0.1687\end{aligned}$ & $\begin{array}{c}\mathrm{R}_{1}=0.0725 \\
\mathrm{wR}_{2}=0.1182\end{array}$ & $\begin{array}{c}\mathrm{R}_{1}=0.0760, \mathrm{wR}_{2}= \\
0.0956\end{array}$ \\
\hline Largest diff. peak/hole/e $\AA^{-3}$ & $6.32 /-2.52$ & $1.64 /-2.77$ & $1.09 /-1.24$ \\
\hline
\end{tabular}


Table 6.7 Structural parameters, register conditions and refinement of $\mathbf{d}$ and $\mathbf{f}$

\begin{tabular}{|c|c|c|}
\hline & d & $\mathbf{f}$ \\
\hline Empirical formula & $\mathrm{C}_{24} \mathrm{H}_{26} \mathrm{Cl}_{2} \mathrm{~N}_{2} \mathrm{Pd}$ & $\mathrm{C}_{32} \mathrm{H}_{36} \mathrm{~N}_{4} \mathrm{O}_{4} \mathrm{Pd}_{2}$ \\
\hline Formula weight & 519.77 & 753.45 \\
\hline Temperature/K & $199.95(10)$ & $219.95(10)$ \\
\hline Crystal system & Monoclinic & Orthorhombic \\
\hline Space group & $\mathrm{C} 2$ & $\mathrm{P} 2{ }_{1} 2_{1} 2_{1}$ \\
\hline $\mathrm{a} / \AA$ & $35.2455(18)$ & $12.4821(3)$ \\
\hline $\mathrm{b} / \AA$ & $6.7435(3)$ & $12.8515(3)$ \\
\hline $\mathrm{c} / \AA$ & $9.6250(6)$ & $20.5376(5)$ \\
\hline$\alpha /^{\circ}$ & 90.00 & 90.00 \\
\hline$\beta /{ }^{\circ}$ & $102.636(5)$ & 90.00 \\
\hline$\gamma /{ }^{\circ}$ & 90.00 & 90.00 \\
\hline Volume $/ \AA^{3}$ & $2232.2(2)$ & $3294.51(15)$ \\
\hline $\mathrm{Z}$ & 4 & 4 \\
\hline$\rho_{\text {calc }} \mathrm{mg} / \mathrm{mm}^{3}$ & 1.547 & 1.519 \\
\hline$\mu / \mathrm{mm}^{-1}$ & 1.084 & 1.131 \\
\hline $\mathrm{F}(000)$ & 1056.0 & 1520 \\
\hline Crystal size $/ \mathrm{mm}^{3}$ & $\begin{array}{c}0.2415 \times 0.1028 \times \\
0.0749\end{array}$ & $0.09 \times 0.08 \times 0.07$ \\
\hline $2 \Theta$ range for data collection & 5.68 to $58.7^{\circ}$ & 6.04 to $58.7^{\circ}$ \\
\hline Index ranges & $\begin{array}{c}-47 \leq \mathrm{h} \leq 48,-9 \leq \mathrm{k} \leq \\
9,-13 \leq 1 \leq 12\end{array}$ & $\begin{array}{c}-15 \leq \mathrm{h} \leq 16,-13 \leq \mathrm{k} \leq \\
16,-27 \leq 1 \leq 27\end{array}$ \\
\hline Reflections collected & 13643 & 21116 \\
\hline Independent reflections & $5264[\mathrm{R}(\mathrm{int})=0.0325]$ & $7870[\mathrm{R}(\mathrm{int})=0.0528]$ \\
\hline Data/restraints/parameters & $5264 / 1 / 264$ & $7870 / 15 / 385$ \\
\hline Goodness-of-fit on $\mathrm{F}^{2}$ & 1.077 & 1.060 \\
\hline Final $\mathrm{R}$ indexes $[\mathrm{I}>=2 \sigma(\mathrm{I})]$ & $\begin{array}{c}\mathrm{R}_{1}=0.0282, \mathrm{wR}_{2}= \\
0.0538\end{array}$ & $\begin{array}{c}\mathrm{R}_{1}=0.0453, w \mathrm{R}_{2}= \\
0.0762\end{array}$ \\
\hline Final $\mathrm{R}$ indexes [all data] & $\begin{array}{c}\mathrm{R}_{1}=0.0324, w \mathrm{R}_{2}= \\
0.0571\end{array}$ & $\begin{array}{c}\mathrm{R}_{1}=0.0815, \mathrm{wR}_{2}= \\
0.0914\end{array}$ \\
\hline Largest diff. peak/hole / e $\AA^{-3}$ & $0.58 /-0.60$ & $0.916 /-0.759$ \\
\hline
\end{tabular}


Table 6.8 Structural parameters, register conditions and refinement of 1D, 3D and 4D

\begin{tabular}{|c|c|c|c|}
\hline & 1D & $3 D$ & 4D \\
\hline Empirical formula & $\mathrm{C}_{15} \mathrm{H}_{22} \mathrm{IN}_{3} \mathrm{Pd}$ & $\mathrm{C}_{17} \mathrm{H}_{21} \mathrm{I}_{2} \mathrm{~N}_{3} \mathrm{Pd}$ & $\mathrm{C}_{17} \mathrm{H}_{20} \mathrm{IN}_{3} \mathrm{Pd}$ \\
\hline Formula weight & 477.66 & 627.57 & 499.66 \\
\hline Temperature/K & 293(2) & $199.95(10)$ & $150(2)$ \\
\hline Crystal system & Orthorhombic & Orthorhombic & Monoclinic \\
\hline Space group & $\mathrm{P} 2{ }_{1} 2_{1} 2_{1}$ & $\mathrm{P} 22_{1} 2_{1} 2_{1}$ & $\mathrm{P} 2_{1}$ \\
\hline $\mathrm{a} / \AA$ & $11.8164(4)$ & $7.8067(5)$ & $8.5601(8)$ \\
\hline $\mathrm{b} / \AA$ & $16.3861(3)$ & $7.9436(5)$ & $7.4175(7)$ \\
\hline$c / \AA$ & $17.9298(4)$ & $33.1475(18)$ & $14.2238(16)$ \\
\hline$\alpha /^{\circ}$ & 90.00 & 90.00 & 90.00 \\
\hline$\beta /^{\circ}$ & 90.00 & 90.00 & $103.859(10)$ \\
\hline$\gamma /{ }^{\circ}$ & 90.00 & 90.00 & 90.00 \\
\hline Volume $/ \AA^{3}$ & $3471.67(14)$ & $2055.6(2)$ & $876.84(16)$ \\
\hline $\mathrm{Z}$ & 8 & 4 & 2 \\
\hline$\rho_{\text {calc }} \mathrm{mg} / \mathrm{mm}^{3}$ & 1.828 & 2.028 & 1.892 \\
\hline$\mu / \mathrm{mm}^{-1}$ & 2.842 & 3.909 & 2.818 \\
\hline $\mathrm{F}(000)$ & 1856.0 & 1184.0 & 484.0 \\
\hline Crystal size $/ \mathrm{mm}^{3}$ & $\begin{array}{c}0.09 \times 0.07 \times \\
0.06\end{array}$ & $\begin{array}{c}0.12 \times 0.11 \times \\
0.09\end{array}$ & $\begin{array}{c}0.19 \times 0.11 \times \\
0.08\end{array}$ \\
\hline $2 \Theta$ range for data collection & 5.7 to $58.94^{\circ}$ & 5.68 to $58.66^{\circ}$ & 5.9 to $57.94^{\circ}$ \\
\hline Index ranges & $\begin{array}{c}-15 \leq \mathrm{h} \leq 13,- \\
22 \leq \mathrm{k} \leq 21,- \\
19 \leq 1 \leq 24\end{array}$ & $\begin{array}{c}-10 \leq \mathrm{h} \leq 10,-10 \\
\leq \mathrm{k} \leq 10,-43 \leq 1 \\
\leq 42\end{array}$ & $\begin{array}{c}-11 \leq \mathrm{h} \leq 11,-9 \\
\leq \mathrm{k} \leq 9,-18 \leq 1 \\
\leq 19\end{array}$ \\
\hline Reflections collected & 15209 & 11976 & 10007 \\
\hline Independent reflections & $\begin{array}{c}7863[\mathrm{R}(\mathrm{int})= \\
0.0349]\end{array}$ & $\begin{array}{c}4907[\mathrm{R}(\mathrm{int})= \\
0.0473]\end{array}$ & $\begin{array}{c}4104[\mathrm{R}(\mathrm{int})= \\
0.0671]\end{array}$ \\
\hline Data/restraints/parameters & $7863 / 0 / 371$ & $4907 / 0 / 211$ & $4104 / 1 / 202$ \\
\hline Goodness-of-fit on $\mathrm{F}^{2}$ & 1.076 & 1.038 & 1.028 \\
\hline Final $R$ indexes $[\mathrm{I}>=2 \sigma(\mathrm{I})]$ & $\begin{array}{c}\mathrm{R}_{1}=0.0377 \\
\mathrm{wR}_{2}=0.0645\end{array}$ & $\begin{array}{c}\mathrm{R}_{1}=0.0444 \\
\mathrm{wR}_{2}=0.0527\end{array}$ & $\begin{array}{c}\mathrm{R}_{1}=0.0430 \\
\mathrm{wR}_{2}=0.0771\end{array}$ \\
\hline Final $R$ indexes [all data] & $\begin{array}{c}\mathrm{R}_{1}=0.0558 \\
\mathrm{wR}_{2}=0.0761\end{array}$ & $\begin{array}{c}\mathrm{R}_{1}=0.0777 \\
\mathrm{wR}_{2}=0.0617\end{array}$ & $\begin{array}{c}\mathrm{R}_{1}=0.0581 \\
\mathrm{wR}_{2}=0.0894\end{array}$ \\
\hline Largest diff. peak/hole/ $\mathrm{e}^{-3}$ & $0.58 /-0.88$ & $0.68 /-0.75$ & $1.13 /-1.02$ \\
\hline
\end{tabular}


Table 6.9 Structural parameters, register conditions and refinement of $6 \mathrm{D}, 6 \mathrm{D} *$ and $3 C^{*}$

\begin{tabular}{|c|c|c|c|}
\hline & $3 \mathrm{C}$ & 6D* & $3 C^{*}$ \\
\hline Empirical formula & $\mathrm{C}_{33} \mathrm{H}_{47} \mathrm{~F}_{6} \mathrm{IrN}_{3} \mathrm{P}$ & $\mathrm{C}_{28} \mathrm{H}_{37} \mathrm{Cl}_{2} \mathrm{~F}_{6} \mathrm{IrN}_{3} \mathrm{P}$ & $\mathrm{C}_{33} \mathrm{H}_{47} \mathrm{~F}_{6} \mathrm{IrN}_{3} \mathrm{P}$ \\
\hline Formula weight & 822.91 & 823.68 & 822.91 \\
\hline Temperature/K & $199(3)$ & $200.00(10)$ & $200.00(14)$ \\
\hline Crystal system & Triclinic & Orthorhombic & Monoclinic \\
\hline Space group & P-1 & $\mathrm{P} 2{ }_{1} 2_{1} 2_{1}$ & $\mathrm{P} 2_{1}$ \\
\hline $\mathrm{a} / \AA$ & $8.71445(14)$ & $11.9558(3)$ & $11.2778(2)$ \\
\hline $\mathrm{b} / \AA$ & $11.7851(2)$ & $15.5372(3)$ & $9.17011(16)$ \\
\hline $\mathrm{c} / \AA$ & $17.6537(3)$ & $18.1992(5)$ & $16.3880(3)$ \\
\hline$\alpha /^{\circ}$ & $101.7413(16)$ & 90.00 & 90.00 \\
\hline$\beta /{ }^{\circ}$ & $102.1693(14)$ & 90.00 & $91.3639(16)$ \\
\hline$\gamma /{ }^{\circ}$ & $101.9854(16)$ & 90.00 & 90.00 \\
\hline Volume $/ \AA^{3}$ & 1674.71(5) & $3380.66(15)$ & $1694.34(5)$ \\
\hline $\mathrm{Z}$ & 2 & 4 & 2 \\
\hline$\rho_{\text {calc }} \mathrm{mg} / \mathrm{mm}^{3}$ & 1.632 & 1.618 & 1.613 \\
\hline$\mu / \mathrm{mm}^{-1}$ & 4.095 & 4.210 & 4.047 \\
\hline $\mathrm{F}(000)$ & 824.0 & 1624.0 & 824.0 \\
\hline Crystal size $/ \mathrm{mm}^{3}$ & $\begin{array}{c}0.4759 \times 0.2029 \\
\times 0.0637\end{array}$ & $0.12 \times 0.11 \times 0.08$ & $\begin{array}{c}0.1888 \times 0.1282 \\
\times 0.0851\end{array}$ \\
\hline $2 \Theta$ range for data collection & 5.72 to $60.22^{\circ}$ & 5.62 to $56^{\circ}$ & 6.08 to $58.7^{\circ}$ \\
\hline Index ranges & $\begin{array}{c}-11 \leq \mathrm{h} \leq 11,- \\
16 \leq \mathrm{k} \leq 16,-24 \\
\leq 1 \leq 23\end{array}$ & $\begin{array}{l}-15 \leq \mathrm{h} \leq 15,-20 \leq \\
\mathrm{k} \leq 19,-23 \leq 1 \leq 20\end{array}$ & $\begin{array}{c}-15 \leq \mathrm{h} \leq 15,- \\
12 \leq \mathrm{k} \leq 11,-21 \\
\leq 1 \leq 22\end{array}$ \\
\hline Reflections collected & 74491 & 22883 & 38409 \\
\hline Independent reflections & $\begin{array}{c}8920[\mathrm{R}(\mathrm{int})= \\
0.0536]\end{array}$ & $\begin{array}{c}7775[\mathrm{R}(\text { int })= \\
0.0331]\end{array}$ & $\begin{array}{c}8581[\mathrm{R}(\text { int })= \\
0.0303]\end{array}$ \\
\hline Data/restraints/parameters & $8920 / 0 / 405$ & $7775 / 19 / 378$ & $8581 / 1 / 475$ \\
\hline Goodness-of-fit on $\mathrm{F}^{2}$ & 1.086 & 1.056 & 1.042 \\
\hline Final $R$ indexes $[\mathrm{I}>=2 \sigma(\mathrm{I})]$ & $\begin{array}{c}\mathrm{R}_{1}=0.0332, \\
\mathrm{wR}_{2}=0.0849\end{array}$ & $\begin{array}{c}\mathrm{R}_{1}=0.0421, w \mathrm{R}_{2}= \\
0.1074\end{array}$ & $\begin{array}{c}\mathrm{R}_{1}=0.0195 \\
\mathrm{wR}_{2}=0.0354\end{array}$ \\
\hline Final $R$ indexes [all data] & $\begin{array}{c}\mathrm{R}_{1}=0.0376 \\
\mathrm{wR}_{2}=0.0885\end{array}$ & $\begin{array}{c}\mathrm{R}_{1}=0.0555, \mathrm{w} \mathrm{R}_{2}= \\
0.1170\end{array}$ & $\begin{array}{c}\mathrm{R}_{1}=0.0246 \\
\mathrm{wR}_{2}=0.0376\end{array}$ \\
\hline 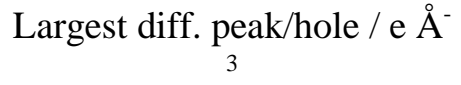 & $2.49 /-1.86$ & $1.77 /-0.88$ & $0.50 /-0.75$ \\
\hline
\end{tabular}


Table 6.10 Structural parameters, register conditions and refinement of $\mathbf{1 H}$ and $\mathbf{2 I}$

\begin{tabular}{|c|c|c|}
\hline & $1 \mathrm{H}$ & $2 I$ \\
\hline Empirical formula & $\mathrm{C}_{22} \mathrm{H}_{26} \mathrm{~N}_{4} \mathrm{O}_{5} \mathrm{~F}_{3} \mathrm{~S} \mathrm{Cl} \mathrm{Ru}$ & $\mathrm{C}_{39} \mathrm{H}_{50} \mathrm{~N}_{8} \mathrm{Cl}_{10} \mathrm{Ru}_{2}$ \\
\hline Formula weight & 652.05 & 1187.51 \\
\hline Temperature/K & 293(2) & $100(2)$ \\
\hline Crystal system & Monoclinic & Monoclinic \\
\hline Space group & $\mathrm{P} 2_{1} / \mathrm{n}(\# 14)$ & $\mathrm{P} 2{ }_{1} / \mathrm{n}(\# 14)$ \\
\hline $\mathrm{a} / \mathrm{A}$ & $11.8088(1)$ & $13.4316(2)$ \\
\hline $\mathrm{b} / \AA$ & $14.5983(2)$ & $20.1958(2)$ \\
\hline $\mathrm{c} / \AA ̊ \AA$ & $15.4772(2)$ & $18.1570(2)$ \\
\hline$\alpha /^{\circ}$ & 90.00 & 90.00 \\
\hline$\beta /^{\circ}$ & $93.6884(7)$ & $101.5298(11)$ \\
\hline$\gamma /{ }^{\circ}$ & 90.00 & 90.00 \\
\hline Volume $/ \AA^{3}$ & $2662.56(5) \AA^{3}$ & $4825.91(10) \AA^{3}$ \\
\hline Z & 4 & 4 \\
\hline$\rho_{\text {calc }} \mathrm{mg} / \mathrm{mm}^{3}$ & 1.627 & 1.634 \\
\hline$\mu / \mathrm{mm}^{-1}$ & 0.828 & 10.470 \\
\hline $\mathrm{F}(000)$ & 1320 & 2392 \\
\hline Crystal size $/ \mathrm{mm}^{3}$ & $0.3031 \times 0.2414 \times 0.1652$ & $\begin{array}{c}0.2567 \times 0.0775 \times \\
0.0413\end{array}$ \\
\hline $2 \Theta$ range for data collection & 2.98 to $28.29^{\circ}$ & 3.31 to $76.90^{\circ}$ \\
\hline Index ranges & $\begin{array}{c}-15<=\mathrm{h}<=15,- \\
19<=\mathrm{k}<=19,-20<=1<=20\end{array}$ & $\begin{array}{c}-12<=\mathrm{h}<=16,- \\
25<=\mathrm{k}<=25,- \\
22<=\mathrm{l}<=18\end{array}$ \\
\hline Reflections collected & 64804 & 56186 \\
\hline Independent reflections & $6600[\mathrm{R}($ int $)=0.0183]$ & $10104[\mathrm{R}(\mathrm{int})=0.0483]$ \\
\hline Data/restraints/parameters & 6600 / 0 / 339 & 10104 / 0 / 550 \\
\hline Goodness-of-fit on $\mathrm{F}^{2}$ & 1.047 & 1.090 \\
\hline Final $R$ indexes $[\mathrm{I}>=2 \sigma(\mathrm{I})]$ & $\begin{array}{c}\mathrm{R} 1=0.0329, \mathrm{wR} 2= \\
0.0964\end{array}$ & $\begin{array}{c}\mathrm{R} 1=0.0555, \mathrm{wR} 2= \\
0.1444\end{array}$ \\
\hline Final $\mathrm{R}$ indexes [all data] & $\begin{array}{c}\mathrm{R} 1=0.0365, \mathrm{wR} 2= \\
0.1002\end{array}$ & $\begin{array}{c}\mathrm{R} 1=0.0634, \mathrm{wR} 2= \\
0.1505\end{array}$ \\
\hline Largest diff. peak/hole / e $\AA^{-3}$ & 0.656 and -0.592 e. $\AA^{-3}$ & 2.463 and -2.243 e. $\AA^{-3}$ \\
\hline
\end{tabular}


Table 6.11 Structural parameters, register conditions and refinement of 1G, 2G, and 3G

\begin{tabular}{|c|c|c|c|}
\hline & $1 G$ & $2 G$ & $3 G$ \\
\hline Empirical formula & $\mathrm{C}_{30} \mathrm{H}_{28} \mathrm{BrN}_{3} \mathrm{Pd}$ & $\mathrm{C}_{21} \mathrm{H}_{16} \mathrm{AuClN}_{2}$ & $\mathrm{C}_{36} \mathrm{H}_{39} \mathrm{Cl}_{8} \mathrm{~N}_{2} \mathrm{Ru}$ \\
\hline Formula weight & 616.86 & 528.77 & 884.36 \\
\hline Temperature/K & 293(2) & 293(2) & $293(2)$ \\
\hline Crystal system & Monoclinic & Monoclinic & Triclinic \\
\hline Space group & $\mathrm{C} 2 / \mathrm{c}$ & $\mathrm{P} 2{ }_{1} / \mathrm{c}$ & P-1 \\
\hline $\mathrm{a} / \AA$ & $35.108(5)$ & $12.2750(3)$ & $11.3681(4)$ \\
\hline $\mathrm{b} / \AA$ & $12.2811(7)$ & $11.5743(3)$ & $14.3155(7)$ \\
\hline $\mathrm{c} / \AA ̊ \AA$ & $18.782(3)$ & $12.3319(3)$ & $14.8357(7)$ \\
\hline$\alpha /^{\circ}$ & 90.00 & 90.00 & $62.367(5)$ \\
\hline$\beta /{ }^{\circ}$ & $140.71(3)$ & $96.802(2)$ & $84.064(4)$ \\
\hline$\gamma /{ }^{\circ}$ & 90.00 & 90.00 & $77.725(4)$ \\
\hline Volume $/ \AA^{3}$ & $5128.6(11)$ & 1739.71(7) & $2090.06(17)$ \\
\hline $\mathrm{Z}$ & 8 & 4 & 2 \\
\hline$\rho_{\text {calc }} \mathrm{mg} / \mathrm{mm}^{3}$ & 1.598 & 2.019 & 1.405 \\
\hline$\mu / \mathrm{mm}^{-1}$ & 2.306 & 8.615 & 0.913 \\
\hline $\mathrm{F}(000)$ & 2480.0 & 1008.0 & 898.0 \\
\hline Crystal size $/ \mathrm{mm}^{3}$ & $\begin{array}{c}0.14 \times 0.11 \times \\
0.10\end{array}$ & $0.12 \times 0.1 \times 0.09$ & $0.13 \times 0.1 \times 0.09$ \\
\hline $2 \Theta$ range for data collection & 5.98 to $59.04^{\circ}$ & 5.66 to $52^{\circ}$ & 5.82 to 59.06 \\
\hline & $-44 \leq \mathrm{h} \leq 45,-15$ & $-15 \leq \mathrm{h} \leq 15,-14$ & $-15 \leq \mathrm{h} \leq 15,-19$ \\
\hline Index ranges & $\begin{aligned} & \leq \mathrm{k} \leq 16,-25 \leq 1 \\
& \leq 24\end{aligned}$ & $\begin{aligned} \leq \mathrm{k} \leq & 14,-15 \leq 1 \\
& \leq 15\end{aligned}$ & $\begin{array}{c}\leq \mathrm{k} \leq 19,-20 \leq 1 \\
\leq 20\end{array}$ \\
\hline Reflections collected & 29553 & 33714 & 45813 \\
\hline Independent reflections & $\begin{array}{c}6473[\mathrm{R}(\text { int })= \\
0.0306]\end{array}$ & $\begin{array}{c}3420[\mathrm{R}(\mathrm{int})= \\
0.0338]\end{array}$ & $\begin{array}{c}10614\left[\mathrm{R}_{\text {int }}=\right. \\
0.0570, \mathrm{R}_{\text {sigma }}= \\
0.0449]\end{array}$ \\
\hline Data/restraints/parameters & $6473 / 0 / 319$ & $3420 / 0 / 275$ & $10614 / 81 / 491$ \\
\hline Goodness-of-fit on $\mathrm{F}^{2}$ & 1.066 & 1.066 & 1.069 \\
\hline Final $R$ indexes $[I>=2 \sigma(I)]$ & $\begin{aligned} \mathrm{R}_{1}= & 0.0380, \mathrm{wR}_{2} \\
& =0.0956\end{aligned}$ & $\begin{aligned} \mathrm{R}_{1}= & 0.0210, \mathrm{wR}_{2} \\
& =0.0563\end{aligned}$ & $\begin{aligned} \mathrm{R}_{1}= & 0.0613, \mathrm{wR}_{2} \\
= & 0.1725\end{aligned}$ \\
\hline Final $\mathrm{R}$ indexes [all data] & $\begin{array}{c}\mathrm{R}_{1}=0.0526, \mathrm{wR}_{2} \\
=0.1048\end{array}$ & $\begin{array}{c}\mathrm{R}_{1}=0.0253, \mathrm{wR}_{2} \\
=0.0590\end{array}$ & $\begin{array}{c}\mathrm{R}_{1}=0.0766, \mathrm{wR} \mathrm{R}_{2} \\
=0.1917\end{array}$ \\
\hline Largest diff. peak/hole /e $\AA^{-3}$ & $2.44 /-0.51$ & $1.09 /-0.42$ & $1.80 /-0.98$ \\
\hline
\end{tabular}



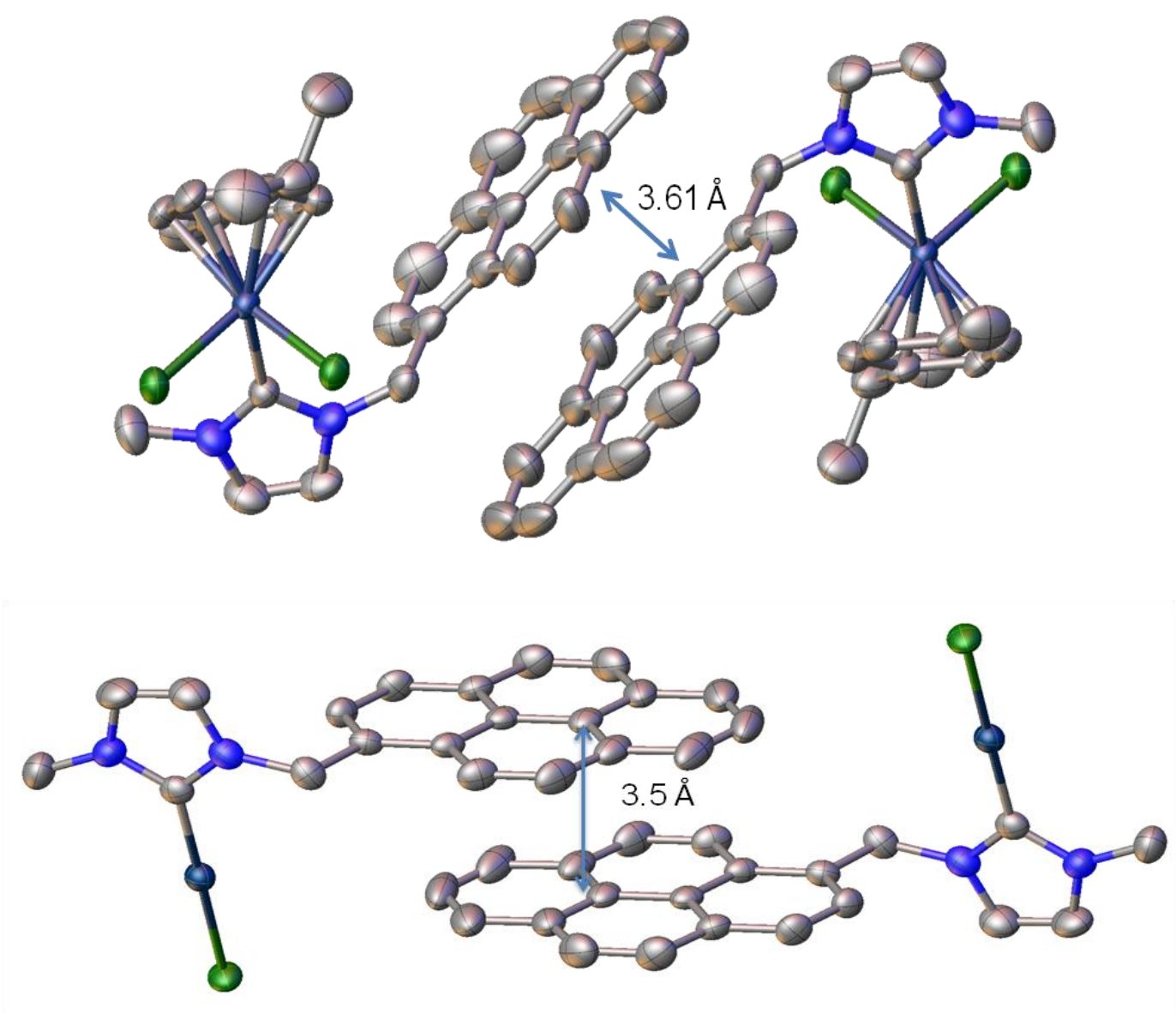

Figure 6.24 Packing diagrams showing the $\pi$-stacking of complexes 2G (top) and 3G (bottom). Ellipsoids are shown at 50\% probability level. Hydrogens are omitted for clarity. 


\section{REFERENCES}

(1) Bennett, M. A.; Smith, A. K. J. Chem. Soc.-Dalton Trans. 1974, 233-241.

(2) Ball, R. G.; Graham, W. A. G.; Heinekey, D. M.; Hoyano, J. K.; McMaster, A. D.; Mattson, B. M.; Michel, S. T. Inorg. Chem. 1990, 29, 2023-2025.

(3) Uson, R.; Laguna, A.; Laguna, M.; Briggs, D. A.; Murray, H. H.; Fackler, J. P. Inorg. Synth. 1989, 26, 85-91.

(4) Ryabov, A. D.; Sakodinskaya, I. K.; Yatsimirsky, A. K. J. Chem. Soc.-Dalton Trans. 1985, 2629-2638.

(5) Fuchita, Y.; Yoshinaga, K.; Ikeda, Y.; KinoshitaKawashima, J. J. Chem. Soc.Dalton Trans. 1997, 2495-2499.

(6) Curphey, T. J.; Prasad, K. S. J. Org. Chem. 1972, 37, 2259.

(7) Sala, A.; Ferrario, F.; Rizzi, E.; Catinella, S.; Traldi, P. Rapid Commun. Mass Sp. 1992, 6, 388-393.

(8) Chu, Y.; Deng, H.; Cheng, J.-P. J. Org. Chem. 2007, 72, 7790-7793.

(9) Blackburn, G. M.; Lockwood, G.; Solan, V. J. Chem. Soc.-Perkin Trans. 2 1976, 1452-1456.

(10) Chattopadhyay, B.; Vera, C. I. R.; Chuprakov, S.; Gevorgyan, V. Org. Lett. 2010, 12, 2166-2169.

(11) Zanardi, A.; Corberan, R.; Mata, J. A.; Peris, E. Organometallics 2008, 27, 35703576.

(12) Viciano, M.; Sanau, M.; Peris, E. Organometallics 2007, 26, 6050-6054.

(13) Mercs, L.; Neels, A.; Albrecht, M. Dalton Trans. 2008, 5570-5576.

(14) Corberan, R.; Sanau, M.; Peris, E. J. Am. Chem. Soc. 2006, 128, 3974-3979.

(15) Xiao, X.-Q.; Jin, G.-X. J. Organomet. Chem. 2008, 693, 3363-3368.

(16) Hummers, W. S.; Offeman, R. E. J. Am. Chem. Soc. 1958, 80, 1339-1339.

(17) Stankovich, S.; Dikin, D. A.; Piner, R. D.; Kohlhaas, K. A.; Kleinhammes, A.; Jia, Y.; Wu, Y.; Nguyen, S. T.; Ruoff, R. S. Carbon 2007, 45, 1558-1565.

(18) Tani, K.; Brown, L. D.; Ahmed, J.; Ibers, J. A.; Yokota, M.; Nakamura, A.; Otsuka, S. J. Am. Chem. Soc. 1977, 99, 7876-7886.

(19) Gul, N.; Nelson, J. H. J. Mol. Struct. 1999, 475, 121-130. 
(20) Crotti, C.; Farnetti, E.; Filipuzzi, S.; Stener, M.; Zangrando, E.; Moras, P. Dalton Trans. 2007, 133-142.

(21) Ackermann, L.; Althammer, A.; Born, R. Tetrahedron 2008, 64, 6115-6124.

(22) Ackermann, L.; Althammer, A.; Born, R. Angew. Chem. Int. Edit. 2006, 45, 2619-2622.

(23) Frisch, M. J.; et al. Gaussian 09, revision D.02: Gaussian, Inc., Wallingford CT, 2009.

(24) Zhao, Y.; Truhlar, D. G. Theor. Chem. Acc. 2008, 120, 215-241.

(25) Andrae, D.; Häussermann, U.; Dolg, M.; Stoll, H.; Preuss, H. Theor. Chim. Acta 1990, 77, 123-141.

(26) Ehlers, A. W.; Böhme, M.; Dapprich, S.; Gobbi, A.; Höllwarth, A.; Jonas, V.; Köhler, K. F.; Stegmann, R.; Veldkamp, A.; Frenking, G. Chem. Phys. Lett. 1993, 208, 111-114.

(27) Harihara, P. C.; Pople, J. A. Theor. Chim. Acta 1973, 28, 213-222.

(28) Francl, M. M.; Pietro, W. J.; Hehre, W. J.; Binkley, J. S.; Gordon, M. S.; Defrees, D. J.; Pople, J. A. J. Chem. Phys. 1982, 77, 3654-3665.

(29) Marenich, A. V.; Cramer, C. J.; Truhlar, D. G. J. Phys. Chem. B 2009, 113, 63786396.

(30) Clark, R. C.; Reid, J. S. Acta Crystallogr. A 1995, 51, 887-897.

(31) Dolomanov, O. V.; Bourhis, L. J.; Gildea, R. J.; Howard, J. A. K.; Puschmann, H. J. Appl. Crystallogr. 2009, 42, 339-341.

(32) Sheldrick, G. M. Acta Crystallogr. A 2008, 64, 112-122. 



\section{CHAPTER 7}

COMPUESTOS MULTIFUNCIONALES CON LIGANDOS NHC PARA EL DISEÑO DE PROCESOS CATALÍTICOS 

En aplicación de la normativa de estudios de doctorado sobre la elaboración de Tesis Doctorales según el programa RD 99/2011, por la que se establece que: la Tesis Doctoral escrita en una lengua diferente del valenciano o del castellano, en el momento de ser depositada, debe contener un apartado suficientemente amplio en una de estas dos lenguas, y debe formar parte de la encuadernación de la tesis; el siguiente capítulo contiene un resumen en castellano del trabajo recogido en la presente Tesis Doctoral.

\section{INTRODUCCIÓN}

En la búsqueda de alternativas sintéticas que sean más eficientes en la preparación de arquitecturas orgánicas complejas y que a su vez minimicen el impacto medioambiental, los procesos catalíticos juegan un papel esencial. En este contexto los catalizadores multifuncionales despiertan un gran interés ya que su objetivo final es el desarrollo de procesos sintéticos que minimicen tiempos de reacción, con mayores selectividades y actividades mejoradas. ${ }^{1,2}$ En este tipo de catalizadores la acción combinada de más de un centro activo (metal o grupo funcional), presente en una misma molécula, promueve eficazmente una amplia gama de transformaciones orgánicas.

Así pues, catalizadores que sean activos en procesos diferentes (Esquema 7.1), presentan ventajas evidentes, ya que permiten diseñar procesos más rentables desde el punto de vista químico y económico, por varios motivos: ${ }^{3-7}$

- La utilización de un solo catalizador en procesos sucesivos o concertados simplifica en gran medida el tratamiento experimental

- Favorece el diseño de procesos sostenibles

- Disminuye el consumo-preparación del catalizador 


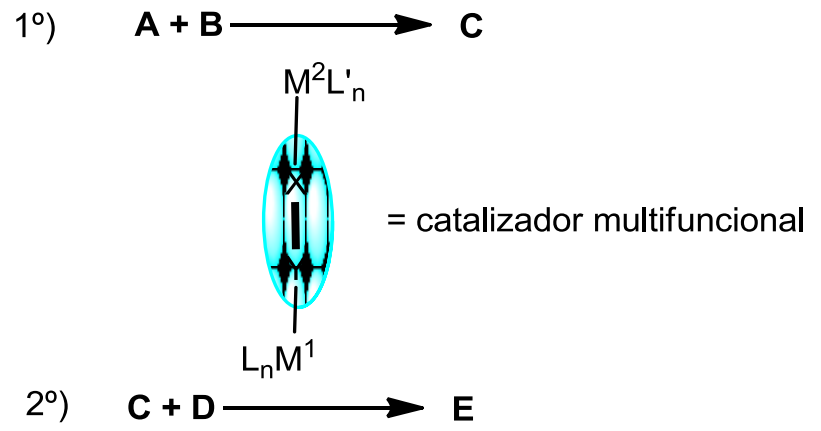

Esquema 7.1

En los últimos años, en nuestro grupo de investigación, se han desarrollado diferentes métodos para la preparación de compuestos homo y heterobimetálicos utilizando como ligando, que actúa como puente entre los dos centros metálicos, el ligando 1,2,4trimetiltriazolil-di-lideno (ditz) (Esquema 7.2). ${ }^{8-15}$ Estos compuestos han demostrado ser altamente versátiles y activos en una amplia gama de procesos catalíticos tándem. La catálisis tándem puede definirse como una estrategia sintética que consiste en el uso secuencial de reacciones catalíticas, con el fin de simplificar los procedimientos experimentales. ${ }^{6}$

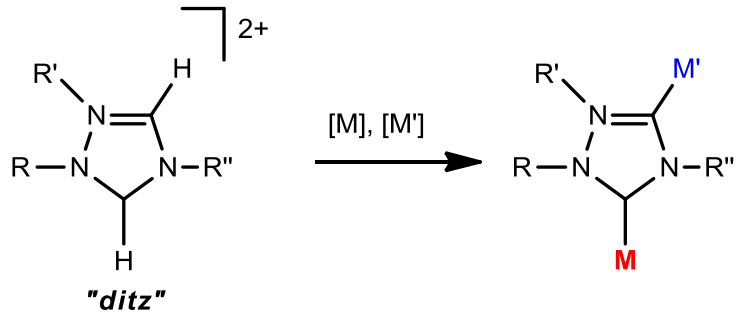

Esquema 7.2

Por otra parte, la introducción de ligandos "no inocentes", es decir que jueguen un papel activo en los ciclos catalíticos, constituye también una estrategia de síntesis de catalizadores multifuncionales. ${ }^{16-19}$ En estos sistemas, la capacidad catalítica intrínseca de los centros metálicos se une la acción asistida de dichos ligandos para dar lugar a una transformación orgánica más eficaz. 
Finalmente, con vistas a aplicaciones industriales, sistemas catalíticos que puedan ser reciclados sin pérdida aparente de actividad constituye uno de los grandes retos de la química actual. ${ }^{20}$ En este contexto, la combinación de la actividad y selectividad que ofrecen catalizadores homogéneos con la reciclabilidad y robustez que ofrecen sistemas heterogéneos, la preparación de materiales híbridos multifuncionales surge como una alternativa altamente eficaz. ${ }^{21-26}$

\section{RESULTADOS Y DISCUSIÓN}

\subsection{Síntesis y aplicaciones catalíticas de compuestos bimetálicos}

La síntesis de los compuestos heterobimetálicos se llevó a cabo mediante estrategias convencionales de coordinación, a partir de los complejos monometálicos $\mathbf{1} \mathbf{A H}^{10}$ y $\mathbf{2} \mathbf{A H}$. Ambos compuestos poseen un enlace $\mathrm{CH}$ que puede ser activado/deprotonado para generar un segundo enlace metal-carbeno (Esquema 7.3)

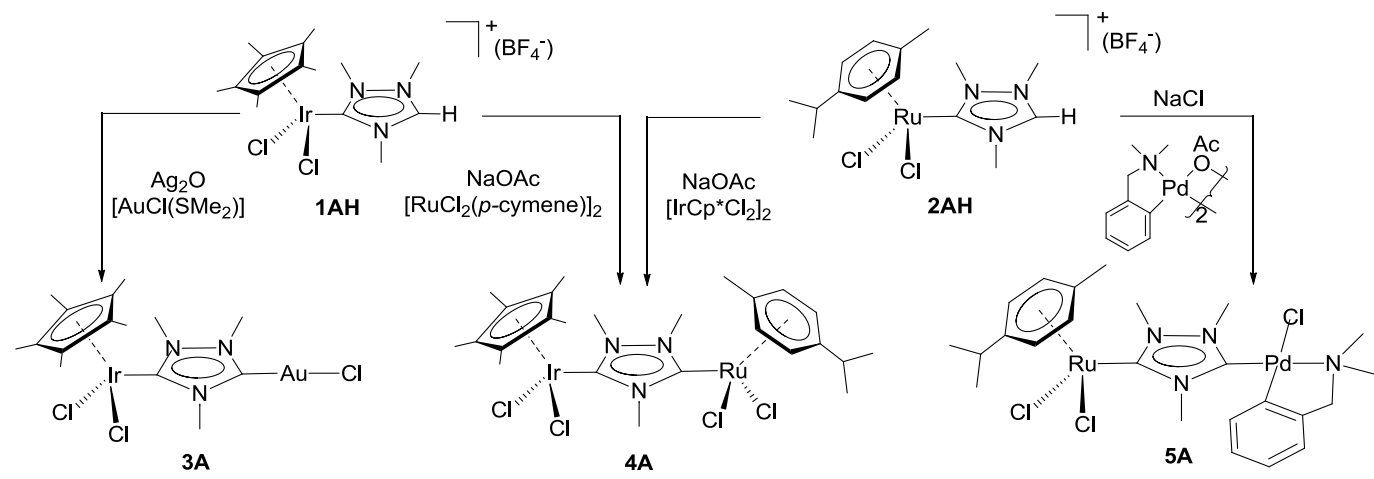

Esquema 7.3

En el estudio de la actividad catalítica de los complejos heterobimetálicos es importante determinar si la naturaleza heterobimetálica de los complejos en cuestión supone algún beneficio con respecto a sus análogos homobimetálicos. Por esta razón, con el objetivo de realizar estudios comparativos, se sintetizaron los complejos homobimetálicos representados en el Esquema 7.4. 

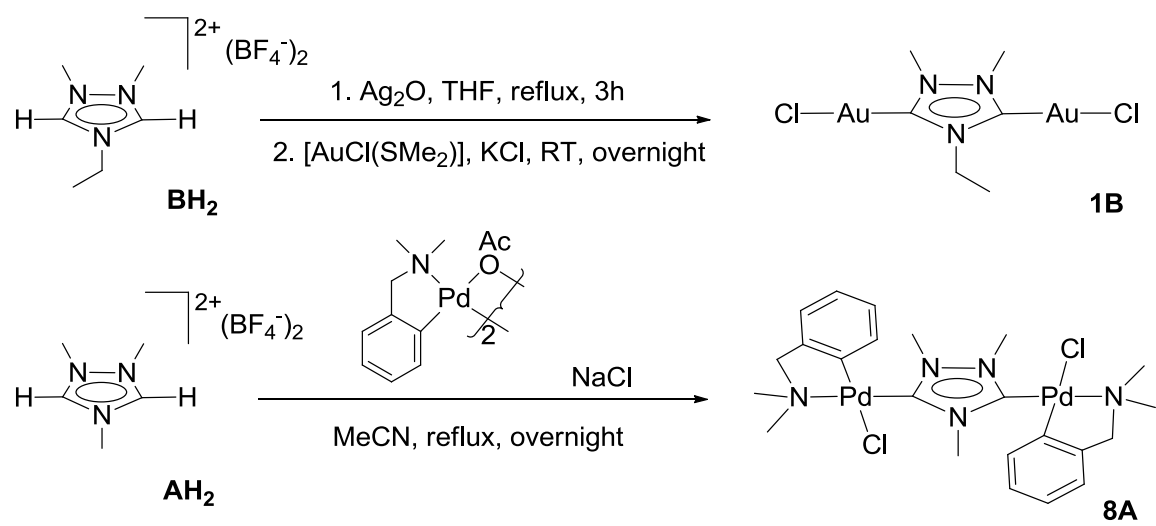

\section{Esquema 7.4}

El principal objetivo de la síntesis de compuestos bimetálicos es su uso como catalizadores multifuncionales. La obtención de complejos heterobimetálicos permite el diseño de procesos catalíticos tándem o duales en los cuales se combina la actividad de los dos centros metálicos en una secuencia de reacciones catalíticas de manera "onepot". ${ }^{27}$

\subsubsection{Reducción de nitroarenos mediante transferencia de hidrógeno usando alcoholes primarios}

Las propiedades catalíticas del compuesto heterobimetálico de Au/Ir 3A, se estudiaron en la reducción catalítica de nitroarenos mediante transferencia de hidrógeno usando alcoholes primarios. ${ }^{13,28-30}$ A efectos comparativos también se estudió la actividad de los compuestos homobimetálicos de oro (1B) e iridio (6A). (Esquema 7.5). 

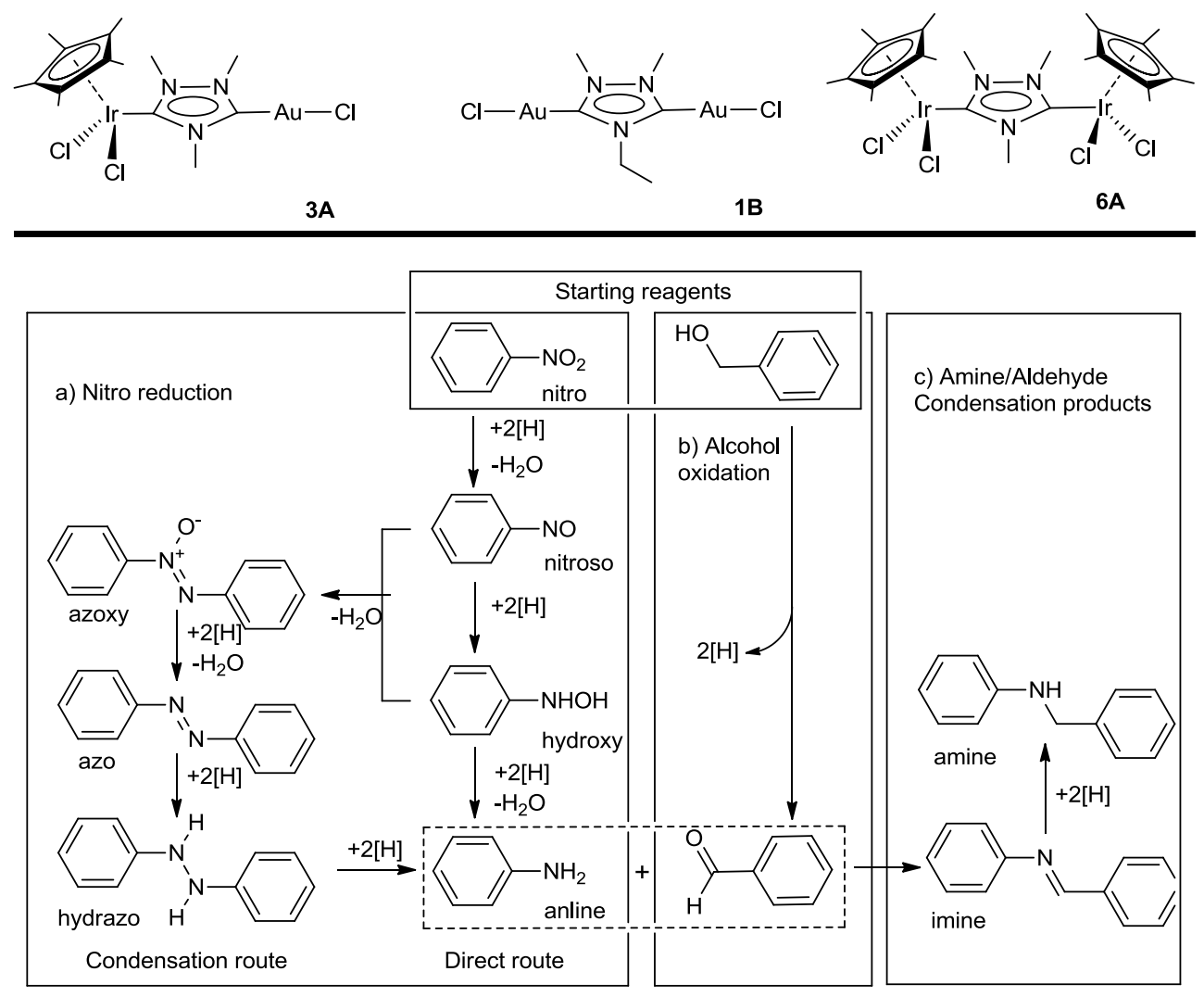

\section{Esquema 7.5}

Los resultados catalíticos indican diferentes actividades y productos dependiendo del catalizador empleado. El compuesto homobimetálicos de oro $\mathbf{1 B}$ presentó actividades moderadas hacia la formación de hidroxi y azo compuestos, mientras que los compuestos que contienen iridio $3 \mathbf{A}$ y $\mathbf{6 A}$ presentaron alta actividad hacia la formación de la imina. Independientemente del substrato empleado y bajo las mismas condiciones de reacción la actividad del compuesto heterobimetálico 3A fue mayor que la actividad de los compuestos homobimetálicos 1B y 6A. Estos resultados son un ejemplo de un sistema catalítico dual en el cual los centros metálicos de oro e iridio contribuyen cooperativamente a la transformación global. Por otra parte, la actividad catalítica de la suma de los dos compuestos homobimetálicos fue menor que la mostrada por el 
compuesto heterobimetálico 3A. Este resultado también indica una cooperación entre los dos centros metálicos unidos mediante el anillo de triazol.

\subsubsection{Activación C-H asistida por quelación y oxidación Oppenauer de alcoholes}

La actividad catalítica del compuesto heterobimetálico 4A fue evaluada en la arilación asistida por quelación de arilpiridinas con 1-(4-halofenil)etanol (reacción típicamente catalizada por $\mathrm{Ru})^{31-33}$ y la oxidación Oppenauer de alcoholes (reacción típicamente catalizada por Ir y Ru). ${ }^{34,35}$ Con fines comparativos también se decidió estudiar la actividad catalítica de los compuestos homobimetálicos de $\operatorname{Ir}(\mathbf{6 A})$ y $\mathrm{Ru}(\mathbf{7 A})$. Así mismo, se evaluó la actividad del compuesto1C, el cual ha mostrado ser un catalizador efectivo en la arilación de diferentes arilpiridinas ${ }^{36}$ (Esquema 7.6).
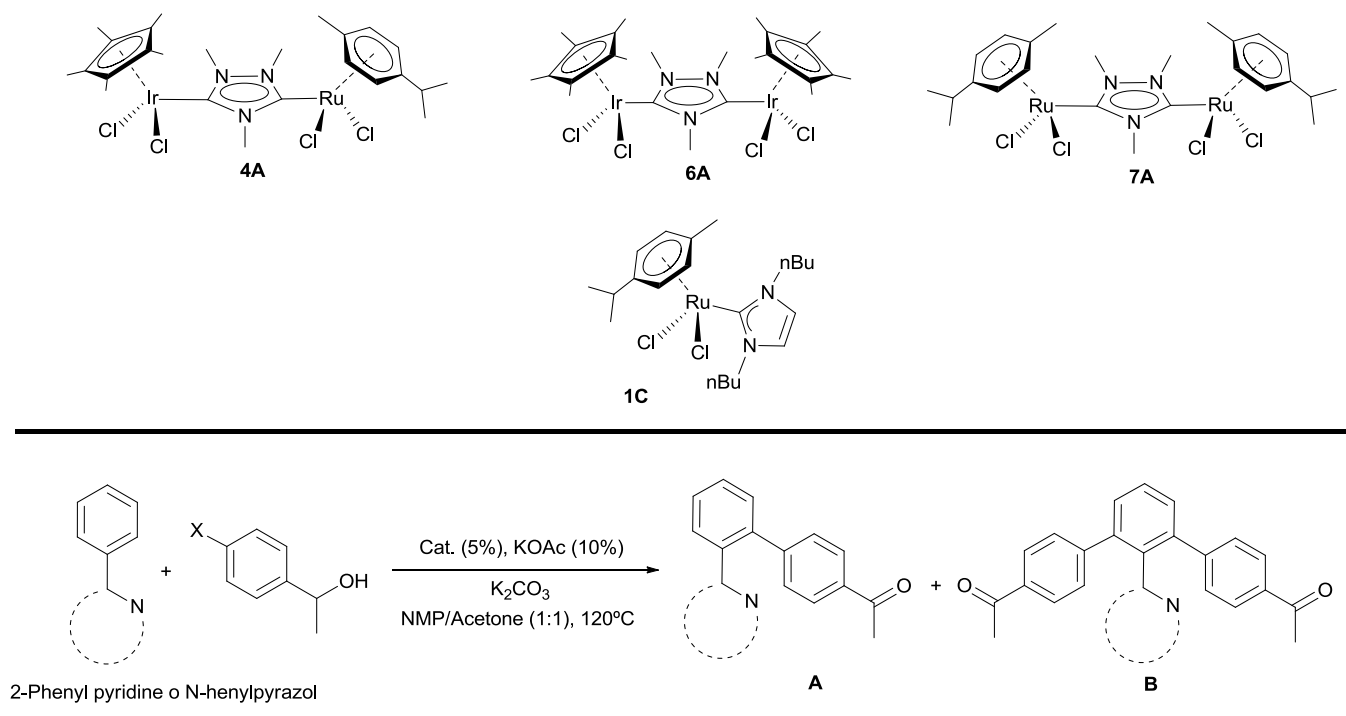

Esquema 7.6

En un estudio preliminar, se estudiaron ambos procesos por separado, la arilación de 2fenilpiridina con clorobenceno y la oxidación de 1-feniletanol utilizando acetona como disolvente. En la arilación de 2-fenilpiridina el complejo homobimetálico 7A resultó ser el más efectivo en términos de actividad y selectividad hacia el producto bisarilado (Esquema 7.6, B). Bajo las mismas condiciones de reacción, el compuesto 7A resultó más activo que el heterobimetálico 4A. Esta mayor actividad es debida probablemente a la 
presencia de dos centros de rutenio en 7A mientras $\mathbf{4 A}$ solo contiene uno. Este resultado no solo indica que el iridio es formalmente inactivo en el proceso, sino también que los dos metales en 7A son activos, indicando pues, que la situación en que un metal está actuando como ligando espectador mientras el otro está catalizando el proceso puede ser descartada.

En la oxidación Oppenauer de 1-feniletanol, el compuesto 1C dio los mejores resultados catalíticos, dando lugar a rendimientos cuantitativos en $1 \mathrm{~h}$ de reacción, mientras los compuestos bimetálicos necesitaron tiempos más prolongados $(9 \mathrm{~h})$ para obtener rendimientos similares.

La actividad de los catalizadores se evaluó en la combinación de ambas reacciones en un mismo matraz de reacción. Los resultados muestran que en este proceso catalítico la arilación es precedida de la transformación del alcohol en la cetona correspondiente, en presencia de acetona a través de un proceso oxidativo Oppenauer. El compuesto 1C presentó una mayor actividad catalítica. El compuesto homobimetálico 7A también presentó una excelente actividad catalítica, mejor que el compuesto heterobimetálico Ir$\mathrm{Ru}$ 4A. La mayor actividad del compuesto 1C puede explicarse si se tiene en cuenta que la oxidación del alcohol es el paso determinante de la reacción, en el que este compuesto es mucho más activo.

Finalmente, se realizaron estudios electroquímicos de los anteriores compuestos bimetálicos. Estos estudios muestran que los compuestos 4A y 7A presentan una interacción electrónica entre los dos centros metálicos enlazados por el anillo triazol. Aunque esta interacción se considera débil (clase II según la clasificación de Robin and Day $)^{37}$ es una de las interacciones más fuertes mostradas para este tipo de compuestos NHC tipo Janus-Head. ${ }^{38-41}$

\subsubsection{Activación de enlaces C-F via hidrodefluoración}

La activación eficaz y en condiciones suaves de los enlaces C-F sigue representando un reto para la química organometálica actual. ${ }^{42,43}$ La posibilidad de poder activar este tipo de enlaces presenta muchas ventajas a nivel industrial y ambiental. Las propiedades catalíticas del compuesto $\mathbf{5 A}$ se evaluaron en procesos de activación carbono-fluor via 
hidrodefluoración (HDF). A modo de comparación también se evaluó las propiedades catalíticas de los compuestos homobimetálicos de Ru (7A) y Pd (8A) (Esquema 7.7)
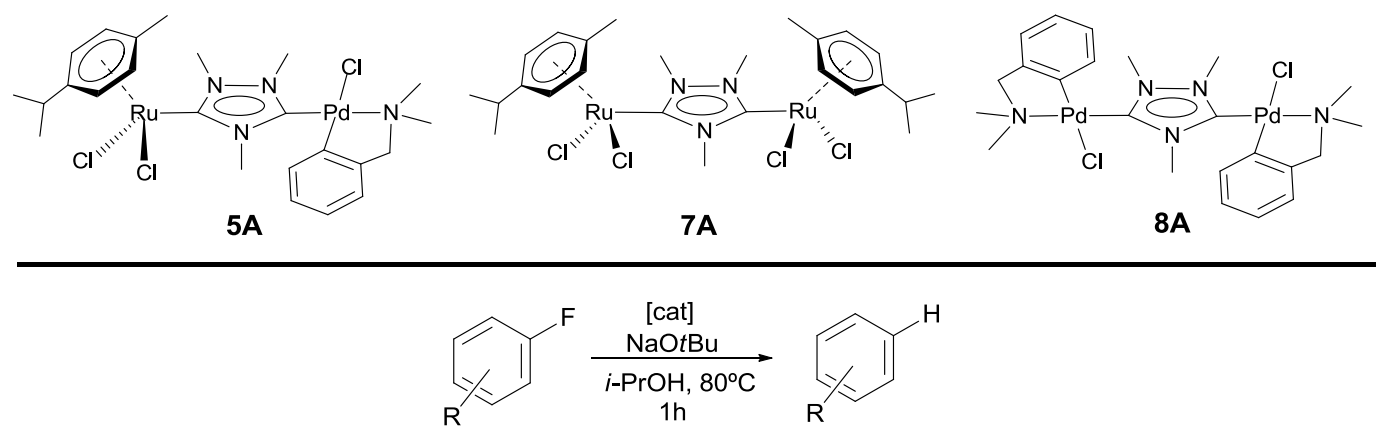

Esquema 7.7

El complejo heterobimetálico 5A fue altamente activo en la HDF de una amplia gama de substratos fluorados, independientemente de sus grupos funcionales, mientras que los complejos homobimetálicos 7A y $\mathbf{8 A}$ por separado no fueron activos en el proceso, es decir es necesaria la presencia de los dos centros metálicos para que tenga lugar la reacción catalítica. Además si comparamos la actividad del compuesto 5A con la actividad de la suma de complejos homobimetálicos 7A y $\mathbf{8 A}$, el compuesto $\mathbf{5 A}$ es el que presentó los mejores resultados. Este hecho sugiere la existencia de una cooperación catalítica entre los dos centros metálicos

A la vista de los buenos resultados obtenidos en la HDF de fluoroarenos, se estudió también la actividad del compuesto 5A en la hidrodefluoración de trifluorotoluenos, los cuales son considerados las moléculas más inertes dentro de los compuestos organofluorados (Esquema 7.8). El compuesto 5A también fue capaz de llevar a cabo la HDF de diferentes trifluorotoluenos en buenos rendimientos y en condiciones suaves de reacción.
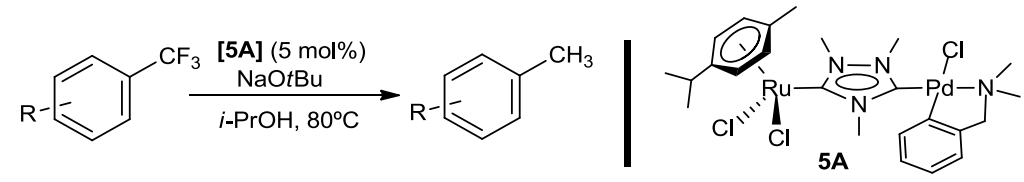

Esquema 7.8 
Los resultados obtenidos en la activación de enlaces $\mathrm{C}-\mathrm{F}$, tanto $\mathrm{C}_{\mathrm{sp} 2}-\mathrm{F}$ como $\mathrm{C}_{\mathrm{sp} 3}-\mathrm{F}$, indican el complejo heterobimetálico $\mathbf{5 A}$ se comporta como un sistema activo de dos componentes que es capaz de activar enlaces C-F en una gran serie de substratos diferentes, incluso trifluorotoluenos.

Aunque la mezcla de los dos diferentes complejos homometalicos también es activa en el proceso, la reacción se ve claramente beneficiada en el caso de utilizar el compuesto heterobimetálico $\mathbf{5 A}$, es decir cuando los dos diferentes centros metálicos están unidos a través de un ligando puente. Teniendo en cuenta los resultados catalíticos y estudios cinéticos realizados, se pueden extraer las siguientes conclusiones:

- 2-Propanol es la fuente de hidrógeno

- El paso determinante de la reacción es la reducción

- Bajo las condiciones de reacción la HDF tiene lugar de manera homogénea

- El ratio $[\mathrm{Pd}]:[\mathrm{Ru}]$ óptimo es 1:1

- La reacción es de primer orden tanto con respecto al catalizador 5A como la suma de 7A y $\mathbf{8 A}$

Con estas conclusiones en mente, se propone un mecanismo de reacción en el que el paladio se encarga de la activación de enlace C-F mediante una adición oxidante del fluoroareno a $\operatorname{Pd}(0)$. En un ciclo paralelo, el rutenio completa la reacción por medio de una transferencia de hidrógeno generando un complejo Ru-hidruro. Este hidruro se transfiere desde el rutenio hacia el paladio. Finalmente una eliminación reductora del paladio da lugar a la obtención del areno defluorado (Esquema 7.9).

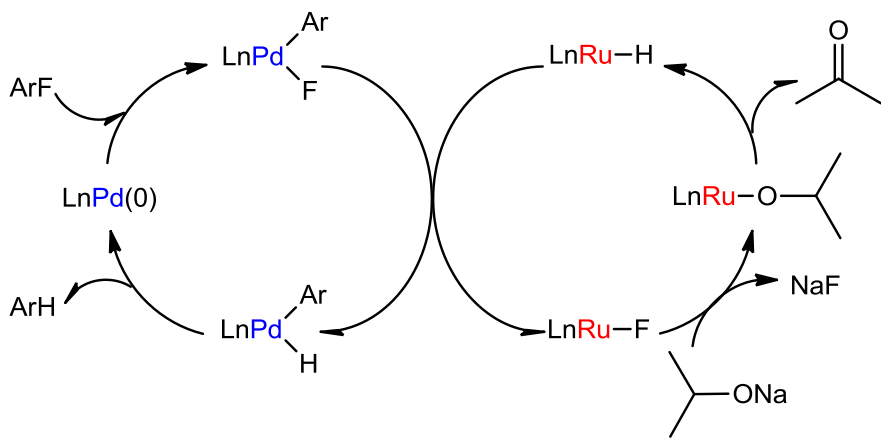

Esquema 7.9 


\subsection{Catálisis asimétrica usando complejos NHC con aminas orto-paladadas enantiopuras}

\subsubsection{Catalizadores quirales de paladio con ligandos NHC}

Los compuestos ciclopaladados ópticamente activos que contienen ligandos de tipo Ndadores son de gran interés debido a sus aplicaciones en diferentes campos como la síntesis orgánica, catálisis homogénea o la química médica. ${ }^{44-46}$ Muchas de las propiedades de este tipo de compuestos se han atribuido a la labilidad del enlace Pd-C que permite la activación de moléculas pequeñas. Por otra parte el uso de paladaciclos es una forma fácil y efectiva de introducir un centro quiral en el diseño de catalizadores enantiopuros que pueden ser aplicados en procesos de catálisis asimétrica. ${ }^{44,45}$

Basándonos en la experiencia de nuestro grupo de investigación en la preparación de compuestos NHC-Pd y su aplicación en procesos catalíticos homogéneos, nos propusimos la preparación de compuestos de paladio con ligandos NHCs y bencilaminas ortometaladas enantiomericamente puras.

El diseño de paladaciclos quirales con ligandos carbeno N-heterocíclicos se llevó a cabo a partir de bencilaminas enantiopuras comerciales. De este modo, a partir de ligandos quirales comerciales se obtuvieron catalizadores enantioméricamente puros en un único paso de reacción. En el caso de aminas terciarias la metodología consiste en una primera formación de dímeros de paladio (a y c, Esquema 7.10) con las bencilaminas ortometaladas. Los dímeros se coordinaron fácilmente a las correspondientes sales de imidazolio en condiciones básicas dando lugar a los compuestos enantiopuros deseados con altos rendimientos. 

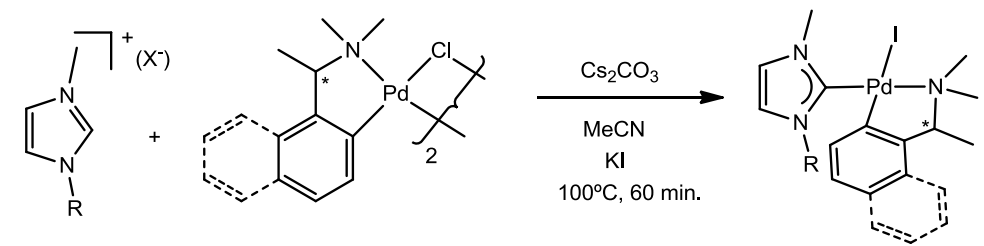

$$
\begin{array}{ll}
\text { D; } \mathrm{R}=\mathrm{CH}_{3}, \mathrm{X}=\mathrm{I} & \text { a; (S,S)-Phenyl } \\
\text { E; } \mathrm{R}=\mathrm{tBu}, \mathrm{X}=\mathrm{I} & \mathbf{c} ;(\mathrm{R}, \mathrm{R}) \text {-Naphthy } \\
\text { F; } \mathrm{R}=9 \text {-antr, } \mathrm{X}=\mathrm{Cl} &
\end{array}
$$

1D; $\mathrm{R}=\mathrm{CH}_{3}$, (S)-Phenyl

1E; $R=$ tBu, (S)-Phenyl

1F; $R=9$-antr, (S)-Phenyl

2D; $\mathrm{R}=\mathrm{CH}_{3}$, (R)-Naphthyl

2E; $R=$ tBu, (R)-Naphthyl

2F; $R=9$-antr, (R)-Naphthyl

\section{Esquema 7.10}

En el caso de aminas primarias la formación de los paladaciclos no es directa, empleando la misma metodología descrita anteriormente se observaron productos de descomposición. Por eso fue necesario el diseño de una ruta diferente para estos casos. La reacción de las sales de imidazolio con las aminas primarias en acetonitrilo en condiciones básicas dio lugar a los compuestos NHC-Pd-amina (3D y 3F, Esquema 7.11). Estos compuestos en presencia de acetato de plata ortometalan dando lugar a los correspondientes compuestos NHC ortopaladados 4D y $4 \mathbf{F}$ en buenos rendimientos.

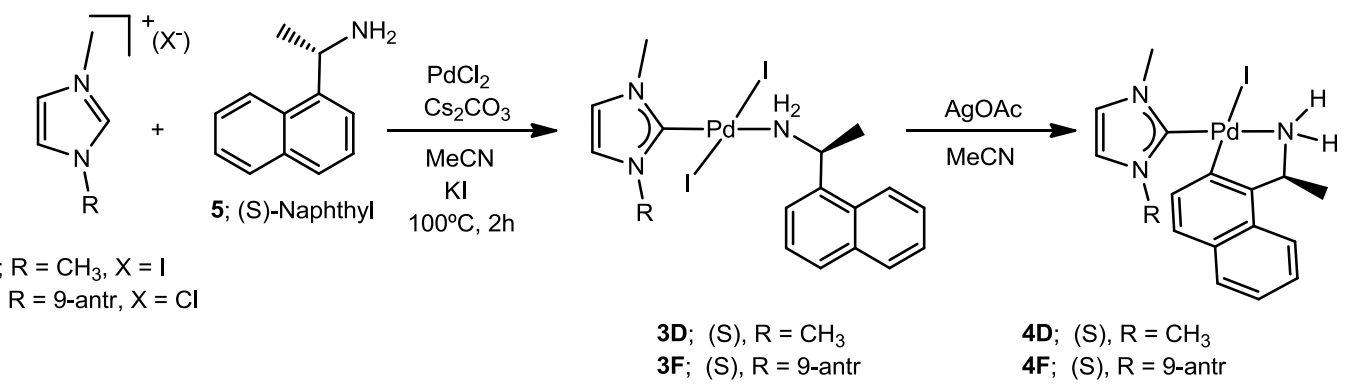

\section{Esquema 7.11}

Los nuevos complejos de paladio ortometalados con bencilaminas quirales se estudiaron en la reacción de hidrofosfinación asimétrica de olefinas. Esta reacción es una manera directa, y de alta economía atómica, de obtener compuestos organofosforados quirales.

Concretamente los nuevos complejos se estudiaron en la hidrofosfinación de 1,2-difenil2-(E)-propen-1-ona (trans-chalcona) usando difenilfosfina (Esquema 7.12). Los 
resultados obtenidos mostraron que los catalizadores sintetizados presentan una gran actividad en condiciones suaves de reacción. La reacción de hidrofosfinación tiene lugar con una excelente regioselectividad (la adición del fosforo se da en el carbono 3) aunque los excesos enantioméricos fueron moderados.

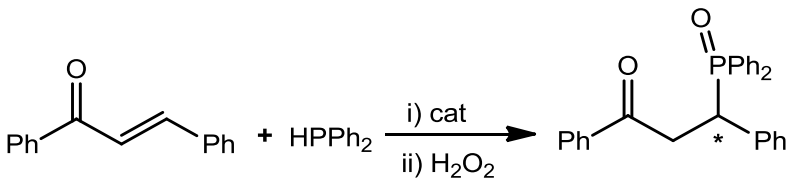

Esquema 7.12

\subsubsection{Complejos heterobimetálicos de Ir/Pd quirales}

A pesar de la gran versatilidad que ha demostrado tener el ligando ditz en el diseño de diversos procesos tándem, sus aplicaciones en el campo de la catálisis asimétrica se han visto limitadas debido a la dificultad de modificación del ligando. Por este motivo, nos propusimos la síntesis de complejos heterobimetálicos de Ir/Pd coordinando $\mathbf{1 A H}$ a fragmentos metálicos con co-ligandos quirales. (Esquema 7.13).

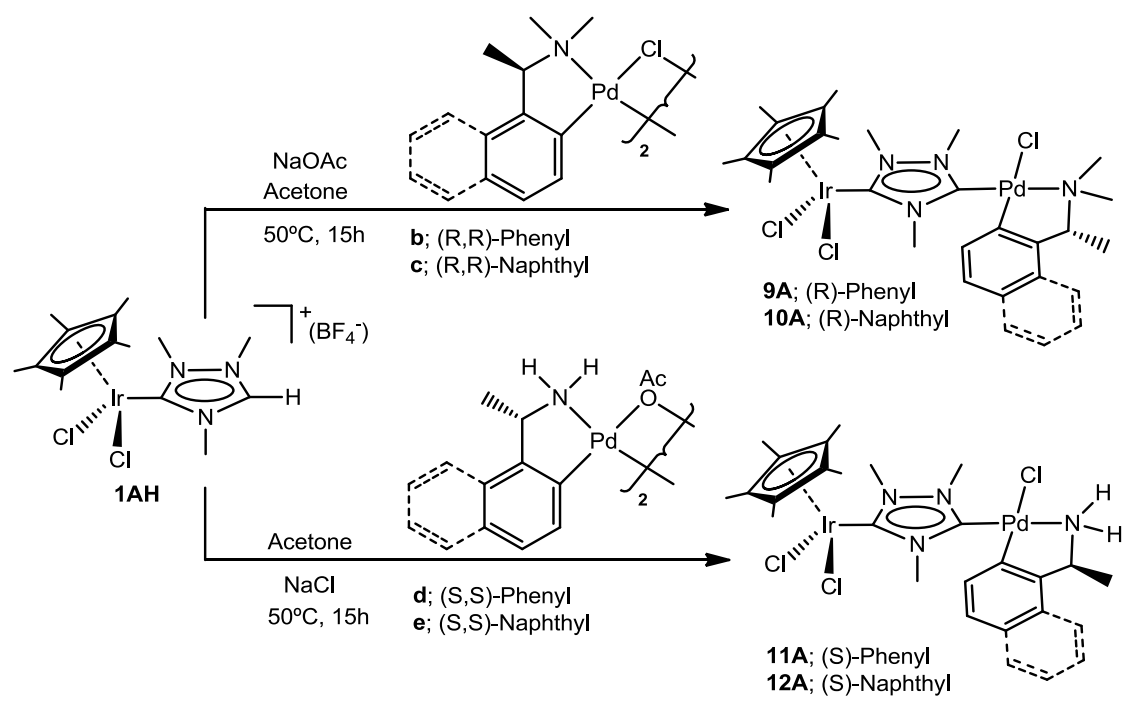

Esquema 7.13 
En el Esquema 7.13 se describe la síntesis de compuestos heterobimetálicos de $\mathrm{Ir} / \mathrm{Pd}$ quirales. La reacción de la sal de triazolio monometálica $\mathbf{1 A H}$ con los correspondientes dímeros de paladio con puentes cloro y acetato sódico, en el caso de aminas terciarias, o dímeros con puentes acetato, en el caso de aminas primarias, dio lugar a los correspondientes compuestos heterobimetálicos con aminas enantiomericamente puras.

Los compuestos heterobimetálicos quirales 9A-12A presentan quiralidad axial, contienen dos ejes de quiralidad. Uno de los ejes está definido por el enlace $\mathrm{Ir}-\mathrm{C}_{\text {carbeno }} \mathrm{y}$ el otro por el enlace $\mathrm{Pd}-\mathrm{C}_{\text {carbeno. }}$. Debido a la imposibilidad de rotación del enlace $\mathrm{M}-\mathrm{C}_{\text {carbeno }}(\mathrm{M}=\mathrm{Ir}$, Pd) por cuestiones estéricas, pueden obtenerse cuatro atropoisomeros diferentes (Esquema 7.14).

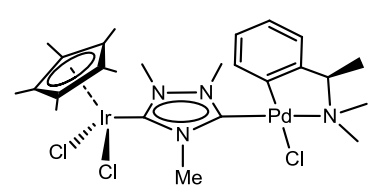

a) $S_{a l r}, S_{a P d}, R_{C}$

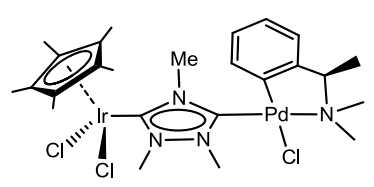

c) $R_{\mathrm{alr}}, R_{\mathrm{aPd}}, R_{\mathrm{C}}$
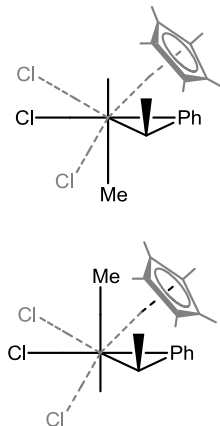

Esquema 7.14

La estructura molecular del compuesto 9A se confirmó por difracción de rayos X. la estructura cristalina contiene dos de los cuatro posibles atropoisomeros, (b y c, Esquema 7.14).

Con objetivo de estudiar la actividad catalítica de los complejos heterobimetálicos se propuso estudiarlos en dos reacciones catalíticas de manera secuencial como se representa en el Esquema 7.15: la isomerización, catalizada por iridio, de 1,3difenilpropargil alcohol que da como producto la trans-chalcona y seguidamente su hidrofosfinación asimétrica, catalizada por paladio. 


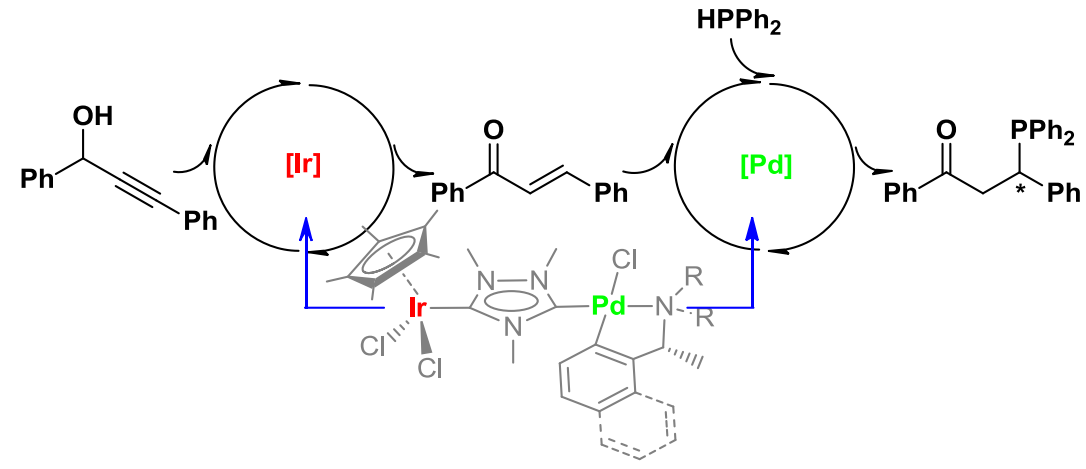

Esquema 7.15

En todos los casos los complejos mostraron ser activos en la reacción de isomerización, en la cual en todos los casos se obtuvieron rendimientos cuantitativos en condiciones de reacción suaves. La hidrofosfinación también tuvo lugar, en todos los casos, en rendimientos de moderados a altos, con regioselectividades excelentes (la adición del fosforo siempre tuvo lugar en el C3 de trans-chalcone). Los excesos enantioméricos, no obstante, fueron bajos.

\subsection{Complejos de iridio con aminas quirales orto-metaladas. Aplicaciones en transferencia de hidrógeno}

Como se ha demostrado en el apartado anterior, el uso de aminas enantiomericamente puras constituye una manera fácil y eficaz de introducir quiralidad en complejos organometálicos que posteriormente puedan ser utilizados en catálisis asimétrica. Por otra parte, diversos estudios han demostrado la capacidad de los compuestos del tipo Ir-amina en procesos de transferencia de hidrógeno. ${ }^{47-54}$ Por estos motivos, en base a la experiencia de nuestro grupo de investigación en la síntesis de complejos de Ir-NHC y su estudio en procesos de transferencia de hidrógeno, nos propusimos la síntesis de complejos de IrNHC con aminas enantiomericamente puras orto-metaladas (Ir-NH-NHC) y el estudio de sus propiedades catalíticas en transferencia de hidrógeno asimétrica. ${ }^{55}$

La síntesis de los compuestos racémicos 6D y 3C se llevó a cabo haciendo reaccionar $\left[\operatorname{IrCp} *(\mathrm{Cl})_{2}\left(\mathrm{Me}_{2} \operatorname{Im}\right)\right](\mathbf{5 D})$ o $\left[\operatorname{IrCp} *(\mathrm{Cl})_{2}\left(n \mathrm{Bu}_{2} \mathrm{Im}\right)\right](\mathbf{2 C})$ con la amina primaria racémica [1-(1-naftil)etil]amina en la presencia de dos equivalentes de AgOAc. Los complejos de 
iridio enantiopuros $6 D^{*}$ y $3 C^{*}$ se obtuvieron usando la enantiomericamente pura (S)-[1(1-naftil)etil]amina (Esquema 7.16). Los compuestos fueron purificados por columna cromatográfica usando acetona- $\mathrm{KPF}_{6}$ como eluyente.

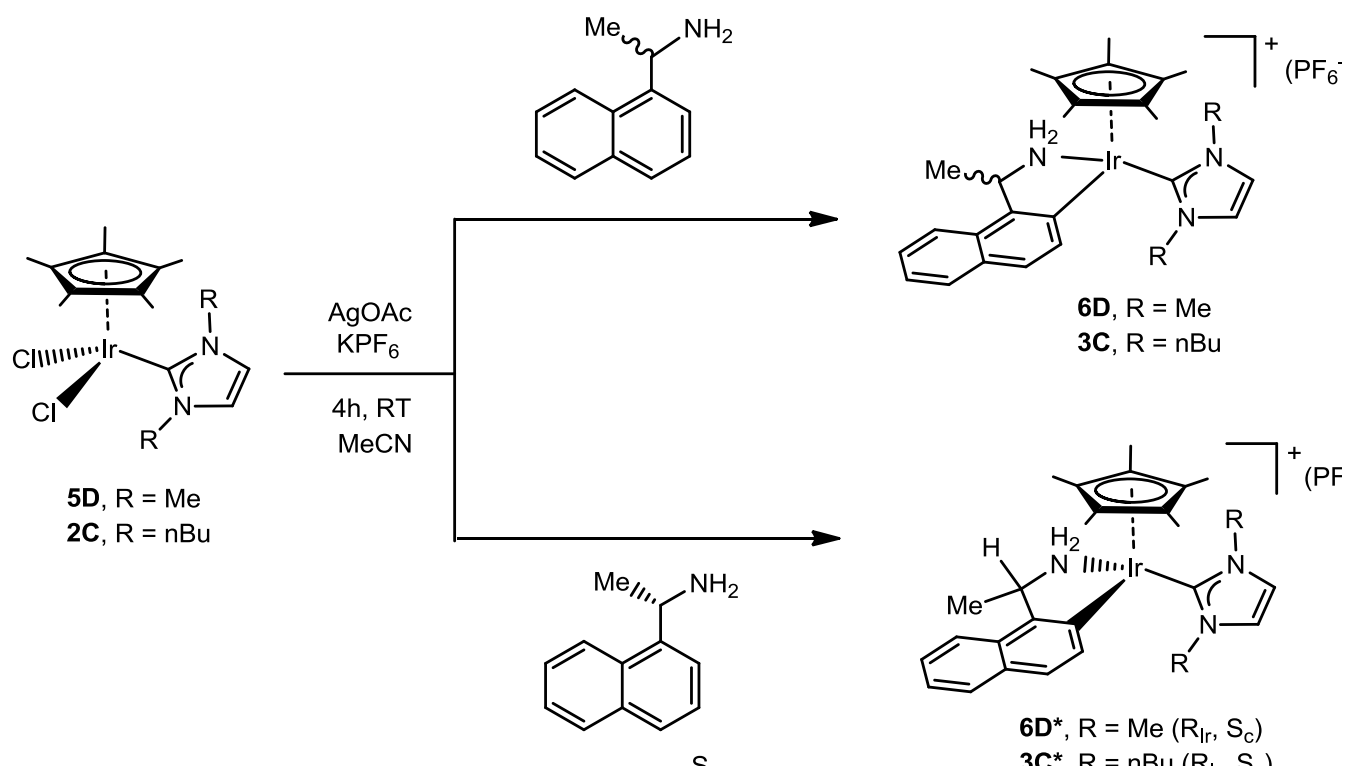

Esquema 7.16

Los complejos orto-metalados 6D y 3C presentan quiralidad centrada en el metal y poseen un carbono estereogénico, por lo tanto podrían esperarse dos diastereómeros. La caracterización espectroscópica por RMN y la difracción de rayos X en monocristal indica que en ambos casos la síntesis es diastereoselectiva.

Los complejos racémicos 6D y $3 \mathrm{C}$ fueron evaluados como catalizadores en la transferencia de hidrógeno de cetonas (Esquema 7.17). Así mismo, la actividad de los complejos enantiopuros $6 \mathrm{D}^{*}$ y $3 \mathrm{C}^{*}$ se estudió en su versión asimétrica. Para ser activos en el proceso, todos los compuestos necesitaron una cantidad catalítica de base. Los resultados muestran que todos los compuestos fueron activos en el proceso, donde carbonilos aromáticos y alifáticos fueron reducidos a los correspondientes alcoholes con rendimientos cuantitativos en $2 \mathrm{~h}$ a $50^{\circ} \mathrm{C}$. En el caso de la transferencia de hidrógeno 
asimétrica, fueron necesarias temperaturas inferiores $\left(30^{\circ} \mathrm{C}\right)$ y por tanto tiempos de reacción más largos (8h) para obtener excesos enantioméricos moderados.

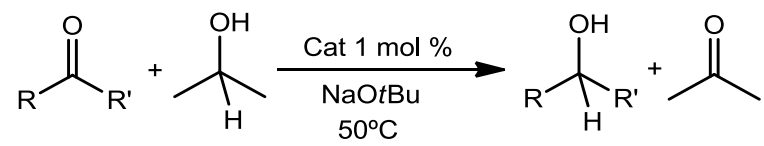

Esquema 7.17

Los resultados experimentales indican que los complejos de $\operatorname{Ir}(\mathrm{III})$ son altamente activos en la transferencia de hidrógeno a bajas temperaturas, a pesar de que los complejos no presentas vacantes libres de coordinación. Así pues, se realizaron estudios mecanisticos por medio de cálculos teóricos DFT para poder determinar un posible mecanismo. Los cálculos teóricos en concordancia de los datos experimentales sugieren un mecanismo de transferencia de hidrógeno que tiene lugar de manera concertada via un estado de transición altamente ordenado centrado en el grupo iridio-amido, sin la participación de ningún intermedio hidruro.

\subsection{Síntesis y aplicaciones catalíticas de complejos de Ru con ligandos carbeno mesoiónicos (MIC) funcionalizados}

La funcionalización de ligandos puede jugar un papel importante en la activación de moléculas pequeñas en un ciclo catalítico, donde ambos, el metal y el ligando simultáneamente participan en proceso de formación y rotura de enlaces. ${ }^{17,56-58}$

La facilidad de modular estereoelectronicamente ligandos del tipo 1,2,3 triazolilidenos facilita el diseño de nuevos complejos organometálicos en los cuales exista un comportamiento sinérgico metal-ligando. Con este objetivo, se decidió preparar una serie de complejos de $\mathrm{Ru}$ con ligandos triazolilidenos funcionalizados con un grupo carboxilato.

La síntesis de los compuestos 1I, 2I y 3I fue llevada a cabo por transmetalación a partir del carbeno de plata formado por reacción de la sal de triazolio I con $\operatorname{Ag}_{2} \mathrm{O}$ (Esquema 7.18). En función de las condiciones de reacción empleadas en la etapa de formación del carbeno de plata, la reacción resultó ser selectiva hacia la formación de los compuestos $\mathbf{1 I}$ (y 2I) o $3 \mathbf{I}$. 

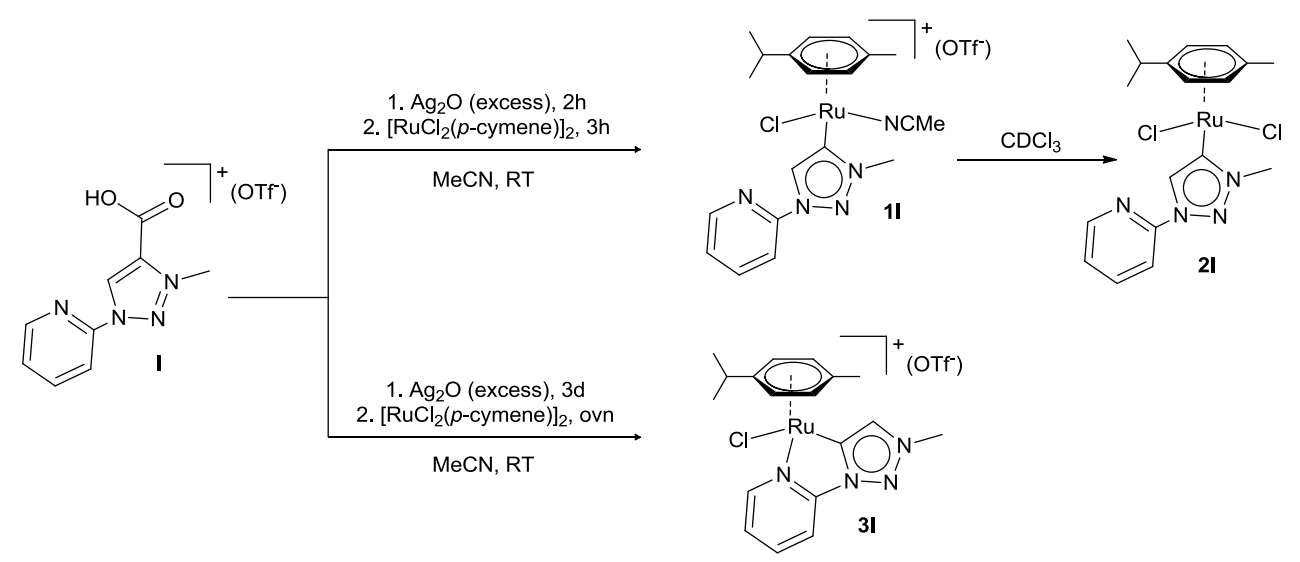

\section{Esquema 7.18}

A partir de la sal de triazolio $\mathbf{H}$ se obtuvieron una serie de compuestos funcionalizados en el anillo de triazol con grupos éster, carboxilato y ácido carboxílico (Esquema 7.19). El compuesto $1 \mathrm{H}$ se obtuvo a partir del carbeno de plata obtenido mediante la reacción de la sal de triazolio $\mathbf{H}$ con $\mathrm{Ag}_{2} \mathrm{O}$. Los compuestos $2 \mathbf{H}$ y $\mathbf{3 H}$ se obtuvieron por hidrólisis básica del grupo éster presente en 1H. La substitución del ligando $p$-cimeno para dar lugar al solvento-complejo $\mathbf{4 H}$ se llevó a cabo mediante tratamiento térmico del compuesto $\mathbf{1 H}$ en acetonitrilo a reflujo. ${ }^{40,59-62} \mathrm{La}$ reacción de $\mathbf{4 H}$ con 2.5 equivalentes de $\mathrm{PPh}_{3}$, en $o$ diclorobenzeno $(o-\mathrm{DCB})$ a $110^{\circ} \mathrm{C}$ durante 3 días, dio lugar a la formación del compuesto $\mathbf{5 H}$. De manera análoga a la obtención de $\mathbf{2 H}$ y $\mathbf{3 H}$, la hidrolisis básica del grupo éster presente en $\mathbf{5 H}$ resultó en la formación de los compuestos funcionalizados con carboxilato $(\mathbf{6 H})$ y ácido carboxílico $(\mathbf{7 H})$. 


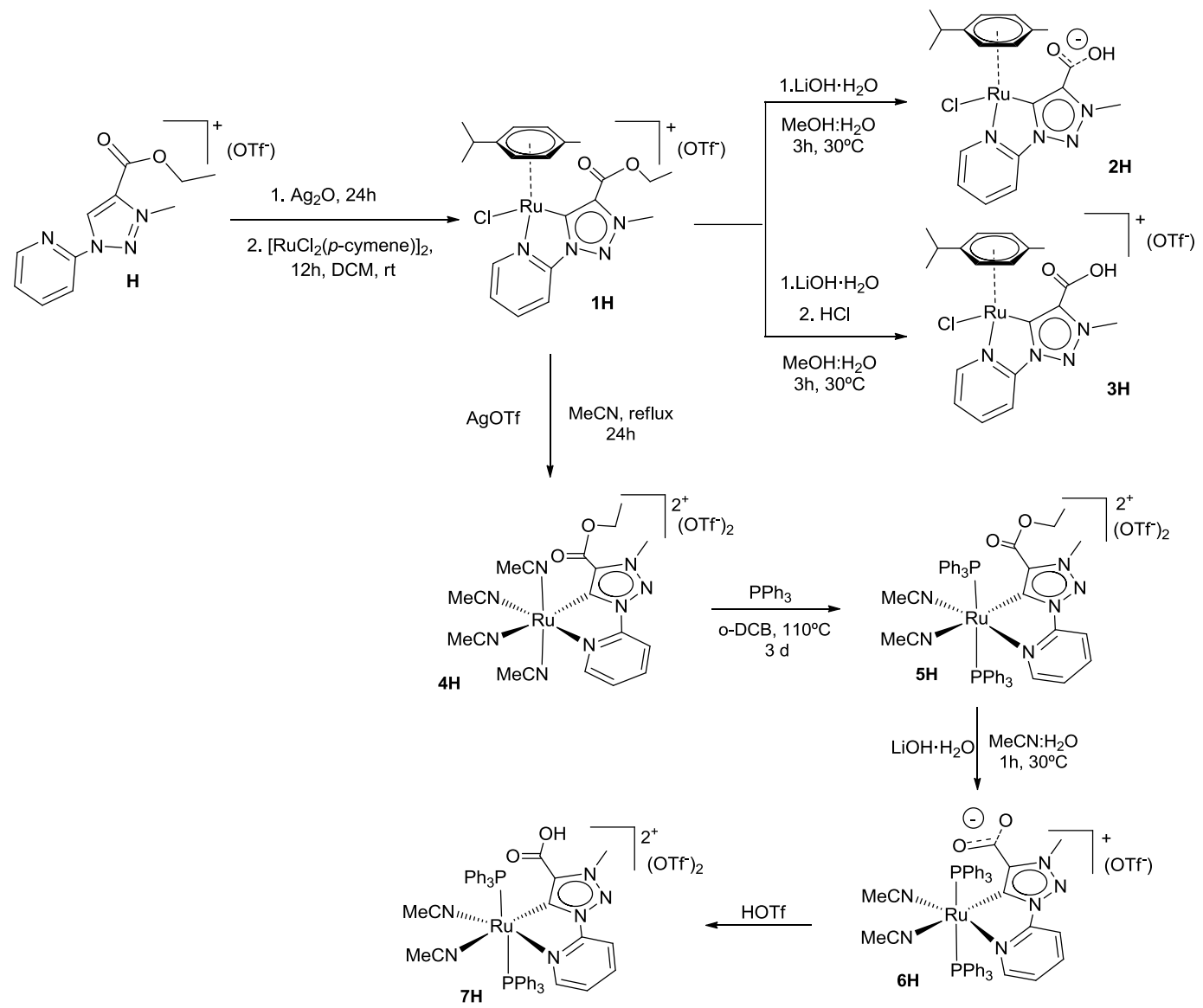

Esquema 7.19

Las propiedades catalíticas de los compuestos 1I-3I y 1H-7H fueron estudiadas en dos reacciones típicamente catalizadas por rutenio: la oxidación deshidrogenativa de alcoholes y en transferencia de hidrógeno. Los resultados catalíticos sugieren una influencia positiva tanto del grupo piridil en 1I-3I como del grupo carboxilato en 1H-7H en los procesos de transferencia de hidrógeno. Estos resultados, sugieren una cooperación metal-ligando activa, y representan un prometedor punto de partida para la obtención de una amplia gama de catalizadores bifuncionales basados en un sinergismo metal-ligando. 


\subsection{Inmovilización de catalizadores sobre superficies de grafeno}

Con el objetivo de soportar catalizadores moleculares sobre la superficie de grafeno, más concretamente, oxido de grafeno reducido (rGO), se sintetizó la sal de imidazolio G, la cual contiene en su estructura un grupo pireno capaz de interaccionar mediante apilamiento $\pi$ sobre la superficie de rGO. Mediante estrategias convencionales de coordinación se obtuvieron los complejos de $\mathrm{Pd}, \mathrm{Ru}, \mathrm{Au}$ e Ir que se representan en el Esquema 7.20.

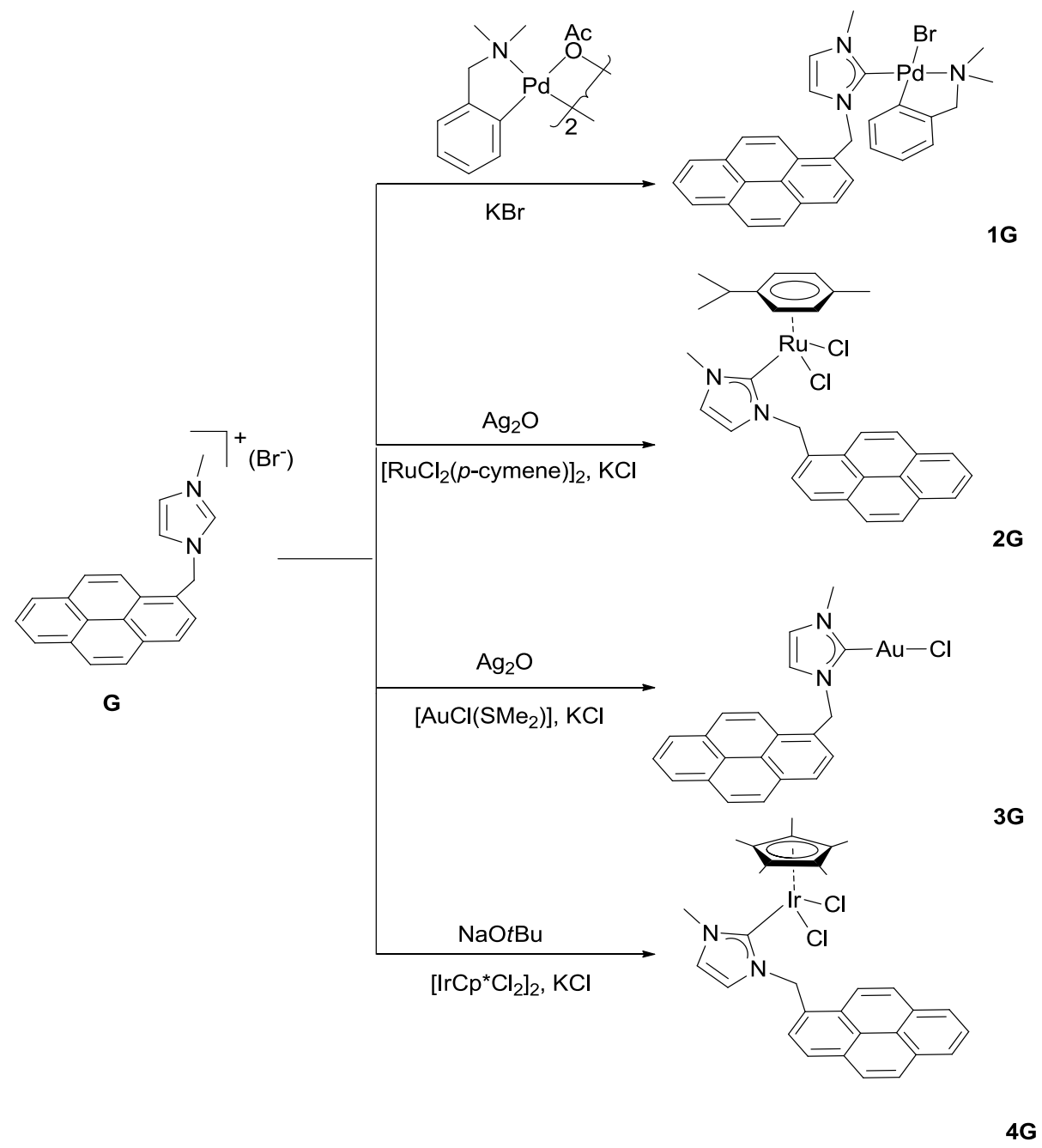

Esquema 7.20 
Los complejos 1G-4G fueron soportados sobre rGO para dar lugar a los materiales híbridos 1G-rGO, 2G-rGO, 3G-rGO, 4G-rGO, y 5rGO los cuales contienen Pd, Ru, $\mathrm{Au}$, Ir, y $\mathrm{Pd} / \mathrm{Ru}$ respectivamente (Esquema 7.21). En general, la preparación de los materiales híbridos se llevó a cabo por agitación de una suspensión de rGO en una disolución del correspondiente compuesto en diclorometano. En todos los casos, la primera evidencia de inmovilización de los compuestos moleculares sobre la superficie de rGO fue a desaparición del color en la disolución de diclorometano. Los sólidos negros resultantes fueron caracterizados por los métodos convencionales de caracterización de materiales, espectroscopía UV/Vis, FTIR y Raman, Scanning Electron Microscopy (SEM), High Resolution Transmission Electron Microscopy (HRTEM) y Brunauer-Emmett-Teller (BET) Surface Area Analysis. El contenido exacto de metal en cada uno de los materiales fue determinado por análisis de ICP-MS.

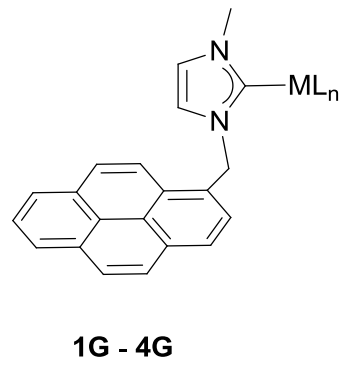

$1 G-4 G$

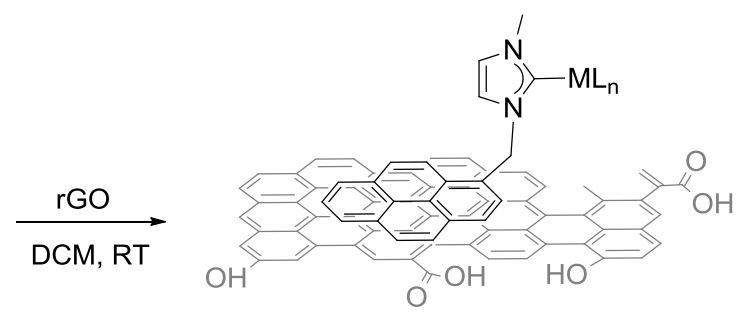

1G-rGO - 4G-rGO

$5 \mathrm{rGO}$

Esquema 7.21

Las propiedades catalíticas de los nuevos materiales de Pd (1G-rGO), Ru (2G-rGO) y $\mathrm{Pd} / \mathrm{Ru}$ (5rGO), así como de los correspondientes compuestos moleculares, fueron evaluadas en procesos típicamente catalizados por Pd (hidrogenación de alquenos y reducción de nitroarenos), $\mathrm{Ru}$ (oxidación de alcoholes) y por la combinación de $\mathrm{Pd} / \mathrm{Ru}$ (hidrodefluoración de fluoroarenos).

En todos los casos, los resultados indican un aumento de la actividad catalítica de los materiales híbridos sobre los compuestos moleculares. Además los sistemas estudiados pudieron ser reutilizados hasta diez veces sin pérdida aparente de actividad de los catalizadores, incluso bajo condiciones en gran escala, utilizando cargas de catalizador de $0.01 \%$ y $0.03 \%$. 


\section{CONCLUSIONES}

En la presente Tesis Doctoral se ha descrito la síntesis de una serie de compuestos homoy hetero-bimetalicos, en los cuales los dos centros metálicos están conectados mediante un ligando triazoldiylideno (ditz) formando complejos biscarbeno. Estos nuevos compuestos han permitido el diseño y el estudio de procesos multimetálicos. Los resultados muestran que:

- $\mathrm{Au} / \mathrm{Ir}$ es activo en un proceso cooperativo dual

- $\mathrm{Ru} / \mathrm{Ir}$ es activo en un proceso tándem en cascada

- $\mathrm{Ru} / \mathrm{Pd}$ es activo en la activación de enlaces C-F comportándose como un sistema sinergético

- $\mathrm{Ir} / \mathrm{Pd}^{*}$ es activo en un proceso tándem asimétrico

En general, la actividad de los complejos heterobimetálicos es mayor que aquella mostrada por la suma de los análogos homobimetálicos. Esta mejora de la actividad catalítica se atribuye a una cooperación mutua entre los dos centros metálicos unidos mediante el ligando ditz.

También se ha sintetizado una serie de compuestos de Pd, Ir y Ru funcionalizados. En el caso de los compuestos de Pd e Ir, la funcionalización se llevó a cabo con aminas enantiomericamente puras obteniéndose compuestos quirales. Los complejos de paladio quirales fueron activos en hidrofosfinación asimétrica. La hidrofosfinación tuvo lugar con excelente regioseletividad y excesos enantioméricos moderados. Los complejos de iridio quirales preparados son complejos inertes de 18 electrones que fueron activos en transferencia de hidrógeno. Mediante estudios DFT se propone un mecanismo de reacción que transcurre de manera concertada via un estado de transición ordenado centrado en el grupo iridio-amido, sin la participación de intermedios de tipo hidruro. Los compuestos de rutenio obtenidos fueron preparados utilizando ligandos triazolylidenos mesoionicos (MICs) funcionalizados con grupos carboxilato. Los compuestos fueron estudiados en procesos de transferencia de hidrógeno y oxidación de alcoholes. Los resultados sugieren una cooperación activa metal-ligando. 
Finalmente, se prepararon compuestos de $\mathrm{Pd}, \mathrm{Ru}, \mathrm{Au}$ e Ir funcionalizados con un ligando NHC que contiene un grupo pireno en su estructura. La presencia del grupo pireno permitió la inmovilización de los compuestos moleculares sobre superficies de oxido de grafeno reducido (rGO), mediante interacciones de tipo no covalente ( $\pi$-stacking) obteniéndose materiales híbridos. Las aplicaciones catalíticas de los nuevos materiales fueron evaluadas en procesos de hidrogenación de alquenos, reducción de nitroarenos, oxidación de alcoholes y hidrodefluoración de fluoroarenos. Estos materiales no solo pudieron ser reutilizados hasta diez veces sin pérdida aparente de actividad de los catalizadores, sino que también presentan una actividad mayor que los compuestos moleculares, indicando que el soporte juega un papel fundamental en el ciclo catalítico. 


\section{REFERENCIAS}

(1) Rowlands, G. J. Tetrahedron 2001, 57, 1865-1882.

(2) Wei, Y.; Shi, M. Accounts Chem. Res. 2010, 43, 1005-1018.

(3) van den Beuken, E. K.; Feringa, B. L. Tetrahedron 1998, 54, 12985-13011.

(4) Ajamian, A.; Gleason, J. L. Angew. Chem. Int. Edit. 2004, 43, 3754-3760.

(5) Fogg, D. E.; dos Santos, E. N. Coord. Chem. Rev. 2004, 248, 2365-2379.

(6) Wasilke, J. C.; Obrey, S. J.; Baker, R. T.; Bazan, G. C. Chem. Rev. 2005, 105, 1001-1020.

(7) Yamada, Y.; Tsung, C.-K.; Huang, W.; Huo, Z.; Habas, S. E.; Soejima, T.; Aliaga, C. E.; Somorjai, G. A.; Yang, P. Nat. Chem. 2011, 3, 372-376.

(8) Mas-Marza, E.; Mata, J. A.; Peris, E. Angew. Chem. Int. Edit. 2007, 46, 37293731.

(9) Viciano, M.; Sanau, M.; Peris, E. Organometallics 2007, 26, 6050-6054.

(10) Zanardi, A.; Corberan, R.; Mata, J. A.; Peris, E. Organometallics 2008, 27, 35703576.

(11) Zanardi, A.; Mata, J. A.; Peris, E. Organometallics 2009, 28, 1480-1483.

(12) Zanardi, A.; Mata, J. A.; Peris, E. J. Am. Chem. Soc. 2009, 131, 14531-14537.

(13) Zanardi, A.; Mata, J. A.; Peris, E. Chem. Eur. J. 2010, 16, 10502-10506.

(14) Zanardi, A.; Mata, J. A.; Peris, E. Chem. Eur. J. 2010, 16, 13109-13115.

(15) Mata, J. A.; Hahn, F. E.; Peris, E. Chem. Sci. 2014, 5, 1723-1732.

(16) Yamakawa, M.; Ito, H.; Noyori, R. J. Am. Chem. Soc. 2000, 122, 1466-1478.

(17) Ikariya, T.; Murata, K.; Noyori, R. Org. Biomol. Chem. 2006, 4, 393-406.

(18) Ikariya, T.; Blacker, A. J. Accounts Chem. Res. 2007, 40, 1300-1308.

(19) Bifunctional Transition metal-based molecular catalysts for asymmetric synthesis; Ikariya, T., Ed.; Springer-Verlag Berlin Heidelberg, 2011; Vol. 37, pp 31-53.

(20) Corma, A.; Garcia, H. Adv. Synth. Catal. 2006, 348, 1391-1412.

(21) Bond, G. C. Chem. Soc. Rev. 1991, 20, 441-475.

(22) Gladysz, J. A. Chem. Rev. 2002, 102, 3215-3216.

(23) Coperet, C.; Chabanas, M.; Saint-Arroman, R. P.; Basset, J. M. Angew. Chem. Int. Edit. 2003, 42, 156-181. 
(24) Fraile, J. M.; Garcia, J. I.; Mayoral, J. A. Chem. Rev. 2009, 109, 360-417.

(25) Wang, Z.; Chen, G.; Ding, K. L. Chem. Rev. 2009, 109, 322-359.

(26) Jones, C. W. Top. Catal. 2010, 53, 942-952.

(27) Buchwalter, P.; Rosé, J.; Braunstein, P. Chem. Rev. 2014, DOI: $10.1021 /$ cr500208k.

(28) Wang, X.; Kawanami, H.; Dapurkar, S. E.; Venkataramanan, N. S.; Chatterjee, M.; Yokoyama, T.; Ikushima, Y. Appl. Catal. A-Gen. 2008, 349, 86-90.

(29) Grirrane, A.; Corma, A.; Garcia, H. Science 2008, 322, 1661-1664.

(30) Corma, A.; Gonzalez-Arellano, C.; Iglesias, M.; Sanchez, F. Appl. Catal. A-Gen. 2009, 356, 99-102.

(31) Ackermann, L.; Vicente, R. C-H Activation 2010, 292, 211-229.

(32) Ackermann, L. Chem. Rev. 2011, 111, 1315-1345.

(33) Arockiam, P. B.; Bruneau, C.; Dixneuf, P. H. Chem. Rev. 2012, 112, 5879-5918.

(34) Fujita, K.; Yamaguchi, R. Synlett 2005, 560-571.

(35) Dobereiner, G. E.; Crabtree, R. H. Chem. Rev. 2010, 110, 681-703.

(36) Prades, A.; Poyatos, M.; Peris, E. Adv. Synth. Catal. 2010, 352, 1155-1162.

(37) Robin, M. B.; Day, P. Adv. Inorg. Chem. Radiochem 1967, 10, 247.

(38) Tennyson, A. G.; Rosen, E. L.; Collins, M. S.; Lynch, V. M.; Bielawski, C. W. Inorg. Chem. 2009, 48, 6924-6933.

(39) Schuster, O.; Mercs, L.; Albrecht, M. Chimia 2010, 64, 184-187.

(40) Nussbaum, M.; Schuster, O.; Albrecht, M. Chem. Eur. J. 2013, 19, 17517-17527.

(41) Prades, A.; Peris, E.; Alcarazo, M. Organometallics 2012, 31, 4623-4626.

(42) Douvris, C.; Ozerov, O. V. Science 2008, 321, 1188-1190.

(43) Grushin, V. V. Accounts Chem. Res. 2010, 43, 160-171.

(44) Elgazwy, A. S. S. H.; Ismail, N. S. M.; Atta-Allah, S. R.; Sarg, M. T.; Soliman, D. H. S.; Zaki, M. Y.; Elgamas, M. A. Curr. Med. Chem 2012, 19, 3967-3981.

(45) Vicente, J.; Saura-Llamas, I. Comments Inorg. Chem. 2007, 28, 39-72.

(46) Albert, J.; Granell, J.; Muller, G. J. Organomet. Chem. 2006, 691, 2101-2106.

(47) Arita, S.; Koike, T.; Kayaki, Y.; Ikariya, T. Organometallics 2008, 27, $2795-$ 2802.

(48) Arita, S.; Koike, T.; Kayaki, Y.; Ikariya, T. Chem.-Asian J. 2008, 3, 1479-1485. 
(49) Watanabe, M.; Kashiwame, Y.; Kuwata, S.; Ikariya, T. Eur. J. Inorg. Chem. 2012, 504-511.

(50) Sato, Y.; Kayaki, Y.; Ikariya, T. Chem. Commun. 2012, 48, 3635-3637.

(51) Kashiwame, Y.; Kuwata, S.; Ikariya, T. Organometallics 2012, 31, 8444-8455.

(52) Barloy, L.; Issenhuth, J.-T.; Weaver, M. G.; Pannetier, N.; Sirlin, C.; Pfeffer, M. Organometallics 2011, 30, 1168-1174.

(53) Jerphagnon, T.; Haak, R.; Berthiol, F.; Gayet, A. J. A.; Ritleng, V.; Holuigue, A.; Pannetier, N.; Pfeffer, M.; Voelklin, A.; Lefort, L.; Verzijl, G.; Tarabiono, C.; Janssen, D. B.; Minnaard, A. J.; Feringa, B. L.; de Vries, J. G. Top. Catal. 2010, 53, 1002-1008.

(54) Pannetier, N.; Sortais, J.-B.; Issenhuth, J.-T.; Barloy, L.; Sirlin, C.; Holuigue, A.; Lefort, L.; Panella, L.; de Vries, J. G.; Pfeffer, M. Adv. Synth. Catal. 2011, 353, 2844-2852.

(55) Cross, W. B.; Daly, C. G.; Boutadla, Y.; Singh, K. Dalton Trans. 2011, 40, 97229730.

(56) Noyori, R.; Yamakawa, M.; Hashiguchi, S. J. Org. Chem. 2001, 66, 7931-7944.

(57) Grützmacher, H. Angew. Chem. Int. Edit. 2008, 47, 1814-1818.

(58) Askevold, B.; Roesky, H. W.; Schneider, S. ChemCatChem 2012, 4, 307-320.

(59) Le Lagadec, R.; Rubio, L.; Alexandrova, L.; Toscano, R. A.; Ivanova, E. V.; Meskys, R.; Laurinavicius, V.; Pfeffer, M.; Ryabov, A. D. J. Organomet. Chem. 2004, 689, 4820-4832.

(60) Ghattas, W.; Bunz, H. M.; Albrecht, M. Organometallics 2010, 29, 6782-6789.

(61) Leigh, V.; Ghattas, W.; Lalrempuia, R.; Mueller-Bunz, H.; Pryce, M. T.; Albrecht, M. Inorg. Chem. 2013, 52, 5395-5402.

(62) Delgado-Rebollo, M.; Canseco-Gonzalez, D.; Hollering, M.; Mueller-Bunz, H.; Albrecht, M. Dalton Trans. 2014, 43, 4462-4473. 